\title{
Savannah River Site Environmental Data for 1999
}

by

M. Arnett

Westinghouse Savannah River Company

Savannah River Site

Aiken, South Carolina 29808

A. Mamatey

This paper was prepared in connection with work done under the above contract number with the U. S. Department of Energy. By acceptance of this paper, the publisher and/or recipient acknowledges the U. S.

Government's right to retain a nonexclusive, royalty-free license in and to any copyright covering this paper, along with the right to reproduce and to authorize others to reproduce all or part of the copyrighted paper. 


\section{RECEIVED \\ OCT 132000 \\ OSTI}

\section{Savannah River Site Environmental Data for 1999}

\section{Editors}

Margaret W. Arnett

Albert R. Mamatey 


\section{DISCLAIMER}

This report was prepared as an account of work sponsored by an agency of the United States Government. Neither the United States Government nor any agency thereof, nor any of their employees, makes any warranty, express or implied, or assumes any legal liability or responsibility for the accuracy, completeness, or usefulness of any information, apparatus, product or process disclosed, or represents that its use would not infringe privately owned rights. Reference herein to any specific commercial product, process or service by trade name, trademark, manufacturer, or otherwise does not necessarily constitute or imply its endorsement, recommendation, or favoring by the United States Government or any agency thereof. The views and opinions of authors expressed herein do not necessarily state or reflect those of the United States Government or any agency thereof.

This report has been reproduced directly from the best available copy.

Available for sale to the public, in paper, from: U.S. Department of Commerce, National Technical Information Service, 5285 Port Royal Road, Springfield, VA 22161 phone: (800) 553-6847

fax: (703) 605-6900

email: orders@ntis.fedworld.gov

online ordering: http://www.ntis.gov/ordering.htm

Available electronically at http://www.doe.gov/bridge Available for a processing fee to U.S. Department of Energy and its contractors, in paper, from: U.S. Department of Energy, Office of Scientific and Technical Information, P.O. Box 62, Oak Ridge, TN 37831-0062

phone: (865)576-8401

fax: (865)576-5728

email: reports@adonis.osti.gov 


\section{DISCLAIMER}

Portions of this document may be illegible in electronic image products. Images are produced from the best available original document. 


\section{Acknowledgments}

The editors acknowledge with appreciation the efforts of the following individuals and groups:

Environmental Monitoring Section/Environmental Protection Department (for technical expertise, review, oversight, and clerical support during the preparation of this book)
Brenda Alejo
Sandra Boynton
Carl Cook
Larry Eldridge
Dave Filler
Pete Fledderman
Lynne Geary
June Hall
Jim Heffner
Bob Henderson

\author{
Tracey Humphrey \\ Moheb Khalil \\ Bill Littrell \\ Bob Lorenz \\ Phil Miller \\ Donald Padgett \\ Priscilla Patterson \\ Stuart Stinson \\ Robin Young
}

Westinghouse Savannah River Company

Timothy Jannik and Ali Simpkins (for dose estimates)

John Ellinger, Karl Bergmann, and Chuck Harvel (for computer and software support)

Marvin Stewart (for support with Internet)

Larry Koffman (for map production)

Brent Blunt (for authorized derivative classification)

WSRC Management Services (for customer service, illustrating and design, classification, printing, and quality assurance support-Bernadette Hobbs; Eleanor Justice; Trish Baughman; Randy Collins, Tom Coughenour; Pat Dominey; Juli Hearn; Sharon Lybrand; Bob Shankle; Alan Clayton; Stephanie Doetsch; Lisa McCullough; Ann Moser; Yvonne Nixon; and Joan Toole)

\section{Department of Energy-Savannah River}

Mary Baranek, coordinator, and Ben Gould (for DOE-SR review)

Mina Perrin (for DOE-SR Scientific and Technical Information review/approval)

\section{Members of Environmental Advisory Committee (for independent technical review)}

Dr. Edgar Berkey, National Environmental Technology Applications Corporation, University of Pittsburgh

Dr. Bernd Kahn, Environmental Research Center, Georgia Institute of Technology

Dr. Dennis Paustenbach, McLaren/Hart Environmental Engineering Group

Dr. Bernard Sweeney, Stroud Water Research Center, Academy of Natural Sciences 


\section{Preface}

This document presents data from Savannah River Site routine effluent monitoring and environmental surveillance programs. Information in the book is summarized in the Savannah River Site Environmental Report for 1999 (WSRC-TR-99-00299). Information about the environmental monitoring program, including a complete description of Environmental Monitoring Section sampling and analytical procedures, can be found in sections 1101-1111 (SRS EM Program) of the Savannah River Site Environmental Monitoring Section Plans and Procedures, WSRC-3Q1-2, Volume 1.

Because of the data table columns' space requirements, many abbreviations have been used. To assist the reader, lists of radionuclide and chemical nomenclature and sampling location abbreviations are included following this introduction. Units of measure, scientific notation, and conversion charts also should help the reader. A collection of maps showing radiological and nonradiological sampling locations completes this introductory section.

The first few tables include lists of the media sampled and of minimum detectable concentrations for various analyses/media. A table of nonradiological environmental surveillance detection/report limits also is included. Following these explanatory tables are data tables with radiological and nonradiological effluent monitoring results, radiological and nonradiological environmental surveillance results, dose estimates, and quality assurance data.

Data tables sometimes present fewer results than would be expected according to the frequency described in the sampling and analysis schedules. There are several reasons for these discrepancies. Sample collection problems, such as loss of power to the sampling site or inaccessibility to the sampling site (because of locked gates, flooding, etc.) may have occurred. Also, results for collected samples can be rejected for reasons such as insufficient sample volume, low chemical yield, or equipment failure. The "number of samples" columns in the tables refer to the number of results used to determine maximum, minimum, and arithmetic mean concentrations.

The following should aid the reader in interpreting the data:

- Analytical results and their corresponding uncertainties generally are reported with up to three significant figures. This is a function of the computer software used and may imply greater accuracy in the reported results than the analyses would allow. However, EMS attempts to report the correct number of significant figures that are appropriate for the result measurement uncertainty.

- An uncertainty quoted with means represents the standard deviation of the mean value. This number is calculated from the results themselves and is not weighted by the uncertainties of the individual results.

- All values represent the weighted average of all acceptable analyses of a sample for a particular analyte. Samples may have undergone multiple analyses for quality assurance purposes or to determine if radionuclides are present. For certain radionuclides, quantifiable concentrations may be below the minimum detectable activity of the analysis, in which case the actual concentration value is presented to satisfy DOE reporting guidelines.

- The reported uncertainty of a single measurement reflects only the counting error-not other components of random and systematic error in the measurement process-so some results may imply a greater confidence than the determination would suggest.

- The generic term "dose," as used in the report, refers to the committed effective dose equivalent (50-year committed dose) from internal deposition of radionuclides and to the effective dose equivalent attributable to beta/gamma radiation from sources external to the body.

Copies of environmental reports may be obtained by contacting

Bob Lorenz, Manager, Environmental Sampling and Reporting

Building 735-16A

Westinghouse Savannah River Company

Aiken, SC 29808

Telephone: 803-725-3556

E-mail address: robert.lorenz@srs.gov 



\section{Contents}

List of Sampling Location Maps $\ldots \ldots \ldots \ldots \ldots \ldots \ldots \ldots \ldots \ldots \ldots \ldots \ldots \ldots \ldots \ldots$

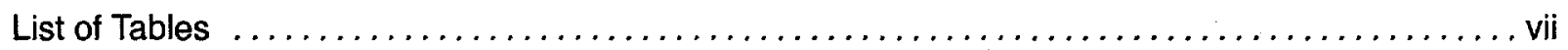

Radionuclide and Chemical Nomenclature $\ldots \ldots \ldots \ldots \ldots \ldots \ldots \ldots \ldots \ldots \ldots \ldots \ldots \ldots \ldots \ldots$

Sampling Location Abbreviations and Other Abbreviations $\ldots \ldots \ldots \ldots \ldots \ldots \ldots \ldots \ldots \ldots$

Units of Measure, Scientific Notation, and Conversion Tables $\ldots \ldots \ldots \ldots \ldots \ldots \ldots \ldots \ldots$ xvii 



\section{List of Sampling Location Maps}

Figure 1 Radiological Sampling Locations - Air Surveillance $\ldots \ldots \ldots \ldots \ldots \ldots \ldots \ldots \ldots \ldots \ldots$

Figure 2 Radiological Sampling Locations - Surface Water

(SRS Stream and Savannah River Water)

$\mathrm{xx}$

Figure 3 Radiological and Nonradiological Sampling Locations - Fish $\ldots \ldots \ldots \ldots \ldots \ldots \ldots$

Figure 4 Radiological Sampling Locations - Soil $\ldots \ldots \ldots \ldots \ldots \ldots \ldots \ldots \ldots \ldots \ldots \ldots \ldots \ldots \ldots \ldots \ldots$

Figure 5 Radiological Sampling Locations - Sediment $\ldots \ldots \ldots \ldots \ldots \ldots \ldots \ldots \ldots \ldots \ldots \ldots \ldots \ldots \ldots \ldots \ldots$

Figure 6 Radiological Sampling Locations - Vegetation $\ldots \ldots \ldots \ldots \ldots \ldots \ldots \ldots \ldots \ldots \ldots \ldots \ldots$

Figure 7 Nonradiological Sampling Locations - Liquid Effluent (NPDES) $\ldots \ldots \ldots \ldots \ldots \ldots \ldots$ xxv

Figure 8 Nonradiological Sampling Locations - Surface Water

(SRS Stream and Savannah River Water) $\ldots \ldots \ldots \ldots \ldots \ldots \ldots \ldots \ldots \ldots \ldots \ldots \ldots \ldots \ldots \ldots \ldots$

Figure 9 Drinking Water Systems $\ldots \ldots \ldots \ldots \ldots \ldots \ldots \ldots \ldots \ldots \ldots \ldots \ldots \ldots \ldots \ldots \ldots \ldots \ldots \ldots \ldots \ldots \ldots$

Figure 10 Nonradiological Sampling Locations — SRS Stream and Savannah River Sediment ... xxviii 



\section{. List of Tables}

\section{Sampling}

Table 1

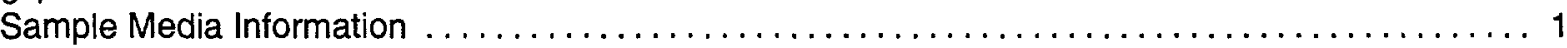

Table 2

Representative Minimum Detectable Concentrations for Radiological Analyses $\ldots \ldots \ldots \ldots \ldots \ldots$

Table 3

Nonradiological Environmental Surveillance Detection/Report Limits $\ldots \ldots \ldots \ldots \ldots \ldots \ldots \ldots 11$

\section{Radiological Effluent Monitoring}

Table 4

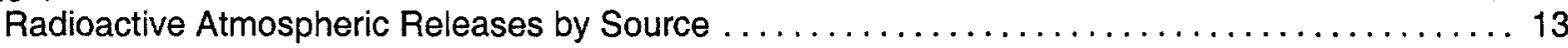

Table 5

Radioactive Atmospheric Releases by Stack/Facility and Comparison

of Annual Average Concentrations to DOE Derived Concentration Guides $\ldots \ldots \ldots \ldots \ldots \ldots \ldots$

Table 6

Radioactive Liquid Releases by Source

(Including Direct and Seepage Basin Migration Releases) . . . . . . . . . . . . . . . . . . 22

Table 7

Liquid Radioactive Releases by Outfall/Facility and Comparison of Annual

Average Radionuclide Concentrations to DOE Derived Concentration Guides $\ldots \ldots \ldots \ldots \ldots .23$

\section{Radiological Environmental Surveillance}

Table 8

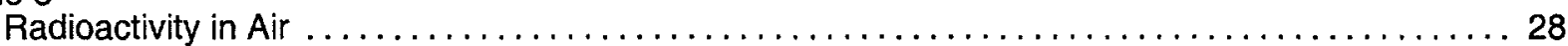

Table 9

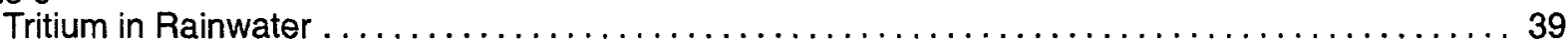

Table 10

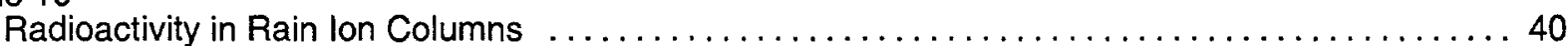

Table 11

Thermoluminescent Dosimeter (TLD) Results - Site Perimeter Stations . . . . . . . . . . . . . . 45

Table 12

Thermoluminescent Dosimeter (TLD) Results - Environmental Surveillance

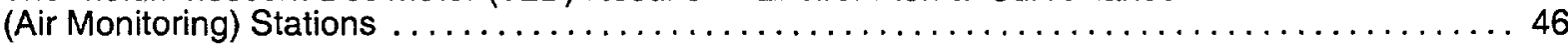

Table 13

Thermoluminescent Dosimeter (TLD) Results — Population Centers $\ldots \ldots \ldots \ldots \ldots \ldots \ldots \ldots .47$

Table 14

Thermoluminescent Dosimeter (TLD) Results — Vogtle Electric Generating Plant Vicinity ...... . 48

Table 15

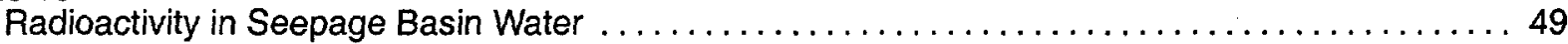

Table 16

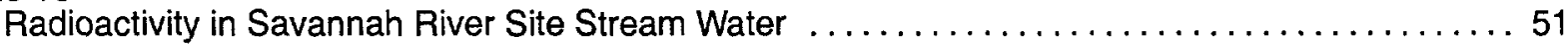

Table 17

Calculated Migration of Radioactivity from Seepage Basins $\ldots \ldots \ldots \ldots \ldots \ldots \ldots \ldots \ldots \ldots, 65$

Table 18

Estimated Tritium Transport in SRS Streams and the Savannah River $\ldots \ldots \ldots \ldots \ldots \ldots \ldots 66$

Table 19

Transport of Actinides in Savannah River Site Streams ..........................69 
Table 20

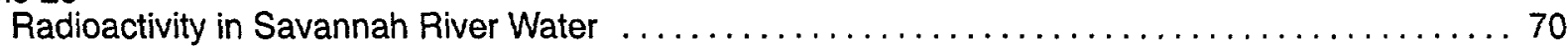

Table 21

Summary of Savannah River Site Tritium Transport, $1960-1999 \ldots \ldots \ldots \ldots \ldots \ldots \ldots \ldots \ldots 73$

Table 22

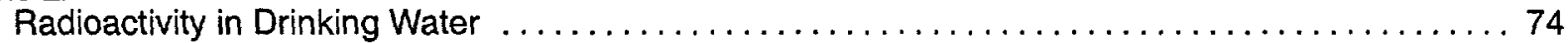

Table 23

Radioactivity in Terrestrial Food Products - Greens, Fruit, and Beef $\ldots \ldots \ldots \ldots \ldots \ldots \ldots . . \ldots 0$

Table 24

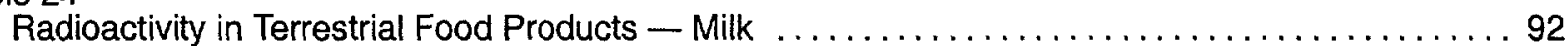

Table 25

Radioactivity in Aquatic Food Products - Freshwater Fish $\ldots \ldots \ldots \ldots \ldots \ldots \ldots \ldots \ldots$

Table 26

Radioactivity in Aquatic Food Products - Saltwater Fish $\ldots \ldots \ldots \ldots \ldots \ldots \ldots \ldots \ldots \ldots$

Table 27

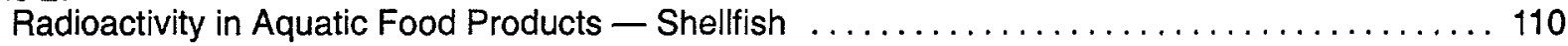

Table 28

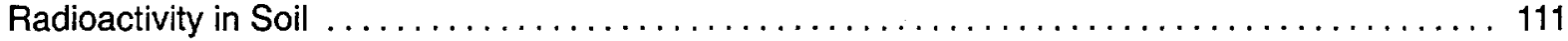

Table 29

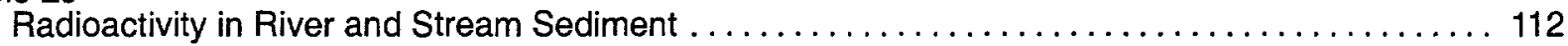

Table 30

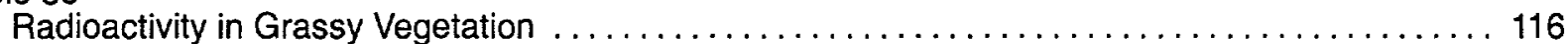

\section{Potential Radiation Doses}

Table 31

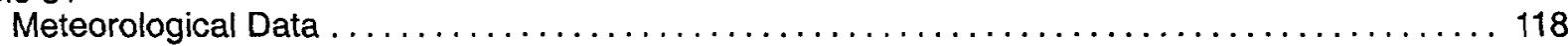

Table 32

80-km-Radius (50-Mile) Population Distribution Around SRS (1990 Census) $\ldots \ldots \ldots \ldots \ldots .125$

Table 33

80-km-Radius (50-Mile) Milk, Meat, and Vegetation Production Around SRS as of $1991 \ldots \ldots \ldots 126$

Table 34

Airborne Release Locations for Maximally Exposed Individual Dose $\ldots \ldots \ldots \ldots \ldots \ldots \ldots \ldots 128$

Table 35

Parameters Used for Adult Consumption Rates and for Atmospheric Dose Calculations $\ldots \ldots \ldots 129$

Table 36

Site-Specific Parameters Used with CAP88 Code Used for NESHAPS Calculations $\ldots \ldots \ldots \ldots 130$

Table 37

Parameters Used for Adult Consumption Rates and for Liquid Dose Calculations . . . . . . . . 131

Table 38

Site-Specific Parameters Used in Liquid Dose Calculations $\ldots \ldots \ldots \ldots \ldots \ldots \ldots \ldots \ldots \ldots 132$

Table 39

Committed Dose to the Maximally Exposed Individual from Atmospheric Releases

(MAXIGASP Code - Using Consumption of Cow Milk Pathway) $\ldots \ldots \ldots \ldots \ldots \ldots \ldots \ldots \ldots$

Table 40

Committed Dose to the Maximally Exposed Individual from Atmospheric Releases

(MAXIGASP Code - Using Consumption of Goat Milk Pathway) $\ldots \ldots \ldots \ldots \ldots \ldots \ldots \ldots \ldots$

Table 41

80-km (50-Mile) Collective Dose from Atmospheric Releases (POPGASP Code) . . . . . . ... 135

Table 42

Total Site Airborne Releases and Maximally Exposed Individual Effective Dose Equivalent

by Radionuclide (CAP88 Dose Calculations for 1999 NESHAP Report to EPA) 
Table 43

NESHAP Airborne-Dose Report Data — CAP88 Compared with MAXDOSE-SR $\ldots \ldots \ldots \ldots 139$

Table 44

NESHAP Airborne-Dose Report Data — CAP88 Compared with POPGASP $\ldots \ldots \ldots \ldots \ldots \ldots 140$

Table 45

Committed Dose to Maximally Exposed Individual from Liquid Releases $\ldots \ldots \ldots \ldots \ldots \ldots \ldots 141$

Table 46

Committed Dose to Maximally Exposed Individual from Public Water Supplies

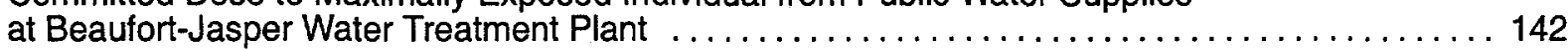

Table 47

Committed Dose to Maximally Exposed Individual from Public Water Supplies

at the City of Savannah Industrial and Domestic Water Supply Plant

(near Port Wentworth, Georgia)

Table 48

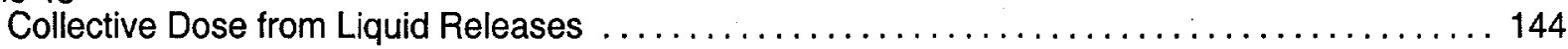

Table 49

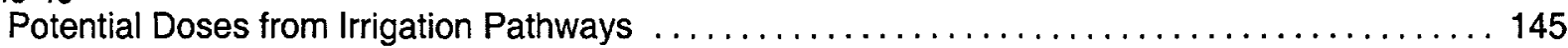

Table 50

Dose from Consumption of Fish from New Savannah Bluff Lock and Dam, SRS Creek Mouths,

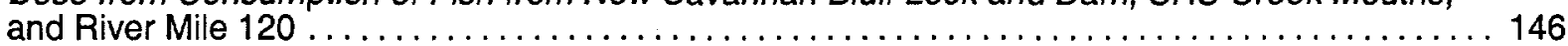

Table 51

Calculated Doses to Aquatic Biota from SRS Releases $\ldots \ldots \ldots \ldots \ldots \ldots \ldots \ldots \ldots \ldots \ldots \ldots 148$

\section{Nonradiological Effluent Monitoring}

Table 52

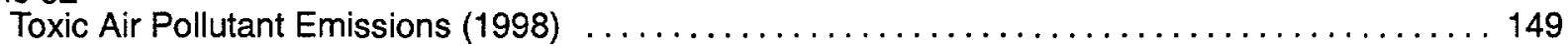

Table 53

National Pollutant Discharge Elimination System Monitoring Data $\ldots \ldots \ldots \ldots \ldots \ldots \ldots \ldots 156$

Table 54

National Pollutant Discharge Elimination System Toxicity Monitoring Data $\ldots \ldots \ldots \ldots \ldots \ldots \ldots 6$

Table 55

National Pollutant Discharge Elimination System Stormwater Monitoring Data $\ldots \ldots \ldots \ldots \ldots 167$

Table 56

National Pollutant Discharge Elimination System Land Application Monitoring Data $\ldots \ldots \ldots \ldots 184$

\section{Nonradiological Environmental Surveillance}

Table 57

Surface Water Surveillance - Inorganic Contaminants, Pesticides, and Herbicides ........ 186

Table 58

Sediment Surveillance - Inorganic Contaminants, Pesticides, and Herbicides $\ldots \ldots \ldots \ldots \ldots 218$

Table 59

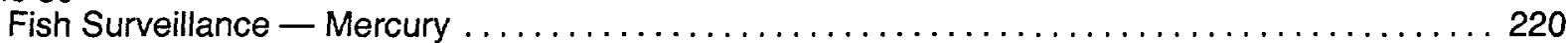

\section{Quality Assurance}

Table 60

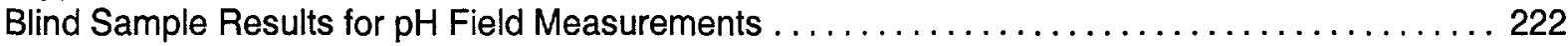

Table 61

Blind Sample Results for Tritium $\ldots \ldots \ldots \ldots \ldots \ldots \ldots \ldots \ldots \ldots \ldots \ldots \ldots \ldots \ldots \ldots \ldots \ldots \ldots \ldots \ldots \ldots \ldots \ldots, 223$

Table 62

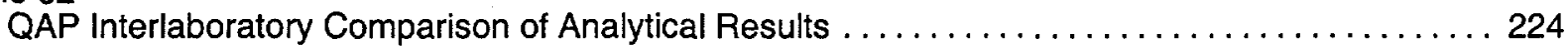

Table 63

Quality Assurance/Quality Control Standards 


\section{Table 64}

NPDES Duplicate Sample Results

230

Table 65

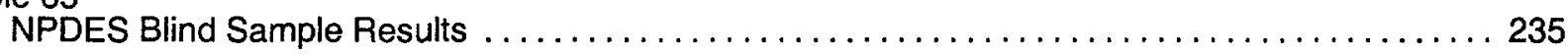

Table 66

SRS Stream and Savannah River Water Quality Duplicate Sample Results

239 


\section{Radionuclide and Chemical Nomenclature}

\begin{tabular}{|c|c|c|c|c|c|}
\hline \multirow[b]{2}{*}{ Radionuclide } & \multicolumn{4}{|c|}{ Nomenclature and Half-Life for Radionuclides } & \multirow[b]{2}{*}{ Half-Life $e^{a, b}$} \\
\hline & Symbol & Half-Life ${ }^{a, b}$ & Radionuclide & Symbol & \\
\hline Actinium-228 & $A C-228$ & $6.15 h$ & Niobium-94 & $\mathrm{Nb}-94$ & $2.0 \mathrm{E} 4 \mathrm{y}$ \\
\hline Americium-241 & Am-241 & $432.7 y$ & Niobium-95 & $\mathrm{Nb}-95$ & $34.97 \mathrm{~d}$ \\
\hline Americium-243 & Am-243 & $7370 y$ & Plutonium-238 & Pu-238 & $87.7 y$ \\
\hline Antimony-124 & Sb-124 & $60.2 \mathrm{~d}$ & Plutonium-239 & Pu-239 & 2.41E4 y \\
\hline Antimony-125 & Sb-125 & $2.758 \mathrm{y}$ & Plutonium-240 & Pu-240 & $6560 y$ \\
\hline Beryllium-7 & $\mathrm{Be}-7$ & $53.28 d$ & Plutonium-241 & Pu-241 & $14.4 y$ \\
\hline Bismuth-212 & $\mathrm{Bi}-212$ & $2.14 \mathrm{~m}$ & Plutonium-242 & Pu-242 & $3.75 E 5 y$ \\
\hline Bismuth-214 & $\mathrm{Bi}-214$ & $19.9 \mathrm{~m}$ & Potassium-40 & $K-40$ & 1.27E9 y \\
\hline Carbon-14 & C-14 & $5714 y$ & Praseodymium-144 & Pr-144 & $17.28 \mathrm{~m}$ \\
\hline Cerium-141 & Ce-141 & $32.5 \mathrm{~d}$ & Promethium-147 & $\mathrm{Pm}-147$ & $2.6234 y$ \\
\hline Cerium-144 & $\mathrm{Ce}-144$ & $284.6 \mathrm{~d}$ & Protactinium-231 & $\mathrm{Pa}-231$ & $3.28 E 4 y$ \\
\hline Cesium-134 & Cs-134 & $2.065 y$ & Protactinium-233 & $\mathrm{Pa}-233$ & $27.0 \mathrm{~d}$ \\
\hline Cesium-137 & Cs-137 & $30.07 y$ & Radium-226 & $\mathrm{Ra}-226$ & $1599 y$ \\
\hline Chromium-51 & $\mathrm{Cr}-51$ & $27.702 d$ & Radium-228 & $\mathrm{Ra}-228$ & $5.76 y$ \\
\hline Cobalt-57 & $\mathrm{Co}-57$ & $271.8 d$ & Radon-222 & Rn-222 & $3.8235 d$ \\
\hline Cobalt-58 & Co-58 & $\begin{array}{l}70.88 d \\
5.271 v\end{array}$ & Ruthenium-103 & $R u-103$ & $39.27 \mathrm{~d}$ \\
\hline $\begin{array}{l}\text { Cobalt-60 } \\
\text { Curium-242 }\end{array}$ & $\begin{array}{l}\text { Co-60 } \\
\text { Cm-242 }\end{array}$ & $\begin{array}{l}5.271 \mathrm{y} \\
162.8 \mathrm{~d}\end{array}$ & Ruthenium-106 & Ru-106 & $1.020 \mathrm{y}$ \\
\hline Curium-244 & $\mathrm{Cm}-244$ & $18.1 \mathrm{y}$ & Selenium-75 & Se-75 & $119.78 \mathrm{~d}$ \\
\hline Curium-246 & $\mathrm{Cm}-246$ & $4.76 E 3 y$ & Selenium-79 & Se-79 & $6.5 \mathrm{E} 5 \mathrm{y}$ \\
\hline Europium-152 & Eu-152 & $13.54 \mathrm{y}$ & Sodium-22 & $\mathrm{Na}-22$ & $2.604 y$ \\
\hline Europium-154 & Eu-154 & $8.593 y$ & Strontium-89 & Sr-89 & $50.52 d$ \\
\hline Europium-155 & Eu-155 & $4.75 y$ & Strontium-90 & Sr-90 & $28.78 y$ \\
\hline lodine- 129 & $1-129$ & $1.57 E 7 y$ & Technetium-99 & Tc-99 & $2.13 E 5 y$ \\
\hline lodine-131 & $\mid-131$ & $8.0207 d$ & Thallium-208 & TI-208 & $3.053 \mathrm{~m}$ \\
\hline Krypton-85 & $\mathrm{Kr}-85$ & $10.76 y$ & Thorium-228 & Th-228 & $1.913 y$ \\
\hline Lead-212 & $\mathrm{Pb}-212$ & $10.64 \mathrm{~h}$ & Thorium-230 & Th-230 & 7.54E4 y \\
\hline Lead-214 & $\mathrm{Pb}-214$ & $27 \mathrm{~m}$ & Thorium-232 & Th-232 & $1.40 \mathrm{E} 10 \mathrm{y}$ \\
\hline Manganese-54 & $M n-54$ & $312.1 \mathrm{~d}$ & Thorium-234 & Th-234 & $24.10 \mathrm{~d}$ \\
\hline Mercury-203 & $\mathrm{Hg}-203$ & $46.61 \mathrm{~d}$ & $\operatorname{Tin}-126$ & Sn-126 & $2.5 \mathrm{E} 5 \mathrm{y}$ \\
\hline Neptunium-237 & $\mathrm{Np}-237$ & $2.14 E 6 y$ & Tritium (Hydrogen-3) & $H-3$ & $12.32 y$ \\
\hline Neptunium-239 & Np-239 & $2.355 \mathrm{~d}$ & Uranium-232 & U-232 & $69.8 y$ \\
\hline Nickel-59 & $\mathrm{Ni}-59$ & $7.6 E 4 y$ & Uranium-233 & U-233 & $1.592 \mathrm{E} 5 \mathrm{y}$ \\
\hline Nickel-63 & $\mathrm{Ni}-63$ & $100 y$ & Uranium-234 & $U-234$ & $2.46 E 5 y$ \\
\hline
\end{tabular}

a $m=$ minute; $h=$ hour; $d=$ day; $y=$ year

b Reference: Chart of the Nuclides, 15th edition, revised 1996, General Electric Company 
Nomenclature and Half-Life for Radionuclides, Continued

\begin{tabular}{llrlrr} 
Radionuclide & Symbol & Half-life $^{\mathbf{a}, \mathrm{b}}$ & Radionuclide & Symbol & Half-life $^{\mathrm{a}, \mathrm{b}}$ \\
\hline Uranium-235 & $\mathrm{U}-235$ & $7.04 \mathrm{E8} \mathrm{y}$ & Yttrium-90 & Y-90 & $2.67 \mathrm{~d}$ \\
Uranium-236 & $\mathrm{U}-236$ & $2.342 \mathrm{E} 7 \mathrm{y}$ & Zinc-65 & Zn-65 & $243.8 \mathrm{~d}$ \\
Uranium-238 & $\mathrm{U}-238$ & $4.47 E 9 \mathrm{y}$ & Zirconium-95 & Zr-95 & $64.02 \mathrm{~d}$ \\
Xenon-135 & $\mathrm{Xe-135}$ & $9.10 \mathrm{~h}$ & & &
\end{tabular}

a minute; $h=$ hour; $d=$ day; $y=$ year

b Reference: Chart of the Nuclides, 15th edition, revised 1996, General Electric Company 
Constituent Nomenclature for Elements and Chemical Constituent Analyses

Note: Some of the symbols listed in this table came from various databases used to format the data tables in this book and are included here to assist the reader in understanding the tables.

\begin{tabular}{|c|c|c|c|}
\hline Aluminum & $\mathrm{Al}$ (or $\mathrm{AL}$ ) & Oil and Grease & O\&G \\
\hline Ammonia & $\mathrm{NH}_{3}$ & $\mathrm{pH}$ & $\mathrm{pH}($ or $\mathrm{PH})$ \\
\hline Ammonia as Nitrogen & $\mathrm{NH}_{3}-\mathrm{N}$ (or AN) & Phenol & PHE \\
\hline Antimony & $\mathrm{Sb}$ (or SB) & Phosphorus & $P$ \\
\hline Arsenic & As (or AS) & \multirow[t]{2}{*}{ Phosphate } & \multirow{2}{*}{$\begin{array}{l}\mathrm{PO}_{4} \text { (or } \mathrm{PO} 4-\mathrm{P} \text { or } \\
\mathrm{PO}_{4}-\mathrm{P} \text { ) }\end{array}$} \\
\hline Barium & $\mathrm{Ba}$ (or $\mathrm{BA})$ & & \\
\hline Biological Oxygen Demand & BOD & Polychlorinated Biphenyl & $\mathrm{PCB}$ \\
\hline Benzene & BEN & Potassium & $\mathrm{K}$ \\
\hline Beryllium & $\mathrm{Be}$ & Radium & $\mathrm{Ra}$ \\
\hline Boron & B & Selenium & Se (or SE) \\
\hline Cadmium & Cd (or CD) & Silver & $\mathrm{Ag}($ or $\mathrm{AG})$ \\
\hline Calcium & $\begin{array}{l}\mathrm{Ca} \\
\mathrm{C}\end{array}$ & Sodium & $\mathrm{Na}$ \\
\hline $\begin{array}{l}\text { Carbon } \\
\text { Chemical Oxygen Demand }\end{array}$ & COD & Sulfate & $\mathrm{SO}_{4}$ (or SO4) \\
\hline Chlorine & $\mathrm{Cl}$ (or $\mathrm{CHL})$ & Temperature & TMP (or T or \\
\hline Chromium & $\mathrm{Cr}$ (or $\mathrm{CR})$ & & \multirow{2}{*}{ PERCL } \\
\hline cis-1,2-dichloroethene & $1,2-D C E$ & $\begin{array}{l}\text { Tetrachloroethylene } \\
\text { (Perchloroethylene) }\end{array}$ & \\
\hline Cobalt & Co & Trichloroethylene & TRICL \\
\hline Copper & $\mathrm{Cu}$ (or CU) & 1,1,1-Trichloroethane & TCE \\
\hline Cyanide & $\mathrm{CN}$ & Tin & SN \\
\hline Dissolved Oxygen & $\begin{array}{l}\text { DO } \\
\text { FFC }\end{array}$ & Total Dissolved Solids & TDS \\
\hline $\begin{array}{l}\text { Fecal Coliform } \\
\text { Fixed Residues }\end{array}$ & $\begin{array}{l}\text { FEC } \\
\text { FR }\end{array}$ & Total Kjeldahl Nitrogen & TKN \\
\hline Flow & FLO & Total Organic Carbon & TOC \\
\hline Iron & $\mathrm{Fe}$ (or FE) & Total Organic Halogens & $\mathrm{TOH}$ \\
\hline Lead & $\mathrm{Pb}$ (or $\mathrm{PB}$ ) & Total Phosphates & \multirow{2}{*}{$\begin{array}{l}\mathrm{TPO}_{4} \text { (or Total } \\
\text { Phos) }\end{array}$} \\
\hline Lithium & $\mathrm{Li}$ & & \\
\hline Magnesium & $M g($ or $M G)$ & Total Phosphorus & TP \\
\hline Manganese & $\mathrm{Mn}$ (or MN) & Total Suspended Solids & TSS \\
\hline Mercury & $\mathrm{Hg}$ (or HG) & Total Volatile Solids & TVS \\
\hline Nickel & $\mathrm{Ni}($ or $\mathrm{NI})$ & Uranium & $U$ \\
\hline Nitrate as Nitrogen & $\mathrm{NO}_{3}-\mathrm{N}$ & Vinyl Chloride & VC \\
\hline Nitrite as Nitrogen' & $\mathrm{NO}_{2}-\mathrm{N}$ & Volatile Organic Compound & voc \\
\hline \multirow[t]{2}{*}{ Nitrite, Nitrate } & \multirow{2}{*}{$\begin{array}{l}\mathrm{NO} 2, \mathrm{NO} 3 \text { (or } \mathrm{NO}_{2}, \\
\mathrm{NO}_{3} \text { or } \\
\mathrm{NO}_{2} / \mathrm{NO} 3 \text { )) }\end{array}$} & Volatile Solids & vs \\
\hline & & Zinc & $\mathrm{Zn}($ or $\mathrm{ZN})$ \\
\hline
\end{tabular}





\section{Sampling Location Abbreviations and Other Abbreviations}

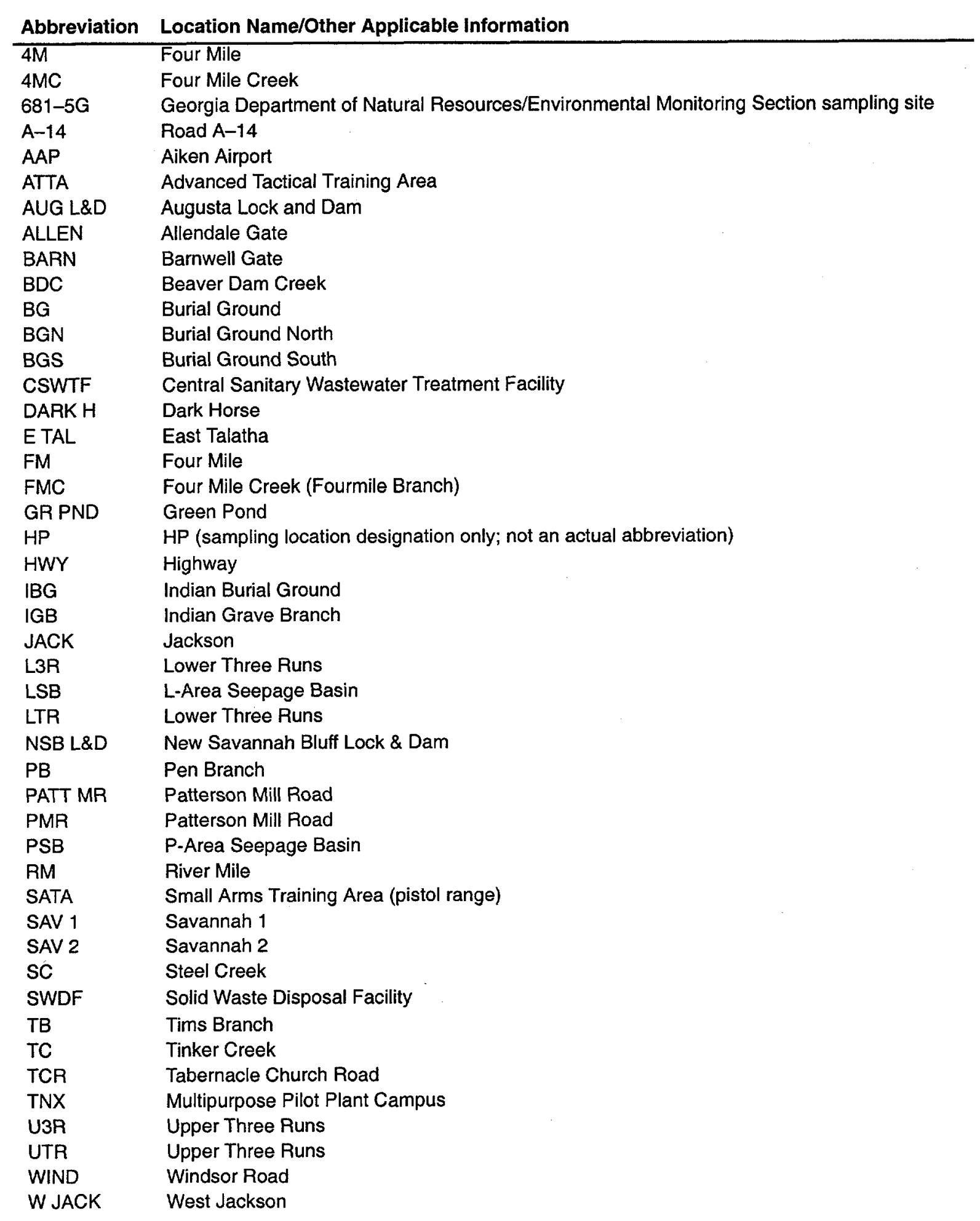


Sample Locations Known By More Than One Abbreviation

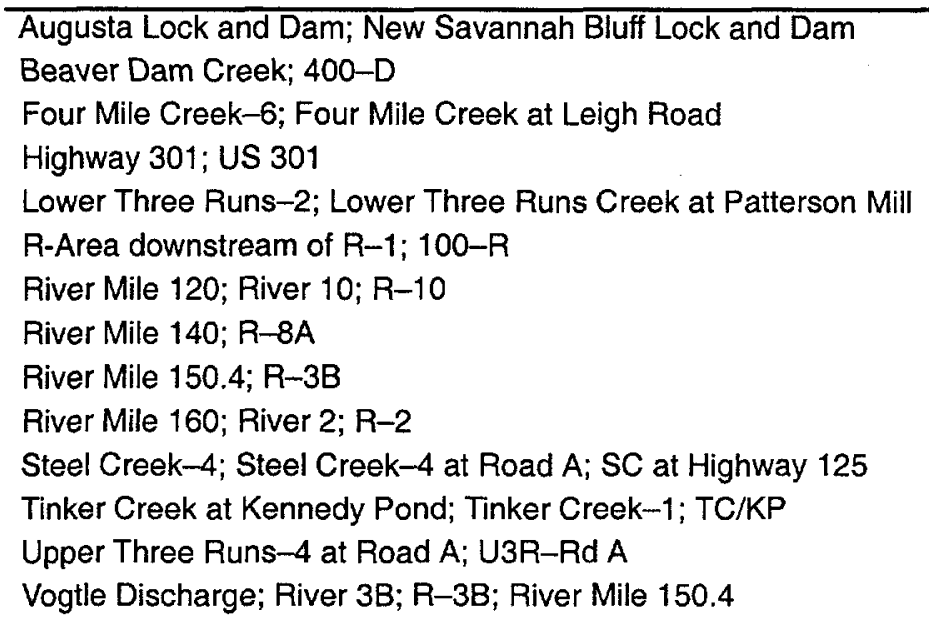

Other Abbreviations and Acronyms Used in This Book

\begin{tabular}{|c|c|}
\hline AMAD & Activity Median Aerodynamic Diameter \\
\hline DCG & Derived Concentration Guide \\
\hline DEG C & Degrees Centigrade \\
\hline DOE & Department of Energy \\
\hline Dup, DUP & Duplicate \\
\hline EML & Department of Energy's Environmental Measurements Laboratory \\
\hline ETF & Effluent Treatment Facility \\
\hline GP & Georgia Power \\
\hline MDA & Minimum Detectable Activity \\
\hline MDC & Minimum Detectable Concentration \\
\hline MDL & Minimum Detectable Limit \\
\hline MGD & Million Gallons Per Day \\
\hline ND & Not Detected \\
\hline NF & No Flow \\
\hline NPDES & National Pollutant Discharge Elimination System \\
\hline NRC & Nuclear Regulatory Commission \\
\hline ORA & Operations Recreation Association \\
\hline PAR & "P and R" Pond \\
\hline PQL & Practical Quantitation Limit \\
\hline QAP & Quality Assurance Program \\
\hline RBOF & Receiving Basin for Offsite Fuel \\
\hline Rep, REP & Replicate \\
\hline RR & Railroad Yard \\
\hline SCDHEC & South Carolina Department of Health and Environmental Control \\
\hline SRS & Savannah River Site \\
\hline SRTC & Savannah River Technology Center \\
\hline SU & Standard Units \\
\hline SWDF & Solid Waste Disposal Facility \\
\hline TCLP & Toxicity Characteristic Leaching Procedure \\
\hline TLD & Thermoluminescent Dosimeter \\
\hline USGS & United States Geological Survey \\
\hline WSRC & Westinghouse Savannah River Company \\
\hline
\end{tabular}




\section{Units of Measure, Scientific Notation, and Conversion Tables}

\begin{tabular}{|c|c|c|c|}
\hline \multicolumn{2}{|c|}{ Units of Measure } & \multicolumn{2}{|c|}{ Units of Measure } \\
\hline Symbol & Name & Symbol & Name \\
\hline Temperature & & Concentration & \\
\hline${ }^{\circ} \mathrm{C}$ & degrees Centigrade & $\mathrm{ppb}$ & parts per billion \\
\hline${ }^{\circ} \mathrm{F}$ & degrees Fahrenheit & ppm & parts per million \\
\hline \multicolumn{4}{|l|}{ Time } \\
\hline d & day & Rate & \\
\hline$h$ & hour & cfs & cubic feet per second \\
\hline$y$ & year & gpm & gallons per minute \\
\hline \multicolumn{4}{|l|}{ Length } \\
\hline $\mathrm{cm}$ & centimeter & Conductivity & \\
\hline $\mathrm{ft}$ & foot & $\mu \mathrm{mho}$ & micromho \\
\hline in. & inch & & \\
\hline $\mathrm{km}$ & kilometer & & \\
\hline $\mathrm{m}$ & meter & Radioactivity & \\
\hline $\mathrm{mm}$ & millimeter & $\mathrm{Ci}$ & curie \\
\hline \multirow[t]{2}{*}{$\mu \mathrm{m}$} & micrometer & $\mathrm{cpm}$ & counts per minute \\
\hline & & $\mathrm{mCi}$ & millicurie \\
\hline Mass & & $\mu \mathrm{Ci}$ & microcurie \\
\hline g & gram & $\mathrm{pCi}$ & picocurie \\
\hline $\mathrm{kg}$ & kilogram & $\mathrm{Bq}$ & becquerel \\
\hline $\mathrm{mg}$ & milligram & & \\
\hline \multirow[t]{2}{*}{$\mu \mathrm{g}$} & microgram & Radiation Dose & \\
\hline & & mrad & millirad \\
\hline Area & & mrem & millirem \\
\hline$m i^{2}$ & square mile & Sv & sievert \\
\hline \multirow[t]{2}{*}{$\mathrm{ft}^{2}$} & square foot & $\mathrm{mSv}$ & millisievert \\
\hline & & $\mu \mathrm{Sv}$ & microsievert \\
\hline Volume & & $\mathbf{R}$ & roentgen \\
\hline gal & gallon & $\mathrm{mR}$ & milliroentgen \\
\hline L & liter & $\mu \mathrm{R}$ & microroentgen \\
\hline $\mathrm{mL}$ & milliliter & Gy & gray \\
\hline
\end{tabular}




\begin{tabular}{|ccccc|}
\hline \multicolumn{5}{c|}{ Fractions and Multiples of Units } \\
\hline Multiple & Decimal Equivalent & Prefix & Symbol & Report \\
\hline $10^{6}$ & $1,000,000$ & mega- & $\mathrm{M}$ & $\mathrm{E}+06$ \\
$10^{3}$ & 1,000 & kilo- & $\mathrm{k}$ & $\mathrm{E}+03$ \\
$10^{2}$ & 100 & hecto- & $\mathrm{h}$ & $\mathrm{E}+02$ \\
10 & 10 & deka- & $\mathrm{da}$ & $\mathrm{E}+01$ \\
$10^{-1}$ & 0.1 & deci- & $\mathrm{d}$ & $\mathrm{E}-01$ \\
$10^{-2}$ & 0.01 & centi- & $\mathrm{c}$ & $\mathrm{E}-02$ \\
$10^{-3}$ & 0.001 & milli- & $\mathrm{m}$ & $\mathrm{E}-03$ \\
$10^{-6}$ & 0.000001 & micro- & $\mu$ & $\mathrm{E}-06$ \\
$10^{-9}$ & 0.000000001 & nano- & $\mathrm{n}$ & $\mathrm{E}-09$ \\
$10^{-12}$ & 0.000000000001 & pico- & $\mathrm{p}$ & $\mathrm{E}-12$ \\
$10^{-15}$ & 0.000000000000001 & femto- & $\mathrm{f}$ & $\mathrm{E}-15$ \\
$10^{-18}$ & 0.000000000000000001 & atto- & $\mathrm{a}$ & $\mathrm{E}-18$ \\
\hline
\end{tabular}

\begin{tabular}{|lll|}
\hline \multicolumn{3}{|c|}{ Conversion Table (Units of Radiation Measure) } \\
\hline Current System & Systéme international & Conversion \\
\hline curie $(\mathrm{Ci})$ & becquerel $(\mathrm{Bq})$ & $1 \mathrm{Ci}=3.7 \times 10^{10} \mathrm{~Bq}$ \\
rad (radiation absorbed dose) & gray (Gy) & $1 \mathrm{rad}=0.01 \mathrm{~Gy}$ \\
rem (roentgen equivalent man) & sievert (Sv) & $1 \mathrm{rem}=0.01 \mathrm{~Sv}$ \\
\hline
\end{tabular}

\begin{tabular}{|lll|lll|}
\hline \multicolumn{7}{c|}{ Conversion Table } \\
\hline Multiply & By & To Obtain & Multiply & By & To Obtain \\
\hline in. & 2.54 & $\mathrm{~cm}$ & $\mathrm{~cm}$ & 0.394 & $\mathrm{in}$. \\
$\mathrm{ft}$ & 0.305 & $\mathrm{~m}$ & $\mathrm{~m}$ & 3.28 & $\mathrm{ft}$ \\
$\mathrm{mi}$ & 1.61 & $\mathrm{~km}$ & $\mathrm{~km}$ & 0.621 & $\mathrm{mi}$ \\
$\mathrm{lb}$ & 0.4536 & $\mathrm{~kg}$ & $\mathrm{~kg}$ & 2.205 & $\mathrm{lb}$ \\
liq qt-U.S. & 0.946 & $\mathrm{~L}$ & $\mathrm{~L}$ & 1.057 & $\mathrm{liq} \mathrm{qt}-\mathrm{U} . \mathrm{S}$. \\
$\mathrm{ft}^{2}$ & 0.093 & $\mathrm{~m}^{2}$ & $\mathrm{~m}^{2}$ & 10.764 & $\mathrm{ft}^{2}$ \\
$\mathrm{mi}^{2}$ & 2.59 & $\mathrm{~km}^{2}$ & $\mathrm{~km}$ & 0.386 & $\mathrm{mi}^{2}$ \\
$\mathrm{ft}^{3}$ & 0.028 & $\mathrm{~m}^{3}$ & $\mathrm{~m}$ & 35.31 & $\mathrm{ft}^{3}$ \\
$\mathrm{~d} / \mathrm{m}$ & 0.450 & $\mathrm{pCi}$ & $\mathrm{mCi}$ & 2.22 & $\mathrm{~d} / \mathrm{m}$ \\
$\mathrm{pCi}$ & $10^{-6}$ & $\mu \mathrm{Ci}$ & $\mu \mathrm{Ci}$ & $10^{6}$ & $\mathrm{pCi}$ \\
$\mathrm{pCi} / \mathrm{L}$ (water) & $10^{-9}$ & $\mu \mathrm{Ci} / \mathrm{mL}$ (water) & $\mu \mathrm{Ci} / \mathrm{mL}$ (water) & $10^{9}$ & $\mathrm{pCi} / \mathrm{L}$ (water) \\
$\mathrm{pCi} / \mathrm{m}^{3}$ (air) & $10^{-12}$ & $\mu \mathrm{Ci} / \mathrm{mL}$ (air) & $\mu \mathrm{Ci} / \mathrm{mL}$ (air) & $10^{12}$ & $\mathrm{pCi} / \mathrm{m}^{3}$ (air) \\
\hline
\end{tabular}




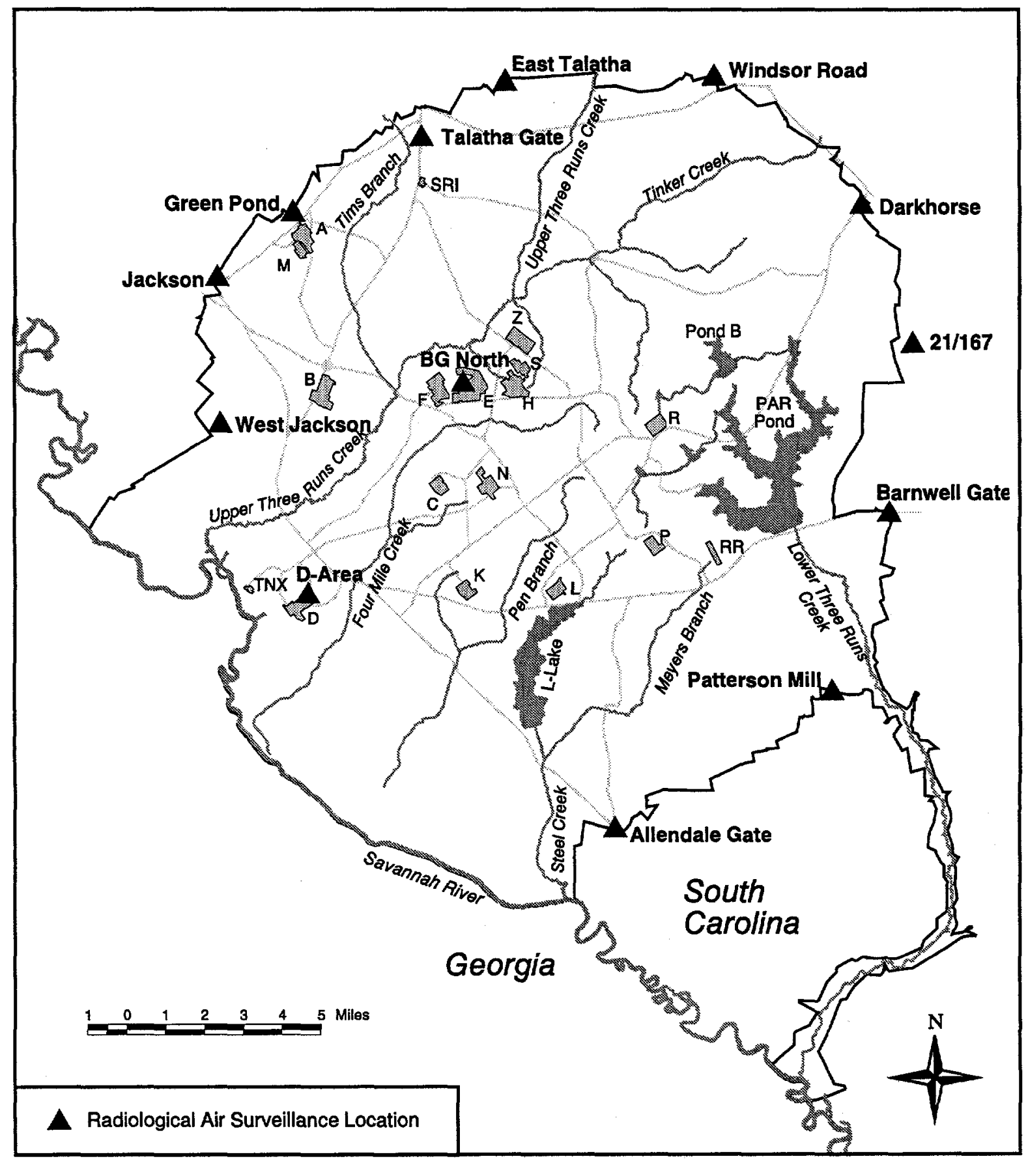

EPD/GIS Map

Figure 1 Radiological Sampling Locations - Air Surveillance 


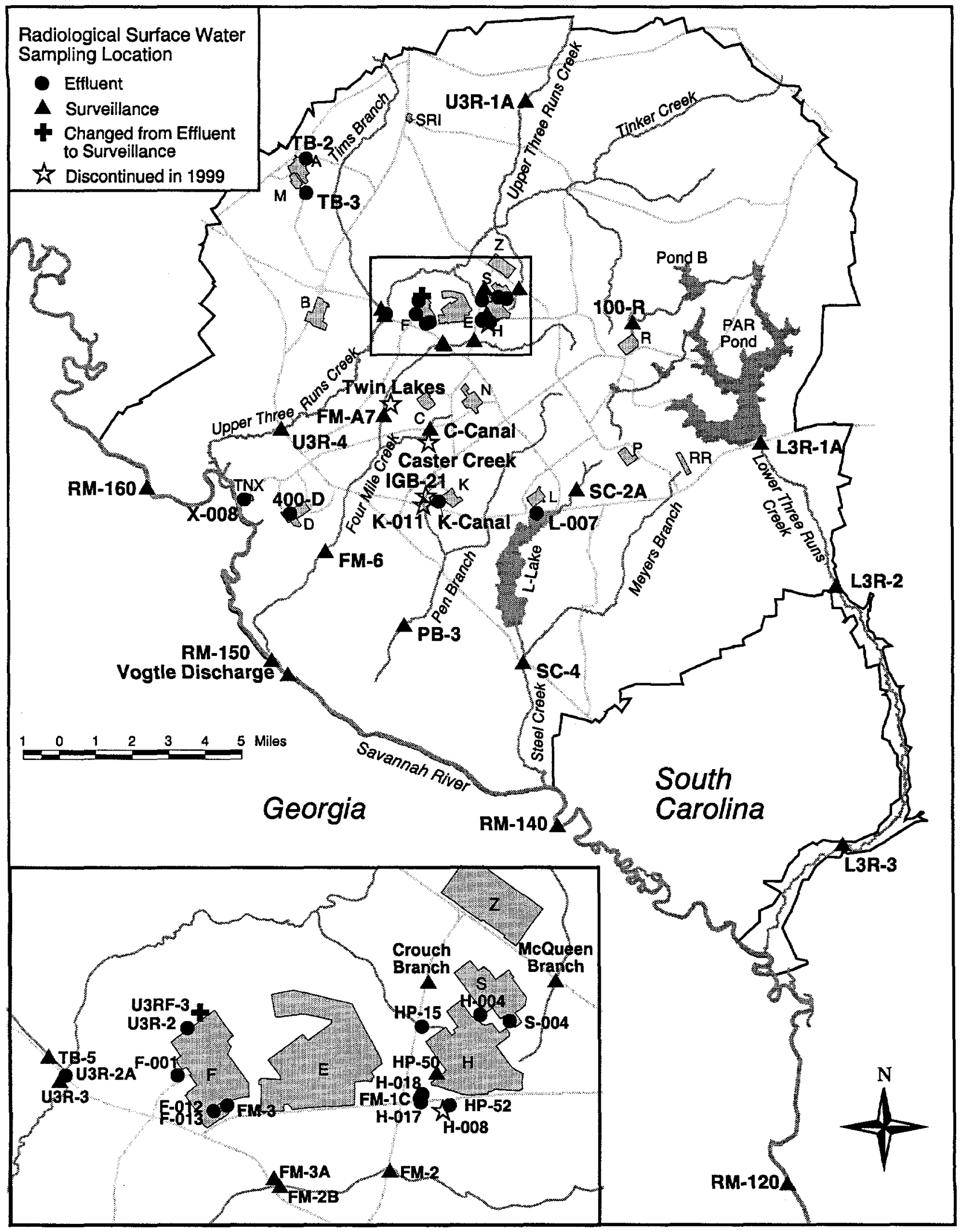

EPD/GIS Map

Figure 2 Radiological Sampling Locations - Surface Water (SRS Stream and Savannah River Water) 


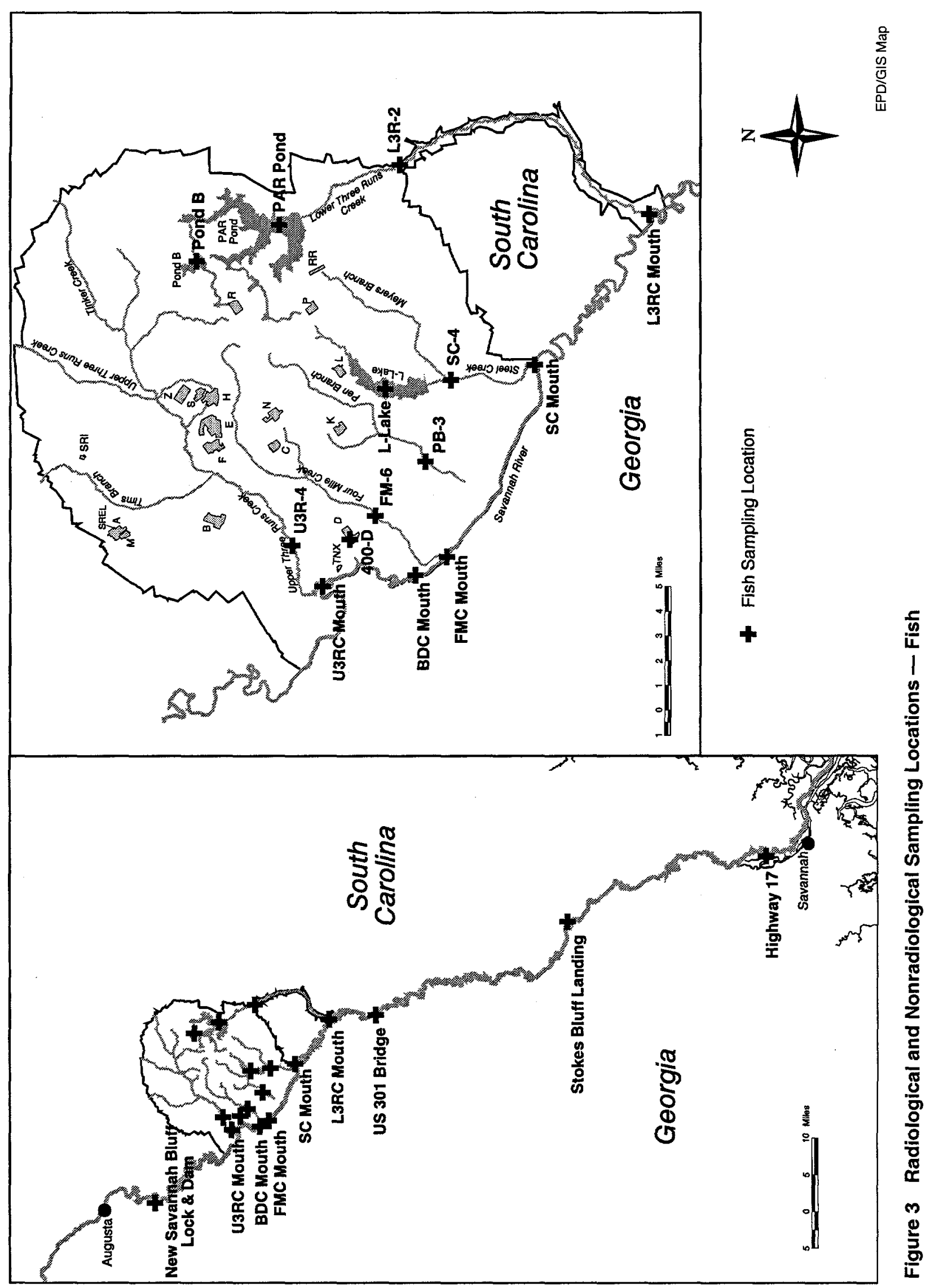




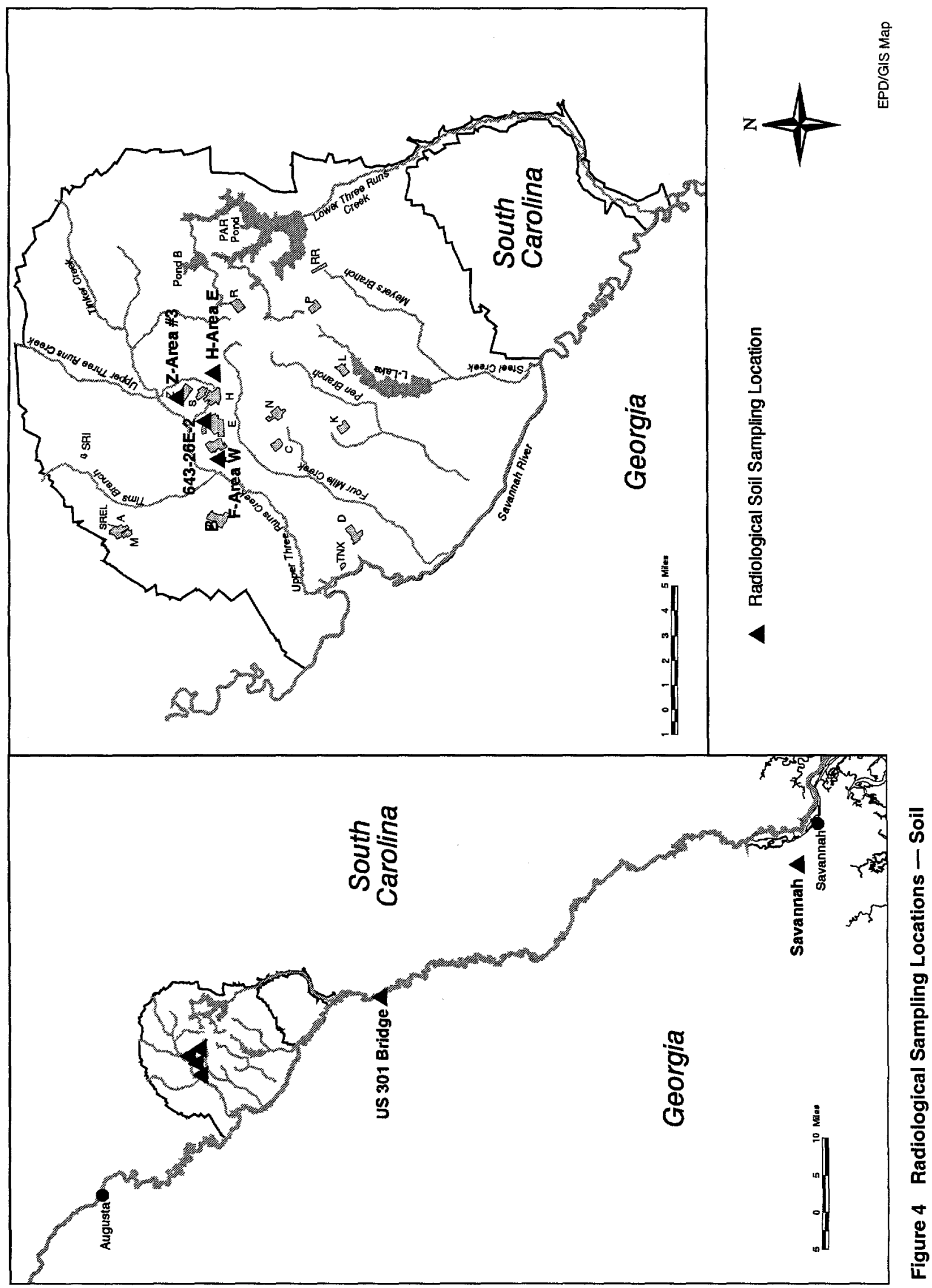




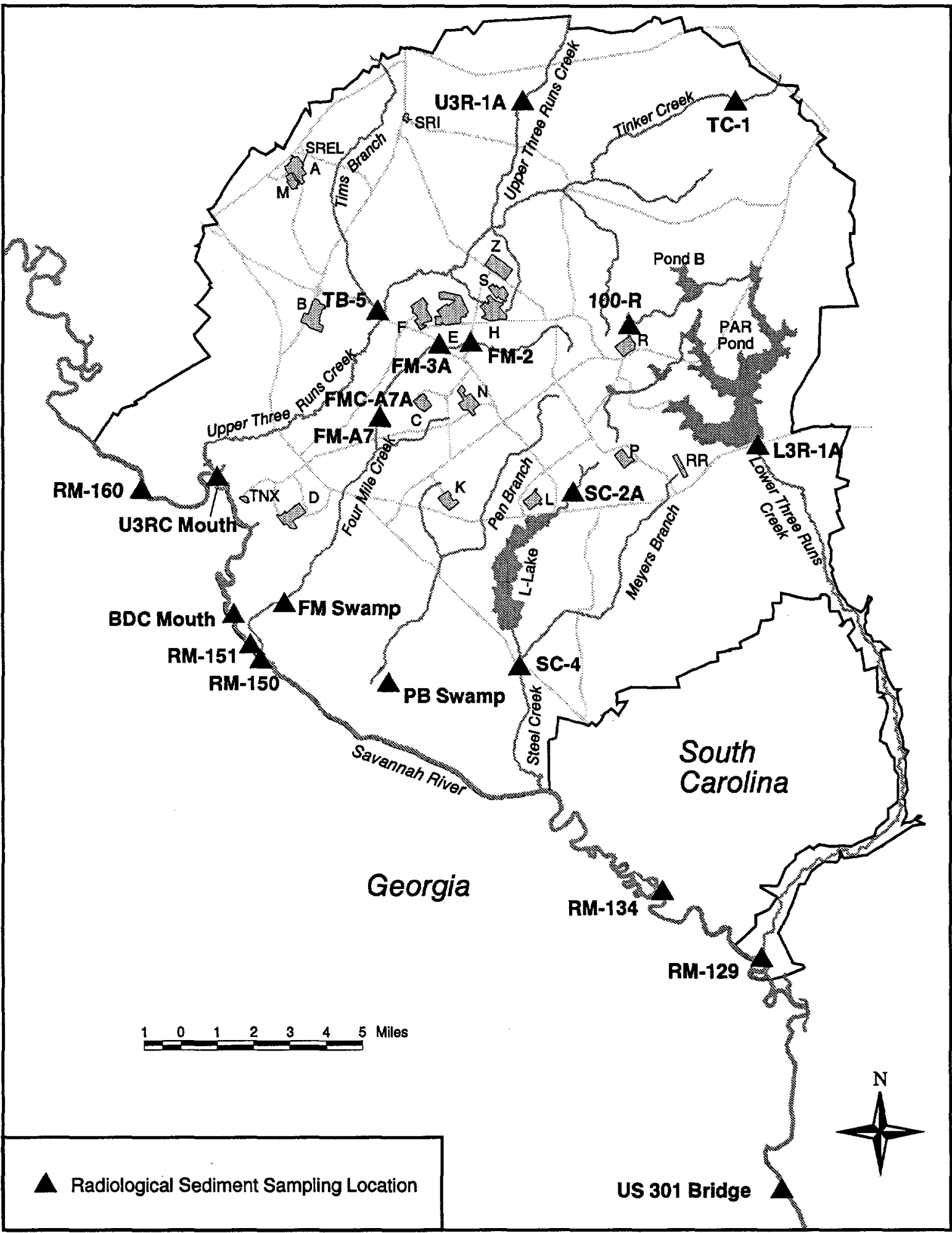

EPD/GIS Map

Figure 5 Radiological Sampling Locations - Sediment 


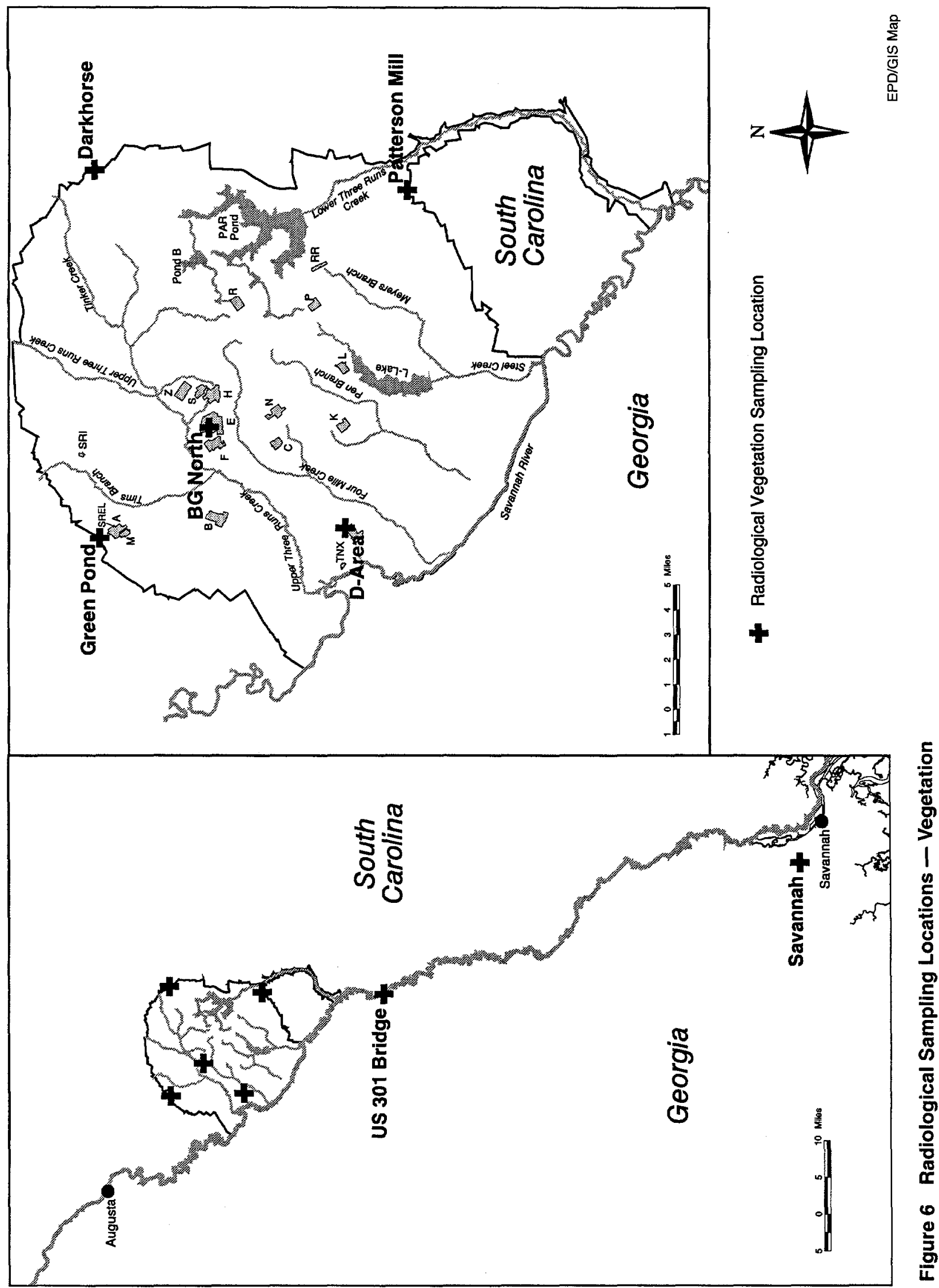




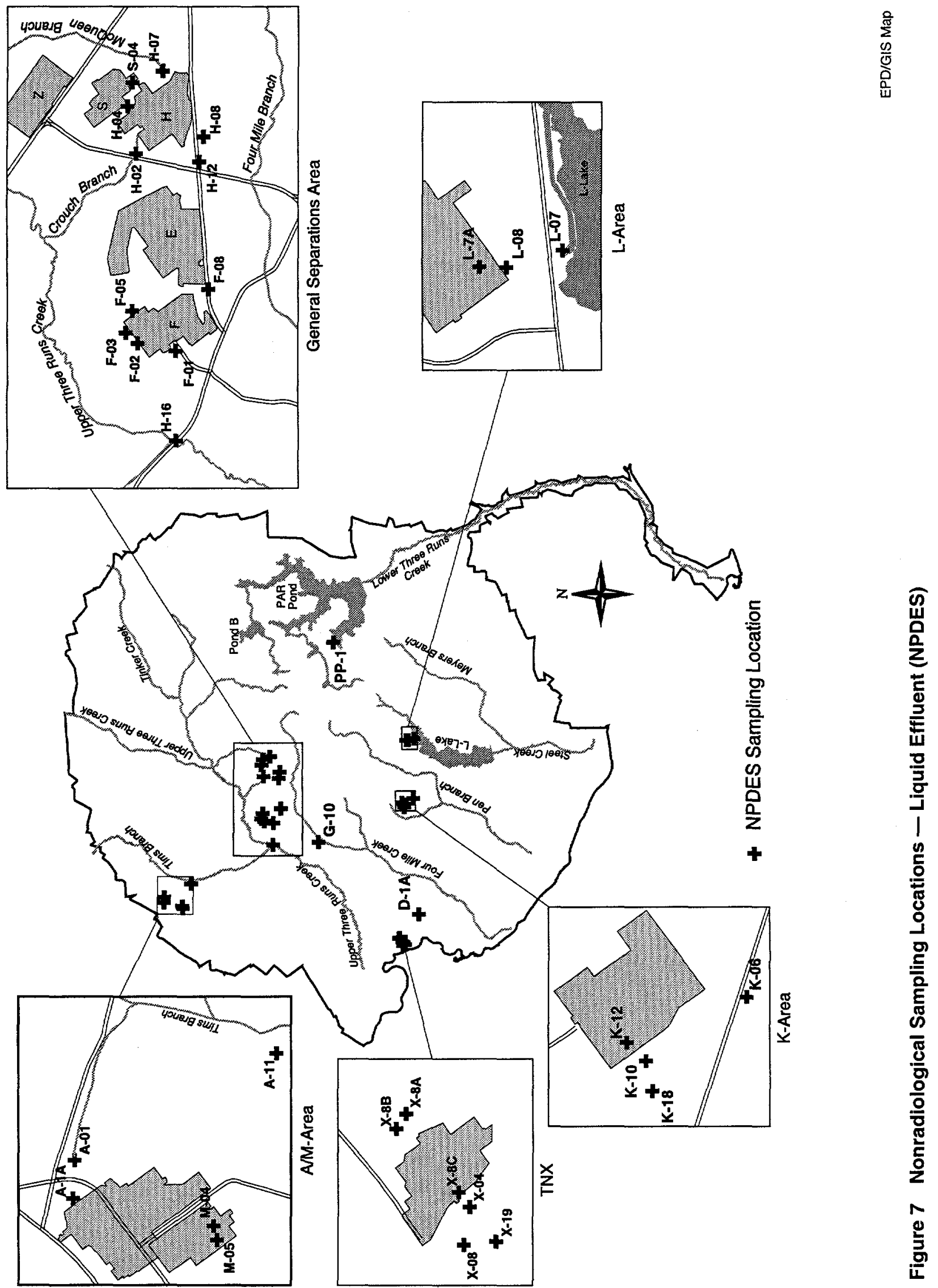




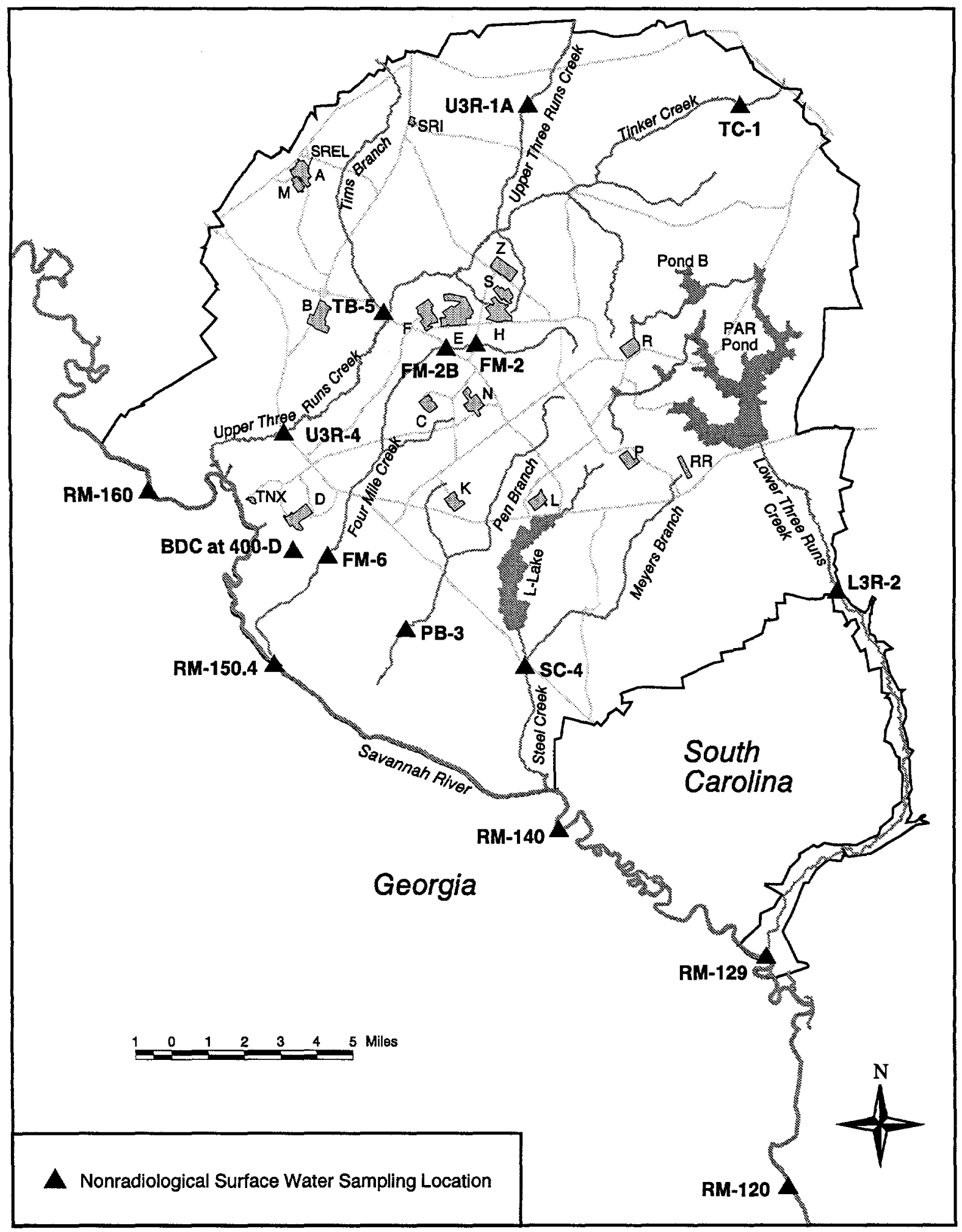

EPD/GIS Map

Figure 8 Nonradiological Sampling Locations - Surface Water (SRS Stream and Savannah River Water) 


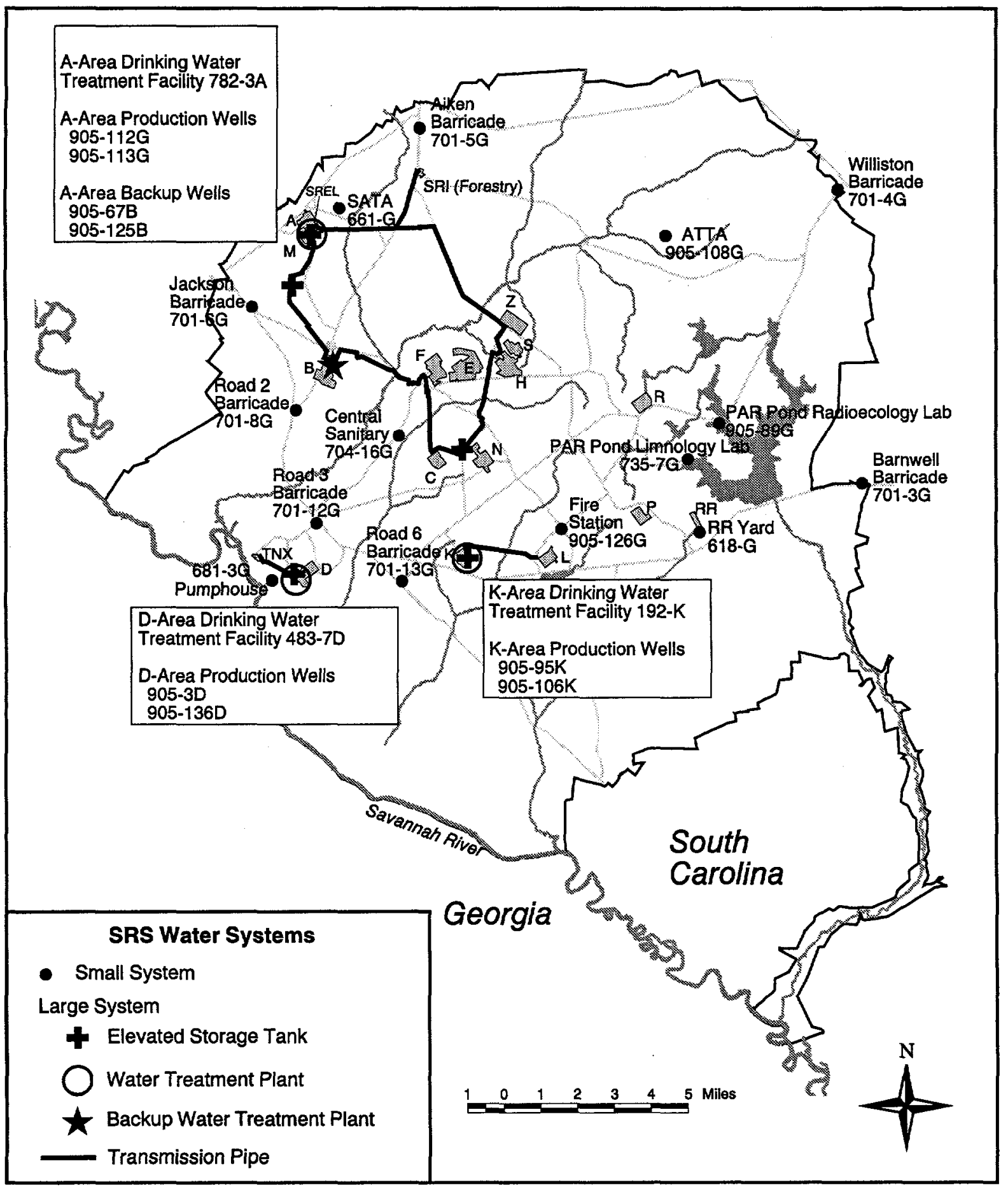

Figure 9 Drinking Water Systems

EPD/GIS Map 


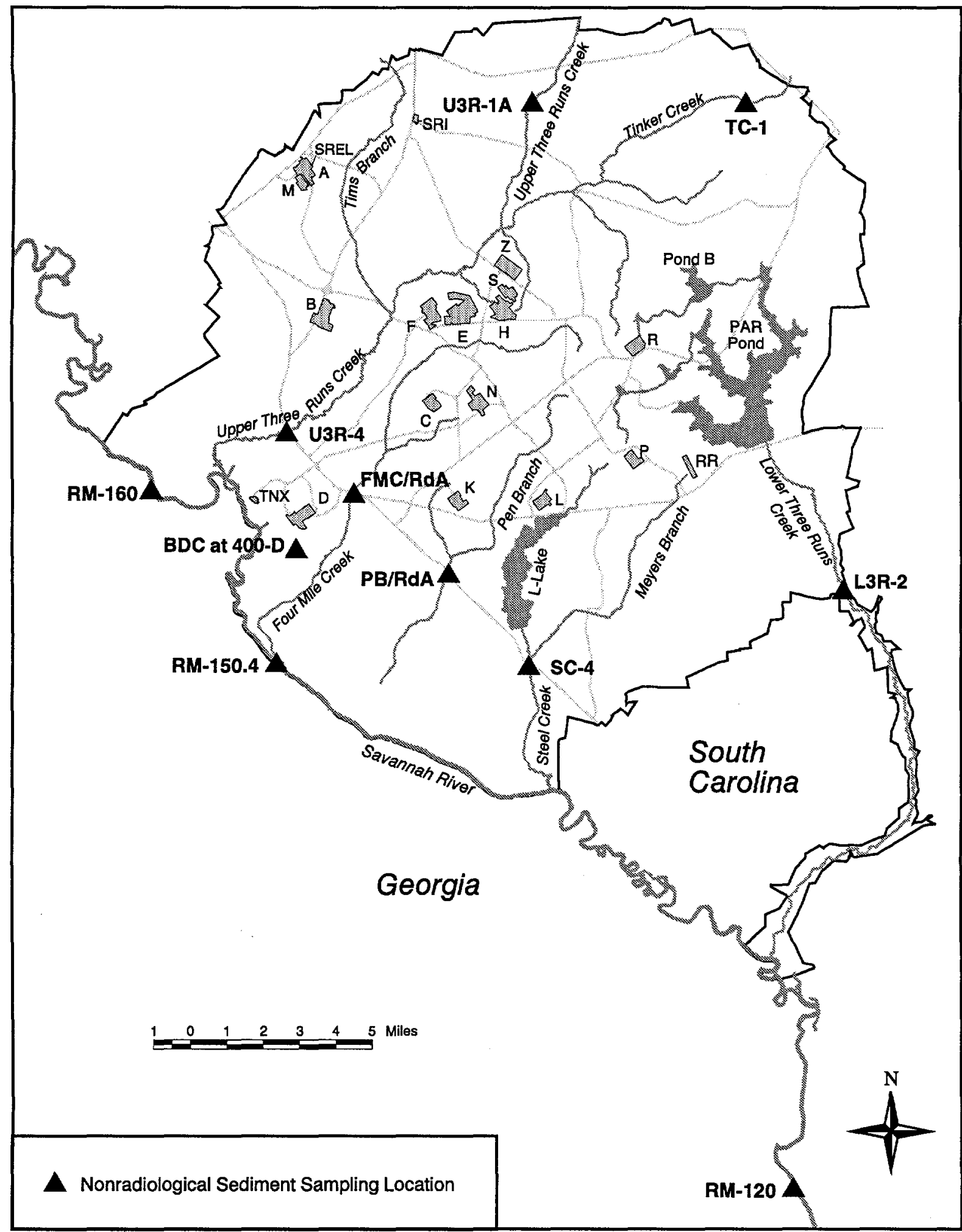

EPD/GIS Map

Figure 10 Nonradiological Sampling Locations - SRS Stream and Savannah River Sediment 
Table 1

Sample Media Information

Page 1 of 4

\begin{tabular}{|c|c|c|}
\hline Matrix & Analytical Parameter & Representative Aliquot \\
\hline \multicolumn{3}{|l|}{ Surveillance Air } \\
\hline \multirow[t]{6}{*}{ On site } & Gamma & $746 \mathrm{~m}^{3}$ \\
\hline & Gross alpha & $746 \mathrm{~m}^{3}$ \\
\hline & Gross beta & $746 \mathrm{~m}^{3}$ \\
\hline & Tritium & $3 \mathrm{~m}^{3}$ \\
\hline & Strontium-89,90 & $746 \mathrm{~m}^{3}$ \\
\hline & Plutonium-238,239 & $746 \mathrm{~m}^{3}$ \\
\hline \multirow[t]{6}{*}{ Site perimeter } & Gamma & $746 \mathrm{~m}^{3}$ \\
\hline & Gross alpha & $746 \mathrm{~m}^{3}$ \\
\hline & Gross beta & $746 \mathrm{~m}^{3}$ \\
\hline & Tritium & $3 \mathrm{~m}^{3}$ \\
\hline & Strontium-89,90 & $746 \mathrm{~m}^{3}$ \\
\hline & Plutonium-238,239 & $746 \mathrm{~m}^{3}$ \\
\hline \multirow[t]{6}{*}{ 25-mile radius } & Gamma & $746 \mathrm{~m}^{3}$ \\
\hline & Gross alpha & $746 \mathrm{~m}^{3}$ \\
\hline & Gross beta & $746 \mathrm{~m}^{3}$ \\
\hline & Tritium & $3 m^{3}$ \\
\hline & Strontium-89,90 & $746 \mathrm{~m}^{3}$ \\
\hline & Plutonium-238,239 & $746 \mathrm{~m}^{3}$ \\
\hline \multirow[t]{6}{*}{ 100-mile radius } & Gamma & $746 \mathrm{~m}^{3}$ \\
\hline & Gross alpha & $746 \mathrm{~m}^{3}$ \\
\hline & Gross beta & $746 \mathrm{~m}^{3}$ \\
\hline & Tritium & $3 \mathrm{~m}^{3}$ \\
\hline & Strontium-89,90 & $746 \mathrm{~m}^{3}$ \\
\hline & Plutonium-238,239 & $746 \mathrm{~m}^{3}$ \\
\hline
\end{tabular}


Table 1

Sample Media Information

Page 2 of 4

Matrix

\section{Effluent Water}

Gamma

Gross alpha

Gross beta

Tritium

Strontium-89,90

Actinides

Stream Water

Gamma
Gross alpha
Gross beta
Tritium
Strontium-89,90
Actinides

River Water

Gamma
Gross alpha
Gross beta
Tritium

Strontium-89,90

Plutonium 238,239

Drinking Water

Gamma
Gross alpha
Gross beta
Tritium
Strontium-89,90
Actinides

Groundwater

$$
\text { Total activity }
$$

Wet/Dry Deposition (Rainwater)

$$
\begin{aligned}
& \text { Gamma } \\
& \text { Gross alpha }
\end{aligned}
$$

\section{Representative Aliquot}

$1 L$

$1 \mathrm{~L}$

$1 \mathrm{~L}$

$5 \mathrm{~mL}$

$1 \mathrm{~L}$

$1 \mathrm{~L}$

$1 \mathrm{~L}$

$1 \mathrm{~L}$

$1 \mathrm{~L}$

$5 \mathrm{~mL}$

$1 \mathrm{~L}$

$1 \mathrm{~L}$

5 L

$1 \mathrm{~L}$

$1 \mathrm{~L}$

$5 \mathrm{~mL}$

$1 \mathrm{~L}$

$1 \mathrm{~L}$

$1 \mathrm{~L}$

$1 \mathrm{~L}$

$1 \mathrm{~L}$

$5 \mathrm{~mL}$

$1 \mathrm{~L}$

$1 \mathrm{~L}$

$5 \mathrm{~mL}$

$0.37 \mathrm{~m}^{2}$

$0.093 \mathrm{~m}^{2}$ (1/4 sample) 
Table 1

Sample Media Information

Page 3 of 4

Matrix

Analytical Parameter

Representative Aliquot

Wet/Dry Deposition (Rainwater), cont.

Gross beta

$0.093 \mathrm{~m}^{2}$ (1/4 sample)

Tritium

Strontium-89,90

$5 \mathrm{~mL}$

Plutonium 238,239

$0.031 \mathrm{~m}^{2}$ (1/12 sample)

$0.031 \mathrm{~m}^{2}$ (1/12 sample)

Soil

$\begin{array}{ll}\text { Gamma } & 650 \mathrm{~g} \\ \text { Gross alpha } & 0.5 \mathrm{~g} \\ \text { Gross beta } & 0.5 \mathrm{~g} \\ \text { Strontium-89,90 } & 20 \mathrm{~g} \\ \text { Plutonium-238,239 } & 10 \mathrm{~g}\end{array}$

Sediment

$\begin{array}{ll}\text { Gamma } & 650 \mathrm{~g} \\ \text { Gross alpha } & 0.1 \mathrm{~g} \\ \text { Gross beta } & 0.1 \mathrm{~g} \\ \text { Strontium-89,90 } & 20 \mathrm{~g} \\ \text { Plutonium-238,239 } & 10 \mathrm{~g}\end{array}$

Vegetation

$\begin{array}{ll}\text { Gamma } & 200 \mathrm{~g} \\ \text { Gross alpha } & 0.5 \mathrm{~g} \\ \text { Gross beta } & 0.5 \mathrm{~g} \\ \text { Tritium } & 5 \mathrm{~mL} \\ \text { Strontium-89,90 } & 5 \mathrm{~g} \\ \text { Plutonium-238,239 } & 10 \mathrm{~g} \\ \text { Actinides } & 10 \mathrm{~g}\end{array}$

Fish

Edible

$\begin{array}{ll}\text { Gamma } & 200 \mathrm{~g} \\ \text { Gross alpha } & 1.5 \mathrm{~g} \\ \text { Gross beta } & 1.5 \mathrm{~g} \\ \text { Tritium } & 5 \mathrm{~mL} \\ \text { Strontium-89,90 } & 200 \mathrm{~g} \\ \text { Plutonium-238,239 } & 200 \mathrm{~g}\end{array}$


Table 1

Sample Media Information

Page 4 of 4

\begin{tabular}{|c|c|c|}
\hline Matrix & Analytical Parameter & Representative Aliquot \\
\hline \multicolumn{3}{|l|}{ Fish, cont. } \\
\hline \multirow[t]{4}{*}{ Nonedible } & Gamma & $200 \mathrm{~g}$ \\
\hline & Gross alpha & $0.5 \mathrm{~g}$ \\
\hline & Gross beta & $0.5 \mathrm{~g}$ \\
\hline & Strontium-89,90 & $25 \mathrm{~g}$ \\
\hline \multirow[t]{6}{*}{ Panfish } & Gamma & $200 \mathrm{~g}$ \\
\hline & Gross alpha & $1.5 \mathrm{~g}$ \\
\hline & Gross beta & $1.5 \mathrm{~g}$ \\
\hline & Tritium & $5 \mathrm{~mL}$ \\
\hline & Strontium-89,90 & $100 \mathrm{~g}$ \\
\hline & Plutonium-238,239 & $100 \mathrm{~g}$ \\
\hline \multicolumn{3}{|l|}{ Deer/Hogs } \\
\hline \multirow[t]{2}{*}{ Muscle } & Gamma & $200 \mathrm{~g}$ \\
\hline & Strontium-89,90 & $100 \mathrm{~g}$ \\
\hline Bone & Strontium-89,90 & $1 \mathrm{~g}$ \\
\hline \multicolumn{3}{|l|}{ Oysters/Crabs } \\
\hline & Gamma & $200 \mathrm{~g}$ \\
\hline & Gross alpha & $0.5 \mathrm{~g}$ \\
\hline & Gross beta & $0.5 \mathrm{~g}$ \\
\hline & Strontium-89,90 & $100 \mathrm{~g}$ \\
\hline \multicolumn{3}{|l|}{ Foods } \\
\hline & Gamma & $1000 \mathrm{~g}$ \\
\hline & Strontium- 89,90 & $20 \mathrm{~g}$ \\
\hline & Tritium & $5 \mathrm{~mL}$ \\
\hline & Plutonium-238,239 & $100 \mathrm{~g}$ \\
\hline \multicolumn{3}{|l|}{ Milk } \\
\hline & Gamma & $1 \mathrm{~L}$ \\
\hline & Tritium & $5 \mathrm{~mL}$ \\
\hline & Strontium-90 & $500 \mathrm{~mL}$ \\
\hline
\end{tabular}


Table 2

Representative Minimum Detectable Concentrations for Radiological Analyses

Page 1 of 6

Note: The minimum detectable concentrations (MDCs) are calculated at the 95-percent confidence level using instrument background measurements and typical detector efficiencies, decay times, and counting intervals. Chemical recoveries and air filter collection efficiencies are assumed to be 100 percent, except for $\mathrm{Pu}, U, \mathrm{Am}$, and $\mathrm{Cm}$ species for which typical chemical recoveries are used. Sample sizes are given in table 1, "Sample Media Information." The MDCs for actual samples may be different because of variations in the sample preparation, size, and content, and because of variations in the chemical recoveries, counting efficiencies, reagent blanks, decay time, counting time, and instrument backgrounds.

\begin{tabular}{|c|c|c|c|c|}
\hline & Onsite Air & Site Perimeter Air & 25-Mile Radius Air & Savannah Air \\
\hline Nuclide & $\mathrm{pCi} / \mathrm{m}^{3}$ & $\mathrm{pCi} / \mathrm{m}^{3}$ & $\mathrm{pCi} / \mathrm{m}^{3}$ & $\mathrm{pCi} / \mathrm{m}^{3}$ \\
\hline $\mathrm{H}-3$ & $1.50 \mathrm{E}+01$ & $1.50 \mathrm{E}+01$ & $1.50 \mathrm{E}+01$ & $1.50 \mathrm{E}+01$ \\
\hline $\mathrm{Be}-7$ & $5.63 \mathrm{E}-02$ & $5.76 \mathrm{E}-02$ & $5.70 E-02$ & $5.70 \mathrm{E}-02$ \\
\hline$K-40$ & $1.41 \mathrm{E}-01$ & $1.34 \mathrm{E}-01$ & $1.40 \mathrm{E}-01$ & $1.40 E-01$ \\
\hline Co-58 & $1.05 \mathrm{E}-02$ & $1.03 E-02$ & $1.00 \mathrm{E}-02$ & $1.00 \mathrm{E}-02$ \\
\hline Co-60 & $1.05 \mathrm{E}-02$ & 1.07E-02 & $1.10 \mathrm{E}-02$ & $1.10 E-02$ \\
\hline$R u-103$ & $1.01 \mathrm{E}-02$ & $1.02 E-02$ & $1.00 E-02$ & $1.00 \mathrm{E}-02$ \\
\hline Ru-106 & $8.85 E-02$ & 8.71E-02 & $8.80 E-02$ & $8.80 E-02$ \\
\hline $\mid-131$ & $1.65 \mathrm{E}-02$ & $1.61 E-02$ & $1.60 \mathrm{E}-02$ & $1.60 \mathrm{E}-02$ \\
\hline Cs-134 & $8.44 \mathrm{E}-03$ & $8.45 E-03$ & $8.40 E-03$ & $8.40 E-03$ \\
\hline Cs-137 & $8.04 E-03$ & 8.18E-03 & $8.20 E-03$ & $8.20 E-03$ \\
\hline $\mathrm{Ce}-141$ & $1.17 E-02$ & $1.15 E-02$ & $1.20 E-02$ & $1.20 E-02$ \\
\hline Ce-144 & $3.26 E-02$ & $3.22 E-02$ & $3.30 E-02$ & $3.30 \mathrm{E}-02$ \\
\hline Sr-89,90 & $1.85 E-03$ & $1.85 E-03$ & $1.85 \mathrm{E}-03$ & $1.85 \mathrm{E}-03$ \\
\hline Sr-90 & & & & \\
\hline \multicolumn{5}{|l|}{ U-234 } \\
\hline \multicolumn{5}{|l|}{$U-235$} \\
\hline \multicolumn{5}{|l|}{$U-238$} \\
\hline Pu-238 & $2.79 E-05$ & $2.79 E-05$ & $2.79 E-05$ & $2.79 E-05$ \\
\hline Pu-239 & $2.82 \mathrm{E}-05$ & $2.82 E-05$ & $2.82 E-05$ & $2.82 E-05$ \\
\hline \multicolumn{5}{|l|}{ Am-241 } \\
\hline \multicolumn{5}{|l|}{$\mathrm{Cm}-244$} \\
\hline Gross alpha & $1.10 E-03$ & $1.10 \mathrm{E}-03$ & $1.10 E-03$ & $1.10 E-03$ \\
\hline Gross beta & $1.90 \mathrm{E}-03$ & $1.90 \mathrm{E}-03$ & $1.90 \mathrm{E}-03$ & $1.90 \mathrm{E}-03$ \\
\hline
\end{tabular}


Table 2

Representative Minimum Detectable Concentrations for Radiological Analyses

Page 2 of 6

\begin{tabular}{|c|c|c|c|}
\hline & Rainwatera & $\begin{array}{c}\text { Stream/Effluent } \\
\text { Water }\end{array}$ & River Water \\
\hline Nuclide & $\mathrm{pCi} / \mathrm{m}^{2}$ & $\overline{p C i / L}$ & $\overline{\mathrm{pCi} / \mathrm{L}}$ \\
\hline $\mathrm{H}-3$ & $1.23 E+03$ & $1.20 \mathrm{E}+03$ & $4.40 \mathrm{E}+02$ \\
\hline $\mathrm{Be}-7$ & $1.70 E+02$ & $4.70 \mathrm{E}+01$ & $1.10 \mathrm{E}+01$ \\
\hline$K-40$ & $4.10 E+02$ & $1.30 \mathrm{E}+01$ & $2.50 \mathrm{E}+01$ \\
\hline Co-58 & $3.00 E+01$ & $7.40 \mathrm{E}+00$ & $1.20 E+00$ \\
\hline Co-60 & $3.20 \mathrm{E}+01$ & $9.10 E+00$ & $1.90 \mathrm{E}+00$ \\
\hline Ru-103 & $2.80 \mathrm{E}+01$ & $6.70 E+00$ & $1.30 E+00$ \\
\hline Ru-106 & $2.80 \mathrm{E}+02$ & $5.90 \mathrm{E}+01$ & $1.10 \mathrm{E}+01$ \\
\hline $\mid-131$ & $7.20 \mathrm{E}+01$ & $1.60 \mathrm{E}+01$ & $2.30 \mathrm{E}+00$ \\
\hline Cs-134 & $2.70 \mathrm{E}+01$ & $5.80 \mathrm{E}+00$ & $1.30 \mathrm{E}+00$ \\
\hline Cs-137 & $2.60 \mathrm{E}+01$ & $7.10 \mathrm{E}+00$ & $1.40 E+00$ \\
\hline $\mathrm{Ce}-141$ & $3.60 \mathrm{E}+01$ & $8.50 \mathrm{E}+00$ & $1.50 E+00$ \\
\hline $\mathrm{Ce}-144$ & $1.30 E+02$ & $2.90 E+01$ & $5.90 \mathrm{E}+00$ \\
\hline Sr-89,90 & $4.50 \mathrm{E}+01$ & $1.40 E+00^{b}$ & $1.40 \mathrm{E}+00$ \\
\hline \multicolumn{4}{|l|}{ Sr-90 } \\
\hline U-234 & & $1.80 \mathrm{E}-02$ & \\
\hline U-235 & & $1.00 E-02$ & \\
\hline U-238 & & $1.80 \mathrm{E}-02$ & \\
\hline Pu-238 & $6.40 \mathrm{E}-01$ & $8.56 E-03$ & $1.92 \mathrm{E}-02$ \\
\hline Pu-239 & $6.80 \mathrm{E}-01$ & $8.00 E-03$ & $2.10 \mathrm{E}-02$ \\
\hline$A m-241$ & & 8.87E-03 & \\
\hline $\mathrm{Cm}-244$ & & $7.80 E-03$ & \\
\hline Gross alpha & $8.60 \mathrm{E}+00$ & $8.00 E-01$ & $8.00 E-01$ \\
\hline Gross beta & $1.50 E+01$ & $1.40 \mathrm{E}+00$ & $1.40 E+00$ \\
\hline Total activity & & & \\
\hline
\end{tabular}

a Values, except for tritium, reflect wet/dry deposition.

b The $\mathrm{Sr}-89,90$ value is for a 1 -liter sample. 
Table 2

Representative Minimum Detectable Concentrations for Radiological Analyses

Page 3 of 6

\begin{tabular}{|c|c|c|c|c|}
\hline & Groundwater & Drinking Water & Soil/Sediment & Vegetation \\
\hline Nuclide & $\overline{p C i / L}$ & $\mathrm{pCi} / \mathrm{L}$ & $\mathrm{pCi} / \mathrm{g}$ & $\mathrm{pCi} / \mathrm{g}$ \\
\hline $\mathrm{H}-3$ & & $4.40 \mathrm{E}+02$ & & $1.80 \mathrm{E}-01$ \\
\hline $\mathrm{Be}-7$ & & $4.70 E+01$ & $3.40 \mathrm{E}-01$ & $4.20 \mathrm{E}-01$ \\
\hline$K-40$ & & $1.30 \mathrm{E}+01$ & $5.50 \mathrm{E}-01$ & $1.40 \mathrm{E}+00$ \\
\hline Co-58 & & $7.40 \mathrm{E}+00$ & $4.40 \mathrm{E}-02$ & $5.50 \mathrm{E}-02$ \\
\hline $\mathrm{Co}-60$ & & $9.10 E+00$ & $4.70 E-02$ & $5.70 E-02$ \\
\hline Ru-103 & & $6.70 E+00$ & $5.00 \mathrm{E}-02$ & $5.00 \mathrm{E}-02$ \\
\hline Ru-106 & & $5.90 E+01$ & $3.20 E-01$ & $4.50 E-01$ \\
\hline $1-131$ & & $1.60 \mathrm{E}+01$ & $8.00 \mathrm{E}-02$ & $8.80 E-02$ \\
\hline Cs-134 & & $5.80 \mathrm{E}+00$ & $3.80 \mathrm{E}-02$ & $4.40 E-02$ \\
\hline Cs-137 & & $7.10 \mathrm{E}+00$ & $4.20 \mathrm{E}-02$ & $4.90 E-02$ \\
\hline Ce-141 & & $8.50 \mathrm{E}+00$ & $7.80 \mathrm{E}-02$ & $8.40 E-02$ \\
\hline Ce-144 & & $2.90 E+01$ & $3.00 \mathrm{E}-01$ & $3.40 E-01$ \\
\hline Sr $-89,90$ & & $1.40 \mathrm{E}+00$ & $7.20 \mathrm{E}-02$ & $1.40 E-02$ \\
\hline \multicolumn{5}{|l|}{ Sr-90 } \\
\hline U-234 & & & & $2.60 \mathrm{E}-03$ \\
\hline U-235 & & & & $1.00 E-03$ \\
\hline U-238 & & & & $4.60 E-03$ \\
\hline Pu-238 & & & $1.96 \mathrm{E}-03$ & $2.00 E-03$ \\
\hline Pu-239 & & & $1.90 E-03$ & $2.10 E-03$ \\
\hline Am-241 & & & & $8.90 E-04$ \\
\hline $\mathrm{Cm}-244$ & & & & $7.80 E-04$ \\
\hline Gross alpha & & $8.00 \mathrm{E}-01$ & $8.00 \mathrm{E}+00$ & $1.60 E+00$ \\
\hline Gross beta & & $1.40 \mathrm{E}+00$ & $1.40 E+01$ & $2.90 E+00$ \\
\hline Total activity & $2.30 \mathrm{E}+03$ & & & \\
\hline
\end{tabular}


Table 2

Representative Minimum Detectable Concentrations for Radiological Analyses

Page 4 of 6

\begin{tabular}{|c|c|c|c|c|}
\hline & Highway 301 Fish & River Mouth Fish & Onsite Fish & Panfish \\
\hline Nuclide & $\mathrm{pCi} / \mathrm{g}$ & $\mathrm{pCi} / \mathrm{g}$ & $\mathrm{pCi} / \mathrm{g}$ & $\mathrm{pCi} / \mathrm{g}$ \\
\hline $\mathrm{H}-3$ & $5.72 E-02$ & $5.72 \mathrm{E}-02$ & $5.72 E-02$ & $5.72 \mathrm{E}-02$ \\
\hline $\mathrm{Be}-7$ & $7.20 E-01$ & $1.50 \mathrm{E}+00$ & $5.00 E-01$ & \\
\hline$K-40$ & $3.50 E-01$ & $9.30 \mathrm{E}-01$ & $6.70 E-01$ & \\
\hline Co-58 & $5.50 E-02$ & $1.10 E-01$ & $7.40 E-02$ & \\
\hline Co-60 & $2.40 E-02$ & $4.60 E-02$ & $5.20 E-02$ & \\
\hline Ru-103 & $1.40 \mathrm{E}-01$ & $3.00 E-01$ & $1.00 \mathrm{E}-01$ & \\
\hline$R u-106$ & $2.10 \mathrm{E}-01$ & $4.20 E-01$ & $4.90 E-01$ & \\
\hline $\mid-131$ & & & $6.80 E+00$ & \\
\hline Cs-134 & $2.20 E-02$ & $3.80 \mathrm{E}-02$ & $4.50 E-02$ & \\
\hline $\mathrm{Cs}-137$ & $2.10 E-02$ & $4.00 \mathrm{E}-02$ & $4.20 E-02$ & \\
\hline Ce-141 & $3.80 E-01$ & $7.10 E-01$ & $1.60 E-01$ & \\
\hline $\mathrm{Ce}-144$ & $1.70 \mathrm{E}-01$ & $3.70 \mathrm{E}-01$ & $2.30 \mathrm{E}-01$ & \\
\hline Sr-89,90 & & & $7.20 E-03$ & \\
\hline \multicolumn{5}{|l|}{ Sr-90 } \\
\hline \multicolumn{5}{|l|}{$U-234$} \\
\hline U-235 & & & & \\
\hline \multicolumn{5}{|l|}{ U-238 } \\
\hline Pu-238 & $1.00 \mathrm{E}-04$ & $1.00 \mathrm{E}-04$ & $1.00 E-04$ & $2.00 E-04$ \\
\hline Pu-239 & $1.00 \mathrm{E}-04$ & $1.00 \mathrm{E}-04$ & $1.00 E-04$ & 2.10 E-04 \\
\hline \multicolumn{5}{|l|}{ Am-241 } \\
\hline \multicolumn{5}{|l|}{$\mathrm{Cm}-244$} \\
\hline Gross alpha & & & $9.54 \mathrm{E}-01$ & $5.30 E-01$ \\
\hline Gross beta & & & $1.83 \mathrm{E}-01$ & $9.5 \mathrm{E}-01$ \\
\hline
\end{tabular}




\section{Table 2}

Representative Minimum Detectable Concentrations for Radiological Analyses

Page 5 of 6

\begin{tabular}{|c|c|c|c|c|}
\hline \multirow[b]{2}{*}{ Nuclide } & \multirow{2}{*}{$\begin{array}{c}\text { Nonedible Fish } \\
\mathrm{pCi} / \mathrm{g}\end{array}$} & \multirow{2}{*}{$\frac{\text { Oysters/Crabs }}{\mathrm{pCi/g}}$} & \multirow{2}{*}{$\frac{\text { Food Products }}{\text { pCi/g }}$} & \multirow{2}{*}{$\frac{\text { Milk }}{p C i / L}$} \\
\hline & & & & \\
\hline $\mathrm{H}-3$ & & & $1.80 \mathrm{E}-01$ & $4.40 \mathrm{E}+02$ \\
\hline $\mathrm{Be}-7$ & & $5.00 \mathrm{E}-01$ & $2.30 E-01$ & $1.80 E+01$ \\
\hline$K-40$ & & $6.70 \mathrm{E}-01$ & $1.20 \mathrm{E}-01$ & $4.30 E+01$ \\
\hline Co-58 & & $7.40 E-02$ & $2.10 E-02$ & $2.50 E+00$ \\
\hline Co-60 & & $5.20 E-02$ & 7.50E-03 & $2.80 E+00$ \\
\hline Ru-103 & & $1.00 \mathrm{E}-01$ & $5.00 \mathrm{E}-02$ & $2.40 E+00$ \\
\hline$R u-106$ & & $4.90 E-01$ & $7.20 E-02$ & $2.30 E+01$ \\
\hline 1.131 & & $6.80 E+00$ & & $2.90 E+00$ \\
\hline Cs-134 & & $4.50 E-02$ & $7.30 \mathrm{E}-03$ & $2.40 E+00$ \\
\hline Cs-137 & & $4.20 E-02$ & $7.20 \mathrm{E}-03$ & $2.70 E+00$ \\
\hline $\mathrm{Ce}-141$ & & $1.60 \mathrm{E}-01$ & $9.80 E-02$ & $2.80 E+00$ \\
\hline $\mathrm{Ce}-144$ & & $2.30 E-01$ & $4.00 \mathrm{E}-02$ & $1.20 \mathrm{E}+01$ \\
\hline Sr-89,90 & $5.60 \mathrm{E}-02$ & $1.40 E-02$ & $7.20 E-02$ & $3.95 E+00$ \\
\hline Sr-90 & & & & $5.80 E+00$ \\
\hline \multicolumn{5}{|l|}{ U-234 } \\
\hline \multicolumn{5}{|l|}{ U-235 } \\
\hline \multicolumn{5}{|l|}{ U-238 } \\
\hline Pu-238 & & & $2.00 \mathrm{E}-04$ & \\
\hline Pu-239 & & & $2.10 E-04$ & \\
\hline$A m-241$ & & & & \\
\hline \multicolumn{5}{|l|}{$\mathrm{Cm}-244$} \\
\hline Gross alpha & $1.60 \mathrm{E}+00$ & $1.60 \mathrm{E}+00$ & & $4.40 \mathrm{E}-01$ \\
\hline Gross beta & $2.90 \mathrm{E}+00$ & $2.90 E+00$ & & $2.90 E-03$ \\
\hline Total activity & & & & \\
\hline
\end{tabular}




\section{Table 2}

Representative Minimum Detectable Concentrations for Radiological Analyses

Page 6 of 6

\begin{tabular}{|c|c|c|}
\hline & Deer Muscle & Deer Bone \\
\hline Nuclide & $\mathrm{pCi} / \mathrm{g}$ & $\mathrm{pCi} / \mathrm{g}$ \\
\hline \multicolumn{3}{|l|}{$\mathrm{H}-3$} \\
\hline $\mathrm{Be}-7$ & $5.00 \mathrm{E}-01$ & \\
\hline$K-40$ & $6.70 \mathrm{E}-01$ & \\
\hline Co-58 & $7.40 \mathrm{E}-02$ & \\
\hline Co-60 & $5.20 \mathrm{E}-02$ & \\
\hline Ru-103 & $1.00 \mathrm{E}-01$ & \\
\hline Ru-106 & $4.90 \mathrm{E}-01$ & \\
\hline $\mid-131$ & $6.80 E+00$ & \\
\hline Cs-134 & $4.50 E-02$ & \\
\hline Cs-137 & $4.20 \mathrm{E}-02$ & \\
\hline Ce-141 & $1.60 E-01$ & \\
\hline Ce-144 & $2.30 \mathrm{E}-01$ & \\
\hline Sr-89,90 & $1.40 E-02$ & $1.40 \mathrm{E}+00$ \\
\hline \multicolumn{3}{|l|}{ Sr-90 } \\
\hline \multicolumn{3}{|l|}{ U-234 } \\
\hline \multicolumn{3}{|l|}{ U-235 } \\
\hline \multicolumn{3}{|l|}{ U-238 } \\
\hline \multicolumn{3}{|l|}{ Pu-238 } \\
\hline \multicolumn{3}{|l|}{ Pu-239 } \\
\hline \multicolumn{3}{|l|}{ Am-241 } \\
\hline \multicolumn{3}{|l|}{$\mathrm{Cm}-244$} \\
\hline \multicolumn{3}{|l|}{ Gross alpha } \\
\hline \multicolumn{3}{|l|}{ Gross beta } \\
\hline Total activity & & \\
\hline
\end{tabular}


Table 3

Nonradiological Environmental Surveillance Detection/Report Limits

Page 1 of 2

Parameter

\begin{tabular}{|c|c|c|c|}
\hline \multicolumn{4}{|l|}{ Field Measurements } \\
\hline Temperature & ${ }^{\circ} \mathrm{C}$ & NA & 0.1 \\
\hline $\mathrm{pH}$ & SU & NA & 0.1 \\
\hline Dissolved oxygen (DO) & $\mathrm{mg} / \mathrm{L}$ & 1.0 & 0.1 \\
\hline \multicolumn{4}{|l|}{ General Water Quality Parameters } \\
\hline Conductivity & $\mu \mathrm{mhos} / \mathrm{cm}$ & NA & 1 \\
\hline Chemical Oxygen Demand (COD) & $\mathrm{mg} / \mathrm{L}$ & 20 & 1 \\
\hline Mercury in Fish Flesh & $\mu g / g$ & 0.008 & 0.01 \\
\hline Total Suspended Solids (TSS) & $\mathrm{mg} / \mathrm{L}$ & 3 & 1 \\
\hline Nitrogen-Nitrite $\left(\mathrm{NO}_{3}-\mathrm{N}\right)$ & $\mathrm{mg} / \mathrm{L}$ & 0.05 & 0.01 \\
\hline Nitrogen-Nitrate $\left(\mathrm{NO}_{2}-\mathrm{N}\right)$ & $\mathrm{mg} / \mathrm{L}$ & 0.02 & 0.01 \\
\hline Total Phosphorus & $\mathrm{mg} / \mathrm{L}$ & 0.01 & 0.01 \\
\hline Total Organic Carbon (TOC) & $\mathrm{mg} / \mathrm{L}$ & 1.0 & 0.1 \\
\hline Total Oil and Grease & $\mathrm{mg} / \mathrm{L}$ & 1.0 & 0.1 \\
\hline Biological Oxygen Demand (BOD) & $\mathrm{mg} / \mathrm{L}$ & 2.0 & 1 \\
\hline \multicolumn{4}{|l|}{ Metals } \\
\hline Aluminum (Al) & $\mathrm{mg} / \mathrm{L}$ & 0.05 & 0.01 \\
\hline Cadmium (Cd) & $\mathrm{mg} / \mathrm{L}$ & 0.002 & 0.0001 \\
\hline Calcium (Ca) & $\mathrm{mg} / \mathrm{L}$ & 0.100 & 0.1 \\
\hline Chromium (Cr) & $\mathrm{mg} / \mathrm{L}$ & 0.005 & 0.0001 \\
\hline Copper $(\mathrm{Cu})$ & $\mathrm{mg} / \mathrm{L}$ & 0.005 & 0.0001 \\
\hline Iron (Fe) & $\mathrm{mg} / \mathrm{L}$ & 0.020 & 0.1 \\
\hline Lead $(\mathrm{Pb})$ & $\mathrm{mg} / \mathrm{L}$ & 0.003 & 0.0001 \\
\hline Magnesium (Mg) & $\mathrm{mg} / \mathrm{L}$ & 0.050 & 0.1 \\
\hline Manganese (Mn) & $\mathrm{mg} / \mathrm{L}$ & 0.005 & 0.01 \\
\hline Mercury $(\mathrm{Hg})$ & $\mathrm{mg} / \mathrm{L}$ & 0.0001 & 0.00001 \\
\hline Nickel (Ni) & $\mathrm{mg} / \mathrm{L}$ & 0.010 & 0.001 \\
\hline Sodium (Na) & $\mathrm{mg} / \mathrm{L}$ & 0.100 & 1 \\
\hline $\operatorname{Zinc}(Z n)$ & $\mathrm{mg} / \mathrm{L}$ & 0.005 & 0.001 \\
\hline \multicolumn{4}{|l|}{ Pesticides } \\
\hline Aldrin & $\mu \mathrm{g} / \mathrm{L}$ & 0.03 & 0.01 \\
\hline Alpha-BHC & $\mu \mathrm{g} / \mathrm{L}$ & 0.03 & 0.01 \\
\hline
\end{tabular}




\section{Table 3}

Nonradiological Environmental Surveillance Detection/Report Limits

Page 2 of 2

\begin{tabular}{|c|c|c|c|}
\hline Parameter & Units & Quantitation Value & $\begin{array}{l}\text { Report Value } \\
\text { Round to the nearest }\end{array}$ \\
\hline \multicolumn{4}{|l|}{ Pesticides (cont.) } \\
\hline Beta-BHC & $\mu \mathrm{g} / \mathrm{L}$ & 0.03 & 0.01 \\
\hline Delta-BHC & $\mu g / L$ & 0.03 & 0.01 \\
\hline Lindane & $\mu g / L$ & 0.03 & 0.01 \\
\hline Chlordane & $\mu \mathrm{g} / \mathrm{L}$ & 0.06 & 0.01 \\
\hline 4,4-DDD & $\mu g / L$ & 0.03 & 0.01 \\
\hline 4,4-DDE & $\mu g / L$ & 0.03 & 0.01 \\
\hline 4,4-DDT & $\mu g / L$ & 0.03 & 0.01 \\
\hline Dieldrin & $\mu g / L$ & 0.03 & 0.01 \\
\hline Endosulfan I & $\mu g / L$ & 0.03 & 0.01 \\
\hline Endosulfan II & $\mu \mathrm{g} / \mathrm{L}$ & 0.03 & 0.01 \\
\hline Endosulfan sulfate & $\mu \mathrm{g} / \mathrm{L}$ & 0.03 & 0.01 \\
\hline Endrin & $\mu g / L$ & 0.03 & 0.01 \\
\hline Endrin aldehyde & $\mu g / L$ & 0.03 & 0.01 \\
\hline Endrin ketone & $\mu g / L$ & 0.05 & 0.01 \\
\hline Heptachlor & $\mu \mathrm{g} / \mathrm{L}$ & 0.03 & 0.01 \\
\hline Heptachlor epoxide & $\mu g / L$ & 0.03 & 0.01 \\
\hline Methoxyclor & $\mu g / L$ & 0.12 & 0.01 \\
\hline Toxaphene & $\mu \mathrm{g} / \mathrm{L}$ & 0.30 & 0.01 \\
\hline \multicolumn{4}{|l|}{ Herbicides } \\
\hline $2,4-D$ & $\mu g / L$ & 2.4 & 0.1 \\
\hline 2,4,5-TP (Silvex) & $\mu \mathrm{g} / \mathrm{L}$ & 0.61 & 0.01 \\
\hline \multicolumn{4}{|c|}{ Volatile Organic Compounds } \\
\hline Cis-1,2-Dichloroethene & $\mu g / L$ & 2.0 & 0.1 \\
\hline Tetrachloroethene & $\mu g / L$ & 2.0 & 0.1 \\
\hline 1,1,1-Trichloroethane & $\mu g / L$ & 2.0 & 0.1 \\
\hline Trichloroethene & $\mu g / L$ & 2.0 & 0.1 \\
\hline Vinyl Chloride & $\mu \mathrm{g} / \mathrm{L}$ & 2.0 & 0.1 \\
\hline
\end{tabular}


Table 4

Radioactive Atmospheric Releases by Source

Page 1 of 3

\begin{tabular}{|c|c|c|c|c|c|c|c|}
\hline \multirow[b]{2}{*}{$\begin{array}{l}\text { Radio- } \\
\text { nuclide }\end{array}$} & \multicolumn{7}{|c|}{ Curies ${ }^{a}$} \\
\hline & Reactors & $\begin{array}{c}\text { Separa- } \\
\text { tions }\end{array}$ & $\begin{array}{c}\text { Reactor } \\
\text { Materials }\end{array}$ & $\begin{array}{l}\text { Heavy } \\
\text { Water }\end{array}$ & SRTCC & $\begin{array}{c}\text { Diffuse and } \\
\text { Fugitive }^{d}\end{array}$ & Total \\
\hline \multicolumn{8}{|c|}{ Note: Blank spaces indicate no quantifiable activity. } \\
\hline \multicolumn{8}{|c|}{ GASES AND VAPORS } \\
\hline H-3(oxide) & $3.04 E+03$ & $3.02 E+04$ & & $2.31 E+02$ & & 4.71E+02 & $3.39 E+04$ \\
\hline $\mathrm{H}-3$ (elem.) & & $1.77 \mathrm{E}+04$ & & & & & $1.77 E+04$ \\
\hline H-3 Total & $3.04 \mathrm{E}+03$ & $4.79 \mathrm{E}+04$ & & $2.31 E+02$ & & $4.71 \mathrm{E}+02$ & $5.16 \mathrm{E}+04$ \\
\hline C-14 & & $2.50 \mathrm{E}-02$ & & & & $4.92 \mathrm{E}-04$ & $2.55 E-02$ \\
\hline $\mathrm{Kr}-85$ & & $3.74 \mathrm{E}+04$ & & & & & $3.74 \mathrm{E}+04$ \\
\hline $\mathrm{Xe}-135$ & & $1.94 \mathrm{E}-02$ & & & & & $1.94 \mathrm{E}-02$ \\
\hline $1-129$ & & 4.77E-03 & & & & $2.50 \mathrm{E}-03$ & 7.27E-03 \\
\hline $\mid-131$ & & & & & $1.01 E-05$ & & $1.01 E-05$ \\
\hline $1-133$ & & & & & $1.25 E-04$ & & $1.25 \mathrm{E}-04$ \\
\hline \multicolumn{8}{|c|}{ PARTICULATES } \\
\hline Cr-51 & & & & & & $1.21 \mathrm{E}-04$ & $1.21 E-04$ \\
\hline Co-57 & & $4.69 E-08$ & & & & $2.01 E-10$ & $4.71 E-08$ \\
\hline Co-58 & & & & & & $1.27 \mathrm{E}-04$ & $1.27 \mathrm{E}-04$ \\
\hline $\mathrm{Co}-60$ & & $1.00 E-06$ & & $1.18 \mathrm{E}-06$ & & $1.28 \mathrm{E}-04$ & $1.30 E-04$ \\
\hline NI-59 & & & & & & $1.02 \mathrm{E}-09$ & $1.02 E-09$ \\
\hline NI-63 & & & & & & $5.89 E-07$ & $5.89 \mathrm{E}-07$ \\
\hline $\mathrm{Zn}-65$ & & & & & & $2.23 E-05$ & $2.23 E-05$ \\
\hline Sr-89,90 & & 3.11E-04 & & & & $7.02 E-04$ & $1.01 E-03$ \\
\hline Zr-95 & & & & & & $1.71 E-05$ & $1.71 E-05$ \\
\hline $\mathrm{Nb}-94$ & & & & & & $3.95 \mathrm{E}-10$ & $3.95 E-10$ \\
\hline $\mathrm{Nb}-95$ & & & & & & 1.13E-04 & 1.13E-04 \\
\hline Tc-99 & & & & & & $6.22 \mathrm{E}-05$ & $6.22 E-05$ \\
\hline$R u-103$ & & & & & & 4.23E-05 & 4.23E-05 \\
\hline$S b-124$ & & & & & & $2.23 E-10$ & $2.23 E-10$ \\
\hline Sb-125 & & & & & & 5.27E-0. & $5.27 \mathrm{E}-05$ \\
\hline Sn-126 & & & & & & $3.13 E-15$ & $3.13 E-15$ \\
\hline Cs-134 & & $5.72 E-08$ & & & & $1.31 E-04$ & $1.31 E-04$ \\
\hline Cs-137 & $2.32 E-05$ & $8.41 E-03$ & $3.36 E-07$ & & & $6.11 E-03$ & $1.45 E-02$ \\
\hline
\end{tabular}

One curie equals $3.7 \mathrm{E}+10$ Becquerels.

Includes separations, waste management, and tritium facilities

Savannah River Technology Center

Estimated releases from minor unmonitored diffuse and fugitive sources 
Table 4

Radioactive Atmospheric Releases by Source

Page 2 of 3

\begin{tabular}{|c|c|c|c|c|c|c|c|}
\hline \multirow[b]{2}{*}{$\begin{array}{l}\text { Radio- } \\
\text { nuclide }\end{array}$} & \multicolumn{7}{|c|}{ Curies $^{a}$} \\
\hline & Reactors & $\begin{array}{c}\text { Separa- } \\
\text { tions }^{b}\end{array}$ & $\begin{array}{l}\text { Reactor } \\
\text { Materials }\end{array}$ & $\begin{array}{l}\text { Heavy } \\
\text { Water }\end{array}$ & SRTCC & $\begin{array}{l}\text { Diffuse and } \\
\text { Fugitive }^{d}\end{array}$ & Total \\
\hline $\mathrm{Ce}-141$ & & & & & & $4.16 \mathrm{E}-05$ & $4.16 \mathrm{E}-05$ \\
\hline Ce-144 & & & & & & $1.45 \mathrm{E}-04$ & $1.45 E-04$ \\
\hline Pr-144 & & & & & & $3.45 \mathrm{E}-09$ & $3.45 \mathrm{E}-09$ \\
\hline Pm-147 & & & & & & $3.49 \mathrm{E}-09$ & $3.49 \mathrm{E}-09$ \\
\hline Eu-152 & & & & & & $1.21 \mathrm{E}-10$ & $1.21 \mathrm{E}-10$ \\
\hline Eu-154 & & & & & & $5.74 \mathrm{E}-06$ & $5.74 \mathrm{E}-06$ \\
\hline Eu-155 & & & & & & $1.10 \mathrm{E}-06$ & $1.10 E-06$ \\
\hline $\mathrm{Hg}-203$ & & & & & & $2.23 E-10$ & $2.23 E-10$ \\
\hline $\mathrm{Pb}-214$ & & & & & & $2.23 E-10$ & $2.23 \mathrm{E}-10$ \\
\hline Ra-226 & & & & & & $1.25 E-05$ & $1.25 E-05$ \\
\hline Ra-228 & & & & & & $1.87 E-05$ & $1.87 \mathrm{E}-05$ \\
\hline$A C-228$ & & & & & & $1.66 \mathrm{E}-06$ & $1.66 \mathrm{E}-06$ \\
\hline Th-228 & & & & & & $2.75 E-07$ & $2.75 E-07$ \\
\hline Th-230 & & & & & & $1.22 E-05$ & $1.22 E-05$ \\
\hline Th-232 & & & & & & $1.64 \mathrm{E}-06$ & $1.64 \mathrm{E}-06$ \\
\hline Th-234 & & & & & & 4.10E-06 & $4.10 E-06$ \\
\hline $\mathrm{Pa}-233$ & & & & & & $2.23 \mathrm{E}-10$ & $2.23 E-10$ \\
\hline U-232 & & & $1.33 E-08$ & & & & $1.33 E-08$ \\
\hline U-233 & & & & & & $2.38 \mathrm{E}-06$ & $2.38 \mathrm{E}-06$ \\
\hline U-234 & & $2.02 E-05$ & $1.41 E-05$ & & & $5.29 \mathrm{E}-05$ & $8.72 E-05$ \\
\hline$U-235$ & & $1.34 \mathrm{E}-06$ & $2.68 \mathrm{E}-06$ & & & $5.89 E-06$ & $9.91 E-06$ \\
\hline U-236 & & & & & & $5.20 \mathrm{E}-09$ & $5.20 \mathrm{E}-09$ \\
\hline U-238 & & $3.61 \mathrm{E}-05$ & $1.07 E-05$ & & & $9.49 E-05$ & $1.42 E-04$ \\
\hline $\mathrm{Np}-237$ & & & & & & $2.23 \mathrm{E}-10$ & $2.23 E-10$ \\
\hline Np-239 & & & & & & 4.51E-09 & $4.51 E-09$ \\
\hline Pu-238 & & $5.27 \mathrm{E}-04$ & $7.16 \mathrm{E}-09$ & & & $1.45 E-03$ & $1.98 E-03$ \\
\hline Pu-239 & & $1.34 \mathrm{E}-04$ & $2.39 E-08$ & & & $1.68 \mathrm{E}-05$ & $1.51 E-04$ \\
\hline Pu-240 & & & & & & $1.46 E-06$ & $1.46 E-06$ \\
\hline Pu-241 & & & & & & $6.47 E-05$ & $6.47 E-05$ \\
\hline Pu-242 & & & & & & $1.53 E-08$ & $1.53 \mathrm{E}-08$ \\
\hline
\end{tabular}

a One curie equals $3.7 E+10$ Becquerels.

b Includes separations, waste management, and tritium facilities

c Savannah River Technology Center

d Estimated releases from minor unmonitored diffuse and fugitive sources 


\section{Table 4}

\section{Radioactive Atmospheric Releases by Source}

Page 3 of 3

\begin{tabular}{|c|c|c|c|c|c|c|c|}
\hline \multirow[b]{2}{*}{$\begin{array}{l}\text { Radio- } \\
\text { nuclide }\end{array}$} & \multicolumn{7}{|c|}{ Curies $^{a}$} \\
\hline & Reactors & $\begin{array}{l}\text { Separa- } \\
\text { tions }^{b}\end{array}$ & $\begin{array}{c}\text { Reactor } \\
\text { Materials }\end{array}$ & $\begin{array}{l}\text { Heavy } \\
\text { Water }\end{array}$ & SRTCC & $\begin{array}{c}\text { Diffuse and } \\
\text { Fugitive }^{\mathrm{d}}\end{array}$ & Total \\
\hline Am-241 & & $3.01 \mathrm{E}-05$ & $1.46 E-08$ & & & $8.44 \mathrm{E}-06$ & $3.86 \mathrm{E}-05$ \\
\hline Am-243 & & & & & & $4.28 \mathrm{E}-06$ & $4.28 E-06$ \\
\hline $\mathrm{Cm}-242$ & & & & & & $3.10 \mathrm{E}-07$ & $3.10 \mathrm{E}-07$ \\
\hline $\mathrm{Cm}-244$ & & $2.59 \mathrm{E}-05$ & $1.69 E-08$ & & & $6.74 E-06$ & $3.27 E-05$ \\
\hline $\mathrm{Cm}-246$ & & & & & & 2.91E-06 & $2.91 E-06$ \\
\hline Alpha & $5.09 \mathrm{E}-04$ & $4.46 \mathrm{E}-05$ & $7.23 E-05$ & $1.05 \mathrm{E}-05$ & $1.75 \mathrm{E}-06$ & $1.47 E-03$ & $2.11 E-03$ \\
\hline $\begin{array}{l}\text { Nonvolatile } \\
\text { Beta }\end{array}$ & $1.19 \mathrm{E}-03$ & $3.27 \mathrm{E}-04$ & $1.84 \mathrm{E}-03$ & $1.23 E-04$ & & $2.74 \mathrm{E}-02$ & $3.09 E-02$ \\
\hline
\end{tabular}

a One curie equals $3.7 E+10$ Becquerels.

b Includes separations, waste management, and tritium facilities

c Savannah River Technology Center

d Estimated releases from minor unmonitored diffuse and fugitive sources 


\section{Table 5}

\section{Radioactive Atmospheric Releases by Stack/Facility and Comparison of Annual Average Concentrations to DOE Derived Concentration Guides}

Page 1 of 6

\begin{tabular}{|c|c|c|c|c|c|}
\hline Stack/Facility & Radionuclide & $\begin{array}{c}\text { Quantity } \\
\text { Released } \\
\text { During } 1999 \\
(\mu \mathrm{Ci})\end{array}$ & $\begin{array}{l}\text { Atmospheric } \\
\text { Release } \\
\text { Volume }(\mathrm{mL})\end{array}$ & $\begin{array}{c}\text { Average } \\
\text { Effluent } \\
\text { Concentration } \\
\text { During 1999 } \\
(\mu \mathrm{Ci} / \mathrm{mL})\end{array}$ & $\begin{array}{c}\text { DOE DCGsa } \\
(\mu \mathrm{Ci} / \mathrm{mL})\end{array}$ \\
\hline Note: $\quad$ Blank spaces i & dicate no quantif & ble activity. & & & \\
\hline \multicolumn{6}{|c|}{ A-Area (Savannah River Technology Center) } \\
\hline \multirow{2}{*}{$\begin{array}{l}\text { 794-A Sandfilter } \\
\text { Discharge }\end{array}$} & $1-131$ & $1.01 \mathrm{E}+01$ & $9.54 \mathrm{E}+14$ & $3.45 \mathrm{E}-14$ & $4.00 \mathrm{E}-10$ \\
\hline & $1-133$ & $1.25 \mathrm{E}+02$ & $9.54 \mathrm{E}+14$ & $2.27 E-12$ & $2.00 E-09$ \\
\hline \multicolumn{6}{|l|}{ C-Area (C-Reactor) } \\
\hline $728-N$ Stack & Cs-137 & $4.96 \mathrm{E}-01$ & $7.09 E+13$ & $9.67 \mathrm{E}-15$ & $4.00 E-10$ \\
\hline $\begin{array}{l}\text { C-Area Main Stack } \\
\left(148^{\prime}\right)\end{array}$ & $H-3$ (oxide) & $2.63 E+08$ & $9.94 \mathrm{E}+14$ & 2.64E-07 & $1.00 \mathrm{E}-07$ \\
\hline \multicolumn{6}{|c|}{ D-Area (Heavy Water Rework) } \\
\hline 420-D Stack & $\mathrm{H}-3$ (oxide) & $2.00 E+08$ & $1.47 \mathrm{E}+14$ & $1.36 \mathrm{E}-06$ & $1.00 E-07$ \\
\hline \multirow[t]{2}{*}{ 421-2D Stack } & $\mathrm{H}-3$ (oxide) & $3.05 E+07$ & $1.11 E+14$ & $2.75 \mathrm{E}-07$ & $1.00 E-07$ \\
\hline & Co-60 & $1.18 \mathrm{E}+00$ & $1.11 \mathrm{E}+14$ & 7.01E-14 & $8.00 E-11$ \\
\hline 772-D Stack & $H-3$ (oxide) & $8.53 E+06$ & $1.53 \mathrm{E}+14$ & $5.58 \mathrm{E}-08$ & $1.00 \mathrm{E}-07$ \\
\hline \multicolumn{6}{|c|}{ F-Area (Separations and Waste Management) } \\
\hline \multirow{5}{*}{$\begin{array}{l}\text { 235-F Sandfilter } \\
\text { Discharge }\end{array}$} & $U-234$ & $5.40 \mathrm{E}-01$ & $5.64 \mathrm{E}+14$ & $9.90 \mathrm{E}-16$ & $9.00 \mathrm{E}-14$ \\
\hline & U-235 & $1.02 E-02$ & $5.64 \mathrm{E}+14$ & $4.08 E-18$ & $1.00 E-13$ \\
\hline & U-238 & $6.46 \mathrm{E}-01$ & $5.64 \mathrm{E}+14$ & $1.15 \mathrm{E}-15$ & $1.00 E-13$ \\
\hline & Pu-239 & $3.39 E-03$ & $5.64 \mathrm{E}+14$ & $1.99 \mathrm{E}-17$ & $2.00 E-14$ \\
\hline & Am-241 & $1.58 \mathrm{E}-02$ & $5.64 \mathrm{E}+14$ & $-2.71 E-17$ & $2.00 E-14$ \\
\hline $\begin{array}{l}\text { 241-F Miscellaneous } \\
\text { Exhaust }\end{array}$ & Cs-137 & $1.11 E+00$ & $2.23 E+13$ & $5.38 \mathrm{E}-14$ & $4.00 E-10$ \\
\hline 241-F Purge Exhaust & Cs-137 & $3.61 \mathrm{E}+00$ & $4.91 E+13$ & $8.30 E-14$ & $4.00 E-10$ \\
\hline $\begin{array}{l}\text { 241-F Tank Annulus } \\
\text { Exhaust }\end{array}$ & Cs-137 & $5.10 E+00$ & $2.82 E+14$ & $2.79 E-14$ & $4.00 \mathrm{E}-10$ \\
\hline \multirow[t]{4}{*}{ 291-F Stack Isokinetic } & Sr-89,90 & $1.59 \mathrm{E}+02$ & $3.19 \mathrm{E}+15$ & $5.69 E-14$ & $9.00 \mathrm{E}-12$ \\
\hline & $\mid-131$ & $2.11 E+01$ & $3.19 \mathrm{E}+15$ & $6.75 E-14$ & $4.00 E-10$ \\
\hline & $X e-135$ & $1.94 \mathrm{E}+04$ & $3.19 E+15$ & $1.24 \mathrm{E}-10$ & $8.00 E-08$ \\
\hline & Cs- 137 & $3.20 E+01$ & $3.19 E+15$ & $2.22 E-14$ & $4.00 \mathrm{E}-10$ \\
\hline
\end{tabular}

a Source: DOE Order 5400.5. In cases where different chemical forms have different DCGs, the lowest DCG for the radionuclide is given. These DCGs are defined as the air concentration of that radionuclide that will give a 50-year committed effective dose equivalent of 100 mrem under conditions of continuous exposure for one year. DCGs are reference values only and are not considered release limits or standards. 
Table 5

Radioactive Atmospheric Releases by Stack/Facility and Comparison of Annual Average Concentrations to DOE Derived Concentration Guides

Page 2 of 6

\begin{tabular}{|c|c|c|c|c|c|}
\hline Stack/Facility & Radionuclide & $\begin{array}{c}\text { Quantity } \\
\text { Released } \\
\text { During } 1999 \\
(\mu \mathrm{Ci})\end{array}$ & $\begin{array}{l}\text { Atmospheric } \\
\text { Release } \\
\text { Volume (mL) }\end{array}$ & $\begin{array}{c}\text { Average } \\
\text { Effluent } \\
\text { Concentration } \\
\text { During } 1999 \\
(\mu \mathrm{Cl} / \mathrm{mL})\end{array}$ & $\begin{array}{l}\text { DOE DCGs } \\
(\mu \mathrm{Ci} / \mathrm{mL})\end{array}$ \\
\hline & U-234 & $1.05 \mathrm{E}+01$ & $3.19 \mathrm{E}+15$ & $3.41 \mathrm{E}-15$ & $9.00 E-14$ \\
\hline & U-235 & $1.11 E+00$ & $3.19 E+15$ & $3.82 E-16$ & $1.00 \mathrm{E}-13$ \\
\hline & U-238 & $2.74 \mathrm{E}+01$ & $3.19 E+15$ & $8.60 E-15$ & $1.00 \mathrm{E}-13$ \\
\hline & Pu-238 & $3.89 E+00$ & $3.19 E+15$ & $1.25 \mathrm{E}-15$ & $3.00 E-14$ \\
\hline & Pu-239 & $9.58 \mathrm{E}+01$ & $3.19 E+15$ & $3.03 E-14$ & $2.00 E-14$ \\
\hline & Am-241 & $9.28 \mathrm{E}+00$ & $3.19 E+15$ & $2.96 \mathrm{E}-15$ & $2.00 E-14$ \\
\hline & $\mathrm{Cm}-244$ & $1.87 E+00$ & $3.19 E+15$ & $6.08 E-16$ & $4.00 \mathrm{E}-14$ \\
\hline \multirow[t]{2}{*}{ 6.1 Dissolver Stack } & Cs-137 & $7.24 \mathrm{E}-01$ & $2.15 E+12$ & $2.48 E-13$ & $4.00 \mathrm{E}-10$ \\
\hline & Am-241 & 2.97E-01 & $2.15 E+12$ & $9.75 E-13$ & $2.00 E-14$ \\
\hline \multirow[t]{5}{*}{ 6.4 Dissolver Stack } & Cs- 137 & $3.34 \mathrm{E}-01$ & $2.14 \mathrm{E}+12$ & $2.14 E-13$ & $4.00 E-10$ \\
\hline & $\mathrm{Pu}-238$ & $3.26 \mathrm{E}-01$ & $2.14 \mathrm{E}+12$ & $5.69 E-12$ & $3.00 E-14$ \\
\hline & $\mathrm{Pu}-239$ & $1.07 E+01$ & $2.14 \mathrm{E}+12$ & $1.87 E-10$ & $2.00 E-14$ \\
\hline & Am-241 & $1.59 E+01$ & $2.14 \mathrm{E}+12$ & $1.12 E-11$ & $2.00 E-14$ \\
\hline & $\mathrm{Cm}-244$ & $3.53 E-04$ & $2.14 \mathrm{E}+12$ & $6.17 \mathrm{E}-15$ & $4.00 \mathrm{E}-14$ \\
\hline \multirow[t]{6}{*}{ 772-4F Stack } & $U-234$ & $1.44 E-01$ & $2.32 E+15$ & $7.20 \mathrm{E}-17$ & $9.00 E-14$ \\
\hline & $U-238$ & $1.72 E-01$ & $2.32 E+15$ & $8.92 E-17$ & $1.00 E-13$ \\
\hline & Pu-238 & $3.02 \mathrm{E}-02$ & $2.32 E+15$ & $1.11 E-17$ & $3.00 E-14$ \\
\hline & Pu-239 & 3.17E-03 & $2.32 \mathrm{E}+15$ & $6.56 \mathrm{E}-17$ & $2.00 E-14$ \\
\hline & $A m-241$ & $1.11 \mathrm{E}-02$ & $2.32 E+15$ & $-4.00 E-18$ & $2.00 E-14$ \\
\hline & $\mathrm{Cm}-244$ & $4.81 E-03$ & $2.32 E+15$ & $-6.74 E-18$ & $4.00 E-14$ \\
\hline \multirow{2}{*}{$\begin{array}{l}800 \text { Cell Stack Exhaust } \\
(211-F)\end{array}$} & Sr-89,90 & $4.62 \mathrm{E}+00$ & $8.91 E+13$ & $1.47 \mathrm{E}-13$ & $9.00 E-12$ \\
\hline & Cs-137 & 8.37E-01 & $8.91 E+13$ & $4.50 E-14$ & $4.00 E-10$ \\
\hline \multirow[t]{5}{*}{ A Line Stack $(221-1 F)$} & U-234 & $1.23 E+00$ & $3.56 \mathrm{E}+13$ & $3.45 E-14$ & $9.00 E-14$ \\
\hline & U-235 & $7.21 E-02$ & $3.56 \mathrm{E}+13$ & $2.39 E-15$ & $1.00 E-13$ \\
\hline & U-238 & $1.72 E+00$ & $3.56 \mathrm{E}+13$ & $4.84 E-14$ & $1.00 \mathrm{E}-13$ \\
\hline & Pu-238 & $4.23 E-02$ & $3.56 E+13$ & $1.40 \mathrm{E}-15$ & $3.00 \mathrm{E}-14$ \\
\hline & Pu-239 & $5.30 E-01$ & $3.56 \mathrm{E}+13$ & $1.52 E-14$ & $2.00 E-14$ \\
\hline
\end{tabular}

a Source: DOE Order 5400.5. In cases where different chemical forms have different DCGs, the lowest DCG for the radionuclide is given. These DCGs are defined as the air concentration of that radionuclide that will give a 50 -year committed effective dose equivalent of 100 mrem under conditions of continuous exposure for one year. DCGs are reference values only and are not considered release limits or standards. 
Table 5

Radioactive Atmospheric Releases by Stack/Facility and Comparison of Annual Average Concentrations to DOE Derived Concentration Guides

Page 3 of 6

\begin{tabular}{|c|c|c|c|c|c|}
\hline Stack/Facility & Radionuclide & $\begin{array}{c}\text { Quantity } \\
\text { Released } \\
\text { During } 1999 \\
(\mu \mathrm{Ci})\end{array}$ & $\begin{array}{c}\text { Atmospheric } \\
\text { Release } \\
\text { Volume (mL) }\end{array}$ & $\begin{array}{c}\text { Average } \\
\text { Effluent } \\
\text { Concentration } \\
\text { During } 1999 \\
(\mu \mathrm{Ci} / \mathrm{mL})\end{array}$ & $\begin{array}{l}\text { DOE DCGsa } \\
(\mu \mathrm{Ci} / \mathrm{mL})\end{array}$ \\
\hline & Am-241 & $9.73 E-02$ & $3.56 \mathrm{E}+13$ & $3.12 E-15$ & $2.00 E-14$ \\
\hline & $\mathrm{Cm}-244$ & $1.16 \mathrm{E}-01$ & $3.56 E+13$ & $3.50 E-15$ & $4.00 E-14$ \\
\hline \multicolumn{6}{|c|}{ H-Area (Separations and Waste Management) } \\
\hline $\begin{array}{l}\text { 241-H Miscellaneous } \\
\text { Exhaust }\end{array}$ & Cs-137 & $1.29 \mathrm{E}+01$ & $3.02 E+14$ & $4.61 \mathrm{E}-14$ & $4.00 \mathrm{E}-10$ \\
\hline \multirow[t]{2}{*}{ 241-H Purge Exhaust } & Cs-134 & $5.72 \mathrm{E}-02$ & $1.06 \mathrm{E}+14$ & $4.26 \mathrm{E}-14$ & $2.00 E-10$ \\
\hline & Cs-137 & $3.23 E+01$ & $1.06 E+14$ & $3.03 E-13$ & $4.00 E-10$ \\
\hline $\begin{array}{l}\text { 241-H Tank Annulus } \\
\text { Exhaust }\end{array}$ & Cs-137 & $3.85 E+01$ & $7.29 E+14$ & 4.77E-14 & $4.00 E-10$ \\
\hline \multirow{3}{*}{$\begin{array}{l}\text { 244-H Vessel Vent } \\
\text { Exhaust }\end{array}$} & Co-57 & $4.69 E-02$ & $5.78 E+12$ & $4.00 E-13$ & $2.00 E-09$ \\
\hline & Co-60 & $1.00 E+00$ & $5.78 E+12$ & $2.55 E-13$ & $8.00 E-11$ \\
\hline & Cs-137 & $1.64 \mathrm{E}+01$ & $5.78 \mathrm{E}+12$ & $2.92 E-12$ & $4.00 E-10$ \\
\hline \multirow[t]{8}{*}{ 291-H Stack Isokinetic } & Sr-89,90 & $5.55 E+00$ & $3.96 E+15$ & $1.02 E-14$ & $9.00 E-12$ \\
\hline & Cs-137 & $2.06 E+00$ & $3.96 E+15$ & $2.18 E-14$ & $4.00 E-10$ \\
\hline & $U-234$ & $1.05 \mathrm{E}-01$ & $3.96 \mathrm{E}+15$ & $1.17 E-17$ & $9.00 E-14$ \\
\hline & U-238 & $5.17 E-02$ & $3.96 \mathrm{E}+15$ & $1.34 \mathrm{E}-18$ & $1.00 E-13$ \\
\hline & Pu-238 & $8.02 E+01$ & $3.96 E+15$ & $2.03 E-14$ & $3.00 E-14$ \\
\hline & Pu-239 & $1.34 \mathrm{E}+01$ & $3.96 \mathrm{E}+15$ & $3.49 E-15$ & $2.00 E-14$ \\
\hline & Am-241 & $2.23 E+00$ & $3.96 \mathrm{E}+15$ & $5.74 E-16$ & $2.00 E-14$ \\
\hline & $\mathrm{Cm}-244$ & $1.48 E-01$ & $3.96 \mathrm{E}+15$ & $5.45 \mathrm{E}-17$ & $4.00 E-14$ \\
\hline $\begin{array}{l}\text { 299-H Bldg. Stack \& } \\
\text { HP Hood }\end{array}$ & Cs-137 & $1.59 \mathrm{E}+00$ & $2.66 \mathrm{E}+14$ & $1.40 E-14$ & $4.00 E-10$ \\
\hline \multirow[t]{6}{*}{ H-CIF 261-H (Off Gas) } & Cs-137 & $8.25 E+03$ & $1.78 E+14$ & $4.60 E-11$ & $4.00 E-10$ \\
\hline & $U-234$ & $3.98 E+00$ & $1.78 \mathrm{E}+14$ & $2.44 \mathrm{E}-14$ & $9.00 E-14$ \\
\hline & U-235 & $2.24 \mathrm{E}-02$ & $1.78 E+14$ & $9.64 \mathrm{E}-16$ & $1.00 E-13$ \\
\hline & U-238 & $1.60 \mathrm{E}+00$ & $1.78 E+14$ & $9.74 E-15$ & $1.00 E-13$ \\
\hline & $\mathrm{Pu}-238$ & $4.40 E+02$ & $1.78 \mathrm{E}+14$ & $2.54 \mathrm{E}-12$ & $3.00 E-14$ \\
\hline & Pu-239 & $1.20 \mathrm{E}+01$ & $1.78 \mathrm{E}+14$ & $6.86 \mathrm{E}-14$ & $2.00 E-14$ \\
\hline
\end{tabular}

a Source: DOE Order 5400.5. In cases where different chemical forms have different DCGs, the lowest DCG for the radionuclide is given. These DCGs are defined as the air concentration of that radionuclide that will give a 50-year committed effective dose equivalent of $100 \mathrm{mrem}$ under conditions of continuous exposure for one year. DCGs are reference values only and are not considered release limits or standards. 


\section{Table 5}

\section{Radioactive Atmospheric Releases by Stack/Facility and Comparison of Annual Average Concentrations to DOE Derived Concentration Guides}

Page 4 of 6

\begin{tabular}{|c|c|c|c|c|c|}
\hline Stack/Facility & Radionuclide & $\begin{array}{c}\text { Quantity } \\
\text { Released } \\
\text { During } 1999 \\
(\mu \mathrm{Ci})\end{array}$ & $\begin{array}{l}\text { Atmospheric } \\
\text { Release } \\
\text { Volume (mL) }\end{array}$ & $\begin{array}{c}\text { Average } \\
\text { Effluent } \\
\text { Concentration } \\
\text { During 1999 } \\
(\mu \mathrm{Ci} / \mathrm{mL})\end{array}$ & $\begin{array}{c}\text { DOE DCGsa } \\
(\mu \mathrm{Ci} / \mathrm{mL})\end{array}$ \\
\hline & Am-241 & $1.16 \mathrm{E}+00$ & $1.78 E+14$ & $1.03 E-14$ & $2.00 E-14$ \\
\hline & $\mathrm{Cm}-244$ & $2.38 \mathrm{E}+01$ & $1.78 E+14$ & $1.37 E-13$ & $4.00 E-14$ \\
\hline \multicolumn{6}{|l|}{ K-Area (K-Reactor) } \\
\hline K-Area Disassembly & Cs-137 & $1.63 \mathrm{E}+01$ & $1.35 \mathrm{E}+15$ & $2.67 \mathrm{E}-14$ & $4.00 \mathrm{E}-10$ \\
\hline $\begin{array}{l}\text { K-Area Main Stack } \\
\left(148^{\prime}\right)\end{array}$ & $\mathrm{H}-3$ (oxide) & $1.16 E+09$ & $1.87 E+15$ & $6.21 \mathrm{E}-07$ & $1.00 \mathrm{E}-07$ \\
\hline \multicolumn{6}{|l|}{ L-Area (L-Reactor) } \\
\hline L-Area Disassembly & Cs-137 & $6.38 \mathrm{E}+00$ & $1.18 \mathrm{E}+15$ & $3.81 \mathrm{E}-14$ & $4.00 \mathrm{E}-10$ \\
\hline $\begin{array}{l}\text { L-Area Main Stack } \\
\left(148^{\prime}\right)\end{array}$ & $\mathrm{H}-3$ (oxide) & $5.53 \mathrm{E}+08$ & $2.61 \mathrm{E}+15$ & $2.12 E-07$ & $1.00 \mathrm{E}-07$ \\
\hline \multicolumn{6}{|c|}{ M-Area (Reactor Materials) } \\
\hline \multirow{5}{*}{$\begin{array}{l}\text { 321-M Machining Room } \\
\text { Stack }\end{array}$} & $\mathrm{U}-234$ & $8.15 E+00$ & $4.80 E+13$ & $2.07 \mathrm{E}-13$ & $9.00 \mathrm{E}-14$ \\
\hline & U-235 & $1.66 \mathrm{E}+00$ & $4.80 E+13$ & $4.42 \mathrm{E}-14$ & $1.00 E-13$ \\
\hline & $U-238$ & $3.50 \mathrm{E}-02$ & $4.80 E+13$ & $1.02 \mathrm{E}-15$ & $1.00 \mathrm{E}-13$ \\
\hline & Pu-239 & $3.46 \mathrm{E}-03$ & $4.80 E+13$ & $-1.43 E-17$ & $2.00 \mathrm{E}-14$ \\
\hline & Am-241 & $5.38 \mathrm{E}-03$ & $4.80 E+13$ & $1.56 \mathrm{E}-16$ & $2.00 \mathrm{E}-14$ \\
\hline \multirow[t]{7}{*}{ 321-M Stacks } & $U-234$ & $4.28 \mathrm{E}+00$ & $4.91 \mathrm{E}+14$ & $9.06 E-15$ & $9.00 \mathrm{E}-14$ \\
\hline & $U-235$ & 8.04E-01 & $4.91 E+14$ & $1.72 E-15$ & $1.00 \mathrm{E}-13$ \\
\hline & $\mathrm{U}-238$ & 4.87E-01 & $4.91 E+14$ & $1.03 E-15$ & $1.00 \mathrm{E}-13$ \\
\hline & Pu-238 & $7.16 \mathrm{E}-03$ & $4.91 E+14$ & $1.91 \mathrm{E}-17$ & $3.00 E-14$ \\
\hline & Pu-239 & $2.04 \mathrm{E}-02$ & $4.91 E+14$ & $2.60 E-17$ & $2.00 \mathrm{E}-14$ \\
\hline & Am-241 & $8.01 E-03$ & $4.91 E+14$ & $-1.59 \mathrm{E}-18$ & $2.00 E-14$ \\
\hline & $\mathrm{Cm}-244$ & $1.62 E-02$ & $4.91 E+14$ & $2.48 E-17$ & $4.00 \mathrm{E}-14$ \\
\hline \multirow[t]{5}{*}{ M-Area VTF } & Cs-137 & $3.36 \mathrm{E}-01$ & $8.95 E+12$ & $5.15 E-13$ & $4.00 E-10$ \\
\hline & U-232 & $1.33 E-02$ & $8.95 E+12$ & $2.03 E-14$ & $2.70 E-18$ \\
\hline & U-234 & $1.71 E+00$ & $8.95 E+12$ & $2.24 E-13$ & $9.00 \mathrm{E}-14$ \\
\hline & U-235 & $2.19 \mathrm{E}-01$ & $8.95 E+12$ & $2.63 E-14$ & $1.00 \mathrm{E}-13$ \\
\hline & $\mathrm{U}-238$ & $1.02 E+01$ & $8.95 E+12$ & $1.27 E-12$ & $1.00 E-13$ \\
\hline
\end{tabular}

a Source: DOE Order 5400.5. In cases where different chemical forms have different DCGs, the lowest DCG for the radionuclide is given. These DCGs are defined as the air concentration of that radionuclide that will give a 50-year committed effective dose equivalent of 100 mrem under conditions of continuous exposure for one year. DCGs are reference values only and are not considered release limits or standards. 


\section{Table 5}

\section{Radioactive Atmospheric Releases by Stack/Facility and Comparison of Annual Average Concentrations to DOE Derived Concentration Guides}

Page 5 of 6

\begin{tabular}{|c|c|c|c|c|c|}
\hline Stack/Facility & Radionuclide & $\begin{array}{c}\text { Quantity } \\
\text { Released } \\
\text { During } 1999 \\
\quad(\mu \mathrm{Ci})\end{array}$ & $\begin{array}{c}\text { Atmospheric } \\
\text { Release } \\
\text { Volume }(\mathrm{mL})\end{array}$ & $\begin{array}{c}\text { Average } \\
\text { Effluent } \\
\text { Concentration } \\
\text { During } 1999 \\
(\mu \mathrm{Ci} / \mathrm{mL})\end{array}$ & $\begin{array}{c}\text { DOE DCGsa } \\
(\mu \mathrm{Ci} / \mathrm{mL})\end{array}$ \\
\hline & $\mathrm{Am}-241$ & $1.19 E-03$ & $8.95 \mathrm{E}+12$ & $-4.92 E-17$ & $2.00 \mathrm{E}-14$ \\
\hline & Cm-244 & $7.21 E-04$ & $8.95 \mathrm{E}+12$ & $7.80 E-16$ & $4.00 \mathrm{E}-14$ \\
\hline \multicolumn{6}{|c|}{ S-Area (Defense Waste Processing Facility) } \\
\hline \multirow{8}{*}{$\begin{array}{l}\text { 221-S Personnel Area } \\
\text { (Zone 2) }\end{array}$} & Sr-89,90 & $2.26 \mathrm{E}+01$ & $1.64 \mathrm{E}+15$ & $9.61 \mathrm{E}-14$ & $9.00 \mathrm{E}-12$ \\
\hline & Cs-137 & $1.02 E+01$ & $1.64 \mathrm{E}+15$ & 1.35E-14 & $4.00 E-10$ \\
\hline & U-234 & $1.73 E+00$ & $1.64 \mathrm{E}+15$ & $1.38 \mathrm{E}-15$ & $9.00 \mathrm{E}-14$ \\
\hline & U-235 & $7.86 \mathrm{E}-02$ & $1.64 \mathrm{E}+15$ & $2.81 \mathrm{E}-17$ & $1.00 E-13$ \\
\hline & U-238 & $1.74 E+00$ & 1.64E+15 & $1.40 \mathrm{E}-15$ & $1.00 \mathrm{E}-13$ \\
\hline & Pu-238 & $1.47 \mathrm{E}+00$ & $1.64 \mathrm{E}+15$ & $9.79 E-16$ & $3.00 E-14$ \\
\hline & Pu-239 & $1.82 E-01$ & $1.64 \mathrm{E}+15$ & $1.85 \mathrm{E}-16$ & $2.00 \mathrm{E}-14$ \\
\hline & Am-241 & $3.99 E-01$ & $1.64 E+15$ & $1.04 \mathrm{E}-16$ & $2.00 \mathrm{E}-14$ \\
\hline \multirow{5}{*}{$\begin{array}{l}\text { 250-S Glass Waste } \\
\text { Bldg \#1488 }\end{array}$} & U-234 & $5.43 E-04$ & $1.89 \mathrm{E}+11$ & $4.99 \mathrm{E}-15$ & $9.00 \mathrm{E}-14$ \\
\hline & U-238 & $1.44 \mathrm{E}-03$ & $1.89 \mathrm{E}+11$ & $8.08 \mathrm{E}-15$ & $1.00 E-13$ \\
\hline & Pu-238 & $3.13 E-04$ & $1.89 \mathrm{E}+11$ & $3.13 E-15$ & $3.00 \mathrm{E}-14$ \\
\hline & Am-241 & $2.55 \mathrm{E}-04$ & $1.89 E+11$ & $9.08 E-16$ & $2.00 \mathrm{E}-14$ \\
\hline & $\mathrm{Cm}-244$ & $9.13 E-06$ & $1.89 \mathrm{E}+11$ & $9.84 \mathrm{E}-16$ & $4.00 E-14$ \\
\hline \multirow{5}{*}{$\begin{array}{l}\text { 250-S Glass Waste } \\
\text { Bldg \#1509 }\end{array}$} & U-234 & $7.64 \mathrm{E}-04$ & $1.89 \mathrm{E}+11$ & $7.36 \mathrm{E}-15$ & $9.00 \mathrm{E}-14$ \\
\hline & U-238 & $8.91 E-04$ & $1.89 E+11$ & $6.05 \mathrm{E}-15$ & $1.00 E-13$ \\
\hline & Pu-238 & $4.79 \mathrm{E}-04$ & $1.89 E+11$ & $7.30 \mathrm{E}-16$ & $3.00 E-14$ \\
\hline & Pu-239 & $9.28 \mathrm{E}-05$ & $1.89 E+11$ & $9.41 \mathrm{E}-16$ & $2.00 E-14$ \\
\hline & Am-241 & $5.95 E-05$ & $1.89 E+11$ & 2.37E-16 & $2.00 \mathrm{E}-14$ \\
\hline \multirow{4}{*}{$\begin{array}{l}\text { 250-S Glass Waste } \\
\text { Bldg \#3928 }\end{array}$} & Sr-89,90 & $1.30 \mathrm{E}-02$ & $1.86 \mathrm{E}+11$ & $5.32 E-13$ & $9.00 \mathrm{E}-12$ \\
\hline & U-234 & $5.20 \mathrm{E}-04$ & $1.86 \mathrm{E}+11$ & $4.75 \mathrm{E}-15$ & $9.00 \mathrm{E}-14$ \\
\hline & $U-238$ & $1.20 \mathrm{E}-03$ & $1.86 E+11$ & $6.18 \mathrm{E}-15$ & $1.00 \mathrm{E}-13$ \\
\hline & Pu-238 & $6.04 E-04$ & $1.86 E+11$ & $1.84 \mathrm{E}-14$ & $3.00 E-14$ \\
\hline
\end{tabular}

a Source: DOE Order 5400.5. In cases where different chemical forms have different DCGs, the lowest DCG for the radionuclide is given. These DCGs are defined as the air concentration of that radionuclide that will give a 50-year committed effective dose equivalent of 100 mrem under conditions of continuous exposure for one year. DCGs are reference values only and are not considered release limits or standards. 


\section{Table 5}

Radioactive Atmospheric Releases by Stack/Facility and Comparison of Annual Average Concentrations to DOE Derived Concentration Guides

Page 6 of 6

\begin{tabular}{|c|c|c|c|c|c|}
\hline Stack/Facility & Radionuclide & $\begin{array}{c}\text { Quantity } \\
\text { Released } \\
\text { During } 1999 \\
(\mu \mathrm{Ci})\end{array}$ & $\begin{array}{c}\text { Atmospheric } \\
\text { Release } \\
\text { Volume }(\mathrm{mL})\end{array}$ & $\begin{array}{c}\text { Average } \\
\text { Effluent } \\
\text { Concentration } \\
\text { During 1999 } \\
(\mu \mathrm{Ci} / \mathrm{mL})\end{array}$ & $\begin{array}{c}\text { DOE DCGsa } \\
(\mu \mathrm{Ci} / \mathrm{mL})\end{array}$ \\
\hline & Am-241 & $1.21 \mathrm{E}-04$ & $1.86 \mathrm{E}+11$ & $1.09 \mathrm{E}-15$ & $2.00 \mathrm{E}-14$ \\
\hline \multirow{6}{*}{$\begin{array}{l}\text { 250-S Glass Waste } \\
\text { Bldg \#3940 }\end{array}$} & $U-234$ & $8.72 E-04$ & $1.85 E+11$ & $5.41 E-15$ & $9.00 E-14$ \\
\hline & U-235 & $1.38 \mathrm{E}-05$ & $1.85 \mathrm{E}+11$ & $8.55 \mathrm{E}-16$ & $1.00 \mathrm{E}-13$ \\
\hline & $U-238$ & $1.11 E-03$ & $1.85 E+11$ & $6.06 \mathrm{E}-15$ & $1.00 E-13$ \\
\hline & Pu-238 & $4.05 \mathrm{E}-04$ & $1.85 E+11$ & $3.41 \mathrm{E}-15$ & $3.00 E-14$ \\
\hline & Pu-239 & $1.68 \mathrm{E}-05$ & $1.85 E+11$ & $-9.84 E-17$ & $2.00 \mathrm{E}-14$ \\
\hline & Am-241 & $4.43 E-04$ & $1.85 \mathrm{E}+11$ & $2.57 \mathrm{E}-15$ & $2.00 \mathrm{E}-14$ \\
\hline \multirow{7}{*}{$\begin{array}{l}291-S \text { Vit. Process } \\
\text { (Zone 1) }\end{array}$} & Sr-89,90 & $1.15 E+02$ & $1.54 \mathrm{E}+15$ & $1.92 \mathrm{E}-13$ & $9.00 \mathrm{E}-12$ \\
\hline & $U-234$ & $1.74 \mathrm{E}+00$ & $1.54 \mathrm{E}+15$ & $1.70 \mathrm{E}-15$ & $9.00 E-14$ \\
\hline & U.235 & $4.96 \mathrm{E}-02$ & $1.54 \mathrm{E}+15$ & $-1.14 \mathrm{E}-16$ & $1.00 \mathrm{E}-13$ \\
\hline & $U-238$ & $2.48 \mathrm{E}+00$ & $1.54 \mathrm{E}+15$ & $2.04 \mathrm{E}-15$ & $1.00 \mathrm{E}-13$ \\
\hline & Pu-238 & $8.84 \mathrm{E}-01$ & $1.54 \mathrm{E}+15$ & $7.14 \mathrm{E}-16$ & $3.00 \mathrm{E}-14$ \\
\hline & Pu-239 & $1.01 E+00$ & $1.54 E+15$ & $7.17 E-16$ & $2.00 E-14$ \\
\hline & Am-241 & $6.72 E-01$ & $1.54 \mathrm{E}+15$ & 4.23E-16 & $2.00 E-14$ \\
\hline \multirow[t]{6}{*}{ 511-S Low Pt. Pump Pit } & Sr-89,90 & $3.76 E+00$ & $2.26 E+14$ & $7.93 E-14$ & $9.00 E-12$ \\
\hline & U-234 & $2.24 \mathrm{E}-01$ & $2.26 E+14$ & $1.18 E-15$ & $9.00 \mathrm{E}-14$ \\
\hline & U-238 & $2.70 E-01$ & $2.26 E+14$ & $1.47 \mathrm{E}-15$ & $1.00 E-13$ \\
\hline & Pu-238 & $2.60 \mathrm{E}-02$ & $2.26 \mathrm{E}+14$ & $1.81 \mathrm{E}-16$ & $3.00 E-14$ \\
\hline & Pu-239 & $2.39 \mathrm{E}-02$ & $2.26 \mathrm{E}+14$ & $8.34 \mathrm{E}-17$ & $2.00 E-14$ \\
\hline & Am-241 & $7.08 E-02$ & $2.26 \mathrm{E}+14$ & $3.34 \mathrm{E}-16$ & $2.00 E-14$ \\
\hline \multicolumn{6}{|l|}{ Tritium } \\
\hline \multirow{2}{*}{$\begin{array}{l}\text { 232-H Line 1\&2 Stack } \\
(200 \mathrm{ft})\end{array}$} & $H-3(\mathrm{el})$ & $1.14 \mathrm{E}+10$ & $1.33 E+15$ & $8.59 E-06$ & $2.00 \mathrm{E}-02$ \\
\hline & $H-3$ (oxide) & $6.70 \mathrm{E}+09$ & $1.33 E+15$ & $5.02 E-06$ & $1.00 E-07$ \\
\hline \multirow{2}{*}{$\begin{array}{l}232-H \text { Line } 3 \text { Stack } \\
\text { (200ft) }\end{array}$} & $\mathrm{H}-3(\mathrm{el})$ & $4.61 E+08$ & $2.46 \mathrm{E}+14$ & $1.87 E-06$ & $2.00 E-02$ \\
\hline & H-3(oxide) & $4.72 E+09$ & $2.46 E+14$ & $1.92 E-05$ & $1.00 E-07$ \\
\hline
\end{tabular}

a Source: DOE Order 5400.5. In cases where different chemical forms have different DCGs, the lowest DCG for the radionuclide is given. These DCGs are defined as the air concentration of that radionuclide that will give a 50-year committed effective dose equivalent of $100 \mathrm{mrem}$ under conditions of continuous exposure for one year. DCGs are reference values only and are not considered release limits or standards. 


\section{Table 6}

\section{Radioactive Liquid Releases by Source (Including Direct and Seepage Basin Migration Releases)}

Page 1 of 1

\begin{tabular}{|c|c|c|c|c|c|c|}
\hline \multirow[b]{2}{*}{$\begin{array}{l}\text { Radio- } \\
\text { nuclide }\end{array}$} & \multicolumn{6}{|c|}{ Curies $^{a}$} \\
\hline & Reactors & Separations ${ }^{b}$ & $\begin{array}{l}\text { Reactor } \\
\text { Materials }\end{array}$ & $\begin{array}{c}\text { Heavy } \\
\text { Water } / \mathrm{TNX}\end{array}$ & $\begin{array}{c}\text { Savannah } \\
\text { River } \\
\text { Technology } \\
\text { Center }\end{array}$ & Total \\
\hline \multicolumn{7}{|c|}{ Blank spaces indicate no quantifiable activity. } \\
\hline $\mathrm{H}-3$ & $1.40 \mathrm{E}+03$ & $4.68 \mathrm{E}+03$ & & $2.13 E+02$ & $1.46 \mathrm{E}+00$ & $6.29 \mathrm{E}+03$ \\
\hline Sr-89,90 & 1.37E-02 & $1.20 \mathrm{E}-01$ & & & & $1.34 \mathrm{E}-01$ \\
\hline Co-60 & & $4.94 \mathrm{E}-04$ & & & & $4.94 E-04$ \\
\hline I-129 & & 7.82E-02 & & & & $7.82 \mathrm{E}-02$ \\
\hline Cs-137 & $3.24 \mathrm{E}-04$ & $1.02 \mathrm{E}-01$ & & & & $1.02 E-01^{c}$ \\
\hline U-234 & $3.93 E-03$ & $8.60 \mathrm{E}-02$ & $1.24 \mathrm{E}-02$ & $4.88 \mathrm{E}-06$ & $8.39 E-05$ & $1.02 E-01$ \\
\hline U-235 & $2.50 \mathrm{E}-04$ & $6.33 E-04$ & & & $2.99 \mathrm{E}-06$ & $8.86 \mathrm{E}-04$ \\
\hline U-238 & $3.10 \mathrm{E}-03$ & $1.08 E-02$ & $1.37 \mathrm{E}-02$ & $1.00 \mathrm{E}-05$ & $7.92 \mathrm{E}-05$ & $2.77 E-02$ \\
\hline Pu-238 & & $9.98 E-05$ & & $1.14 \mathrm{E}-06$ & $7.73 E-06$ & $1.09 E-04$ \\
\hline Pu-239 & $9.96 \mathrm{E}-05$ & 1.97E-06 & & & & $1.02 \mathrm{E}-04$ \\
\hline$A m-241$ & & $1.83 E-06$ & $1.16 \mathrm{E}-05$ & & & $1.34 \mathrm{E}-05$ \\
\hline $\mathrm{Cm}-244$ & & $1.26 \mathrm{E}-06$ & & & & $1.26 \mathrm{E}-06$ \\
\hline Alpha & $6.45 \mathrm{E}-04$ & $2.05 E-02$ & $3.56 \mathrm{E}-03$ & $1.04 \mathrm{E}-03$ & $5.25 \mathrm{E}-03$ & $3.10 E-02$ \\
\hline $\begin{array}{l}\text { Nonvolatile } \\
\text { Beta }\end{array}$ & $2.40 E-02$ & $2.23 E-02$ & $9.97 E-04$ & $3.21 E-03$ & 4.63E-03 & $5.51 \mathrm{E}-02$ \\
\hline
\end{tabular}

a One curie equals 3.7E+10 Becquerels.

b Includes separations, waste management, and tritium facilities

c For conservatism, the higher release value (2.40E-01 Ci) calculated from River Mile 120 fish concentrations was used for dose calculations [see chapter 7, "Potential Radiation Doses," of the SRS Environmental Report for 1999 (WSRC-TR-99-00299)]. 


\section{Table 7 \\ Liquid Radioactive Releases by Outfall/Facility and Comparison of Annual Average Radionuclide Concentrations to DOE Derived Concentration Guides}

Page 1 of 5

\begin{tabular}{|c|c|c|c|c|c|}
\hline $\begin{array}{l}\text { Outfall or } \\
\text { Facility }\end{array}$ & Radionuclide & $\begin{array}{l}\text { Quantity of } \\
\text { Radionuclides } \\
\text { Released } \\
\text { During } 1999 \\
\text { (Ci) }\end{array}$ & $\begin{array}{c}\text { Average } \\
\text { Effluent } \\
\text { Concentration } \\
\text { During 1999 } \\
(\mu \mathrm{Ci} / \mathrm{mL})\end{array}$ & $\begin{array}{c}\text { DOE DCGsa } \\
(\mu \mathrm{Ci} / \mathbf{m L})\end{array}$ & $\begin{array}{l}\text { Fraction of } \\
\text { DOE DCG }\end{array}$ \\
\hline Note: "MDL" denotes" & minimum detect & able level." & & & \\
\hline \multicolumn{6}{|c|}{ A-Area (Savannah River Technology Center) } \\
\hline \multirow[t]{7}{*}{ TB-2 Outfall at Road $1 \mathrm{~A}$} & $\mathrm{H}-3$ & $1.46 \mathrm{E}+00$ & $1.75 E-06$ & $2.00 \mathrm{E}-03$ & $8.76 \mathrm{E}-04$ \\
\hline & Cs-137 & Below MDL & $4.02 E-10$ & $3.00 \mathrm{E}-06$ & $1.34 E-04$ \\
\hline & U-234 & 8.39E-05 & $1.10 \mathrm{E}-10$ & $6.00 E-07$ & $1.84 \mathrm{E}-04$ \\
\hline & U-235 & $2.99 E-06$ & $5.22 E-12$ & $6.00 \mathrm{E}-07$ & $8.70 \mathrm{E}-06$ \\
\hline & U-238 & $7.92 \mathrm{E}-05$ & $1.07 \mathrm{E}-10$ & $6.00 \mathrm{E}-07$ & $1.78 \mathrm{E}-04$ \\
\hline & Pu-238 & $7.73 E-06$ & $1.07 E-11$ & $4.00 E-08$ & 2.67E-04 \\
\hline & & & & \multicolumn{2}{|c|}{ Sum of Fractions $-1.65 E-03$} \\
\hline \multicolumn{6}{|c|}{ D-Area (Heavy Water Rework) } \\
\hline \multirow{4}{*}{$\begin{array}{l}\text { 400-D Effluent } \\
\text { Discharge }\end{array}$} & $\mathrm{H}-3$ & $2.13 \mathrm{E}+02$ & $1.07 E-04$ & $2.00 \mathrm{E}-03$ & $5.33 \mathrm{E}-02$ \\
\hline & Sr-89,90 & Below MDL & 2.87E-10 & $1.00 \mathrm{E}-06$ & 2.87E-04 \\
\hline & Cs-137 & Below MDL & $3.48 \mathrm{E}-10$ & $3.00 \mathrm{E}-06$ & $1.16 \mathrm{E}-04$ \\
\hline & & & & Sum of Fracti & $-5.37 \mathrm{E}-02$ \\
\hline \multicolumn{6}{|c|}{ F-Area (Separations and Waste Management) } \\
\hline \multirow[t]{4}{*}{$\mathrm{F}-01$} & $\mathrm{H}-3$ & $4.60 \mathrm{E}-02$ & $5.76 \mathrm{E}-07$ & $2.00 \mathrm{E}-03$ & $2.88 E-04$ \\
\hline & Sr-89,90 & $3.19 \mathrm{E}-06$ & $2.90 \mathrm{E}-10$ & $1.00 \mathrm{E}-06$ & $2.90 E-04$ \\
\hline & Cs-137 & Below MDL & $2.56 \mathrm{E}-10$ & $3.00 \mathrm{E}-06$ & $8.54 E-05$ \\
\hline & & & & \multicolumn{2}{|c|}{ Sum of Fractions $-6.63 \mathrm{E}-04$} \\
\hline \multirow{4}{*}{$\begin{array}{l}\mathrm{F}-012 \text { 281-8F } \\
\text { Retention Basin }\end{array}$} & $\mathrm{H}-3$ & $8.66 \mathrm{E}-01$ & $1.60 \mathrm{E}-05$ & $2.00 E-03$ & $8.02 \mathrm{E}-03$ \\
\hline & Sr-89,90 & Below MDL & $3.50 \mathrm{E}-09$ & $1.00 \mathrm{E}-06$ & $3.50 E-03$ \\
\hline & Cs-137 & $2.41 E-03$ & $3.39 E-08$ & $3.00 \mathrm{E}-06$ & $1.13 E-02$ \\
\hline & & & & \multicolumn{2}{|c|}{ Sum of Fractions $-2.28 \mathrm{E}-02$} \\
\hline \multirow{4}{*}{$\begin{array}{l}\mathrm{F}-013 \text { 200-F Cooling } \\
\text { Basin }\end{array}$} & $\mathrm{H}-3$ & $5.23 E-02$ & $3.60 E-06$ & $2.00 \mathrm{E}-03$ & $1.80 E-03$ \\
\hline & Sr-89,90 & $4.24 E-05$ & $1.85 E-08$ & $1.00 E-06$ & $1.85 E-02$ \\
\hline & Cs-137 & $1.71 \mathrm{E}-04$ & $1.09 E-08$ & $3.00 \mathrm{E}-06$ & $3.62 E-03$ \\
\hline & & & & \multicolumn{2}{|c|}{ Sum of Fractions $-2.39 E-02$} \\
\hline
\end{tabular}

a Source: DOE Order 5400.5. In cases where different chemical forms have different DCGs, the lowest DCG for the radionuclide is given. DCGs are defined as the concentration of that radionuclide that will give a 50-year committed effective dose equivalent of 100 mrem under conditions of continuous exposure for one year. DCGs are reference values only and are not considered release limits or standards. 
Table 7

Liquid Radioactive Releases by Outfall/Facility and Comparison of Annual Average Radionuclide Concentrations to DOE Derived Concentration Guides

Page 2 of 5

\begin{tabular}{|c|c|c|c|c|c|}
\hline $\begin{array}{l}\text { Outfall or } \\
\text { Facility }\end{array}$ & Radionuclide & $\begin{array}{c}\text { Quantity of } \\
\text { Radionuclides } \\
\text { Released } \\
\text { During } 1999 \\
\text { (Ci) }\end{array}$ & $\begin{array}{c}\text { Average } \\
\text { Effluent } \\
\text { Concentration } \\
\text { During } 1999 \\
(\mu \mathrm{Ci} / \mathrm{mL})\end{array}$ & $\begin{array}{c}\text { DOE DCGsa } \\
(\mu \mathrm{Ci} / \mathrm{mL})\end{array}$ & $\begin{array}{c}\text { Fraction of } \\
\text { DOE DCG }\end{array}$ \\
\hline \multirow[t]{4}{*}{ FM-3 F-Area Effluent } & $\mathrm{H}-3$ & $1.18 \mathrm{E}+00$ & $6.47 \mathrm{E}-07$ & $2.00 \mathrm{E}-03$ & $3.23 E-04$ \\
\hline & Sr-89,90 & Below MDL & $7.16 \mathrm{E}-10$ & $1.00 \mathrm{E}-06$ & $7.16 \mathrm{E}-04$ \\
\hline & Cs-137 & $3.48 E-04$ & $1.22 E-09$ & $3.00 E-06$ & $4.08 \mathrm{E}-04$ \\
\hline & & & & \multicolumn{2}{|c|}{ Sum of Fractions $-1.45 E-03$} \\
\hline \multirow[t]{11}{*}{ U3R-2 F Storm Sewer } & $\mathrm{H}-3$ & $4.66 \mathrm{E}-02$ & 4.34E-07 & $2.00 \mathrm{E}-03$ & 2.17E-04 \\
\hline & Sr- 89,90 & Below MDL & $4.46 \mathrm{E}-10$ & $1.00 \mathrm{E}-06$ & $4.46 \mathrm{E}-04$ \\
\hline & Cs-137 & Below MDL & $-1.06 \mathrm{E}-11$ & $3.00 E-06$ & $0.00 E+00$ \\
\hline & U-234 & $1.10 \mathrm{E}-05$ & $9.68 \mathrm{E}-11$ & $6.00 \mathrm{E}-07$ & $1.61 E-04$ \\
\hline & $\mathrm{U}-235$ & $6.89 \mathrm{E}-07$ & $9.36 \mathrm{E}-12$ & $6.00 \mathrm{E}-07$ & $1.56 \mathrm{E}-05$ \\
\hline & U-238 & $2.80 E-05$ & $2.74 \mathrm{E}-10$ & $6.00 \mathrm{E}-07$ & $4.56 \mathrm{E}-04$ \\
\hline & Pu-238 & $3.23 E-06$ & $2.88 E-11$ & $4.00 E-08$ & $7.20 \mathrm{E}-04$ \\
\hline & Pu-239 & $1.96 \mathrm{E}-06$ & $1.90 \mathrm{E}-11$ & $3.00 \mathrm{E}-08$ & $6.33 E-04$ \\
\hline & Am-241 & $1.83 \mathrm{E}-06$ & $1.76 \mathrm{E}-11$ & $3.00 \mathrm{E}-08$ & $5.85 E-04$ \\
\hline & $\mathrm{Cm}-244$ & $1.26 \mathrm{E}-06$ & $1.40 \mathrm{E}-11$ & $6.00 \mathrm{E}-08$ & $2.34 \mathrm{E}-04$ \\
\hline & & & & Sum of Fractic & $-3.47 E-03$ \\
\hline \multicolumn{6}{|c|}{ H-Area (Separations and Waste Management) } \\
\hline \multirow[t]{4}{*}{ FM-1C H-Area Effluent } & $\mathrm{H}-3$ & $8.61 E+00$ & $9.04 \mathrm{E}-06$ & $2.00 \mathrm{E}-03$ & $4.52 \mathrm{E}-03$ \\
\hline & Sr-89,90 & $1.31 \mathrm{E}-04$ & $9.46 \mathrm{E}-10$ & $1.00 \mathrm{E}-06$ & $9.46 \mathrm{E}-04$ \\
\hline & Cs-137 & $1.30 \mathrm{E}-03$ & $4.34 \mathrm{E}-09$ & $3.00 E-06$ & $1.45 \mathrm{E}-03$ \\
\hline & & & & \multicolumn{2}{|c|}{ Sum of Fractions $-6.91 \mathrm{E}-03$} \\
\hline \multirow[t]{8}{*}{$\mathrm{H}-004$} & $\mathrm{H}-3$ & $3.25 E-01$ & $9.62 E-06$ & $2.00 E-03$ & $4.81 \mathrm{E}-03$ \\
\hline & Sr-89,90 & Below MDL & $4.90 \mathrm{E}-10$ & $1.00 \mathrm{E}-06$ & $4.90 E-04$ \\
\hline & Cs-137 & Below MDL & $3.80 \mathrm{E}-10$ & $3.00 \mathrm{E}-06$ & $1.27 E-04$ \\
\hline & $U-234$ & $1.86 \mathrm{E}-06$ & $6.20 E-11$ & $6.00 \mathrm{E}-07$ & $1.03 E-04$ \\
\hline & U-238 & $1.88 \mathrm{E}-07$ & $1.51 \mathrm{E}-11$ & $6.00 \mathrm{E}-07$ & $2.51 E-05$ \\
\hline & Pu-238 & 7.97E-07 & $3.20 E-11$ & $4.00 E-08$ & $8.00 E-04$ \\
\hline & Pu-239 & 8.97E-09 & $5.33 E-13$ & $3.00 E-08$ & $1.78 \mathrm{E}-05$ \\
\hline & & & & \multicolumn{2}{|c|}{ Sum of Fractions $-6.37 \mathrm{E}-03$} \\
\hline
\end{tabular}

a Source: DOE Order 5400.5. In cases where different chemical forms have different DCGs, the lowest DCG for the radionuclide is given. DCGs are defined as the concentration of that radionuclide that will give a 50-year committed effective dose equivalent of 100 mrem under conditions of continuous exposure for one year. DCGs are reference values only and are not considered release limits or standards. 
Table 7

Liquid Radioactive Releases by Outfall/Facility and Comparison of Annual Average Radionuclide Concentrations to DOE Derived Concentration Guides

Page 3 of 5

\begin{tabular}{|c|c|c|c|c|c|}
\hline $\begin{array}{l}\text { Outfall or } \\
\text { Facility }\end{array}$ & Radionuclide & $\begin{array}{l}\text { Quantity of } \\
\text { Radionuclides } \\
\text { Released } \\
\text { During } 1999 \\
\text { (Ci) }\end{array}$ & $\begin{array}{c}\text { Average } \\
\text { Effluent } \\
\text { Concentration } \\
\text { During 1999 } \\
(\mu \mathrm{Ci} / \mathrm{mL})\end{array}$ & $\begin{array}{c}\text { DOE DCGsa } \\
(\mu \mathrm{Ci} / \mathrm{mL})\end{array}$ & $\begin{array}{l}\text { Fraction of } \\
\text { DOE DCG }\end{array}$ \\
\hline \multirow{4}{*}{$\begin{array}{l}\mathrm{H}-017281-8 \mathrm{H} \\
\text { Retention Basin }\end{array}$} & $\mathrm{H}-3$ & $2.29 \mathrm{E}-01$ & $6.20 \mathrm{E}-06$ & $2.00 E-03$ & $3.10 \mathrm{E}-03$ \\
\hline & Sr-89,90 & $1.72 E-03$ & 3.73E-08 & $1.00 \mathrm{E}-06$ & $3.73 E-02$ \\
\hline & Cs-137 & $2.12 E-02$ & $3.40 E-07$ & $3.00 E-06$ & $1.13 E-01$ \\
\hline & & & & \multicolumn{2}{|c|}{ Sum of Fractions $-1.54 E-01$} \\
\hline \multirow{4}{*}{$\begin{array}{l}\mathrm{H}-018 \text { 200-H Cooling } \\
\text { Basin }\end{array}$} & $\mathrm{H}-3$ & $5.15 \mathrm{E}-01$ & $1.83 E-05$ & $2.00 E-03$ & $9.13 \mathrm{E}-03$ \\
\hline & Sr-89,90 & $4.71 \mathrm{E}-04$ & $3.68 E-08$ & $1.00 \mathrm{E}-06$ & $3.68 E-02$ \\
\hline & Cs-137 & $4.35 \mathrm{E}-03$ & $2.18 \mathrm{E}-07$ & $3.00 E-06$ & $7.25 \mathrm{E}-02$ \\
\hline & & & & \multicolumn{2}{|c|}{ Sum of Fractions $-1.18 E-01$} \\
\hline \multirow{4}{*}{$\begin{array}{l}\text { HP-52 H-Area Tank } \\
\text { Farm }\end{array}$} & $H-3$ & $9.33 E-01$ & $1.17 E-06$ & $2.00 E-03$ & $5.87 E-04$ \\
\hline & Sr-89,90 & Below MDL & $2.73 E-10$ & $1.00 \mathrm{E}-06$ & $2.73 \mathrm{E}-04$ \\
\hline & Cs-137 & $8.96 \mathrm{E}-04$ & $2.48 E-09$ & $3.00 E-06$ & $8.26 E-04$ \\
\hline & & & & \multicolumn{2}{|c|}{ Sum of Fractions $-1.69 E-03$} \\
\hline \multicolumn{6}{|l|}{ H-ETF } \\
\hline \multirow{5}{*}{$\begin{array}{l}\text { U3R-2A ETF Outfall at } \\
\text { Road C }\end{array}$} & $\mathrm{H}-3$ & $7.93 E+02$ & $1.07 E-02$ & $2.00 \mathrm{E}-03$ & $5.34 \mathrm{E}+00$ \\
\hline & Co-0 & $4.94 \mathrm{E}-06$ & $7.79 \mathrm{E}-10$ & $5.00 \mathrm{E}-02$ & $1.56 \mathrm{E}-08$ \\
\hline & Sr-89,90 & $3.29 \mathrm{E}-05$ & $3.74 E-09$ & $1.00 E-06$ & $3.74 \mathrm{E}-03$ \\
\hline & Cs-137 & $1.84 \mathrm{E}-02$ & $2.38 \mathrm{E}-07$ & $3.00 E-06$ & $7.95 E-02$ \\
\hline & & & & \multicolumn{2}{|c|}{ Sum of Fractions $-5.42 E+00$} \\
\hline \multicolumn{6}{|l|}{ K-Area (K-Reactor) } \\
\hline \multirow[t]{3}{*}{ K-Canal } & $\mathrm{H}-3$ & $3.26 \mathrm{E}-01$ & $5.94 \mathrm{E}-07$ & $2.00 \mathrm{E}-03$ & $2.97 \mathrm{E}-04$ \\
\hline & Sr-89,90 & Below MDL & $3.26 \mathrm{E}-10$ & $1.00 E-06$ & $3.26 \mathrm{E}-04$ \\
\hline & Cs-137 & Below MDL & $3.46 \mathrm{E}-10$ & $3.00 E-06$ & 1.15E-04 \\
\hline
\end{tabular}

a Source: DOE Order 5400.5. In cases where different chemical forms have different DCGs, the lowest DCG for the radionuclide is given. DCGs are defined as the concentration of that radionuclide that will give a 50 -year committed effective dose equivalent of 100 mrem under conditions of continuous exposure for one year. DCGs are reference values only and are not considered release limits or standards. 
Table 7

Liquid Radioactive Releases by Outfall/Facility and Comparison of Annual Average Radionuclide Concentrations to DOE Derived Concentration Guides

Page 4 of 5

\begin{tabular}{|c|c|c|c|c|c|}
\hline $\begin{array}{l}\text { Outfall or } \\
\text { Facility }\end{array}$ & Radionuclide & $\begin{array}{c}\text { Quantity of } \\
\text { Radionuclides } \\
\text { Released } \\
\text { During } 1999 \\
\text { (Ci) }\end{array}$ & $\begin{array}{c}\text { Average } \\
\text { Effluent } \\
\text { Concentration } \\
\text { During } 1999 \\
(\mu \mathrm{Ci} / \mathrm{mL})\end{array}$ & $\begin{array}{l}\text { DOE DCGsa } \\
(\mu \mathrm{Ci} / \mathrm{mL})\end{array}$ & $\begin{array}{c}\text { Fraction of } \\
\text { DOE DCG }\end{array}$ \\
\hline \multicolumn{6}{|l|}{ L-Area (L-Reactor) } \\
\hline \multirow[t]{4}{*}{$L-07$} & $\mathrm{H}-3$ & $6.24 \mathrm{E}+00$ & $7.23 E-07$ & $2.00 \mathrm{E}-03$ & $3.62 E-04$ \\
\hline & Sr-89,90 & Below MDL & $2.65 \mathrm{E}-10$ & $1.00 \mathrm{E}-06$ & $2.65 E-04$ \\
\hline & Cs-137 & Below MDL & $-6.87 E-11$ & $3.00 E-06$ & $0.00 \mathrm{E}+00$ \\
\hline & & & & \multicolumn{2}{|c|}{ Sum of Fractions $-6.26 E-04$} \\
\hline \multicolumn{6}{|c|}{ M-Area (Reactor Materials) } \\
\hline \multirow{5}{*}{$\begin{array}{l}\text { TB-3 M-Area Effluent } \\
\text { at Road D }\end{array}$} & Cs-137 & Below MDL & $2.52 E-10$ & $3.00 \mathrm{E}-06$ & $8.39 E-05$ \\
\hline & U-234 & $1.28 \mathrm{E}-05$ & $1.34 E-11$ & $6.00 \mathrm{E}-07$ & $2.24 E-05$ \\
\hline & $\mathrm{U}-238$ & $6.20 \mathrm{E}-06$ & $9.18 \mathrm{E}-12$ & $6.00 \mathrm{E}-07$ & $1.53 E-05$ \\
\hline & Am-241 & $1.16 \mathrm{E}-05$ & $1.07 \mathrm{E}-11$ & $3.00 \mathrm{E}-08$ & $3.57 E-04$ \\
\hline & & & & \multicolumn{2}{|c|}{ Sum of Fractions $-4.78 E-04$} \\
\hline \multicolumn{6}{|l|}{ P-Area (P-Reactor) } \\
\hline \multirow[t]{4}{*}{ 105-R Sumps } & $\mathrm{H}-3$ & $6.09 \mathrm{E}-01$ & $2.12 E-04$ & $2.00 \mathrm{E}-03$ & $1.06 \mathrm{E}-01$ \\
\hline & Sr-89,90 & 4.67E-05 & $1.83 E-08$ & $1.00 \mathrm{E}-06$ & $1.83 E-02$ \\
\hline & Cs-137 & $3.24 \mathrm{E}-04$ & $1.22 \mathrm{E}-07$ & $3.00 E-06$ & $4.05 E-02$ \\
\hline & & & & \multicolumn{2}{|c|}{ Sum of Fractions $-1.65 E-01$} \\
\hline \multicolumn{6}{|l|}{ S-Area } \\
\hline \multirow[t]{7}{*}{$\mathrm{S}-004$} & $\mathrm{H}-3$ & $3.25 \mathrm{E}-01$ & $1.30 \mathrm{E}-05$ & $2.00 \mathrm{E}-03$ & $6.52 \mathrm{E}-03$ \\
\hline & Sr-89,90 & Below MDL & $4.82 E-10$ & $1.00 \mathrm{E}-06$ & $4.82 E-04$ \\
\hline & Cs-137 & $3.02 E-06$ & $5.87 E-10$ & $3.00 E-06$ & $1.96 \mathrm{E}-04$ \\
\hline & $U-234$ & $3.07 \mathrm{E}-07$ & $2.33 \mathrm{E}-11$ & $6.00 \mathrm{E}-07$ & $3.89 \mathrm{E}-05$ \\
\hline & U-238 & $1.40 E-07$ & $1.72 E-11$ & $6.00 \mathrm{E}-07$ & $2.87 E-05$ \\
\hline & Pu-238 & $5.81 E-07$ & $2.63 E-11$ & $4.00 E-08$ & $6.57 \mathrm{E}-04$ \\
\hline & & & & \multicolumn{2}{|c|}{ Sum of Fractions $-7.92 \mathrm{E}-03$} \\
\hline
\end{tabular}

a Source: DOE Order 5400.5. In cases where different chemical forms have different DCGs, the lowest DCG for the radionuclide is given. DCGs are defined as the concentration of that radionuclide that will give a 50-year committed effective dose equivalent of 100 mrem under conditions of continuous exposure for one year. DCGs are reference values only and are not considered release limits or standards. 
Table 7

Liquid Radioactive Releases by Outfall/Facility and Comparison of Annual Average Radionuclide Concentrations to DOE Derived Concentration Guides

Page 5 of 5

\begin{tabular}{|c|c|c|c|c|c|}
\hline $\begin{array}{l}\text { Outfall or } \\
\text { Facility }\end{array}$ & Radionuclide & $\begin{array}{c}\text { Quantity of } \\
\text { Radionuclides } \\
\text { Released } \\
\text { During } 1999 \\
\text { (Ci) }\end{array}$ & $\begin{array}{c}\text { Average } \\
\text { Effluent } \\
\text { Concentration } \\
\text { During 1999 } \\
(\mu \mathrm{Ci} / \mathrm{mL})\end{array}$ & $\begin{array}{l}\text { DOE DCGsa } \\
(\mu \mathrm{Ci} / \mathrm{mL})\end{array}$ & $\begin{array}{l}\text { Fraction of } \\
\text { DOE DCG }\end{array}$ \\
\hline \multicolumn{6}{|l|}{ TNX Area } \\
\hline \multirow[t]{6}{*}{ TNX-008 } & $\mathrm{H}-3$ & $3.48 \mathrm{E}-01$ & $8.36 \mathrm{E}-07$ & $2.00 E-03$ & $4.18 E-04$ \\
\hline & Cs-137 & Below MDL & $2.41 E-10$ & $3.00 \mathrm{E}-06$ & 8.03E-05 \\
\hline & $U-234$ & $4.88 E-06$ & $1.21 \mathrm{E}-11$ & $6.00 \mathrm{E}-07$ & $2.02 E-05$ \\
\hline & $U-238$ & $1.00 E-05$ & $2.41 E-11$ & $6.00 E-07$ & 4.01E-05 \\
\hline & Pu-238 & $1.14 \mathrm{E}-06$ & 4.43E-12 & $4.00 E-08$ & $1.11 E-04$ \\
\hline & & & & \multicolumn{2}{|c|}{ Sum of Fractions $-6.70 E-04$} \\
\hline \multicolumn{6}{|l|}{ Tritium } \\
\hline \multirow{4}{*}{$\begin{array}{l}\text { HP-15 Tritium Facility } \\
\text { Outfall }\end{array}$} & $\mathrm{H}-3$ & $1.34 \mathrm{E}+00$ & $1.31 \mathrm{E}-05$ & $2.00 \mathrm{E}-03$ & $6.55 \mathrm{E}-03$ \\
\hline & Sr-89,90 & $3.21 E-06$ & $3.56 \mathrm{E}-10$ & $1.00 E-06$ & $3.56 \mathrm{E}-04$ \\
\hline & Cs-137 & Below MDL & $5.24 \mathrm{E}-11$ & $3.00 \mathrm{E}-06$ & $1.75 \mathrm{E}-05$ \\
\hline & & & & \multicolumn{2}{|c|}{ Sum of Fractions $-6.93 \mathrm{E}-03$} \\
\hline
\end{tabular}

a Source: DOE Order 5400.5. In cases where different chemical forms have different DCGs, the lowest DCG for the radionuclide is given. DCGs are defined as the concentration of that radionuclide that will give a 50-year committed effective dose equivalent of 100 mrem under conditions of continuous exposure for one year. DCGs are reference values only and are not considered release limits or standards. 


\section{Table 8}

\section{Radioactivity in Air}

Page 1 of 11

\begin{tabular}{|c|c|c|c|c|}
\hline Location & $\begin{array}{c}\text { No. of } \\
\text { Samples }\end{array}$ & Arithmetic Mean $\pm \sigma$ & Maximum $\pm \sigma$ & Minimum $\pm \sigma$ \\
\hline \multicolumn{5}{|c|}{ Silica Gel } \\
\hline \multicolumn{5}{|l|}{$\mathrm{H}-3, \mathrm{pCi} / \mathrm{cu} \mathrm{m}$} \\
\hline \multicolumn{5}{|l|}{ On Site } \\
\hline Burial Ground North & 26 & $(1.48 \pm 0.17) E+02$ & $(4.23 \pm 0.15) E+02$ & $(1.04 \pm 0.90) E+01$ \\
\hline \multicolumn{5}{|l|}{ Site Perimeter } \\
\hline Allendale Gate & 26 & $(6.80 \pm 1.45) E+00$ & $(2.10 \pm 0.19) E+01$ & $(-0.76 \pm 1.11) \mathrm{E}+01$ \\
\hline Barnwell Gate & 26 & $(1.24 \pm 0.17) E+01$ & $(3.62 \pm 1.17) E+01$ & $(2.41 \pm 2.59) E+00$ \\
\hline D-Area & 26 & $(2.17 \pm 0.24) E+01$ & $(4.37 \pm 0.90) E+01$ & $(3.06 \pm 1.04) \mathrm{E}+00$ \\
\hline Darkhorse @ Williston Gate & 26 & $(1.22 \pm 0.17) E+01$ & $(3.09 \pm 0.90) E+01$ & $(-0.28 \pm 1.14) E+01$ \\
\hline East Talatha & 26 & $(1.93 \pm 0.54) E+01$ & $(1.45 \pm 0.07) E+02$ & $(0.70 \pm 2.28) E+00$ \\
\hline Green Pond & 26 & $(1.45 \pm 0.18) E+01$ & $(4.79 \pm 0.93) E+01$ & $(-2.30 \pm 2.40) E+00$ \\
\hline Highway $21 / 167$ & 26 & $(1.34 \pm 0.15) E+01$ & $(3.09 \pm 1.48) E+01$ & $(1.70 \pm 9.87) E+00$ \\
\hline Jackson & 26 & $(1.59 \pm 0.24) E+01$ & $(5.12 \pm 1.03) E+01$ & $(-0.25 \pm 2.09) E+00$ \\
\hline Patterson Mill Road & 25 & $(9.28 \pm 1.82) E+00$ & $(3.09 \pm 0.86) E+01$ & $(-1.04 \pm 1.22) E+01$ \\
\hline Talatha Gate & 26 & $(1.56 \pm 0.21) E+01$ & $(4.75 \pm 1.04) E+01$ & $(1.75 \pm 5.24) \mathrm{E}+00$ \\
\hline West Jackson & 26 & $(1.16 \pm 0.19) E+01$ & $(4.03 \pm 1.19) E+01$ & $(-1.18 \pm 1.24) E+01$ \\
\hline Windsor Road & 26 & $(1.73 \pm 0.45) E+01$ & $(1.23 \pm 0.05) E+02$ & $(0.79 \pm 2.12) E+00$ \\
\hline \multicolumn{5}{|l|}{ 25-Mile Radius } \\
\hline Aiken Airport & 25 & $(1.00 \pm 0.30) E+01$ & $(7.42 \pm 0.54) E+01$ & $(-7.93 \pm 7.28) E+00$ \\
\hline $\begin{array}{l}\text { Augusta Lock and Dam } \\
614\end{array}$ & 26 & $(1.33 \pm 0.41) E+01$ & $(1.00 \pm 0.06) E+02$ & $(-0.16 \pm 1.85) E-01$ \\
\hline Highway $301 @$ State Line & 25 & $(4.67 \pm 1.07) E+00$ & $(1.63 \pm 1.05) E+01$ & $(-0.55 \pm 1.16) E+01$ \\
\hline \multicolumn{5}{|l|}{ 100-Mile Radius } \\
\hline Savannah, Ga. & 12 & $(5.75 \pm 2.45) E+00$ & $(2.30 \pm 0.87) E+01$ & $(-0.89 \pm 1.25) E+01$ \\
\hline
\end{tabular}

Filter Paper

\begin{tabular}{lcccc}
\hline Co-60, pCi/cu m & & & & \\
\hline $\begin{array}{l}\text { On Site } \\
\quad \begin{array}{l}\text { Burial Ground North } \\
\text { Site Perimeter }\end{array}\end{array}$ & 52 & $(5.94 \pm 5.69) \mathrm{E}-04$ & $(7.08 \pm 3.28) \mathrm{E}-03$ & $(-1.05 \pm 0.49) \mathrm{E}-02$ \\
$\quad$ Allendale Gate & 52 & $(6.20 \pm 6.25) \mathrm{E}-04$ & $(1.10 \pm 0.36) \mathrm{E}-02$ & $(-1.16 \pm 2.21) \mathrm{E}-02$ \\
$\quad$ Barnwell Gate & 52 & $(1.28 \pm 0.54) \mathrm{E}-03$ & $(1.10 \pm 0.48) \mathrm{E}-02$ & $(-7.94 \pm 3.07) \mathrm{E}-03$ \\
$\quad$ D-Area & 52 & $(4.93 \pm 8.00) \mathrm{E}-04$ & $(2.34 \pm 0.55) \mathrm{E}-02$ & $(-1.99 \pm 3.47) \mathrm{E}-02$ \\
$\quad$ Darkhorse @ Williston Gate & 52 & $(1.41 \pm 5.61) \mathrm{E}-04$ & $(1.16 \pm 1.34) \mathrm{E}-02$ & $(-7.89 \pm 3.31) \mathrm{E}-03$
\end{tabular}


Table 8

Radioactivity in Air

Page 2 of 11

\begin{tabular}{|c|c|c|c|c|}
\hline Location & $\begin{array}{l}\text { No. of } \\
\text { Samples }\end{array}$ & Arithmetic Mean $\pm \sigma$ & Maximum $\pm \sigma$ & Minimum $\pm \sigma$ \\
\hline East Talatha & 52 & $(2.86 \pm 5.49) E-04$ & $(8.78 \pm 3.34) \mathrm{E}-03$ & $(-8.25 \pm 4.35) \mathrm{E}-03$ \\
\hline Green Pond & 52 & $(1.18 \pm 0.60) E-03$ & $(1.52 \pm 2.08) E-02$ & $(-9.00 \pm 4.49) E-03$ \\
\hline Highway 21/167 & 52 & $(5.35 \pm 4.78) \mathrm{E}-04$ & $(8.49 \pm 3.93) E-03$ & $(-6.53 \pm 3.31) E-03$ \\
\hline Jackson & 52 & $(-2.20 \pm 6.35) E-04$ & $(8.77 \pm 4.71) \mathrm{E}-03$ & $(-9.01 \pm 4.06) E-03$ \\
\hline Patterson Mill Road & 52 & $(2.43 \pm 5.54) E-04$ & $(8.35 \pm 2.22) E-03$ & $(-1.03 \pm 0.32) E-02$ \\
\hline Talatha Gate & 52 & $(1.85 \pm 6.94) E-04$ & $(9.65 \pm 5.22) \mathrm{E}-03$ & $(-1.16 \pm 0.59) \mathrm{E}-02$ \\
\hline West Jackson & 50 & $(4.98 \pm 6.26) E-04$ & $(1.04 \pm 0.43) \mathrm{E}-02$ & $(-6.45 \pm 4.59) E-03$ \\
\hline Windsor Road & 52 & $(1.20 \pm 0.58) E-03$ & $(8.36 \pm 5.91) \mathrm{E}-03$ & $(-7.48 \pm 4.40) E-03$ \\
\hline \multicolumn{5}{|l|}{ 25-Mile Radius } \\
\hline Aiken Airport & 52 & $(2.27 \pm 5.06) E-04$ & $(1.37 \pm 0.46) \mathrm{E}-02$ & $(-7.55 \pm 5.04) E-03$ \\
\hline $\begin{array}{l}\text { Augusta Lock and Dam } \\
614\end{array}$ & 52 & $(3.28 \pm 4.93) E-04$ & $(9.20 \pm 3.57) E-03$ & $(-7.03 \pm 4.20) E-03$ \\
\hline Highway $301 @$ State Line & 52 & $(5.71 \pm 8.00) E-04$ & $(2.61 \pm 5.38) E-02$ & $(-1.05 \pm 0.48) E-02$ \\
\hline \multicolumn{5}{|l|}{ 100-Mile Radius } \\
\hline Savannah, Ga. & 24 & $(1.27 \pm 1.04) E-03$ & $(1.22 \pm 0.49) \mathrm{E}-02$ & $(-7.20 \pm 3.56) E-03$ \\
\hline \multicolumn{5}{|l|}{ Cs-137, pCi/cu m } \\
\hline \multicolumn{5}{|l|}{ On Site } \\
\hline Burial Ground North & 52 & $(-0.76 \pm 4.12) E-04$ & $(5.22 \pm 4.00) E-03$ & $(-1.04 \pm 0.43) E-02$ \\
\hline \multicolumn{5}{|l|}{ Site Perimeter } \\
\hline Allendale Gate & 52 & $(3.49 \pm 4.83) E-04$ & $(9.22 \pm 3.07) \mathrm{E}-03$ & $(-1.06 \pm 1.65) E-02$ \\
\hline Barnwell Gate & 52 & $(0.97 \pm 5.10) E-04$ & $(1.02 \pm 0.42) E-02$ & $(-8.18 \pm 3.76) E-03$ \\
\hline D-Area & 52 & $(5.96 \pm 4.39) E-04$ & $(8.16 \pm 3.65) E-03$ & $(-5.69 \pm 4.47) E-03$ \\
\hline Darkhorse @ Williston Gate & 52 & $(-8.58 \pm 4.24) E-04$ & $(6.27 \pm 3.74) E-03$ & $(-6.66 \pm 3.37) E-03$ \\
\hline East Talatha & 52 & $(4.98 \pm 4.65) E-04$ & $(7.36 \pm 3.16) E-03$ & $(-8.45 \pm 3.39) E-03$ \\
\hline Green Pond & 52 & $(9.49 \pm 5.62) E-04$ & $(1.32 \pm 0.43) E-02$ & $(-8.30 \pm 3.38) E-03$ \\
\hline Highway $21 / 167$ & 52 & $(2.57 \pm 4.68) E-04$ & $(6.88 \pm 3.48) E-03$ & $(-9.48 \pm 4.70) E-03$ \\
\hline Jackson & 52 & $(8.11 \pm 5.69) E-04$ & $(1.18 \pm 0.37) E-02$ & $(-1.04 \pm 0.36) E-02$ \\
\hline Patterson Mill Road & 52 & $(0.16 \pm 4.29) E-04$ & $(7.60 \pm 4.17) \mathrm{E}-03$ & $(-6.85 \pm 3.55) E-03$ \\
\hline Talatha Gate & 52 & $(-0.85 \pm 5.53) E-04$ & $(9.96 \pm 2.92) E-03$ & $(-7.10 \pm 4.00) E-03$ \\
\hline West Jackson & 50 & $(3.28 \pm 5.43) E-04$ & $(1.21 \pm 0.30) E-02$ & $(-5.33 \pm 3.37) E-03$ \\
\hline Windsor Road & 52 & $(-6.18 \pm 4.73) E-04$ & $(6.34 \pm 3.91) E-03$ & $(-1.04 \pm 0.52) E-02$ \\
\hline \multicolumn{5}{|l|}{ 25-Mile Radius } \\
\hline Aiken Airport & 52 & $(6.67 \pm 4.65) E-04$ & $(7.07 \pm 4.10) E-03$ & $(-7.45 \pm 2.85) E-03$ \\
\hline $\begin{array}{l}\text { Augusta Lock and Dam } \\
614\end{array}$ & 52 & $(-2.57 \pm 4.43) E-04$ & $(7.42 \pm 3.27) E-03$ & $(-8.56 \pm 3.35) E-03$ \\
\hline
\end{tabular}


Table 8

Radioactivity in Air

Page 3 of 11

\begin{tabular}{|c|c|c|c|c|}
\hline Location & $\begin{array}{l}\text { No. of } \\
\text { Samples }\end{array}$ & Arithmetic Mean $\pm \sigma$ & Maximum $\pm \sigma$ & Minimum $\pm \sigma$ \\
\hline Highway 301 @ State Line & 52 & $(-7.55 \pm 4.96) E-04$ & $(6.30 \pm 5.65) E-03$ & $(-1.61 \pm 4.49) \mathrm{E}-02$ \\
\hline \multicolumn{5}{|l|}{ 100-Mile Radius } \\
\hline Savannah, Ga. & 24 & $(-0.85 \pm 6.22) \mathrm{E}-04$ & $(4.49 \pm 4.36) \mathrm{E}-03$ & $(-6.19 \pm 4.14) E-03$ \\
\hline \multicolumn{5}{|l|}{ Sr-89,90, pCi/cu m } \\
\hline \multicolumn{5}{|l|}{ On Site } \\
\hline Burial Ground North & 1 & $(9.84 \pm 8.74) \mathrm{E}-04$ & & \\
\hline \multicolumn{5}{|l|}{ Site Perimeter } \\
\hline Allendale Gate & 1 & $(2.02 \pm 8.24) E-04$ & & \\
\hline Barnwell Gate & 1 & $(1.69 \pm 8.60) E-04$ & & \\
\hline D-Area & 1 & $(0.78 \pm 1.02) E-03$ & & \\
\hline Darkhorse @ Williston Gate & 1 & $(1.07 \pm 0.95) E-03$ & & \\
\hline East Talatha & 1 & $(2.19 \pm 0.92) E-03$ & & \\
\hline Green Pond & 1 & $(0.50 \pm 1.33) E-03$ & & \\
\hline Highway $21 / 167$ & 1 & $(6.96 \pm 9.31) E-04$ & & \\
\hline Jackson & 1 & $(-5.59 \pm 8.11) E-04$ & & \\
\hline Patterson Mill Road & 1 & $(1.00 \pm 0.91) E-03$ & & \\
\hline Talatha Gate & 1 & $(1.51 \pm 1.02) E-03$ & & \\
\hline West Jackson & 1 & $(3.73 \pm 0.24) E-02$ & & \\
\hline Windsor Road & 1 & $(5.42 \pm 9.22) E-04$ & & \\
\hline \multicolumn{5}{|l|}{ 25-Mile Radius } \\
\hline Aiken Airport & 1 & $(2.09 \pm 0.95) E-03$ & & \\
\hline $\begin{array}{l}\text { Augusta Lock and Dam } \\
614\end{array}$ & 1 & $(5.71 \pm 9.22) \mathrm{E}-04$ & & \\
\hline Highway $301 @$ State Line & 1 & $(6.01 \pm 9.71) E-04$ & & \\
\hline \multicolumn{5}{|l|}{ 100-Mile Radius } \\
\hline Savannah, Ga. & 1 & $(1.85 \pm 9.42) E-04$ & & \\
\hline \multicolumn{5}{|l|}{ U-234, pCi/cu m } \\
\hline \multicolumn{5}{|l|}{ On Site } \\
\hline Burial Ground North & 1 & $(1.59 \pm 6.51) \mathrm{E}-06$ & & \\
\hline \multicolumn{5}{|l|}{ Site Perimeter } \\
\hline Allendale Gate & 1 & $(-4.49 \pm 5.36) E-06$ & & \\
\hline Barnwell Gate & 1 & $(2.40 \pm 0.91) E-05$ & & \\
\hline D-Area & 1 & $(1.64 \pm 0.86) E-05$ & & \\
\hline Darkhorse @ Williston Gate & 1 & $(-3.29 \pm 4.63) E-06$ & & \\
\hline
\end{tabular}


Table 8

Radioactivity in Air

Page 4 of 11

\begin{tabular}{|c|c|c|c|c|}
\hline Location & $\begin{array}{l}\text { No. of } \\
\text { Samples }\end{array}$ & Arithmetic Mean $\pm \sigma$ & Maximum $\pm \sigma$ & Minimum $\pm \sigma$ \\
\hline East Talatha & 1 & $(6.44 \pm 5.68) E-06$ & & \\
\hline Green Pond & 1 & $(1.03 \pm 3.53) E-05$ & & \\
\hline Highway $21 / 167$ & 1 & $(4.58 \pm 7.39) E-06$ & & \\
\hline Jackson & 1 & $(2.70 \pm 0.89) E-05$ & & \\
\hline Patterson Mill Road & 1 & $(1.45 \pm 0.82) E-05$ & & \\
\hline Talatha Gate & 1 & $(-1.59 \pm 0.35) E-05$ & & \\
\hline West Jackson & 1 & $(-3.53 \pm 5.73) E-06$ & & \\
\hline Windsor Road & 1 & $(-0.34 \pm 6.25) E-06$ & & \\
\hline \multicolumn{5}{|l|}{ 25-Mile Radius } \\
\hline Aiken Airport & 1 & $(5.56 \pm 6.58) \mathrm{E}-06$ & & \\
\hline $\begin{array}{l}\text { Augusta Lock and Dam } \\
614\end{array}$ & 1 & $(0.52 \pm 7.01) \mathrm{E}-06$ & & \\
\hline Highway $301 @$ State Line & 1 & $(8.44 \pm 8.07) E-06$ & & \\
\hline \multicolumn{5}{|l|}{ 100-Mile Radius } \\
\hline Savannah, Ga. & 1 & $(7.23 \pm 7.07) E-06$ & & \\
\hline \multicolumn{5}{|l|}{$\mathrm{U}-235, \mathrm{pci} / \mathrm{cu} \mathrm{m}$} \\
\hline \multicolumn{5}{|l|}{ On Site } \\
\hline Burial Ground North & 1 & $(7.20 \pm 4.19) \mathrm{E}-06$ & & \\
\hline \multicolumn{5}{|l|}{ Site Perimeter } \\
\hline Allendale Gate & 1 & $(2.14 \pm 2.14) \mathrm{E}-06$ & & \\
\hline Barnwell Gate & 1 & $(1.10 \pm 0.50) E-05$ & & \\
\hline D-Area & 1 & $(-1.67 \pm 1.19) E-06$ & & \\
\hline Darkhorse @ Williston Gate & 1 & $(5.68 \pm 3.18) E-06$ & & \\
\hline East Talatha & 1 & $(1.05 \pm 0.43) E-05$ & & \\
\hline Green Pond & 1 & $(2.47 \pm 1.76) E-05$ & & \\
\hline Highway $21 / 167$ & 1 & $(0.73 \pm 2.66) E-06$ & & \\
\hline Jackson & 1 & $(1.61 \pm 0.58) \mathrm{E}-05$ & & \\
\hline Patterson Mill Road & 1 & $(1.14 \pm 0.51) \mathrm{E}-05$ & & \\
\hline Talatha Gate & 1 & $(7.95 \pm 4.02) E-06$ & & \\
\hline West Jackson & 1 & $(1.51 \pm 2.46) E-06$ & & \\
\hline Windsor Road & 1 & $(5.75 \pm 3.85) E-06$ & & \\
\hline \multicolumn{5}{|l|}{ 25-Mile Radius } \\
\hline Aiken Airport & 1 & $(2.06 \pm 2.07) E-06$ & & \\
\hline $\begin{array}{l}\text { Augusta Lock and Dam } \\
614\end{array}$ & 1 & $(-9.39 \pm 9.43) E-07$ & & \\
\hline
\end{tabular}




\section{Table 8}

Radioactivity in Air

Page 5 of 11

\begin{tabular}{|c|c|c|c|c|}
\hline Location & $\begin{array}{l}\text { No. of } \\
\text { Samples }\end{array}$ & Arithmetic Mean $\pm \sigma$ & Maximum $\pm \sigma$ & Minimum $\pm \sigma$ \\
\hline Highway $301 @$ State Line & 1 & $(2.73 \pm 2.74) E-06$ & & \\
\hline \multicolumn{5}{|l|}{ 100-Mile Radius } \\
\hline Savannah, Ga. & 1 & $(6.95 \pm 4.05) E-06$ & & \\
\hline \multicolumn{5}{|l|}{ U-238, pCi/cu m } \\
\hline \multicolumn{5}{|l|}{ On Site } \\
\hline Burial Ground North & 1 & $(1.79 \pm 0.79) E-05$ & & \\
\hline \multicolumn{5}{|l|}{ Site Perimeter } \\
\hline Allendale Gate & 1 & $(9.72 \pm 6.30) E-06$ & & \\
\hline Barnwell Gate & 1 & $(1.77 \pm 0.76) E-05$ & & \\
\hline D-Area & 1 & $(3.31 \pm 5.85) E-06$ & & \\
\hline Darkhorse @ Williston Gate & 1 & $(0.78 \pm 4.20) E-06$ & & \\
\hline East Talatha & 1 & $(2.19 \pm 0.68) E-05$ & & \\
\hline Green Pond & 1 & $(3.11 \pm 3.37) E-05$ & & \\
\hline Highway 21/167 & 1 & $(-0.10 \pm 5.11) E-06$ & & \\
\hline Jackson & 1 & $(9.11 \pm 5.92) E-06$ & & \\
\hline Patterson Mill Road & 1 & $(1.13 \pm 0.70) E-05$ & & \\
\hline Talatha Gate & 1 & $(-2.95 \pm 4.27) E-06$ & & \\
\hline West Jackson & 1 & $(5.62 \pm 5.95) E-06$ & & \\
\hline Windsor Road & 1 & $(1.02 \pm 0.64) E-05$ & & \\
\hline \multicolumn{5}{|l|}{ 25-Mile Radius } \\
\hline Aiken Airport & 1 & $(1.28 \pm 0.65) E-05$ & & \\
\hline $\begin{array}{l}\text { Augusta Lock and Dam } \\
614\end{array}$ & 1 & $(6.95 \pm 6.99) E-06$ & & \\
\hline Highway $301 @$ State Line & 1 & $(6.43 \pm 6.64) \mathrm{E}-06$ & & \\
\hline \multicolumn{5}{|l|}{ 100-Mile Radius } \\
\hline Savannah, Ga. & 1 & $(3.78 \pm 5.64) E-06$ & & \\
\hline \multicolumn{5}{|l|}{ Pu-238, pCi/cu m } \\
\hline \multicolumn{5}{|l|}{ On Site } \\
\hline Burial Ground North & 1 & $(-6.81 \pm 1.49) E-06$ & & \\
\hline \multicolumn{5}{|l|}{ Site Perimeter } \\
\hline Allendale Gate & 1 & $(-3.41 \pm 2.18) E-06$ & & \\
\hline Barnwell Gate & 1 & $(-3.82 \pm 2.49) E-06$ & & \\
\hline D-Area & 1 & $(-2.38 \pm 2.32) E-06$ & & \\
\hline Darkhorse @ Williston Gate & 1 & $(-4.90 \pm 0.79) E-06$ & & \\
\hline
\end{tabular}




\section{Table 8}

\section{Radioactivity in Air}

Page 6 of 11

\begin{tabular}{|c|c|c|c|c|}
\hline Location & $\begin{array}{l}\text { No. of } \\
\text { Samples }\end{array}$ & Arithmetic Mean $\pm \sigma$ & Maximum $\pm \sigma$ & Minimum $\pm \sigma$ \\
\hline East Talatha & 1 & $(-0.46 \pm 3.01) E-06$ & & \\
\hline Green Pond & 1 & $(-2.63 \pm 0.40) E-0.5$ & & \\
\hline Highway $21 / 167$ & 1 & $(-5.39 \pm 2.79) E-06$ & & \\
\hline Jackson & 1 & $(-4.02 \pm 0.24) E-06$ & & \\
\hline Patterson Mill Road & 1 & $(-2.21 \pm 2.11) E-06$ & & \\
\hline Talatha Gate & 1 & $(-2.25 \pm 2.47) E-06$ & & \\
\hline West Jackson & 1 & $(-4.37 \pm 0.26) E-06$ & & \\
\hline Windsor Road & 1 & $(-6.44 \pm 1.35) E-06$ & & \\
\hline \multicolumn{5}{|l|}{ 25-Mile Radius } \\
\hline Aiken Airport & 1 & $(-4.02 \pm 0.24) E-06$ & & \\
\hline $\begin{array}{l}\text { Augusta Lock and Dam } \\
614\end{array}$ & 1 & $(-2.37 \pm 2.34) E-06$ & & \\
\hline Highway $301 @$ State Line & 1 & $(-4.42 \pm 0.27) E-06$ & & \\
\hline \multicolumn{5}{|l|}{ 100-Mile Radius } \\
\hline Savannah, Ga. & 1 & $(-0.78 \pm 3.15) E-06$ & & \\
\hline \multicolumn{5}{|l|}{ Pu-239, pCi/cu m } \\
\hline \multicolumn{5}{|l|}{ On Site } \\
\hline Burial Ground North & 1 & $(-0.93 \pm 2.89) E-06$ & & \\
\hline \multicolumn{5}{|l|}{ Site Perimeter } \\
\hline Allendale Gate & 1 & $(7.09 \pm 3.97) E-06$ & & \\
\hline Barnwell Gate & 1 & $(0.00 \pm 0.00) E+00$ & & \\
\hline D-Area & 1 & $(0.71 \pm 2.57) E-06$ & & \\
\hline Darkhorse @ Williston Gate & 1 & $(5.61 \pm 3.74) E-06$ & & \\
\hline East Talatha & 1 & $(3.42 \pm 3.01) \mathrm{E}-06$ & & \\
\hline Green Pond & 1 & $(0.00 \pm 0.00) E+00$ & & \\
\hline Highway $21 / 167$ & 1 & $(0.00 \pm 0.00) E+00$ & & \\
\hline Jackson & 1 & $(6.28 \pm 3.63) E-06$ & & \\
\hline Patterson Mill Road & 1 & $(3.47 \pm 3.08) E-06$ & & \\
\hline Talatha Gate & 1 & $(4.93 \pm 3.50) E-06$ & & \\
\hline West Jackson & 1 & $(0.00 \pm 0.00) E+00$ & & \\
\hline Windsor Road & 1 & $(0.66 \pm 2.39) E-06$ & & \\
\hline \multicolumn{5}{|l|}{ 25-Mile Radius } \\
\hline Aiken Airport & 1 & $(0.67 \pm 2.42) E-06$ & & \\
\hline $\begin{array}{l}\text { Augusta Lock and Dam } \\
614\end{array}$ & 1 & $(8.53 \pm 4.77) E-06$ & & \\
\hline
\end{tabular}


Table 8

Radioactivity in Air

Page 7 of 11

\begin{tabular}{|c|c|c|c|c|}
\hline Location & $\begin{array}{l}\text { No. of } \\
\text { Samples }\end{array}$ & Arithmetic Mean $\pm \sigma$ & Maximum $\pm \sigma$ & Minimum $\pm \sigma$ \\
\hline Highway 301 @ State Line & 1 & $(1.44 \pm 2.34) \mathrm{E}-06$ & & \\
\hline \multicolumn{5}{|l|}{ 100-Mile Radius } \\
\hline Savannah, Ga. & 1 & $(1.41 \pm 2.29) E-06$ & & \\
\hline \multicolumn{5}{|l|}{$\mathrm{Am}-241, \mathrm{pCi} / \mathrm{cu} \mathrm{m}$} \\
\hline \multicolumn{5}{|l|}{ On Site } \\
\hline Burial Ground North & 1 & $(-0.04 \pm 2.32) E-06$ & & \\
\hline \multicolumn{5}{|l|}{ Site Perimeter } \\
\hline Allendale Gate & 1 & $(1.08 \pm 0.50) E-05$ & & \\
\hline Barnwell Gate & 1 & $(4.22 \pm 3.83) E-06$ & & \\
\hline D-Area & 1 & $(6.91 \pm 4.25) \mathrm{E}-06$ & & \\
\hline Darkhorse @ Williston Gate & 1 & $(1.35 \pm 0.60) E-05$ & & \\
\hline East Talatha & 1 & $(5.59 \pm 4.30) E-06$ & & \\
\hline Green Pond & 1 & $(1.53 \pm 1.62) \mathrm{E}-05$ & & \\
\hline Highway 21/167 & 1 & $(1.63 \pm 0.67) E-05$ & & \\
\hline Jackson & 1. & $(1.20 \pm 0.51) \mathrm{E}-05$ & & \\
\hline Patterson Mill Road & 1 & $(7.51 \pm 4.49) E-06$ & & \\
\hline Talatha Gate & 1 & $(1.04 \pm 0.58) E-05$ & & \\
\hline West Jackson & 1 & $(1.27 \pm 0.58) E-05$ & & \\
\hline Windsor Road & 1 & $(1.58 \pm 0.65) E-05$ & & \\
\hline \multicolumn{5}{|l|}{ 25-Mile Radius } \\
\hline Aiken Airport & 1 & $(9.28 \pm 4.78) E-06$ & & \\
\hline $\begin{array}{l}\text { Augusta Lock and Dam } \\
614\end{array}$ & 1 & $(3.19 \pm 4.47) \mathrm{E}-06$ & & \\
\hline Highway $301 @$ State Line & 1 & $(1.44 \pm 0.61) E-05$ & & \\
\hline \multicolumn{5}{|l|}{ 100-Mile Radius } \\
\hline Savannah, Ga. & 1 & $(6.64 \pm 4.52) \mathrm{E}-06$ & & \\
\hline \multicolumn{5}{|l|}{$\mathrm{Cm}-244, \mathrm{pCi} / \mathrm{cu} \mathrm{m}$} \\
\hline \multicolumn{5}{|l|}{ On Site } \\
\hline Burial Ground North & 1 & $(4.40 \pm 3.10) E-06$ & & \\
\hline \multicolumn{5}{|l|}{ Site Perimeter } \\
\hline Allendale Gate & 1 & $(0.00 \pm 0.00) E+00$ & & \\
\hline Barnwell Gate & 1 & $(-1.50 \pm 1.07) E-06$ & & \\
\hline D-Area & 1 & $(0.00 \pm 0.00) E+00$ & & \\
\hline Darkhorse @ Williston Gate & 1 & $(0.00 \pm 0.00) E+00$ & & \\
\hline
\end{tabular}




\section{Table 8}

\section{Radioactivity in Air}

Page 8 of 11

\begin{tabular}{|c|c|c|c|c|}
\hline Location & $\begin{array}{l}\text { No. of } \\
\text { Samples }\end{array}$ & Arithmetic Mean $\pm \sigma$ & Maximum $\pm \sigma$ & Minimum $\pm \sigma$ \\
\hline East Talatha & 1 & $(0.00 \pm 0.00) E+00$ & & \\
\hline Green Pond & 1 & $(1.15 \pm 1.15) E-05$ & & \\
\hline Highway $21 / 167$ & 1 & $(2.34 \pm 2.34) \mathrm{E}-06$ & & \\
\hline Jackson & 1 & $(0.00 \pm 0.00) E+00$ & & \\
\hline Patterson Mill Road & 1 & $(1.46 \pm 2.38) E-06$ & & \\
\hline Talatha Gate & 1 & $(1.68 \pm 2.72) E-06$ & & \\
\hline West Jackson & 1 & $(0.00 \pm 0.00) E+00$ & & \\
\hline Windsor Road & 1 & $(4.49 \pm 3.19) E-06$ & & \\
\hline \multicolumn{5}{|l|}{ 25-Mile Radius } \\
\hline Aiken Airport & 1 & $(0.00 \pm 0.00) E+00$ & & \\
\hline $\begin{array}{l}\text { Augusta Lock and Dam } \\
614\end{array}$ & 1 & $(4.04 \pm 3.56) E-06$ & & \\
\hline Highway $301 @$ State Line & 1 & $(0.70 \pm 2.54) E-06$ & & \\
\hline \multicolumn{5}{|l|}{ 100-Mile Radius } \\
\hline Savannah, Ga. & 1 & $(-1.54 \pm 1.09) E-06$ & & \\
\hline \multicolumn{5}{|l|}{ Gross Alpha, pCi/cu m } \\
\hline \multicolumn{5}{|l|}{ On Site } \\
\hline Burial Ground North & 52 & $(1.94 \pm 0.13) E-03$ & $(4.61 \pm 0.75) \mathrm{E}-03$ & $(3.53 \pm 3.08) E-04$ \\
\hline \multicolumn{5}{|l|}{ Site Perimeter } \\
\hline Allendale Gate & 52 & $(2.01 \pm 0.15) E-03$ & $(5.11 \pm 0.77) E-03$ & $(1.98 \pm 2.81) E-04$ \\
\hline Barnwell Gate & 52 & $(2.02 \pm 0.15) E-03$ & $(4.87 \pm 0.95) E-03$ & $(4.17 \pm 3.49) E-04$ \\
\hline D-Area & 52 & $(1.92 \pm 0.16) E-03$ & $(6.68 \pm 3.18) E-03$ & $(-0.46 \pm 2.96) E-04$ \\
\hline Darkhorse @ Williston Gate & 52 & $(2.10 \pm 0.14) E-03$ & $(4.62 \pm 0.72) E-03$ & $(3.00 \pm 2.16) E-04$ \\
\hline East Talatha & 52 & $(1.83 \pm 0.13) E-03$ & $(4.37 \pm 0.77) \mathrm{E}-03$ & $(-0.92 \pm 2.23) \mathrm{E}-04$ \\
\hline Green Pond & 52 & $(2.20 \pm 0.16) E-03$ & $(5.17 \pm 0.83) E-03$ & $(6.07 \pm 3.88) E-04$ \\
\hline Highway $21 / 167$ & 52 & $(2.03 \pm 0.14) E-03$ & $(4.96 \pm 0.81) E-03$ & $(5.34 \pm 3.82) E-04$ \\
\hline Jackson & 52 & $(1.82 \pm 0.15) E-03$ & $(4.43 \pm 0.74) E-03$ & $(0.11 \pm 3.39) E-04$ \\
\hline Patterson Mill Road & 52 & $(2.04 \pm 0.15) E-03$ & $(5.54 \pm 1.45) E-03$ & $(3.38 \pm 3.22) E-04$ \\
\hline Talatha Gate & 52 & $(2.03 \pm 0.15) E-03$ & $(5.15 \pm 0.86) E-03$ & $(3.86 \pm 3.92) E-04$ \\
\hline West Jackson & 50 & $(2.19 \pm 0.15) E-03$ & $(5.45 \pm 0.95) E-03$ & $(2.85 \pm 3.21) E-04$ \\
\hline Windsor Road & 52 & $(2.11 \pm 0.13) E-03$ & $(4.39 \pm 0.54) \mathrm{E}-03$ & $(4.94 \pm 2.55) E-04$ \\
\hline \multicolumn{5}{|l|}{ 25-Mile Radius } \\
\hline Aiken Airport & 52 & $(1.83 \pm 0.13) E-03$ & $(4.58 \pm 0.91) E-03$ & $(2.37 \pm 2.89) \mathrm{E}-04$ \\
\hline $\begin{array}{l}\text { Augusta Lock and Dam } \\
614\end{array}$ & 52 & $(1.98 \pm 0.15) E-03$ & $(5.14 \pm 0.84) E-03$ & $(4.24 \pm 3.71) E-04$ \\
\hline
\end{tabular}




\section{Table 8}

Radioactivity in Air

Page 9 of 11

\begin{tabular}{|c|c|c|c|c|}
\hline Location & $\begin{array}{l}\text { No. of } \\
\text { Samples }\end{array}$ & Arithmetic Mean $\pm \sigma$ & Maximum $\pm \sigma$ & Minimum $\pm \sigma$ \\
\hline Highway $301 @$ State Line & 52 & $(2.10 \pm 0.18) E-03$ & $(6.51 \pm 1.01) \mathrm{E}-03$ & $(2.25 \pm 3.35) \mathrm{E}-04$ \\
\hline \multicolumn{5}{|l|}{ 100-Mile Radius } \\
\hline Savannah, Ga. & 24 & $(2.07 \pm 0.20) E-03$ & $(3.74 \pm 0.74) E-03$ & $(2.94 \pm 3.15) E-04$ \\
\hline \multicolumn{5}{|l|}{ Gross Beta, pCi/cu m } \\
\hline \multicolumn{5}{|l|}{ On Site } \\
\hline Burial Ground North & 52 & $(1.90 \pm 0.09) E-02$ & $(3.67 \pm 0.17) E-02$ & $(9.18 \pm 0.94) E-03$ \\
\hline \multicolumn{5}{|l|}{ Site Perimeter } \\
\hline Allendale Gate & 52 & $(1.95 \pm 0.08) E-02$ & $(3.75 \pm 0.16) E-02$ & $(8.86 \pm 0.89) E-03$ \\
\hline Barnwell Gate & 52 & $(1.96 \pm 0.09) E-02$ & $(3.89 \pm 0.17) \mathrm{E}-02$ & $(7.71 \pm 0.91) E-03$ \\
\hline D-Area & 52 & $(1.95 \pm 0.10) E-02$ & $(3.67 \pm 0.18) E-02$ & $(7.47 \pm 0.95) E-03$ \\
\hline Darkhorse@ Williston Gate & 52 & $(1.92 \pm 0.09) E-02$ & $(3.71 \pm 0.12) E-02$ & $(6.99 \pm 0.58) E-03$ \\
\hline East Talatha & 52 & $(1.91 \pm 0.09) E-02$ & $(3.43 \pm 0.16) E-02$ & $(2.98 \pm 0.69) E-03$ \\
\hline Green Pond & 52 & $(1.96 \pm 0.08) E-02$ & $(4.08 \pm 0.19) E-02$ & $(1.04 \pm 0.11) E-02$ \\
\hline Highway $21 / 167$ & 52 & $(1.93 \pm 0.09) E-02$ & $(3.82 \pm 0.18) E-02$ & $(7.25 \pm 0.89) E-03$ \\
\hline Jackson & 52 & $(1.74 \pm 0.08) E-02$ & $(3.30 \pm 0.17) E-02$ & $(8.02 \pm 0.92) E-03$ \\
\hline Patterson Mill Road & 52 & $(1.88 \pm 0.09) E-02$ & $(4.29 \pm 0.30) E-02$ & $(8.81 \pm 0.93) E-03$ \\
\hline Talatha Gate & 52 & $(1.93 \pm 0.09) E-02$ & $(3.82 \pm 0.20) E-02$ & $(1.00 \pm 0.12) \mathrm{E}-02$ \\
\hline West Jackson & 50 & $(2.09 \pm 0.10) E-02$ & $(4.04 \pm 0.17) E-02$ & $(9.91 \pm 0.98) E-03$ \\
\hline Windsor Road & 52 & $(1.98 \pm 0.08) E-02$ & $(3.60 \pm 0.12) E-02$ & $(1.01 \pm 0.07) E-02$ \\
\hline \multicolumn{5}{|l|}{ 25-Mile Radius } \\
\hline Aiken Airport & 52 & $(1.87 \pm 0.09) E-02$ & $(3.95 \pm 0.16) E-02$ & $(8.52 \pm 0.87) \mathrm{E}-03$ \\
\hline $\begin{array}{l}\text { Augusta Lock and Dam } \\
614\end{array}$ & 52 & $(1.86 \pm 0.09) E-02$ & $(4.20 \pm 0.18) E-02$ & $(9.54 \pm 0.96) E-03$ \\
\hline Highway $301 @$ State Line & 52 & $(2.04 \pm 0.11) E-02$ & $(4.77 \pm 0.19) \mathrm{E}-02$ & $(7.95 \pm 0.94) E-03$ \\
\hline \multicolumn{5}{|l|}{ 100-Mile Radius } \\
\hline Savannah, Ga. & 24 & $(1.88 \pm 0.15) E-02$ & $(3.16 \pm 0.16) E-02$ & $(6.11 \pm 0.85) E-03$ \\
\hline \multicolumn{5}{|c|}{ Charcoal } \\
\hline \multicolumn{5}{|l|}{$\mathrm{Co}-60, \mathrm{pCi} / \mathrm{cu} \mathrm{m}$} \\
\hline \multicolumn{5}{|l|}{ On Site } \\
\hline Burial Ground North & 1 & $(4.39 \pm 3.55) E-03$ & & \\
\hline \multicolumn{5}{|l|}{ Site Perimeter } \\
\hline Allendale Gate & 1 & $(0.55 \pm 3.32) E-03$ & & \\
\hline Barnwell Gate & 1 & $(2.76 \pm 3.10) E-03$ & & \\
\hline D-Area & 1 & $(-1.15 \pm 3.85) E-03$ & & \\
\hline
\end{tabular}


Table 8

Radioactivity in Air

Page 10 of 11

\begin{tabular}{|c|c|c|c|c|}
\hline Location & $\begin{array}{l}\text { No. of } \\
\text { Samples }\end{array}$ & Arithmetic Mean $\pm \sigma$ & Maximum $\pm \sigma$ & Minimum $\pm \sigma$ \\
\hline Darkhorse @ Williston Gate & 1 & $(-0.86 \pm 3.35) \mathrm{E}-03$ & & \\
\hline East Talatha & 1 & $(1.31 \pm 2.71) \mathrm{E}-03$ & & \\
\hline Green Pond & 1 & $(-1.18 \pm 0.56) E-02$ & & \\
\hline Highway 21/167 & 1 & $(-4.52 \pm 3.43) E-03$ & & \\
\hline Jackson & 1 & $(-7.16 \pm 3.55) E-03$ & & \\
\hline Patterson Mill Road & 1 & $(-1.27 \pm 3.17) E-03$ & & \\
\hline Talatha Gate & 1 & $(-3.91 \pm 3.78) E-03$ & & \\
\hline West Jackson & 1 & $(7.57 \pm 3.26) E-03$ & & \\
\hline Windsor Road & 1 & $(4.39 \pm 3.84) E-03$ & & \\
\hline \multicolumn{5}{|l|}{ 25-Mile Radius } \\
\hline Aiken Airport & 1 & $(1.98 \pm 3.64) E-03$ & & \\
\hline $\begin{array}{l}\text { Augusta Lock and Dam } \\
614\end{array}$ & 1 & $(1.91 \pm 3.72) E-03$ & & \\
\hline Highway $301 @$ State Line & 1 & $(-0.25 \pm 3.66) E-03$ & & \\
\hline \multicolumn{5}{|l|}{ 100-Mile Radius } \\
\hline Savannah, Ga. & 1 & $(-1.49 \pm 3.95) E-03$ & & \\
\hline \multicolumn{5}{|l|}{ Cs-137, pCi/cu m } \\
\hline \multicolumn{5}{|l|}{ On Site } \\
\hline Burial Ground North & 1 & $(2.52 \pm 3.24) E-03$ & & \\
\hline \multicolumn{5}{|l|}{ Site Perimeter } \\
\hline Allendale Gate & 1 & $(6.51 \pm 3.11) \mathrm{E}-03$ & & \\
\hline Barnwell Gate & 1 & $(7.62 \pm 3.87) E-03$ & & \\
\hline D-Area & 1 & $(-1.64 \pm 3.68) E-03$ & & \\
\hline Darkhorse @ Williston Gate & 1 & $(-3.02 \pm 3.59) E-03$ & & \\
\hline East Talatha & 1 & $(1.79 \pm 2.84) E-03$ & & \\
\hline Green Pond & 1 & $(1.11 \pm 4.78) E-03$ & & \\
\hline Highway 21/167 & 1 & $(2.39 \pm 3.44) E-03$ & & \\
\hline Jackson & 1 & $(8.50 \pm 3.32) E-03$ & & \\
\hline Patterson Mill Road & 1 & $(3.50 \pm 3.29) E-03$ & & \\
\hline Talatha Gate & 1 & $(2.10 \pm 4.09) \mathrm{E}-03$ & & \\
\hline West Jackson & 1 & $(3.34 \pm 3.70) E-03$ & & \\
\hline Windsor Road & 1 & $(0.35 \pm 3.99) E-03$ & & \\
\hline \multicolumn{5}{|l|}{ 25-Mile Radius } \\
\hline Aiken Airport & 1 & $(0.26 \pm 3.59) E-03$ & & \\
\hline
\end{tabular}


Table 8

Radioactivity in Air

Page 11 of 11

No. of

Location

Samples

Arithmetic Mean $\pm \sigma$

Maximum $\pm \sigma$

Minimum $\pm \sigma$

Augusta Lock and Dam

$(2.33 \pm 3.31) E-03$

614

Highway 301 @ State Line

100-Mile Radius

Savannah, Ga.

$1 \quad(-1.61 \pm 3.54) E-03$ 


\section{Table 9}

\section{Tritium in Rainwater}

Page 1 of 1

\begin{tabular}{|c|c|c|c|c|}
\hline Location & $\begin{array}{l}\text { No. of } \\
\text { Samples }\end{array}$ & Arithmetic Mean $\pm \sigma$ & Maximum $\pm \sigma$ & Minimum $\pm \sigma$ \\
\hline \multicolumn{5}{|l|}{$\mathrm{H}-3, \mathrm{pCi} / \mathrm{L}$} \\
\hline \multicolumn{5}{|l|}{ On Site } \\
\hline Burial Ground North & 25 & $(2.73 \pm 0.53) E+03$ & $(1.23 \pm 0.05) E+04$ & $(0.64 \pm 3.80) E+02$ \\
\hline \multicolumn{5}{|l|}{ Site Perimeter } \\
\hline Allendale Gate & 26 & $(1.69 \pm 1.00) E+02$ & $(2.15 \pm 0.30) E+03$ & $(-4.80 \pm 3.00) E+02$ \\
\hline Barnwell Gate & 25 & $(4.92 \pm 3.20) E+02$ & $(8.03 \pm 0.31) E+03$ & $(-5.11 \pm 2.85) E+02$ \\
\hline D-Area & 26 & $(4.83 \pm 1.04) E+02$ & $(1.43 \pm 0.31) E+03$ & $(-5.99 \pm 3.71) E+02$ \\
\hline $\begin{array}{l}\text { Darkhorse @ Williston } \\
\text { Gate }\end{array}$ & 26 & $(1.14 \pm 0.70) E+02$ & $(8.94 \pm 3.59) E+02$ & $(-5.35 \pm 2.84) E+02$ \\
\hline East Talatha & 25 & $(2.99 \pm 0.91) E+02$ & $(1.36 \pm 0.37) E+03$ & $(-6.07 \pm 4.18) E+02$ \\
\hline Green Pond & 25 & $(1.62 \pm 0.67) \mathrm{E}+02$ & $(7.45 \pm 1.82) \mathrm{E}+02$ & $(-3.99 \pm 4.95) E+02$ \\
\hline Highway $21 / 167$ & 25 & $(2.94 \pm 1.04) E+02$ & $(2.03 \pm 0.47) E+03$ & $(-6.89 \pm 3.82) E+02$ \\
\hline Jackson & 25 & $(2.61 \pm 0.94) E+02$ & $(1.28 \pm 0.41) E+03$ & $(-4.52 \pm 3.41) E+02$ \\
\hline Patterson Mill Road & 25 & $(1.87 \pm 0.63) E+02$ & $(1.01 \pm 0.33) E+03$ & $(-2.82 \pm 4.27) E+02$ \\
\hline Talatha Gate & 25 & $(3.29 \pm 1.21) E+02$ & $(2.47 \pm 0.30) E+03$ & $(-4.36 \pm 2.76) E+02$ \\
\hline West Jackson & 25 & $(1.91 \pm 0.74) \mathrm{E}+02$ & $(9.21 \pm 3.04) E+02$ & $(-5.69 \pm 4.23) E+02$ \\
\hline Windsor Road & 26 & $(3.17 \pm 1.08) E+02$ & $(1.86 \pm 0.36) E+03$ & $(-5.47 \pm 3.71) E+02$ \\
\hline \multicolumn{5}{|l|}{ 25-Mile Radius } \\
\hline Aiken Airport & 25 & $(3.08 \pm 4.95) E+01$ & $(7.27 \pm 4.44) E+02$ & $(-4.15 \pm 2.73) E+02$ \\
\hline $\begin{array}{l}\text { Augusta Lock and Dam } \\
614\end{array}$ & 25 & $(8.01 \pm 5.30) E+01$ & $(5.74 \pm 4.00) E+02$ & $(-3.53 \pm 3.90) E+02$ \\
\hline Highway $301 @$ State Line & 25 & $(7.39 \pm 5.90) E+01$ & $(7.49 \pm 3.51) E+02$ & $(-4.71 \pm 3.43) E+02$ \\
\hline \multicolumn{5}{|l|}{ 100-Mile Radius } \\
\hline Savannah, Ga. & 25 & $(-0.86 \pm 5.56) E+01$ & $(5.42 \pm 3.47) E+02$ & $(-6.31 \pm 2.76) E+02$ \\
\hline
\end{tabular}


Table 10

Radioactivity in Rain Ion Columns

Page 1 of 5

\begin{tabular}{|c|c|c|c|c|}
\hline Location & $\begin{array}{l}\text { No. of } \\
\text { Samples }\end{array}$ & Arithmetic Mean $\pm \sigma$ & Maximum $\pm \sigma$ & Minimum $\pm \sigma$ \\
\hline \multicolumn{5}{|l|}{ Co-60, pCi/sq m } \\
\hline \multicolumn{5}{|l|}{ On Site } \\
\hline Burial Ground North & 13 & $(2.64 \pm 2.87) E+00$ & $(1.96 \pm 0.75) E+01$ & $(-1.77 \pm 0.97) E+01$ \\
\hline \multicolumn{5}{|l|}{ Site Perimeter } \\
\hline D-Area & 13 & $(-1.11 \pm 2.60) E+00$ & $(1.29 \pm 0.96) E+01$ & $(-1.57 \pm 0.85) E+01$ \\
\hline Darkhorse @ Williston Gate & 13 & $(3.89 \pm 2.60) \mathrm{E}+00$ & $(1.82 \pm 0.99) E+01$ & $(-1.30 \pm 0.85) E+01$ \\
\hline Green Pond & 13 & $(1.45 \pm 2.61) \mathrm{E}+00$ & $(1.82 \pm 0.82) E+01$ & $(-1.23 \pm 1.06) E+01$ \\
\hline Patterson Mill Road & 13 & $(2.68 \pm 2.58) E+00$ & $(1.98 \pm 0.94) E+01$ & $(-1.94 \pm 0.91) \mathrm{E}+01$ \\
\hline \multicolumn{5}{|l|}{ 25-Mile Radius } \\
\hline Highway $301 @$ State Line & 13 & $(1.66 \pm 3.04) E+00$ & $(2.62 \pm 1.16) E+01$ & $(-1.47 \pm 0.76) E+01$ \\
\hline \multicolumn{5}{|l|}{ 100-Mile Radius } \\
\hline Savannah, Ga. & 13 & $(-2.87 \pm 2.04) E+00$ & $(1.37 \pm 1.25) E+01$ & $(-1.27 \pm 1.00) E+01$ \\
\hline \multicolumn{5}{|l|}{ Cs-137, pCi/sq m } \\
\hline \multicolumn{5}{|l|}{ On Site } \\
\hline Burial Ground North & 13 & $(-2.35 \pm 2.14) E+00$ & $(1.35 \pm 0.88) E+01$ & $(-1.42 \pm 0.96) E+01$ \\
\hline \multicolumn{5}{|l|}{ Site Perimeter } \\
\hline D-Area & 13 & $(0.98 \pm 2.09) E+00$ & $(1.34 \pm 0.85) E+01$ & $(-1.03 \pm 0.93) E+01$ \\
\hline Darkhorse @ Williston Gate & 13 & $(0.30 \pm 2.40) E+00$ & $(1.52 \pm 0.92) E+01$ & $(-1.68 \pm 0.85) E+01$ \\
\hline Green Pond & 13 & $(0.23 \pm 2.82) E+00$ & $(1.64 \pm 1.00) \mathrm{E}+01$ & $(-1.55 \pm 0.85) E+01$ \\
\hline Patterson Mill Road & 13 & $(9.04 \pm 1.92) E+00$ & $(1.89 \pm 0.82) \mathrm{E}+01$ & $(-5.52 \pm 8.86) E+00$ \\
\hline \multicolumn{5}{|l|}{ 25-Mile Radius } \\
\hline Highway $301 @$ State Line & 13 & $(2.73 \pm 3.13) \mathrm{E}+00$ & $(2.47 \pm 0.96) E+01$ & $(-1.51 \pm 0.90) E+01$ \\
\hline \multicolumn{5}{|l|}{ 100-Mile Radius } \\
\hline Savannah, Ga. & 13 & $(-0.07 \pm 3.33) E+00$ & $(1.58 \pm 0.86) E+01$ & $(-2.02 \pm 0.94) E+01$ \\
\hline \multicolumn{5}{|l|}{ Sr-89,90, pCi/sq m } \\
\hline \multicolumn{5}{|l|}{ On Site } \\
\hline Burial Ground North & 1 & $(-0.30 \pm 1.41) E+00$ & & \\
\hline \multicolumn{5}{|l|}{ Site Perimeter } \\
\hline D-Area & 1 & $(0.21 \pm 1.32) E+00$ & & \\
\hline Darkhorse @ Williston Gate & 1 & $(1.25 \pm 1.40) \mathrm{E}+00$ & & \\
\hline Green Pond & 1 & $(0.59 \pm 1.05) E+00$ & & \\
\hline Patterson Mill Road & 1 & $(-1.17 \pm 1.33) E+00$ & & \\
\hline \multicolumn{5}{|l|}{ 25-Mile Radius } \\
\hline Highway 301 @ State Line & 1 & $(-0.82 \pm 1.39) E+00$ & & \\
\hline
\end{tabular}


Table 10

Radioactivity in Rain Ion Columns

Page 2 of 5

No. of

\begin{tabular}{|c|c|c|c|c|}
\hline Location & $\begin{array}{l}\text { No. of } \\
\text { Samples }\end{array}$ & Arithmetic Mean $\pm \sigma$ & Maximum $\pm \sigma$ & Minimum $\pm \sigma$ \\
\hline \multicolumn{5}{|l|}{ 100-Mile Radius } \\
\hline Savannah, Ga. & 1 & $(-0.69 \pm 1.38) E+00$ & & \\
\hline \multicolumn{5}{|l|}{ U-234, pCi/sq m } \\
\hline \multicolumn{5}{|l|}{ On Site } \\
\hline Burial Ground North & 1 & $(1.83 \pm 0.66) \mathrm{E}-01$ & & \\
\hline \multicolumn{5}{|l|}{ Site Perimeter } \\
\hline D-Area & 1 & $(2.22 \pm 3.26) \mathrm{E}-02$ & & \\
\hline Darkhorse @ Williston Gate & 1 & $(8.62 \pm 4.85) E-02$ & & \\
\hline Green Pond & 1 & $(1.16 \pm 0.55) E-01$ & & \\
\hline Patterson Mill Road & 1 & $(9.93 \pm 4.80) E-02$ & & \\
\hline \multicolumn{5}{|l|}{ 25-Mile Radius } \\
\hline Highway $301 @$ State Line & 1 & $(1.32 \pm 0.59) E-01$ & & \\
\hline \multicolumn{5}{|l|}{ 100-Mile Radius } \\
\hline Savannah, Ga. & 1 & $(8.97 \pm 3.51) E-02$ & & \\
\hline
\end{tabular}

\section{On Site}

Burial Ground North

$(4.57 \pm 3.26) \mathrm{E}-02$

\section{Site Perimeter}

D-Area

Darkhorse @ Williston Gate

Green Pond

Patterson Mill Road

-Mile Radius

Highway 301 @ State Line 11 (1.33 10.59$) E-01$

100-Mile Radius

Savannah, Ga.

$(2.27 \pm 2.28) \mathrm{E}-02$

$(1.44 \pm 2.34) E-02$

$(4.42 \pm 3.13) \mathrm{E}-02$

$(-1.54 \pm 1.09) \mathrm{E}-02$

$\mathrm{J}-238$, pCi/sq $\mathrm{m}$

On Site

Burial Ground North

$(1.14 \pm 0.59) E-01$

Site Perimeter

D-Area

$(4.58 \pm 4.51) E-02$

Darkhorse @ Williston Gate

$(-3.51 \pm 2.61) E-02$

Green Pond

$(0.10 \pm 3.62) E-02$

Patterson Mill Road

$(0.74 \pm 3.59) \mathrm{E}-02$ 
Table 10

Radioactivity in Rain Ion Columns

Page 3 of 5

\begin{tabular}{|c|c|c|c|c|}
\hline Location & $\begin{array}{l}\text { No. of } \\
\text { Samples }\end{array}$ & Arithmetic Mean $\pm \sigma$ & $\operatorname{Maximum} \pm \sigma$ & Minimum $\pm \sigma$ \\
\hline \multicolumn{5}{|l|}{ 25-Mile Radius } \\
\hline Highway 301 @ State Line & 1 & $(3.05 \pm 4.26) E-02$ & & \\
\hline \multicolumn{5}{|l|}{ 100-Mile Radius } \\
\hline Savannah, Ga. & 1 & $(4.22 \pm 3.19) \mathrm{E}-02$ & & \\
\hline \multicolumn{5}{|l|}{ Pu-238, pCi/sq m } \\
\hline \multicolumn{5}{|l|}{ On Site } \\
\hline Burial Ground North & 1 & $(-3.58 \pm 0.22) E-02$ & & \\
\hline \multicolumn{5}{|l|}{ Site Perimeter } \\
\hline D-Area & 1 & $(-3.05 \pm 1.98) E-02$ & & \\
\hline Darkhorse @ Williston Gate & 1 & $(-0.67 \pm 2.56) E-02$ & & \\
\hline Green Pond & 1 & $(-3.05 \pm 1.97) E-02$ & & \\
\hline Patterson Mill Road & 1 & $(-1.94 \pm 1.64) \mathrm{E}-02$ & & \\
\hline \multicolumn{5}{|l|}{ 25-Mile Radius } \\
\hline Highway $301 @$ State Line & 1 & $(-1.82 \pm 1.76) E-02$ & & \\
\hline \multicolumn{5}{|l|}{ 100-Mile Radius } \\
\hline Savannah, Ga. & 1 & $(-3.95 \pm 0.67) E-02$ & & \\
\hline \multicolumn{5}{|l|}{ Pu-239, pCi/sq m } \\
\hline \multicolumn{5}{|l|}{ On Site } \\
\hline Burial Ground North & 1 & $(-3.54 \pm 2.92) E-02$ & & \\
\hline \multicolumn{5}{|l|}{ Site Perimeter } \\
\hline D-Area & 1 & $(-2.45 \pm 2.58) E-02$ & & \\
\hline Darkhorse @ Williston Gate & 1 & $(-1.85 \pm 2.48) E-02$ & & \\
\hline Green Pond & 1 & $(-6.01 \pm 0.71) E-02$ & & \\
\hline Patterson Mill Road & 1 & $(1.17 \pm 3.27) E-02$ & & \\
\hline \multicolumn{5}{|l|}{ 25-Mile Radius } \\
\hline Highway $301 @$ State Line & 1 & $(-4.21 \pm 1.88) E-02$ & & \\
\hline \multicolumn{5}{|l|}{ 100-Mile Radius } \\
\hline Savannah, Ga. & 1 & $(-5.78 \pm 0.69) E-02$ & & \\
\hline \multicolumn{5}{|l|}{ Am-241, pCi/sq m } \\
\hline \multicolumn{5}{|l|}{ On Site } \\
\hline Burial Ground North & 1 & $(-2.86 \pm 2.98) E-02$ & & \\
\hline \multicolumn{5}{|l|}{ Site Perimeter } \\
\hline D-Area & 1 & $(4.10 \pm 5.01) E-02$ & & \\
\hline Darkhorse @ Williston Gate & 1 & $(-4.55 \pm 2.91) E-02$ & & \\
\hline
\end{tabular}


Table 10

Radioactivity in Rain Ion Columns

Page 4 of 5

\begin{tabular}{|c|c|c|c|c|}
\hline Location & $\begin{array}{c}\text { No. of } \\
\text { Samples }\end{array}$ & Arithmetic Mean $\pm \sigma$ & Maximum $\pm \sigma$ & Minimum $\pm \sigma$ \\
\hline Green Pond & 1 & $(3.16 \pm 4.98) E-02$ & & \\
\hline Patterson Mill Road & 1 & $(1.44 \pm 4.26) E-02$ & & \\
\hline \multicolumn{5}{|l|}{ 25-Mile Radius } \\
\hline Highway $301 @$ State Line & 1 & $(4.48 \pm 4.72) E-02$ & & \\
\hline \multicolumn{5}{|l|}{ 100-Mile Radius } \\
\hline Savannah, Ga. & 1 & $(1.60 \pm 3.15) E-02$ & & \\
\hline \multicolumn{5}{|l|}{ Cm-244, pci/sq m } \\
\hline \multicolumn{5}{|l|}{ On Site } \\
\hline Burial Ground North & 1 & $(2.11 \pm 2.11) \mathrm{E}-02$ & & \\
\hline \multicolumn{5}{|l|}{ Site Perimeter } \\
\hline D-Area & 1 & $(-7.77 \pm 7.79) E-03$ & & \\
\hline Darkhorse @ Williston Gate & 1 & $(0.00 \pm 0.29) E+00$ & & \\
\hline Green Pond & 1 & $(-1.53 \pm 1.09) E-02$ & & \\
\hline Patterson Mill Road & 1 & $(0.00 \pm 0.33) E+00$ & & \\
\hline \multicolumn{5}{|l|}{ 25-Mile Radius } \\
\hline Highway $301 @$ State Line & 1 & $(-6.71 \pm 6.71) E-03$ & & \\
\hline \multicolumn{5}{|l|}{ 100-Mile Radius } \\
\hline Savannah, Ga. & 1 & $(1.96 \pm 1.39) E-02$ & & \\
\hline \multicolumn{5}{|l|}{ Gross Alpha, pCi/sq m } \\
\hline \multicolumn{5}{|l|}{ On Site } \\
\hline Burial Ground North & 13 & $(2.84 \pm 1.19) E+00$ & $(1.45 \pm 0.47) E+01$ & $(-1.73 \pm 2.08) E+00$ \\
\hline \multicolumn{5}{|l|}{ Site Perimeter } \\
\hline D-Area & 13 & $(2.07 \pm 0.92) E+00$ & $(8.24 \pm 4.27) E+00$ & $(-3.14 \pm 1.46) E+00$ \\
\hline Darkhorse @ Williston Gate & 13 & $(1.31 \pm 0.52) E+00$ & $(4.60 \pm 3.07) E+00$ & $(-2.22 \pm 1.84) E+00$ \\
\hline Green Pond & 13 & $(1.17 \pm 0.64) E+\infty$ & $(5.17 \pm 2.91) E+\infty 0$ & $(-1.44 \pm 1.65) E+\infty 0$ \\
\hline Patterson Mill Road & 13 & $(1.14 \pm 1.12) E+00$ & $(1.22 \pm 0.47) E+01$ & $(-2.71 \pm 2.19) E+00$ \\
\hline \multicolumn{5}{|l|}{ 25-Mile Radius } \\
\hline Highway $301 @$ State Line & 13 & $(1.76 \pm 1.23) E+00$ & $(1.22 \pm 0.51) E+01$ & $(-1.38 \pm 2.10) E+00$ \\
\hline \multicolumn{5}{|l|}{ 100-Mile Radius } \\
\hline Savannah, Ga. & 13 & $(2.58 \pm 1.47) E+00$ & $(1.45 \pm 0.32) E+01$ & $(-3.44 \pm 1.85) E+00$ \\
\hline \multicolumn{5}{|l|}{ Gross Beta, pCi/sq m } \\
\hline \multicolumn{5}{|l|}{ On Site } \\
\hline Burial Ground North & 13 & $(1.08 \pm 0.32) E+02$ & $(4.21 \pm 0.17) E+02$ & $(2.43 \pm 0.56) E+01$ \\
\hline
\end{tabular}




\section{Table 10}

Radioactivity in Rain Ion Columns

Page 5 of 5

\begin{tabular}{|c|c|c|c|c|}
\hline Location & $\begin{array}{c}\text { No. of } \\
\text { Samples }\end{array}$ & Arithmetic Mean $\pm \sigma$ & Maximum $\pm \sigma$ & Minimum $\pm \sigma$ \\
\hline \multicolumn{5}{|l|}{ Site Perimeter } \\
\hline D-Area & 13 & $(6.84 \pm 2.45) E+01$ & $(3.39 \pm 0.13) E+02$ & $(5.65 \pm 4.86) \mathrm{E}+00$ \\
\hline Darkhorse @ Williston Gate & 13 & $(5.55 \pm 1.32) E+01$ & $(1.67 \pm 0.10) E+02$ & $(4.29 \pm 3.69) \mathrm{E}+00$ \\
\hline Green Pond & 13 & $(5.87 \pm 1.60) E+01$ & $(2.08 \pm 0.08) E+02$ & $(4.66 \pm 2.67) E+00$ \\
\hline Patterson Mill Road & 13 & $(5.87 \pm 1.33) E+01$ & $(1.56 \pm 0.10) E+02$ & $(9.38 \pm 4.73) E+00$ \\
\hline \multicolumn{5}{|l|}{ 25-Mile Radius } \\
\hline Highway 301 @ State Line & 13 & $(6.73 \pm 2.22) E+01$ & $(3.15 \pm 0.13) E+02$ & $(1.14 \pm 0.49) E+01$ \\
\hline \multicolumn{5}{|l|}{ 100-Mile Radius } \\
\hline Savannah, Ga. & 13 & $(8.81 \pm 2.36) E+01$ & $(3.39 \pm 0.13) E+02$ & $(2.08 \pm 3.68) E+00$ \\
\hline
\end{tabular}


Table 11

Thermoluminescent Dosimeter (TLD) Results -

Site Perimeter Stations

Page 1 of 1

Quarterly Exposure in mR/Day

Yearly Exposure in $\mathrm{mR} /$ Year

\begin{tabular}{llllll}
\hline Location & Quarter 1 & Quarter 2 & Quarter 3 & Quarter 4 & mR/Year \\
\hline
\end{tabular}

Notes: $1 . m R=$ milliRoentgen, exposure unit for gamma radiation. One $m R$ is approximately equal to $1 \mathrm{mrem}$. Although the TLD is an integrating device, the derived unit $m R /$ day is used to facilitate data comparison between locations or through time at a single location.

2. An $18 \%$ uncertainty is associated with each result.

3. Blank spaces indicate missing TLD results.

\begin{tabular}{llllll}
\hline PP-15 & 0.24 & 0.21 & 0.22 & & 80 \\
PP-42 & 0.26 & 0.22 & 0.24 & 0.22 & 84 \\
PP-48 & 0.23 & 0.19 & 0.21 & 0.20 & 75 \\
PP-51 & 0.24 & 0.19 & 0.21 & 0.20 & 75 \\
PP-57D & 0.23 & 0.20 & 0.21 & 0.19 & 74 \\
PP-61B & 0.24 & 0.20 & 0.21 & 0.20 & 75 \\
PP-65D & 0.27 & 0.24 & 0.25 & 0.23 & 88 \\
PP-72B & 0.24 & 0.19 & 0.22 & 0.20 & 76 \\
PP-75D & 0.21 & 0.18 & 0.19 & 0.17 & 67
\end{tabular}


Table 12

Thermoluminescent Dosimeter (TLD) Results -

Environmental Surveillance (Air Monitoring) Stations

Page 1 of 1

Quarterly Exposure in mR/Day

Yearly Exposure in $\mathrm{mR} /$ Year

\begin{tabular}{llllll}
\hline Location & Quarter 1 & Quarter 2 & Quarter 3 & Quarter 4 & $\mathrm{mR} /$ Year \\
\hline
\end{tabular}

Notes: 1. $m R=$ milliRoentgen, exposure unit for gamma radiation. One $m R$ is approximately equal to $1 \mathrm{mrem}$. Although the TLD is an integrating device, the derived unit $m R /$ day is used to facilitate data comparison between locations or through time at a single location.

2. An $18 \%$ uncertainty is associated with each result.

3. Blank spaces indicate missing TLD results.

\begin{tabular}{llllll}
\hline Burial Ground North & 0.36 & 0.33 & 0.36 & 0.34 & 124 \\
Aiken Gate & 0.26 & 0.23 & 0.24 & 0.22 & 85 \\
Allendale Gate & 0.21 & 0.18 & 0.21 & 0.17 & 68 \\
Barnwell Gate & 0.25 & 0.22 & 0.23 & 0.22 & 81 \\
D-Area & 0.23 & 0.21 & & 0.21 & 77 \\
Dark Horse & 0.24 & 0.21 & 0.23 & 0.21 & 79 \\
East Talatha & 0.23 & 0.19 & 0.20 & 0.19 & 72 \\
Green Pond & 0.23 & 0.21 & 0.22 & 0.19 & 76 \\
Hwy 21/167 & 0.30 & 0.28 & 0.29 & 0.27 & 101 \\
Jackson & 0.25 & 0.22 & 0.23 & 0.22 & 82 \\
Patterson Mill Rd & 0.21 & 0.19 & 0.20 & 0.18 & 70 \\
West Jackson & 0.29 & 0.26 & 0.27 & 0.25 & 95 \\
Windsor Rd & 0.18 & 0.17 & 0.18 & 0.17 & 61 \\
Aiken Airport & 0.22 & 0.17 & 0.19 & 0.17 & 67 \\
Augusta Lock \& Dam & 0.24 & 0.19 & 0.22 & 0.21 & 77 \\
Hwy 301 & 0.29 & 0.23 & & 0.23 & 87 \\
Savannah 1 & 0.22 & 0.20 & 0.23 & 0.19 & 77 \\
Savannah 2 & 0.17 & 0.16 & 0.21 & 0.17 & 64
\end{tabular}


Table 13

Thermoluminescent Dosimeter (TLD) Results -

Population Centers

Page 1 of 1

Quarterly Exposure in mR/Day

Yearly Exposure in mR/Year

\begin{tabular}{llllll}
\hline Location & Quarter 1 & Quarter 2 & Quarter 3 & Quarter 4 & mR/Year
\end{tabular}

Notes: 1. $m R=$ milliRoentgen, exposure unit for gamma radiation. One $m R$ is approximately equal to $1 \mathrm{mrem}$. Although the $T L D$ is an integrating device, the derived unit $m R /$ day is used to facilitate data comparison between locations or through time at a single location.

2. An $18 \%$ uncertainty is associated with each result.

3. Blank spaces indicate missing TLD results.

\begin{tabular}{lllllr}
\hline Barnwell & 0.25 & 0.19 & 0.29 & 0.25 & 86 \\
Beech Island & 0.35 & 0.31 & 0.37 & 0.32 & 117 \\
Girard & 0.36 & & & 0.32 & 106 \\
Jackson & 0.27 & 0.22 & 0.29 & 0.25 & 88 \\
Martin & 0.20 & 0.16 & 0.23 & 0.19 & 68 \\
McBean & 0.34 & & & 0.30 & 99 \\
New Ellenton & 0.30 & 0.25 & 0.33 & 0.29 & 101 \\
Williston & 0.34 & 0.29 & 0.36 & 0.31 & 113 \\
Windsor & 0.17 & 0.14 & 0.20 & 0.16 & 59
\end{tabular}


Table 14

Thermoluminescent Dosimeter (TLD) Results -

Vogtle Electric Generating Plant Vicinity

Page 1 of 1

\section{Quarterly Exposure in mR/Day}

Yearly Exposure in $\mathrm{mR} / \mathrm{Year}$

\begin{tabular}{llllll}
\hline Location & Quarter 1 & Quarter 2 & Quarter 3 & Quarter 4 & mR/Year \\
\hline
\end{tabular}

Notes: 1. $m R=$ milliRoentgen, exposure unit for gamma radiation. One $m R$ is approximately equal to $1 \mathrm{mrem}$. Although the TLD is an integrating device, the derived unit $m R /$ day is used to facilitate data comparison between locations or through time at a single location.

2. An $18 \%$ uncertainty is associated with each result.

3. Blank spaces indicate missing TLD results.

\begin{tabular}{llllll}
\hline GAP 1H & 0.21 & 0.18 & 0.23 & 0.19 & 74 \\
GAP 1L & 0.21 & 0.18 & 0.22 & 0.19 & 73 \\
GAP 2H & 0.23 & 0.20 & 0.24 & 0.21 & 79 \\
GAP 2L & 0.23 & 0.20 & 0.24 & 0.21 & 80 \\
GAP 3H & 0.23 & 0.21 & 0.26 & 0.22 & 84 \\
GAP 3L & & 0.21 & 0.25 & 83 \\
GAP 4H & 0.28 & 0.25 & 0.29 & 0.26 & 98 \\
GAP 4L & 0.26 & 0.24 & 0.28 & 0.25 & 94 \\
GAP 5H & 0.22 & 0.19 & 0.23 & 0.19 & 76 \\
GAP 5L & 0.17 & 0.15 & 0.19 & 0.16 & 61 \\
NRC 1 & 0.30 & 0.21 & 0.20 & 0.37 & 89 \\
NRC 2 & 0.24 & 0.16 & 0.15 & 0.26 & 67 \\
NRC 3 & 0.24 & 0.17 & 0.15 & 0.27 & 68 \\
NRC 4 & 0.28 & 0.20 & 0.18 & 0.32 & 8.39 \\
NRC 5 & 0.34 & 0.24 & 0.22 & 0.19 &
\end{tabular}


Table 15

Radioactivity in Seepage Basin Water

Page 1 of 2

\begin{tabular}{|c|c|c|c|c|}
\hline Location & $\begin{array}{l}\text { No. of } \\
\text { Samples }\end{array}$ & Arithmetic Mean $\pm \sigma$ & Maximum $\pm \sigma$ & Minimum $\pm \sigma$ \\
\hline \multicolumn{5}{|l|}{$\mathrm{H}-3, \mathrm{pCi} / \mathrm{L}$} \\
\hline $\mathrm{E}-05$ & 12 & $(6.24 \pm 2.63) E+03$ & $(3.04 \pm 0.08) E+04$ & $(7.52 \pm 3.97) \mathrm{E}+02$ \\
\hline$E-06$ & 1 & $(1.20 \pm 0.43) E+03$ & & \\
\hline EAV Basin North $(E-004)$ & 12 & $(5.03 \pm 0.80) E+03$ & $(1.04 \pm 0.03) E+04$ & $(1.82 \pm 0.38) E+03$ \\
\hline EAV Basin South (E-003) & 1 & $(7.29 \pm 0.53) \mathrm{E}+03$ & & \\
\hline $\begin{array}{l}\text { SWDF Basin North } \\
(E-002)\end{array}$ & 12 & $(8.18 \pm 0.76) \mathrm{E}+03$ & $(1.19 \pm 0.05) E+04$ & $(2.67 \pm 0.39) E+03$ \\
\hline $\begin{array}{l}\text { SWDF Basin South } \\
(E-001)\end{array}$ & 12 & $(1.19 \pm 0.22) \mathrm{E}+04$ & $(2.92 \pm 0.05) \mathrm{E}+04$ & $(3.89 \pm 0.29) E+03$ \\
\hline \multicolumn{5}{|l|}{ Co-60, pCi/L } \\
\hline$E-05$ & 12 & $(1.54 \pm 0.62) \mathrm{E}+00$ & $(4.20 \pm 2.16) E+00$ & $(-3.79 \pm 2.21) E+00$ \\
\hline$E-06$ & 1 & $(-1.71 \pm 1.74) E+00$ & & \\
\hline EAV Basin North (E-004) & 12 & $(7.29 \pm 7.46) E-01$ & $(5.07 \pm 2.65) E+00$ & $(-3.47 \pm 2.47) \mathrm{E}+00$ \\
\hline EAV Basin South (E-003) & 1 & $(2.46 \pm 1.08) \mathrm{E}+00$ & & \\
\hline $\begin{array}{l}\text { SWDF Basin North } \\
(E-002)\end{array}$ & 12 & $(1.29 \pm 0.61) \mathrm{E}+00$ & $(5.87 \pm 2.39) E+00$ & $(-2.06 \pm 2.32) E+00$ \\
\hline $\begin{array}{l}\text { SWDF Basin South } \\
(E-001)\end{array}$ & 12 & $(1.33 \pm 0.80) E+00$ & $(7.17 \pm 2.81) E+00$ & $(-2.41 \pm 2.43) E+00$ \\
\hline \multicolumn{5}{|l|}{ Cs-137, pCi/L } \\
\hline$E-05$ & 12 & $(3.19 \pm 6.35) \mathrm{E}-01$ & $(3.81 \pm 2.40) E+00$ & $(-2.27 \pm 2.10) \mathrm{E}+00$ \\
\hline$E-06$ & 1 & $(-0.64 \pm 1.87) E+00$ & & \\
\hline EAV Basin North (E-004) & 12 & $(1.85 \pm 3.64) E-01$ & $(2.89 \pm 2.19) E+00$ & $(-1.36 \pm 1.96) \mathrm{E}+00$ \\
\hline EAV Basin South (E-003) & 1 & $(0.11 \pm 1.15) \mathrm{E}+00$ & & \\
\hline $\begin{array}{l}\text { SWDF Basin North } \\
(E-002)\end{array}$ & 12 & $(1.51 \pm 0.52) \mathrm{E}+00$ & $(4.73 \pm 1.91) E+00$ & $(-0.89 \pm 2.34) E+00$ \\
\hline $\begin{array}{l}\text { SWDF Basin South } \\
(E-001)\end{array}$ & 12 & $(1.37 \pm 0.96) E+00$ & $(1.06 \pm 0.58) E+01$ & $(-2.02 \pm 2.01) E+00$ \\
\hline \multicolumn{5}{|l|}{ Gross Alpha, pCi/L } \\
\hline$E-05$ & 12 & $(7.26 \pm 1.98) \mathrm{E}-01$ & $(1.67 \pm 0.83) E+00$ & $(-4.59 \pm 5.01) \mathrm{E}-01$ \\
\hline$E-06$ & 1 & $(1.97 \pm 0.85) \mathrm{E}+00$ & & \\
\hline EAV Basin North (E-004) & 12 & $(1.15 \pm 0.21) E+00$ & $(2.80 \pm 0.82) E+00$ & $(1.62 \pm 3.47) \mathrm{E}-01$ \\
\hline EAV Basin South (E-003) & 1 & $(2.41 \pm 0.89) E+00$ & & \\
\hline $\begin{array}{l}\text { SWDF Basin North } \\
(E-002)\end{array}$ & 12 & $(9.35 \pm 2.44) E-01$ & $(2.80 \pm 1.02) E+00$ & $(-2.95 \pm 4.06) E-01$ \\
\hline $\begin{array}{l}\text { SWDF Basin South } \\
(E-001)\end{array}$ & 12 & $(6.20 \pm 1.97) E-01$ & $(1.73 \pm 0.69) E+00$ & $(-2.21 \pm 4.85) E-01$ \\
\hline
\end{tabular}


Table 15

Radioactivity in Seepage Basin Water

Page 2 of 2

No. of

\begin{tabular}{lrrrr} 
Location & $\begin{array}{c}\text { No. of } \\
\text { Samples }\end{array}$ & Arithmetic Mean $\pm \sigma$ & Maximum $\pm \sigma$ & Minimum $\pm \sigma$ \\
\hline Gross Beta, pCi/L & 12 & $(4.09 \pm 0.55) \mathrm{E}+00$ & $(7.33 \pm 1.04) \mathrm{E}+00$ & $(1.88 \pm 1.06) \mathrm{E}+00$ \\
\hline E-05 & 1 & $(3.39 \pm 0.95) \mathrm{E}+00$ & & \\
E-06 & 12 & $(3.25 \pm 0.30) \mathrm{E}+00$ & $(5.13 \pm 0.82) \mathrm{E}+00$ & $(1.68 \pm 1.02) \mathrm{E}+00$ \\
EAV Basin North (E-004) & 1 & $(2.31 \pm 0.88) \mathrm{E}+00$ & & $(3.53 \pm 0.96) \mathrm{E}+00$ \\
EAV Basin South (E-003) & 12 & $(5.61 \pm 0.32) \mathrm{E}+00$ & $(7.71 \pm 1.29) \mathrm{E}+00$ & \\
SWDF Basin North & 12 & $(4.10 \pm 0.26) \mathrm{E}+00$ & $(5.56 \pm 1.14) \mathrm{E}+00$ & $(2.44 \pm 0.87) \mathrm{E}+00$ \\
(E-002) & & &
\end{tabular}




\section{Table 16}

\section{Radioactivity in Savannah River Site Stream Water}

Page 1 of 14

\begin{tabular}{|c|c|c|c|c|}
\hline Location & $\begin{array}{c}\text { No. of } \\
\text { Samples }\end{array}$ & Arithmetic Mean $\pm \sigma$ & Maximum $\pm \sigma$ & Minimum $\pm \sigma$ \\
\hline \multicolumn{5}{|l|}{$\mathrm{H}-3, \mathrm{pCi} / \mathrm{L}$} \\
\hline \multicolumn{5}{|l|}{ Tims Branch } \\
\hline TB-5 near Road C & 26 & $(8.07 \pm 0.70) E+02$ & $(1.52 \pm 0.33) E+03$ & $(1.48 \pm 3.61) E+02$ \\
\hline \multicolumn{5}{|l|}{ Upper Three Runs } \\
\hline Crouch Branch at Road 4 & 26 & $(1.12 \pm 0.04) E+04$ & $(1.43 \pm 0.06) E+04$ & $(7.53 \pm 0.50) E+03$ \\
\hline $\begin{array}{l}\text { McQueen Branch } \\
\text { at Road F }\end{array}$ & 26 & $(1.08 \pm 0.08) E+04$ & $(2.94 \pm 0.04) E+04$ & $(6.66 \pm 0.49) E+03$ \\
\hline $\begin{array}{l}\text { U3R-1A Treadway Bridge } \\
\text { Road 8-1 }\end{array}$ & 52 & $(3.82 \pm 0.39) E+02$ & $(1.01 \pm 0.37) E+03$ & $(-2.55 \pm 2.04) E+02$ \\
\hline U3R-3 at Road C & 26 & $(5.65 \pm 1.01) E+03$ & $(2.02 \pm 0.07) E+04$ & $(8.59 \pm 3.71) E+02$ \\
\hline U3R-4 at Road A & 26 & $(6.65 \pm 1.44) \mathrm{E}+03$ & $(3.32 \pm 0.08) \mathrm{E}+04$ & $(8.78 \pm 3.15) E+02$ \\
\hline U3R-F3 & 10 & $(9.49 \pm 2.97) E+02$ & $(2.75 \pm 0.44) E+03$ & $(-0.79 \pm 3.53) E+02$ \\
\hline \multicolumn{5}{|l|}{ Four Mile Creek } \\
\hline C-Canal & 26 & $(2.62 \pm 0.28) E+03$ & $(9.40 \pm 0.27) \mathrm{E}+03$ & $(1.76 \pm 0.28) E+03$ \\
\hline $\begin{array}{l}\text { Caster Creek-southeast } \\
\text { of C-Area }\end{array}$ & 6 & $(2.98 \pm 0.20) E+03$ & $(3.63 \pm 0.37) \mathrm{E}+03$ & $(2.37 \pm 0.25) E+03$ \\
\hline FM-2 at Road 4 & 26 & $(1.49 \pm 0.12) E+04$ & $(2.91 \pm 0.07) E+04$ & $(5.49 \pm 0.19) \mathrm{E}+03$ \\
\hline $\begin{array}{l}\text { FM-2B above } \\
\text { F-Area effluent }\end{array}$ & 26 & $(5.25 \pm 0.25) E+04$ & $(7.35 \pm 0.52) \mathrm{E}+04$ & $(2.92 \pm 0.44) E+04$ \\
\hline $\begin{array}{l}\text { FM-3A below } \\
\text { F-Area effluent }\end{array}$ & 26 & $(1.08 \pm 0.04) E+06$ & $(1.69 \pm 0.02) E+06$ & $(8.68 \pm 0.12) E+05$ \\
\hline FM-6 at Road A-12.2 & 26 & $(1.91 \pm 0.03) E+05$ & $(2.27 \pm 0.02) E+05$ & $(1.65 \pm 0.01) E+05$ \\
\hline FM-A7 at Road A-7 & 26 & $(3.27 \pm 0.10) E+05$ & $(4.27 \pm 0.03) E+05$ & $(2.37 \pm 0.02) E+05$ \\
\hline $\mathrm{H}-008$ Outfall & 6 & $(1.49 \pm 0.24) E+03$ & $(2.42 \pm 0.25) E+03$ & $(6.93 \pm 4.24) \mathrm{E}+02$ \\
\hline $\begin{array}{l}\text { HP-50 Tritium Facility } \\
\text { Outfall }\end{array}$ & 26 & $(2.49 \pm 0.37) E+04$ & $(7.85 \pm 0.09) E+04$ & $(6.12 \pm 0.40) E+03$ \\
\hline $\begin{array}{l}\text { Twin Lakes-west } \\
\text { of C-Area }\end{array}$ & 6 & $(1.48 \pm 0.05) E+04$ & $(1.58 \pm 0.04) E+04$ & $(1.31 \pm 0.06) \mathrm{E}+04$ \\
\hline \multicolumn{5}{|l|}{ Pen Branch } \\
\hline $\begin{array}{l}\text { IGB-21 } 800 \text { feet south } \\
\text { of Road } 6-1\end{array}$ & 6 & $(3.75 \pm 0.75) E+05$ & $(4.81 \pm 0.10) E+05$ & $(4.35 \pm 9.82) E+03$ \\
\hline $\mathrm{K}-011$ Outfall at B Road & 6 & $(2.56 \pm 0.07) E+05$ & $(2.79 \pm 0.03) E+05$ & $(2.41 \pm 0.02) \mathrm{E}+05$ \\
\hline PB-3 at Road A-13.2 & 26 & $(1.03 \pm 0.03) E+05$ & $(1.35 \pm 0.02) E+05$ & $(7.15 \pm 0.14) E+04$ \\
\hline \multicolumn{5}{|l|}{ Steel Creek } \\
\hline $\begin{array}{l}\text { SC-2A } 1 \text { mile } \\
\text { above Road B }\end{array}$ & 26 & $(2.80 \pm 0.06) E+05$ & $(3.21 \pm 0.02) E+05$ & $(1.90 \pm 0.03) E+05$ \\
\hline $\begin{array}{l}\text { SC-4 Steel Creek } \\
\text { at Road A }\end{array}$ & 26 & $(6.26 \pm 0.45) E+03$ & $(1.18 \pm 0.05) E+04$ & $(3.03 \pm 0.41) E+03$ \\
\hline
\end{tabular}




\section{Table 16}

\section{Radioactivity in Savannah River Site Stream Water}

Page 2 of 14

\begin{tabular}{|c|c|c|c|c|}
\hline Location & $\begin{array}{l}\text { No. of } \\
\text { Samples }\end{array}$ & Arithmetic Mean $\pm \sigma$ & Maximum $\pm \sigma$ & Minimum $\pm \sigma$ \\
\hline \multicolumn{5}{|l|}{ Lower Three Runs Creek } \\
\hline L3R-1A at Road B & 26 & $(1.06 \pm 0.09) E+03$ & $(2.15 \pm 0.38) E+03$ & $(1.13 \pm 3.72) E+02$ \\
\hline L3R-2 Patterson Mill Road & 26 & $(2.36 \pm 0.17) E+03$ & $(4.80 \pm 0.30) E+03$ & $(1.11 \pm 0.37) E+03$ \\
\hline L3R-3 at Highway 125 & 20 & $(1.32 \pm 0.09) E+03$ & $(1.92 \pm 0.41) E+03$ & $(4.84 \pm 3.90) E+02$ \\
\hline R-Area downstream of R-1 & 26 & $(4.05 \pm 0.85) E+04$ & $(2.16 \pm 0.01) E+05$ & $(3.30 \pm 0.47) E+03$ \\
\hline \multicolumn{5}{|l|}{$\overline{\mathrm{Co}}-60, \mathrm{pCi} / \mathrm{L}$} \\
\hline \multicolumn{5}{|l|}{ Tims Branch } \\
\hline TB-5 near Road C & 20 & $(6.58 \pm 6.76) E-01$ & $(6.67 \pm 2.91) E+00$ & $(-4.32 \pm 2.09) E+00$ \\
\hline \multicolumn{5}{|l|}{ Upper Three Runs } \\
\hline Crouch Branch at Road 4 & 26 & $(5.13 \pm 5.35) E-01$ & $(5.19 \pm 2.22) E+00$ & $(-5.46 \pm 2.16) E+00$ \\
\hline $\begin{array}{l}\text { McQueen Branch } \\
\text { at Road F }\end{array}$ & 26 & $(8.41 \pm 4.62) E-01$ & $(5.94 \pm 2.57) E+00$ & $(-3.32 \pm 2.43) E+00$ \\
\hline $\begin{array}{l}\text { U3R-1A Treadway Bridge } \\
\text { Road 8-1 }\end{array}$ & 38 & $(3.16 \pm 3.85) E-01$ & $(4.62 \pm 2.72) E+\infty 0$ & $(-4.58 \pm 2.48) E+00$ \\
\hline U3R-3 at Road C & 26 & $(6.08 \pm 5.52) E-01$ & $(6.60 \pm 2.57) E+00$ & $(-4.88 \pm 2.80) E+00$ \\
\hline U3R-4 at Road A & 26 & $(8.20 \pm 4.55) E-01$ & $(5.50 \pm 2.44) E+00$ & $(-2.33 \pm 2.41) E+00$ \\
\hline U3R-F3 & 10 & $(9.82 \pm 7.74) E-01$ & $(6.59 \pm 2.50) E+00$ & $(-0.19 \pm 1.28) E+01$ \\
\hline \multicolumn{5}{|l|}{ Four Mile Creek } \\
\hline C-Canal & 26 & $(6.48 \pm 4.74) E-01$ & $(6.51 \pm 2.75) E+00$ & $(-4.23 \pm 2.21) E+00$ \\
\hline FM-2 at Road 4 & 26 & $(7.25 \pm 3.63) E-01$ & $(4.18 \pm 2.17) E+\infty 0$ & $(-2.34 \pm 2.50) E+00$ \\
\hline $\begin{array}{l}\text { FM-2B above } \\
\text { F-Area effluent }\end{array}$ & 26 & $(-0.52 \pm 5.24) E-01$ & $(4.99 \pm 2.19) E+00$ & $(-7.54 \pm 2.30) E+00$ \\
\hline $\begin{array}{l}\text { FM-3A below } \\
\text { F-Area effluent }\end{array}$ & 26 & $(4.55 \pm 4.02) \mathrm{E}-01$ & $(4.92 \pm 1.97) \mathrm{E}+00$ & $(-4.31 \pm 2.45) E+00$ \\
\hline FM-6 at Road A-12.2 & 26 & $(-1.38 \pm 3.82) E-01$ & $(3.13 \pm 2.87) E+00$ & $(-4.32 \pm 2.37) E+00$ \\
\hline FM-A7 at Road $A-7$ & 26 & $(7.70 \pm 4.89) \mathrm{E}-01$ & $(4.73 \pm 2.52) E+00$ & $(-5.84 \pm 2.44) E+00$ \\
\hline $\mathrm{H}-008$ Outfall & 6 & $(1.47 \pm 0.99) E+00$ & $(4.42 \pm 2.23) E+00$ & $(-2.72 \pm 1.37) E+00$ \\
\hline $\begin{array}{l}\text { HP-50 Tritium Facility } \\
\text { Outfall }\end{array}$ & 26 & $(7.30 \pm 4.47) E-01$ & $(6.84 \pm 2.05) E+00$ & $(-3.45 \pm 2.52) E+00$ \\
\hline \multicolumn{5}{|l|}{ Pen Branch } \\
\hline $\mathrm{K}-011$ Outfall at B Road & 6 & $(3.94 \pm 7.63) E-01$ & $(2.31 \pm 1.99) E+00$ & $(-2.59 \pm 2.48) E+00$ \\
\hline PB-3 at Road A-13.2 & 26 & $(7.19 \pm 4.96) E-01$ & $(5.10 \pm 2.10) E+00$ & $(-4.59 \pm 1.76) E+00$ \\
\hline \multicolumn{5}{|l|}{ Steel Creek } \\
\hline $\begin{array}{l}\text { SC-2A } 1 \text { mile } \\
\text { above Road B }\end{array}$ & 26 & $(7.17 \pm 4.97) E-01$ & $(5.03 \pm 2.26) E+00$ & $(-4.99 \pm 2.51) E+00$ \\
\hline SC-4 Steel Creek & 26 & $(5.31 \pm 4.30) E-01$ & $(5.21 \pm 1.77) \mathrm{E}+00$ & $(-3.13 \pm 2.40) E+00$ \\
\hline
\end{tabular}


Table 16

Radioactivity in Savannah River Site Stream Water

Page 3 of 14

\begin{tabular}{|c|c|c|c|c|}
\hline Location & $\begin{array}{l}\text { No. of } \\
\text { Samples }\end{array}$ & Arithmetic Mean $\pm \sigma$ & Maximum $\pm \sigma$ & Minimum $\pm \sigma$ \\
\hline \multicolumn{5}{|l|}{ Lower Three Runs Creek } \\
\hline L3R-1A at Road B & 20 & $(1.09 \pm 0.52) E+00$ & $(6.51 \pm 3.45) E+00$ & $(-2.54 \pm 2.07) E+00$ \\
\hline L3R-2 Patterson Mill Road & 26 & $(-4.29 \pm 4.96) E-01$ & $(4.96 \pm 2.37) \mathrm{E}+00$ & $(-5.13 \pm 2.16) E+00$ \\
\hline L3R-3 at Highway 125 & 20 & $(4.98 \pm 5.77) E-01$ & $(6.18 \pm 2.47) E+00$ & $(-4.81 \pm 2.39) E+00$ \\
\hline R-Area downstream of $R-1$ & 26 & $(2.08 \pm 5.35) E-01$ & $(6.18 \pm 2.84) E+00$ & $(-4.01 \pm 2.83) E+00$ \\
\hline \multicolumn{5}{|l|}{ Cs-137, pCi/L } \\
\hline \multicolumn{5}{|l|}{ Tims Branch } \\
\hline TB-5 near Road C & 20 & $(-0.04 \pm 5.85) E-01$ & $(4.03 \pm 2.28) E+00$ & $(-4.98 \pm 2.72) E+00$ \\
\hline \multicolumn{5}{|l|}{ Upper Three Runs } \\
\hline Crouch Branch at Road 4 & 26 & $(1.17 \pm 0.48) E+00$ & $(5.19 \pm 2.32) E+00$ & $(-3.35 \pm 2.00) E+00$ \\
\hline $\begin{array}{l}\text { McQueen Branch } \\
\text { at Road F }\end{array}$ & 26 & $(3.92 \pm 3.81) \mathrm{E}+00$ & $(9.85 \pm 0.14) E+01$ & $(-5.06 \pm 2.34) E+00$ \\
\hline $\begin{array}{l}\text { U3R-1A Treadway Bridge } \\
\text { Road 8-1 }\end{array}$ & 38 & $(5.08 \pm 4.82) E-01$ & $(1.26 \pm 0.40) E+01$ & $(-4.63 \pm 2.57) E+00$ \\
\hline U3R-3 at Road C & 26 & $(5.46 \pm 4.64) \mathrm{E}-01$ & $(5.49 \pm 2.05) E+00$ & $(-2.87 \pm 2.36) E+00$ \\
\hline U3R-4 at Road A & 26 & $(0.77 \pm 3.69) E-01$ & $(3.62 \pm 2.34) E+00$ & $(-5.03 \pm 2.43) E+00$ \\
\hline U3R-F3 & 10 & $(1.54 \pm 2.19) \mathrm{E}+00$ & $(2.03 \pm 1.30) E+01$ & $(-3.19 \pm 1.87) E+00$ \\
\hline \multicolumn{5}{|l|}{ Four Mile Creek } \\
\hline C-Canal & 26 & $(-1.72 \pm 4.40) E-01$ & $(5.57 \pm 2.32) E+00$ & $(-4.19 \pm 2.39) E+00$ \\
\hline FM-2 at Road 4 & 26 & $(1.20 \pm 0.15) E+01$ & $(2.95 \pm 0.49) E+01$ & $(2.13 \pm 2.54) \mathrm{E}+00$ \\
\hline $\begin{array}{l}\text { FM-2B above } \\
\text { F-Area effluent }\end{array}$ & 26 & $(1.17 \pm 0.16) E+01$ & $(3.63 \pm 0.48) E+01$ & $(3.68 \pm 1.80) E+00$ \\
\hline $\begin{array}{l}\text { FM-3A below } \\
\text { F-Area effluent }\end{array}$ & 26 & $(9.55 \pm 3.90) E-01$ & $(4.08 \pm 2.35) E+00$ & $(-2.98 \pm 2.21) E+00$ \\
\hline FM-6 at Road A-12.2 & 26 & $(4.65 \pm 0.77) E+00$ & $(1.15 \pm 0.32) \mathrm{E}+01$ & $(-2.39 \pm 2.43) E+00$ \\
\hline FM-A7 at Road A-7 & 26 & $(8.95 \pm 1.09) E+00$ & $(2.61 \pm 0.39) E+01$ & $(0.15 \pm 3.02) E+00$ \\
\hline $\mathrm{H}-008$ Outfall & 6 & $(3.59 \pm 1.09) \mathrm{E}+00$ & $(6.30 \pm 1.37) \mathrm{E}+00$ & $(-1.37 \pm 1.99) E+00$ \\
\hline $\begin{array}{l}\text { HP-50 Tritium Facility } \\
\text { Outfall }\end{array}$ & 26 & $(2.63 \pm 0.53) \mathrm{E}+00$ & $(9.09 \pm 2.90) E+00$ & $(-3.76 \pm 2.09) E+00$ \\
\hline \multicolumn{5}{|l|}{ Pen Branch } \\
\hline $\mathrm{K}-011$ Outfall at B Road & 6 & $(-0.79 \pm 1.12) E+00$ & $(4.77 \pm 2.44) E+00$ & $(-2.24 \pm 1.23) E+00$ \\
\hline PB-3 at Road A-13.2 & 26 & $(1.85 \pm 4.80) E-01$ & $(4.44 \pm 2.29) E+00$ & $(-5.57 \pm 2.56) E+00$ \\
\hline \multicolumn{5}{|l|}{ Steel Creek } \\
\hline $\begin{array}{l}\text { SC- } 2 A 1 \text { mile } \\
\text { above Road B }\end{array}$ & 26 & $(3.08 \pm 0.56) E+00$ & $(9.63 \pm 3.76) \mathrm{E}+00$ & $(-1.85 \pm 2.49) E+00$ \\
\hline SC-4 Steel Creek & 26 & $(1.43 \pm 4.13) E-01$ & $(3.76 \pm 2.47) \mathrm{E}+00$ & $(-5.76 \pm 2.36) E+00$ \\
\hline
\end{tabular}


Table 16

Radioactivity in Savannah River Site Stream Water

Page 4 of 14

\begin{tabular}{|c|c|c|c|c|}
\hline Location & $\begin{array}{l}\text { No. of } \\
\text { Samples }\end{array}$ & Arithmetic Mean $\pm \sigma$ & Maximum $\pm \sigma$ & Minimum $\pm \sigma$ \\
\hline \multicolumn{5}{|l|}{ Lower Three Runs Creek } \\
\hline L3R-1A at Road $B$ & 20 & $(3.36 \pm 1.02) E+00$ & $(2.10 \pm 0.50) E+01$ & $(-2.01 \pm 2.34) E+00$ \\
\hline L3R-2 Patterson Mill Road & 26 & $(1.73 \pm 0.47) E+00$ & $(5.23 \pm 1.98) E+00$ & $(-2.76 \pm 2.59) E+00$ \\
\hline L3R-3 at Highway 125 & 20 & $(9.54 \pm 3.91) E-01$ & $(4.71 \pm 2.65) E+00$ & $(-1.71 \pm 2.61) E+00$ \\
\hline R-Area downstream of R-1 & 26 & $(2.19 \pm 0.18) E+01$ & $(4.19 \pm 0.48) E+01$ & $(6.20 \pm 2.58) \mathrm{E}+00$ \\
\hline \multicolumn{5}{|l|}{ Sr-89,90, pCi/l } \\
\hline \multicolumn{5}{|l|}{ Tims Branch } \\
\hline TB-5 near Road C & 1 & $(0.46 \pm 1.22) E+00$ & & \\
\hline \multicolumn{5}{|l|}{ Upper Three Runs } \\
\hline Crouch Branch at Road 4 & 1 & $(9.09 \pm 4.83) E-01$ & & \\
\hline $\begin{array}{l}\text { McQueen Branch } \\
\text { at Road F }\end{array}$ & 1 & $(3.89 \pm 5.04) E-01$ & & \\
\hline $\begin{array}{l}\text { U3R-1 A Treadway Bridge } \\
\text { Road 8-1 }\end{array}$ & 1 & $(-0.66 \pm 5.93) \mathrm{E}-01$ & & \\
\hline U3R-3 at Road C & 1 & $(3.56 \pm 5.04) \mathrm{E}-01$ & & \\
\hline U3R-4 at Road A & 1 & $(1.25 \pm 0.47) E+00$ & & \\
\hline U3R-F3 & 9 & $(1.42 \pm 2.69) E-01$ & $(1.33 \pm 1.12) E+00$ & $(-1.02 \pm 0.97) E+00$ \\
\hline \multicolumn{5}{|l|}{ Four Mile Creek } \\
\hline C-Canal & 6 & $(0.51 \pm 1.34) E-01$ & $(4.69 \pm 3.65) E-01$ & $(-4.59 \pm 3.35) E-01$ \\
\hline C-Canal & 1 & $(0.91 \pm 1.26) \mathrm{E}+00$ & & \\
\hline FM-2 at Road 4 & 20 & $(4.16 \pm 0.37) E+00$ & $(7.64 \pm 0.90) E+00$ & $(1.64 \pm 0.66) E+00$ \\
\hline FM-2 at Road 4 & 1 & $(1.07 \pm 0.40) E+00$ & & \\
\hline $\begin{array}{l}\text { FM-2B above } \\
\text { F-Area effluent }\end{array}$ & 20 & $(7.98 \pm 1.88) E+00$ & $(3.39 \pm 0.35) E+01$ & $(2.49 \pm 0.68) E+00$ \\
\hline $\begin{array}{l}\text { FM-2B above } \\
\text { F-Area effluent }\end{array}$ & 1 & $(4.52 \pm 1.47) E+00$ & & \\
\hline $\begin{array}{l}\text { FM-3A below } \\
\text { F-Area effluent }\end{array}$ & 20 & $(7.41 \pm 0.93) E-01$ & $(1.80 \pm 0.48) E+00$ & $(1.89 \pm 5.47) \mathrm{E}-01$ \\
\hline $\begin{array}{l}\text { FM-3A below } \\
\text { F-Area effluent }\end{array}$ & 1 & $(2.82 \pm 4.12) E-01$ & & \\
\hline FM-6 at Road A-12.2 & 1 & $(3.88 \pm 1.44) E+00$ & & \\
\hline FM-A7 at Road $A-7$ & 20 & $(8.17 \pm 1.10) E+00$ & $(2.30 \pm 0.19) E+01$ & $(2.49 \pm 0.47) \mathrm{E}+00$ \\
\hline FM-A7 at Road A-7 & 1 & $(1.05 \pm 0.18) E+01$ & & \\
\hline $\begin{array}{l}\text { HP-50 Tritium Facility } \\
\text { Outfall }\end{array}$ & 1 & $(3.33 \pm 2.94) E-01$ & & \\
\hline
\end{tabular}


Table 16

Radioactivity in Savannah River Site Stream Water

Page 5 of 14

\begin{tabular}{lcr} 
Location & $\begin{array}{c}\text { No. of } \\
\text { Samples }\end{array}$ & Arithmetic Mean $\pm \sigma$ \\
\hline $\begin{array}{l}\text { Pen Branch } \\
\text { IGB-21 } 800 \text { feet south } \\
\text { of Road 6-1 }\end{array}$ & 1 & $(0.97 \pm 3.75) E-01$ \\
$\quad$ PB-3 at Road A-13.2 & 1 & $(-0.03 \pm 1.19) E+00$ \\
Steel Creek & & \\
$\quad \begin{array}{l}\text { SC-2A } 1 \text { mile } \\
\text { above Road B }\end{array}$ & 1 & $(-0.58 \pm 4.19) E-01$ \\
$\begin{array}{l}\text { SC-4 Steel Creek } \\
\text { at Road A }\end{array}$ & 1 & $(-0.36 \pm 1.17) E+00$ \\
Lower Three Runs Creek & & \\
L3R-1A at Road B & 1 & $(1.43 \pm 1.29) E+00$ \\
L3R-2 Patterson Mill Road & 1 & $(-0.80 \pm 3.58) E-01$ \\
L3R-3 at Highway 125 & 1 & $(2.37 \pm 3.86) E-01$ \\
R-Area downstream of R-1 & 1 & $(5.22 \pm 4.06) E-01$
\end{tabular}

U-234, pCi/L

Tims Branch

TB-5 near Road C

$(1.78 \pm 0.22) E-01$

Upper Three Runs

Crouch Branch at Road 4

$(1.83 \pm 4.23) \mathrm{E}-03$

McQueen Branch

$(1.30 \pm 0.66) \mathrm{E}-02$

at Road $F$

U3R-1A Treadway Bridge

$(5.63 \pm 5.74) E-03$

Road 8-1

$(2.39 \pm 0.81) \mathrm{E}-02$

U3R-3 at Road C

$(5.32 \pm 0.94) \mathrm{E}-02$

Maximum $\pm \sigma$

Minimum $\pm \sigma$

U3R-4 at Road $A$

$(1.28 \pm 0.70) \mathrm{E}-02$

U3R-4 at Road A

$(2.92 \pm 0.54) \mathrm{E}-01$

$(9.30 \pm 1.59) E-02$

$(2.73 \pm 0.80) E-02$

U3R-F3

Four Mile Creek

C-Canal

FM-2 at Road 4

FM-2B above

F-Area effluent

FM-3A below

F-Area effluent

FM-6 at Road A-12.2

FM-6 at Road A-12.2

FM-A7 at Road A-7
$(2.54 \pm 0.81) \mathrm{E}-02$

(3.66 \pm 6.04$) \mathrm{E}-03$

$(-8.13 \pm 2.98) E-03$

$(-1.87 \pm 4.09) E-03$

$(4.02 \pm 2.52) E-01$

$(1.65 \pm 0.12) E+00$

$(5.56 \pm 1.06) E-02$

$(9.66 \pm 5.71) \mathrm{E}-03$

$(2.96 \pm 0.89) E-02$ 


\section{Table 16}

\section{Radioactivity in Savannah River Site Stream Water}

Page 6 of 14

\begin{tabular}{|c|c|c|c|c|}
\hline Location & $\begin{array}{l}\text { No. of } \\
\text { Samples }\end{array}$ & Arithmetic Mean $\pm \sigma$ & Maximum $\pm \sigma$ & Minimum $\pm \sigma$ \\
\hline $\begin{array}{l}\text { HP-50 Tritium Facility } \\
\text { Outfall }\end{array}$ & 1 & $(2.14 \pm 4.25) E-03$ & & \\
\hline \multicolumn{5}{|l|}{ Pen Branch } \\
\hline PB-3 at Road A-13.2 & 6 & $(3.95 \pm 1.19) \mathrm{E}-02$ & $(9.50 \pm 1.49) \mathrm{E}-02$ & $(1.75 \pm 0.74) E-02$ \\
\hline PB-3 at Road A-13.2 & 1 & $(-8.53 \pm 2.44) E-03$ & & \\
\hline \multicolumn{5}{|l|}{ Steel Creek } \\
\hline $\begin{array}{l}\text { SC-2A } 1 \text { mile } \\
\text { above Road B }\end{array}$ & 1 & $(9.06 \pm 6.31) E-03$ & & \\
\hline $\begin{array}{l}\text { SC-4 Steel Creek } \\
\text { at Road A }\end{array}$ & 1 & $(1.98 \pm 4.31) \mathrm{E}-03$ & & \\
\hline \multicolumn{5}{|l|}{ Lower Three Runs Creek } \\
\hline L3R-1A at Road B & 1 & $(7.98 \pm 5.86) E-03$ & & \\
\hline L3R-2 Patterson Mill Road & 6 & $(5.72 \pm 0.90) \mathrm{E}-02$ & $(9.72 \pm 1.47) \mathrm{E}-02$ & $(3.98 \pm 0.85) \mathrm{E}-02$ \\
\hline L3R-2 Patterson Mill Road & 1 & $(1.39 \pm 0.65) E-02$ & & \\
\hline L3R-3 at Highway 125 & 1 & $(8.48 \pm 6.12) E-03$ & & \\
\hline R-Area downstream of R-1 & 1 & $(3.98 \pm 4.36) E-03$ & & \\
\hline \multicolumn{5}{|l|}{ U-235, pCi/L } \\
\hline \multicolumn{5}{|l|}{ Tims Branch } \\
\hline TB-5 near Road C & 1 & $(1.28 \pm 0.62) E-02$ & & \\
\hline \multicolumn{5}{|l|}{ Upper Three Runs } \\
\hline Crouch Branch at Road 4 & 1 & $(-1.45 \pm 0.81) E-03$ & & \\
\hline $\begin{array}{l}\text { McQueen Branch } \\
\text { at Road F }\end{array}$ & 1 & $(6.41 \pm 3.73) \mathrm{E}-03$ & & \\
\hline $\begin{array}{l}\text { U3R-1A Treadway Bridge } \\
\text { Road 8-1 }\end{array}$ & 1 & $(-4.33 \pm 0.79) E-03$ & & \\
\hline U3R-3 at Road C & 1 & $(-5.88 \pm 1.38) E-03$ & & \\
\hline U3R-4 at Road A & 6 & $(3.08 \pm 2.16) E-03$ & $(7.90 \pm 4.43) E-03$ & $(-6.57 \pm 2.74) E-03$ \\
\hline U3R-4 at Road A & 1 & $(1.42 \pm 2.31) E-03$ & & \\
\hline U3R-F3 & 8 & $(1.11 \pm 0.20) E-02$ & $(1.87 \pm 1.12) \mathrm{E}-02$ & $(4.10 \pm 4.45) \mathrm{E}-03$ \\
\hline \multicolumn{5}{|l|}{ Four Mile Creek } \\
\hline C-Canal & 1 & $(0.64 \pm 2.32) E-03$ & & \\
\hline FM-2 at Road 4 & 1 & $(1.57 \pm 2.56) E-03$ & & \\
\hline $\begin{array}{l}\text { FM-2B above } \\
\text { F-Area effluent }\end{array}$ & 1 & $(-6.88 \pm 6.89) E-04$ & & \\
\hline $\begin{array}{l}\text { FM-3A below } \\
\text { F-Area effluent }\end{array}$ & 1 & $(1.33 \pm 2.16) \mathrm{E}-03$ & & \\
\hline FM-6 at Road A-12.2 & 6 & $(3.48 \pm 2.53) \mathrm{E}-02$ & $(1.59 \pm 0.22) E-01$ & $(-7.27 \pm 7.30) E-04$ \\
\hline
\end{tabular}


Table 16

Radioactivity in Savannah River Site Stream Water

Page 7 of 14

\begin{tabular}{|c|c|c|c|c|}
\hline Location & $\begin{array}{c}\text { No. of } \\
\text { Samples }\end{array}$ & Arithmetic Mean $\pm \sigma$ & Maximum $\pm \sigma$ & Minimum $\pm \sigma$ \\
\hline FM-6 at Road A-12.2 & 1 & $(-5.11 \pm 1.13) E-03$ & & \\
\hline FM-A7 at Road A-7 & 1 & $(5.74 \pm 3.84) E-03$ & & \\
\hline $\begin{array}{l}\text { HP-50 Tritium Facility } \\
\text { Outfall }\end{array}$ & 1 & $(-0.01 \pm 2.33) E-03$ & & \\
\hline \multicolumn{5}{|l|}{ Pen Branch } \\
\hline PB-3 at Road A-13.2 & 6 & $(2.84 \pm 1.94) E-03$ & $(6.69 \pm 3.89) \mathrm{E}-03$ & $(-5.09 \pm 2.49) E-03$ \\
\hline PB-3 at Road A-13.2 & 1 & $(1.32 \pm 2.14) E-03$ & & \\
\hline \multicolumn{5}{|l|}{ Steel Creek } \\
\hline $\begin{array}{l}\text { SC-2A } 1 \text { mile } \\
\text { above Road B }\end{array}$ & 1 & $(0.00 \pm 0.03) E+00$ & & \\
\hline $\begin{array}{l}\text { SC-4 Steel Creek } \\
\text { at Road A }\end{array}$ & 1 & $(-1.56 \pm 2.85) E-03$ & & \\
\hline \multicolumn{5}{|l|}{ Lower Three Runs Creek } \\
\hline L3R-1A at Road B & 1 & $(0.07 \pm 3.23) E-03$ & & \\
\hline L3R-2 Patterson Mill Road & 6 & $(5.17 \pm 2.17) E-03$ & $(1.49 \pm 0.57) E-02$ & $(-0.24 \pm 3.72) E-03$ \\
\hline L3R-2 Patterson Mill Road & 1 & $(1.61 \pm 2.27) E-03$ & & \\
\hline L.3R-3 at Highway 125 & 1 & $(-0.74 \pm 2.16) E-03$ & & \\
\hline R-Area downstream of R-1 & 1 & $(-3.60 \pm 0.22) E-03$ & & \\
\hline \multicolumn{5}{|l|}{$\overline{U-238, p C i / L}$} \\
\hline \multicolumn{5}{|l|}{ Tims Branch } \\
\hline TB-5 near Road C & 1 & $(2.16 \pm 0.24) E-01$ & & \\
\hline \multicolumn{5}{|l|}{ Upper Three Runs } \\
\hline Crouch Branch at Road 4 & 1 & $(-0.77 \pm 3.29) E-03$ & & \\
\hline $\begin{array}{l}\text { McQueen Branch } \\
\text { at Road F }\end{array}$ & 1 & $(2.01 \pm 0.66) E-02$ & & \\
\hline $\begin{array}{l}\text { U3R-1 A Treadway Bridge } \\
\text { Road 8-1 }\end{array}$ & 1 & $(1.49 \pm 0.64) E-02$ & & \\
\hline U3R-3 at Road C & 1 & $(3.23 \pm 0.86) E-02$ & & \\
\hline U3R-4 at Road A & 6 & $(6.16 \pm 0.43) E-02$ & $(7.86 \pm 1.40) E-02$ & $(4.61 \pm 1.01) \mathrm{E}-02$ \\
\hline U3R-4 at Road A & 1 & $(9.29 \pm 5.45) \mathrm{E}-03$ & & \\
\hline U3R-F3 & 8 & $(9.74 \pm 1.31) \mathrm{E}-02$ & $(1.52 \pm 0.20) E-01$ & $(4.23 \pm 3.45) E-02$ \\
\hline \multicolumn{5}{|l|}{ Four Mile Creek } \\
\hline C-Canal & 1 & $(7.16 \pm 4.52) E-03$ & & \\
\hline FM-2 at Road 4 & 1 & $(-0.87 \pm 2.84) E-03$ & & \\
\hline $\begin{array}{l}\text { FM-2B above } \\
\text { F-Area effluent }\end{array}$ & 1 & $(5.50 \pm 3.94) E-03$ & & \\
\hline
\end{tabular}


Table 16

Radioactivity in Savannah River Site Stream Water

Page 8 of 14

\begin{tabular}{|c|c|c|c|c|}
\hline Location & $\begin{array}{l}\text { No. of } \\
\text { Samples }\end{array}$ & Arithmetic Mean $\pm \sigma$ & Maximum $\pm \sigma$ & Minimum $\pm \sigma$ \\
\hline $\begin{array}{l}\text { FM-3A below } \\
\text { F-Area effluent }\end{array}$ & 1 & $(1.24 \pm 0.53) E-02$ & & \\
\hline$F M-6$ at Road A-12.2 & 6 & $(5.02 \pm 2.94) E-01$ & $(1.96 \pm 0.14) E+00$ & $(9.32 \pm 1.42) E-02$ \\
\hline FM-6 at Road A-12.2 & 1 & $(2.43 \pm 0.77) \mathrm{E}-02$ & & \\
\hline FM-A7 at Road A-7 & 1 & $(5.18 \pm 1.04) E-02$ & & \\
\hline $\begin{array}{l}\text { HP-50 Tritium Facility } \\
\text { Outfall }\end{array}$ & 1 & $(8.34 \pm 5.17) E-03$ & & \\
\hline \multicolumn{5}{|l|}{ Pen Branch } \\
\hline PB-3 at Road A-13.2 & 6 & $(3.02 \pm 0.85) E-02$ & $(5.59 \pm 1.07) E-02$ & $(4.99 \pm 4.55) E-03$ \\
\hline PB-3 at Road A-13.2 & 1 & $(5.14 \pm 4.05) E-03$ & & \\
\hline \multicolumn{5}{|l|}{ Steel Creek } \\
\hline $\begin{array}{l}\text { SC-2A } 1 \text { mile } \\
\text { above Road B }\end{array}$ & 1 & $(1.02 \pm 0.51) E-02$ & & \\
\hline $\begin{array}{l}\text { SC-4 Steel Creek } \\
\text { at Road A }\end{array}$ & 1 & $(-2.58 \pm 2.76) E-03$ & & \\
\hline \multicolumn{5}{|l|}{ Lower Three Runs Creek } \\
\hline L3R-1A at Road B & 1 & $(-2.28 \pm 3.18) E-03$ & & \\
\hline L3R-2 Patterson Mill Road & 6 & $(4.66 \pm 0.70) E-02$ & $(7.93 \pm 1.27) \mathrm{E}-02$ & $(2.99 \pm 0.74) \mathrm{E}-02$ \\
\hline L3R-2 Patterson Mill Road & 1 & $(2.83 \pm 4.15) E-03$ & & \\
\hline L3R-3 at Highway 125 & 1 & $(1.41 \pm 4.99) E-03$ & & \\
\hline R-Area downstream of R-1 & 1 & $(2.22 \pm 4.07) E-03$ & & \\
\hline \multicolumn{5}{|l|}{$\mathrm{Pu}-238, \mathrm{pCi} / \mathrm{L}$} \\
\hline \multicolumn{5}{|l|}{ Tims Branch } \\
\hline TB-5 near Road C & 1 & $(4.29 \pm 3.22) E-03$ & & \\
\hline \multicolumn{5}{|l|}{ Upper Three Runs } \\
\hline Crouch Branch at Road 4 & 1 & $(9.39 \pm 1.48) E-02$ & & \\
\hline $\begin{array}{l}\text { McQueen Branch } \\
\text { at Road F }\end{array}$ & 1 & $(2.85 \pm 0.30) E-01$ & & \\
\hline $\begin{array}{l}\text { U3R-1A Treadway Bridge } \\
\text { Road 8-1 }\end{array}$ & 1 & $(4.03 \pm 2.98) E-03$ & & \\
\hline U3R-3 at Road C & 1 & $(0.67 \pm 2.85) E-03$ & & \\
\hline U3R-4 at Road A & 6 & $(-0.04 \pm 2.03) E-03$ & $(7.16 \pm 5.07) \mathrm{E}-03$ & $(-6.75 \pm 0.35) E-03$ \\
\hline U3R-4 at Road A & 1 & $(6.23 \pm 1.16) E-02$ & & \\
\hline U3R-F3 & 8 & $(1.64 \pm 0.43) \mathrm{E}-02$ & $(3.88 \pm 1.01) \mathrm{E}-02$ & $(0.48 \pm 1.38) E-02$ \\
\hline
\end{tabular}




\section{Table 16}

\section{Radioactivity in Savannah River Site Stream Water}

Page 9 of 14

\begin{tabular}{|c|c|c|c|c|}
\hline Location & $\begin{array}{l}\text { No. of } \\
\text { Samples }\end{array}$ & Arithmetic Mean $\pm \sigma$ & Maximum $\pm \sigma$ & Minimum $\pm \sigma$ \\
\hline \multicolumn{5}{|l|}{ Four Mile Creek } \\
\hline C-Canal & 1 & $(2.00 \pm 0.23) E-01$ & & \\
\hline FM-2 at Road 4 & 1 & $(2.20 \pm 0.25) E-01$ & & \\
\hline $\begin{array}{l}\text { FM-2B above } \\
\text { F-Area effluent }\end{array}$ & 1 & $(3.28 \pm 0.81) E-02$ & & \\
\hline $\begin{array}{l}\text { FM-3A below } \\
\text { F-Area effluent }\end{array}$ & 1 & $(2.93 \pm 0.29) E-01$ & & \\
\hline FM-6 at Road A-12.2 & 6 & $(5.28 \pm 2.33) E-03$ & $(1.38 \pm 0.66) E-02$ & $(0.32 \pm 3.93) E-03$ \\
\hline FM-6 at Road A-12.2 & 1 & $(-1.10 \pm 0.07) E-03$ & & \\
\hline FM-A7 at Road A-7 & 1 & $(1.03 \pm 0.15) E-01$ & & \\
\hline $\begin{array}{l}\text { HP-50 Tritium Facility } \\
\text { Outfall }\end{array}$ & 1 & $(3.22 \pm 0.22) E+00$ & & \\
\hline \multicolumn{5}{|l|}{ Pen Branch } \\
\hline PB-3 at Road A-13.2 & 6 & $(-0.39 \pm 2.28) E-03$ & $(8.31 \pm 4.84) E-03$ & $(-7.43 \pm 0.78) E-03$ \\
\hline PB-3 at Road A-13.2 & 1 & $(2.86 \pm 0.29) E-01$ & & \\
\hline \multicolumn{5}{|l|}{ Steel Creek } \\
\hline $\begin{array}{l}\text { SC-2A } 1 \text { mile } \\
\text { above Road B }\end{array}$ & 1 & $(2.87 \pm 0.29) E-01$ & & \\
\hline $\begin{array}{l}\text { SC-4 Steel Creek } \\
\text { at Road A }\end{array}$ & 1 & $(1.84 \pm 0.23) E-01$ & & \\
\hline \multicolumn{5}{|l|}{ Lower Three Runs Creek } \\
\hline L3R-1A at Road B & 1 & $(4.02 \pm 2.97) E-03$ & & \\
\hline L3R-2 Patterson Mill Road & 6 & $(-2.40 \pm 1.02) E-03$ & $(2.16 \pm 2.17) \mathrm{E}-03$ & $(-5.44 \pm 2.15) E-03$ \\
\hline L3R-2 Patterson Mill Road & 1 & $(7.47 \pm 3.76) E-03$ & & \\
\hline L3R-3 at Highway 125 & 1 & $(2.31 \pm 2.53) E-03$ & & \\
\hline R-Area downstream of $R-1$ & 1 & $(-1.73 \pm 0.63) E-03$ & & \\
\hline \multicolumn{5}{|l|}{ Pu-239, pCi/L } \\
\hline \multicolumn{5}{|l|}{ Tims Branch } \\
\hline TB-5 near Road C & 1 & $(0.00 \pm 0.03) E+00$ & & \\
\hline \multicolumn{5}{|l|}{ Upper Three Runs } \\
\hline Crouch Branch at Road 4 & 1 & $(-0.49 \pm 2.04) E-03$ & & \\
\hline $\begin{array}{l}\text { McQueen Branch } \\
\text { at Road F }\end{array}$ & 1 & $(1.09 \pm 2.76) E-03$ & & \\
\hline $\begin{array}{l}\text { U3R-1A Treadway Bridge } \\
\text { Road 8-1 }\end{array}$ & 1 & $(5.14 \pm 2.99) E-03$ & & \\
\hline U3R-3 at Road C & 1 & $(3.07 \pm 2.70) E-03$ & & \\
\hline U3R-4 at Road A & 6 & $(-7.45 \pm 3.51) E-04$ & $(0.00 \pm 0.03) E+00$ & $(-1.98 \pm 1.15) E-03$ \\
\hline
\end{tabular}


Table 16 Radioactivity in Savannah River Site Stream Water

Page 10 of 14

\begin{tabular}{|c|c|c|c|c|}
\hline Location & $\begin{array}{l}\text { No. of } \\
\text { Samples }\end{array}$ & Arithmetic Mean $\pm \sigma$ & Maximum $\pm \sigma$ & Minimum $\pm \sigma$ \\
\hline U3R-4 at Road A & 1 & $(1.05 \pm 2.73) E-03$ & & \\
\hline U3R-F3 & 8 & $(7.79 \pm 2.42) E-03$ & $(1.97 \pm 0.99) E-02$ & $(-0.01 \pm 1.12) E-02$ \\
\hline \multicolumn{5}{|l|}{ Four Mile Creek } \\
\hline C-Canal & 1 & $(4.83 \pm 3.84) E-03$ & & \\
\hline FM-2 at Road 4 & 1 & $(-0.07 \pm 1.97) E-03$ & & \\
\hline $\begin{array}{l}\text { FM-2B above } \\
\text { F-Area effluent }\end{array}$ & 1 & $(-0.92 \pm 1.84) E-03$ & & \\
\hline $\begin{array}{l}\text { FM-3A below } \\
\text { F-Area effluent }\end{array}$ & 1 & $(7.89 \pm 4.38) E-03$ & & \\
\hline FM-6 at Road A-12.2 & 6 & $(0.89 \pm 1.16) E-03$ & $(3.63 \pm 3.19) E-03$ & $(-4.63 \pm 1.21) \mathrm{E}-03$ \\
\hline FM-6 at Road A-12.2 & 1 & $(3.58 \pm 2.54) \mathrm{E}-03$ & & \\
\hline FM-A7 at Road A-7 & 1 & $(5.17 \pm 3.86) E-03$ & & \\
\hline $\begin{array}{l}\text { HP-50 Tritium Facility } \\
\text { Outfall }\end{array}$ & 1 & $(5.05 \pm 1.01) E-02$ & & \\
\hline \multicolumn{5}{|l|}{ Pen Branch } \\
\hline PB-3 at Road A-13.2 & 6 & $(1.22 \pm 0.57) E-03$ & $(3.26 \pm 2.87) E-03$ & $(-6.90 \pm 6.92) E-04$ \\
\hline PB-3 at Road A-13.2 & 1 & $(4.32 \pm 3.55) E-03$ & & \\
\hline \multicolumn{5}{|l|}{ Steel Creek } \\
\hline $\begin{array}{l}\text { SC-2A } 1 \text { mile } \\
\text { above Road B }\end{array}$ & 1 & $(6.65 \pm 3.91) E-03$ & & \\
\hline $\begin{array}{l}\text { SC-4 Steel Creek } \\
\text { at Road A }\end{array}$ & 1 & $(-1.09 \pm 2.39) E-03$ & & \\
\hline \multicolumn{5}{|l|}{ Lower Three Runs Creek } \\
\hline L3R-1A at Road $B$ & 1 & $(4.54 \pm 3.03) E-03$ & & \\
\hline L3R-2 Patterson Mill Road & 6 & $(2.84 \pm 2.20) E-03$ & $(1.30 \pm 0.53) E-02$ & $(-1.43 \pm 1.01) E-03$ \\
\hline L3R-2 Patterson Mill Road & 1 & $(-3.04 \pm 0.94) E-03$ & & \\
\hline L3R-3 at Highway 125 & 1 & $(2.33 \pm 2.68) E-03$ & & \\
\hline R-Area downstream of $R-1$ & 1 & $(3.61 \pm 2.56) \mathrm{E}-03$ & & \\
\hline \multicolumn{5}{|l|}{$\overline{A m-241, p C i / L}$} \\
\hline Tims Branch & & 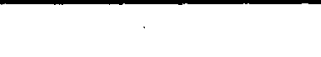 & & \\
\hline TB-5 near Road C & 1 & $(-5.22 \pm 4.73) E-03$ & & \\
\hline \multicolumn{5}{|l|}{ Upper Three Runs } \\
\hline Crouch Branch at Road 4 & 1 & $(2.56 \pm 4.32) E-03$ & & \\
\hline $\begin{array}{l}\text { McQueen Branch } \\
\text { at Road F }\end{array}$ & 1 & $(4.02 \pm 5.80) E-03$ & & \\
\hline $\begin{array}{l}\text { U3R-1A Treadway Bridge } \\
\text { Road 8-1 }\end{array}$ & 1 & $(1.97 \pm 0.70) E-02$ & & \\
\hline
\end{tabular}


Table 16

Radioactivity in Savannah River Site Stream Water

Page 11 of 14

\begin{tabular}{|c|c|c|c|c|}
\hline Location & $\begin{array}{l}\text { No. of } \\
\text { Samples }\end{array}$ & Arithmetic Mean $\pm \sigma$ & Maximum $\pm \sigma$ & Minimum $\pm \sigma$ \\
\hline U3R-3 at Road C & 1 & $(-3.00 \pm 1.85) E-03$ & & \\
\hline U3R-4 at Road A & 1 & $(1.67 \pm 4.41) \mathrm{E}-03$ & & \\
\hline U3R-F3 & 8 & $(6.75 \pm 2.42) E-03$ & $(2.04 \pm 1.31) E-02$ & $(-2.05 \pm 4.54) E-03$ \\
\hline \multicolumn{5}{|l|}{ Four Mile Creek } \\
\hline C-Canal & 1 & $(0.13 \pm 4.14) E-03$ & & \\
\hline FM-2 at Road 4 & 1 & $(-5.93 \pm 2.82) E-03$ & & \\
\hline $\begin{array}{l}\text { FM-2B above } \\
\text { F-Area effluent }\end{array}$ & 1 & $(-3.21 \pm 3.26) E-03$ & & \\
\hline $\begin{array}{l}\text { FM-3A below } \\
\text { F-Area effluent }\end{array}$ & 1 & $(-2.42 \pm 3.36) E-03$ & & \\
\hline FM-6 at Road A-12.2 & 1 & $(1.70 \pm 0.64) E-02$ & & \\
\hline $\mathrm{FM}-\mathrm{A} 7$ at Road $\mathrm{A}-7$ & 1 & $(9.80 \pm 5.73) \mathrm{E}-03$ & & \\
\hline $\begin{array}{l}\text { HP-50 Tritium Facility } \\
\text { Outfall }\end{array}$ & 1 & $(3.16 \pm 0.84) E-02$ & & \\
\hline \multicolumn{5}{|l|}{ Pen Branch } \\
\hline $\mathrm{PB}-3$ at Road A-13.2 & 1 & $(-5.98 \pm 2.22) \mathrm{E}-03$ & & \\
\hline \multicolumn{5}{|l|}{ Steel Creek } \\
\hline $\begin{array}{l}\text { SC-2A } 1 \text { mile } \\
\text { above Road B }\end{array}$ & 1 & $(-0.32 \pm 3.93) E-03$ & & \\
\hline $\begin{array}{l}\text { SC-4 Steel Creek } \\
\text { at Road A }\end{array}$ & 1 & $(2.04 \pm 0.74) E-02$ & & \\
\hline \multicolumn{5}{|l|}{ Lower Three Runs Creek } \\
\hline L3R-1A at Road B & 1 & $(4.03 \pm 3.99) E-03$ & & \\
\hline L3R-2 Patterson Mill Road & 1 & $(1.93 \pm 0.69) E-02$ & & \\
\hline L3R-3 at Highway 125 & 1 & $(-7.52 \pm 4.53) E-03$ & & \\
\hline R-Area downstream of $R-1$ & 1 & $(8.70 \pm 5.15) \mathrm{E}-03$ & & \\
\hline \multicolumn{5}{|l|}{$\mathrm{Cm}-244, \mathrm{pCi} / \mathrm{L}$} \\
\hline \multicolumn{5}{|l|}{ Tims Branch } \\
\hline TB-5 near Road C & 1 & $(0.83 \pm 3.30) E-03$ & & \\
\hline \multicolumn{5}{|l|}{ Upper Three Runs } \\
\hline Crouch Branch at Road 4 & 1 & $(-0.08 \pm 2.24) E-03$ & & \\
\hline $\begin{array}{l}\text { McQueen Branch } \\
\text { at Road F }\end{array}$ & 1 & $(4.06 \pm 3.51) E-03$ & & \\
\hline $\begin{array}{l}\text { U3R-1A Treadway Bridge } \\
\text { Road 8-1 }\end{array}$ & 1 & $(1.90 \pm 3.20) E-03$ & & \\
\hline U3R-3 at Road C & 1 & $(5.59 \pm 4.14) E-03$ & & \\
\hline
\end{tabular}


Table 16

Radioactivity in Savannah River Site Stream Water

Page 12 of 14

\begin{tabular}{|c|c|c|c|c|}
\hline Location & $\begin{array}{l}\text { No. of } \\
\text { Samples }\end{array}$ & Arithmetic Mean $\pm \sigma$ & Maximum $\pm \sigma$ & Minimum $\pm \sigma$ \\
\hline U3R-4 at Road A & 1 & $(1.16 \pm 2.69) \mathrm{E}-03$ & & \\
\hline U3R-F3 & 8 & $(2.72 \pm 2.77) E-02$ & $(2.21 \pm 0.31) E-01$ & $(-2.88 \pm 0.73) E-03$ \\
\hline \multicolumn{5}{|l|}{ Four Mile Creek } \\
\hline C-Canal & 1 & $(0.66 \pm 1.85) E-03$ & & \\
\hline FM-2 at Road 4 & 1 & $(6.50 \pm 3.85) E-03$ & & \\
\hline $\begin{array}{l}\text { FM-2B above } \\
\text { F-Area effluent }\end{array}$ & 1 & $(-1.18 \pm 0.05) E-03$ & & \\
\hline $\begin{array}{l}\text { FM-3A below } \\
\text { F-Area effluent }\end{array}$ & 1 & $(2.22 \pm 0.63) E-02$ & & \\
\hline FM-6 at Road A-12.2 & 1 & $(-1.83 \pm 1.82) E-03$ & & \\
\hline FM-A7 at Road A-7 & 1 & $(5.68 \pm 3.44) E-03$ & & $\cdot$ \\
\hline $\begin{array}{l}\text { HP-50 Tritium Facility } \\
\text { Outfall }\end{array}$ & 1 & $(6.99 \pm 3.52) E-03$ & & \\
\hline \multicolumn{5}{|l|}{ Pen Branch } \\
\hline PB-3 at Road A-13.2 & 1 & $(1.93 \pm 2.20) E-03$ & & \\
\hline \multicolumn{5}{|l|}{ Steel Creek } \\
\hline $\begin{array}{l}\text { SC-2A } 1 \text { mile } \\
\text { above Road B }\end{array}$ & 1 & $(3.46 \pm 3.10) E-03$ & & \\
\hline $\begin{array}{l}\text { SC-4 Steel Creek } \\
\text { at Road A }\end{array}$ & 1 & $(5.88 \pm 3.39) E-03$ & & \\
\hline \multicolumn{5}{|l|}{ Lower Three Runs Creek } \\
\hline L3R-1A at Road $B$ & 1 & $(-1.88 \pm 1.78) E-03$ & & \\
\hline L3R-2 Patterson Mill Road & 1 & $(1.69 \pm 0.56) E-02$ & & \\
\hline L3R-3 at Highway 125 & 1 & $(-1.63 \pm 2.86) E-03$ & & \\
\hline R-Area downstream of $R-1$ & 1 & $(2.16 \pm 3.36) E-03$ & & \\
\hline \multicolumn{5}{|l|}{ Gross Alpha, pCi/L } \\
\hline \multicolumn{5}{|l|}{ Tims Branch } \\
\hline TB-5 near Road C & 26 & $(3.13 \pm 0.29) E+00$ & $(6.69 \pm 1.13) \mathrm{E}+00$ & $(7.92 \pm 3.68) \mathrm{E}-01$ \\
\hline \multicolumn{5}{|l|}{ Upper Three Runs } \\
\hline Crouch Branch at Road 4 & 26 & $(3.70 \pm 0.62) E-01$ & $(1.20 \pm 0.47) E+00$ & $(-3.12 \pm 1.79) E-01$ \\
\hline $\begin{array}{l}\text { McQueen Branch } \\
\text { at Road F }\end{array}$ & 26 & $(2.27 \pm 0.29) E+00$ & $(6.63 \pm 1.12) E+00$ & $(-1.14 \pm 2.26) E-01$ \\
\hline $\begin{array}{l}\text { U3R-1A Treadway Bridge } \\
\text { Road 8-1 }\end{array}$ & 52 & $(3.67 \pm 0.17) E+00$ & $(6.30 \pm 0.98) E+00$ & $(1.68 \pm 0.48) E+00$ \\
\hline U3R-3 at Road C & 26 & $(2.46 \pm 0.15) E+00$ & $(4.19 \pm 0.71) E+00$ & $(9.66 \pm 4.06) E-01$ \\
\hline U3R-4 at Road A & 26 & $(3.04 \pm 0.35) E+00$ & $(8.94 \pm 1.04) E+00$ & $(8.15 \pm 3.68) E-01$ \\
\hline U3R-F3 & 10 & $(1.40 \pm 0.43) E+00$ & $(3.59 \pm 1.30) E+00$ & $(-1.35 \pm 1.98) E+00$ \\
\hline
\end{tabular}




\section{Table 16}

\section{Radioactivity in Savannah River Site Stream Water}

\section{Page 13 of 14}

\begin{tabular}{|c|c|c|c|c|}
\hline Location & $\begin{array}{l}\text { No. of } \\
\text { Samples }\end{array}$ & Arithmetic Mean $\pm \sigma$ & Maximum $\pm \sigma$ & Minimum $\pm \sigma$ \\
\hline \multicolumn{5}{|l|}{ Four Mile Creek } \\
\hline C-Canal & 26 & $(9.97 \pm 0.78) E-01$ & $(1.98 \pm 0.53) E+00$ & $(1.30 \pm 3.75) E-01$ \\
\hline FM-2 at Road 4 & 26 & $(2.40 \pm 0.38) E+00$ & $(8.83 \pm 1.17) \mathrm{E}+00$ & $(2.97 \pm 2.01) E-01$ \\
\hline $\begin{array}{l}\text { FM-2B above } \\
\text { F-Area effluent }\end{array}$ & 26 & $(1.01 \pm 0.52) E+01$ & $(1.07 \pm 0.08) E+02$ & $(-0.58 \pm 6.13) E-01$ \\
\hline $\begin{array}{l}\text { FM-3A below } \\
\text { F-Area effluent }\end{array}$ & 26 & $(2.37 \pm 0.26) E+\infty 0$ & $(7.57 \pm 1.05) E+\infty 0$ & $(7.68 \pm 4.64) E-01$ \\
\hline FM-6 at Road A-12.2 & 26 & $(4.84 \pm 1.10) E+00$ & $(2.09 \pm 0.24) E+01$ & $(1.50 \pm 3.29) E-01$ \\
\hline FM-A7 at Road A-7 & 26 & $(9.18 \pm 2.26) E+00$ & $(5.13 \pm 0.29) E+01$ & $(7.37 \pm 4.46) \mathrm{E}-01$ \\
\hline $\mathrm{H}-008$ Outfall & 6 & $(3.64 \pm 0.58) E+00$ & $(5.63 \pm 0.70) E+00$ & $(1.98 \pm 0.39) E+00$ \\
\hline $\begin{array}{l}\text { HP-50 Tritium Facility } \\
\text { Outfall }\end{array}$ & 26 & $(8.52 \pm 0.84) E+00$ & $(1.95 \pm 0.32) E+01$ & $(-1.25 \pm 2.20) E-01$ \\
\hline \multicolumn{5}{|l|}{ Pen Branch } \\
\hline $\begin{array}{l}\text { IGB-21 } 800 \text { feet south } \\
\text { of Road 6-1 }\end{array}$ & 1 & $(6.53 \pm 5.21) E-01$ & & \\
\hline K-011 Outfall at B Road & 6 & $(8.87 \pm 9.26) E-02$ & $(4.34 \pm 2.45) E-01$ & $(-1.29 \pm 1.66) \mathrm{E}-01$ \\
\hline PB-3 at Road A-13.2 & 26 & $(3.47 \pm 0.77) \mathrm{E}-01$ & $(1.27 \pm 0.53) E+00$ & $(-1.50 \pm 2.84) E-01$ \\
\hline \multicolumn{5}{|l|}{ Steel Creek } \\
\hline $\begin{array}{l}\text { SC-2A } 1 \text { mile } \\
\text { above Road B }\end{array}$ & 26 & $(8.30 \pm 1.00) E-01$ & $(2.33 \pm 0.39) E+00$ & $(-0.26 \pm 2.35) E-01$ \\
\hline $\begin{array}{l}\mathrm{SC}-4 \text { Steel Creek } \\
\text { at Road A }\end{array}$ & 26 & $(1.49 \pm 0.23) E+00$ & $(4.04 \pm 0.75) E+00$ & $(-0.08 \pm 2.66) E-01$ \\
\hline \multicolumn{5}{|l|}{ Lower Three Runs Creek } \\
\hline L3R-1A at Road B & 26 & $(1.55 \pm 0.74) E+00$ & $(1.81 \pm 0.25) E+01$ & $(-3.32 \pm 2.76) E-01$ \\
\hline L3R-2 Patterson Mill Road & 26 & $(9.15 \pm 1.01) \mathrm{E}-01$ & $(2.14 \pm 0.77) \mathrm{E}+00$ & $(1.94 \pm 2.49) \mathrm{E}-01$ \\
\hline L3R-3 at Highway 125 & 20 & $(1.46 \pm 0.28) E+00$ & $(4.58 \pm 0.94) E+00$ & $(-2.31 \pm 1.53) E-01$ \\
\hline R-Area downstream of R-1 & 26 & $(8.36 \pm 1.39) E-01$ & $(3.08 \pm 0.98) E+00$ & $(-1.85 \pm 3.25) E-01$ \\
\hline \multicolumn{5}{|l|}{ Gross Beta, pCi/L } \\
\hline \multicolumn{5}{|l|}{ Tims Branch } \\
\hline TB-5 near Road C & 26 & $(1.80 \pm 0.16) E+00$ & $(4.65 \pm 0.72) E+00$ & $(7.98 \pm 4.52) \mathrm{E}-01$ \\
\hline \multicolumn{5}{|l|}{ Upper Three Runs } \\
\hline Crouch Branch at Road 4 & 26 & $(1.04 \pm 0.09) E+00$ & $(1.98 \pm 0.59) E+00$ & $(-0.15 \pm 4.52) E-01$ \\
\hline $\begin{array}{l}\text { McQueen Branch } \\
\text { at Road F }\end{array}$ & 26 & $(2.71 \pm 0.21) \mathrm{E}+00$ & $(4.79 \pm 0.82) E+00$ & $(1.05 \pm 0.41) E+00$ \\
\hline $\begin{array}{l}\text { U3R-1A Treadway Bridge } \\
\text { Road 8-1 }\end{array}$ & 52 & $(1.93 \pm 0.24) E+00$ & $(1.31 \pm 0.10) E+01$ & $(6.78 \pm 5.50) E-01$ \\
\hline U3R-3 at Road C & 26 & $(1.44 \pm 0.13) E+00$ & $(2.64 \pm 0.57) E+00$ & $(2.05 \pm 4.62) E-01$ \\
\hline
\end{tabular}


Table 16

Radioactivity in Savannah River Site Stream Water

Page 14 of 14

\begin{tabular}{|c|c|c|c|c|}
\hline Location & $\begin{array}{l}\text { No. of } \\
\text { Samples }\end{array}$ & Arithmetic Mean $\pm \sigma$ & Maximum $\pm \sigma$ & Minimum $\pm \sigma$ \\
\hline U3R-4 at Road A & 26 & $(1.68 \pm 0.19) E+00$ & $(5.48 \pm 0.71) E+00$ & $(6.58 \pm 5.24) \mathrm{E}-01$ \\
\hline U3R-F3 & 10 & $(2.36 \pm 0.89) \mathrm{E}+00$ & $(5.24 \pm 1.47) \mathrm{E}+00$ & $(-5.01 \pm 4.20) E+00$ \\
\hline \multicolumn{5}{|l|}{ Four Mile Creek } \\
\hline C-Canal & 26 & $(9.85 \pm 0.83) E-01$ & $(2.06 \pm 0.58) E+00$ & $(1.27 \pm 4.18) E-01$ \\
\hline FM-2 at Road 4 & 26 & $(1.53 \pm 0.14) E+01$ & $(3.21 \pm 0.14) E+01$ & $(5.15 \pm 0.44) E+00$ \\
\hline $\begin{array}{l}\text { FM-2B above } \\
\text { F-Area effluent }\end{array}$ & 26 & $(3.11 \pm 0.88) E+01$ & $(1.72 \pm 0.07) E+02$ & $(6.59 \pm 3.59) \mathrm{E}+00$ \\
\hline $\begin{array}{l}\text { FM-3A below } \\
\text { F-Area effluent }\end{array}$ & 26 & $(4.92 \pm 0.25) \mathrm{E}+00$ & $(8.78 \pm 0.86) E+00$ & $(2.93 \pm 0.48) E+00$ \\
\hline FM-6 at Road A-12.2 & 26 & $\langle 1.83 \pm 0.20) E+01$ & $(4.64 \pm 0.20) E+01$ & $(7.21 \pm 0.76) E+00$ \\
\hline FM-A7 at Road A-7 & 26 & $(2.41 \pm 0.20) E+01$ & $(5.34 \pm 0.22) E+01$ & $(3.18 \pm 0.93) E+00$ \\
\hline $\mathrm{H}-008$ Outfall & 6 & $(6.15 \pm 0.55) E+00$ & $(8.68 \pm 0.56) E+00$ & $(4.91 \pm 0.45) E+00$ \\
\hline $\begin{array}{l}\text { HP-50 Tritium Facility } \\
\text { Outfall }\end{array}$ & 26 & $(5.05 \pm 0.40) E+00$ & $(1.19 \pm 0.11) E+01$ & $(1.14 \pm 0.49) E+00$ \\
\hline \multicolumn{5}{|l|}{ Pen Branch } \\
\hline $\begin{array}{l}\text { IGB-21 } 800 \text { feet south } \\
\text { of Road } 6-1\end{array}$ & 1 & $(2.27 \pm 0.66) E+00$ & & \\
\hline $\mathrm{K}-011$ Outfall at B Road & 6 & $(1.28 \pm 0.19) E+00$ & $(1.74 \pm 0.44) E+00$ & $(5.13 \pm 2.76) \mathrm{E}-01$ \\
\hline PB-3 at Road A-13.2 & 26 & $(9.58 \pm 1.02) E-01$ & $(1.90 \pm 0.52) E+00$ & $(-0.20 \pm 4.34) E-01$ \\
\hline \multicolumn{5}{|l|}{ Steel Creek } \\
\hline $\begin{array}{l}\text { SC-2A } 1 \text { mile } \\
\text { above Road B }\end{array}$ & 26 & $(3.35 \pm 0.19) E+00$ & $(5.02 \pm 0.47) E+00$ & $(1.94 \pm 0.41) E+00$ \\
\hline $\begin{array}{l}\text { SC-4 Steel Creek } \\
\text { at Road A }\end{array}$ & 26 & $(1.54 \pm 0.12) E+00$ & $(2.54 \pm 0.58) E+00$ & $(4.46 \pm 4.15) E-01$ \\
\hline \multicolumn{5}{|l|}{ Lower Three Runs Creek } \\
\hline L3R-1A at Road B & 26 & $(4.60 \pm 1.05) E+00$ & $(2.93 \pm 0.15) E+01$ & $(1.68 \pm 0.44) E+00$ \\
\hline L3R-2 Patterson Mill Road & 26 & $(2.06 \pm 0.16) E+00$ & $(3.99 \pm 0.79) E+00$ & $(6.58 \pm 5.05) \mathrm{E}-01$ \\
\hline L3R-3 at Highway 125 & 20 & $(2.16 \pm 0.28) E+00$ & $(5.39 \pm 0.75) E+00$ & $(8.53 \pm 4.61) \mathrm{E}-01$ \\
\hline R-Area downstream of $R-1$ & 26 & $(2.19 \pm 0.19) E+01$ & $(4.72 \pm 0.19) E+01$ & $(6.52 \pm 0.65) E+00$ \\
\hline
\end{tabular}


Table 17

\section{Calculated Migration of Radioactivity from Seepage Basins}

Page 1 of 1

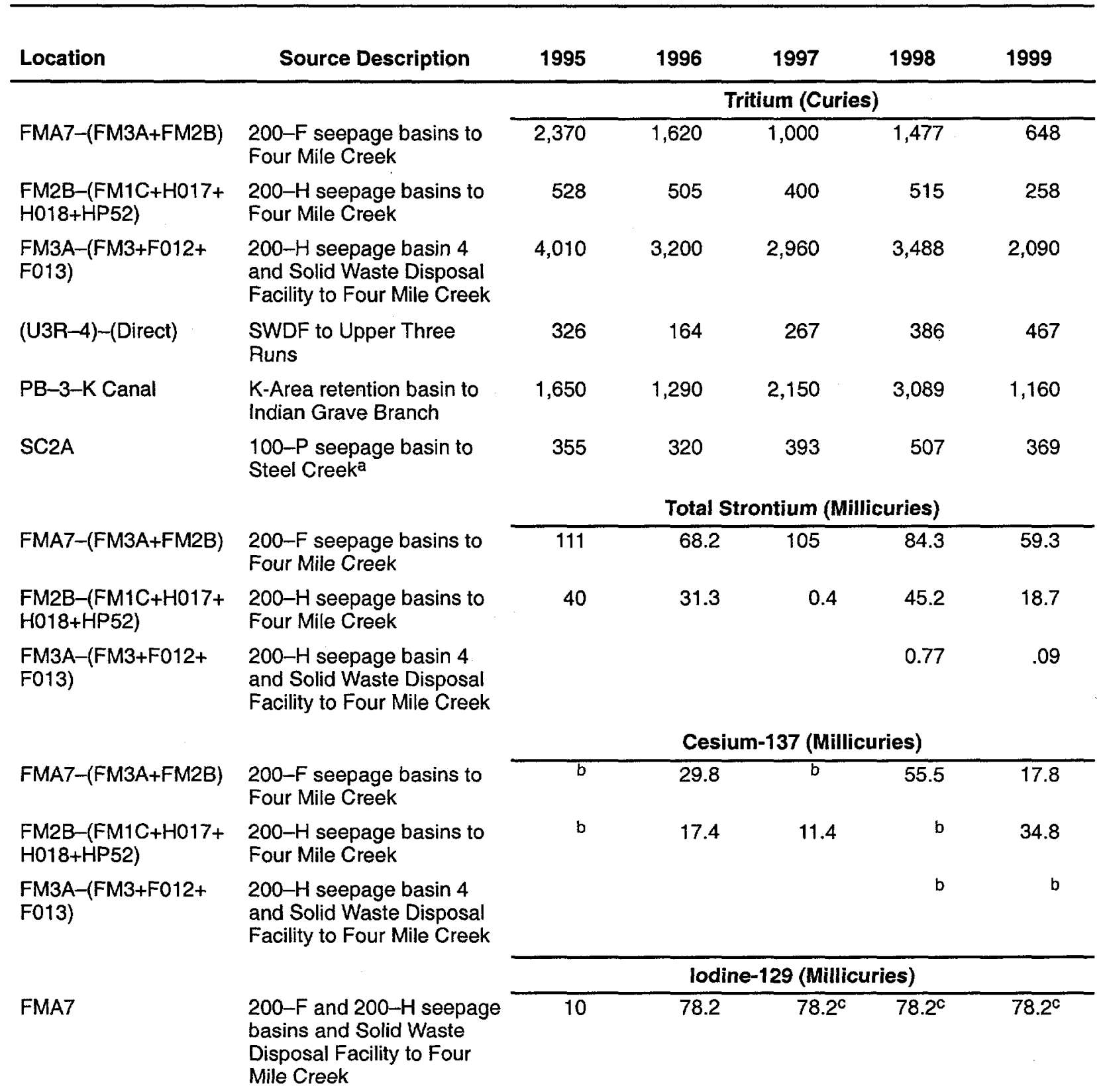

\footnotetext{
Includes some secondary effluent releases from P-Area when effluent was diverted from PAR Pond

Measured iodine-129 results were not available for 1997, 1998, and 1999 from the Savannah River Technology Laboratory. This value was measured in 1996.
} 
Table 18

Estimated Tritium Transport in SRS Streams and the Savannah River

Page 1 of 3

\begin{tabular}{|c|c|c|c|c|c|c|}
\hline \multirow[b]{2}{*}{ Area } & \multirow[b]{2}{*}{ Release Point } & \multicolumn{5}{|c|}{ Direct Releases (Curies) } \\
\hline & & 1995 & 1996 & 1997 & 1998 & 1999 \\
\hline \multicolumn{7}{|c|}{ Reactor } \\
\hline $100-P$ & $\begin{array}{l}\text { PAR Pond overflow to Lower } \\
\text { Three Runs Creek (L3R2) }\end{array}$ & 56 & 47 & 46 & 110 & 87 \\
\hline $100-R$ & 105-R sumps to PAR Pond & & & & & 1 \\
\hline \multirow[t]{4}{*}{$100-L$} & $\begin{array}{l}\text { L-Lake overflow to Steel Creek } \\
\text { (SC4) }\end{array}$ & 472 & 376 & 715 & $(243)^{a}$ & $(152)^{\mathrm{a}}$ \\
\hline & ${ }^{*}$ Process sewer to L-Lake & & & & & \\
\hline & $\begin{array}{l}{ }^{*} \text { Reactor heat exchanger cool- } \\
\text { ing water to L-Lake }\end{array}$ & & & & & \\
\hline & ${ }^{*}$ Combined in 1992 (L007) & $(11)^{a}$ & $(10)^{\mathrm{a}}$ & $(6)^{\mathrm{a}}$ & $(3)^{\mathrm{a}}$ & 6 \\
\hline $100-K$ & (K-Canal) & 11 & 12 & 2 & 1 & 1 \\
\hline \multirow[t]{2}{*}{$100-C$} & $\begin{array}{l}\text { Process Sewer to Four Mile } \\
\text { Creek (C-Canal) }\end{array}$ & 3 & 2 & 1 & b & b \\
\hline & Subtotal & 542 & 437 & 764 & 111 & 95 \\
\hline \multicolumn{7}{|c|}{ Separations } \\
\hline \multirow[t]{2}{*}{$200-F$} & Effluent to Four Mile Creek & 2 & 1 & 1 & 1 & 2 \\
\hline & Effluent to Upper Three Runs & 1 & 2 & 1 & 2 & 1 \\
\hline \multirow[t]{4}{*}{$200-\mathrm{H}$} & Effluent to Four Mile Creek & 9 & 7 & 9 & 8 & 10 \\
\hline & Effluent to Upper Three Runs & 14 & 11 & 15 & 2 & 2 \\
\hline & Effluent Treatment Facility & 142 & 308 & 382 & 567 & 793 \\
\hline & Subtotal & 168 & 329 & 408 & 580 & 808 \\
\hline
\end{tabular}

D-Area and TNX

\begin{tabular}{llrrrrr}
\hline 400-D & $\begin{array}{l}\text { Process sewer to Beaver Dam } \\
\text { Creek (400-D) } \\
\text { TNX }\end{array}$ & 628 & 183 & 402 & 398 & 213 \\
& $\begin{array}{l}\text { TNX-008 } \\
\text { Subtotal }\end{array}$ & 628 & 183 & 402 & 399 & 1 \\
A-Area & & & & & & \\
\hline $700-A$ & $\begin{array}{l}\text { Effluent to Tims Branch (TB-2) } \\
\text { Subtotal } \\
\quad \text { Total Direct Releasesc }\end{array}$ & 1,340 & 949 & 1,570 & 1092 & 1,119
\end{tabular}

a Not used in totals because release was counted elsewhere

b Discontinued in 1998

c Because of rounding, sums of individual columns might not equal totals. 
Table 18

Estimated Tritium Transport in SRS Streams and the Savannah River

Page 2 of 3

\begin{tabular}{|c|c|c|c|c|c|c|}
\hline \multirow[b]{2}{*}{ Area } & \multirow[b]{2}{*}{ Release Point } & \multicolumn{5}{|c|}{ Migration (Curies) } \\
\hline & & 1995 & 1996 & 1997 & 1998 & 1999 \\
\hline $\begin{array}{l}200- \\
F \& H\end{array}$ & $\begin{array}{l}\text { Solid Waste Disposal Facility } \\
\text { and General Separations Area } \\
\text { to Upper Three Runs }\end{array}$ & $(326)^{a}$ & $(164)^{\mathrm{a}}$ & 267 & 386 & 467 \\
\hline \multirow[t]{3}{*}{$\begin{array}{l}200- \\
\text { F\&H }\end{array}$} & $\begin{array}{l}\text { Solid Waste Disposal Facility } \\
\text { (FM3A-FM3) and H-Area seep- } \\
\text { age basin to Four Mile Creek }\end{array}$ & 4,010 & 3,200 & 2,960 & 3,488 & 2,090 \\
\hline & $\begin{array}{l}200-F \text { seepage basin to Four } \\
\text { Mile Creek }\end{array}$ & 2,370 & 1,620 & 1,000 & 1,477 & 648 \\
\hline & $\begin{array}{l}200-H \text { seepage basin to Four } \\
\text { Mile Creek }\end{array}$ & 528 & 505 & 400 & 515 & 258 \\
\hline $100-K$ & $\begin{array}{l}\text { 904-88G to Indian Grave } \\
\text { Branch and Pen Branch }\end{array}$ & $1,650^{\mathrm{b}}$ & $1,290^{b}$ & 2,150 & 3,090 & 1,160 \\
\hline \multirow[t]{4}{*}{$100-P$} & Seepage basin to Steel Creek & $(355)^{c}$ & $(320)^{c}$ & $(393)^{c}$ & 507 & 369 \\
\hline & Subtotal & 8,560 & 6,610 & 6,780 & 9,463 & 4,992 \\
\hline & $\begin{array}{l}\text { Total Direct Releases } \\
\text { and Migrationd }\end{array}$ & 9,900 & 7,560 & 8,350 & 10,555 & 6,111 \\
\hline & & \multicolumn{5}{|c|}{ Stream Transport (Curies) } \\
\hline Area & Release Point & 1995 & 1996 & 1997 & 1998 & 1999 \\
\hline $\mathrm{F} \& \mathrm{H}$ & $\begin{array}{l}\text { Upper Three Runs at Road A } \\
\text { (U3R4) }\end{array}$ & 483 & 485 & 665 & 957 & 1,260 \\
\hline $400-0$ & Beaver Dam Creek at swamp & 628 & 183 & 402 & 398 & 213 \\
\hline$F \& H$ & $\begin{array}{l}\text { Four Mile Creek at Road A13 } \\
\text { (FM6) }\end{array}$ & 7,350 & 4,620 & 4,570 & 5,790 & 3,420 \\
\hline $100-K$ & Pen Branch at Road A (PB3) & 2,440 & 2,310 & 2,150 & 3,090 & 1,160 \\
\hline $100-\mathrm{L}$ & Steel Creek at Road A (SC4) & 472 & 376 & 715 & 243 & 152 \\
\hline $100-P$ & $\begin{array}{l}\text { Lower Three Runs at Road B } \\
\text { (L3R2) }\end{array}$ & 56 & 47 & 46 & 110 & 87 \\
\hline & Totald & 11,430 & 8,020 & 8,550 & 10,588 & 6,292 \\
\hline
\end{tabular}

a Not used in totals because migration release was counted as part of transport total

b Beginning in 1995, this value was determined from K018-K-Canal. The K018 location was discontinued in 1997. The value is now (PB-3) - (K-Canal).

c Not used in totals because release was counted as part of direct release

d Because of rounding, sums of individual columns might not equal totals. 
Table 18

Estimated Tritium Transport in SRS Streams and the Savannah River

Page 3 of 3

\begin{tabular}{lrrrrr}
\hline & \multicolumn{5}{c}{ River Transport (Curies) } \\
\cline { 2 - 5 } & 1995 & 1996 & $\mathbf{1 9 9 7}$ & $\mathbf{1 9 9 8}$ & $\mathbf{1 9 9 9}$ \\
\hline $\begin{array}{l}\text { Tritium measured in the Savannah River } \\
\text { below SRS (RM 120) }\end{array}$ & 12,600 & 10,090 & 9,030 & 10,600 & 6,420 \\
$\begin{array}{l}\text { Tritium measured in the Savannah River } \\
\text { above SRS (RM 160) }\end{array}$ & 1,940 & 1,140 & 1,330 & 1,180 & 608 \\
$\begin{array}{l}\text { Tritium measured in the Savannah River } \\
\text { below SRS (downriver minus upriver) }\end{array}$ & $\mathbf{1 0 , 6 6 0}$ & $\mathbf{8 , 9 5 0}$ & $\mathbf{7 , 7 0 0}$ & $\mathbf{9 , 4 2 0}$ & $\mathbf{5 , 8 1 0}$
\end{tabular}

a Because of rounding, sums of individual columns might not equal totals. 
Table 19

Transport of Actinides in Savannah River Site Streams

Page 1 of 1

\begin{tabular}{|c|c|c|c|c|c|}
\hline Location & Source Description & 1996 & 1997 & 1998 & 1999 \\
\hline & & \multicolumn{4}{|c|}{ Uranium-234 (Curies) } \\
\hline FM-6 & stream transport & $6.08 \mathrm{E}-04$ & $4.35 \mathrm{E}-03$ & $1.62 \mathrm{E}-02$ & $8.60 \mathrm{E}-02$ \\
\hline U3R-4 & stream transport & $6.38 \mathrm{E}-03$ & $1.88 \mathrm{E}-02$ & $2.38 E-02$ & $1.24 \mathrm{E}-02$ \\
\hline PB-3 & stream transport & $5.47 \mathrm{E}-04$ & $2.94 \mathrm{E}-03$ & $1.74 \mathrm{E}-03$ & $7.28 \mathrm{E}-04$ \\
\hline$L 3 R-2$ & stream transport & $6.45 E-04$ & $1.51 \mathrm{E}-03$ & $4.96 E-03$ & $3.20 E-03$ \\
\hline \multirow[t]{2}{*}{$\mathrm{SC}-4$} & stream transport & a & a & a & a \\
\hline & & \multicolumn{4}{|c|}{ Uranium-235 (Curies) } \\
\hline $\mathrm{FM}-6$ & stream transport & $5.47 \mathrm{E}-06$ & $1.63 \mathrm{E}-04$ & $1.07 \mathrm{E}-03$ & $6.32 E-04$ \\
\hline U3R-4 & stream transport & $2.04 E-04$ & $5.63 E-04$ & $6.37 \mathrm{E}-04$ & $b$ \\
\hline PB-3 & stream transport & $5.31 E-06$ & $2.24 \mathrm{E}-05$ & $4.60 E-05$ & $b$ \\
\hline L3R-2 & stream transport & $1.28 E-05$ & $2.67 \mathrm{E}-05$ & $2.56 \mathrm{E}-05$ & $2.50 E-04$ \\
\hline \multirow[t]{2}{*}{$S C-4$} & stream transport & a & a & a & a \\
\hline & & \multicolumn{4}{|c|}{ Uranium-238 (Curies) } \\
\hline FM-6 & stream transport & $8.09 E-04$ & $5.27 E-03$ & $2.00 \mathrm{E}-02$ & $1.08 \mathrm{E}-02$ \\
\hline U3R-4 & stream transport & $8.89 E-03$ & $2.06 \mathrm{E}-02$ & $2.79 E-02$ & $1.37 \mathrm{E}-02$ \\
\hline PB-3 & stream transport & $4.66 \mathrm{E}-04$ & $2.76 \mathrm{E}-03$ & $1.32 E-03$ & $4.92 E-04$ \\
\hline L3R-2 & stream transport & $3.55 E-04$ & $1.07 E-03$ & $3.77 \mathrm{E}-03$ & $2.61 E-03$ \\
\hline \multirow[t]{2}{*}{$\mathrm{SC}-4$} & stream transport & a & a & a & a \\
\hline & & \multicolumn{4}{|c|}{ Plutonium-238 (Curies) } \\
\hline$F M-6$ & stream transport & $1.38 \mathrm{E}-04$ & $1.11 \mathrm{E}-04$ & $1.99 \mathrm{E}-04$ & $9.52 E-05$ \\
\hline U3R-4 & stream transport & $2.53 E-03$ & $8.48 \mathrm{E}-04$ & $7.99 E-04$ & $b$ \\
\hline PB-3 & stream transport & $5.29 E-05$ & $1.74 \mathrm{E}-05$ & $4.00 E-05$ & b \\
\hline L3R-2 & stream transport & $8.30 \mathrm{E}-05$ & $2.50 E-05$ & $4.50 E-04$ & $b$ \\
\hline \multirow[t]{2}{*}{$S C-4$} & stream transport & a & $a$ & a & a \\
\hline & & \multicolumn{4}{|c|}{ Plutonium-239 (Curies) } \\
\hline$F M-6$ & stream transport & b & $\bar{b}$ & $1.15 \mathrm{E}-05$ & $\bar{b}$ \\
\hline U3R-4 & stream transport & $\mathrm{b}$ & $b$ & $6.71 E-05$ & b \\
\hline $\mathrm{PB}-3$ & stream transport & $b$ & $b$ & $2.96 \mathrm{E}-05$ & b \\
\hline L3R-2 & stream transport & b & $\mathrm{b}$ & $1.28 \mathrm{E}-05$ & $9.96 E-05$ \\
\hline$S C-4$ & stream transport & a & a & $\mathbf{a}$ & a \\
\hline
\end{tabular}


Table 20

Radioactivity in Savannah River Water

Page 1 of 3

\begin{tabular}{|c|c|c|c|c|}
\hline Location & $\begin{array}{l}\text { No. of } \\
\text { Samples }\end{array}$ & Arithmetic Mean $\pm \sigma$ & Maximum $\pm \sigma$ & Minimum $\pm \sigma$ \\
\hline \multicolumn{5}{|l|}{$\mathrm{H}-3, \mathrm{pCi} / \mathrm{L}$} \\
\hline River Mile 120 & 53 & $(1.19 \pm 0.06) \mathrm{E}+03$ & $(2.21 \pm 0.12) E+03$ & $(2.85 \pm 0.80) E+02$ \\
\hline River Mile 140 & 53 & $(1.46 \pm 0.07) E+03$ & $(2.76 \pm 0.12) \mathrm{E}+03$ & $(3.37 \pm 0.90) E+02$ \\
\hline River Mile 150.0 & 53 & $(2.02 \pm 0.06) E+03$ & $(3.27 \pm 0.11) E+03$ & $(9.09 \pm 0.95) E+02$ \\
\hline River Mile 150.4 & 53 & $(1.92 \pm 0.25) E+03$ & $(8.42 \pm 0.10) E+03$ & $(-9.25 \pm 7.75) E+01$ \\
\hline River Mile 160 & 53 & $(8.72 \pm 1.64) E+01$ & $(3.56 \pm 1.27) E+02$ & $(-1.50 \pm 0.77) E+02$ \\
\hline \multicolumn{5}{|l|}{$\mathrm{Co}-60, \mathrm{pCi} / \mathrm{L}$} \\
\hline River Mile 120 & 53 & $(1.99 \pm 0.55) \mathrm{E}-01$ & $(9.93 \pm 7.32) \mathrm{E}-01$ & $(-6.22 \pm 3.86) E-01$ \\
\hline River Mile 140 & 53 & $(2.42 \pm 0.78) E-01$ & $(1.68 \pm 0.67) E+00$ & $(-1.88 \pm 1.30) E+00$ \\
\hline River Mile 150.0 & 53 & $(1.36 \pm 0.69) \mathrm{E}-01$ & $(1.26 \pm 0.73) E+00$ & $(-1.03 \pm 0.41) E+00$ \\
\hline River Mile 150.4 & 53 & $(1.45 \pm 0.78) E-01$ & $(2.71 \pm 0.79) E+00$ & $(-1.06 \pm 1.01) E+00$ \\
\hline River Mile 160 & 53 & $(1.29 \pm 0.55) E-01$ & $(1.58 \pm 0.63) E+00$ & $(-1.09 \pm 0.89) E+00$ \\
\hline \multicolumn{5}{|l|}{$\mathrm{Cs}-137, \mathrm{pCi} / \mathrm{L}$} \\
\hline River Mile 120 & 53 & $(2.58 \pm 4.17) \mathrm{E}-02$ & $(8.03 \pm 3.88) E-01$ & $(-5.32 \pm 3.85) E-01$ \\
\hline River Mile 140 & 53 & $(1.62 \pm 0.64) E-01$ & $(8.58 \pm 5.70) E-01$ & $(-8.43 \pm 3.96) E-01$ \\
\hline River Mile 150.0 & 53 & $(2.10 \pm 3.70) E-02$ & $(5.05 \pm 3.29) E-01$ & $(-9.61 \pm 6.07) E-01$ \\
\hline \multicolumn{5}{|l|}{$\overline{\mathrm{Sr}-89,90, \mathrm{pCi} / \mathrm{L}}$} \\
\hline River Mile 120 & 1 & $(2.61 \pm 1.65) \mathrm{E}-01$ & & \\
\hline River Mile 140 & 1 & $(2.17 \pm 0.74) E-01$ & & \\
\hline River Mile 150.0 & 1 & $(7.96 \pm 8.58) E-02$ & & \\
\hline River Mile 150.4 & 1 & $(4.51 \pm 7.71) \mathrm{E}-02$ & & \\
\hline River Mile 150.4 & 53 & $(4.95 \pm 6.29) \mathrm{E}-02$ & $(1.36 \pm 0.62) E+00$ & $(-0.98 \pm 1.34) E+00$ \\
\hline River Mile 160 & 1 & $(0.85 \pm 1.00) E-01$ & & \\
\hline River Mile 160 & 53 & $(8.37 \pm 5.18) E-02$ & $(1.07 \pm 0.60) E+00$ & $(-1.33 \pm 0.83) E+00$ \\
\hline \multicolumn{5}{|l|}{$\mathrm{U}-234, \mathrm{pCi} / \mathrm{L}$} \\
\hline River Mile 120 & 1 & $(8.81 \pm 2.04) \mathrm{E}-03$ & & \\
\hline River Mile 140 & 1 & $(5.25 \pm 1.24) E-03$ & & \\
\hline River Mile 150.0 & 1 & $(9.21 \pm 2.36) E-03$ & & \\
\hline River Mile 150.4 & 1 & $(1.42 \pm 0.34) E-02$ & & \\
\hline River Mile 160 & 1 & $(1.04 \pm 0.25) E-02$ & & \\
\hline
\end{tabular}


Table 20

Radioactivity in Savannah River Water

Page 2 of 3

\begin{tabular}{|c|c|c|c|c|}
\hline Location & $\begin{array}{c}\text { No. of } \\
\text { Samples }\end{array}$ & Arithmetic Mean $\pm \sigma$ & Maximum $\pm \sigma$ & Minimum $\pm \sigma$ \\
\hline \multicolumn{5}{|l|}{$\mathrm{U}-235, \mathrm{pCi} / \mathrm{L}$} \\
\hline River Mile 120 & 1 & $(-3.19 \pm 3.80) E-04$ & & \\
\hline River Mile 140 & 1 & $(3.05 \pm 4.89) E-04$ & & \\
\hline River Mile 150.0 & 1 & $(1.36 \pm 0.97) E-03$ & & \\
\hline River Mile 150.4 & 1 & $(5.80 \pm 9.41) E-04$ & & \\
\hline River Mile 160 & 1 & $(4.13 \pm 6.70) E-04$ & & \\
\hline \multicolumn{5}{|l|}{ U-238, pCi/L } \\
\hline River Mile 120 & $\overline{1}$ & $(7.39 \pm 1.93) E-03$ & & \\
\hline River Mile 140 & 1 & $(4.75 \pm 1.28) E-03$ & & \\
\hline River Mile 150.0 & 1 & $(6.64 \pm 2.24) E-03$ & & \\
\hline River Mile 150.4 & 1 & $(1.17 \pm 0.33) E-02$ & & \\
\hline River Mile 160 & 1 & $(5.33 \pm 2.02) E-03$ & & \\
\hline \multicolumn{5}{|l|}{ Pu-238, pCi/L } \\
\hline River Mile 120 & 1 & $(-5.96 \pm 1.96) \mathrm{E}-04$ & & \\
\hline River Mile 140 & 1 & $(-1.94 \pm 0.10) E-04$ & & \\
\hline River Mile 150.0 & 1 & $(-0.80 \pm 5.09) E-04$ & & \\
\hline River Mile 150.4 & 1 & $(-3.94 \pm 0.21) E-04$ & & \\
\hline River Mile 160 & 1 & $(-3.94 \pm 0.19) E-04$ & & \\
\hline \multicolumn{5}{|l|}{$\mathrm{Pu}-239, \mathrm{pCi} / \mathrm{L}$} \\
\hline River Mile 120 & 1 & $(-2.37 \pm 7.09) E-04$ & & \\
\hline River Mile 140 & 1 & $(-3.39 \pm 3.98) E-04$ & & \\
\hline River Mile 150.0 & 1 & $(0.03 \pm 1.00) E-03$ & & \\
\hline River Mile 150.4 & 1 & $(-1.18 \pm 0.77) E-03$ & & \\
\hline River Mile 160 & 1 & $(-9.04 \pm 4.90) E-04$ & & \\
\hline \multicolumn{5}{|l|}{ Am-241, pCi/L } \\
\hline River Mile 120 & 1 & $(6.17 \pm 1.62) E-03$ & & \\
\hline River Mile 140 & 1 & $(2.48 \pm 4.19) E-04$ & & \\
\hline River Mile 150.0 & 1 & $(1.61 \pm 1.16) E-03$ & & \\
\hline River Mile 150.4 & 1 & $(1.62 \pm 1.51) E-03$ & & \\
\hline River Mile 160 & 1 & $(1.82 \pm 1.31) E-03$ & & \\
\hline
\end{tabular}




\section{Table 20}

\section{Radioactivity in Savannah River Water}

Page 3 of 3

\begin{tabular}{|c|c|c|c|c|}
\hline Location & $\begin{array}{l}\text { No. of } \\
\text { Samples }\end{array}$ & Arithmetic Mean $\pm \sigma$ & Maximum $\pm \sigma$ & Minimum $\pm \sigma$ \\
\hline \multicolumn{5}{|l|}{$\mathrm{Cm-244,} \mathrm{pCi} / \mathrm{L}$} \\
\hline River Mile 120 & 1 & $(9.55 \pm 7.49) E-04$ & & \\
\hline River Mile 140 & 1 & $(-3.16 \pm 2.58) E-04$ & & \\
\hline River Mile 150.0 & 1 & $(0.76 \pm 7.35) E-04$ & & \\
\hline River Mile 150.4 & 1 & $(-1.19 \pm 0.24) E-03$ & & \\
\hline River Mile 160 & 1 & $(-8.00 \pm 5.90) E-04$ & & \\
\hline \multicolumn{5}{|l|}{ Gross Alpha, pCi/L } \\
\hline River Mile 120 & 53 & $(2.08 \pm 0.38) E-01$ & $(9.78 \pm 4.18) \mathrm{E}-01$ & $(-2.58 \pm 1.65) E-01$ \\
\hline River Mile 140 & 53 & $(1.18 \pm 0.43) E-01$ & $(6.96 \pm 4.40) E-01$ & $(-4.17 \pm 3.66) \mathrm{E}-01$ \\
\hline River Mile 150.0 & 53 & $(1.27 \pm 0.46) E-01$ & $(1.14 \pm 0.53) E+00$ & $(-3.75 \pm 3.28) E-01$ \\
\hline River Mile 150.4 & 53 & $(5.50 \pm 0.93) E-01$ & $(2.67 \pm 0.91) E+00$ & $(-5.52 \pm 2.76) E-01$ \\
\hline River Mile 160 & 53 & $(5.93 \pm 4.90) E-02$ & $(9.83 \pm 5.93) E-01$ & $(-5.89 \pm 3.05) E-01$ \\
\hline \multicolumn{5}{|l|}{ Gross Beta, pCi/L } \\
\hline River Mile 120 & 53 & $(2.12 \pm 0.08) \mathrm{E}+00$ & $(3.50 \pm 0.52) E+00$ & $(6.61 \pm 3.64) \mathrm{E}-01$ \\
\hline River Mile 140 & 53 & $(2.35 \pm 0.09) E+00$ & $(3.81 \pm 0.74) E+00$ & $(5.01 \pm 3.64) E-01$ \\
\hline River Mile 150.0 & 53 & $(2.12 \pm 0.09) E+00$ & $(3.69 \pm 0.83) E+00$ & $(9.54 \pm 4.59) E-01$ \\
\hline River Mile 150.4 & 53 & $(2.58 \pm 0.11) E+00$ & $(4.32 \pm 0.74) E+00$ & $(7.16 \pm 5.04) E-01$ \\
\hline River Mile 160 & 53 & $(2.22 \pm 0.07) E+00$ & $(3.53 \pm 0.70) E+00$ & $(9.21 \pm 3.85) \mathrm{E}-01$ \\
\hline
\end{tabular}


Table 21

Summary of Savannah River Site Tritium Transport, 1960-1999

Page 1 of 1

\begin{tabular}{|c|c|c|c|}
\hline \multirow[b]{2}{*}{ Year } & \multicolumn{3}{|c|}{ Estimated Tritium Transport (Ci) } \\
\hline & $\begin{array}{l}\text { Based on Point-of- } \\
\text { Release } \\
\text { Concentrations } \\
\text { and Flow Rates }\end{array}$ & $\begin{array}{l}\text { Based on Stream } \\
\text { Concentrations and } \\
\text { Flow Rates }\end{array}$ & $\begin{array}{c}\text { Based on Savannah River } \\
\text { Concentrations and } \\
\text { Flow Rates }\end{array}$ \\
\hline 1960 & $64,000^{b}$ & 69,600 & 73,700 \\
\hline 1961 & $69,000^{b}$ & 83,000 & 77,000 \\
\hline 1962 & $58,000^{b}$ & 64,000 & 63,000 \\
\hline 1963 & $97,000^{b}$ & 96,900 & 122,800 \\
\hline 1964 & $111,000^{b}$ & 131,600 & 143,000 \\
\hline 1965 & 108,400 & 109,200 & 100,200 \\
\hline 1966 & 84,900 & 97,800 & 78,300 \\
\hline 1967 & 70,600 & 77,000 & 68,500 \\
\hline 1968 & 63,800 & 67,200 & 61,800 \\
\hline 1969 & 64,600 & 64,000 & 58,100 \\
\hline 1970 & 36,900 & 43,200 & 31,800 \\
\hline 1971 & 38,200 & 44,700 & 39,100 \\
\hline 1972 & 46,800 & 47,300 & 45,300 \\
\hline 1973 & 71,100 & 62,800 & 61,100 \\
\hline 1974 & 59,900 & 54,600 & 46,000 \\
\hline 1975 & 55,600 & 50,000 & 49,500 \\
\hline 1976 & 59,600 & 47,400 & 51,100 \\
\hline 1977 & 43,800 & 39,700 & 42,500 \\
\hline 1978 & 37,600 & 35,300 & 36,600 \\
\hline 1979 & 29,400 & 27,100 & 30,600 \\
\hline 1980 & 24,900 & 28,800 & 30,700 \\
\hline 1981 & 23,900 & 22,100 & 25,100 \\
\hline 1982 & 32,200 & 31,300 & 30,600 \\
\hline 1983 & 34,200 & 33,000 & 33,000 \\
\hline 1984 & 32,800 & 32,600 & 33,200 \\
\hline 1985 & 25,000 & 22,300 & 24,100 \\
\hline 1986 & 27,800 & 22,300 & 22,100 \\
\hline 1987 & 22,700 & 20,500 & 26,200 \\
\hline 1988 & 19,300 & 18,300 & 14,600 \\
\hline 1989 & 17,300 & 17,800 & 15,600 \\
\hline 1990 & 16,100 & 15,600 & 14,500 \\
\hline 1991 & 27,400 & 26,600 & 26,300 \\
\hline 1992 & 13,800 & 13,100 & 13,800 \\
\hline 1993 & 11,300 & 12,700 & 12,200 \\
\hline 1994 & 8,800 & 10,400 & 10,900 \\
\hline 1995 & 9,900 & 11,400 & 10,700 \\
\hline 1996 & 7,560 & 8,020 & 8,950 \\
\hline 1997 & 8,350 & 8,550 & 7,700 \\
\hline 1998 & 10,555 & 10,588 & 9,420 \\
\hline 1999 & 6,111 & 6,292 & 5,810 \\
\hline
\end{tabular}




\section{Table 22}

\section{Radioactivity in Drinking Water}

Page 1 of 16

No. of

Location Samples Arithmetic Mean $\pm \sigma \quad$ Maximum $\pm \sigma \quad$ Minimum $\pm \sigma$

Note: Results of special analyses of radon and radium in drinking water are discussed in chapter 6, "Radiological Environmental Surveillance," of the SRS Environmental Report for 1999 (WSRC-TR-99-00299).

\begin{tabular}{|c|c|c|c|c|}
\hline $\mathrm{H}-3, \mathrm{pCi} / \mathrm{L}$ & & & & \\
\hline On Site & & & & \\
\hline 192-K Treatment Facility & 4 & $(1.09 \pm 0.87) E+02$ & $(2.91 \pm 1.37) \mathrm{E}+02$ & $(-1.22 \pm 0.77) E+02$ \\
\hline 192-K Treatment Facility & 1 & $(7.28 \pm 9.85) E+01$ & & \\
\hline 483-7D Treatment Facility & 4 & $(-0.45 \pm 2.78) E+01$ & $(7.01 \pm 9.79) E+01$ & $(-6.22 \pm 7.69) E+01$ \\
\hline 483-7D Treatment Facility & 1 & $(-4.45 \pm 9.76) \mathrm{E}+01$ & & \\
\hline $\begin{array}{l}617-G \text { Advanced Tactical } \\
\text { Training Area }\end{array}$ & 1 & $(0.28 \pm 1.23) E+02$ & & \\
\hline $\begin{array}{l}\text { 618-G Class. Yard } \\
\text { Lunchroom }\end{array}$ & 1 & $(-8.32 \pm 9.68) E+01$ & & \\
\hline $\begin{array}{l}\text { 661-G Firing Range } \\
\text { (pumphouse) }\end{array}$ & 1 & $(7.82 \pm 1.27) \mathrm{E}+02$ & & \\
\hline $\begin{array}{l}681-3 G \text { Domestic } \\
\text { Water Faucet }\end{array}$ & 1 & $(0.15 \pm 1.23) E+02$ & & \\
\hline 701-12G Patrol Gate 7 & 1 & $(2.66 \pm 1.25) E+02$ & & \\
\hline 701-13G Patrol Gate 6 & 1 & $(2.44 \pm 0.12) E+03$ & & \\
\hline 701-3G Barnwell Gate & 1 & $(6.68 \pm 9.67) E+01$ & & \\
\hline 701-4G Williston Gate & 1 & $(1.11 \pm 1.23) E+02$ & & \\
\hline 701-5G Talatha Gate & 4 & $(7.58 \pm 0.42) E+02$ & $(8.44 \pm 1.17) \mathrm{E}+02$ & $(6.52 \pm 0.82) E+02$ \\
\hline 701-6G Jackson Gate & 1 & $(5.65 \pm 9.84) E+01$ & & \\
\hline 701-8G Patrol Gate 8 & 1 & $(1.45 \pm 0.11) E+03$ & & \\
\hline $\begin{array}{l}\text { 704-16G Central Sanitary } \\
\text { Wastewater Treatment } \\
\text { Facility }\end{array}$ & 1 & $(0.24 \pm 1.22) E+02$ & & \\
\hline 709-1G L-Area Fire Demo & 1 & $(-8.37 \pm 9.74) E+01$ & & \\
\hline 730-4B Lunchroom & 2 & $(3.90 \pm 1.01) E+01$ & $(0.49 \pm 1.22) E+02$ & $(2.89 \pm 5.48) E+01$ \\
\hline $\begin{array}{l}\text { 735-7G PAR Pond } \\
\text { Limnology Lab }\end{array}$ & 1 & $(-1.33 \pm 1.23) E+02$ & & \\
\hline $\begin{array}{l}\text { 737-G PAR Pond } \\
\text { Radioecology Lab }\end{array}$ & 1 & $(-0.61 \pm 1.22) E+02$ & & \\
\hline 782-3A Treatment Facility & 4 & $(-1.49 \pm 3.69) E+01$ & $(5.49 \pm 9.90) E+01$ & $(-8.59 \pm 7.80) E+01$ \\
\hline 782-3A Treatment Facility & 1 & $(2.42 \pm 9.69) E+01$ & & \\
\hline 905-106K Well & 1 & $(0.22 \pm 1.22) E+02$ & & \\
\hline 905-112G Well & 1 & $(-1.41 \pm 1.34) E+02$ & & \\
\hline 905-113G Well & 1 & $(2.04 \pm 1.37) E+02$ & & \\
\hline
\end{tabular}


Table 22

Radioactivity in Drinking Water

Page 2 of 16

\begin{tabular}{|c|c|c|c|c|}
\hline Location & $\begin{array}{l}\text { No. of } \\
\text { Samples }\end{array}$ & Arithmetic Mean $\pm \sigma$ & $\operatorname{Maximum} \pm \sigma$ & Minimum $\pm \sigma$ \\
\hline 905-125B Well & 1 & $(0.46 \pm 1.36) E+02$ & & \\
\hline 905-136D Well & 1 & $(0.55 \pm 1.22) E+02$ & & \\
\hline 905-3D Well & 1 & $(-0.00 \pm 1.21) E+02$ & & \\
\hline 905-67B Well & 1 & $(2.55 \pm 0.85) E+02$ & & \\
\hline $905-95 \mathrm{~K}$ Well & 1 & $(-1.35 \pm 0.86) E+02$ & & \\
\hline \multicolumn{5}{|l|}{ EMS Sampler } \\
\hline Beaufort Water Works & 25 & $(1.01 \pm 0.06) \mathrm{E}+03$ & $(1.61 \pm 0.08) E+03$ & $(4.09 \pm 0.82) E+02$ \\
\hline \multicolumn{5}{|l|}{ Treatment Plants - Raw } \\
\hline $\begin{array}{l}\text { Beaufort Public Water } \\
\text { Works }\end{array}$ & 12 & $(1.06 \pm 0.08) E+03$ & $(1.47 \pm 0.11) E+03$ & $(5.70 \pm 0.57) \mathrm{E}+02$ \\
\hline $\begin{array}{l}\text { N. Augusta Public Water } \\
\text { Works }\end{array}$ & 12 & $(6.03 \pm 3.38) E+01$ & $(2.91 \pm 1.42) E+02$ & $(-1.53 \pm 1.51) E+02$ \\
\hline $\begin{array}{l}\text { Savannah Public Water } \\
\text { Works }\end{array}$ & 12 & $(9.95 \pm 0.96) E+02$ & $(1.63 \pm 0.12) E+03$ & $(6.14 \pm 0.81) \mathrm{E}+02$ \\
\hline \multicolumn{5}{|l|}{ Treatment Plants - Finished } \\
\hline $\begin{array}{l}\text { Beaufort Public Water } \\
\text { Works }\end{array}$ & 12 & $(9.72 \pm 0.67) E+02$ & $(1.33 \pm 0.12) E+03$ & $(4.96 \pm 0.80) E+02$ \\
\hline $\begin{array}{l}\text { N. Augusta Public Water } \\
\text { Works }\end{array}$ & 12 & $(3.43 \pm 3.34) E+01$ & $(1.83 \pm 0.76) E+02$ & $(-2.18 \pm 1.51) E+02$ \\
\hline $\begin{array}{l}\text { Savannah Public Water } \\
\text { Works }\end{array}$ & 12 & $(9.65 \pm 0.62) E+02$ & $(1.27 \pm 0.16) E+03$ & $(6.03 \pm 0.92) \mathrm{E}+02$ \\
\hline \multicolumn{5}{|l|}{$\mathrm{Co}-60, \mathrm{pCi} / \mathrm{L}$} \\
\hline \multicolumn{5}{|l|}{ On Site } \\
\hline 192-K Treatment Facility & 4 & $(1.43 \pm 1.25) E+00$ & $(3.74 \pm 2.77) \mathrm{E}+00$ & $(-2.03 \pm 2.17) E+00$ \\
\hline 192-K Treatment Facility & 1 & $(-1.05 \pm 2.75) E+00$ & & $\because$ \\
\hline 483-7D Treatment Facility & 4 & $(-1.30 \pm 9.84) E-01$ & $(2.27 \pm 2.10) E+00$ & $(-2.41 \pm 1.85) E+00$ \\
\hline 483-7D Treatment Facility & 1 & $(2.76 \pm 2.32) E+\infty 0$ & & \\
\hline $\begin{array}{l}\text { 617-G Advanced Tactical } \\
\text { Training Area }\end{array}$ & 1 & $(2.65 \pm 2.46) E+00$ & & \\
\hline $\begin{array}{l}\text { 618-G Class. Yard } \\
\text { Lunchroom }\end{array}$ & 1 & $(-1.04 \pm 2.40) E+00$ & & \\
\hline $\begin{array}{l}661-G \text { Firing Range } \\
\text { (pumphouse) }\end{array}$ & 1 & $(-0.94 \pm 2.49) E+00$ & & \\
\hline $\begin{array}{l}\text { 681-3G Domestic } \\
\text { Water Faucet }\end{array}$ & 1 & $(-0.79 \pm 2.54) E+00$ & & \\
\hline 701-12G Patrol Gate 7 & 1 & $(1.17 \pm 2.42) E+00$ & & \\
\hline 701-13G Patrol Gate 6 & 1 & $(4.73 \pm 2.47) E+00$ & & \\
\hline 701-3G Barnwell Gate & 1 & $(0.70 \pm 1.97) E+00$ & & \\
\hline
\end{tabular}




\section{Table 22}

\section{Radioactivity in Drinking Water}

Page 3 of 16

\begin{tabular}{|c|c|c|c|c|}
\hline Location & $\begin{array}{l}\text { No. of } \\
\text { Samples }\end{array}$ & Arithmetic Mean $\pm \sigma$ & Maximum $\pm \sigma$ & Minimum $\pm \sigma$ \\
\hline 701-4G Williston Gate & 1 & $(-4.44 \pm 2.46) E+00$ & & \\
\hline 701-5G Talatha Gate & 4 & $(3.12 \pm 1.56) E+00$ & $(5.79 \pm 1.43) E+00$ & $(-0.82 \pm 2.23) E+00$ \\
\hline 701-6G Jackson Gate & 1 & $(0.20 \pm 1.37) E+00$ & & \\
\hline 701-8G Patrol Gate 8 & 1 & $(0.62 \pm 1.33) E+00$ & & \\
\hline $\begin{array}{l}\text { 704-16G Central Sanitary } \\
\text { Wastewater Treatment } \\
\text { Facility }\end{array}$ & 1 & $(4.83 \pm 2.40) E+00$ & & \\
\hline 709-1G L-Area Fire Demo & 1 & $(2.64 \pm 2.32) E+00$ & & \\
\hline 730-4B Lunchroom & 2 & $(1.53 \pm 0.78) E+00$ & $(2.31 \pm 2.54) E+00$ & $(0.75 \pm 2.51) E+00$ \\
\hline $\begin{array}{l}\text { 735-7G PAR Pond } \\
\text { Limnology Lab }\end{array}$ & 1 & $(1.57 \pm 2.56) E+00$ & & \\
\hline $\begin{array}{l}\text { 737-G PAR Pond } \\
\text { Radioecology Lab }\end{array}$ & 1 & $(1.12 \pm 2.57) E+00$ & & \\
\hline 782-3A Treatment Facility & 4 & $(1.98 \pm 1.51) \mathrm{E}+00$ & $(5.08 \pm 2.46) E+00$ & $(-2.11 \pm 2.34) E+00$ \\
\hline 782-3A Treatment Facility & 1 & $(3.40 \pm 2.36) E+00$ & & \\
\hline 905-106K Well & 1 & $(-0.78 \pm 2.52) E+00$ & & \\
\hline 905-112G Well & 1 & $(0.21 \pm 1.97) E+00$ & & \\
\hline 905-113G Well & 1 & $(1.69 \pm 1.25) E+00$ & & \\
\hline 905-125B Well & 1 & $(2.17 \pm 2.35) E+00$ & & \\
\hline 905-136D Well & 1 & $(2.32 \pm 2.50) E+00$ & & \\
\hline 905-3D Well & 1 & $(0.57 \pm 2.52) E+00$ & & \\
\hline 905-67B Well & 1 & $(1.70 \pm 1.97) E+00$ & & \\
\hline 905-95K Well & 1 & $(2.11 \pm 2.33) E+00$ & & \\
\hline \multicolumn{5}{|l|}{ EMS Sampler } \\
\hline Beaufort Water Works & 25 & $(1.33 \pm 0.69) E+00$ & $(1.17 \pm 1.05) E+01$ & $(-3.99 \pm 1.90) E+00$ \\
\hline \multicolumn{5}{|l|}{ Treatment Plants - Raw } \\
\hline $\begin{array}{l}\text { Beaufort Public Water } \\
\text { Works }\end{array}$ & 12 & $(1.36 \pm 0.77) E+00$ & $(4.85 \pm 2.26) E+00$ & $(-2.56 \pm 2.06) E+00$ \\
\hline $\begin{array}{l}\text { N. Augusta Public Water } \\
\text { Works }\end{array}$ & 12 & $(-2.77 \pm 7.72) E-01$ & $(5.63 \pm 2.42) E+00$ & $(-4.14 \pm 2.56) E+00$ \\
\hline $\begin{array}{l}\text { Savannah Public Water } \\
\text { Works }\end{array}$ & 12 & $(5.59 \pm 8.32) E-01$ & $(4.70 \pm 2.40) E+00$ & $(-5.05 \pm 2.21) E+00$ \\
\hline \multicolumn{5}{|l|}{ Treatment Plants - Finished } \\
\hline $\begin{array}{l}\text { Beaufort Public Water } \\
\text { Works }\end{array}$ & 12 & $(0.73 \pm 6.70) E-01$ & $(3.17 \pm 2.25) E+00$ & $(-4.70 \pm 1.99) E+00$ \\
\hline
\end{tabular}


Table 22

Radioactivity in Drinking Water

Page 4 of 16

\begin{tabular}{|c|c|c|c|c|}
\hline Location & $\begin{array}{l}\text { No. of } \\
\text { Samples }\end{array}$ & Arithmetic Mean $\pm \sigma$ & Maximum $\pm \sigma$ & Minimum $\pm \sigma$ \\
\hline $\begin{array}{l}\text { N. Augusta Public Water } \\
\text { Works }\end{array}$ & 12 & $(2.49 \pm 8.20) \mathrm{E}-01$ & $(3.91 \pm 2.39) E+00$ & $(-5.52 \pm 2.14) E+00$ \\
\hline $\begin{array}{l}\text { Savannah Public Water } \\
\text { Works }\end{array}$ & 12 & $(1.34 \pm 1.06) E+00$ & $(8.65 \pm 3.80) \mathrm{E}+00$ & $(-3.38 \pm 2.61) E+00$ \\
\hline \multicolumn{5}{|l|}{ Cs-137, pCi/L } \\
\hline \multicolumn{5}{|l|}{ On Site } \\
\hline 192-K Treatment Facility & 4 & $(0.21 \pm 1.25) E+00$ & $(2.90 \pm 2.22) \mathrm{E}+00$ & $(-3.17 \pm 2.15) E+\infty 0$ \\
\hline 192-K Treatment Facility & 1 & $(0.60 \pm 2.28) E+00$ & & \\
\hline 483-7D Treatment Facility & 4 & $(9.31 \pm 3.03) E-01$ & $(1.40 \pm 2.18) \mathrm{E}+00$ & $(0.09 \pm 2.23) E+00$ \\
\hline 483-7D Treatment Facility & 1 & $(1.40 \pm 2.37) E+00$ & & \\
\hline $\begin{array}{l}617-G \text { Advanced Tactical } \\
\text { Training Area }\end{array}$ & 1 & $(1.57 \pm 2.55) E+00$ & & \\
\hline $\begin{array}{l}\text { 618-G Class. Yard } \\
\text { Lunchroom }\end{array}$ & 1 & $(1.57 \pm 1.94) E+00$ & & \\
\hline $\begin{array}{l}\text { 661-G Firing Range } \\
\text { (pumphouse) }\end{array}$ & 1 & $(0.66 \pm 1.88) E+00$ & & \\
\hline $\begin{array}{l}\text { 681-3G Domestic } \\
\text { Water Faucet }\end{array}$ & 1 & $(1.05 \pm 2.12) E+00$ & & \\
\hline 701-12G Patrol Gate 7 & 1 & $(1.18 \pm 2.13) E+00$ & & \\
\hline 701-13G Patrol Gate 6 & 1 & $(1.00 \pm 2.08) E+00$ & & \\
\hline 701-3G Barnwell Gate & 1 & $(3.87 \pm 2.41) E+00$ & & \\
\hline $701-4 G$ Williston Gate & 1 & $(-3.06 \pm 2.14) E+00$ & & \\
\hline 701-5G Talatha Gate & 4 & $(3.77 \pm 1.78) E+00$ & $(8.38 \pm 3.41) E+00$ & $(-0.29 \pm 1.38) \mathrm{E}+00$ \\
\hline $701-6$ G Jackson Gate & 1 & $(1.03 \pm 1.31) E+00$ & & \\
\hline 701-8G Patrol Gate 8 & 1 & $(-0.49 \pm 1.31) E+00$ & & \\
\hline $\begin{array}{l}704-16 G \text { Central Sanitary } \\
\text { Wastewater Treatment } \\
\text { Facility }\end{array}$ & 1 & $(1.54 \pm 2.61) E+00$ & & \\
\hline 709-1G L-Area Fire Demo & 1 & $(0.10 \pm 2.35) E+00$ & & \\
\hline 730-4B Lunchroom & 2 & $(1.64 \pm 0.60) E+00$ & $(2.23 \pm 2.17) \mathrm{E}+00$ & $(1.04 \pm 1.93) E+00$ \\
\hline $\begin{array}{l}\text { 735-7G PAR Pond } \\
\text { Limnology Lab }\end{array}$ & 1 & $(-0.63 \pm 2.58) E+00$ & & \\
\hline $\begin{array}{l}\text { 737-G PAR Pond } \\
\text { Radioecology Lab }\end{array}$ & 1 & $(0.01 \pm 2.56) E+00$ & & \\
\hline 782-3A Treatment Facility & 4 & $(0.51 \pm 1.18) E+00$ & $(3.60 \pm 2.33) E+00$ & $(-1.78 \pm 2.14) E+00$ \\
\hline 782-3A Treatment Facility & 1 & $(0.44 \pm 1.93) E+00$ & & \\
\hline 905-106K Well & 1 & $(-0.34 \pm 2.20) E+00$ & & \\
\hline 905-112G Well & 1 & $(-2.87 \pm 2.31) E+00$ & & \\
\hline
\end{tabular}


Table 22

Radioactivity in Drinking Water

Page 5 of 16

\begin{tabular}{|c|c|c|c|c|}
\hline Location & $\begin{array}{l}\text { No. of } \\
\text { Samples }\end{array}$ & Arithmetic Mean $\pm \sigma$ & Maximum $\pm \sigma$ & Minimum $\pm \sigma$ \\
\hline 905-113G Well & 1 & $(-1.33 \pm 1.20) E+00$ & & \\
\hline 905-125B Well & 1 & $(1.76 \pm 2.52) E+00$ & & \\
\hline 905-136D Well & 1 & $(0.47 \pm 2.49) E+00$ & & \\
\hline 905-3D Well & 1 & $(-4.04 \pm 2.38) E+00$ & & \\
\hline 905-67B Well & 1 & $(4.14 \pm 2.25) E+00$ & & \\
\hline 905-95K Well & 1 & $(1.06 \pm 2.24) E+00$ & & \\
\hline \multicolumn{5}{|l|}{ EMS Sampler } \\
\hline Beaufort Water Works & 25 & $(2.11 \pm 4.22) E-01$ & $(7.63 \pm 9.17) \mathrm{E}+00$ & $(-2.61 \pm 1.99) E+00$ \\
\hline \multicolumn{5}{|l|}{ Treatment Plants - Raw } \\
\hline $\begin{array}{l}\text { Beaufort Public Water } \\
\text { Works }\end{array}$ & 12 & $(2.06 \pm 5.47) E-01$ & $(3.14 \pm 1.77) E+00$ & $(-3.77 \pm 2.17) E+00$ \\
\hline $\begin{array}{l}\text { N. Augusta Public Water } \\
\text { Works }\end{array}$ & 12 & $(-4.27 \pm 5.96) E-01$ & $(3.44 \pm 2.46) E+00$ & $(-3.79 \pm 2.08) E+00$ \\
\hline $\begin{array}{l}\text { Savannah Public Water } \\
\text { Works }\end{array}$ & 12 & $(-7.59 \pm 7.50) E-01$ & $(3.71 \pm 2.15) E+00$ & $(-5.04 \pm 3.33) E+00$ \\
\hline \multicolumn{5}{|l|}{ Treatment Plants - Finished } \\
\hline $\begin{array}{l}\text { Beaufort Public Water } \\
\text { Works }\end{array}$ & 12 & $(-3.06 \pm 5.37) E-01$ & $(3.87 \pm 2.02) E+00$ & $(-3.63 \pm 2.09) E+00$ \\
\hline $\begin{array}{l}\text { N. Augusta Public Water } \\
\text { Works }\end{array}$ & 12 & $(-5.00 \pm 7.91) E-01$ & $(4.25 \pm 2.12) E+00$ & $(-4.41 \pm 2.30) E+00$ \\
\hline $\begin{array}{l}\text { Savannah Public Water } \\
\text { Works }\end{array}$ & 12 & $(9.95 \pm 7.03) E-01$ & $(5.33 \pm 2.57) E+00$ & $(-2.73 \pm 2.32) E+00$ \\
\hline \multicolumn{5}{|l|}{$\mathrm{Sr}-89,90, \mathrm{pCi} / \mathrm{L}$} \\
\hline \multicolumn{5}{|l|}{ On Site } \\
\hline 192-K Treatment Facility & 1 & $(5.84 \pm 4.17) E-01$ & & \\
\hline 483-7D Treatment Facility & 1 & $(0.05 \pm 3.78) E-01$ & & \\
\hline $\begin{array}{l}\text { 617-G Advanced Tactical } \\
\text { Training Area }\end{array}$ & 1 & $(-0.58 \pm 3.22) E-01$ & & \\
\hline $\begin{array}{l}\text { 618-G Class. Yard } \\
\text { Lunchroom }\end{array}$ & 1 & $(-0.07 \pm 2.74) E-01$ & & \\
\hline $\begin{array}{l}661-G \text { Firing Range } \\
\text { (pumphouse) }\end{array}$ & 1 & $(1.16 \pm 2.49) \mathrm{E}-01$ & & \\
\hline $\begin{array}{l}\text { 681-3G Domestic } \\
\text { Water Faucet }\end{array}$ & 1 & $(2.75 \pm 3.27) E-01$ & & \\
\hline $701-12 \mathrm{G}$ Patrol Gate 7 & 1 & $(1.21 \pm 0.42) E+00$ & & \\
\hline 701-13G Patrol Gate 6 & 1 & $(3.30 \pm 4.08) E-01$ & & \\
\hline 701-3G Barnwell Gate & 1 & $(1.63 \pm 3.91) E-01$ & & \\
\hline 701-4G Williston Gate & 1 & $(3.32 \pm 3.79) \mathrm{E}-01$ & & \\
\hline
\end{tabular}




\section{Table 22}

\section{Radioactivity in Drinking Water}

Page 6 of 16

\begin{tabular}{|c|c|c|c|c|}
\hline Location & $\begin{array}{c}\text { No. of } \\
\text { Samples }\end{array}$ & Arithmetic Mean $\pm \sigma$ & Maximum $\pm \sigma$ & Minimum $\pm \sigma$ \\
\hline 701-5G Talatha Gate & 4 & $(1.32 \pm 2.53) E-01$ & $(7.93 \pm 5.61) E-01$ & $(-4.34 \pm 3.73) E-01$ \\
\hline 701-6G Jackson Gate & 1 & $(5.36 \pm 2.88) E-01$ & & \\
\hline 701-8G Patrol Gate 8 & 1 & $(0.10 \pm 3.42) E-01$ & & \\
\hline $\begin{array}{l}\text { 704-16G Central Sanitary } \\
\text { Wastewater Treatment } \\
\text { Facility }\end{array}$ & 1 & $(-1.71 \pm 2.97) E-01$ & & \\
\hline 709-1G L-Area Fire Demo & 1 & $(-0.60 \pm 3.57) E-01$ & & \\
\hline $\begin{array}{l}\text { 735-7G PAR Pond } \\
\text { Limnology Lab }\end{array}$ & 1 & $(-1.35 \pm 3.43) E-01$ & & \\
\hline $\begin{array}{l}\text { 737-G PAR Pond } \\
\text { Radioecology Lab }\end{array}$ & 1 & $(0.63 \pm 3.22) E-01$ & & \\
\hline 782-3A Treatment Facility & 1 & $(0.99 \pm 3.91) E-01$ & & \\
\hline 905-106K Well & 1 & $(1.41 \pm 3.95) E-01$ & & \\
\hline 905-112G Well & 1 & $(3.12 \pm 2.35) E-01$ & & \\
\hline 905-113G Well & 1 & $(3.24 \pm 3.34) E-01$ & & \\
\hline 905-125B Well & 1 & $(1.53 \pm 3.16) E-01$ & & \\
\hline 905-136D Well & 1 & $(8.01 \pm 4.46) E-01$ & & \\
\hline 905-3D Well & 1 & $(3.96 \pm 4.00) \mathrm{E}-01$ & & \\
\hline 905-67B Well & 1 & $(0.04 \pm 3.15) E-01$ & & \\
\hline 905-95K Well & 1 & $(-0.84 \pm 3.51) E-01$ & & \\
\hline \multicolumn{5}{|l|}{ U-234, pCi/L } \\
\hline \multicolumn{5}{|l|}{ On Site } \\
\hline 192-K Treatment Facility & 1 & $(2.24 \pm 2.58) E-03$ & & \\
\hline 483-7D Treatment Facility & 1 & $(5.17 \pm 4.25) E-03$ & & \\
\hline $\begin{array}{l}617-G \text { Advanced Tactical } \\
\text { Training Area }\end{array}$ & 1 & $(1.26 \pm 0.59) E-02$ & & \\
\hline $\begin{array}{l}\text { 618-G Class. Yard } \\
\text { Lunchroom }\end{array}$ & 1 & $(6.08 \pm 0.83) E-02$ & & \\
\hline $\begin{array}{l}\text { 661-G Firing Range } \\
\text { (pumphouse) }\end{array}$ & 1 & $(-0.71 \pm 3.78) \mathrm{E}-03$ & & \\
\hline $\begin{array}{l}\text { 681-3G Domestic } \\
\text { Water Faucet }\end{array}$ & 1 & $(-1.93 \pm 2.85) E-03$ & & \\
\hline 701-12G Patrol Gate 7 & 1 & $(-1.37 \pm 3.80) E-03$ & & \\
\hline 701-13G Patrol Gate 6 & 1 & $(1.00 \pm 0.36) E-02$ & & \\
\hline 701-3G Barnwell Gate & 1 & $(1.10 \pm 1.78) E-03$ & & \\
\hline 701-4G Williston Gate & 1 & $(-7.29 \pm 0.99) E-03$ & & \\
\hline 701-5G Talatha Gate & 4 & $(0.02 \pm 3.16) E-03$ & $(6.08 \pm 4.20) E-03$ & $(-6.09 \pm 1.75) E-03$ \\
\hline
\end{tabular}




\section{Table 22}

Radioactivity in Drinking Water

Page 7 of 16

\begin{tabular}{|c|c|c|c|c|}
\hline Location & $\begin{array}{l}\text { No. of } \\
\text { Samples }\end{array}$ & Arithmetic Mean $\pm \sigma$ & Maximum $\pm \sigma$ & Minimum $\pm \sigma$ \\
\hline 701-6G Jackson Gate & 1 & $(-5.10 \pm 3.25) E-03$ & & \\
\hline 701-8G Patrol Gate 8 & 1 & $(2.37 \pm 3.78) E-03$ & & \\
\hline $\begin{array}{l}\text { 704-16G Central Sanitary } \\
\text { Wastewater Treatment } \\
\text { Facility }\end{array}$ & 1 & $(-1.85 \pm 3.99) E-03$ & & \\
\hline 709-1G L-Area Fire Demo & 1 & $(-1.57 \pm 4.24) E-03$ & & \\
\hline $\begin{array}{l}\text { 735-7G PAR Pond } \\
\text { Limnology Lab }\end{array}$ & 1 & $(-1.59 \pm 4.50) E-03$ & & \\
\hline $\begin{array}{l}\text { 737-G PAR Pond } \\
\text { Radioecology Lab }\end{array}$ & 1 & $(4.75 \pm 5.21) E-03$ & & \\
\hline 782-3A Treatment Facility & 1 & $(1.01 \pm 0.42) E-02$ & & \\
\hline 905-106K Well & 1 & $(3.90 \pm 3.70) E-03$ & & \\
\hline 905-112G Well & 1 & $(3.56 \pm 3.77) E-03$ & & \\
\hline 905-113G Well & 1 & $(1.47 \pm 0.63) E-02$ & & \\
\hline $905-125 B$ Well & 1 & $(4.84 \pm 1.01) \mathrm{E}-02$ & & \\
\hline 905-136D Well & 1 & $(8.16 \pm 4.64) E-03$ & & \\
\hline 905-3D Well & 1 & $(1.46 \pm 3.09) E-03$ & & \\
\hline 905-67B Well & 1 & $(3.58 \pm 0.95) E-02$ & & \\
\hline 905-95K Well & 1 & $(6.51 \pm 3.84) E-03$ & & \\
\hline \multicolumn{5}{|l|}{$\mathrm{U}-235, \mathrm{pCi} / \mathrm{L}$} \\
\hline \multicolumn{5}{|l|}{ On Site } \\
\hline 192-K Treatment Facility & 1 & $(-7.34 \pm 7.37) E-04$ & & \\
\hline 483-7D Treatment Facility & 1 & $(3.60 \pm 3.19) E-03$ & & \\
\hline $\begin{array}{l}617-G \text { Advanced Tactical } \\
\text { Training Area }\end{array}$ & 1 & $(-6.28 \pm 2.27) E-03$ & & \\
\hline $\begin{array}{l}\text { 618-G Class. Yard } \\
\text { Lunchroom }\end{array}$ & 1 & $(6.19 \pm 3.08) E-03$ & & \\
\hline $\begin{array}{l}\text { 661-G Firing Range } \\
\text { (pumphouse) }\end{array}$ & 1 & $(-1.47 \pm 0.78) E-03$ & & \\
\hline $\begin{array}{l}\text { 681-3G Domestic } \\
\text { Water Faucet }\end{array}$ & 1 & $(-7.04 \pm 2.45) E-03$ & & \\
\hline 701-12G Patrol Gate 7 & 1 & $(2.20 \pm 3.32) E-03$ & & \\
\hline 701-13G Patrol Gate 6 & 1 & $(1.17 \pm 1.99) E-03$ & & \\
\hline 701-3G Barnwell Gate & 1 & $(0.00 \pm 0.03) E+00$ & & \\
\hline 701-4G Williston Gate & 1 & $(0.75 \pm 2.34) E-03$ & & \\
\hline 701-5G Talatha Gate & 4 & $(0.57 \pm 1.14) E-03$ & $(2.52 \pm 3.65) \mathrm{E}-03$ & $(-2.40 \pm 0.80) E-03$ \\
\hline $701-6$ G Jackson Gate & 1 & $(-0.07 \pm 2.26) E-03$ & & \\
\hline
\end{tabular}


Table 22

Radioactivity in Drinking Water

Page 8 of 16

No. of

Location

Samples Arithmetic Mean $\pm \sigma$

Maximum $\pm \sigma$

Minimum $\pm \sigma$

\begin{tabular}{|c|c|c|c|c|}
\hline 701-8G Patrol Gate 8 & 1 & $(-2.23 \pm 2.64) \mathrm{E}-03$ & & \\
\hline $\begin{array}{l}\text { 704-16G Central Sanitary } \\
\text { Wastewater Treatment } \\
\text { Facility }\end{array}$ & 1 & $(5.41 \pm 4.07) \mathrm{E}-03$ & & \\
\hline 709-1G L-Area Fire Demo & 1 & $(3.55 \pm 3.97) E-03$ & & \\
\hline $\begin{array}{l}\text { 735-7G PAR Pond } \\
\text { Limnology Lab }\end{array}$ & 1 & $(-8.53 \pm 0.53) E-03$ & & \\
\hline $\begin{array}{l}\text { 737-G PAR Pond } \\
\text { Radioecology Lab }\end{array}$ & 1 & $(-7.04 \pm 2.46) E-03$ & & \\
\hline 782-3A Treatment Facility & 1 & $(4.14 \pm 2.95) E-03$ & & \\
\hline 905-106K Well & 1 & $(0.65 \pm 2.37) \mathrm{E}-03$ & & \\
\hline 905-112G Well & 1 & $(1.47 \pm 2.39) E-03$ & & \\
\hline 905-113G Well & 1 & $(6.28 \pm 4.21) \mathrm{E}-03$ & & \\
\hline 905-125B Well & 1 & $(3.62 \pm 3.20) E-03$ & & \\
\hline 905-136D Well & 1 & $(0.00 \pm 0.03) E+00$ & & \\
\hline 905-3D Well & 1 & $(6.51 \pm 4.08) E-03$ & & \\
\hline 905-67B Well & 1 & $(-7.48 \pm 7.50) E-04$ & & \\
\hline 905-95K Well & 1 & $(0.00 \pm 0.03) E+00$ & & \\
\hline \multicolumn{5}{|l|}{ U-238, pCi/L } \\
\hline \multicolumn{5}{|l|}{ On Site } \\
\hline 192-K Treatment Facility & 1 & $(3.26 \pm 3.53) \mathrm{E}-03$ & & \\
\hline 483-7D Treatment Facility & 1 & $(-0.14 \pm 3.36) E-03$ & & \\
\hline $\begin{array}{l}\text { 617-G Advanced Tactical } \\
\text { Training Area }\end{array}$ & 1 & $(6.58 \pm 4.08) \mathrm{E}-03$ & & \\
\hline $\begin{array}{l}\text { 618-G Class. Yard } \\
\text { Lunchroom }\end{array}$ & 1 & $(4.78 \pm 0.74) \mathrm{E}-02$ & & \\
\hline $\begin{array}{l}\text { 661-G Firing Range } \\
\text { (pumphouse) }\end{array}$ & 1 & $(2.03 \pm 0.70) E-02$ & & \\
\hline $\begin{array}{l}\text { 681-3G Domestic } \\
\text { Water Faucet }\end{array}$ & 1 & $(-1.26 \pm 1.95) E-03$ & & \\
\hline 701-12G Patrol Gate 7 & 1 & $(2.92 \pm 3.17) \mathrm{E}-03$ & & \\
\hline 701-13G Patrol Gate 6 & 1 & $(5.92 \pm 3.00) E-03$ & & \\
\hline 701-3G Barnwell Gate & 1 & $(4.33 \pm 3.37) \mathrm{E}-03$ & & \\
\hline 701-4G Williston Gate & 1 & $(2.91 \pm 3.16) \mathrm{E}-03$ & & \\
\hline 701-5G Talatha Gate & 4 & $(-2.31 \pm 0.90) E-03$ & $(0.35 \pm 1.46) E-03$ & $(-3.61 \pm 2.70) E-03$ \\
\hline 701-6G Jackson Gate & 1 & $(0.89 \pm 2.39) E-03$ & & \\
\hline 701-8G Patrol Gate 8 & 1 & $(6.00 \pm 3.84) E-03$ & & \\
\hline
\end{tabular}


Table 22

Radioactivity in Drinking Water

Page 9 of 16

\begin{tabular}{|c|c|c|c|c|}
\hline Location & $\begin{array}{l}\text { No. of } \\
\text { Samples }\end{array}$ & Arithmetic Mean $\pm \sigma$ & Maximum $\pm \sigma$ & Minimum $\pm \sigma$ \\
\hline $\begin{array}{l}\text { 704-16G Central Sanitary } \\
\text { Wastewater Treatment } \\
\text { Facility }\end{array}$ & 1 & $(1.88 \pm 2.63) E-03$ & & \\
\hline 709-1G L-Area Fire Demo & 1 & $(5.17 \pm 3.52) E-03$ & & \\
\hline $\begin{array}{l}\text { 735-7G PAR Pond } \\
\text { Limnology Lab }\end{array}$ & 1 & $(3.44 \pm 3.69) E-03$ & & \\
\hline $\begin{array}{l}\text { 737-G PAR Pond } \\
\text { Radioecology Lab }\end{array}$ & 1 & $(1.72 \pm 3.97) E-03$ & & \\
\hline 782-3A Treatment Facility & 1 & $(1.27 \pm 0.51) E-02$ & & \\
\hline 905-106K Well & 1 & $(6.16 \pm 4.36) E-03$ & & \\
\hline 905-112G Well & 1 & $(1.49 \pm 4.52) \mathrm{E}-03$ & & \\
\hline 905-113G Well & 1 & $(3.07 \pm 0.90) E-02$ & & \\
\hline 905-125B Well & 1 & $(6.68 \pm 1.24) E-02$ & & \\
\hline 905-136D Well & 1 & $(-1.30 \pm 1.75) E-03$ & & \\
\hline 905-3D Well & 1 & $(-4.03 \pm 0.99) E-03$ & & \\
\hline 905-67B Well & 1 & $(5.32 \pm 1.11) E-02$ & & \\
\hline 905-95K Well & 1 & $(5.28 \pm 3.74) E-03$ & & \\
\hline \multicolumn{5}{|l|}{ Pu-238, pCi/L } \\
\hline \multicolumn{5}{|l|}{ On Site } \\
\hline 192-K Treatment Facility & 1 & $(1.53 \pm 3.44) \mathrm{E}-03$ & & \\
\hline 483-7D Treatment Facility & 1 & $(-1.54 \pm 2.14) E-03$ & & \\
\hline $\begin{array}{l}617-G \text { Advanced Tactical } \\
\text { Training Area }\end{array}$ & 1 & $(7.27 \pm 6.22) E-03$ & & \\
\hline $\begin{array}{l}\text { 618-G Class. Yard } \\
\text { Lunchroom }\end{array}$ & 1 & $(2.36 \pm 1.83) \mathrm{E}-03$ & & \\
\hline $\begin{array}{l}661-G \text { Firing Range } \\
\text { (pumphouse) }\end{array}$ & 1 & $(1.21 \pm 1.97) E-03$ & & \\
\hline $\begin{array}{l}\text { 681-3G Domestic } \\
\text { Water Faucet }\end{array}$ & 1 & $(5.65 \pm 6.77) \mathrm{E}-03$ & & \\
\hline 701-12G Patrol Gate 7 & 1 & $(2.29 \pm 2.29) E-03$ & & \\
\hline 701-13G Patrol Gate 6 & 1 & $(0.95 \pm 1.84) E-03$ & & \\
\hline 701-3G Barnwell Gate & 1 & $(-2.13 \pm 0.11) E-03$ & & \\
\hline 701-4G Williston Gate & 1 & $(4.67 \pm 3.31) E-03$ & & \\
\hline 701-5G Talatha Gate & 4 & $(-1.63 \pm 0.75) E-03$ & $(0.12 \pm 2.11) E-03$ & $(-3.43 \pm 0.17) E-03$ \\
\hline 701-6G Jackson Gate & 1 & $(-2.60 \pm 1.51) E-03$ & & \\
\hline 701-8G Patrol Gate 8 & 1 & $(-7.38 \pm 7.39) E-04$ & & \\
\hline
\end{tabular}


Table 22

Radioactivity in Drinking Water

Page 10 of 16

\begin{tabular}{|c|c|c|c|c|}
\hline Location & $\begin{array}{l}\text { No. of } \\
\text { Samples }\end{array}$ & Arithmetic Mean $\pm \sigma$ & Maximum $\pm \sigma$ & Minimum $\pm \sigma$ \\
\hline $\begin{array}{l}\text { 704-16G Central Sanitary } \\
\text { Wastewater Treatment } \\
\text { Facility }\end{array}$ & 1 & $(1.32 \pm 2.13) \mathrm{E}-03$ & & \\
\hline 709-1G L-Area Fire Demo & 1 & $(-2.27 \pm 1.32) E-03$ & & \\
\hline $\begin{array}{l}\text { 735-7G PAR Pond } \\
\text { Limnology Lab }\end{array}$ & 1 & $(1.10 \pm 0.71) \mathrm{E}-02$ & & \\
\hline $\begin{array}{l}\text { 737-G PAR Pond } \\
\text { Radioecology Lab }\end{array}$ & 1 & $(2.03 \pm 0.80) E-02$ & & \\
\hline 782-3A Treatment Facility & 1 & $(1.18 \pm 2.92) E-03$ & & \\
\hline 905-106K Well & 1 & $(1.76 \pm 2.76) \mathrm{E}-03$ & & \\
\hline 905-112G Well & 1 & $(8.53 \pm 4.68) E-03$ & & \\
\hline 905-113G Well & 1 & $(5.84 \pm 4.43) E-03$ & & \\
\hline 905-125B Well & 1 & $(3.16 \pm 0.88) E-02$ & & \\
\hline 905-136D Well & 1 & $(6.65 \pm 4.68) E-03$ & & \\
\hline 905-3D Well & 1 & $(0.73 \pm 2.52) E-03$ & & \\
\hline 905-67B Well & 1 & $(9.35 \pm 5.61) E-03$ & & \\
\hline 905-95K Well & 1 & $(7.33 \pm 4.62) \mathrm{E}-03$ & & \\
\hline \multicolumn{5}{|l|}{ Pu-239, pCi/L } \\
\hline \multicolumn{5}{|l|}{ On Site } \\
\hline 192-K Treatment Facility & 1 & $(-2.12 \pm 2.28) E-03$ & & \\
\hline 483-7D Treatment Facility & 1 & $(-0.81 \pm 2.13) E-03$ & & \\
\hline $\begin{array}{l}617-G \text { Advanced Tactical } \\
\text { Training Area }\end{array}$ & 1 & $(-0.79 \pm 2.82) E-03$ & & \\
\hline $\begin{array}{l}\text { 618-G Class. Yard } \\
\text { Lunchroom }\end{array}$ & 1 & $(-7.38 \pm 4.27) E-04$ & & \\
\hline $\begin{array}{l}\text { 661-G Firing Range } \\
\text { (pumphouse) }\end{array}$ & 1 & $(-6.44 \pm 6.46) \mathrm{E}-04$ & & \\
\hline $\begin{array}{l}\text { 681-3G Domestic } \\
\text { Water Faucet }\end{array}$ & 1 & $(-5.58 \pm 1.55) E-03$ & & \\
\hline 701-12G Patrol Gate 7 & 1 & $(0.00 \pm 0.04) E+00$ & & \\
\hline 701-13G Patrol Gate 6 & 1 & $(3.04 \pm 2.25) E-03$ & & \\
\hline 701-3G Barnwell Gate & 1 & $(0.48 \pm 1.87) E-03$ & & \\
\hline 701-4G Williston Gate & 1 & $(8.51 \pm 4.75) \mathrm{E}-03$ & & \\
\hline 701-5G Talatha Gate & 4 & $(-4.49 \pm 8.84) E-04$ & $(1.45 \pm 3.15) E-03$ & $(-2.79 \pm 0.73) E-03$ \\
\hline 701-6G Jackson Gate & 1 & $(4.14 \pm 3.64) \mathrm{E}-03$ & & \\
\hline 701-8G Patrol Gate 8 & 1 & $(0.65 \pm 2.37) E-03$ & & \\
\hline
\end{tabular}


Table 22

Radioactivity in Drinking Water

Page 11 of 16

\begin{tabular}{|c|c|c|c|c|}
\hline Location & $\begin{array}{l}\text { No. of } \\
\text { Samples }\end{array}$ & Arithmetic Mean $\pm \sigma$ & Maximum $\pm \sigma$ & Minimum $\pm \sigma$ \\
\hline $\begin{array}{l}\text { 704-16G Central Sanitary } \\
\text { Wastewater Treatment } \\
\text { Facility }\end{array}$ & $\overline{1}$ & $(-1.40 \pm 0.99) E-03$ & & \\
\hline 709-1G L-Area Fire Demo & 1 & $(-3.02 \pm 1.52) E-03$ & & \\
\hline $\begin{array}{l}\text { 735-7G PAR Pond } \\
\text { Limnology Lab }\end{array}$ & 1 & $(-0.01 \pm 3.81) E-03$ & & \\
\hline $\begin{array}{l}\text { 737-G PAR Pond } \\
\text { Radioecology Lab }\end{array}$ & 1 & $(-0.71 \pm 1.85) E-03$ & & \\
\hline 782-3A Treatment Facility & 1 & $(1.22 \pm 3.00) \mathrm{E}-03$ & & \\
\hline $905-106 \mathrm{~K}$ Well & 1 & $(0.55 \pm 1.95) E-03$ & & \\
\hline 905-112G Well & 1 & $(-0.08 \pm 2.19) E-03$ & & \\
\hline 905-113G Well & 1 & $(2.17 \pm 2.18) E-03$ & & \\
\hline 905-125B Well & 1 & $(-7.17 \pm 7.19) E-04$ & & \\
\hline 905-136D Well & 1 & $(0.56 \pm 3.13) E-03$ & & \\
\hline 905-3D Well & 1 & $(-1.99 \pm 0.61) E-03$ & & \\
\hline 905-67B Well & 1 & $(0.00 \pm 0.03) E+00$ & & \\
\hline 905-95K Well & 1 & $(-1.48 \pm 2.37) E-03$ & & \\
\hline \multicolumn{5}{|l|}{$\mathrm{Am}-241, \mathrm{pCi} / \mathrm{L}$} \\
\hline \multicolumn{5}{|l|}{ On Site } \\
\hline 192-K Treatment Facility & 1 & $(0.03 \pm 3.21) E-03$ & & \\
\hline 483-7D Treatment Facility & 1 & $(0.47 \pm 3.51) E-03$ & & \\
\hline $\begin{array}{l}617-G \text { Advanced Tactical } \\
\text { Training Area }\end{array}$ & 1 & $(1.66 \pm 0.72) \mathrm{E}-02$ & & \\
\hline $\begin{array}{l}\text { 618-G Class. Yard } \\
\text { Lunchroom }\end{array}$ & 1 & $(-1.90 \pm 4.32) E-03$ & & \\
\hline $\begin{array}{l}\text { 661-G Firing Range } \\
\text { (pumphouse) }\end{array}$ & 1 & $(-3.33 \pm 3.56) E-03$ & & \\
\hline $\begin{array}{l}\text { 681-3G Domestic } \\
\text { Water Faucet }\end{array}$ & 1 & $(1.49 \pm 0.66) E-02$ & & \\
\hline 701-12G Patrol Gate 7 & 1 & $(2.64 \pm 0.81) E-02$ & & \\
\hline 701-13G Patrol Gate 6 & 1 & $(0.84 \pm 2.59) E-03$ & & \\
\hline 701-3G Barnwell Gate & 1 & $(3.21 \pm 4.26) \mathrm{E}-03$ & & \\
\hline 701-4G Williston Gate & 1 & $(-4.47 \pm 3.44) E-03$ & & \\
\hline 701-5G Talatha Gate & 4 & $(5.57 \pm 4.20) \mathrm{E}-03$ & $(1.34 \pm 0.62) E-02$ & $(-6.04 \pm 2.30) E-03$ \\
\hline $701-6 G$ Jackson Gate & 1 & $(1.07 \pm 4.70) E-03$ & & \\
\hline 701-8G Patrol Gate 8 & 1 & $(-5.79 \pm 3.65) \mathrm{E}-03$ & & \\
\hline
\end{tabular}


Table 22

Radioactivity in Drinking Water

Page 12 of 16

\begin{tabular}{|c|c|c|c|c|}
\hline Location & $\begin{array}{l}\text { No. of } \\
\text { Samples }\end{array}$ & Arithmetic Mean $\pm \sigma$ & Maximum $\pm \sigma$ & Minimum $\pm \sigma$ \\
\hline $\begin{array}{l}\text { 704-16G Central Sanitary } \\
\text { Wastewater Treatment } \\
\text { Facility }\end{array}$ & 1 & $(-6.83 \pm 2.57) E-03$ & & \\
\hline 709-1G L-Area Fire Demo & 1 & $(-3.05 \pm 3.69) E-03$ & & \\
\hline $\begin{array}{l}\text { 735-7G PAR Pond } \\
\text { Limnology Lab }\end{array}$ & 1 & $(1.32 \pm 0.61) E-02$ & & \\
\hline $\begin{array}{l}\text { 737-G PAR Pond } \\
\text { Radioecology Lab }\end{array}$ & 1 & $(2.96 \pm 3.93) E-03$ & & \\
\hline 782-3A Treatment Facility & 1 & $(-0.89 \pm 2.77) E-03$ & & \\
\hline 905-106K Well & 1 & $(1.19 \pm 0.59) E-02$ & & \\
\hline 905-112G Well & 1 & $(3.00 \pm 5.03) E-03$ & & \\
\hline 905-113G Well & 1 & $(-3.43 \pm 3.43) E-03$ & & \\
\hline 905-125B Well & 1 & $(0.79 \pm 4.53) \mathrm{E}-03$ & & \\
\hline 905-136D Well & 1 & $(0.34 \pm 3.43) E-03$ & & \\
\hline 905-3D Well & 1 & $(-1.51 \pm 2.90) E-03$ & & \\
\hline 905-67B Well & 1 & $(1.12 \pm 0.69) E-02$ & & \\
\hline 905-95K Well & 1 & $(4.73 \pm 4.65) E-03$ & & \\
\hline \multicolumn{5}{|l|}{$\mathrm{Cm-244, \textrm {pCi } / \mathrm { L }}$} \\
\hline \multicolumn{5}{|l|}{ On Site } \\
\hline $192-K$ Treatment Facility & 1 & $(-2.32 \pm 1.82) E-03$ & & \\
\hline 483-7D Treatment Facility & 1 & $(-0.86 \pm 2.89) E-03$ & & \\
\hline $\begin{array}{l}617-G \text { Advanced Tactical } \\
\text { Training Area }\end{array}$ & 1 & $(6.68 \pm 3.86) E-03$ & & \\
\hline $\begin{array}{l}\text { 618-G Class. Yard } \\
\text { Lunchroom }\end{array}$ & 1 & $(2.82 \pm 2.42) E-03$ & & \\
\hline $\begin{array}{l}\text { 661-G Firing Range } \\
\text { (pumphouse) }\end{array}$ & 1 & $(-1.21 \pm 0.62) E-03$ & & \\
\hline $\begin{array}{l}681-3 G \text { Domestic } \\
\text { Water Faucet }\end{array}$ & 1 & $(2.05 \pm 2.05) E-03$ & & \\
\hline 701-12G Patrol Gate 7 & 1 & $(9.30 \pm 4.35) \mathrm{E}-03$ & & \\
\hline 701-13G Patrol Gate 6 & 1 & $(-4.18 \pm 0.17) E-03$ & & \\
\hline 701-3G Barnwell Gate & 1 & $(-4.14 \pm 0.17) E-03$ & & \\
\hline 701-4G Williston Gate & 1 & $(5.36 \pm 3.45) \mathrm{E}-03$ & & \\
\hline 701-5G Talatha Gate & 4 & $(4.87 \pm 7.05) E-04$ & $(2.56 \pm 2.80) E-03$ & $(-5.99 \pm 0.24) E-04$ \\
\hline 701-6G Jackson Gate & 1 & $(1.32 \pm 1.91) \mathrm{E}-03$ & & \\
\hline 701-8G Patrol Gate 8 & 1 & $(-5.97 \pm 0.25) E-04$ & & \\
\hline
\end{tabular}


Table 22

Radioactivity in Drinking Water

Page 13 of 16

\begin{tabular}{|c|c|c|c|c|}
\hline Location & $\begin{array}{l}\text { No. of } \\
\text { Samples }\end{array}$ & Arithmetic Mean $\pm \sigma$ & Maximum $\pm \sigma$ & Minimum $\pm \sigma$ \\
\hline $\begin{array}{l}\text { 704-16G Central Sanitary } \\
\text { Wastewater Treatment } \\
\text { Facility }\end{array}$ & 1 & $(-5.97 \pm 0.24) E-04$ & & \\
\hline 709-1G L-Area Fire Demo & 1 & $(-1.24 \pm 0.64) E-03$ & & \\
\hline $\begin{array}{l}\text { 735-7G PAR Pond } \\
\text { Limnology Lab }\end{array}$ & 1 & $(0.00 \pm 0.03) E+00$ & & \\
\hline $\begin{array}{l}\text { 737-G PAR Pond } \\
\text { Radioecology Lab }\end{array}$ & 1 & $(0.00 \pm 0.03) E+00$ & & \\
\hline 782-3A Treatment Facility & 1 & $(-0.24 \pm 2.76) E-03$ & & \\
\hline 905-106K Well & 1 & $(-4.87 \pm 0.75) E-03$ & & \\
\hline $905-112 \mathrm{G}$ Well & 1 & $(-4.08 \pm 0.17) E-03$ & & \\
\hline 905-113G Well & 1 & $(-4.08 \pm 0.17) E-03$ & & \\
\hline 905-125B Well & 1 & $(-1.05 \pm 2.68) E-03$ & & \\
\hline 905-136D Well & 1 & $(-4.82 \pm 0.70) E-03$ & & \\
\hline 905-3D Well & 1 & $(-0.86 \pm 2.89) E-03$ & & \\
\hline 905-67B Well & 1 & $(6.97 \pm 4.85) E-03$ & & \\
\hline 905-95K Well & 1 & $(-4.14 \pm 0.18) E-03$ & & \\
\hline \multicolumn{5}{|l|}{ Gross Alpha, pCi/L } \\
\hline \multicolumn{5}{|l|}{ On Site } \\
\hline 192-K Treatment Facility & 4 & $(1.31 \pm 0.96) E-01$ & $(4.02 \pm 4.26) E-01$ & $(-0.27 \pm 4.87) E-01$ \\
\hline 192-K Treatment Facility & 1 & $(6.58 \pm 5.42) E-01$ & & \\
\hline 483-7D Treatment Facility & 4 & $(6.71 \pm 1.04) E-01$ & $(8.66 \pm 6.21) E-01$ & $(3.76 \pm 6.02) E-01$ \\
\hline 483-7D Treatment Facility & 1 & $(1.75 \pm 6.68) E-01$ & & \\
\hline $\begin{array}{l}617-G \text { Advanced Tactical } \\
\text { Training Area }\end{array}$ & 1 & $(4.31 \pm 3.61) E-01$ & & \\
\hline $\begin{array}{l}\text { 618-G Class. Yard } \\
\text { Lunchroom }\end{array}$ & 1 & $(1.26 \pm 0.53) E+00$ & & \\
\hline $\begin{array}{l}661-G \text { Firing Range } \\
\text { (pumphouse) }\end{array}$ & 1 & $(2.88 \pm 0.57) E+00$ & & \\
\hline $\begin{array}{l}\text { 681-3G Domestic } \\
\text { Water Faucet }\end{array}$ & 1 & $(4.11 \pm 2.96) E-01$ & & \\
\hline 701-12G Patrol Gate 7 & 1 & $(1.00 \pm 0.59) E+00$ & & \\
\hline 701-13G Patrol Gate 6 & 1 & $(1.49 \pm 0.59) E+00$ & & \\
\hline 701-3G Barnwell Gate & 1 & $(1.64 \pm 4.09) E-01$ & & \\
\hline 701-4G Williston Gate & 1 & $(-0.16 \pm 3.10) E-01$ & & \\
\hline 701-5G Talatha Gate & 4 & $(9.23 \pm 0.73) E+00$ & $(1.08 \pm 0.07) E+01$ & $(7.86 \pm 0.94) E+00$ \\
\hline $701-6$ G Jackson Gate & 1 & $(2.85 \pm 0.67) E+00$ & & \\
\hline
\end{tabular}


Table 22

Radioactivity in Drinking Water

Page 14 of 16

\begin{tabular}{|c|c|c|c|c|}
\hline Location & $\begin{array}{l}\text { No. of } \\
\text { Samples }\end{array}$ & Arithmetic Mean $\pm \sigma$ & Maximum $\pm \sigma$ & Minimum $\pm \sigma$ \\
\hline 701-8G Patrol Gate 8 & 1 & $(3.44 \pm 3.71) \mathrm{E}-01$ & & \\
\hline $\begin{array}{l}\text { 704-16G Central Sanitary } \\
\text { Wastewater Treatment } \\
\text { Facility }\end{array}$ & 1 & $(9.77 \pm 4.42) E-01$ & & \\
\hline 709-1G L-Area Fire Demo & 1 & $(-2.29 \pm 4.08) E-01$ & & \\
\hline 730-4B Lunchroom & 2 & $(6.29 \pm 0.73) E-01$ & $(7.01 \pm 4.02) E-01$ & $(5.56 \pm 5.78) E-01$ \\
\hline $\begin{array}{l}\text { 735-7G PAR Pond } \\
\text { Limnology Lab }\end{array}$ & 1 & $(2.73 \pm 3.50) E-01$ & & \\
\hline $\begin{array}{l}\text { 737-G PAR Pond } \\
\text { Radioecology Lab }\end{array}$ & 1 & $(3.01 \pm 3.45) E-01$ & & \\
\hline 782-3A Treatment Facility & 4 & $(2.15 \pm 1.27) \mathrm{E}+00$ & $(5.91 \pm 1.28) E+00$ & $(4.32 \pm 4.88) E-01$ \\
\hline 782-3A Treatment Facility & 1 & $(7.83 \pm 6.42) E-01$ & & \\
\hline 905-106K Well & 1 & $(1.73 \pm 0.59) E+00$ & & \\
\hline 905-112G Well & 1 & $(1.13 \pm 0.66) E+00$ & & \\
\hline 905-113G Well & 1 & $(3.10 \pm 0.92) E+00$ & & \\
\hline 905-125B Well & 1 & $(5.91 \pm 1.19) E+00$ & & \\
\hline 905-136D Well & 1 & $(4.07 \pm 4.54) E-01$ & & \\
\hline 905-3D Well & 1 & $(2.44 \pm 4.20) E-01$ & & \\
\hline 905-67B Well & 1 & $(-0.13 \pm 7.94) E-01$ & & \\
\hline 905-95K Well & 1 & $(1.32 \pm 0.58) E+00$ & & \\
\hline \multicolumn{5}{|l|}{ EMS Sampler } \\
\hline Beaufort Water Works & 25 & $(2.83 \pm 1.02) E-01$ & $(1.64 \pm 0.93) E+00$ & $(-5.67 \pm 5.17) \mathrm{E}-01$ \\
\hline \multicolumn{5}{|l|}{ Treatment Plants - Raw } \\
\hline $\begin{array}{l}\text { Beaufort Public Water } \\
\text { Works }\end{array}$ & 12 & $(2.87 \pm 1.20) \mathrm{E}-01$ & $(1.10 \pm 0.73) E+00$ & $(-1.85 \pm 3.03) E-01$ \\
\hline $\begin{array}{l}\text { N. Augusta Public Water } \\
\text { Works }\end{array}$ & 12 & $(2.87 \pm 1.59) E-01$ & $(1.52 \pm 0.71) E+00$ & $(-3.88 \pm 2.47) E-01$ \\
\hline $\begin{array}{l}\text { Savannah Public Water } \\
\text { Works }\end{array}$ & 12 & $(1.96 \pm 0.62) E-01$ & $(5.94 \pm 4.10) \mathrm{E}-01$ & $(-1.36 \pm 2.33) E-01$ \\
\hline \multicolumn{5}{|l|}{ Treatment Plants - Finished } \\
\hline $\begin{array}{l}\text { Beaufort Public Water } \\
\text { Works }\end{array}$ & 12 & $(1.18 \pm 0.98) \mathrm{E}-01$ & $(7.20 \pm 7.60) E-01$ & $(-4.75 \pm 3.04) E-01$ \\
\hline $\begin{array}{l}\text { N. Augusta Public Water } \\
\text { Works }\end{array}$ & 12 & $(-1.80 \pm 0.46) E-01$ & $(2.28 \pm 3.09) \mathrm{E}-01$ & $(-4.09 \pm 2.49) E-01$ \\
\hline $\begin{array}{l}\text { Savannah Public Water } \\
\text { Works }\end{array}$ & 12 & $(-0.26 \pm 1.27) E-01$ & $(8.78 \pm 6.01) \mathrm{E}-01$ & $(-6.39 \pm 3.37) \mathrm{E}-01$ \\
\hline
\end{tabular}


Table 22

Radioactivity in Drinking Water

Page 15 of 16

\begin{tabular}{|c|c|c|c|c|}
\hline Location & $\begin{array}{c}\text { No. of } \\
\text { Samples }\end{array}$ & Arithmetic Mean $\pm \sigma$ & Maximum $\pm \sigma$ & Minimum $\pm \sigma$ \\
\hline \multicolumn{5}{|l|}{ Gross Beta, pCi/L } \\
\hline \multicolumn{5}{|l|}{ On Site } \\
\hline 192-K Treatment Facility & 4 & $(2.32 \pm 0.53) E+00$ & $(3.86 \pm 0.70) E+00$ & $(1.54 \pm 0.56) E+00$ \\
\hline 192-K Treatment Facility & 1 & $(3.44 \pm 0.69) E+00$ & & \\
\hline 483-7D Treatment Facility & 4 & $(3.77 \pm 0.54) E+00$ & $(5.39 \pm 0.94) E+00$ & $(3.13 \pm 0.69) E+00$ \\
\hline 483-7D Treatment Facility & 1 & $(4.48 \pm 0.85) E+00$ & & \\
\hline $\begin{array}{l}\text { 617-G Advanced Tactical } \\
\text { Training Area }\end{array}$ & 1 & $(1.26 \pm 0.47) E+00$ & & \\
\hline $\begin{array}{l}\text { 618-G Class. Yard } \\
\text { Lunchroom }\end{array}$ & 1 & $(2.38 \pm 0.46) E+00$ & & \\
\hline $\begin{array}{l}\text { 661-G Firing Range } \\
\text { (pumphouse) }\end{array}$ & 1 & $(1.47 \pm 0.47) E+00$ & & \\
\hline $\begin{array}{l}\text { 681-3G Domestic } \\
\text { Water Faucet }\end{array}$ & 1 & $(2.75 \pm 0.53) E+00$ & & \\
\hline 701-12G Patrol Gate 7 & 1 & $(2.13 \pm 0.62) E+00$ & & \\
\hline 701-13G Patrol Gate 6 & 1 & $(1.19 \pm 0.63) E+00$ & & \\
\hline 701-3G Barnwell Gate & 1 & $(2.50 \pm 5.66) E-01$ & & \\
\hline 701-4G Williston Gate & 1 & $(1.50 \pm 0.54) E+00$ & & \\
\hline 701-5G Talatha Gate & 4 & $(2.59 \pm 0.28) E+00$ & $(2.98 \pm 0.58) E+00$ & $(1.81 \pm 0.61) E+00$ \\
\hline 701-6G Jackson Gate & 1 & $(2.19 \pm 0.59) E+00$ & & \\
\hline $701-8 G$ Patrol Gate 8 & 1 & $(2.13 \pm 0.58) \mathrm{E}+00$ & & \\
\hline $\begin{array}{l}\text { 704-16G Central Sanitary } \\
\text { Wastewater Treatment } \\
\text { Facility }\end{array}$ & 1 & $(2.70 \pm 4.15) E-01$ & & \\
\hline 709-1G L-Area Fire Demo & 1 & $(2.51 \pm 0.64) E+00$ & & \\
\hline 730-4B Lunchroom & 2 & $(8.80 \pm 0.16) E-01$ & $(8.96 \pm 5.53) E-01$ & $(8.64 \pm 4.50) E-01$ \\
\hline $\begin{array}{l}\text { 735-7G PAR Pond } \\
\text { Limnology Lab }\end{array}$ & 1 & $(2.86 \pm 0.56) E+00$ & & \\
\hline $\begin{array}{l}\text { 737-G PAR Pond } \\
\text { Radioecology Lab }\end{array}$ & 1 & $(1.27 \pm 0.48) E+00$ & & \\
\hline 782-3A Treatment Facility & 4 & $(1.89 \pm 0.44) E+00$ & $(3.20 \pm 0.96) E+00$ & $(1.39 \pm 0.56) E+00$ \\
\hline 782-3A Treatment Facility & 1 & $(2.41 \pm 0.65) E+00$ & & \\
\hline $905-106 \mathrm{~K}$ Well & 1 & $(2.57 \pm 0.61) E+00$ & & \\
\hline $905-112 \mathrm{G}$ Well & 1 & $(1.28 \pm 0.79) E+00$ & & \\
\hline 905-113G Well & 1 & $(2.81 \pm 0.89) \mathrm{E}+00$ & & \\
\hline 905-125B Well & 1 & $(3.10 \pm 0.92) E+00$ & & \\
\hline 905-136D Well & 1 & $(4.38 \pm 0.71) E+00$ & & \\
\hline
\end{tabular}


Table 22

Radioactivity in Drinking Water

Page 16 of 16

\begin{tabular}{|c|c|c|c|c|}
\hline Location & $\begin{array}{l}\text { No. of } \\
\text { Samples }\end{array}$ & Arithmetic Mean $\pm \sigma$ & Maximum $\pm \sigma$ & Minimum $\pm \sigma$ \\
\hline 905-3D Well & 1 & $(4.07 \pm 0.69) E+00$ & & \\
\hline 905-67B Well & 1 & $(1.33 \pm 0.88) E+00$ & & \\
\hline 905-95K Well & 1 & $(3.13 \pm 0.66) E+00$ & & \\
\hline \multicolumn{5}{|l|}{ EMS Sampler } \\
\hline Beaufort Water Works & 25 & $(1.96 \pm 0.22) E+00$ & $(4.79 \pm 1.28) E+00$ & $(-1.16 \pm 9.35) E-01$ \\
\hline \multicolumn{5}{|l|}{ Treatment Plants - Raw } \\
\hline $\begin{array}{l}\text { Beaufort Public Water } \\
\text { Works }\end{array}$ & 12 & $(1.75 \pm 0.12) E+00$ & $(2.44 \pm 0.66) E+00$ & $(1.05 \pm 0.60) E+00$ \\
\hline $\begin{array}{l}\text { N. Augusta Public Water } \\
\text { Works }\end{array}$ & 12 & $(1.75 \pm 0.21) E+00$ & $(2.75 \pm 0.51) E+00$ & $(5.12 \pm 4.76) \mathrm{E}-01$ \\
\hline $\begin{array}{l}\text { Savannah Public Water } \\
\text { Works }\end{array}$ & 12 & $(2.11 \pm 0.19) E+00$ & $(3.00 \pm 0.71) E+00$ & $(1.23 \pm 0.48) E+00$ \\
\hline \multicolumn{5}{|l|}{ Treatment Plants - Finished } \\
\hline $\begin{array}{l}\text { Beaufort Public Water } \\
\text { Works }\end{array}$ & 12 & $(1.72 \pm 0.14) E+00$ & $(2.36 \pm 0.69) E+00$ & $(8.47 \pm 5.92) E-01$ \\
\hline $\begin{array}{l}\text { N. Augusta Public Water } \\
\text { Works }\end{array}$ & 12 & $(1.54 \pm 0.13) E+00$ & $(2.29 \pm 0.64) E+00$ & $(7.99 \pm 3.17) \mathrm{E}-01$ \\
\hline $\begin{array}{l}\text { Savannah Public Water } \\
\text { Works }\end{array}$ & 12 & $(2.05 \pm 0.15) E+00$ & $(2.75 \pm 0.65) E+00$ & $(1.34 \pm 0.46) \mathrm{E}+00$ \\
\hline
\end{tabular}




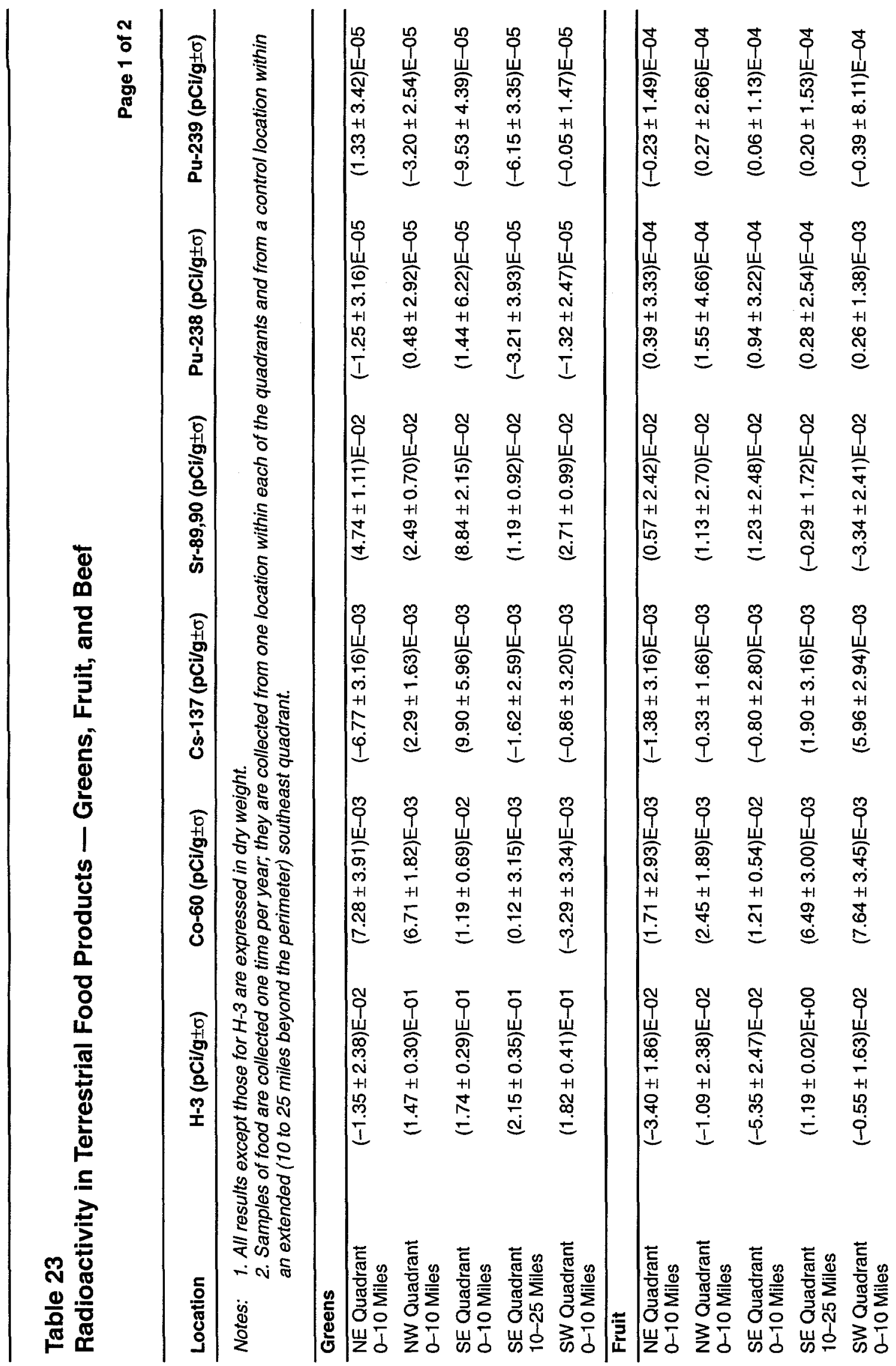




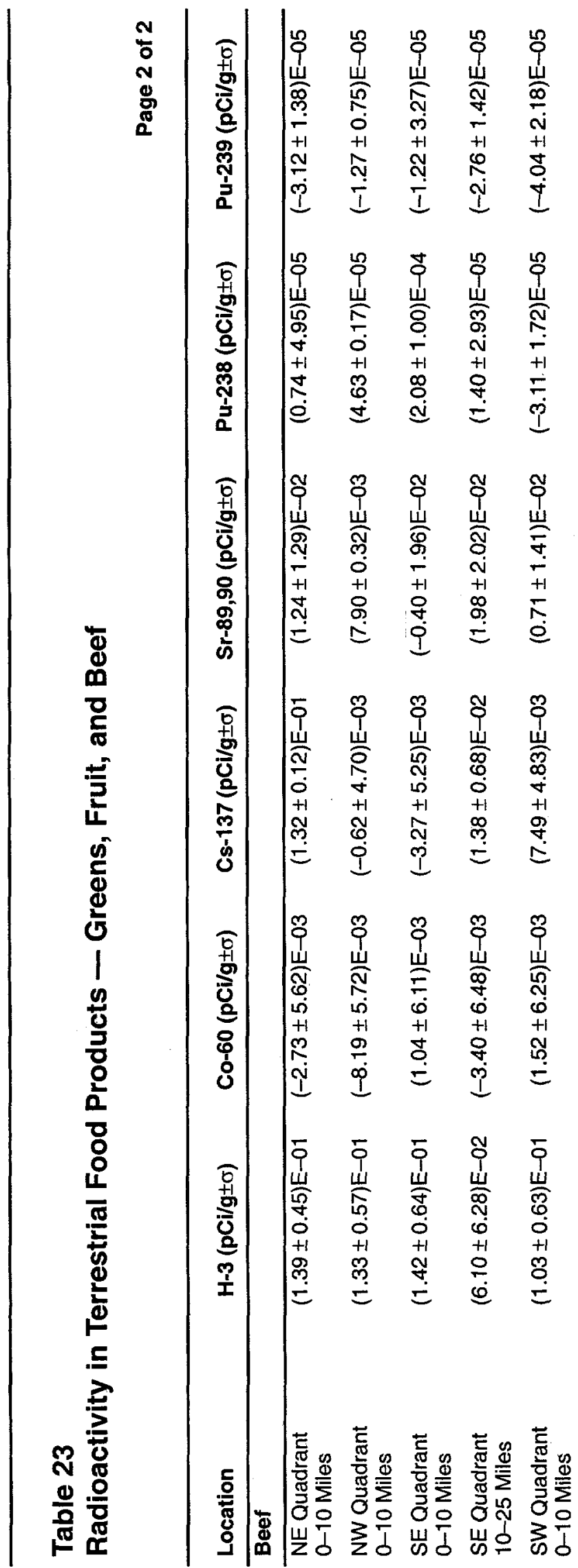




\section{Table 24}

Radioactivity in Terrestrial Food Products - Milk

Page 1 of 1

\begin{tabular}{|c|c|c|c|c|}
\hline Location & $\begin{array}{c}\text { No. of } \\
\text { Samples }\end{array}$ & Arithmetic Mean $\pm \sigma$ & Maximum $\pm \sigma$ & Minimum $\pm \sigma$ \\
\hline \multicolumn{5}{|l|}{$\mathrm{H}-3, \mathrm{pCi} / \mathrm{L}$} \\
\hline Dairy - major distributor & 12 & $(6.24 \pm 4.37) E+01$ & $(2.21 \pm 0.87) E+02$ & $(-1.64 \pm 1.26) \mathrm{E}+02$ \\
\hline Denmark, S.C. & 12 & $(8.27 \pm 2.18) E+01$ & $(1.89 \pm 1.10) E+02$ & $(-0.45 \pm 1.34) E+02$ \\
\hline Girard, Ga., dairy & 11 & $(1.28 \pm 0.39) E+02$ & $(3.18 \pm 1.30) E+02$ & $(-1.11 \pm 0.71) E+02$ \\
\hline Gracewood, Ga., dairy & 12 & $(1.01 \pm 0.33) E+02$ & $(2.72 \pm 1.33) E+02$ & $(-0.78 \pm 1.50) E+02$ \\
\hline Jackson, S.C., dairy & 5 & $(5.45 \pm 0.46) E+02$ & $(6.66 \pm 1.25) \mathrm{E}+02$ & $(4.12 \pm 1.30) E+02$ \\
\hline Waynesboro, Ga., dairy & 12 & $(5.89 \pm 3.35) E+01$ & $(1.80 \pm 1.32) E+02$ & $(-1.95 \pm 1.35) E+02$ \\
\hline \multicolumn{5}{|l|}{ Co-60, pCi/L } \\
\hline Dairy - major distributor & 12 & $(9.24 \pm 5.18) E-01$ & $(3.76 \pm 1.28) E+00$ & $(-1.98 \pm 1.43) E+00$ \\
\hline Denmark, S.C. & 12 & $(-1.09 \pm 5.16) \mathrm{E}-01$ & $(3.22 \pm 1.13) E+00$ & $(-2.90 \pm 1.89) E+00$ \\
\hline Girard, Ga., dairy & 11 & $(9.87 \pm 5.98) E-01$ & $(3.76 \pm 1.29) E+00$ & $(-3.03 \pm 1.63) E+00$ \\
\hline Gracewood, Ga., dairy & 12 & $(9.00 \pm 6.57) E-01$ & $(6.08 \pm 1.77) E+00$ & $(-3.22 \pm 1.66) E+00$ \\
\hline Jackson, S.C., dairy & 5 & $(1.03 \pm 0.70) E+00$ & $(3.47 \pm 1.22) E+00$ & $(-0.72 \pm 1.25) E+00$ \\
\hline Waynesboro, Ga., dairy & 12 & $(2.12 \pm 6.25) E-01$ & $(2.82 \pm 1.30) E+00$ & $(-3.81 \pm 1.70) E+00$ \\
\hline \multicolumn{5}{|l|}{$\mathrm{Cs}-137, \mathrm{pCi} / \mathrm{L}$} \\
\hline Dairy - major distributor & 12 & $(1.57 \pm 0.48) E+00$ & $(4.08 \pm 1.40) E+00$ & $(-1.62 \pm 1.13) E+00$ \\
\hline Denmark, S.C. & 12 & $(4.27 \pm 0.56) E+00$ & $(7.14 \pm 1.43) E+00$ & $(6.05 \pm 8.67) E-01$ \\
\hline Girard, Ga., dairy & 11 & $(1.39 \pm 0.50) E+00$ & $(4.54 \pm 1.36) E+00$ & $(-0.84 \pm 1.10) E+00$ \\
\hline Gracewood, Ga., dairy & 12 & $(1.59 \pm 0.36) E+00$ & $(4.49 \pm 1.33) E+00$ & $(-1.98 \pm 9.82) E-01$ \\
\hline Jackson, S.C., dairy & 5 & $(1.41 \pm 1.03) E+00$ & $(3.70 \pm 1.62) E+00$ & $(-2.45 \pm 1.14) E+00$ \\
\hline Waynesboro, Ga., dairy & 12 & $(1.19 \pm 0.32) E+00$ & $(2.44 \pm 0.96) E+00$ & $(-5.81 \pm 8.63) \mathrm{E}-01$ \\
\hline \multicolumn{5}{|l|}{ Sr-89,90, pCi/L } \\
\hline Dairy - major distributor & 1 & $(2.11 \pm 1.03) \mathrm{E}+00$ & & \\
\hline Denmark, S.C. & 1 & $(2.55 \pm 1.05) \mathrm{E}+00$ & & \\
\hline Girard, Ga., dairy & 1 & $(2.15 \pm 1.03) E+00$ & & \\
\hline Gracewood, Ga., dairy & 1 & $(2.63 \pm 1.05) E+00$ & & \\
\hline Jackson, S.C., dairy & 1 & $(1.71 \pm 1.01) E+00$ & & \\
\hline Waynesboro, Ga., dairy & 1 & $(5.52 \pm 1.65) E+00$ & & \\
\hline
\end{tabular}


Table 25

Radioactivity in Aquatic Food Products - Freshwater Fish

Page 1 of 16

No. of

Location

Samples

Arithmetic Mean $\pm \sigma$

Maximum $\pm \sigma$

Minimum $\pm \sigma$

\begin{tabular}{l} 
H-3, pCi/g \\
\hline Off Site (Edible) \\
New Savannah Bluff Lock and Dam \\
Bass \\
Bream \\
Catfish \\
Shad \\
Beaver Dam Creek River Mouth \\
Bass \\
Bream \\
Catfish
\end{tabular}

Four Mile Creek River Mouth

Bass
Bream
Catfish
Shad

Hwy-301 Bridge Area

Bass

Bream

$2.23 \pm 0.09) \mathrm{E}-01$

(2.38 \pm 0.34$) E-01$

$(0.13 \pm 2.59) \mathrm{E}-02$

$(1.81 \pm 0.96) \mathrm{E}-02$

$(3.43 \pm 3.69) \mathrm{E}-02$

$(1.94 \pm 3.38) E-02$

3

$(2.93 \pm 0.85) \mathrm{E}-02$

$(4.62 \pm 3.55) \mathrm{E}-02$

$(2.15 \pm 2.45) \mathrm{E}-02$

3

$(3.88 \pm 0.91) \mathrm{E}-02$

$(5.22 \pm 3.40) \mathrm{E}-02$

$(0.92 \pm 3.21) E-02$

Catfish

$(2.55 \pm 0.03) \mathrm{E}-01$

$(4.83 \pm 2.97) E-02$

$(6.68 \pm 1.43) \mathrm{E}-02$

$(9.45 \pm 6.14) \mathrm{E}-02$

(4.65 \pm 5.55$) \mathrm{E}-02$

$(1.10 \pm 0.26) \mathrm{E}-01$

$(1.59 \pm 0.61) \mathrm{E}-01$

$(6.96 \pm 6.04) \mathrm{E}-02$

3

$(1.95 \pm 0.62) E-01$

$(1.57 \pm 0.62) \mathrm{E}-01$

Shad

$(2.56 \pm 0.33) E-01$

$(9.43 \pm 0.56) \mathrm{E}-01$

$(5.57 \pm 0.35) E-01$

$(6.84 \pm 1.18) \mathrm{E}-01$

$(9.10 \pm 0.55) \mathrm{E}-01$

$(5.15 \pm 0.51) E-01$

$(1.39 \pm 0.03) \mathrm{E}+00$

$(1.14 \pm 0.04) \mathrm{E}+00$

$(7.75 \pm 0.59) \mathrm{E}-01$

$(5.91 \pm 0.60) \mathrm{E}-01$

\section{L3R Creek River Mouth}

Bass
Bream
Catfish

Catfish

$(2.52 \pm 0.08) \mathrm{E}-01$

(3.21 \pm 0.24$) E-01$

$(2.06 \pm 0.30) \mathrm{E}-01$

3

$(2.64 \pm 0.49) E-01$

$(2.51 \pm 0.31) \mathrm{E}-01$

(2.18 \pm 0.37$) E-01$

3

$2.79+0.56) E-01$

$(2.37 \pm 0.49) \mathrm{E}-01$

Steel Creek River Mouth

Bass
Bream
Catfish
Shad

$3 \quad(2.54 \pm 0.13) E-01$

$(2.79 \pm 0.56) \mathrm{E}-01$

$(2.38 \pm 0.49) E-01$

$3 \quad(2.52 \pm 0.18) \mathrm{E}-01$

$(2.82 \pm 0.35) \mathrm{E}-01$

$(2.20 \pm 0.27) \mathrm{E}-01$

3

$(2.02 \pm 0.26) \mathrm{E}-01$

$(2.33 \pm 0.33) \mathrm{E}-01$

$(1.51 \pm 0.24) \mathrm{E}-01$

U3R Creek River Mouth

Bass
Bream
Catfish

$3 \quad(5.03 \pm 0.07) E-01$

$(5.17 \pm 0.36) \mathrm{E}-01$

(4.94 \pm 0.36$) \mathrm{E}-01$

3

$(6.32 \pm 0.11) \mathrm{E}-01$

$(6.53 \pm 0.37) E-01$

$(6.17 \pm 0.37) \mathrm{E}-01$

3

$(9.11 \pm 7.34) \mathrm{E}-01$

$(2.38 \pm 0.04) E+00$

(1.74 \pm 0.31$) \mathrm{E}-01$

3

$(5.24 \pm 0.10) E-01$

$(5.38 \pm 0.53) E-01$

$(5.04 \pm 0.55) E-01$

$(3.09 \pm 1.60) E-02$

$(6.28 \pm 4.79) \mathrm{E}-02$

$(1.39 \pm 3.44) \mathrm{E}-02$

$3 \quad(7.32 \pm 3.09) \mathrm{E}-02$

$(1.10 \pm 0.41) E-01$

$(1.18 \pm 3.44) \mathrm{E}-02$

3

$(1.35 \pm 0.72) E-01$

$(2.79 \pm 0.34) \mathrm{E}-01$

$(4.96 \pm 3.43) \mathrm{E}-02$ 
Table 25

Radioactivity in Aquatic Food Products - Freshwater Fish

Page 2 of 16

No. of

Location

Samples

Arithmetic Mean $\pm \sigma$

Maximum $\pm \sigma$

Minimum $\pm \sigma$

\begin{tabular}{|c|c|c|c|c|}
\hline \multicolumn{5}{|c|}{$\mathrm{Co-60,pCi/g}$} \\
\hline \multicolumn{5}{|c|}{ Off Site (Edible) } \\
\hline \multicolumn{5}{|c|}{ New Savannah Bluff Lock and Dam (formerly Augusta Lock and Dam) } \\
\hline Bass & 3 & $(0.32 \pm 1.10) E-02$ & $(2.52 \pm 1.40) E-02$ & $(-0.88 \pm 1.45) E-02$ \\
\hline Bream & 3 & $(1.49 \pm 2.14) \mathrm{E}-03$ & $(0.49 \pm 1.47) E-02$ & $(-0.25 \pm 1.36) E-02$ \\
\hline Catfish & 3 & $(5.94 \pm 9.48) E-03$ & $(2.18 \pm 1.46) E-02$ & $(-1.10 \pm 1.37) E-02$ \\
\hline Shad & 3 & $(1.19 \pm 1.34) \mathrm{E}-02$ & $(3.35 \pm 1.47) \mathrm{E}-02$ & $(-1.27 \pm 1.47) \mathrm{E}-02$ \\
\hline \multicolumn{5}{|c|}{ Beaver Dam Creek River Mouth } \\
\hline Bass & 3 & $(2.16 \pm 0.54) E-02$ & $(2.93 \pm 1.43) E-02$ & $(1.11 \pm 1.45) E-02$ \\
\hline Bream & 3 & $(-0.83 \pm 7.45) E-03$ & $(0.89 \pm 1.36) E-02$ & $(-1.55 \pm 0.95) E-02$ \\
\hline Catfish & 3 & $(5.73 \pm 7.81) E-03$ & $(1.57 \pm 1.37) E-02$ & $(-0.97 \pm 1.32) E-02$ \\
\hline \multicolumn{5}{|c|}{ Four Mile Creek River Mouth } \\
\hline Bass & 3 & $(3.58 \pm 7.33) E-03$ & $(1.80 \pm 1.48) \mathrm{E}-02$ & $(-0.58 \pm 1.56) E-02$ \\
\hline Bream & 3 & $(-0.52 \pm 1.25) E-02$ & $(1.65 \pm 1.59) E-02$ & $(-2.69 \pm 1.24) E-02$ \\
\hline Catfish & 3 & $(1.70 \pm 1.09) E-02$ & $(3.80 \pm 1.51) \mathrm{E}-02$ & $(0.17 \pm 1.37) \mathrm{E}-02$ \\
\hline Shad & 3 & $(1.10 \pm 0.79) E-02$ & $(2.26 \pm 1.36) \mathrm{E}-02$ & $(-0.40 \pm 1.39) E-02$ \\
\hline \multicolumn{5}{|c|}{ Hwy-17 Bridge Area } \\
\hline Bass & 3 & $(3.08 \pm 1.05) E-02$ & $(4.41 \pm 1.90) E-02$ & $(1.01 \pm 1.85) E-02$ \\
\hline Bream & 3 & $(1.18 \pm 0.19) E-02$ & $(1.55 \pm 1.92) E-02$ & $(0.94 \pm 2.10) E-02$ \\
\hline Catfish & 3 & $(2.14 \pm 0.45) E-02$ & $(3.03 \pm 2.16) E-02$ & $(1.58 \pm 2.09) E-02$ \\
\hline \multicolumn{5}{|c|}{ Hwy-301 Bridge Area } \\
\hline Bass & 3 & $(-0.29 \pm 3.04) E-03$ & $(4.33 \pm 7.65) \mathrm{E}-03$ & $(-6.03 \pm 4.66) E-03$ \\
\hline Bream & 3 & $(4.59 \pm 3.05) E-03$ & $(1.06 \pm 0.63) \mathrm{E}-02$ & $(0.63 \pm 4.88) E-03$ \\
\hline Catfish & 3 & $(-0.90 \pm 2.06) E-03$ & $(3.20 \pm 6.15) \mathrm{E}-03$ & $(-3.27 \pm 4.81) \mathrm{E}-03$ \\
\hline Shad & 3 & $(-0.58 \pm 4.98) \mathrm{E}-03$ & $(9.26 \pm 4.69) \mathrm{E}-03$ & $(-6.89 \pm 4.86) \mathrm{E}-03$ \\
\hline \multicolumn{5}{|c|}{ L3R Creek River Mouth } \\
\hline Bass & 3 & $(-3.27 \pm 7.77) E-03$ & $(0.80 \pm 1.49) E-02$ & $(-1.82 \pm 1.38) E-02$ \\
\hline Bream & 3 & $(2.63 \pm 0.17) E-02$ & $(2.96 \pm 1.14) E-02$ & $(2.37 \pm 1.39) \mathrm{E}-02$ \\
\hline Cattish & 3 & $(1.27 \pm 1.60) E-02$ & $(4.41 \pm 1.85) E-02$ & $(-0.85 \pm 1.29) E-02$ \\
\hline \multicolumn{5}{|c|}{ Steel Creek River Mouth } \\
\hline Bass & 3 & $(4.01 \pm 0.83) E-03$ & $(0.53 \pm 1.39) E-02$ & $(0.24 \pm 1.40) E-02$ \\
\hline Bream & 3 & $(9.50 \pm 3.55) E-03$ & $(1.61 \pm 1.45) \mathrm{E}-02$ & $(0.40 \pm 1.33) E-02$ \\
\hline Catfish & 3 & $(2.01 \pm 7.30) E-03$ & $(1.29 \pm 1.14) E-02$ & $(-1.19 \pm 1.55) E-02$ \\
\hline Shad & 3 & $(-4.05 \pm 9.70) E-03$ & $(1.36 \pm 1.27) \mathrm{E}-02$ & $(-1.98 \pm 1.49) E-02$ \\
\hline
\end{tabular}




\section{Table 25}

Radioactivity in Aquatic Food Products - Freshwater Fish

Page 3 of 16

\begin{tabular}{|c|c|c|c|c|}
\hline Location & $\begin{array}{l}\text { No. of } \\
\text { Samples }\end{array}$ & Arithmetic Mean $\pm \sigma$ & Maximum $\pm \sigma$ & Minimum $\pm \sigma$ \\
\hline \multicolumn{5}{|c|}{ Stokes Bluff Landing } \\
\hline Bass & 3 & $(8.69 \pm 8.25) E-03$ & $(2.35 \pm 1.77) E-02$ & $(-0.50 \pm 2.16) E-02$ \\
\hline Bream & 3 & $(2.11 \pm 0.26) E-02$ & $(2.39 \pm 2.43) \mathrm{E}-02$ & $(1.59 \pm 1.67) E-02$ \\
\hline Catfish & 3 & $(0.96 \pm 5.23) E-03$ & $(1.13 \pm 1.97) E-02$ & $(-0.58 \pm 2.12) E-02$ \\
\hline \multicolumn{5}{|c|}{ U3R Creek River Mouth } \\
\hline Bass & 3 & $(-0.84 \pm 1.24) E-02$ & $(0.79 \pm 1.61) \mathrm{E}-02$ & $(-3.27 \pm 1.77) \mathrm{E}-02$ \\
\hline Bream & 3 & $(1.62 \pm 0.93) E-02$ & $(3.48 \pm 1.57) E-02$ & $(0.60 \pm 1.60) E-02$ \\
\hline Catfish & 3 & $(-1.05 \pm 0.90) E-02$ & $(0.72 \pm 1.60) E-02$ & $(-2.20 \pm 1.67) E-02$ \\
\hline \multicolumn{5}{|c|}{ Off Site (Nonedible) } \\
\hline \multicolumn{5}{|c|}{ New Savannah Bluff Lock and Dam (formerly Augusta Lock and Dam) } \\
\hline Bass & 3 & $(0.78 \pm 6.58) E-03$ & $(1.15 \pm 1.30) \mathrm{E}-02$ & $(-1.11 \pm 1.52) E-02$ \\
\hline Bream & 3 & $(1.95 \pm 0.50) E-02$ & $(2.56 \pm 1.17) \mathrm{E}-02$ & $(0.97 \pm 1.52) \mathrm{E}-02$ \\
\hline Catfish & 3 & $(8.54 \pm 2.02) E-03$ & $(1.23 \pm 1.35) \mathrm{E}-02$ & $(0.54 \pm 1.36) E-02$ \\
\hline Shad & 3 & $(1.74 \pm 0.69) E-02$ & $(3.13 \pm 1.48) \mathrm{E}-02$ & $(1.00 \pm 1.55) \mathrm{E}-02$ \\
\hline \multicolumn{5}{|c|}{ Beaver Dam Creek River Mouth } \\
\hline Bass & 3 & $(0.08 \pm 1.13) E-02$ & $(1.36 \pm 1.32) E-02$ & $(-2.17 \pm 1.30) E-02$ \\
\hline Bream & 3 & $(1.25 \pm 0.61) \mathrm{E}-02$ & $(1.91 \pm 1.35) \mathrm{E}-02$ & $(0.04 \pm 1.50) E-02$ \\
\hline Catfish & 3 & $(0.98 \pm 1.36) E-02$ & $(3.71 \pm 1.46) \mathrm{E}-02$ & $(-0.55 \pm 1.47) E-02$ \\
\hline \multicolumn{5}{|c|}{ Four Mile Creek River Mouth } \\
\hline Bass & 3 & $(0.26 \pm 1.47) E-02$ & $(2.94 \pm 1.56) \mathrm{E}-02$ & $(-2.13 \pm 1.71) E-02$ \\
\hline Bream & 3 & $(9.33 \pm 7.72) E-03$ & $(1.71 \pm 1.53) \mathrm{E}-02$ & $(-0.61 \pm 1.52) E-02$ \\
\hline Catfish & 3 & $(3.27 \pm 1.59) E-02$ & $(6.42 \pm 1.93) E-02$ & $(1.32 \pm 0.97) \mathrm{E}-02$ \\
\hline Shad & 3 & $(0.18 \pm 1.82) E-02$ & $(3.14 \pm 1.48) E-02$ & $(-3.13 \pm 1.43) E-02$ \\
\hline \multicolumn{5}{|c|}{ Hwy-301 Bridge Area } \\
\hline Bass & 3 & $(5.46 \pm 4.35) \mathrm{E}-03$ & $(1.36 \pm 0.59) \mathrm{E}-02$ & $(-1.18 \pm 4.89) E-03$ \\
\hline Bream & 3 & $(-4.59 \pm 3.74) E-03$ & $(2.65 \pm 4.63) E-03$ & $(-9.84 \pm 5.10) E-03$ \\
\hline Catfish & 3 & $(-8.70 \pm 3.29) E-03$ & $(-2.22 \pm 4.71) E-03$ & $(-1.29 \pm 0.46) E-02$ \\
\hline Shad & 3 & $(4.67 \pm 4.81) \mathrm{E}-03$ & $(1.43 \pm 0.64) \mathrm{E}-02$ & $(-0.66 \pm 5.01) \mathrm{E}-03$ \\
\hline \multicolumn{5}{|c|}{ L3R Creek River Mouth } \\
\hline Bass & 3 & $(2.06 \pm 0.98) E-02$ & $(3.06 \pm 1.44) E-02$ & $(0.11 \pm 1.69) E-02$ \\
\hline Bream & 3 & $(2.14 \pm 0.56) E-02$ & $(3.24 \pm 1.90) \mathrm{E}-02$ & $(1.40 \pm 1.44) \mathrm{E}-02$ \\
\hline Catfish & 3 & $(1.58 \pm 7.08) E-03$ & $(1.54 \pm 1.37) \mathrm{E}-02$ & $(-0.80 \pm 1.11) E-02$ \\
\hline
\end{tabular}


Table 25

Radioactivity in Aquatic Food Products - Freshwater Fish

Page 4 of 16

\begin{tabular}{|c|c|c|c|c|}
\hline Location & $\begin{array}{l}\text { No. of } \\
\text { Samples }\end{array}$ & Arithmetic Mean $\pm \sigma$ & Maximum $\pm \sigma$ & Minimum $\pm \sigma$ \\
\hline \multicolumn{5}{|c|}{ Steel Creek River Mouth } \\
\hline Bass & 3 & $(1.70 \pm 0.46) E-02$ & $(2.61 \pm 1.31) E-02$ & $(1.22 \pm 1.18) \mathrm{E}-02$ \\
\hline Bream & 3 & $(3.74 \pm 7.47) E-03$ & $(1.86 \pm 1.35) \mathrm{E}-02$ & $(-0.48 \pm 1.39) E-02$ \\
\hline Catfish & 3 & $(-8.51 \pm 5.85) E-03$ & $(0.32 \pm 1.36) E-02$ & $(-1.53 \pm 1.44) E-02$ \\
\hline Shad & 3 & $(1.36 \pm 0.53) E-02$ & $(2.35 \pm 1.18) E-02$ & $(0.55 \pm 1.31) E-02$ \\
\hline \multicolumn{5}{|c|}{ U3R Creek River Mouth } \\
\hline Bass & 3 & $(-0.03 \pm 1.27) E-02$ & $(2.29 \pm 1.42) E-02$ & $(-2.10 \pm 1.65) E-02$ \\
\hline Bream & 3 & $(1.25 \pm 1.08) E-02$ & $(3.36 \pm 1.49) E-02$ & $(-0.23 \pm 1.50) E-02$ \\
\hline Catfish & 3 & $(0.81 \pm 1.24) E-02$ & $(2.83 \pm 1.34) \mathrm{E}-02$ & $(-1.46 \pm 1.48) E-02$ \\
\hline \multicolumn{5}{|c|}{ On Site (Edible) } \\
\hline \multicolumn{5}{|l|}{ L-Lake } \\
\hline Bass & 3 & $(2.19 \pm 0.09) \mathrm{E}-02$ & $(2.36 \pm 2.18) E-02$ & $(2.05 \pm 1.80) E-02$ \\
\hline Bream & 3 & $(1.43 \pm 0.50) E-02$ & $(1.96 \pm 1.59) \mathrm{E}-02$ & $(0.43 \pm 1.89) E-02$ \\
\hline \multicolumn{5}{|c|}{ L3R-2 Patterson Mill Road } \\
\hline Bass & 3 & $(0.61 \pm 7.02) E-03$ & $(1.46 \pm 1.90) \mathrm{E}-02$ & $(-0.74 \pm 1.87) \mathrm{E}-02$ \\
\hline Bream & 3 & $(-0.19 \pm 2.30) E-02$ & $(4.28 \pm 2.08) E-02$ & $(-3.36 \pm 1.96) E-02$ \\
\hline \multicolumn{5}{|c|}{ PAR Pond } \\
\hline Bass & 3 & $(1.28 \pm 1.05) E-02$ & $(3.38 \pm 1.89) \mathrm{E}-02$ & $(0.21 \pm 1.97) \mathrm{E}-02$ \\
\hline Bream & 3 & $(1.81 \pm 8.90) E-03$ & $(1.14 \pm 1.98) E-02$ & $(-1.60 \pm 1.78) E-02$ \\
\hline \multicolumn{5}{|l|}{ Pond B } \\
\hline Bass & 3 & $(1.22 \pm 1.91) \mathrm{E}-02$ & $(4.36 \pm 1.89) \mathrm{E}-02$ & $(-2.22 \pm 2.14) \mathrm{E}-02$ \\
\hline Bream & 3 & $(3.70 \pm 1.82) E-03$ & $(0.69 \pm 1.95) E-02$ & $(0.05 \pm 1.84) E-02$ \\
\hline \multicolumn{5}{|c|}{ Cs-137, $\mathrm{pCi} / \mathrm{g}$} \\
\hline \multicolumn{5}{|c|}{ Off Site (Edible) } \\
\hline \multicolumn{5}{|c|}{ New Savannah Bluff Lock and Dam (formerly Augusta Lock and Dam) } \\
\hline Bass & 3 & $(1.31 \pm 0.21) E-02$ & $(1.57 \pm 1.26) \mathrm{E}-02$ & $(0.90 \pm 1.31) E-02$ \\
\hline Bream & 3 & $(2.06 \pm 0.52) E-02$ & $(2.89 \pm 1.27) E-02$ & $(1.11 \pm 1.25) E-02$ \\
\hline Catfish & 3 & $(5.01 \pm 1.32) E-02$ & $(7.60 \pm 2.05) E-02$ & $(3.29 \pm 1.64) \mathrm{E}-02$ \\
\hline Shad & 3 & $(4.05 \pm 1.12) E-02$ & $(5.94 \pm 2.07) E-02$ & $(2.07 \pm 1.24) \mathrm{E}-02$ \\
\hline \multicolumn{5}{|c|}{ Beaver Dam Creek River Mouth } \\
\hline Bass & 3 & $(1.79 \pm 0.66) E-01$ & $(2.49 \pm 0.27) \mathrm{E}-01$ & $(4.73 \pm 1.94) \mathrm{E}-02$ \\
\hline Bream & 3 & $(3.38 \pm 0.38) E-02$ & $(3.89 \pm 1.39) E-02$ & $(2.64 \pm 1.60) \mathrm{E}-02$ \\
\hline Catfish & 3 & $(6.84 \pm 0.95) E-02$ & $(8.31 \pm 2.47) \mathrm{E}-02$ & $(5.07 \pm 2.43) E-02$ \\
\hline
\end{tabular}


Table 25

Radioactivity in Aquatic Food Products - Freshwater Fish

Page 5 of 16

\begin{tabular}{|c|c|c|c|c|}
\hline Location & $\begin{array}{c}\text { No. of } \\
\text { Samples }\end{array}$ & Arithmetic Mean $\pm \sigma$ & Maximum $\pm \sigma$ & Minimum $\pm \sigma$ \\
\hline \multicolumn{5}{|c|}{ Four Mile Creek River Mouth } \\
\hline Bass & 3 & $(2.01 \pm 0.48) E-01$ & $(2.96 \pm 0.30) E-01$ & $(1.51 \pm 0.23) E-01$ \\
\hline Bream & 3 & $(3.02 \pm 2.14) E-02$ & $(6.98 \pm 1.43) \mathrm{E}-02$ & $(-0.34 \pm 1.47) E-02$ \\
\hline Catfish & 3 & $(9.23 \pm 3.68) \mathrm{E}-02$ & $(1.66 \pm 0.23) \mathrm{E}-01$ & $(5.33 \pm 2.36) E-02$ \\
\hline Shad & 3 & $(1.95 \pm 0.70) \mathrm{E}-01$ & $(2.90 \pm 0.36) E-01$ & $(5.84 \pm 1.99) E-02$ \\
\hline \multicolumn{5}{|c|}{ Hwy-17 Bridge Area } \\
\hline Bass & 3 & $(6.94 \pm 1.75) E-02$ & $(9.35 \pm 2.78) \mathrm{E}-02$ & $(3.54 \pm 2.03) \mathrm{E}-02$ \\
\hline Bream & 3 & $(2.00 \pm 1.14) \mathrm{E}-02$ & $(3.97 \pm 2.02) E-02$ & $(0.02 \pm 1.57) E-02$ \\
\hline Catfish & 3 & $(4.50 \pm 0.45) E-02$ & $(5.04 \pm 2.89) E-02$ & $(3.62 \pm 1.94) \mathrm{E}-02$ \\
\hline \multicolumn{5}{|c|}{ Hwy-301 Bridge Area } \\
\hline Bass & 3 & $(3.20 \pm 2.17) E-01$ & $(7.54 \pm 0.41) \mathrm{E}-01$ & $(9.16 \pm 1.05) \mathrm{E}-02$ \\
\hline Bream & 3 & $(3.65 \pm 0.33) E-02$ & $(4.27 \pm 0.80) E-02$ & $(3.15 \pm 0.72) E-02$ \\
\hline Catfish & 3 & $(5.19 \pm 0.27) E-02$ & $(5.72 \pm 0.81) E-02$ & $(4.80 \pm 0.94) \mathrm{E}-02$ \\
\hline Shad & 3 & $(4.21 \pm 0.41) E-02$ & $(4.96 \pm 0.65) E-02$ & $(3.54 \pm 0.77) E-02$ \\
\hline \multicolumn{5}{|c|}{ L3R Creek River Mouth } \\
\hline Bass & 3 & $(8.15 \pm 1.22) E-02$ & $(1.05 \pm 0.20) \mathrm{E}-01$ & $(6.46 \pm 2.27) E-02$ \\
\hline Bream & 3 & $(3.16 \pm 0.28) E-02$ & $(3.66 \pm 2.02) E-02$ & $(2.68 \pm 1.40) E-02$ \\
\hline Catfish & 3 & $(1.17 \pm 0.30) E-01$ & $(1.61 \pm 0.26) \mathrm{E}-01$ & $(6.05 \pm 2.46) E-02$ \\
\hline \multicolumn{5}{|c|}{ Steel Creek River Mouth } \\
\hline Bass & 3 & $(5.98 \pm 1.90) E-01$ & $(9.20 \pm 0.65) \mathrm{E}-01$ & $(2.62 \pm 0.28) \mathrm{E}-01$ \\
\hline Bream & 3 & $(6.07 \pm 1.22) \mathrm{E}-02$ & $(8.51 \pm 2.50) E-02$ & $(4.68 \pm 2.36) \mathrm{E}-02$ \\
\hline Catfish & 3 & $(1.03 \pm 0.08) E-01$ & $(1.19 \pm 0.25) E-01$ & $(9.33 \pm 2.23) \mathrm{E}-02$ \\
\hline Shad & 3 & $(9.56 \pm 1.67) \mathrm{E}-02$ & $(1.26 \pm 0.26) E-01$ & $(6.81 \pm 1.98) E-02$ \\
\hline \multicolumn{5}{|c|}{ Stokes Bluff Landing } \\
\hline Bass & 3 & $(8.65 \pm 4.28) E-02$ & $(1.36 \pm 0.26) \mathrm{E}-01$ & $(0.12 \pm 1.84) \mathrm{E}-02$ \\
\hline Bream & 3 & $(3.32 \pm 1.13) \mathrm{E}-02$ & $(5.34 \pm 2.05) E-02$ & $(1.44 \pm 2.13) \mathrm{E}-02$ \\
\hline Catfish & 3 & $(5.78 \pm 2.63) \mathrm{E}-02$ & $(9.79 \pm 3.48) E-02$ & $(0.82 \pm 2.16) E-02$ \\
\hline \multicolumn{5}{|c|}{ U3R Creek River Mouth } \\
\hline Bass & 3 & $(6.03 \pm 1.58) \mathrm{E}-02$ & $(9.00 \pm 1.74) \mathrm{E}-02$ & $(3.62 \pm 1.66) \mathrm{E}-02$ \\
\hline Bream & 3 & $(4.67 \pm 1.96) \mathrm{E}-02$ & $(7.94 \pm 2.51) E-02$ & $(1.15 \pm 1.33) E-02$ \\
\hline Catfish & 3 & $(3.15 \pm 0.69) E-02$ & $(4.22 \pm 1.74) E-02$ & $(1.86 \pm 1.49) E-02$ \\
\hline
\end{tabular}


Table 25

Radioactivity in Aquatic Food Products - Freshwater Fish

Page 6 of 16

\begin{tabular}{|c|c|c|c|c|}
\hline Location & $\begin{array}{l}\text { No. of } \\
\text { Samples }\end{array}$ & Arithmetic Mean $\pm \sigma$ & Maximum $\pm \sigma$ & Minimum $\pm \sigma$ \\
\hline \multicolumn{5}{|c|}{ Otf Site (Nonedible) } \\
\hline \multicolumn{5}{|c|}{ New Savannah Bluff Lock and Dam (formerly Augusta Lock and Dam) } \\
\hline Bass & 3 & $(1.64 \pm 1.72) E-02$ & $(4.74 \pm 1.90) \mathrm{E}-02$ & $(-1.19 \pm 1.35) E-02$ \\
\hline Bream & 3 & $(1.22 \pm 1.19) E-02$ & $(3.15 \pm 1.82) E-02$ & $(-0.96 \pm 1.23) \mathrm{E}-02$ \\
\hline Catfish & 3 & $(3.92 \pm 0.55) E-02$ & $(4.81 \pm 1.37) \mathrm{E}-02$ & $(2.93 \pm 1.22) \mathrm{E}-02$ \\
\hline Shad & 3 & $(3.13 \pm 0.66) E-02$ & $(4.14 \pm 2.24) E-02$ & $(1.88 \pm 1.33) E-02$ \\
\hline \multicolumn{5}{|c|}{ Beaver Dam Creek River Mouth } \\
\hline Bass & 3 & $(1.00 \pm 0.11) \mathrm{E}-01$ & $(1.21 \pm 0.19) E-01$ & $(8.20 \pm 1.84) E-02$ \\
\hline Bream & 3 & $(3.56 \pm 1.02) E-02$ & $(5.46 \pm 1.39) E-02$ & $(1.96 \pm 1.28) E-02$ \\
\hline Catfish & 3 & $(4.37 \pm 1.50) E-02$ & $(7.33 \pm 1.75) E-02$ & $(2.43 \pm 1.44) E-02$ \\
\hline \multicolumn{5}{|c|}{ Four Mile Creek River Mouth } \\
\hline Bass & 3 & $(1.30 \pm 0.16) E-01$ & $(1.46 \pm 0.27) E-01$ & $(9.85 \pm 2.45) \mathrm{E}-02$ \\
\hline Bream & 3 & $(3.96 \pm 2.20) E-02$ & $(7.18 \pm 1.95) \mathrm{E}-02$ & $(-0.23 \pm 1.36) E-02$ \\
\hline Catfish & 3 & $(5.19 \pm 1.35) \mathrm{E}-02$ & $(7.38 \pm 1.46) E-02$ & $(2.73 \pm 1.45) \mathrm{E}-02$ \\
\hline Shad & 3 & $(7.72 \pm 1.41) \mathrm{E}-02$ & $(1.04 \pm 0.18) E-01$ & $(5.72 \pm 2.16) E-02$ \\
\hline \multicolumn{5}{|c|}{ Hwy-301 Bridge Area } \\
\hline Bass & 3 & $(1.29 \pm 0.05) E-01$ & $(1.39 \pm 0.14) \mathrm{E}-01$ & $(1.22 \pm 0.11) \mathrm{E}-01$ \\
\hline Bream & 3 & $(1.94 \pm 0.14) E-02$ & $(2.12 \pm 0.82) E-02$ & $(1.66 \pm 0.65) E-02$ \\
\hline Catfish & 3 & $(3.05 \pm 0.18) E-02$ & $(3.24 \pm 0.79) \mathrm{E}-02$ & $(2.70 \pm 0.77) E-02$ \\
\hline Shad & 3 & $(2.82 \pm 0.66) E-02$ & $(4.09 \pm 0.72) \mathrm{E}-02$ & $(1.85 \pm 0.61) E-02$ \\
\hline \multicolumn{5}{|c|}{ L3R Creek River Mouth } \\
\hline Bass & 3 & $(4.59 \pm 0.76) E-02$ & $(6.06 \pm 2.01) E-02$ & $(3.55 \pm 1.90) E-02$ \\
\hline Bream & 3 & $(1.17 \pm 0.73) E-02$ & $(2.61 \pm 1.43) E-02$ & $(0.24 \pm 1.37) E-02$ \\
\hline Catfish & 3 & $(6.36 \pm 0.68) E-02$ & $(7.27 \pm 1.85) \mathrm{E}-02$ & $(5.02 \pm 2.04) \mathrm{E}-02$ \\
\hline \multicolumn{5}{|c|}{ Steel Creek River Mouth } \\
\hline Bass & 3 & $(6.48 \pm 0.42) E-01$ & $(7.11 \pm 0.51) \mathrm{E}-01$ & $(5.69 \pm 0.47) \mathrm{E}-01$ \\
\hline Bream & 3 & $(2.82 \pm 0.09) E-02$ & $(2.93 \pm 1.39) E-02$ & $(2.64 \pm 1.42) E-02$ \\
\hline Catfish & 3 & $(5.53 \pm 0.21) E-02$ & $(5.93 \pm 2.17) \mathrm{E}-02$ & $(5.24 \pm 2.20) \mathrm{E}-02$ \\
\hline Shad & 3 & $(5.33 \pm 0.74) E-02$ & $(6.35 \pm 2.82) E-02$ & $(3.90 \pm 2.09) \mathrm{E}-02$ \\
\hline \multicolumn{5}{|c|}{ U3R Creek River Mouth } \\
\hline Bass & 3 & $(1.46 \pm 0.42) \mathrm{E}-02$ & $(2.29 \pm 1.40) \mathrm{E}-02$ & $(0.88 \pm 1.35) E-02$ \\
\hline Bream & 3 & $(3.08 \pm 2.47) E-02$ & $(8.01 \pm 1.95) \mathrm{E}-02$ & $(0.53 \pm 1.49) E-02$ \\
\hline Catfish & 3 & $(3.66 \pm 2.04) E-02$ & $(7.66 \pm 1.96) E-02$ & $(0.97 \pm 1.44) E-02$ \\
\hline
\end{tabular}


Table 25

Radioactivity in Aquatic Food Products - Freshwater Fish

Page 7 of 16

\begin{tabular}{|c|c|c|c|c|}
\hline Location & $\begin{array}{c}\text { No. of } \\
\text { Samples }\end{array}$ & Arithmetic Mean $\pm \sigma$ & Maximum $\pm \sigma$ & Minimum $\pm \sigma$ \\
\hline \multicolumn{5}{|c|}{ On Site (Edible) } \\
\hline \multicolumn{5}{|l|}{ L-Lake } \\
\hline Bass & 3 & $(6.44 \pm 0.17) E-01$ & $(6.60 \pm 0.59) E-01$ & $(6.10 \pm 0.64) E-01$ \\
\hline Bream & 3 & $(2.80 \pm 0.16) E-01$ & $(2.97 \pm 0.41) E-01$ & $(2.48 \pm 0.42) E-01$ \\
\hline \multicolumn{5}{|c|}{ L3R-2 Patterson Mill Road } \\
\hline Bass & 3 & $(5.65 \pm 0.26) E+00$ & $(6.15 \pm 0.34) E+00$ & $(5.27 \pm 0.29) \mathrm{E}+00$ \\
\hline Bream & 3 & $(2.78 \pm 0.18) E+00$ & $(3.07 \pm 0.18) E+00$ & $(2.46 \pm 0.15) E+00$ \\
\hline \multicolumn{5}{|c|}{ PAR Pond } \\
\hline Bass & 3 & $(9.96 \pm 0.21) E+00$ & $(1.03 \pm 0.05) E+01$ & $(9.60 \pm 0.51) \mathrm{E}+00$ \\
\hline Bream & 3 & $(2.64 \pm 0.13) E+00$ & $(2.88 \pm 0.18) E+00$ & $(2.43 \pm 0.15) E+00$ \\
\hline \multicolumn{5}{|l|}{ Pond B } \\
\hline Bass & 3 & $(6.67 \pm 0.04) E+01$ & $(6.73 \pm 0.34) E+01$ & $(6.61 \pm 0.34) E+01$ \\
\hline Bream & 3 & $(2.85 \pm 0.06) E+01$ & $(2.97 \pm 0.15) E+01$ & $(2.78 \pm 0.14) E+01$ \\
\hline \multicolumn{5}{|c|}{$\mathrm{Sr}-89,90, \mathrm{pCi} / \mathrm{g}$} \\
\hline \multicolumn{5}{|c|}{ Off Site (Edible) } \\
\hline \multicolumn{5}{|c|}{ New Savannah Bluff Lock and Dam (formerly Augusta Lock and Dam) } \\
\hline Bass & 3 & $(8.24 \pm 6.66) E-03$ & $(1.83 \pm 0.52) E-02$ & $(-4.36 \pm 5.21) E-03$ \\
\hline Bream & 3 & $(1.72 \pm 0.83) E-02$ & $(3.37 \pm 1.29) \mathrm{E}-02$ & $(7.05 \pm 9.50) E-03$ \\
\hline Catfish & 3 & $(9.96 \pm 2.83) E-03$ & $(1.43 \pm 0.49) \mathrm{E}-02$ & $(4.63 \pm 4.42) E-03$ \\
\hline Shad & 3 & $(5.56 \pm 3.07) E-03$ & $(1.10 \pm 0.48) E-02$ & $(0.38 \pm 4.10) E-03$ \\
\hline \multicolumn{5}{|c|}{ Beaver Dam Creek River Mouth } \\
\hline Bass & 3 & $(5.13 \pm 2.55) E-03$ & $(8.88 \pm 3.87) E-03$ & $(0.25 \pm 4.49) E-03$ \\
\hline Bream & 3 & $(1.78 \pm 0.83) E-02$ & $(3.43 \pm 1.02) E-02$ & $(8.60 \pm 3.92) E-03$ \\
\hline Catfish & 3 & $(3.89 \pm 1.22) E-03$ & $(6.07 \pm 3.71) E-03$ & $(1.86 \pm 3.46) E-03$ \\
\hline \multicolumn{5}{|c|}{ Four Mile Creek River Mouth } \\
\hline Bass & 3 & $(1.28 \pm 0.08) E-02$ & $(1.43 \pm 0.42) E-02$ & $(1.15 \pm 0.28) E-02$ \\
\hline Bream & 3 & $(2.01 \pm 0.63) E-02$ & $(2.96 \pm 1.00) E-02$ & $(8.27 \pm 8.84) E-03$ \\
\hline Catfish & 3 & $(3.52 \pm 1.27) E-03$ & $(4.85 \pm 2.58) E-03$ & $(0.99 \pm 1.85) E-03$ \\
\hline Shad & 3 & $(1.61 \pm 0.50) E-02$ & $(2.44 \pm 0.54) \mathrm{E}-02$ & $(7.26 \pm 2.15) E-03$ \\
\hline \multicolumn{5}{|c|}{ Hwy-301 Bridge Area } \\
\hline Bass & 3 & $(6.66 \pm 1.70) E-03$ & $(8.80 \pm 2.63) E-03$ & $(3.31 \pm 4.02) \mathrm{E}-03$ \\
\hline Bream & 3 & $(1.11 \pm 0.65) E-02$ & $(2.06 \pm 0.92) E-02$ & $(-0.12 \pm 1.09) \mathrm{E}-02$ \\
\hline Catfish & 3 & $(8.16 \pm 2.78) E-03$ & $(1.20 \pm 0.39) E-02$ & $(2.76 \pm 3.35) E-03$ \\
\hline Shad & 3 & $(8.27 \pm 3.93) E-03$ & $(1.61 \pm 0.54) E-02$ & $(3.61 \pm 4.74) \mathrm{E}-03$ \\
\hline
\end{tabular}


Table 25

Radioactivity in Aquatic Food Products - Freshwater Fish

Page 8 of 16

\begin{tabular}{lcccc} 
Location & $\begin{array}{c}\text { No. of } \\
\text { Samples }\end{array}$ & Arithmetic Mean $\pm \sigma$ & Maximum $\pm \sigma$ & Minimum $\pm \sigma$ \\
\hline $\begin{array}{l}\text { L3R Creek River Mouth } \\
\text { Bass }\end{array}$ & 3 & $(3.80 \pm 0.43) \mathrm{E}-03$ & $(4.66 \pm 1.95) \mathrm{E}-03$ & $(3.27 \pm 1.92) \mathrm{E}-03$ \\
$\quad$ Bream & 3 & $(3.61 \pm 0.70) \mathrm{E}-02$ & $(4.66 \pm 1.07) \mathrm{E}-02$ & $(2.28 \pm 0.95) \mathrm{E}-02$ \\
$\quad$ Catfish & 3 & $(8.30 \pm 7.47) \mathrm{E}-04$ & $(2.31 \pm 1.87) \mathrm{E}-03$ & $(-0.06 \pm 1.80) \mathrm{E}-03$ \\
Steel Creek River Mouth & & & & \\
Bass & 3 & $(1.47 \pm 0.32) \mathrm{E}-02$ & $(2.08 \pm 0.53) \mathrm{E}-02$ & $(1.04 \pm 0.33) \mathrm{E}-02$ \\
Bream & 3 & $(1.60 \pm 0.45) \mathrm{E}-02$ & $(2.39 \pm 0.78) \mathrm{E}-02$ & $(8.53 \pm 8.76) \mathrm{E}-03$ \\
Catfish & 3 & $(3.17 \pm 1.48) \mathrm{E}-03$ & $(6.13 \pm 3.17) \mathrm{E}-03$ & $(1.59 \pm 4.22) \mathrm{E}-03$ \\
Shad & 3 & $(9.84 \pm 1.56) \mathrm{E}-03$ & $(1.29 \pm 0.48) \mathrm{E}-02$ & $(7.67 \pm 3.06) \mathrm{E}-03$ \\
U3R Creek River Mouth & & & & \\
Bass & 3 & $(6.82 \pm 0.03) \mathrm{E}-03$ & $(6.85 \pm 1.50) \mathrm{E}-03$ & $(6.77 \pm 3.61) \mathrm{E}-03$ \\
Bream & 3 & $(7.71 \pm 3.28) \mathrm{E}-03$ & $(1.11 \pm 0.71) \mathrm{E}-02$ & $(1.15 \pm 6.48) \mathrm{E}-03$ \\
Catfish & 3 & $(-1.11 \pm 1.43) \mathrm{E}-03$ & $(1.74 \pm 4.15) \mathrm{E}-03$ & $(-2.62 \pm 2.57) \mathrm{E}-03$
\end{tabular}

Off Site (Nonedible)

New Savannah Bluff Lock and Dam (formerly Augusta Lock and Dam)

$\begin{array}{lllll}\text { Bass } & 3 & (1.68 \pm 0.68) \mathrm{E}-01 & (3.04 \pm 0.52) \mathrm{E}-01 & (8.65 \pm 1.28) \mathrm{E}-02 \\ \text { Bream } & 3 & (2.70 \pm 0.29) \mathrm{E}-01 & (3.11 \pm 0.52) \mathrm{E}-01 & (2.14 \pm 0.48) \mathrm{E}-01 \\ \text { Catfish } & 3 & (1.86 \pm 0.10) \mathrm{E}-01 & (2.01 \pm 0.47) \mathrm{E}-01 & (1.66 \pm 0.45) \mathrm{E}-01 \\ \text { Shad } & 3 & (1.33 \pm 0.10) \mathrm{E}-01 & (1.52 \pm 0.31) \mathrm{E}-01 & (1.22 \pm 0.19) \mathrm{E}-01\end{array}$

Beaver Dam Creek River Mouth

Bass

Bream

Catfish

Four Mile Creek River Mouth

Bass
Bream
Shatish

Hwy-301 Bridge Area

Bass
Bream
Shatfish

3

3

3

3

3

3

3

3

3

3
$(1.92 \pm 0.20) \mathrm{E}-01$

$(3.57 \pm 0.58) \mathrm{E}-01$

$(1.99 \pm 0.67) E-01$

$(7.85 \pm 1.96) \mathrm{E}-02$

$(2.33 \pm 0.81) \mathrm{E}-01$

$(2.36 \pm 0.22) \mathrm{E}-01$

$(1.58 \pm 0.55) E-01$

$(2.45 \pm 0.47) \mathrm{E}-01$

(3.26 \pm 0.38$) \mathrm{E}-01$

$(2.53 \pm 0.46) \mathrm{E}-01$

$(6.68 \pm 2.05) E-02$
$(2.29 \pm 0.33) E-01$

$(1.59 \pm 0.42) E-01$

(4.51 \pm 0.56$) E-01$

$(2.51 \pm 0.47) \mathrm{E}-01$

$(3.26 \pm 0.51) E-01$

$(9.93 \pm 3.67) E-02$

$(1.11 \pm 0.20) E-01$

$(4.36 \pm 1.97) \mathrm{E}-02$

(3.47 \pm 0.54$) E-01$

(7.75 \pm 1.75$) E-02$

(2.80 \pm 0.38$) E-01$

$(2.13 \pm 0.32) E-01$

$(2.56 \pm 0.34) E-01$

$(6.54 \pm 2.32) E-02$

(3.30 \pm 0.51$) E-01$

$(1.69 \pm 0.43) E-01$

$(4.02 \pm 0.54) E-01$

$(2.88 \pm 0.49) E-01$

$(3.45 \pm 0.40) E-01$

(2.02 \pm 0.33$) E-01$

$(1.01 \pm 0.42) E-01$ 
Table 25

\section{Radioactivity in Aquatic Food Products - Freshwater Fish}

Page 9 of 16

\begin{tabular}{|c|c|c|c|c|}
\hline Location & $\begin{array}{c}\text { No. of } \\
\text { Samples }\end{array}$ & Arithmetic Mean $\pm \sigma$ & Maximum $\pm \sigma$ & Minimum $\pm \sigma$ \\
\hline \multicolumn{5}{|c|}{ L3R Creek River Mouth } \\
\hline Bass & 3 & $(1.94 \pm 0.41) E-01$ & $(2.45 \pm 0.35) \mathrm{E}-01$ & $(1.13 \pm 0.28) E-01$ \\
\hline Bream & 3 & $(2.19 \pm 0.63) E-01$ & $(3.09 \pm 0.50) E-01$ & $(9.90 \pm 3.67) E-02$ \\
\hline Catfish & 3 & $(1.17 \pm 0.63) E-01$ & $(2.41 \pm 0.47) E-01$ & $(3.96 \pm 1.98) E-02$ \\
\hline \multicolumn{5}{|c|}{ Steel Creek River Mouth } \\
\hline Bass & 3 & $(2.34 \pm 0.47) E-01$ & $(2.94 \pm 0.50) E-01$ & $(1.42 \pm 0.43) E-01$ \\
\hline Bream & 3 & $(1.86 \pm 0.36) E-01$ & $(2.45 \pm 0.48) E-01$ & $(1.20 \pm 0.42) E-01$ \\
\hline Catfish & 3 & $(2.51 \pm 0.49) E-01$ & $(3.50 \pm 0.52) E-01$ & $(2.01 \pm 0.45) E-01$ \\
\hline Shad & 3 & $(1.36 \pm 0.57) E-01$ & $(2.49 \pm 0.33) E-01$ & $(6.98 \pm 2.33) E-02$ \\
\hline \multicolumn{5}{|c|}{ U3R Creek River Mouth } \\
\hline Bass & 3 & $(2.88 \pm 0.46) E-01$ & $(3.67 \pm 0.55) E-01$ & $(2.09 \pm 0.31) E-01$ \\
\hline Bream & 3 & $(2.60 \pm 0.21) E-01$ & $(3.00 \pm 0.49) E-01$ & $(2.27 \pm 0.46) E-01$ \\
\hline Catfish & 3 & $(1.16 \pm 0.33) E-01$ & $(1.74 \pm 0.46) E-01$ & $(6.02 \pm 1.57) E-02$ \\
\hline \multicolumn{5}{|c|}{$\mathrm{Pu}-238, \mathrm{pCi} / \mathrm{g}$} \\
\hline \multicolumn{5}{|c|}{ Off Site (Edible) } \\
\hline \multicolumn{5}{|c|}{ New Savannah Bluff Lock and Dam (formerly Augusta Lock and Dam) } \\
\hline Bass & 3 & $(1.41 \pm 0.94) E-05$ & $(3.07 \pm 2.51) E-05$ & $(-0.19 \pm 4.72) E-05$ \\
\hline Bream & 3 & $(-2.40 \pm 5.75) E-0.5$ & $(4.00 \pm 7.29) E-05$ & $(-1.39 \pm 0.42) E-04$ \\
\hline Catfish & 3 & $(-1.55 \pm 0.72) E-05$ & $(-0.44 \pm 1.71) \mathrm{E}-05$ & $(-2.91 \pm 1.32) E-05$ \\
\hline Shad & 3 & $(7.95 \pm 1.28) E-06$ & $(0.98 \pm 1.57) E-05$ & $(0.55 \pm 1.90) E-05$ \\
\hline \multicolumn{5}{|c|}{ Beaver Dam Creek River Mouth } \\
\hline Bass & 3 & $(-3.52 \pm 0.90) E-05$ & $(-1.72 \pm 5.05) E-05$ & $(-0.45 \pm 1.71) E-04$ \\
\hline Bream & 3 & $(2.93 \pm 3.99) E-05$ & $(1.09 \pm 1.20) \mathrm{E}-04$ & $(-1.46 \pm 4.56) \mathrm{E}-05$ \\
\hline Catfish & 3 & $(1.44 \pm 1.06) E-05$ & $(0.32 \pm 1.71) E-04$ & $(-0.05 \pm 1.07) \mathrm{E}-04$ \\
\hline \multicolumn{5}{|c|}{ Four Mile Creek River Mouth } \\
\hline Bass & 3 & $(-0.21 \pm 1.16) E-05$ & $(2.10 \pm 3.30) E-05$ & $(-0.15 \pm 1.17) E-04$ \\
\hline Bream & 3 & $(2.59 \pm 2.11) \mathrm{E}-05$ & $(4.73 \pm 3.71) E-05$ & $(-1.62 \pm 2.79) E-05$ \\
\hline Catfish & 3 & $(1.98 \pm 1.51) E-05$ & $(4.56 \pm 4.33) \mathrm{E}-05$ & $(-0.68 \pm 7.61) E-05$ \\
\hline Shad & 3 & $(3.61 \pm 3.53) E-05$ & $(1.07 \pm 0.99) E-04$ & $(-0.01 \pm 2.08) E-04$ \\
\hline \multicolumn{5}{|c|}{ Hwy-301 Bridge Area } \\
\hline Bass & 3 & $(-0.45 \pm 1.21) \mathrm{E}-05$ & $(1.56 \pm 2.10) E-05$ & $(-2.63 \pm 1.04) E-05$ \\
\hline Bream & 3 & $(-1.63 \pm 0.25) E-05$ & $(-1.26 \pm 2.85) E-05$ & $(-2.10 \pm 4.51) E-05$ \\
\hline Catfish & 3 & $(-1.66 \pm 0.65) E-05$ & $(-0.40 \pm 6.70) E-05$ & $(-2.59 \pm 8.65) E-05$ \\
\hline Shad & 3 & $(5.35 \pm 3.01) E-05$ & $(0.97 \pm 1.44) E-04$ & $(-0.44 \pm 6.08) E-05$ \\
\hline
\end{tabular}




\section{Table 25}

\section{Radioactivity in Aquatic Food Products - Freshwater Fish}

Page 10 of 16

\begin{tabular}{|c|c|c|c|c|}
\hline Location & $\begin{array}{l}\text { No. of } \\
\text { Samples }\end{array}$ & Arithmetic Mean $\pm \sigma$ & Maximum $\pm \sigma$ & Minimum $\pm \sigma$ \\
\hline \multicolumn{5}{|c|}{ L3R Creek River Mouth } \\
\hline Bass & 3 & $(-0.71 \pm 1.18) E-04$ & $(5.10 \pm 4.15) \mathrm{E}-05$ & $(-3.07 \pm 1.69) E-04$ \\
\hline Bream & 3 & $(3.22 \pm 2.95) E-05$ & $(7.64 \pm 6.00) \mathrm{E}-05$ & $(-2.38 \pm 4.10) E-05$ \\
\hline Catfish & 3 & $(-0.96 \pm 2.74) E-05$ & $(0.36 \pm 3.29) E-04$ & $(-0.59 \pm 1.81) E-04$ \\
\hline \multicolumn{5}{|c|}{ Steel Creek River Mouth } \\
\hline Bass & 3 & $(0.26 \pm 8.50) E-06$ & $(1.72 \pm 2.77) \mathrm{E}-05$ & $(-9.19 \pm 6.71) E-06$ \\
\hline Bream & 3 & $(-0.24 \pm 1.94) E-05$ & $(3.04 \pm 2.68) E-05$ & $(-3.68 \pm 1.66) E-05$ \\
\hline Catfish & 3 & $(-2.32 \pm 0.70) E-05$ & $(-1.38 \pm 1.48) \mathrm{E}-05$ & $(-3.67 \pm 1.67) E-05$ \\
\hline Shad & 3 & $(-1.16 \pm 0.38) E-04$ & $(-7.66 \pm 3.88) E-05$ & $(-1.92 \pm 0.96) E-04$ \\
\hline \multicolumn{5}{|c|}{ U3R Creek River Mouth } \\
\hline Bass & 3 & $(-1.68 \pm 0.60) E-05$ & $(-4.88 \pm 5.09) E-06$ & $(-2.37 \pm 7.30) E-05$ \\
\hline Bream & 3 & $(-0.41 \pm 2.95) E-05$ & $(4.54 \pm 4.02) E-05$ & $(-0.57 \pm 1.61) E-04$ \\
\hline Catfish & 3 & $(-6.01 \pm 7.34) E-05$ & $(4.62 \pm 6.95) E-05$ & $(-2.01 \pm 1.10) E-04$ \\
\hline \multicolumn{5}{|c|}{ Pu-239, pCi/g } \\
\hline \multicolumn{5}{|c|}{ Off Site (Edible) } \\
\hline \multicolumn{5}{|c|}{ New Savannah Bluff Lock and Dam (formerly Augusta Lock and Dam) } \\
\hline Bass & 3 & $(-2.57 \pm 3.55) E-05$ & $(1.56 \pm 1.21) \mathrm{E}-05$ & $(-9.63 \pm 2.44) E-05$ \\
\hline Bream & 3 & $(-1.20 \pm 0.16) E-04$ & $(-8.82 \pm 2.62) E-05$ & $(-1.39 \pm 0.44) E-04$ \\
\hline Catfish & 3 & $(-7.78 \pm 3.63) E-06$ & $(-0.06 \pm 1.83) E-05$ & $(-1.23 \pm 2.11) E-05$ \\
\hline Shad & 3 & $(1.16 \pm 0.82) E-05$ & $(2.54 \pm 2.31) \mathrm{E}-05$ & $(-0.31 \pm 1.19) E-05$ \\
\hline \multicolumn{5}{|c|}{ Beaver Dam Creek River Mouth } \\
\hline Bass & 3 & $(-1.36 \pm 2.53) E-05$ & $(0.31 \pm 2.35) E-04$ & $(-5.70 \pm 4.24) E-05$ \\
\hline Bream & 3 & $(-8.14 \pm 6.37) E-05$ & $(0.98 \pm 6.02) E-05$ & $(-2.04 \pm 0.68) E-04$ \\
\hline Catfish & 3 & $(0.62 \pm 1.41) E-05$ & $(0.22 \pm 1.41) E-04$ & $(-2.19 \pm 9.91) \mathrm{E}-05$ \\
\hline \multicolumn{5}{|c|}{ Four Mile Creek River Mouth } \\
\hline Bass & 3 & $(-0.85 \pm 1.35) E-05$ & $(0.19 \pm 2.17) E-04$ & $(-2.43 \pm 1.89) E-05$ \\
\hline Bream & 3 & $(-1.10 \pm 1.01) \mathrm{E}-05$ & $(-0.08 \pm 2.35) E-05$ & $(-3.13 \pm 2.98) E-05$ \\
\hline Catfish & 3 & $(-1.50 \pm 1.65) \mathrm{E}-05$ & $(0.15 \pm 1.67) E-04$ & $(-4.22 \pm 2.74) E-05$ \\
\hline Shad & 3 & $(-2.58 \pm 6.12) E-06$ & $(0.82 \pm 7.34) E-05$ & $(-0.13 \pm 1.53) E-04$ \\
\hline \multicolumn{5}{|c|}{ Hwy-301 Bridge Area } \\
\hline Bass & 3 & $(0.18 \pm 1.08) E-05$ & $(2.14 \pm 3.05) E-05$ & $(-1.58 \pm 8.32) E-05$ \\
\hline Bream & 3 & $(-4.57 \pm 1.88) E-05$ & $(-1.34 \pm 0.87) E-05$ & $(-7.87 \pm 3.57) E-05$ \\
\hline Cattish & 3 & $(-4.35 \pm 1.03) E-05$ & $(-2.36 \pm 7.04) E-05$ & $(-5.80 \pm 7.95) E-05$ \\
\hline Shad & 3 & $(-1.25 \pm 0.64) \mathrm{E}-04$ & $(-4.12 \pm 2.42) \mathrm{E}-05$ & $(-2.50 \pm 0.93) E-04$ \\
\hline
\end{tabular}


Table 25

\section{Radioactivity in Aquatic Food Products - Freshwater Fish}

Page 11 of 16

\begin{tabular}{lcrrr}
\hline Location & $\begin{array}{c}\text { No. of } \\
\text { Samples }\end{array}$ & Arithmetic Mean $\pm \sigma$ & Maximum $\pm \sigma$ & Minimum $\pm \sigma$ \\
\hline $\begin{array}{l}\text { L3R Creek River Mouth } \\
\text { Bass }\end{array}$ & 3 & $(-1.09 \pm 1.11) \mathrm{E}-04$ & $(4.36 \pm 4.67) \mathrm{E}-05$ & $(-3.25 \pm 2.11) \mathrm{E}-04$ \\
$\quad$ Bream & 3 & $(-4.32 \pm 1.98) \mathrm{E}-05$ & $(-0.42 \pm 2.92) \mathrm{E}-05$ & $(-6.85 \pm 4.41) \mathrm{E}-05$ \\
Catfish & 3 & $(-1.86 \pm 2.18) \mathrm{E}-05$ & $(0.22 \pm 2.09) \mathrm{E}-04$ & $(-0.53 \pm 1.52) \mathrm{E}-04$ \\
Steel Creek River Mouth & & & & \\
$\quad$ Bass & 3 & $(-1.40 \pm 0.28) \mathrm{E}-05$ & $(-1.02 \pm 0.60) \mathrm{E}-05$ & $(-1.94 \pm 2.35) \mathrm{E}-05$ \\
Bream & 3 & $(0.70 \pm 4.24) \mathrm{E}-06$ & $(0.56 \pm 2.04) \mathrm{E}-05$ & $(-0.78 \pm 2.43) \mathrm{E}-05$ \\
Catfish & 3 & $(1.91 \pm 9.93) \mathrm{E}-06$ & $(1.68 \pm 3.80) \mathrm{E}-05$ & $(-1.69 \pm 0.96) \mathrm{E}-05$ \\
$\quad$ Shad & 3 & $(-9.32 \pm 3.07) \mathrm{E}-05$ & $(-5.94 \pm 7.22) \mathrm{E}-05$ & $(-1.54 \pm 1.35) \mathrm{E}-04$ \\
U3R Creek River Mouth & & & & \\
$\quad$ Bass & 3 & $(-3.57 \pm 2.25) \mathrm{E}-05$ & $(2.26 \pm 9.26) \mathrm{E}-06$ & $(-7.58 \pm 7.89) \mathrm{E}-05$ \\
Bream & 3 & $(-5.90 \pm 3.25) \mathrm{E}-05$ & $(-0.93 \pm 2.90) \mathrm{E}-05$ & $(-1.20 \pm 1.31) \mathrm{E}-04$ \\
$\quad$ Catfish & 3 & $(1.73 \pm 2.11) \mathrm{E}-05$ & $(5.70 \pm 8.04) \mathrm{E}-05$ & $(-1.50 \pm 4.07) \mathrm{E}-05$ \\
\hline Gross Alpha, pCi/g & & & &
\end{tabular}

Off Site (Edible)

New Savannah Bluff Lock and Dam (formerly Augusta Lock and Dam)

$\begin{array}{lllll}\text { Bass } & 3 & (-9.14 \pm 9.21) \mathrm{E}-02 & (0.55 \pm 1.57) \mathrm{E}-01 & (-2.61 \pm 1.14) \mathrm{E}-01 \\ \text { Bream } & 3 & (-0.92 \pm 1.18) \mathrm{E}-01 & (1.36 \pm 1.69) \mathrm{E}-01 & (-2.61 \pm 1.14) \mathrm{E}-01 \\ \text { Catfish } & 3 & (-4.02 \pm 7.87) \mathrm{E}-02 & (1.08 \pm 1.76) \mathrm{E}-01 & (-1.61 \pm 1.33) \mathrm{E}-01 \\ \text { Shad } & 3 & (-9.23 \pm 2.39) \mathrm{E}-02 & (-0.59 \pm 1.38) \mathrm{E}-01 & (-1.39 \pm 1.16) \mathrm{E}-01\end{array}$

Beaver Dam Creek River Mouth

Bass
Bream
Catfish

$3 \quad(-0.56 \pm 4.19) \mathrm{E}-02$

$(0.72 \pm 1.85) E-01$

$(-0.72 \pm 1.54) E-01$

$3 \quad(0.19 \pm 4.76) \mathrm{E}-02$

$(0.94 \pm 2.34) E-01$

$(-0.65 \pm 1.62) \mathrm{E}-01$

$3 \quad(0.80 \pm 1.48) E-01$

$(3.75 \pm 2.26) E-01$

$(-0.85 \pm 1.03) E-01$

Four Mile Creek River Mouth

Bass
Bream
Catfish
Shad

Hwy-17 Bridge Area

$\begin{array}{llrrr}\text { Bass } & 3 & (-1.17 \pm 0.16) \mathrm{E}-01 & (-0.96 \pm 1.31) \mathrm{E}-01 & (-1.49 \pm 2.06) \mathrm{E}-01 \\ \text { Bream } & 3 & (-6.28 \pm 1.85) \mathrm{E}-02 & (-0.39 \pm 1.51) \mathrm{E}-01 & (-0.99 \pm 1.34) \mathrm{E}-01 \\ \text { Catfish } & 3 & (-6.87 \pm 5.80) \mathrm{E}-02 & (0.47 \pm 2.06) \mathrm{E}-01 & (-1.33 \pm 1.74) \mathrm{E}-01\end{array}$


Table 25

Radioactivity in Aquatic Food Products - Freshwater Fish

Page 12 of 16

\begin{tabular}{|c|c|c|c|c|}
\hline Location & $\begin{array}{c}\text { No. of } \\
\text { Samples }\end{array}$ & Arithmetic Mean $\pm \sigma$ & Maximum $\pm \sigma$ & Minimum $\pm \sigma$ \\
\hline \multicolumn{5}{|c|}{ Hwy-301 Bridge Area } \\
\hline Bass & 3 & $(-7.63 \pm 8.26) E-02$ & $(0.65 \pm 1.85) E-01$ & $(-2.22 \pm 1.04) E-01$ \\
\hline Bream & 3 & $(-9.91 \pm 2.59) E-02$ & $(-0.59 \pm 1.58) E-01$ & $(-1.48 \pm 1.36) E-01$ \\
\hline Catfish & 3 & $(-1.25 \pm 0.28) E-01$ & $(-0.76 \pm 1.27) E-01$ & $(-1.72 \pm 1.35) E-01$ \\
\hline Shad & 3 & $(2.97 \pm 3.87) E-02$ & $(1.07 \pm 1.24) E-01$ & $(-0.10 \pm 1.49) E-01$ \\
\hline \multicolumn{5}{|c|}{ L3R Creek River Mouth } \\
\hline Bass & 3 & $(-9.17 \pm 1.38) E-02$ & $(-0.72 \pm 1.29) E-01$ & $(-1.18 \pm 1.71) E-01$ \\
\hline Bream & 3 & $(-7.21 \pm 6.63) E-02$ & $(-0.02 \pm 1.95) E-01$ & $(-2.05 \pm 1.02) E-01$ \\
\hline Catfish & 3 & $(-6.15 \pm 5.48) E-02$ & $(0.47 \pm 1.55) E-01$ & $(-1.31 \pm 1.11) \mathrm{E}-01$ \\
\hline \multicolumn{5}{|c|}{ Steel Creek River Mouth } \\
\hline Bass & 3 & $(-1.27 \pm 4.02) E-02$ & $(0.59 \pm 1.95) E-01$ & $(-0.80 \pm 1.68) E-01$ \\
\hline Bream & 3 & $(-4.26 \pm 6.15) E-02$ & $(0.26 \pm 1.95) E-01$ & $(-1.65 \pm 1.50) E-01$ \\
\hline Catfish & 3 & $(-2.91 \pm 4.34) E-02$ & $(0.56 \pm 1.86) E-01$ & $(-0.87 \pm 1.52) E-01$ \\
\hline Shad & 3 & $(2.12 \pm 5.54) E-02$ & $(1.32 \pm 2.38) E-01$ & $(-0.37 \pm 2.03) E-01$ \\
\hline \multicolumn{5}{|c|}{ Stokes Bluff Landing } \\
\hline Bass & 3 & $(-2.42 \pm 9.81) E-02$ & $(1.08 \pm 2.24) E-01$ & $(-2.16 \pm 1.58) E-01$ \\
\hline Bream & 3 & $(4.17 \pm 1.59) E-01$ & $(7.34 \pm 4.48) E-01$ & $(2.50 \pm 2.18) E-01$ \\
\hline Catfish & 3 & $(3.31 \pm 8.90) E-02$ & $(1.40 \pm 1.90) E-01$ & $(-1.43 \pm 1.25) E-01$ \\
\hline \multicolumn{5}{|c|}{ U3R Creek River Mouth } \\
\hline Bass & 3 & $(-0.85 \pm 7.21) E-02$ & $(0.78 \pm 1.96) E-01$ & $(-1.52 \pm 1.22) E-01$ \\
\hline Bream & 3 & $(2.04 \pm 1.32) E-01$ & $(4.47 \pm 2.34) E-01$ & $(-0.08 \pm 1.45) E-01$ \\
\hline Catfish & 3 & $(-1.10 \pm 0.23) E-01$ & $(-0.84 \pm 1.54) E-01$ & $(-1.57 \pm 1.01) E-01$ \\
\hline
\end{tabular}

Off Site (Nonedible)

New Savannah Bluff Lock and Dam (formerly Augusta Lock and Dam)

$\begin{array}{lrrrr}\text { Bass } & 3 & (-3.17 \pm 0.18) \mathrm{E}-01 & (-2.96 \pm 5.05) \mathrm{E}-01 & (-3.52 \pm 6.45) \mathrm{E}-01 \\ \text { Bream } & 3 & (1.10 \pm 3.98) \mathrm{E}-01 & (6.63 \pm 9.54) \mathrm{E}-01 & (-6.63 \pm 6.03) \mathrm{E}-01 \\ \text { Catfish } & 3 & (-3.23 \pm 0.75) \mathrm{E}-01 & (-2.24 \pm 4.33) \mathrm{E}-01 & (-4.69 \pm 4.21) \mathrm{E}-01 \\ \text { Shad } & 3 & (2.92 \pm 6.05) \mathrm{E}-01 & (1.50 \pm 1.02) \mathrm{E}+00 & (-3.71 \pm 4.25) \mathrm{E}-01 \\ \text { Beaver Dam Creek River Mouth } & & & & \\ \text { Bass } & 3 & (-4.10 \pm 1.75) \mathrm{E}-01 & (-0.61 \pm 4.97) \mathrm{E}-01 & (-6.12 \pm 3.57) \mathrm{E}-01 \\ \text { Bream } & 3 & (2.97 \pm 2.57) \mathrm{E}-01 & (7.65 \pm 7.39) \mathrm{E}-01 & (-1.22 \pm 6.25) \mathrm{E}-01 \\ \text { Catfish } & 3 & (-2.21 \pm 4.46) \mathrm{E}-01 & (5.24 \pm 8.63) \mathrm{E}-01 & (-1.02 \pm 0.56) \mathrm{E}+00 \\ \begin{array}{l}\text { Four Mile Creek River Mouth } \\ \text { Bass }\end{array} & & & & \\ & 3 & (-8.65 \pm 2.19) \mathrm{E}-01 & (-4.37 \pm 4.79) \mathrm{E}-01 & (-1.16 \pm 0.61) \mathrm{E}+00\end{array}$


Table 25

Radioactivity in Aquatic Food Products - Freshwater Fish

Page 13 of 16

\begin{tabular}{|c|c|c|c|c|}
\hline Location & $\begin{array}{c}\text { No. of } \\
\text { Samples }\end{array}$ & Arithmetic Mean $\pm \sigma$ & Maximum $\pm \sigma$ & Minimum $\pm \sigma$ \\
\hline Bream & 3 & $(-3.98 \pm 2.97) E-01$ & $(0.19 \pm 1.01) \mathrm{E}+00$ & $(-7.75 \pm 9.09) E-01$ \\
\hline Catfish & 3 & $(9.73 \pm 4.65) E-01$ & $(1.66 \pm 1.12) \mathrm{E}+00$ & $(0.86 \pm 6.98) E-01$ \\
\hline Shad & 3 & $(-2.62 \pm 1.37) E-01$ & $(-0.37 \pm 5.35) E-01$ & $(-5.10 \pm 4.56) \mathrm{E}-01$ \\
\hline \multicolumn{5}{|c|}{ Hwy-301 Bridge Area } \\
\hline Bass & 3 & $(-1.53 \pm 9.73) E-02$ & $(1.78 \pm 6.65) E-01$ & $(-1.27 \pm 6.45) \mathrm{E}-01$ \\
\hline Bream & 3 & $(-1.00 \pm 1.70) E-01$ & $(2.09 \pm 6.25) E-01$ & $(-3.77 \pm 4.89) \mathrm{E}-01$ \\
\hline Catfish & 3 & $(-4.13 \pm 3.30) E-01$ & $(2.44 \pm 6.49) E-01$ & $(-7.95 \pm 4.17) E-01$ \\
\hline Shad & 3 & $(-4.34 \pm 1.06) E-01$ & $(-2.44 \pm 4.86) \mathrm{E}-01$ & $(-6.09 \pm 3.54) E-01$ \\
\hline \multicolumn{5}{|c|}{ L3R Creek River Mouth } \\
\hline Bass & 3 & $(-4.98 \pm 0.85) E-01$ & $(-3.87 \pm 4.76) E-01$ & $(-6.64 \pm 7.82) E-01$ \\
\hline Bream & 3 & $(-3.24 \pm 0.17) E-01$ & $(-3.08 \pm 6.02) E-01$ & $(-3.57 \pm 7.55) E-01$ \\
\hline Catfish & 3 & $(-2.54 \pm 3.62) E-01$ & $(2.01 \pm 5.81) \mathrm{E}-01$ & $(-9.69 \pm 5.05) E-01$ \\
\hline \multicolumn{5}{|c|}{ Steel Creek River Mouth } \\
\hline Bass & 3 & $(-5.48 \pm 1.52) E-01$ & $(-3.02 \pm 5.77) E-01$ & $(-8.25 \pm 4.04) E-01$ \\
\hline Bream & 3 & $(-6.25 \pm 3.87) E-01$ & $(-0.83 \pm 8.87) E-01$ & $(-1.37 \pm 0.72) E+\infty 0$ \\
\hline Catfish & 3 & $(-2.73 \pm 1.51) E-01$ & $(-0.12 \pm 5.77) E-01$ & $(-5.33 \pm 4.76) E-01$ \\
\hline Shad & 3 & $(2.79 \pm 2.64) E-01$ & $(6.63 \pm 6.84) E-01$ & $(-2.28 \pm 3.72) E-01$ \\
\hline \multicolumn{5}{|c|}{ U3R Creek River Mouth } \\
\hline Bass & 3 & $(-5.16 \pm 1.48) E-01$ & $(-2.21 \pm 6.31) \mathrm{E}-01$ & $(-6.64 \pm 3.91) \mathrm{E}-01$ \\
\hline Bream & 3 & $(-4.29 \pm 0.40) E-01$ & $(-3.55 \pm 6.27) E-01$ & $(-4.93 \pm 4.56) \mathrm{E}-01$ \\
\hline Catfish & 3 & $(-0.42 \pm 1.95) E-01$ & $(3.47 \pm 5.59) \mathrm{E}-01$ & $(-2.44 \pm 4.65) E-01$ \\
\hline \multicolumn{5}{|c|}{ On Site (Edible) } \\
\hline \multicolumn{5}{|l|}{ L-Lake } \\
\hline Bass & 3 & $(7.91 \pm 4.90) E-02$ & $(1.65 \pm 1.90) \mathrm{E}-01$ & $(-0.05 \pm 1.57) E-01$ \\
\hline Bream & 3 & $(0.85 \pm 1.12) E-01$ & $(3.03 \pm 3.45) E-01$ & $(-0.70 \pm 1.53) E-01$ \\
\hline \multicolumn{5}{|c|}{ L3R-2 Patterson Mill Road } \\
\hline Bass & 3 & $(1.39 \pm 2.01) E-01$ & $(5.39 \pm 2.82) E-01$ & $(-1.00 \pm 1.75) E-01$ \\
\hline Bream & 3 & $(0.35 \pm 1.06) E-01$ & $(1.69 \pm 2.01) \mathrm{E}-01$ & $(-1.74 \pm 1.72) E-01$ \\
\hline \multicolumn{5}{|c|}{ PAR Pond } \\
\hline Bass & 3 & $(4.84 \pm 0.51) E-01$ & $(5.51 \pm 2.98) E-01$ & $(3.84 \pm 2.69) E-01$ \\
\hline Bream & 3 & $(-4.94 \pm 1.41) E-01$ & $(-3.08 \pm 4.63) E-01$ & $(-7.70 \pm 3.77) E-01$ \\
\hline \multicolumn{5}{|l|}{ Pond B } \\
\hline Bass & 3 & $(1.73 \pm 0.42) E+00$ & $(2.54 \pm 0.65) E+00$ & $(1.13 \pm 0.28) E+00$ \\
\hline Bream & 3 & $(4.08 \pm 0.66) E-01$ & $(5.36 \pm 3.11) E-01$ & $(3.16 \pm 2.92) E-01$ \\
\hline
\end{tabular}




\section{Table 25}

\section{Radioactivity in Aquatic Food Products - Freshwater Fish}

Page 14 of 16

No. of

\begin{tabular}{lllll} 
Location & $\begin{array}{c}\text { No. of } \\
\text { Samples }\end{array}$ & Arithmetic Mean $\pm \sigma$ & Maximum $\pm \sigma$ & Minimum $\pm \sigma$ \\
\hline Gross Beta, pCi/g & & & \\
\hline Off Site (Edible) &
\end{tabular}

New Savannah Bluff Lock and Dam (formerly Augusta Lock and Dam)

$\begin{array}{lllll}\text { Bass } & 3 & (2.39 \pm 0.31) \mathrm{E}+00 & (2.99 \pm 0.43) \mathrm{E}+00 & (1.98 \pm 0.35) \mathrm{E}+00 \\ \begin{array}{l}\text { Bream } \\ \text { Catfish }\end{array} & 3 & (2.27 \pm 0.29) \mathrm{E}+00 & (2.61 \pm 0.41) \mathrm{E}+00 & (1.69 \pm 0.32) \mathrm{E}+00 \\ \text { Shad } & 3 & (2.45 \pm 0.27) \mathrm{E}+00 & (2.93 \pm 0.37) \mathrm{E}+00 & (2.00 \pm 0.34) \mathrm{E}+00 \\ \text { Beaver Dam Creek River Mouth } & 3 & (1.96 \pm 0.21) \mathrm{E}+00 & (2.19 \pm 0.35) \mathrm{E}+00 & (1.54 \pm 0.32) \mathrm{E}+00 \\ \text { Bass } & & & & \\ \text { Bream } & 3 & (2.28 \pm 0.27) \mathrm{E}+00 & (2.77 \pm 0.41) \mathrm{E}+00 & (1.85 \pm 0.37) \mathrm{E}+00 \\ \text { Catfish } & 3 & (2.21 \pm 0.43) \mathrm{E}+00 & (2.95 \pm 0.43) \mathrm{E}+00 & (1.46 \pm 0.35) \mathrm{E}+00 \\ & 3 & (1.42 \pm 0.20) \mathrm{E}+00 & (1.77 \pm 0.26) \mathrm{E}+00 & (1.09 \pm 0.33) \mathrm{E}+00\end{array}$

Four Mile Creek River Mouth

Bass
Bream
Catfish
Shad

$(2.34 \pm 0.32) \mathrm{E}+00$

$(2.66 \pm 0.36) E+00$

$(1.69 \pm 0.32) \mathrm{E}+00$

3

$(1.96 \pm 0.21) \mathrm{E}+00$

$(2.35 \pm 0.35) E+00$

$(1.64 \pm 0.36) \mathrm{E}+00$

3

$(1.44 \pm 0.06) \mathrm{E}+00$

$(1.55 \pm 0.35) \mathrm{E}+00$

$(1.33 \pm 0.38) E+00$

Hwy-17 Bridge Area

3

$(2.07 \pm 0.17) \mathrm{E}+00$

$(2.33 \pm 0.43) \mathrm{E}+00$

$(1.75 \pm 0.40) \mathrm{E}+00$

Bass

$(2.05 \pm 0.17) \mathrm{E}+00$

$(2.28 \pm 0.30) \mathrm{E}+00$

$(1.72 \pm 0.34) \mathrm{E}+00$

Bream

$(1.79 \pm 0.21) \mathrm{E}+00$

$(2.20 \pm 0.32) \mathrm{E}+00$

$(1.51 \pm 0.30) E+00$

Catfish

$(1.93 \pm 0.24) \mathrm{E}+00$

$(2.36 \pm 0.42) \mathrm{E}+00$

$(1.53 \pm 0.38) \mathrm{E}+00$

Hwy-301 Bridge Area

Bass

Bream

$(2.01 \pm 0.29) E+00$

$(2.58 \pm 0.40) E+00$

$(1.61 \pm 0.36) \mathrm{E}+00$

Catfish

$(1.63 \pm 0.17) E+00$

$(1.83 \pm 0.39) E+00$

$(1.29 \pm 0.34) E+00$

Shad

$(2.37 \pm 0.28) E+00$

$(2.80 \pm 0.41) E+00$

$(1.85 \pm 0.33) \mathrm{E}+00$

L3R Creek River Mouth

Bass

$(2.12 \pm 0.18) E+00$

$(2.46 \pm 0.35) E+00$

$(1.84 \pm 0.24) E+00$

Bream

$(2.11 \pm 0.21) \mathrm{E}+00$

$(2.51 \pm 0.36) \mathrm{E}+00$

$(1.78 \pm 0.39) E+00$

Catfish

$(2.32 \pm 0.05) E+00$

$(2.41 \pm 0.41) E+00$

$(2.23 \pm 0.39) E+00$

3

$(2.47 \pm 0.32) \mathrm{E}+00$

$(3.08 \pm 0.43) E+00$

$(2.00 \pm 0.33) E+00$

Steel Creek River Mouth

Bass
Bream
Shatfish

$\begin{array}{llll}3 & (2.59 \pm 0.20) E+00 & (2.79 \pm 0.42) E+00 & (2.19 \pm 0.40) E+00 \\ 3 & (1.59 \pm 0.20) E+00 & (1.86 \pm 0.38) E+00 & (1.21 \pm 0.34) E+00 \\ 3 & (3.16 \pm 0.49) E+00 & (4.09 \pm 0.47) E+00 & (2.41 \pm 0.40) E+00 \\ 3 & (1.54 \pm 0.11) E+00 & (1.69 \pm 0.39) E+00 & (1.34 \pm 0.31) E+00\end{array}$




\section{Table 25}

\section{Radioactivity in Aquatic Food Products - Freshwater Fish}

Page 15 of 16

\begin{tabular}{lcccc} 
Location & $\begin{array}{c}\text { No. of } \\
\text { Samples }\end{array}$ & Arithmetic Mean $\pm \sigma$ & Maximum $\pm \sigma$ & Minimum $\pm \sigma$ \\
\hline $\begin{array}{l}\text { Stokes Bluff Landing } \\
\text { Bass }\end{array}$ & 3 & $(2.29 \pm 0.07) \mathrm{E}+00$ & $(2.36 \pm 0.43) \mathrm{E}+00$ & $(2.16 \pm 0.42) \mathrm{E}+00$ \\
$\quad$ Bream & 3 & $(1.80 \pm 0.22) \mathrm{E}+00$ & $(2.17 \pm 0.38) \mathrm{E}+00$ & $(1.40 \pm 0.33) \mathrm{E}+00$ \\
$\quad \begin{array}{l}\text { Catfish } \\
\text { U3R Creek River Mouth }\end{array}$ & 3 & $(2.20 \pm 0.10) \mathrm{E}+00$ & $(2.34 \pm 0.37) \mathrm{E}+00$ & $(2.00 \pm 0.36) \mathrm{E}+00$ \\
$\quad$ & & & & \\
Bass & 3 & $(2.34 \pm 0.22) \mathrm{E}+00$ & $(2.77 \pm 0.37) \mathrm{E}+00$ & $(2.05 \pm 0.38) \mathrm{E}+00$ \\
$\quad \begin{array}{l}\text { Bream } \\
\text { Catfish }\end{array}$ & 3 & $(2.02 \pm 0.38) \mathrm{E}+00$ & $(2.66 \pm 0.37) \mathrm{E}+00$ & $(1.36 \pm 0.31) \mathrm{E}+00$ \\
& 3 & $(2.91 \pm 0.37) \mathrm{E}+00$ & $(3.64 \pm 0.32) \mathrm{E}+00$ & $(2.43 \pm 0.40) \mathrm{E}+00$
\end{tabular}

Off Site (Nonedible)

New Savannah Bluff Lock and Dam (formerly Augusta Lock and Dam)

$\begin{array}{lllll}\text { Bass } & 3 & (2.18 \pm 0.29) \mathrm{E}+00 & (2.75 \pm 0.95) \mathrm{E}+00 & (1.85 \pm 0.95) \mathrm{E}+00 \\ \text { Bream } & 3 & (1.24 \pm 0.25) \mathrm{E}+00 & (1.68 \pm 0.97) \mathrm{E}+00 & (8.16 \pm 8.98) \mathrm{E}-01 \\ \text { Catfish } & 3 & (1.32 \pm 0.09) \mathrm{E}+00 & (1.43 \pm 0.87) \mathrm{E}+00 & (1.14 \pm 0.64) \mathrm{E}+00 \\ \text { Shad } & 3 & (1.82 \pm 0.35) \mathrm{E}+00 & (2.46 \pm 1.12) \mathrm{E}+00 & (1.27 \pm 0.90) \mathrm{E}+00\end{array}$

Beaver Dam Creek River Mouth

$\begin{array}{lllll}\text { Bass } & 3 & (1.35 \pm 0.70) E+00 & (2.39 \pm 0.69) E+00 & (0.25 \pm 7.90) E-01 \\ \text { Bream } & 3 & (8.67 \pm 2.12) E-01 & (1.27 \pm 0.90) E+00 & (5.61 \pm 8.83) E-01 \\ \text { Catfish } & 3 & (2.59 \pm 0.12) E+00 & (2.73 \pm 1.04) E+00 & (2.35 \pm 1.02) E+00\end{array}$

Four Mile Creek River Mouth

Bass
Bream
Catfish
Shad

$\begin{array}{llll}3 & (2.34 \pm 0.76) \mathrm{E}+00 & (3.54 \pm 1.11) \mathrm{E}+00 & (9.41 \pm 9.42) \mathrm{E}-01 \\ 3 & (2.34 \pm 0.24) \mathrm{E}+00 & (2.66 \pm 1.06) \mathrm{E}+00 & (1.88 \pm 1.04) \mathrm{E}+00 \\ 3 & (3.74 \pm 0.48) \mathrm{E}+00 & (4.66 \pm 1.11) \mathrm{E}+00 & (3.05 \pm 1.07) \mathrm{E}+00 \\ 3 & (1.50 \pm 0.30) \mathrm{E}+00 & (1.89 \pm 0.90) \mathrm{E}+00 & (9.18 \pm 8.53) \mathrm{E}-01\end{array}$

Hwy-301 Bridge Area

Bass
Bream
Catfish
Shad

$(1.27 \pm 0.91) \mathrm{E}+00$

$3 \quad(7.93 \pm 4.74) \mathrm{E}-01$

$(1.70 \pm 0.89) E+00$

$(1.12 \pm 8.22) E-01$

$3 \quad(1.29 \pm 0.28) \mathrm{E}+00$

$(1.80 \pm 0.92) \mathrm{E}+00$

$(8.48 \pm 8.87) \mathrm{E}-01$

3

$(1.69 \pm 0.41) \mathrm{E}+00$

$(2.46 \pm 0.69) \mathrm{E}+00$

$(1.06 \pm 0.89) E+00$

L3R Creek River Mouth

Bass
Bream
Catfish

$\begin{array}{llll}3 & (1.36 \pm 0.20) E+00 & (1.66 \pm 0.94) E+00 & (9.96 \pm 8.75) E-01 \\ 3 & (1.38 \pm 0.41) E+00 & (2.09 \pm 1.00) E+00 & (0.68 \pm 1.05) E+00 \\ 3 & (1.05 \pm 0.41) E+00 & (1.58 \pm 0.94) E+00 & (2.33 \pm 8.38) E-01\end{array}$

Steel Creek River Mouth

Bass

$(1.91 \pm 0.23) \mathrm{E}+00$

$(2.36 \pm 1.00) E+00$

$(1.65 \pm 0.95) \mathrm{E}+00$ 


\section{Table 25}

\section{Radioactivity in Aquatic Food Products - Freshwater Fish}

Page 16 of 16

\begin{tabular}{|c|c|c|c|c|}
\hline Location & $\begin{array}{c}\text { No. of } \\
\text { Samples }\end{array}$ & Arithmetic Mean $\pm \sigma$ & Maximum $\pm \sigma$ & Minimum $\pm \sigma$ \\
\hline Bream & 3 & $(2.29 \pm 0.46) E+00$ & $(2.80 \pm 1.09) E+00$ & $(1.37 \pm 1.03) E+00$ \\
\hline Catfish & 3 & $(1.26 \pm 0.21) E+00$ & $(1.59 \pm 0.95) E+\infty 0$ & $(8.80 \pm 9.04) E-01$ \\
\hline Shad & 3 & $(1.22 \pm 0.29) E+00$ & $(1.78 \pm 0.91) E+00$ & $(9.21 \pm 6.08) E-01$ \\
\hline \multicolumn{5}{|c|}{ U3R Creek River Mouth } \\
\hline Bass & 3 & $(2.21 \pm 0.74) E+00$ & $(3.65 \pm 1.06) E+00$ & $(1.22 \pm 0.89) E+00$ \\
\hline Bream & 3 & $(2.00 \pm 0.55) E+00$ & $(2.60 \pm 1.02) E+00$ & $(9.01 \pm 8.87) E-01$ \\
\hline Catfish & 3 & $(2.01 \pm 0.39) E+00$ & $(2.75 \pm 0.93) E+00$ & $(1.43 \pm 0.90) E+00$ \\
\hline \multicolumn{5}{|c|}{ On Site (Edible) } \\
\hline \multicolumn{5}{|l|}{ L-Lake } \\
\hline Bass & 3 & $(1.94 \pm 0.25) E+00$ & $(2.34 \pm 0.37) E+00$ & $(1.48 \pm 0.33) E+00$ \\
\hline Bream & 3 & $(1.84 \pm 0.32) E+00$ & $(2.39 \pm 0.59) E+00$ & $(1.30 \pm 0.33) E+00$ \\
\hline \multicolumn{5}{|c|}{ L3R-2 Patterson Mill Road } \\
\hline Bass & 3 & $(5.94 \pm 0.31) E+00$ & $(5.94 \pm 0.54) E+00$ & $(5.94 \pm 0.54) E+00$ \\
\hline Bream & 3 & $(4.07 \pm 0.67) E+00$ & $(5.41 \pm 0.52) E+00$ & $(3.39 \pm 0.39) E+00$ \\
\hline \multicolumn{5}{|c|}{ PAR Pond } \\
\hline Bass & 3 & $(8.29 \pm 0.22) E+00$ & $(8.51 \pm 0.58) E+00$ & $(7.84 \pm 0.55) E+00$ \\
\hline Bream & 3 & $(3.31 \pm 0.56) E+00$ & $(4.40 \pm 1.07) E+00$ & $(2.53 \pm 0.98) E+00$ \\
\hline \multicolumn{5}{|l|}{ Pond B } \\
\hline Bass & 3 & $(5.55 \pm 0.66) E+01$ & $(6.83 \pm 0.17) E+01$ & $(4.60 \pm 0.08) E+01$ \\
\hline Bream & 3 & $(2.15 \pm 0.05) E+01$ & $(2.20 \pm 0.08) E+01$ & $(2.06 \pm 0.08) E+01$ \\
\hline
\end{tabular}


Table 26

Radioactivity in Aquatic Food Products - Saltwater Fish

Page 1 of 1

No. of

Location

Samples

Arithmetic Mean $\pm \sigma$

Maximum $\pm \sigma$

Minimum $\pm \sigma$

Note: 1. Only edible portions were analyzed.

2. Saltwater fish are collected downstream of the Highway 17 Bridge.

Co-60, pCi/g

\begin{tabular}{|c|c|c|c|c|}
\hline Marine Mullet & 3 & $(5.45 \pm 6.80) E-03$ & $(1.88 \pm 1.39) E-02$ & $(-0.35 \pm 1.75) E-02$ \\
\hline Red Fish (drum) & 3 & $(0.02 \pm 1.69) E-02$ & $(3.27 \pm 1.54) \mathrm{E}-02$ & $(-2.43 \pm 1.74) E-02$ \\
\hline Sea Trout & 1 & $(1.07 \pm 1.52) \mathrm{E}-02$ & & \\
\hline \multicolumn{5}{|l|}{$\mathrm{Cs}-137, \mathrm{pCi} / \mathrm{g}$} \\
\hline Marine Mullet & 3 & $(1.13 \pm 0.64) E-02$ & $(2.08 \pm 1.23) E-02$ & $(-0.10 \pm 1.33) E-02$ \\
\hline Red Fish (drum) & 3 & $(1.38 \pm 0.95) E-02$ & $(3.11 \pm 1.96) E-02$ & $(-0.17 \pm 1.33) E-02$ \\
\hline Sea Trout & 1 & $(-2.58 \pm 1.30) \mathrm{E}-02$ & & \\
\hline \multicolumn{5}{|l|}{ Gross Alpha, pCi/g } \\
\hline Marine Mullet & 3 & $(3.81 \pm 9.35) E-02$ & $(2.24 \pm 2.21) E-01$ & $(-0.73 \pm 1.56) \mathrm{E}-01$ \\
\hline Red Fish (drum) & 3 & $(-3.67 \pm 9.15) E-02$ & $(1.40 \pm 2.05) E-01$ & $(-1.66 \pm 1.35) E-01$ \\
\hline Sea Trout & 1 & $(-2.06 \pm 0.90) E-01$ & & \\
\hline \multicolumn{5}{|l|}{ Gross Beta, pCi/g } \\
\hline Marine Mullet & 3 & $(2.00 \pm 0.22) E+00$ & $(2.43 \pm 0.40) E+00$ & $(1.73 \pm 0.39) E+00$ \\
\hline Red Fish (drum) & 3 & $(2.49 \pm 0.06) E+00$ & $(2.61 \pm 0.41) \mathrm{E}+00$ & $(2.43 \pm 0.40) E+00$ \\
\hline Sea Trout & 1 & $(1.93 \pm 0.30) E+00$ & & \\
\hline
\end{tabular}




\section{Table 27}

Radioactivity in Aquatic Food Products - Shellfish

Page 1 of 1

\begin{tabular}{|c|c|c|c|c|}
\hline Location & $\begin{array}{l}\text { No. of } \\
\text { Samples }\end{array}$ & Arithmetic Mean $\pm \sigma$ & Maximum $\pm \sigma$ & Minimum $\pm \sigma$ \\
\hline \multicolumn{5}{|l|}{$\mathrm{Co}-60, \mathrm{pCi} / \mathrm{g}$} \\
\hline $\begin{array}{l}\text { Downstream of Highway } 17 \\
\text { Bridge to Savannah River } \\
\text { Mouth }\end{array}$ & 2 & $(0.57 \pm 1.43) E-02$ & $(1.99 \pm 1.50) \mathrm{E}-02$ & $(-0.86 \pm 1.34) \mathrm{E}-02$ \\
\hline \multicolumn{5}{|l|}{$\mathrm{Cs}-137, \mathrm{pCi} / \mathrm{g}$} \\
\hline $\begin{array}{l}\text { Downstream of Highway } 17 \\
\text { Bridge to Savannah River } \\
\text { Mouth }\end{array}$ & 2 & $(4.65 \pm 1.22) E-03$ & $(0.59 \pm 1.30) E-02$ & $(0.34 \pm 1.25) E-02$ \\
\hline \multicolumn{5}{|l|}{$\mathrm{Sr}-89,90, \mathrm{pCi} / \mathrm{g}$} \\
\hline $\begin{array}{l}\text { Downstream of Highway } 17 \\
\text { Bridge to Savannah River } \\
\text { Mouth }\end{array}$ & 2 & $(1.94 \pm 5.46) E-04$ & $(0.74 \pm 3.26) E-03$ & $(-0.35 \pm 3.21) E-03$ \\
\hline \multicolumn{5}{|l|}{ Gross Alpha, pCi/g } \\
\hline $\begin{array}{l}\text { Downstream of Highway } 17 \\
\text { Bridge to Savannah River } \\
\text { Mouth }\end{array}$ & 2 & $(2.26 \pm 0.01) E-01$ & $(2.26 \pm 1.53) \mathrm{E}-01$ & $(2.25 \pm 1.98) E-01$ \\
\hline \multicolumn{5}{|l|}{ Gross Beta, pCi/g } \\
\hline $\begin{array}{l}\text { Downstream of Highway } 17 \\
\text { Bridge to Savannah River } \\
\text { Mouth }\end{array}$ & 2 & $(7.35 \pm 3.12) E-01$ & $(1.05 \pm 0.26) E+00$ & $(4.23 \pm 2.09) E-01$ \\
\hline
\end{tabular}




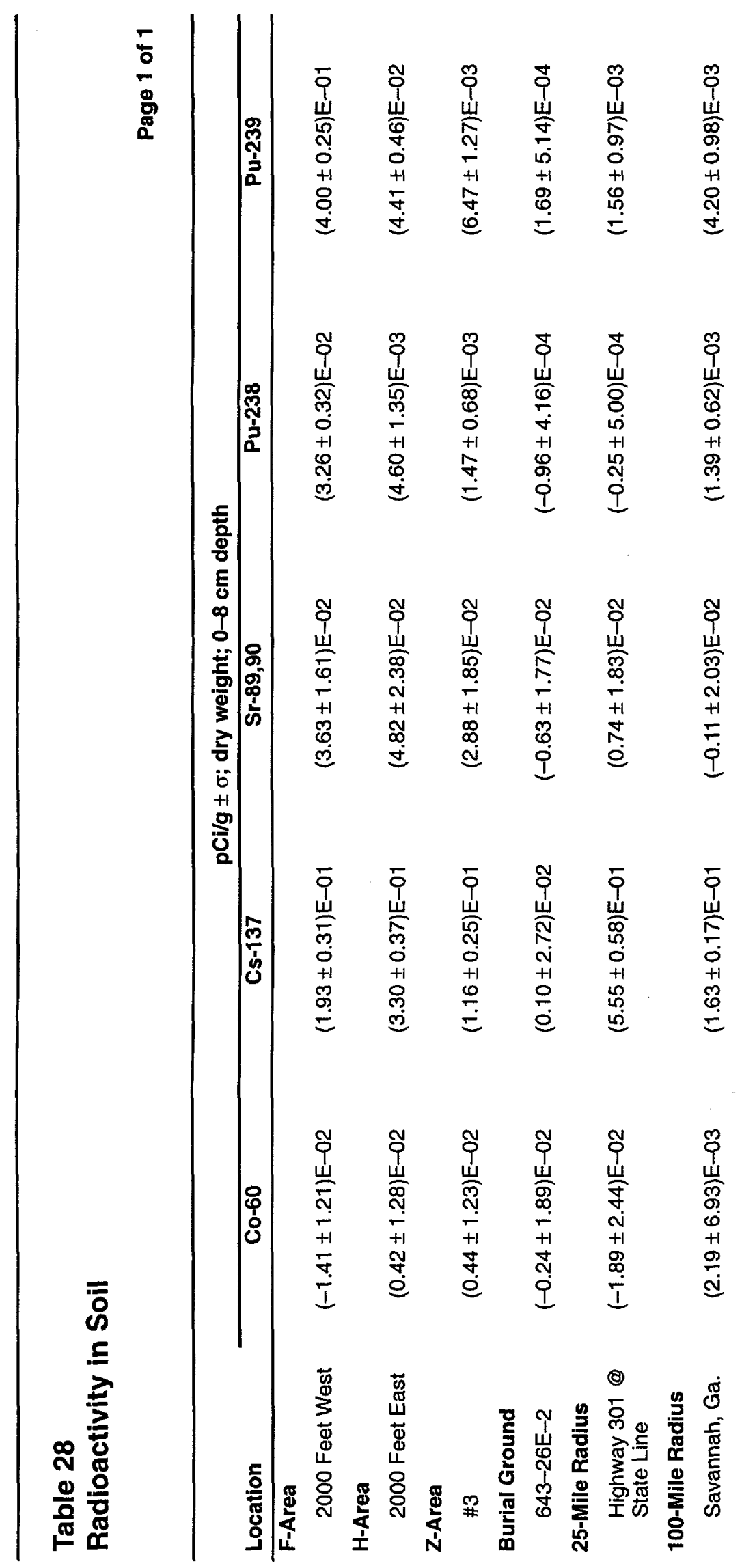




\section{Table 29}

\section{Radioactivity in River and Stream Sediment}

Page 1 of 4

\begin{tabular}{|c|c|c|c|c|}
\hline Location & & 1997 & 1998 & 1999 \\
\hline \multicolumn{5}{|l|}{ Co-60, pCi/g (dry weight) } \\
\hline Savannah River & River Mile & & & \\
\hline Highway 301 & 118.7 & $(9.31 \pm 2.94) E-02$ & $(4.95 \pm 1.26) E-02$ & $(4.35 \pm 1.79) E-02$ \\
\hline Lower 3 Runs Mouth & 129.0 & $(1.90 \pm 1.41) E-02$ & $(-1.34 \pm 1.63) E-02$ & $(3.59 \pm 1.84) \mathrm{E}-02$ \\
\hline Below Little Hell Landing & 134.0 & $(-0.28 \pm 1.11) E-02$ & $(1.38 \pm 1.66) E-02$ & $(0.14 \pm 1.62) E-02$ \\
\hline Below Four Mile Creek & 150.0 & $(0.05 \pm 6.60) E-03$ & $(-0.94 \pm 1.51) \mathrm{E}-02$ & $(-0.48 \pm 1.95) E-02$ \\
\hline R-3A above Vogtle & 151.0 & $(-2.11 \pm 2.32) E-02$ & $(-1.43 \pm 1.51) \mathrm{E}-02$ & $(2.56 \pm 1.83) \mathrm{E}-02$ \\
\hline Beaver Dam Creek Mouth & 152.1 & $(-0.79 \pm 1.08) E-02$ & $(-0.51 \pm 1.78) E-02$ & $(3.97 \pm 2.17) E-02$ \\
\hline Upper 3 Runs Mouth & 157.2 & $(-1.09 \pm 1.28) E-02$ & $(-0.00 \pm 4.31) E-02$ & $(0.81 \pm 2.64) E-02$ \\
\hline Demier Landing & 160.0 & $(-1.93 \pm 1.12) E-02$ & $(-0.88 \pm 1.75) \mathrm{E}-02$ & $(2.17 \pm 2.28) \mathrm{E}-02$ \\
\hline \multicolumn{5}{|l|}{ SRS Streams } \\
\hline FM-2 at Road 4 & & $(1.10 \pm 0.62) E-02$ & $(8.67 \pm 1.13) E-02$ & $(1.95 \pm 1.01) E-02$ \\
\hline FM-3A below F-Area effluent & & $(-1.26 \pm 1.77) E-02$ & $(0.84 \pm 1.08) E-02$ & $(0.68 \pm 1.68) \mathrm{E}-02$ \\
\hline Four Mile A-7A (beaver pond) & & $(1.35 \pm 0.13) E-01$ & $(9.14 \pm 1.72) \mathrm{E}-02$ & $(6.18 \pm 0.36) E-01$ \\
\hline $\begin{array}{l}\text { Four Mile Creek } \\
\text { Swamp discharge }\end{array}$ & & $(1.05 \pm 0.09) E-01$ & $(7.77 \pm 0.90) E-02$ & $(1.74 \pm 1.18) E-02$ \\
\hline Four Mile Creek at Road A-7 & & $(5.66 \pm 0.73) E-02$ & $(-8.07 \pm 9.78) E-03$ & $(6.29 \pm 1.53) E-02$ \\
\hline L3R-1A at Road B & & $(0.86 \pm 1.06) E-02$ & $(-1.89 \pm 0.96) \mathrm{E}-02$ & $(2.01 \pm 1.70) \mathrm{E}-02$ \\
\hline Pen Branch Swamp discharge & & $(-4.69 \pm 3.23) E-02$ & $(5.85 \pm 3.48) \mathrm{E}-02$ & $(5.85 \pm 3.91) E-02$ \\
\hline R-Area downstream of R-1 & & $(1.65 \pm 0.63) E-02$ & $(1.55 \pm 0.14) E-01$ & $(5.14 \pm 0.26) E-01$ \\
\hline SC-2A 1 mile above Road B & & $(0.37 \pm 1.16) E-02$ & $(0.94 \pm 1.02) E-02$ & $(3.22 \pm 0.69) \mathrm{E}-02$ \\
\hline SC-4 Steel Creek at Road A & & $(7.69 \pm 0.92) E-02$ & $(2.30 \pm 0.19) E-01$ & $(7.91 \pm 1.28) \mathrm{E}-02$ \\
\hline TB-4A & & $(1.12 \pm 0.71) E-02$ & $(3.16 \pm 1.37) E-02$ & a \\
\hline TB-4B & & $(-1.39 \pm 1.49) \mathrm{E}-02$ & $(0.15 \pm 1.35) E-02$ & a \\
\hline TB-5 near Road C & & $(0.45 \pm 1.20) E-02$ & $(4.38 \pm 2.85) E-02$ & $(-1.59 \pm 1.81) \mathrm{E}-02$ \\
\hline Tinker Creek 1 & & $(-1.14 \pm 1.11) \mathrm{E}-02$ & $(-0.88 \pm 1.02) E-02$ & $(-2.19 \pm 2.96) E-02$ \\
\hline $\begin{array}{l}\text { U3R-1A Treadway Bridge } \\
\text { Road 8-1 }\end{array}$ & & $(0.22 \pm 1.55) E-02$ & $(-0.09 \pm 1.53) E-02$ & $(-0.04 \pm 2.48) E-02$ \\
\hline \multicolumn{5}{|l|}{ Cs-137, pCi/g (dry weight) } \\
\hline Savannah River & River Mile & & & \\
\hline Highway 301 & 118.7 & $(5.13 \pm 0.37) E-01$ & $(3.63 \pm 0.25) E-01$ & $(8.57 \pm 2.37) \mathrm{E}-02$ \\
\hline Lower 3 Runs Mouth & 129.0 & $(1.24 \pm 0.06) E+00$ & $(1.85 \pm 1.68) E-02$ & $(1.17 \pm 0.21) \mathrm{E}-01$ \\
\hline Below Little Hell Landing & 134.0 & $(2.10 \pm 1.17) \mathrm{E}-02$ & $(6.16 \pm 2.20) E-02$ & $(4.20 \pm 1.51) \mathrm{E}-02$ \\
\hline Below Four Mile Creek & 150.0 & $(-2.19 \pm 8.79) E-03$ & $(2.23 \pm 1.43) \mathrm{E}-02$ & $(1.04 \pm 1.94) E-02$ \\
\hline R-3A above Vogtle & 151.0 & $(1.82 \pm 2.32) E-02$ & $(1.18 \pm 1.39) \mathrm{E}-02$ & $(2.77 \pm 0.33) \mathrm{E}-01$ \\
\hline Beaver Dam Creek Mouth & 152.1 & $(7.42 \pm 1.70) \mathrm{E}-02$ & $(3.02 \pm 2.09) E-02$ & $(7.55 \pm 3.03) E-02$ \\
\hline
\end{tabular}

a Sampling location discontinued in 1999 
Table 29

Radioactivity in River and Stream Sediment

Page 2 of 4

\begin{tabular}{|c|c|c|c|c|}
\hline Location & & 1997 & 1998 & 1999 \\
\hline Upper 3 Runs Mouth & 157.2 & $(1.97 \pm 0.23) \mathrm{E}-01$ & $(2.15 \pm 0.16) E+00$ & $(3.77 \pm 0.43) \mathrm{E}-01$ \\
\hline Demier Landing & 160.0 & $(2.85 \pm 1.02) E-02$ & $(-0.11 \pm 1.51) E-02$ & $(5.90 \pm 2.56) \mathrm{E}-02$ \\
\hline \multicolumn{5}{|l|}{ SRS Streams } \\
\hline FM-2 at Road 4 & & $(6.90 \pm 0.22) E+00$ & $(3.13 \pm 0.08) E+01$ & $(4.08 \pm 0.15) E+01$ \\
\hline FM-3A below F-Area effluent & & $(6.24 \pm 0.50) E-01$ & $(4.29 \pm 0.36) E-01$ & $(1.19 \pm 0.08) E+00$ \\
\hline Four Mile A-7A (beaver pond) & & $(1.89 \pm 0.06) E+01$ & $(1.03 \pm 0.05) E+01$ & $(1.01 \pm 0.04) \mathrm{E}+02$ \\
\hline $\begin{array}{l}\text { Four Mile Creek } \\
\text { Swamp discharge }\end{array}$ & & $(2.62 \pm 0.09) E+00$ & $(1.74 \pm 0.06) E+00$ & $(6.70 \pm 0.48) E-01$ \\
\hline Four Mile Creek at Road A-7 & & $(6.12 \pm 0.20) E+00$ & $(1.66 \pm 0.09) E+00$ & $(1.15 \pm 0.06) E+01$ \\
\hline L3R-1A at Road B & & $(7.05 \pm 0.50) E-01$ & $(1.05 \pm 0.21) E-01$ & $(5.90 \pm 0.47) \mathrm{E}-01$ \\
\hline Pen Branch Swamp discharge & & $(1.47 \pm 0.11) E+00$ & $(2.13 \pm 0.14) E+00$ & $(2.04 \pm 0.15) E+00$ \\
\hline R-Area downstream of $R-1$ & & $(1.39 \pm 0.05) E+00$ & $(4.80 \pm 0.15) E+01$ & $(4.10 \pm 0.15) \mathrm{E}+02$ \\
\hline SC-2A 1 mile above Road B & & $(1.10 \pm 0.06) E+00$ & $(4.65 \pm 0.35) E-01$ & $(1.15 \pm 0.05) E+00$ \\
\hline SC-4 Steel Creek at Road A & & $(1.57 \pm 0.06) E+00$ & $(1.36 \pm 0.05) E+01$ & $(4.51 \pm 0.17) \mathrm{E}+00$ \\
\hline TB-4A & & $(1.97 \pm 1.14) E-02$ & $(8.90 \pm 3.31) E-02$ & a \\
\hline TB-4B & & $(4.55 \pm 1.84) E-02$ & $(-2.83 \pm 1.68) E-02$ & a \\
\hline TB-5 near Road C & & $(-1.32 \pm 1.54) E-02$ & $(1.67 \pm 4.41) E-02$ & $(2.63 \pm 0.32) E-01$ \\
\hline Tinker Creek 1 & & $(7.69 \pm 2.72) \mathrm{E}-02$ & $(7.68 \pm 2.61) E-02$ & $(5.26 \pm 0.63) E-01$ \\
\hline $\begin{array}{l}\text { U3R-1A Treadway Bridge } \\
\text { Road 8-1 }\end{array}$ & & $(8.54 \pm 3.63) E-02$ & $(1.45 \pm 0.25) E-01$ & $(2.85 \pm 0.44) \mathrm{E}-01$ \\
\hline \multicolumn{5}{|l|}{ Sr-89,90, pCi/g (dry weight) } \\
\hline Savannah River & River Mile & & & \\
\hline Highway 301 & 118.7 & $(2.92 \pm 1.38) E-02$ & $(2.93 \pm 2.05) E-02$ & $(2.64 \pm 1.75) E-02$ \\
\hline Lower 3 Runs Mouth & 129.0 & $(1.23 \pm 1.73) E-02$ & $(2.70 \pm 2.20) \mathrm{E}-02$ & $(6.96 \pm 2.68) E-02$ \\
\hline Below Little Hell Landing & 134.0 & $(0.35 \pm 2.03) E-02$ & $(1.00 \pm 2.06) E-02$ & $(9.19 \pm 2.88) E-02$ \\
\hline Below Four Mile Creek & 150.0 & $(3.32 \pm 2.29) E-02$ & $(1.56 \pm 2.14) \mathrm{E}-02$ & $(4.72 \pm 2.38) \mathrm{E}-02$ \\
\hline R-3A above Vogtle & 151.0 & $(2.81 \pm 2.09) E-02$ & $(-0.38 \pm 1.99) E-02$ & $(3.05 \pm 2.54) \mathrm{E}-02$ \\
\hline Beaver Dam Creek Mouth & 152.1 & $(0.92 \pm 1.98) E-02$ & $(1.89 \pm 2.11) \mathrm{E}-02$ & $(2.44 \pm 2.28) \mathrm{E}-02$ \\
\hline Upper 3 Runs Mouth & 157.2 & $(2.48 \pm 2.02) \mathrm{E}-02$ & $(9.67 \pm 3.45) \mathrm{E}-02$ & $(1.29 \pm 2.20) E-02$ \\
\hline Demier Landing & 160.0 & $(0.36 \pm 2.04) E-02$ & $(1.06 \pm 0.35) E-01$ & $(-0.44 \pm 2.02) E-02$ \\
\hline \multicolumn{5}{|l|}{ SRS Streams } \\
\hline FM-2 at Road 4 & & $(2.38 \pm 2.45) E-02$ & $(1.45 \pm 0.18) E-01$ & $(4.41 \pm 0.50) E-01$ \\
\hline FM-3A below F-Area effluent & & $(5.28 \pm 2.63) E-02$ & $(3.33 \pm 2.07) E-02$ & $(1.81 \pm 0.39) \mathrm{E}-01$ \\
\hline Four Mile A-7A (beaver pond) & & $(7.16 \pm 0.53) E-01$ & $(4.37 \pm 0.39) E-01$ & $(1.70 \pm 0.09) E+00$ \\
\hline $\begin{array}{l}\text { Four Mile Creek } \\
\text { Swamp discharge }\end{array}$ & & $(3.14 \pm 0.39) E-01$ & $(2.78 \pm 0.33) E-01$ & $(1.70 \pm 0.38) E-01$ \\
\hline Four Mile Creek at Road A-7 & & $(3.79 \pm 0.30) E-01$ & $(9.26 \pm 2.60) E-02$ & $(9.58 \pm 0.67) E-01$ \\
\hline
\end{tabular}

a Sampling location discontinued in 1999 
Table 29

Radioactivity in River and Stream Sediment

Page 3 of 4

\begin{tabular}{|c|c|c|c|c|}
\hline Location & & 1997 & 1998 & 1999 \\
\hline L3R-1A at Road B & & $(-2.19 \pm 2.13) E-02$ & $(3.88 \pm 2.95) E-02$ & $(1.32 \pm 0.36) \mathrm{E}-01$ \\
\hline Pen Branch Swamp discharge & & $(0.75 \pm 2.34) E-02$ & $(0.37 \pm 1.18) E-02$ & $(1.79 \pm 2.03) \mathrm{E}-02$ \\
\hline R-Area downstream of R-1 & & $(1.39 \pm 2.38) E-02$ & $(1.85 \pm 0.38) E-01$ & $(3.09 \pm 0.45) E-01$ \\
\hline SC-2A 1 mile above Road $B$ & & $(0.74 \pm 2.34) E-02$ & $(2.37 \pm 2.86) E-02$ & $(1.86 \pm 3.01) E-02$ \\
\hline SC-4 Steel Creek at Road A & & $(2.38 \pm 2.45) E-02$ & $(1.59 \pm 0.26) \mathrm{E}-01$ & $(3.96 \pm 3.12) E-02$ \\
\hline TB-4A & & $(1.06 \pm 1.67) E-02$ & $(2.60 \pm 2.89) \mathrm{E}-02$ & a \\
\hline TB-4B & & $(-1.20 \pm 2.19) E-02$ & $(0.44 \pm 2.74) \mathrm{E}-02$ & a \\
\hline TB-5 near Road C & & $(2.07 \pm 2.42) E-02$ & $(0.87 \pm 2.77) E-02$ & $(6.13 \pm 3.26) E-02$ \\
\hline Tinker Creek 1 & & $(-1.56 \pm 2.17) E-02$ & $(1.46 \pm 2.82) \mathrm{E}-02$ & $(2.38 \pm 2.41) \mathrm{E}-02$ \\
\hline $\begin{array}{l}\text { U3R-1A Treadway Bridge } \\
\text { Road 8-1 }\end{array}$ & & $(-0.85 \pm 2.22) E-02$ & $(1.46 \pm 2.82) E-02$ & $(1.23 \pm 2.95) \mathrm{E}-02$ \\
\hline \multicolumn{5}{|l|}{ U-235, pCi/g (dry weight) } \\
\hline \multicolumn{5}{|l|}{ SRS Streams } \\
\hline TB-4A & & $(1.42 \pm 0.07) \mathrm{E}+00$ & $(2.53 \pm 0.12) E+00$ & a \\
\hline TB-5 near Road C & & $(0.00 \pm 0.00) E+00$ & $(0.00 \pm 0.00) E+00$ & $(1.64 \pm 0.11) E+00$ \\
\hline \multicolumn{5}{|l|}{ Pu-238, pCi/g (dry weight) } \\
\hline Savannah River & River Mile & & & \\
\hline Highway 301 & 118.7 & $(-4.53 \pm 1.93) E-03$ & $(2.21 \pm 1.13) \mathrm{E}-03$ & $(0.27 \pm 1.13) E-03$ \\
\hline Lower 3 Runs Mouth & 129.0 & $(6.15 \pm 8.22) \mathrm{E}-04$ & $(1.87 \pm 0.94) E-03$ & $(0.79 \pm 2.18) E-03$ \\
\hline Below Little Hell Landing & 134.0 & $(-2.75 \pm 5.66) E-04$ & $(-0.13 \pm 1.64) E-03$ & $(2.63 \pm 9.03) E-04$ \\
\hline Below Four Mile Creek & 150.0 & $(6.87 \pm 9.02) E-04$ & $(4.55 \pm 1.30) E-03$ & $(0.86 \pm 1.83) E-03$ \\
\hline R-3A above Vogtle & 151.0 & $(2.80 \pm 0.98) E-03$ & $(4.55 \pm 6.85) E-04$ & $(-1.22 \pm 0.56) E-03$ \\
\hline Beaver Dam Creek Mouth & 152.1 & $(0.06 \pm 3.21) E-04$ & $(1.14 \pm 0.58) E-03$ & $(-0.19 \pm 5.21) E-04$ \\
\hline Upper 3 Runs Mouth & 157.2 & $(3.27 \pm 1.21) \mathrm{E}-03$ & $(9.65 \pm 2.63) E-03$ & $(-0.31 \pm 1.16) E-03$ \\
\hline Demier Landing & 160.0 & $(-1.53 \pm 1.04) E-03$ & $(1.39 \pm 1.02) E-03$ & $(1.01 \pm 1.34) E-03$ \\
\hline \multicolumn{5}{|l|}{ SRS Streams } \\
\hline FM-2 at Road 4 & & $(8.53 \pm 1.04) E-02$ & $(7.34 \pm 0.49) \mathrm{E}-01$ & $(4.44 \pm 0.31) \mathrm{E}-01$ \\
\hline FM-3A below F-Area effluent & & $(7.58 \pm 2.27) \mathrm{E}-03$ & $(-5.20 \pm 7.32) \mathrm{E}-04$ & $(3.73 \pm 1.16) E-03$ \\
\hline Four Mile A-7A (beaver pond) & & $(2.60 \pm 0.27) \mathrm{E}-01$ & $(1.92 \pm 0.19) \mathrm{E}-01$ & $(1.60 \pm 0.10) E+00$ \\
\hline $\begin{array}{l}\text { Four Mile Creek } \\
\text { Swamp discharge }\end{array}$ & & $(1.13 \pm 0.24) \mathrm{E}-02$ & $(1.35 \pm 0.26) E-02$ & $(3.46 \pm 1.47) \mathrm{E}-03$ \\
\hline Four Mile Creek at Road A-7 & & $(9.99 \pm 1.14) \mathrm{E}-02$ & $(6.37 \pm 1.63) E-03$ & $(2.37 \pm 0.19) E-01$ \\
\hline L3R-1A at Road B & & $(-1.17 \pm 1.01) E-03$ & $(3.16 \pm 1.48) \mathrm{E}-03$ & $(0.12 \pm 1.05) E-03$ \\
\hline Pen Branch Swamp discharge & & $(1.17 \pm 1.92) E-03$ & $(6.82 \pm 1.44) E-03$ & $(4.15 \pm 1.26) E-03$ \\
\hline R-Area downstream of R-1 & & $(9.53 \pm 5.23) \mathrm{E}-04$ & $(2.76 \pm 0.42) \mathrm{E}-02$ & $(5.47 \pm 0.66) E-02$ \\
\hline SC-2A 1 mile above Road B & & $(4.65 \pm 1.70) E-03$ & $(1.21 \pm 1.14) E-03$ & $(1.72 \pm 0.81) \mathrm{E}-03$ \\
\hline
\end{tabular}

a Sampling location discontinued in 1999 


\section{Table 29}

\section{Radioactivity in River and Stream Sediment}

Page 4 of 4

\begin{tabular}{lrrc}
\hline Location & \multicolumn{1}{c}{1997} & \multicolumn{1}{c}{1998} & 1999 \\
\hline SC-4 Steel Creek at Road A & $(6.59 \pm 1.86) \mathrm{E}-03$ & $(7.88 \pm 0.62) \mathrm{E}-02$ & $(2.02 \pm 0.31) \mathrm{E}-02$ \\
TB-4A & $(-1.68 \pm 6.59) \mathrm{E}-04$ & $(0.61 \pm 1.00) \mathrm{E}-03$ & $\mathrm{a}$ \\
TB-4B & $(3.90 \pm 8.22) \mathrm{E}-04$ & $(7.89 \pm 6.50) \mathrm{E}-04$ & $\mathrm{a}$ \\
TB-5 near Road C & $(3.27 \pm 8.42) \mathrm{E}-04$ & $(-0.47 \pm 4.93) \mathrm{E}-04$ & $(2.21 \pm 1.10) \mathrm{E}-03$ \\
Tinker Creek 1 & $(-0.06 \pm 1.22) \mathrm{E}-03$ & $(7.11 \pm 8.05) \mathrm{E}-04$ & $(0.51 \pm 1.08) \mathrm{E}-03$ \\
U3R-1A Treadway Bridge & $(0.21 \pm 1.08) \mathrm{E}-03$ & $(5.74 \pm 7.14) \mathrm{E}-04$ & $(8.25 \pm 7.86) \mathrm{E}-04$ \\
Road 8-1 & & &
\end{tabular}

Pu-239, pCi/g (dry weight)

Savannah River

Highway 301

Lower 3 Runs Mouth

Below Little Hell Landing

Below Four Mile Creek

R-3A above Vogtle

Beaver Dam Creek Mouth

Upper 3 Runs Mouth

Demier Landing

\section{SRS Streams}

FM-2 at Road 4

FM-3A below F-Area effluent

Four Mile A-7A (beaver pond)

Four Mile Creek

Swamp discharge

Four Mile Creek at Road A-7

L3R-1A at Road B

Pen Branch Swamp discharge

R-Area downstream of $\mathrm{R}-1$

SC-2A 1 mile above Road B

SC-4 Steel Creek at Road A

TB-4A

TB-4B

TB-5 near Road C

Tinker Creek 1

U3R-1A Treadway Bridge

Road 8-1

\section{River Mile}

118.7

129.0

$(2.36 \pm 2.48) \mathrm{E}-03$

(1.36 \pm 1.08$) \mathrm{E}-03$

$(-1.71 \pm 1.53) E-03$

134.0

$(-3.85 \pm 9.78) \mathrm{E}-04$

(2.22 \pm 1.19$) \mathrm{E}-03$

$(-0.76 \pm 1.66) \mathrm{E}-03$

150.0

$(1.07 \pm 0.93) E-03$

$(0.52 \pm 1.27) \mathrm{E}-03$

$(-7.58 \pm 5.84) E-04$

151.0

$(-2.76 \pm 7.25) \mathrm{E}-04$

$(1.84 \pm 0.93) \mathrm{E}-03$

$(-1.21 \pm 0.96) E-03$

152.1

$(1.03 \pm 1.18) \mathrm{E}-03$

$(-3.94 \pm 7.06) \mathrm{E}-04$

$(-1.24 \pm 0.85) E-03$

157.2

(7.28 \pm 3.59$) E-04$

$(-2.03 \pm 3.51) \mathrm{E}-04$

$(1.77 \pm 1.25) \mathrm{E}-03$

$(3.97 \pm 1.95) \mathrm{E}-03$

$(3.33 \pm 0.51) \mathrm{E}-02$

$(1.69 \pm 1.62) \mathrm{E}-03$

160.0

$(-0.47 \pm 1.05) \mathrm{E}-03$

$(-4.62 \pm 5.61) \mathrm{E}-04$

$(1.32 \pm 1.43) \mathrm{E}-03$

$(3.13 \pm 2.11) \mathrm{E}-03$

$(3.03 \pm 0.37) \mathrm{E}-02$

$(4.27 \pm 0.49) \mathrm{E}-02$

$(1.50 \pm 0.33) E-02$

$(5.09 \pm 1.72) \mathrm{E}-03$

$(4.87 \pm 0.49) \mathrm{E}-02$

$(1.24 \pm 0.15) \mathrm{E}-01$

$(7.03 \pm 0.90) E-02$

$(7.74 \pm 0.47) \mathrm{E}-01$

(4.95 \pm 1.48$) E-03$

$(6.81 \pm 1.81) \mathrm{E}-03$

$(2.39 \pm 8.23) E-04$

$(5.58 \pm 0.75) \mathrm{E}-02$

$(6.63 \pm 1.66) \mathrm{E}-03$

$(1.14 \pm 0.11) \mathrm{E}-01$

$(-1.98 \pm 0.96) E-03$

$(-4.54 \pm 5.51) \mathrm{E}-04$

$(2.35 \pm 0.44) \mathrm{E}-02$

(3.22 \pm 0.35$) E-02$

$(-5.68 \pm 6.89) E-04$

$(1.08 \pm 0.55) \mathrm{E}-03$

$(8.56 \pm 0.87) \mathrm{E}-02$

$(1.46 \pm 0.28) \mathrm{E}-02$

(3.91 \pm 1.72$) E-03$

$(0.85 \pm 1.15) \mathrm{E}-03$

$(2.70 \pm 0.22) \mathrm{E}-01$

(3.19 \pm 1.44$) \mathrm{E}-03$

$(7.24 \pm 0.59) \mathrm{E}-02$

$(1.27 \pm 0.93) \mathrm{E}-03$

$(1.64 \pm 1.20) \mathrm{E}-03$

$(3.90 \pm 8.21) E-04$

$(3.00 \pm 5.46) E-04$

(3.80 \pm 1.19$) \mathrm{E}-03$

$(9.94 \pm 2.15) E-03$

$(-0.28 \pm 1.26) \mathrm{E}-03$

$(-1.48 \pm 3.86) E-04$

$(1.60 \pm 1.01) E-03$

$(4.23 \pm 1.58) E-03$ a

a

$(1.02 \pm 0.18) \mathrm{E}-02$

$(7.80 \pm 2.06) \mathrm{E}-03$

$(5.70 \pm 1.57) \mathrm{E}-03$

a Sampling location discontinued in 1999 


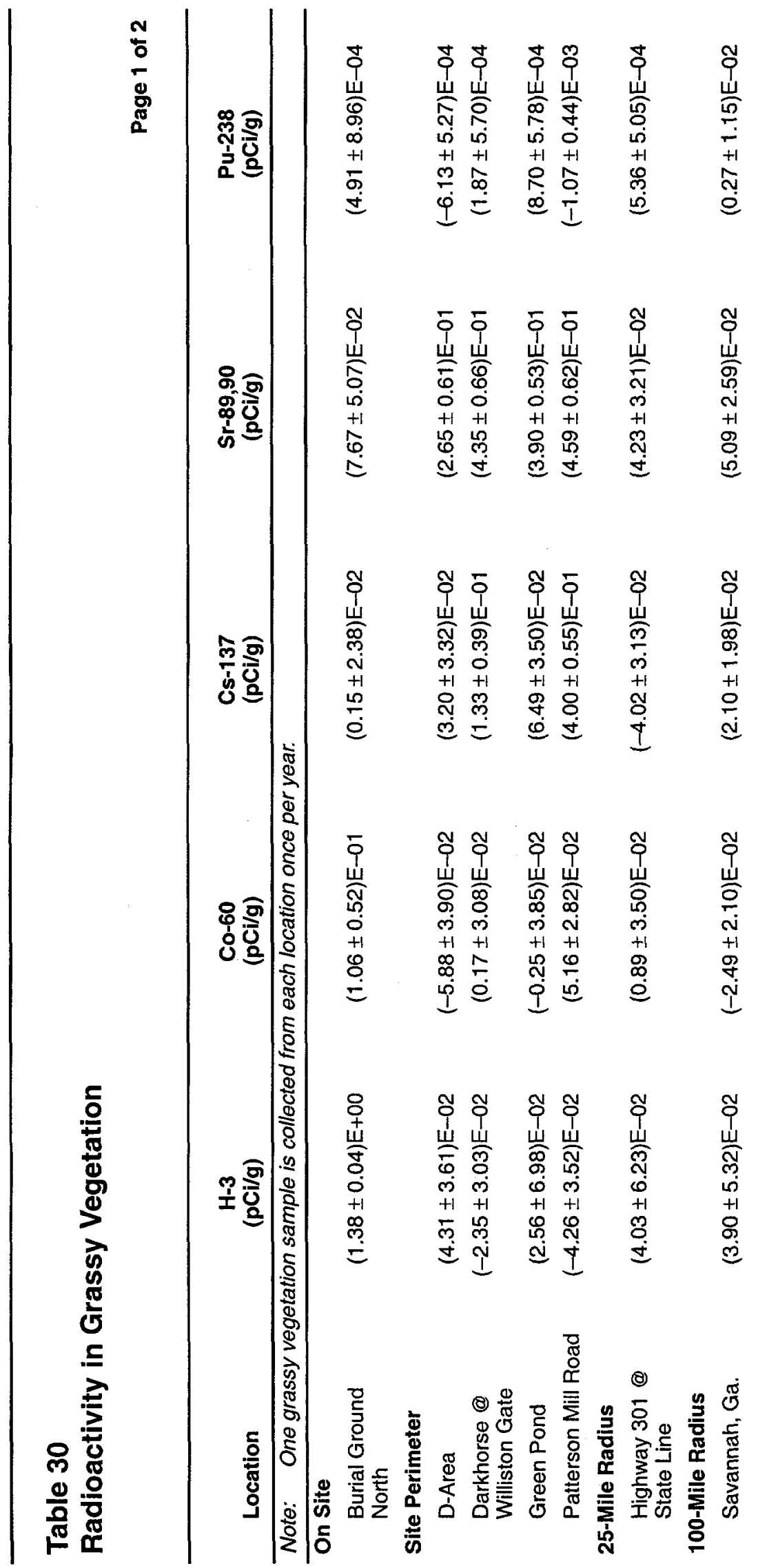




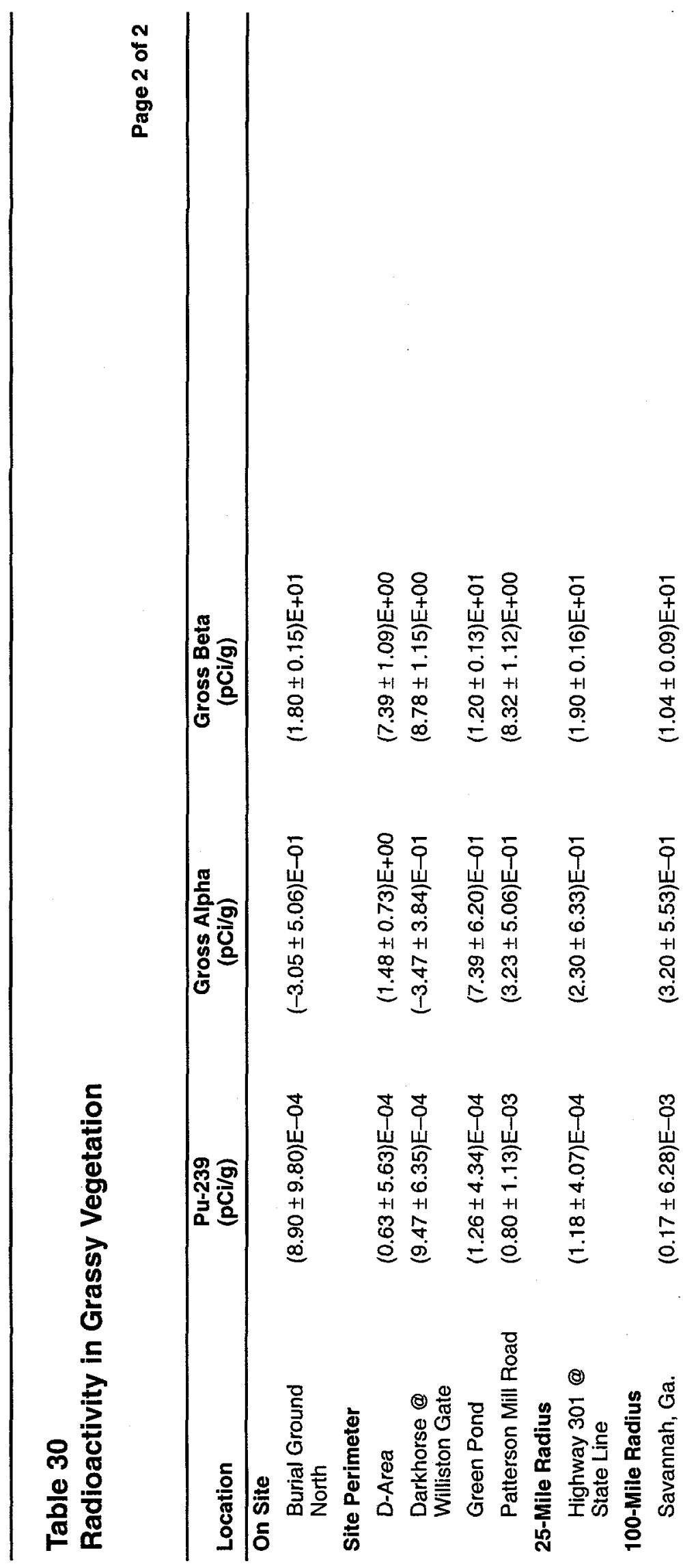


Table 31

Meteorological Data

Page 1 of 7

USNRC Computer Code-XOQDOQ, Version 2.0 43848 Wind Stats H-Area 60 minute

$62 \mathrm{M} \mathrm{92-96}$

Run Date: 98.077 (SRL 6/29/83 VERSION)

Stability from Sigma A

Joint Frequency Distribution of Wind Speed and Direction:

Atmospheric Stability Class A Extremely Unstable Conditions

\begin{tabular}{ccccccccc}
\hline $\begin{array}{c}\text { (M/S) } \\
\text { UMAX }\end{array}$ & N & NNE & NE & ENE & E & ESE & SE & SSE \\
\hline 2.00 & 0.445 & 0.420 & 0.449 & 0.436 & 0.440 & 0.379 & 0.331 & 0.376 \\
4.00 & 0.997 & 0.791 & 0.983 & 1.163 & 1.106 & 0.805 & 0.620 & 0.504 \\
6.00 & 0.787 & 0.465 & 0.292 & 0.244 & 0.160 & 0.132 & 0.146 & 0.096 \\
8.00 & 0.112 & 0.137 & 0.009 & 0.011 & 0.002 & 0.011 & 0.014 & 0.002 \\
12.00 & 0.011 & 0.007 & 0.000 & 0.000 & 0.000 & 0.002 & 0.000 & 0.000 \\
14.10 & 0.000 & 0.000 & 0.000 & 0.000 & 0.000 & 0.000 & 0.000 & 0.000 \\
TOTAL & $\mathbf{2 . 3 5 2}$ & 1.820 & $\mathbf{1 . 7 3 3}$ & $\mathbf{1 . 8 5 4}$ & $\mathbf{1 . 7 0 8}$ & $\mathbf{1 . 3 2 9}$ & $\mathbf{1 . 1 1 1}$ & $\mathbf{0 . 9 7 8}$
\end{tabular}

Joint Frequency Distribution of Wind Speed and Direction:

Atmospheric Stability Class A Extremely Unstable Conditions

\begin{tabular}{cccccccccc}
\hline $\begin{array}{c}\text { (M/S) } \\
\text { UMAX }\end{array}$ & S & SSW & SW & WSW & W & WNW & NW & NNW & TOTAL \\
\hline 2.00 & 0.376 & 0.369 & 0.497 & 0.536 & 0.399 & 0.406 & 0.301 & 0.381 & 6.541 \\
4.00 & 0.689 & 0.862 & 1.090 & 1.473 & 1.305 & 0.844 & 0.509 & 0.579 & 14.320 \\
6.00 & 0.192 & 0.221 & 0.324 & 0.335 & 0.367 & 0.308 & 0.141 & 0.267 & 4.477 \\
8.00 & 0.011 & 0.027 & 0.036 & 0.075 & 0.046 & 0.068 & 0.016 & 0.064 & 0.643 \\
12.00 & 0.000 & 0.002 & 0.002 & 0.009 & 0.021 & 0.023 & 0.000 & 0.009 & 0.087 \\
14.10 & 0.000 & 0.000 & 0.000 & 0.000 & 0.000 & 0.000 & 0.000 & 0.000 & 0.000 \\
TOTAL & $\mathbf{1 . 2 6 8}$ & $\mathbf{1 . 4 8 1}$ & $\mathbf{1 . 9 4 9}$ & $\mathbf{2 . 4 2 8}$ & $\mathbf{2 . 1 3 8}$ & $\mathbf{1 . 6 4 9}$ & $\mathbf{0 . 9 6 7}$ & $\mathbf{1 . 3 0 0}$ & $\mathbf{2 6 . 0 6 8}$
\end{tabular}




\section{Table 31}

Meteorological Data

Page 2 of 7

USNRC Computer Code-XOQDOQ, 43848 Wind Stats H-Area 60 minute
Version 2.0

62M 92-96
Run Date: 98.077

Stability from Sigma A
(SRL 6/29/83 VERSION)

Atmospheric Stability Class B

Joint Frequency Distribution of Wind Speed and Direction: Moderately Unstable Conditions

\begin{tabular}{ccccccccc}
\hline $\begin{array}{c}\text { (M/S) } \\
\text { UMAX }\end{array}$ & N & NNE & NE & ENE & E & ESE & SE & SSE \\
\hline 2.00 & 0.046 & 0.082 & 0.082 & 0.073 & 0.050 & 0.034 & 0.030 & 0.059 \\
4.00 & 0.312 & 0.504 & 0.602 & 0.682 & 0.461 & 0.342 & 0.219 & 0.223 \\
6.00 & 0.123 & 0.440 & 0.481 & 0.422 & 0.180 & 0.137 & 0.109 & 0.151 \\
8.00 & 0.002 & 0.116 & 0.048 & 0.023 & 0.018 & 0.018 & 0.025 & 0.025 \\
12.00 & 0.000 & 0.014 & 0.000 & 0.000 & 0.000 & 0.000 & 0.000 & 0.002 \\
14.10 & 0.000 & 0.000 & 0.000 & 0.000 & 0.000 & 0.000 & 0.000 & 0.000 \\
TOTAL & $\mathbf{0 . 4 8 3}$ & $\mathbf{1 . 1 5 6}$ & $\mathbf{1 . 2 1 3}$ & $\mathbf{1 . 2 0 0}$ & $\mathbf{0 . 7 0 9}$ & $\mathbf{0 . 5 3 1}$ & $\mathbf{0 . 3 8 3}$ & $\mathbf{0 . 4 6 0}$
\end{tabular}

Joint Frequency Distribution of Wind Speed and Direction: Moderately Unstable Conditions

\begin{tabular}{cccccccccc}
\hline $\begin{array}{c}\text { (M/S) } \\
\text { UMAX }\end{array}$ & S & SSW & SW & WSW & W & WNW & NW & NNW & TOTAL \\
\hline 2.00 & 0.048 & 0.071 & 0.068 & 0.089 & 0.032 & 0.062 & 0.064 & 0.052 & 0.942 \\
4.00 & 0.342 & 0.303 & 0.461 & 0.584 & 0.395 & 0.344 & 0.176 & 0.208 & 6.158 \\
6.00 & 0.205 & 0.265 & 0.395 & 0.639 & 0.477 & 0.315 & 0.123 & 0.123 & 4.584 \\
8.00 & 0.014 & 0.046 & 0.132 & 0.189 & 0.353 & 0.246 & 0.043 & 0.009 & 1.309 \\
12.00 & 0.000 & 0.002 & 0.018 & 0.055 & 0.105 & 0.096 & 0.025 & 0.005 & 0.322 \\
14.10 & 0.000 & 0.000 & 0.000 & 0.000 & 0.000 & 0.000 & 0.000 & 0.000 & 0.000 \\
TOTAL & $\mathbf{0 . 6 0 9}$ & $\mathbf{0 . 6 8 7}$ & $\mathbf{1 . 0 7 4}$ & $\mathbf{1 . 5 5 6}$ & $\mathbf{1 . 3 6 2}$ & $\mathbf{1 . 0 6 3}$ & $\mathbf{0 . 4 3 1}$ & $\mathbf{0 . 3 9 7}$ & $\mathbf{1 3 . 3 1 5}$
\end{tabular}




\section{Table 31}

Meteorological Data

Page 3 of 7

USNRC Computer Code-XOQDOQ, 43848 Wind Stats H-Area 60 minute
Version 2.0

62M 92-96
Run Date: 98.077

Stability from Sigma A

(SRL 6/29/83 VERSION)

Atmospheric Stability Class C

Joint Frequency Distribution of Wind Speed and Direction: Slightly Unstable Conditions

\begin{tabular}{ccccccccc}
\hline $\begin{array}{c}\text { (M/S) } \\
\text { UMAX }\end{array}$ & N & NNE & NE & ENE & E & ESE & SE & SSE \\
\hline 2.00 & 0.039 & 0.046 & 0.052 & 0.046 & 0.027 & 0.043 & 0.057 & 0.071 \\
4.00 & 0.198 & 0.600 & 1.234 & 0.842 & 0.618 & 0.360 & 0.342 & 0.388 \\
6.00 & 0.084 & 0.579 & 1.070 & 0.607 & 0.426 & 0.326 & 0.315 & 0.360 \\
8.00 & 0.000 & 0.091 & 0.287 & 0.114 & 0.064 & 0.052 & 0.114 & 0.155 \\
12.00 & 0.000 & 0.009 & 0.007 & 0.011 & 0.007 & 0.007 & 0.014 & 0.048 \\
14.10 & 0.000 & 0.000 & 0.000 & 0.000 & 0.000 & 0.000 & 0.000 & 0.000 \\
TOTAL & $\mathbf{0 . 3 2 1}$ & $\mathbf{1 . 3 2 5}$ & $\mathbf{2 . 6 5 0}$ & $\mathbf{1 . 6 2 0}$ & $\mathbf{1 . 1 4 2}$ & $\mathbf{0 . 7 8 8}$ & $\mathbf{0 . 8 4 2}$ & $\mathbf{1 . 0 2 2}$
\end{tabular}

Joint Frequency Distribution of Wind Speed and Direction: Slightly Unstable Conditions

\begin{tabular}{cccccccccc}
\hline $\begin{array}{c}\text { (M/S) } \\
\text { UMAX }\end{array}$ & S & SSW & SW & WSW & W & WNW & NW & NNW & TOTAL \\
\hline 2.00 & 0.055 & 0.046 & 0.041 & 0.036 & 0.034 & 0.034 & 0.052 & 0.039 & 0.718 \\
4.00 & 0.486 & 0.556 & 0.618 & 0.725 & 0.543 & 0.422 & 0.251 & 0.239 & 8.422 \\
6.00 & 0.411 & 0.447 & 0.607 & 0.627 & 0.666 & 0.598 & 0.230 & 0.064 & 7.417 \\
8.00 & 0.107 & 0.217 & 0.281 & 0.347 & 0.536 & 0.426 & 0.082 & 0.007 & 2.880 \\
12.00 & 0.041 & 0.062 & 0.062 & 0.105 & 0.210 & 0.148 & 0.021 & 0.002 & 0.753 \\
14.10 & 0.000 & 0.000 & 0.000 & 0.002 & 0.005 & 0.000 & 0.000 & 0.000 & 0.007 \\
TOTAL & 1.100 & $\mathbf{1 . 3 2 8}$ & $\mathbf{1 . 6 0 9}$ & $\mathbf{1 . 8 4 2}$ & $\mathbf{1 . 9 9 4}$ & $\mathbf{1 . 6 2 8}$ & $\mathbf{0 . 6 3 6}$ & $\mathbf{0 . 3 5 1}$ & $\mathbf{2 0 . 1 9 7}$
\end{tabular}


Table 31

Meteorological Data

Page 4 of 7

USNRC Computer Code-XOQDOQ,

Version 2.0

Run Date: 98.077

43848 Wind Stats H-Area 60 minute

$62 \mathrm{M} 92-96$

Stability from Sigma A

(SRL 6/29/83 VERSION)

Joint Frequency Distribution of Wind Speed and Direction:

Atmospheric Stability Class D Neutral Conditions

\begin{tabular}{ccccccccc}
\hline $\begin{array}{c}\text { (M/S) } \\
\text { UMAX }\end{array}$ & N & NNE & NE & ENE & E & ESE & SE & SSE \\
\hline 2.00 & 0.009 & 0.032 & 0.027 & 0.032 & 0.036 & 0.034 & 0.036 & 0.048 \\
4.00 & 0.109 & 0.392 & 1.168 & 0.728 & 0.529 & 0.440 & 0.541 & 0.639 \\
6.00 & 0.073 & 0.445 & 1.122 & 0.618 & 0.559 & 0.534 & 0.661 & 1.008 \\
8.00 & 0.000 & 0.034 & 0.080 & 0.034 & 0.023 & 0.025 & 0.039 & 0.296 \\
12.00 & 0.002 & 0.000 & 0.005 & 0.002 & 0.000 & 0.000 & 0.005 & 0.039 \\
14.10 & 0.000 & 0.000 & 0.000 & 0.000 & 0.000 & 0.000 & 0.000 & 0.000 \\
TOTAL & $\mathbf{0 . 1 9 3}$ & $\mathbf{0 . 9 0 3}$ & $\mathbf{2 . 4 0 2}$ & $\mathbf{1 . 4 1 4}$ & $\mathbf{1 . 1 4 7}$ & $\mathbf{1 . 0 3 3}$ & $\mathbf{1 . 2 8 2}$ & $\mathbf{2 . 0 3 0}$
\end{tabular}

Joint Frequency Distribution of Wind Speed and Direction:

Atmospheric Stability Class D Neutral Conditions

\begin{tabular}{cccccccccc}
\hline $\begin{array}{c}\text { (M/S) } \\
\text { UMAX }\end{array}$ & S & SSW & SW & WSW & W & WNW & NW & NNW & TOTAL \\
\hline 2.00 & 0.016 & 0.048 & 0.050 & 0.039 & 0.014 & 0.011 & 0.039 & 0.027 & 0.499 \\
4.00 & 0.759 & 0.789 & 0.716 & 0.730 & 0.600 & 0.483 & 0.381 & 0.294 & 9.298 \\
6.00 & 1.259 & 0.737 & 0.949 & 0.917 & 0.741 & 0.705 & 0.358 & 0.103 & 10.787 \\
8.00 & 0.242 & 0.148 & 0.135 & 0.068 & 0.052 & 0.039 & 0.030 & 0.002 & 1.247 \\
12.00 & 0.032 & 0.023 & 0.018 & 0.002 & 0.027 & 0.005 & 0.000 & 0.000 & 0.160 \\
14.10 & 0.000 & 0.000 & 0.000 & 0.000 & 0.023 & 0.005 & 0.000 & 0.000 & 0.027 \\
TOTAL & $\mathbf{2 . 3 0 8}$ & $\mathbf{1 . 7 4 5}$ & $\mathbf{1 . 8 6 8}$ & $\mathbf{1 . 7 5 6}$ & $\mathbf{1 . 4 5 7}$ & $\mathbf{1 . 2 4 8}$ & $\mathbf{0 . 8 0 8}$ & $\mathbf{0 . 4 2 6}$ & $\mathbf{2 2 . 0 1 8}$
\end{tabular}


Table 31

Meteorological Data

Page 5 of 7

USNRC Computer Code-XOQDOQ, 43848 Wind Stats H-Area 60 minute
Version 2.0

62M 92-96
Run Date: 98.077 (SR
Stability from Sigma A

Run Date: $98.077 \quad$ (SR
Stability from Sigma A

Run Date: 98.077 (SRL
Stability from Sigma A
(SRL 6/29/83 VERSION) 


\section{Table 31}

Meteorological Data

Page 6 of 7

USNRC Computer Code-XOQDOQ,

Version 2.0

Run Date: 98.077

(SRL 6/29/83 VERSION) 43848 Wind Stats H-Area 60 minute

Joint Frequency Distribution of Wind Speed and Direction:

Atmospheric Stability Class F

\begin{tabular}{ccccccccc}
\hline $\begin{array}{c}\text { (M/S) } \\
\text { UMAX }\end{array}$ & N & NNE & NE & ENE & E & ESE & SE & SSE \\
\hline 2.00 & 0.002 & 0.002 & 0.000 & 0.000 & 0.000 & 0.000 & 0.002 & 0.002 \\
4.00 & 0.007 & 0.064 & 0.084 & 0.018 & 0.016 & 0.036 & 0.057 & 0.050 \\
6.00 & 0.016 & 0.212 & 0.130 & 0.100 & 0.096 & 0.178 & 0.155 & 0.155 \\
8.00 & 0.000 & 0.014 & 0.002 & 0.000 & 0.002 & 0.005 & 0.002 & 0.005 \\
12.00 & 0.000 & 0.000 & 0.000 & 0.000 & 0.000 & 0.000 & 0.000 & 0.000 \\
14.10 & 0.000 & 0.000 & 0.000 & 0.000 & 0.000 & 0.000 & 0.000 & 0.000 \\
TOTAL & $\mathbf{0 . 0 2 5}$ & $\mathbf{0 . 2 9 2}$ & $\mathbf{0 . 2 1 6}$ & $\mathbf{0 . 1 1 8}$ & $\mathbf{0 . 1 1 4}$ & $\mathbf{0 . 2 1 9}$ & $\mathbf{0 . 2 1 6}$ & $\mathbf{0 . 2 1 2}$
\end{tabular}

Joint Frequency Distribution of Wind Speed and Direction: Moderately Stable Conditions

\begin{tabular}{cccccccccc}
\hline $\begin{array}{c}\text { (M/S) } \\
\text { UMAX }\end{array}$ & S & ssW & SW & WSW & W & WNW & NW & NNW & TOTAL \\
\hline 2.00 & 0.002 & 0.000 & 0.002 & 0.009 & 0.000 & 0.000 & 0.000 & 0.000 & 0.023 \\
4.00 & 0.087 & 0.071 & 0.062 & 0.103 & 0.021 & 0.027 & 0.041 & 0.068 & 0.812 \\
6.00 & 0.171 & 0.135 & 0.182 & 0.235 & 0.059 & 0.048 & 0.023 & 0.030 & 1.925 \\
8.00 & 0.002 & 0.002 & 0.005 & 0.009 & 0.000 & 0.000 & 0.005 & 0.000 & 0.052 \\
12.00 & 0.000 & 0.000 & 0.000 & 0.000 & 0.000 & 0.000 & 0.000 & 0.000 & 0.000 \\
14.10 & 0.000 & 0.000 & 0.000 & 0.000 & 0.000 & 0.000 & 0.000 & 0.000 & 0.000 \\
TOTAL & $\mathbf{0 . 2 6 2}$ & $\mathbf{0 . 2 0 8}$ & $\mathbf{0 . 2 5 1}$ & $\mathbf{0 . 3 5 6}$ & $\mathbf{0 . 0 8 0}$ & $\mathbf{0 . 0 7 5}$ & $\mathbf{0 . 0 6 9}$ & $\mathbf{0 . 0 9 8}$ & $\mathbf{2 . 8 1 2}$
\end{tabular}




\section{Table 31}

Meteorological Data

Page 7 of 7

USNRC Computer Code-XOQDOQ, 43848 Wind Stats H-Area 60 minute

Version 2.0

Run Date: 98.077

(SRL 6/29/83 VERSION)

62M 92-96 Stability from Sigma A

Joint Frequency Distribution of Wind Speed and Direction:

Atmospheric Stability Class G

\begin{tabular}{ccccccccc}
\hline $\begin{array}{c}\text { (M/S) } \\
\text { UMAX }\end{array}$ & N & NNE & NE & ENE & E & ESE & SE & SSE \\
\hline 2.00 & 0.000 & 0.000 & 0.000 & 0.000 & 0.000 & 0.000 & 0.000 & 0.000 \\
4.00 & 0.000 & 0.007 & 0.002 & 0.000 & 0.000 & 0.002 & 0.000 & 0.000 \\
6.00 & 0.002 & 0.014 & 0.009 & 0.002 & 0.002 & 0.014 & 0.014 & 0.011 \\
8.00 & 0.000 & 0.000 & 0.000 & 0.000 & 0.000 & 0.000 & 0.002 & 0.000 \\
12.00 & 0.000 & 0.000 & 0.000 & 0.000 & 0.000 & 0.000 & 0.000 & 0.000 \\
14.10 & 0.000 & 0.000 & 0.000 & 0.000 & 0.000 & 0.000 & 0.000 & 0.000 \\
TOTAL & $\mathbf{0 . 0 0 2}$ & $\mathbf{0 . 0 2 1}$ & $\mathbf{0 . 0 1 1}$ & $\mathbf{0 . 0 0 2}$ & $\mathbf{0 . 0 0 2}$ & $\mathbf{0 . 0 1 6}$ & $\mathbf{0 . 0 1 6}$ & $\mathbf{0 . 0 1 1}$
\end{tabular}

Joint Frequency Distribution of Wind Speed and Direction:

Atmospheric Stability Class G Extremely Stable Conditions

\begin{tabular}{cccccccccc}
\hline $\begin{array}{c}\text { (M/S) } \\
\text { UMAX }\end{array}$ & S & SSW & SW & WSW & W & WNW & NW & NNW & TOTAL \\
\hline 2.00 & 0.000 & 0.000 & 0.000 & 0.000 & 0.000 & 0.000 & 0.000 & 0.000 & 0.000 \\
4.00 & 0.007 & 0.002 & 0.002 & 0.002 & 0.002 & 0.002 & 0.000 & 0.005 & 0.034 \\
6.00 & 0.011 & 0.014 & 0.007 & 0.016 & 0.002 & 0.000 & 0.002 & 0.002 & 0.123 \\
8.00 & 0.000 & 0.000 & 0.000 & 0.000 & 0.000 & 0.000 & 0.000 & 0.000 & 0.002 \\
12.00 & 0.000 & 0.000 & 0.000 & 0.000 & 0.000 & 0.000 & 0.000 & 0.000 & 0.000 \\
14.10 & 0.000 & 0.000 & 0.000 & 0.000 & 0.000 & 0.000 & 0.000 & 0.000 & 0.000 \\
TOTAL & $\mathbf{0 . 0 1 8}$ & $\mathbf{0 . 0 1 6}$ & $\mathbf{0 . 0 0 9}$ & $\mathbf{0 . 0 1 8}$ & $\mathbf{0 . 0 0 4}$ & $\mathbf{0 . 0 0 2}$ & $\mathbf{0 . 0 0 2}$ & $\mathbf{0 . 0 0 7}$ & $\mathbf{0 . 1 5 9}$
\end{tabular}


Table 32

80-km-Radius (50-Mile) Population Distribution Around SRS (1990 Census)

Page 1 of 1

\begin{tabular}{|c|c|c|c|c|c|c|}
\hline Dir (Miles) ${ }^{\mathrm{a}}$ & $5-10$ & $10-20$ & $20-30$ & $30-40$ & $40-50$ & Total \\
\hline $\mathbf{N}$ & $2.600 \mathrm{E}+01$ & $5.321 E+03$ & $1.002 E+04$ & $5.067 \mathrm{E}+03$ & $1.221 E+04$ & $3.264 \mathrm{E}+04$ \\
\hline NNE & $6.000 E+00$ & $1.320 \mathrm{E}+03$ & $2.066 \mathrm{E}+03$ & $4.445 E+03$ & $1.437 E+04$ & $2.220 \mathrm{E}+04$ \\
\hline NE & $1.000 E+00$ & $2.945 E+03$ & $2.928 E+03$ & $5.269 E+03$ & $1.020 E+04$ & $2.134 \mathrm{E}+04$ \\
\hline ENE & $2.700 E+01$ & $3.126 \mathrm{E}+03$ & $4.483 E+03$ & $5.337 \mathrm{E}+03$ & 4.077E+04 & $5.375 E+04$ \\
\hline $\mathbf{E}$ & $1.550 E+02$ & $6.743 E+03$ & $5.305 E+03$ & $8.812 E+03$ & $4.334 E+03$ & $2.535 E+04$ \\
\hline ESE & $3.600 E+01$ & $1.556 \mathrm{E}+03$ & $1.931 E+03$ & $2.711 E+03$ & $3.253 E+03$ & $9.487 \mathrm{E}+03$ \\
\hline SE & $2.600 E+01$ & $5.470 E+02$ & $6.511 E+03$ & $6.685 E+03$ & $8.577 E+03$ & $2.235 E+04$ \\
\hline SSE & $4.000 E+01$ & $3.910 E+02$ & $7.690 E+02$ & $1.356 \mathrm{E}+03$ & $2.539 E+03$ & $5.095 E+03$ \\
\hline $\mathbf{s}$ & $1.000 E+00$ & $5.580 E+02$ & $1.332 E+03$ & $7.251 E+03$ & $3.335 E+03$ & $1.248 \mathrm{E}+04$ \\
\hline ssw & $2.000 E+00$ & $8.970 E+02$ & $2.008 E+03$ & $4.181 E+03$ & $2.944 E+03$ & $1.003 E+04$ \\
\hline sw & $1.700 E+01$ & $9.440 E+02$ & $2.240 E+03$ & $2.606 \mathrm{E}+03$ & $2.660 E+03$ & $8.467 E+03$ \\
\hline wsw & $6.000 E+01$ & $1.103 E+03$ & $7.112 E+03$ & $2.285 E+03$ & $5.818 E+03$ & $1.638 E+04$ \\
\hline$w$ & $5.500 E+01$ & $3.314 \mathrm{E}+03$ & $7.941 E+03$ & $7.994 E+03$ & $6.780 E+03$ & $2.608 \mathrm{E}+04$ \\
\hline WNW & $4.490 \mathrm{E}+02$ & $3.342 E+03$ & $1.069 \mathrm{E}+05$ & $5.031 E+04$ & $1.155 E+04$ & $1.725 \mathrm{E}+05$ \\
\hline NW & $2.710 E+02$ & $5.899 \mathrm{E}+03$ & $8.793 E+04$ & $2.657 E+04$ & $3.025 E+03$ & $1.237 \mathrm{E}+05$ \\
\hline NNW & $3.63 \mathrm{E}+02$ & $1.803 E+04$ & $2.716 E+04$ & $6.665 E+03$ & $6.079 E+03$ & $5.830 E+04$ \\
\hline Total & $1.535 E+03$ & $5.603 E+04$ & $2.766 \mathrm{E}+05$ & $1.475 E+05$ & $1.384 E+05$ & $6.201 E+05$ \\
\hline
\end{tabular}

a No one lives within 5 miles of the center of SRS because all areas within this distance are inside the site boundary. 
Table 33

80-km-Radius (50-Mile) Milk, Meat, and Vegetation Production Around SRS as of 1991

Page 1 of 2

Site Annual Milk Production (L)

\begin{tabular}{|c|c|c|c|c|c|c|c|}
\hline Dir (miles) & $0-5$ & $5-10$ & $10-20$ & $20-30$ & $30-40$ & $40-50$ & Total \\
\hline$N$ & 0.0 & 0.0 & $4.200 \mathrm{E}+04$ & $6.900 \mathrm{E}+04$ & $1.000 E+06$ & $5.300 E+06$ & $6.411 E+06$ \\
\hline NNE & 0.0 & 0.0 & $4.200 \mathrm{E}+04$ & $6.900 \mathrm{E}+04$ & $2.100 \mathrm{E}+05$ & $5.000 E+05$ & $8.210 E+05$ \\
\hline NE & 0.0 & 0.0 & $3.200 \mathrm{E}+04$ & $1.000 \mathrm{E}+06$ & $2.700 E+06$ & $2.000 E+06$ & $5.732 \mathrm{E}+06$ \\
\hline ENE & 0.0 & 0.0 & $2.500 E+04$ & $1.200 E+06$ & $4.400 E+06$ & $5.200 E+06$ & $1.083 E+07$ \\
\hline E & 0.0 & 0.0 & $2.500 E+04$ & $1.400 \mathrm{E}+06$ & $3.900 E+06$ & $4.900 \mathrm{E}+06$ & $1.023 \mathrm{E}+07$ \\
\hline ESE & 0.0 & 0.0 & $2.500 E+04$ & $5.600 \mathrm{E}+05$ & $3.000 \mathrm{E}+04$ & $4.900 \mathrm{E}+05$ & $1.105 E+06$ \\
\hline SE & 0.0 & 0.0 & $2.500 E+03$ & $0.000 E+00$ & $0.000 E+00$ & $0.000 E+00$ & $2.500 E+03$ \\
\hline SSE & 0.0 & 0.0 & $4.800 E+05$ & $8.600 E+05$ & $1.200 E+06$ & $1.200 \mathrm{E}+06$ & $3.740 E+06$ \\
\hline $\mathrm{S}$ & 0.0 & 0.0 & $1.000 E+06$ & $2.100 E+06$ & $3.000 E+06$ & $3.500 \mathrm{E}+06$ & $9.600 E+06$ \\
\hline SSW & 0.0 & 0.0 & $9.900 E+05$ & $3.800 \mathrm{E}+06$ & $7.400 \mathrm{E}+06$ & $7.600 \mathrm{E}+06$ & $1.979 E+07$ \\
\hline SW & 0.0 & 0.0 & $9.900 E+05$ & $2.200 E+06$ & $5.800 E+06$ & $4.800 E+06$ & $1.379 E+07$ \\
\hline WSW & 0.0 & 0.0 & $9.900 E+05$ & $1.700 E+06$ & $2.400 E+06$ & $3.500 \mathrm{E}+06$ & $8.590 E+06$ \\
\hline W & 0.0 & 0.0 & $6.700 E+05$ & $1.300 E+06$ & $2.200 \mathrm{E}+06$ & $3.600 E+06$ & $7.770 E+06$ \\
\hline WNW & 0.0 & 0.0 & $2.300 E+05$ & $1.100 E+06$ & $1.200 E+06$ & $2.000 E+06$ & $4.530 E+06$ \\
\hline NW & 0.0 & 0.0 & $4.200 E+04$ & $3.800 E+05$ & $1.400 E+06$ & $1.000 E+06$ & $2.822 \mathrm{E}+06$ \\
\hline NNW & 0.0 & 0.0 & $4.200 E+04$ & $6.000 E+04$ & $1.700 E+06$ & $3.400 E+06$ & $5.211 E+06$ \\
\hline Total & 0.0 & 0.0 & $5.62 E+06$ & 1.781E+07 & $3.954 E+07$ & 4.899E+07 & $1.110 E+08$ \\
\hline
\end{tabular}

Site Annual Meat Production (kg)

\begin{tabular}{lccccccc} 
Dir (miles) & $\mathbf{0 - 5}$ & $\mathbf{5 - 1 0}$ & $\mathbf{1 0 - 2 0}$ & $\mathbf{2 0 - 3 0}$ & $\mathbf{3 0 - 4 0}$ & $40-50$ & Total \\
\hline $\mathrm{N}$ & 0.0 & 0.0 & $5.300 \mathrm{E}+04$ & $\mathbf{8 . 8 0 0 \mathrm { E } + 0 4}$ & $2.500 \mathrm{E}+05$ & $9.800 \mathrm{E}+05$ & $1.371 \mathrm{E}+06$ \\
NNE & 0.0 & 0.0 & $5.300 \mathrm{E}+04$ & $\mathbf{8 . 8 0 0 E + 0 4}$ & $2.000 \mathrm{E}+05$ & $4.100 \mathrm{E}+05$ & $7.510 \mathrm{E}+05$ \\
NE & 0.0 & 0.0 & $7.100 \mathrm{E}+04$ & $1.700 \mathrm{E}+05$ & $3.500 \mathrm{E}+05$ & $4.500 \mathrm{E}+05$ & $1.041 \mathrm{E}+06$ \\
ENE & 0.0 & 0.0 & $8.300 \mathrm{E}+04$ & $2.000 \mathrm{E}+05$ & $4.600 \mathrm{E}+05$ & $5.700 \mathrm{E}+05$ & $1.313 \mathrm{E}+06$ \\
E & 0.0 & 0.0 & $8.300 \mathrm{E}+04$ & $1.900 \mathrm{E}+05$ & $3.400 \mathrm{E}+05$ & $5.100 \mathrm{E}+05$ & $1.123 \mathrm{E}+06$ \\
ESE & 0.0 & 0.0 & $8.300 \mathrm{E}+04$ & $1.900 \mathrm{E}+05$ & $2.200 \mathrm{E}+05$ & $2.500 \mathrm{E}+05$ & $7.430 \mathrm{E}+05$ \\
SE & 0.0 & 0.0 & $1.200 \mathrm{E}+05$ & $2.100 \mathrm{E}+05$ & $2.600 \mathrm{E}+05$ & $3.000 \mathrm{E}+05$ & $8.900 \mathrm{E}+05$ \\
SSE & 0.0 & 0.0 & $1.100 \mathrm{E}+05$ & $1.900 \mathrm{E}+05$ & $2.600 \mathrm{E}+05$ & $2.900 \mathrm{E}+05$ & $8.500 \mathrm{E}+05$ \\
S & 0.0 & 0.0 & $9.400 \mathrm{E}+04$ & $1.500 \mathrm{E}+05$ & $2.000 \mathrm{E}+05$ & $2.700 \mathrm{E}+05$ & $7.140 \mathrm{E}+05$ \\
SSW & 0.0 & 0.0 & $9.500 \mathrm{E}+04$ & $1.800 \mathrm{E}+05$ & $2.900 \mathrm{E}+05$ & $3.900 \mathrm{E}+05$ & $9.550 \mathrm{E}+05$ \\
SW & 0.0 & 0.0 & $9.500 \mathrm{E}+04$ & $1.700 \mathrm{E}+05$ & $2.700 \mathrm{E}+05$ & $3.200 \mathrm{E}+05$ & $8.550 \mathrm{E}+05$
\end{tabular}


Table 33

80-km-Radius (50-Mile) Milk, Meat, and Vegetation Production Around SRS as of 1991

Page 2 of 2

Site Annual Meat Production (kg), cont.

\begin{tabular}{|c|c|c|c|c|c|c|c|}
\hline Dir (miles) & $0-5$ & $5-10$ & $10-20$ & $20-30$ & $30-40$ & $40-50$ & Total \\
\hline WSW & 0.0 & 0.0 & $9.500 E+04$ & $1.600 E+05$ & $2.300 \mathrm{E}+05$ & $4.000 E+05$ & $8.850 E+05$ \\
\hline$w$ & 0.0 & 0.0 & $5.800 E+04$ & $1.000 E+05$ & $2.100 E+05$ & $4.100 E+05$ & $7.780 E+05$ \\
\hline WNW & 0.0 & 0.0 & $4.800 E+04$ & $6.200 E+04$ & $1.300 E+05$ & $2.900 \mathrm{E}+05$ & $5.300 E+05$ \\
\hline$N W$ & 0.0 & 0.0 & $5.800 \mathrm{E}+04$ & $8.000 E+04$ & $2.800 E+05$ & $2.700 E+05$ & $6.830 E+05$ \\
\hline NNW & 0.0 & 0.0 & $5.300 E+04$ & $8.800 E+04$ & $3.300 E+05$ & $6.200 \mathrm{E}+05$ & $1.091 E+06$ \\
\hline Total & 0.0 & 0.0 & $1.247 E+06$ & 2.316E+06 & $4.280 E+06$ & $6.730 E+06$ & $1.457 E+07$ \\
\hline
\end{tabular}

Site Annual Vegetation Production (kg)

\begin{tabular}{lccccccc} 
Dir (miles) & $\mathbf{0 - 5}$ & $\mathbf{5 - 1 0}$ & $\mathbf{1 0 - 2 0}$ & $\mathbf{2 0 - 3 0}$ & $30-40$ & $40-50$ & Total \\
\hline N & 0.0 & 0.0 & $3.600 \mathrm{E}+05$ & $6.000 \mathrm{E}+05$ & $8.400 \mathrm{E}+05$ & $8.700 \mathrm{E}+05$ & $2.670 \mathrm{E}+06$ \\
NNE & 0.0 & 0.0 & $3.600 \mathrm{E}+05$ & $6.000 \mathrm{E}+05$ & $5.100 \mathrm{E}+05$ & $6.300 \mathrm{E}+03$ & $1.476 \mathrm{E}+06$ \\
NE & 0.0 & 0.0 & $3.600 \mathrm{E}+05$ & $6.900 \mathrm{E}+05$ & $1.000 \mathrm{E}+06$ & $5.000 \mathrm{E}+05$ & $2.550 \mathrm{E}+06$ \\
ENE & 0.0 & 0.0 & $3.600 \mathrm{E}+05$ & $6.600 \mathrm{E}+05$ & $1.200 \mathrm{E}+06$ & $1.500 \mathrm{E}+06$ & $3.270 \mathrm{E}+06$ \\
E & 0.0 & 0.0 & $3.600 \mathrm{E}+05$ & $5.900 \mathrm{E}+05$ & $8.500 \mathrm{E}+05$ & $1.400 \mathrm{E}+06$ & $3.200 \mathrm{E}+06$ \\
ESE & 0.0 & 0.0 & $3.600 \mathrm{E}+05$ & $2.200 \mathrm{E}+06$ & $1.900 \mathrm{E}+06$ & $1.100 \mathrm{E}+06$ & $5.560 \mathrm{E}+06$ \\
SE & 0.0 & 0.0 & $2.500 \mathrm{E}+06$ & $4.500 \mathrm{E}+06$ & $3.000 \mathrm{E}+06$ & $1.100 \mathrm{E}+06$ & $1.110 \mathrm{E}+07$ \\
SSE & 0.0 & 0.0 & $1.700 \mathrm{E}+06$ & $2.900 \mathrm{E}+06$ & $3.600 \mathrm{E}+06$ & $1.100 \mathrm{E}+06$ & $9.300 \mathrm{E}+06$ \\
S & 0.0 & 0.0 & $7.200 \mathrm{E}+04$ & $5.400 \mathrm{E}+05$ & $8.400 \mathrm{E}+05$ & $9.700 \mathrm{E}+05$ & $2.422 \mathrm{E}+06$ \\
SSW & 0.0 & 0.0 & $3.500 \mathrm{E}+02$ & $1.200 \mathrm{E}+05$ & $2.500 \mathrm{E}+05$ & $1.100 \mathrm{E}+05$ & $4.804 \mathrm{E}+05$ \\
SW & 0.0 & 0.0 & $3.500 \mathrm{E}+02$ & $7.800 \mathrm{E}+02$ & $2.200 \mathrm{E}+03$ & $3.100 \mathrm{E}+05$ & $3.133 \mathrm{E}+05$ \\
WSW & 0.0 & 0.0 & $3.500 \mathrm{E}+02$ & $5.800 \mathrm{E}+02$ & $7.900 \mathrm{E}+03$ & $2.900 \mathrm{E}+03$ & $1.173 \mathrm{E}+04$ \\
W & 0.0 & 0.0 & $4.500 \mathrm{E}+04$ & $2.300 \mathrm{E}+04$ & $1.700 \mathrm{E}+04$ & $5.200 \mathrm{E}+04$ & $1.370 \mathrm{E}+05$ \\
WNW & 0.0 & 0.0 & $2.600 \mathrm{E}+05$ & $3.800 \mathrm{E}+04$ & $6.200 \mathrm{E}+04$ & $1.100 \mathrm{E}+06$ & $1.848 \mathrm{E}+06$ \\
NW & 0.0 & 0.0 & $3.600 \mathrm{E}+05$ & $4.300 \mathrm{E}+05$ & $8.400 \mathrm{E}+05$ & $1.100 \mathrm{E}+06$ & $2.730 \mathrm{E}+06$ \\
NNW & 0.0 & 0.0 & $3.600 \mathrm{E}+05$ & $6.000 \mathrm{E}+05$ & $8.400 \mathrm{E}+05$ & $1.100 \mathrm{E}+06$ & $2.900 \mathrm{E}+06$ \\
Total & 0.0 & 0.0 & $7.458 \mathrm{E}+06$ & $1.449 \mathrm{E}+07$ & $1.614 \mathrm{E}+07$ & $1.232 \mathrm{E}+07$ & $5.041 \mathrm{E}+07$
\end{tabular}


Table 34

Airborne Release Locations for Maximally Exposed Individual Dose

Page 1 of 1

\section{Release Source Area}

\begin{tabular}{lccccc} 
& \multicolumn{5}{c}{ Release Source Area } \\
\cline { 2 - 6 } & F,H,P,K,C & M & D & $\begin{array}{c}\text { Savannah River } \\
\text { Technology Center }\end{array}$ & Diffuse \& Fugitive \\
\cline { 2 - 6 } & 61 & 0 & 16 & 31 & 0 \\
Release height, $m$ & & & & \\
Release location & & 50041 & 20938 & 51863 & 58000 \\
$\quad$ Site coordinate & 58000 & 104828 & 65284 & 106670 & 62000 \\
$\quad$ East & 62000 & & & &
\end{tabular}




\section{Table 35}

Parameters Used for Adult Consumption Rates and for Atmospheric Dose Calculations

Page 1 of 1

\begin{tabular}{|c|c|c|}
\hline Pathway & Maximally Exposed Individual & Population \\
\hline Note: Values were determined by the Savar & Giver Technology Center for SRS. & \\
\hline Fruits, vegetables, and grains $(\mathrm{kg} / \mathrm{yr})$ & 276 & 163 \\
\hline Leafy vegetables $(\mathrm{kg} / \mathrm{yr})$ & 43 & 21 \\
\hline Cow milk (Lyr) & 230 & 120 \\
\hline Goat milk (L/yr) & 230 & $\mathrm{n} / \mathrm{a}$ \\
\hline Meat (beef) (kg/yr) & 81 & 43 \\
\hline Inhalation $\left(\mathrm{m}^{3} / \mathrm{yr}\right)$ & 8,000 & 8,000 \\
\hline $\begin{array}{l}\text { Fraction of external dose received after taking } \\
\text { structural shielding into account }\end{array}$ & 0.7 & 0.5 \\
\hline
\end{tabular}




\section{Table 36}

Site-Specific Parameters Used with CAP88 Code Used for NESHAPS Calculations

Page 1 of 1

\section{Particle size, AMADa}

$$
\mathrm{H}-3, \mathrm{C}-14, \mathrm{Kr}-85
$$

All other

Meteorological data

Plume rise

1992-96; H-Area

Number of stacks None

Stack heights, $m$

Height of lid, $m$

Rainfall, cm/yr

0 and 61

$$
1000
$$

Average air temperature, $\mathrm{C}$

Surface roughness length, $m$

Height of wind measurements, $m$

Average wind speed, $\mathrm{m} / \mathrm{s}$

61

Population size

Food supply fractions (fraction from local sources)

$$
\text { Vegetable }
$$

0.70

Meat

0.44

Milk

0.40

a Activity Median Aerodynamic Diameter 
Table 37

Parameters Used for Adult Consumption Rates and for Liquid Dose Calculations

Page 1 of 1

\section{Pathway}

Maximally Exposed Individual

Population

Note: Values were determined by the Savannah River Technology Center for SRS.

Water consumption (L/yr)

Fish consumption $(\mathrm{kg} / \mathrm{yr})$

Marine invertebrates $(\mathrm{kg} / \mathrm{yr})$

Boating $^{a}$

Swimming ${ }^{a}$

Shoreline recreation ${ }^{a}$

Water treatment plants ${ }^{b}$

Water consumption (L/yr)

730

19

Not applicable

21

23

730

\section{0}

9

2

$1,100,000$

160,000

960,000

2 
Table 38

Site-Specific Parameters Used in Liquid Dose Calculations

Page 1 of 1

Savannah River flow rate at Highway 301 for 1999 (cu ft/sec) $)^{a}$

5,920

River dilution in estuary

Transit time from process areas to river (hr)

Transit time from SRS to water treatment plants ( $\mathrm{hr}$ )

Water treatment time ( $\mathrm{hr}$ )

Edible aquatic food harvest $(\mathrm{kg} / \mathrm{yr})$

Fish - sport

$35,000^{\mathrm{b}}$

Fish - commercial

$2,700^{\mathrm{b}}$

Invertebrates - salt water

$390,000^{\mathrm{b}}$

Irrigation (acres devoted to each of the

Nonec

four major food types)

Shore width factor

0.2

Fish bioaccumulation factor for cesium

$3,000^{b}$

a The effective river flow rate was based on tritium measurements. The 1999 USGS measured river flow rate was 6,160 cfs. Values determined by SRTC for SRS [Hamby, 1991].

Irrigation is considered a "special case" scenario. The food produced on each 1,000-acre parcel is assumed to be eaten by the 80 -kilometer population of 620,100 . 
Table 39

Committed Dose to the Maximally Exposed Individual from Atmospheric Releases (MAXIGASP Code - Using Consumption of Cow Milk Pathway)

Page 1 of 1

\begin{tabular}{|c|c|c|}
\hline By Pathway & Maximally Exposed Individual Dose (mrem) & Percent of Total Dose \\
\hline Plume & $8.45 E-05$ & 0.1 \\
\hline Ground & $5.86 \mathrm{E}-04$ & 1.0 \\
\hline Inhalation & $2.76 E-02$ & 48.3 \\
\hline Vegetation & $2.54 \mathrm{E}-02$ & 44.4 \\
\hline Cow milk & $2.62 E-03$ & 4.6 \\
\hline Meat & $9.75 E-04$ & 1.7 \\
\hline Total & $5.72 E-02$ & \\
\hline By Radionuclide & Maximally Exposed Individual Dose (mrem) ${ }^{a}$ & Percent of Total Dose \\
\hline \multicolumn{3}{|l|}{ Gases and Vapors } \\
\hline $\mathrm{H}-3$ & $1.59 \mathrm{E}-02$ & 27.8 \\
\hline$C-14$ & $5.33 E-06$ & 0.01 \\
\hline Kr-85 & $8.45 \mathrm{E}-05$ & 0.1 \\
\hline $1-129$ & $2.42 E-03$ & 4.2 \\
\hline $1-131$ & $5.95 E-06$ & 0.01 \\
\hline \multicolumn{3}{|l|}{ Particulates } \\
\hline Co-60 & $1.09 E-05$ & 0.02 \\
\hline Sr-90 & $3.00 E-05$ & 0.1 \\
\hline Cs-134 & $5.98 E-06$ & 0.01 \\
\hline Cs-137 & $7.62 E-04$ & 1.3 \\
\hline Th-232 & $1.09 E-05$ & 0.02 \\
\hline U-232 & $3.08 E-06$ & 0.01 \\
\hline U-234 & $6.71 E-04$ & 1.2 \\
\hline U-235 & $1.20 E-04$ & 0.2 \\
\hline U-238 & $5.01 E-04$ & 0.9 \\
\hline Pu-238 & $4.72 E-03$ & 8.2 \\
\hline Pu-239 & $3.00 \mathrm{E}-04$ & 0.5 \\
\hline Pu-240 & $4.36 \mathrm{E}-06$ & 0.01 \\
\hline Pu-241 & $3.80 \mathrm{E}-06$ & 0.01 \\
\hline Am-241 & $8.53 \mathrm{E}-05$ & 0.1 \\
\hline Am-243 & $1.32 \mathrm{E}-05$ & 0.02 \\
\hline $\mathrm{Cm}-244$ & $3.76 \mathrm{E}-05$ & 0.1 \\
\hline Alpha & $2.38 \mathrm{E}-02$ & 41.6 \\
\hline Nonvolatile beta & $7.72 E-03$ & 13.5 \\
\hline Total & $5.72 E-02$ & \\
\hline
\end{tabular}

a Committed effective dose equivalent

b Radionuclides contributing $0.01 \%$ or more of the total dose 


\section{Table 40}

Committed Dose to the Maximally Exposed Individual from Atmospheric Releases (MAXIGASP Code - Using Consumption of Goat Milk Pathway)

Page 1 of 1

\begin{tabular}{|c|c|c|}
\hline By Pathway & Maximally Exposed Individual Dose (mrem)a & Percent of Total Dose \\
\hline Plume & $8.45 \mathrm{E}-05$ & 0.1 \\
\hline Ground & $5.86 E-04$ & 1.0 \\
\hline Inhalation & $2.76 \mathrm{E}-02$ & 45.7 \\
\hline Vegetation & $2.54 \mathrm{E}-02$ & 42.1 \\
\hline Goat milk & $5.73 E-03$ & 9.5 \\
\hline Meat & $9.75 E-04$ & 1.6 \\
\hline Total & $6.04 E-02$ & \\
\hline By Radionuclide & Maximally Exposed Individual Dose (mrem) ${ }^{a}$ & Percent of Total Dose $e^{b}$ \\
\hline \multicolumn{3}{|l|}{ Gases and Vapors } \\
\hline$H-3$ & $1.87 E-02$ & 31.0 \\
\hline C-14 & $5.51 E-06$ & 0.01 \\
\hline Kr-85 & 8.45E-05 & 0.1 \\
\hline $1-129$ & $2.48 E-03$ & 4.1 \\
\hline $1-131$ & $6.70 E-06$ & 0.01 \\
\hline \multicolumn{3}{|l|}{ Particulates } \\
\hline Co-60 & $1.09 \mathrm{E}-05$ & 0.02 \\
\hline Sr-90 & $3.00 \mathrm{E}-05$ & 0.05 \\
\hline Cs-134 & $7.38 E-06$ & 0.01 \\
\hline Cs- 137 & $8.56 \mathrm{E}-04$ & 1.4 \\
\hline Th-232 & $1.09 \mathrm{E}-05$ & 0.02 \\
\hline U-234 & $6.71 E-04$ & 1.1 \\
\hline U-235 & $1.20 E-04$ & 0.2 \\
\hline U-238 & $5.01 E-04$ & 0.8 \\
\hline Pu-238 & $4.72 E-03$ & 7.8 \\
\hline Pu-239 & $3.00 E-04$ & 0.5 \\
\hline Am-241 & $8.53 E-05$ & 0.1 \\
\hline Am-243 & 1.32E-05 & 0.02 \\
\hline $\mathrm{Cm}-244$ & $3.76 \mathrm{E}-05$ & 0.1 \\
\hline Alpha & $2.38 E-02$ & 39.4 \\
\hline Nonvolatile beta & 7.87E-03 & 13.0 \\
\hline Total & $6.04 E-02$ & \\
\hline
\end{tabular}


Table 41

80-km (50-Mile) Collective Dose from Atmospheric Releases (POPGASP Code)

Page 1 of 1

\begin{tabular}{|c|c|c|}
\hline By Pathway & Population Dose (person-rem)a & Percent of Total Dose \\
\hline Plume & $9.53 \mathrm{E}-03$ & 0.4 \\
\hline Ground & $6.93 E-02$ & 2.7 \\
\hline Inhalation & $1.80 \mathrm{E}+00$ & 69.8 \\
\hline Vegetation & $5.06 \mathrm{E}-01$ & 19.6 \\
\hline Cow milk & $1.59 \mathrm{E}-01$ & 6.2 \\
\hline Meat & $3.48 \mathrm{E}-02$ & 1.3 \\
\hline Total & $2.58 E+00$ & \\
\hline By Radionuclide & Population Dose (person-rem) ${ }^{a}$ & Percent of Total Dose ${ }^{b}$ \\
\hline \multicolumn{3}{|l|}{ Gases and Vapors } \\
\hline $\mathrm{H}-3$ & $1.55 \mathrm{E}+00$ & 60.09 \\
\hline C-14 & 2.38E-04 & 0.01 \\
\hline $\mathrm{Kr}-85$ & $9.53 E-03$ & 0.37 \\
\hline $1-129$ & $1.18 \mathrm{E}-01$ & 4.57 \\
\hline \multicolumn{3}{|l|}{ Particulates } \\
\hline Co-60 & $9.84 \mathrm{E}-04$ & 0.04 \\
\hline Sr-90 & $1.68 \mathrm{E}-03$ & 0.07 \\
\hline Cs-134 & 3.97E-04 & 0.02 \\
\hline Cs-137 & $7.66 \mathrm{E}-02$ & 2.97 \\
\hline$R a-228$ & $3.22 E-04$ & 0.01 \\
\hline Th-230 & $1.18 \mathrm{E}-03$ & 0.05 \\
\hline Th-232 & $7.98 E-04$ & 0.03 \\
\hline U-234 & $3.48 E-03$ & 0.13 \\
\hline U-235 & $3.83 E-04$ & 0.01 \\
\hline U-238 & $5.21 E-03$ & 0.20 \\
\hline Pu-238 & $3.29 \mathrm{E}-01$ & 12.76 \\
\hline Pu-239 & $2.79 \mathrm{E}-02$ & 1.08 \\
\hline Pu-240 & $2.70 \mathrm{E}-04$ & 0.01 \\
\hline Pu-241 & $2.35 E-04$ & 0.01 \\
\hline Am-241 & $7.33 \mathrm{E}-03$ & 0.28 \\
\hline Am-243 & $8.15 E-04$ & 0.03 \\
\hline $\mathrm{Cm}-244$ & $3.21 E-03$ & 0.12 \\
\hline $\mathrm{Cm}-246$ & $5.70 \mathrm{E}-04$ & 0.02 \\
\hline Alpha & $3.90 E-01$ & 15.12 \\
\hline Nonvolatile beta & $5.12 E-02$ & 1.99 \\
\hline Total & $2.58 E+00$ & \\
\hline
\end{tabular}

a Committed effective dose equivalent

b Radionuclides contributing $0.1 \%$ or more of the total dose 


\section{Table 42}

Total Site Airborne Releases and Maximally Exposed Individual Effective Dose Equivalent by Radionuclide (CAP88 Dose Calculations for 1999 NESHAP Report to EPA)

Page 1 of 3

\begin{tabular}{|c|c|c|c|}
\hline Radionuclide ${ }^{a}$ & Releases (curies) & Maximally Exposed Individual EDE (mrem) & Percent of Dose \\
\hline $\mathrm{H}-3$ (oxide) & $3.39 \mathrm{E}+04$ & $4.43 \mathrm{E}-02$ & 86.49 \\
\hline Alpha & $2.11 E-03$ & $2.46 \mathrm{E}-03$ & 4.80 \\
\hline Pu-238 & $1.98 E-03$ & $2.15 E-03$ & 4.20 \\
\hline Cs-137 & $1.45 \mathrm{E}-02$ & $8.10 E-04$ & 1.58 \\
\hline Nonvolatile beta & $3.09 \mathrm{E}-02$ & 4.43E-04 & 0.86 \\
\hline $1-129$ & $7.27 \mathrm{E}-03$ & 4.04E-04 & 0.79 \\
\hline $\mathrm{Kr}-85$ & $3.74 E+04$ & $2.24 E-04$ & 0.44 \\
\hline Pu-239 & $1.51 \mathrm{E}-04$ & $1.76 E-04$ & 0.34 \\
\hline Am-241 & $3.86 \mathrm{E}-05$ & $6.41 E-05$ & 0.13 \\
\hline U-238 & $1.42 \mathrm{E}-04$ & $5.52 E-05$ & 0.11 \\
\hline U-234 & $8.72 E-05$ & $3.79 E-05$ & 0.07 \\
\hline $\mathrm{Cm}-244$ & $3.27 \mathrm{E}-05$ & $2.86 E-05$ & 0.06 \\
\hline Sr-89,90 & $1.01 E-03$ & $1.45 E-05$ & 0.03 \\
\hline Th-230 & $1.22 \mathrm{E}-05$ & $1.07 E-05$ & 0.02 \\
\hline Am-243 & $4.28 \mathrm{E}-06$ & $7.88 E-06$ & 0.02 \\
\hline Co-60 & $1.30 \mathrm{E}-04$ & $6.69 E-06$ & 0.01 \\
\hline $\mathrm{Cm}-246$ & $2.91 E-06$ & $5.47 E-06$ & 0.01 \\
\hline U-235 & $9.91 E-06$ & $4.22 E-06$ & 0.01 \\
\hline Cs-134 & $1.31 \mathrm{E}-04$ & $2.33 E-06$ & 0.005 \\
\hline Ra-228 & $1.87 \mathrm{E}-05$ & 2.15E-06 & 0.004 \\
\hline Th-232 & $1.64 \mathrm{E}-06$ & $2.05 E-06$ & 0.004 \\
\hline Pu-240 & $1.46 \mathrm{E}-06$ & $1.77 E-06$ & 0.003 \\
\hline C-14 & $2.55 E-02$ & $1.61 E-06$ & 0.003 \\
\hline Pu-241 & $6.47 \mathrm{E}-05$ & $1.25 E-06$ & 0.002 \\
\hline U-233 & $2.38 E-06$ & $1.08 \mathrm{E}-06$ & 0.002 \\
\hline Ra-226 & $1.25 E-05$ & $9.82 E-07$ & 0.002 \\
\hline
\end{tabular}

a The radionuclides that follow were listed as diffuse and fugitive releases but were not included in the dose calculations; the reasons appear in parentheses.

$\mathrm{Ba}-137 \mathrm{~m}$. Pa-234, $\mathrm{Pb}-212, \mathrm{Pr}-144, \mathrm{Pr}-144 \mathrm{~m}, \mathrm{Rh}-106$, and Y-90 (progeny radionuclides that are included with their parent radionuclides in dose calculations)

$\mathrm{Se}-79, \mathrm{I}-131, \mathrm{Ce}-139, \mathrm{TI}-208, \mathrm{Cf}-249, \mathrm{Cf}-251, \mathrm{Pm}-144, \mathrm{Pm}-146, \mathrm{Y}-88$, and $\mathrm{Zr}$-95 (short-lived radionuclides with very low dose potential and/or not in the CAP88 radionuclide library)

U-233,234 (emission added to U-234)

U-235,236 (emission added to U-235)

$\mathrm{Cm}-243,244$ (emission added to $\mathrm{Cm}-244$ ) 
Table 42

Total Site Airborne Releases and Maximally Exposed Individual

Effective Dose Equivalent by Radionuclide

(CAP88 Dose Calculations for 1999 NESHAP Report to EPA)

Page 2 of 3

\begin{tabular}{|c|c|c|c|}
\hline Radionuclide ${ }^{a}$ & Releases (curies) & Maximally Exposed Individual EDE (mrem) & Percent of Dose \\
\hline H-3 (elem.) & $1.77 \mathrm{E}+04$ & $9.24 \mathrm{E}-07$ & 0.002 \\
\hline Ce-144 & $1.45 E-04$ & $6.69 E-07$ & 0.001 \\
\hline $\mathrm{Sb}-125$ & $5.27 \mathrm{E}-05$ & $2.93 E-07$ & 0.001 \\
\hline Eu-154 & $5.74 \mathrm{E}-06$ & $2.35 \mathrm{E}-07$ & 0.0005 \\
\hline Th-228 & $2.75 \mathrm{E}-07$ & $2.21 E-07$ & 0.0004 \\
\hline Co-58 & $1.27 \mathrm{E}-04$ & $1.34 \mathrm{E}-07$ & 0.0003 \\
\hline TC-99 & $6.22 E-05$ & $9.34 \mathrm{E}-08$ & 0.0002 \\
\hline$Z n-65$ & $2.23 E-05$ & $6.18 \mathrm{E}-08$ & 0.0001 \\
\hline $\mathrm{Nb}-95$ & $1.13 E-04$ & $5.59 E-08$ & 0.0001 \\
\hline U-232 & $1.33 E-08$ & $3.19 \mathrm{E}-08$ & 0.0001 \\
\hline Zr-95 & $1.71 \mathrm{E}-05$ & $2.68 \mathrm{E}-08$ & 0.0001 \\
\hline $\mathrm{Cm}-242$ & $3.10 E-07$ & $1.84 \mathrm{E}-08$ & 0.00004 \\
\hline$P u-242$ & $1.53 E-08$ & $1.77 \mathrm{E}-08$ & 0.00003 \\
\hline$R u-103$ & $4.23 \mathrm{E}-05$ & $1.51 E-08$ & 0.00003 \\
\hline$X e-135$ & $1.94 \mathrm{E}-02$ & $7.98 \mathrm{E}-09$ & 0.00002 \\
\hline $\mid-131$ & $1.01 E-05$ & $6.72 \mathrm{E}-09$ & 0.00001 \\
\hline $\mathrm{Ce}-141$ & $4.16 \mathrm{E}-05$ & $5.30 E-09$ & 0.00001 \\
\hline$U-236$ & $5.20 \mathrm{E}-09$ & $2.21 \mathrm{E}-09$ & 0.000004 \\
\hline $1-133$ & $1.25 \mathrm{E}-04$ & $2.09 E-09$ & 0.000004 \\
\hline Cr-51 & $1.21 \mathrm{E}-04$ & $1.97 \mathrm{E}-09$ & 0.000004 \\
\hline Eu-155 & $1.10 \mathrm{E}-06$ & $1.79 \mathrm{E}-09$ & 0.000003 \\
\hline Th-234 & $4.10 E-06$ & $1.48 E-09$ & 0.000003 \\
\hline$A c-228$ & $1.66 \mathrm{E}-06$ & $3.97 E-10$ & 0.000001 \\
\hline $\mathrm{Np}-237$ & $2.23 E-10$ & $3.74 \mathrm{E}-10$ & 0.000001 \\
\hline $\mathrm{Nb}-94$ & $3.95 E-10$ & $5.63 E-11$ & 0.0000001 \\
\hline Co-57 & $4.71 \mathrm{E}-08$ & $2.55 E-11$ & 0.00000005 \\
\hline $\mathrm{Ni}-63$ & $5.89 E-07$ & $2.27 E-11$ & 0.00000004 \\
\hline Eu-152 & $1.21 \mathrm{E}-10$ & $5.99 \mathrm{E}-12$ & 0.00000001 \\
\hline Pm-147 & $3.49 E-09$ & $5.58 E-13$ & 0.000000001 \\
\hline Sb-124 & $2.23 \mathrm{E}-10$ & $3.88 E-13$ & 0.000000001 \\
\hline$N p-239$ & $4.51 \mathrm{E}-09$ & $6.60 E-14$ & 0.0000000001 \\
\hline $\mathrm{Hg}-203$ & $2.23 \mathrm{E}-10$ & $6.32 E-14$ & 0.0000000001 \\
\hline Ni-59 & $1.02 E-09$ & $5.28 E-14$ & 0.0000000001 \\
\hline
\end{tabular}


Table 42

Total Site Airborne Releases and Maximally Exposed Individual Effective Dose Equivalent by Radionuclide

(CAP88 Dose Calculations for 1999 NESHAP Report to EPA)

Page 3 of 3

\begin{tabular}{lccc}
\hline & & & \\
Radionuclide $^{a}$ & Releases (curies) & Maximally Exposed Individual EDE (mrem) & Percent of Dose \\
\hline $\mathrm{Pa}-233$ & $2.23 \mathrm{E}-10$ & $4.07 \mathrm{E}-14$ & 0.0000000001 \\
$\mathrm{Sn}-126$ & $3.13 \mathrm{E}-15$ & $7.98 \mathrm{E}-16$ & 0.000000000002 \\
$\mathrm{~Pb}-214$ & $2.23 \mathrm{E}-10$ & $3.21 \mathrm{E}-16$ & 0.000000000001 \\
& & & \\
Total & & $5.12 \mathrm{E}-02$ &
\end{tabular}


Table 43

NESHAP Airborne-Dose Report Data - CAP88 Compared with MAXDOSE-SR

Page 1 of 1

\begin{tabular}{|c|c|c|c|c|}
\hline & \multicolumn{4}{|c|}{ Maximally Exposed Individual Dose at Site Boundary } \\
\hline & \multicolumn{2}{|c|}{ CAP88 Code } & \multicolumn{2}{|c|}{ MAXDOSE-SR Code } \\
\hline & Millirema & Percent of Dose & Millirema & Percent of Dose ${ }^{b}$ \\
\hline \multicolumn{5}{|l|}{ By Pathway } \\
\hline Plume & $1.51 E-04$ & 0.3 & $8.45 E-05$ & 0.1 \\
\hline Ground & $8.23 E-04$ & 1.6 & $5.86 \mathrm{E}-04$ & 1.0 \\
\hline Inhalation & $1.69 E-02$ & 33.0 & $2.76 E-02$ & 48.3 \\
\hline Food ${ }^{c}$ & 3.33E-02 & 65.1 & $2.90 \mathrm{E}-02$ & 50.7 \\
\hline Total & $5.12 E-02$ & & $5.72 E-02$ & \\
\hline \multicolumn{5}{|l|}{ By Radionuclide } \\
\hline \multicolumn{5}{|l|}{ Gases and Vapors } \\
\hline$H-3^{d}$ & 4.43E-02 & 86.5 & $1.59 \mathrm{E}-02$ & 27.8 \\
\hline C-14 & $1.61 \mathrm{E}-06$ & 0.003 & $5.33 E-06$ & 0.01 \\
\hline Kr-85 & $2.24 \mathrm{E}-04$ & 0.4 & $8.45 \mathrm{E}-05$ & 0.1 \\
\hline $1-129$ & $4.04 \mathrm{E}-04$ & 0.8 & $2.42 \mathrm{E}-03$ & 4.2 \\
\hline $1-131$ & $6.72 E-09$ & 0.00001 & $5.95 E-06$ & 0.01 \\
\hline \multicolumn{5}{|l|}{ Particulates } \\
\hline Co-60 & $6.69 E-06$ & 0.01 & $1.09 E-05$ & 0.02 \\
\hline Sr-90 & $1.45 \mathrm{E}-05$ & 0.03 & $3.00 E-05$ & 0.1 \\
\hline Cs-134 & $2.33 E-06$ & 0.005 & $5.98 \mathrm{E}-06$ & 0.01 \\
\hline Cs-137 & $8.10 E-04$ & 1.6 & $7.62 \mathrm{E}-04$ & 1.3 \\
\hline Th-232 & $2.05 \mathrm{E}-06$ & 0.004 & $1.09 \mathrm{E}-05$ & 0.02 \\
\hline U-232 & $3.19 \mathrm{E}-08$ & 0.0001 & $3.08 \mathrm{E}-06$ & 0.01 \\
\hline$U-234$ & $3.79 E-05$ & 0.1 & $6.71 \mathrm{E}-04$ & 1.2 \\
\hline$U-235$ & $4.22 E-06$ & 0.01 & $1.20 \mathrm{E}-04$ & 0.2 \\
\hline U-238 & $5.52 E-05$ & 0.1 & $5.01 E-04$ & 0.9 \\
\hline Pu-238 & $2.15 E-03$ & 4.2 & $4.72 E-03$ & 8.2 \\
\hline Pu-239 & $1.76 E-04$ & 0.3 & $3.00 E-04$ & 0.5 \\
\hline Pu-240 & $1.77 \mathrm{E}-06$ & 0.003 & $4.36 \mathrm{E}-06$ & 0.01 \\
\hline Pu-241 & $1.25 \mathrm{E}-06$ & 0.002 & $3.80 \mathrm{E}-06$ & 0.01 \\
\hline Am-241 & $6.41 E-05$ & 0.1 & $8.53 E-05$ & 0.1 \\
\hline Am-243 & $7.88 \mathrm{E}-06$ & 0.02 & $1.32 \mathrm{E}-05$ & 0.02 \\
\hline $\mathrm{Cm}-244$ & $2.86 \mathrm{E}-05$ & 0.1 & $3.76 \mathrm{E}-05$ & 0.1 \\
\hline Alpha & $2.46 E-03$ & 4.8 & $2.38 \mathrm{E}-02$ & 41.6 \\
\hline Nonvolatile beta & 4.43E-04 & 0.9 & $7.72 \mathrm{E}-03$ & 13.5 \\
\hline Total & $5.12 E-02$ & & $5.72 E-02$ & \\
\hline $\begin{array}{ll}\text { a } & \text { Committed effe } \\
\text { b } & \text { Radionuclides } \\
\text { c } & \text { Meat, milk, and } \\
\text { d } & \text { Dose from tritiu }\end{array}$ & $\begin{array}{l}\text { se equivalen } \\
\text { ing } 0.01 \text { per } \\
\text { sles } \\
\text { ds calculatec }\end{array}$ & $\begin{array}{l}\text { ore from MAXDOSE-S } \\
\text { olute humidity of } 11.4\end{array}$ & air & \\
\hline
\end{tabular}


Table 44

NESHAP Airborne-Dose Report Data - CAP88 Compared with POPGASP

Page 1 of 1

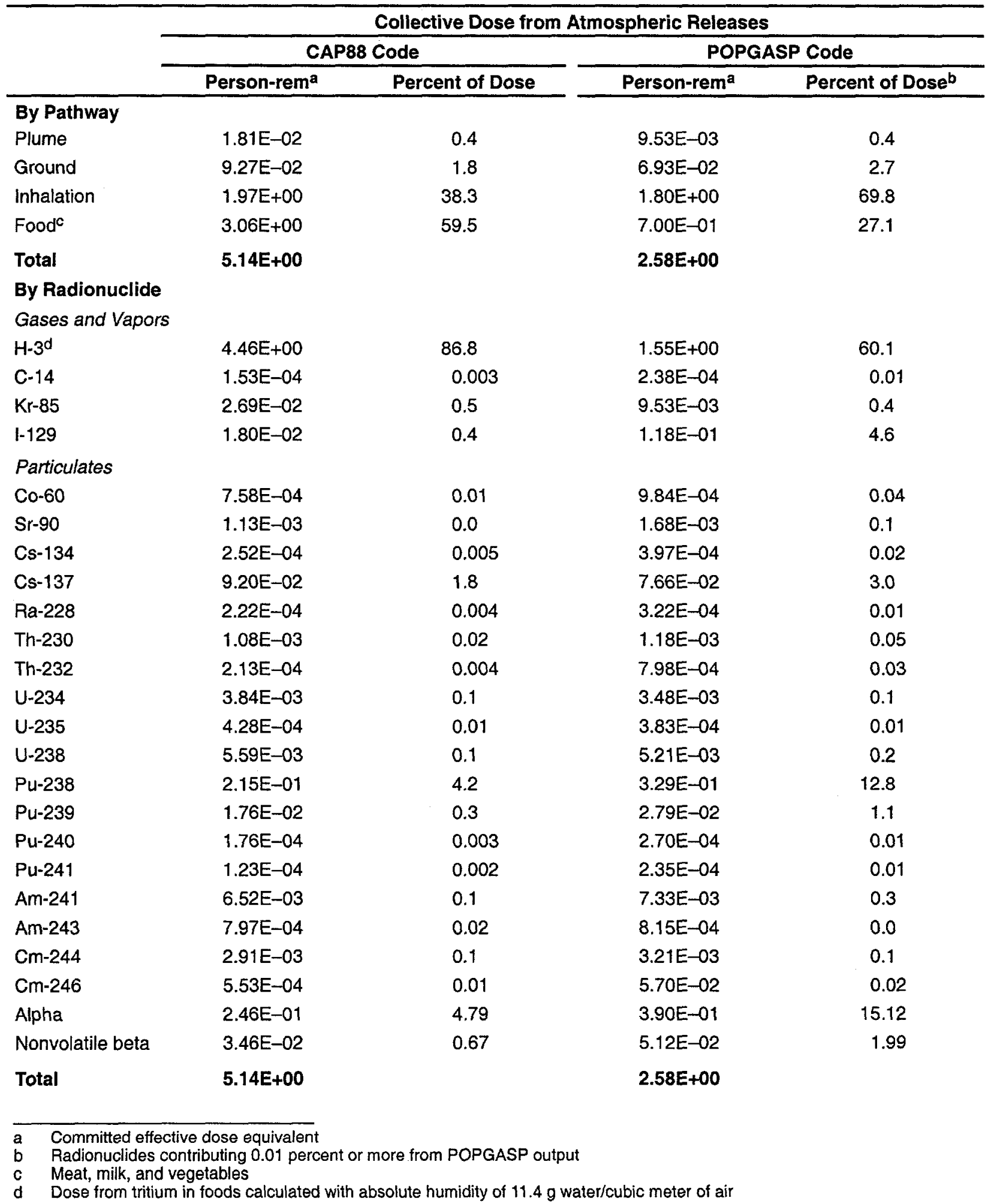


Table 45

Committed Dose to Maximally Exposed Individual from Liquid Releases

Page 1 of 1

\begin{tabular}{lcc}
\hline By Pathway & Individual Dose $(\mathbf{m r e m})^{\mathrm{a}}$ & Percent of Total Dose \\
\hline Fish & $1.36 \mathrm{E}-01$ & 61.03 \\
Water & $8.59 \mathrm{E}-02$ & 38.54 \\
Shoreline & $9.34 \mathrm{E}-04$ & 0.42 \\
Swimming & $2.36 \mathrm{E}-05$ & 0.01 \\
Boating & $3.45 \mathrm{E}-07$ & 0.0002 \\
Total & $2.23 \mathrm{E}-01$ & \\
By Radionuclide & Individual Dose (mrem) & Percent of Total Dose \\
\hline H-3 (oxide) & $5.60 \mathrm{E}-02$ & 25.08 \\
Co-60 & $6.22 \mathrm{E}-06$ & 0.003 \\
Sr-89,90 & $4.28 \mathrm{E}-03$ & 1.91 \\
l-129 & $4.22 \mathrm{E}-03$ & 1.89 \\
Cs-137 & $1.32 \mathrm{E}-01$ & 59.11 \\
U-234 & $3.85 \mathrm{E}-03$ & 1.72 \\
U-235 & $3.37 \mathrm{E}-05$ & 0.015 \\
U-238 & $9.26 \mathrm{E}-04$ & 0.41 \\
Pu-238 & $6.24 \mathrm{E}-05$ & 0.03 \\
Pu-239 & $6.61 \mathrm{E}-05$ & 0.03 \\
Am-241 & $1.37 \mathrm{E}-05$ & 0.006 \\
Cm-244 & $6.60 \mathrm{E}-07$ & 0.0003 \\
Alpha & $2.01 \mathrm{E}-02$ & 9.00 \\
Nonvolatile beta & $1.76 \mathrm{E}-03$ & 0.79 \\
Total & $2.23 \mathrm{E}-01$ & \\
& &
\end{tabular}

$\overline{\text { a Committed effective dose equivalent }}$ 
Table 46

Committed Dose to Maximally Exposed Individual from Public Water Supplies at Beaufort-Jasper Water Treatment Plant

Page 1 of 1

\begin{tabular}{lcc}
\hline Radionuclide & Individual Dose, mrem & \\
\hline H-3 (oxide) & $4.47 \mathrm{E}-02$ & Percent of Total Dose \\
Co-60 & $1.45 \mathrm{E}-06$ & 63.72 \\
Sr-89,90 & $1.96 \mathrm{E}-03$ & 0.002 \\
-129 & $2.47 \mathrm{E}-03$ & 2.80 \\
Cs-137 & $1.35 \mathrm{E}-03$ & 3.52 \\
U-234 & $2.99 \mathrm{E}-03$ & 1.93 \\
U-235 & $2.50 \mathrm{E}-05$ & 4.27 \\
U-238 & $7.19 \mathrm{E}-04$ & 0.04 \\
Pu-238 & $4.67 \mathrm{E}-05$ & 1.03 \\
Pu-239 & $4.95 \mathrm{E}-05$ & 0.07 \\
Am-241 & $6.80 \mathrm{E}-06$ & 0.07 \\
Cm-244 & $3.27 \mathrm{E}-07$ & 0.01 \\
Alpha & $1.50 \mathrm{E}-02$ & 0.0005 \\
Nonvolatile beta & $8.08 \mathrm{E}-04$ & 21.40 \\
Total & $7.01 \mathrm{E}-02$ & 1.15
\end{tabular}

a Committed effective dose equivalent 
Table 47

Committed Dose to Maximally Exposed Individual from Public Water Supplies at the City of Savannah Industrial and Domestic Water Supply Plant

(near Port Wentworth, Georgia)

Page 1 of 1

\begin{tabular}{lcc}
\hline Radionuclide & Individual Dose, mrem & Percent of Total Dose \\
\hline H-3 (oxide) & $4.43 \mathrm{E}-02$ & 63.71 \\
Co-60 & $1.44 \mathrm{E}-06$ & 0.002 \\
Sr-89,90 & $1.95 \mathrm{E}-03$ & 2.80 \\
$\mathrm{H}-129$ & $2.45 \mathrm{E}-03$ & 3.52 \\
Cs-137 & $1.34 \mathrm{E}-03$ & 1.93 \\
U-234 & $2.97 \mathrm{E}-03$ & 4.27 \\
U-235 & $2.48 \mathrm{E}-05$ & 0.04 \\
U-238 & $7.13 \mathrm{E}-04$ & 1.03 \\
Pu-238 & $4.64 \mathrm{E}-05$ & 0.07 \\
Pu-239 & $4.91 \mathrm{E}-05$ & 0.07 \\
Am-241 & $6.75 \mathrm{E}-06$ & 0.01 \\
Cm-244 & $3.24 \mathrm{E}-07$ & 0.0005 \\
Alpha & $1.49 \mathrm{E}-02$ & 21.41 \\
Nonvolatile beta & $8.02 \mathrm{E}-04$ & 1.15 \\
Total & $6.96 \mathrm{E}-02$ &
\end{tabular}

a Committed effective dose equivalent 


\section{Table 48}

Collective Dose from Liquid Releases

Page 1 of 1

\begin{tabular}{|c|c|c|}
\hline By Pathway & Collective Dose (person-rem) ${ }^{a}$ & Percent of Total Dose \\
\hline Sport fish & $2.51 \mathrm{E}-01$ & 6.33 \\
\hline Commercial fish & $1.94 \mathrm{E}-02$ & 0.49 \\
\hline Beaufort-Jasper & $2.67 \mathrm{E}+00$ & 67.30 \\
\hline Port Wentworth & $3.53 E-01$ & 8.91 \\
\hline Saltwater invertebrates & $6.33 E-01$ & 15.97 \\
\hline Shoreline exposure & $3.90 \mathrm{E}-02$ & 0.98 \\
\hline Swimming & $4.25 \mathrm{E}-04$ & 0.01 \\
\hline Boating & $1.81 \mathrm{E}-05$ & 0.0005 \\
\hline Total & $3.96 \mathrm{E}+00$ & \\
\hline By Radionuclide & Collective Dose (person-rem) ${ }^{a}$ & Percent of Total Dose \\
\hline $\mathrm{H}-3$ & $1.93 \mathrm{E}+00$ & 48.79 \\
\hline Co-60 & 4.31E-04 & 0.01 \\
\hline Sr-89,90 & $9.58 \mathrm{E}-02$ & 2.42 \\
\hline $1-129$ & $1.33 E-01$ & 3.36 \\
\hline Cs-137 & $3.59 \mathrm{E}-01$ & 9.08 \\
\hline$U-234$ & $1.35 \mathrm{E}-01$ & 3.41 \\
\hline U-235 & $1.19 \mathrm{E}-03$ & 0.03 \\
\hline$U-238$ & $3.24 E-02$ & 0.82 \\
\hline Pu-238 & $3.80 \mathrm{E}-03$ & 0.1 \\
\hline Pu-239 & $4.02 E-03$ & 0.1 \\
\hline Am-241 & $1.60 \mathrm{E}-03$ & 0.04 \\
\hline $\mathrm{Cm}-244$ & $7.67 \mathrm{E}-05$ & 0.002 \\
\hline Alpha & $1.22 \mathrm{E}+00$ & 30.84 \\
\hline Nonvolatile beta & $3.94 \mathrm{E}-02$ & 1.00 \\
\hline Total & $3.96 E+00$ & \\
\hline
\end{tabular}

a Committed effective dose equivalent 
Table 49

Potential Doses from Irrigation Pathways

Page 1 of 1

\begin{tabular}{lcc}
\hline Food Type $^{\mathrm{a}}$ & Maximally Exposed Individual $(\mathbf{m r e m})^{\mathrm{b}}$ & Population (person-rem) $^{\mathbf{b}}$ \\
\hline Vegetation & $1.01 \mathrm{E}-01$ & $6.33 \mathrm{E}+00$ \\
Leafy vegetable & $1.58 \mathrm{E}-02$ & $4.07 \mathrm{E}-01$ \\
Milk & $2.28 \mathrm{E}-02$ & $3.11 \mathrm{E}+00$ \\
Meat & $6.88 \mathrm{E}-03$ & $3.68 \mathrm{E}-01$ \\
Total & $1.46 \mathrm{E}-01$ & $1.02 \mathrm{E}+01$
\end{tabular}

a Irrigated acreage for each food type assumed to be 1,000 acres

b Committed effective dose equivalent 
Table 50

Dose from Consumption of Fish from New Savannah Bluff Lock and Dam, SRS Creek Mouths, and River Mile 120

Page 1 of 2

Average Concentration in Composites, $\mathrm{pCi}^{\mathrm{a}}$

\begin{tabular}{|c|c|c|c|c|c|c|}
\hline Location & Species & $\mathrm{H}-3$ & Sr-90 & Cs-137 & Pu-238 & Pu-239 \\
\hline \multirow[t]{3}{*}{ NSB L\&D } & Bass & $1.81 \mathrm{E}-02$ & $8.20 E-03$ & $1.31 \mathrm{E}-02$ & $1.41 E-05$ & $-2.57 E-05$ \\
\hline & Catfish & $3.88 \mathrm{E}-02$ & $9.96 \mathrm{E}-03$ & $5.01 E-02$ & $-1.55 E-05$ & $-7.78 E-06$ \\
\hline & Panfish & 2.93E-02 & $1.72 E-02$ & $2.06 \mathrm{E}-02$ & $-2.40 E-05$ & $-1.20 E-04$ \\
\hline \multirow[t]{3}{*}{ U3R Mouth } & Bass & $3.09 E-02$ & $6.82 E-03$ & $6.03 E-02$ & $-1.68 E-05$ & $-3.57 E-05$ \\
\hline & Catfish & $1.35 E-01$ & $-1.11 E-03$ & $3.15 E-02$ & $-6.00 E-05$ & $1.73 E-05$ \\
\hline & Panfish & 7.32E-02 & 7.71E-03 & 4.67E-02 & $-4.10 E-06$ & $-5.90 E-05$ \\
\hline \multirow[t]{3}{*}{ BDC Mouth } & Bass & $6.68 \mathrm{E}-02$ & 5.13E-03 & $1.79 E-01$ & $-3.52 E-05$ & $-1.36 \mathrm{E}-05$ \\
\hline & Catfish & $1.72 E-01$ & $3.89 E-03$ & $6.84 E-02$ & $1.44 \mathrm{E}-05$ & $6.20 E-06$ \\
\hline & Panfish & $1.10 E-01$ & $1.78 E-02$ & $3.38 \mathrm{E}-02$ & 2.93E-05 & $-8.10 E-05$ \\
\hline \multirow[t]{3}{*}{ FMC Mouth } & Bass & $7.38 \mathrm{E}+01$ & $1.28 E-02$ & $2.01 E-01$ & $-2.10 E-06$ & $-8.50 E-06$ \\
\hline & Catfish & $1.29 E-00$ & $3.52 E-03$ & $9.23 \mathrm{E}-02$ & $1.98 E-05$ & $-1.50 E-05$ \\
\hline & Panfish & $6.84 E-01$ & $2.01 E-02$ & $3.02 E-02$ & $2.59 \mathrm{E}-05$ & $-1.10 E-05$ \\
\hline \multirow[t]{3}{*}{ SC Mouth } & Bass & $5.03 E-01$ & $1.47 E-02$ & $5.98 \mathrm{E}-01$ & $3.00 E-07$ & $-1.40 E-05$ \\
\hline & Catfish & $9.10 \mathrm{E}-01$ & $3.81 E-03$ & $1.10 \mathrm{E}-01$ & $-2.32 E-05$ & $1.90 E-06$ \\
\hline & Panfish & $6.32 \mathrm{E}-01$ & $1.60 \mathrm{E}-02$ & $6.07 E-02$ & $-2.40 E-06$ & $6.90 \mathrm{E}-07$ \\
\hline \multirow[t]{3}{*}{ L3R Mouth } & Bass & $2.54 \mathrm{E}-01$ & $3.80 E-03$ & $8.15 E-02$ & $-7.00 E-05$ & $-1.09 E-04$ \\
\hline & Catfish & $2.02 E-01$ & $8.30 E-04$ & 1.17E-01 & $-9.60 E-06$ & $-1.86 \mathrm{E}-05$ \\
\hline & Panfish & $2.52 E-01$ & $3.61 E-02$ & $3.16 \mathrm{E}-02$ & $3.22 E-05$ & $-4.32 E-05$ \\
\hline \multirow[t]{3}{*}{ RM 120} & Bass & $2.23 E-01$ & $6.66 E-03$ & $3.20 E-01$ & $-4.50 E-06$ & $1.80 \mathrm{E}-06$ \\
\hline & Catfish & $2.56 \mathrm{E}-01$ & $8.16 E-03$ & 5.19E-02 & $-1.66 E-05$ & $-4.35 E-05$ \\
\hline & Panfish & $2.55 E-01$ & $1.11 E-02$ & $3.65 E-02$ & $-1.95 E-05$ & $-4.53 E-05$ \\
\hline
\end{tabular}

a Each composite included five fish; three composites of each species type were collected at each sampling location.

b New Savannah Bluff Lock and Dam (formerly Augusta Lock and Dam) 
Table 50

Dose from Consumption of Fish from New Savannah Bluff Lock and Dam, SRS Creek Mouths, and River Mile 120

Page 2 of 2

Dose from Consumption of $19 \mathrm{~kg} /$ year (42 lbs), mrem,b

\begin{tabular}{|c|c|c|c|c|c|c|c|}
\hline Location & Species & $H-3$ & Sr-90 & Cs-137 & Pu-238 & Pu-239 & Total \\
\hline \multirow[t]{3}{*}{ NSB L\&DC } & Bass & $2.17 E-05$ & $2.03 E-02$ & $1.24 \mathrm{E}-02$ & $1.02 E-03$ & $0.00 E+00$ & 3.37E-02 \\
\hline & Catfish & $4.64 E-05$ & $2.46 \mathrm{E}-02$ & 4.76E-02 & $0.00 \mathrm{E}+00$ & $0.00 E+00$ & $7.22 \mathrm{E}-02$ \\
\hline & Panfish & $3.51 E-05$ & $4.25 \mathrm{E}-02$ & $1.96 \mathrm{E}-02$ & $0.00 E+00$ & $0.00 E+00$ & $6.21 \mathrm{E}-02$ \\
\hline \multirow[t]{3}{*}{ U3R Mouth } & Bass & $3.70 E-05$ & $1.68 \mathrm{E}-02$ & $5.73 E-02$ & $0.00 \mathrm{E}+00$ & $0.00 E+00$ & $7.42 E-02$ \\
\hline & Catfish & $1.62 E-04$ & $0.00 E+00$ & $2.99 E-02$ & $0.00 E+00$ & $1.41 E-03$ & $3.15 E-02$ \\
\hline & Panfish & $8.76 E-05$ & $1.90 E-02$ & $4.44 \mathrm{E}-02$ & $0.00 E+00$ & $0.00 E+00$ & $6.35 E-02$ \\
\hline \multirow[t]{3}{*}{ BDC Mouth } & Bass & $8.00 E-05$ & $1.27 E-02$ & $1.70 \mathrm{E}-01$ & $0.00 E+00$ & $0.00 E+00$ & $1.83 E-01$ \\
\hline & Catfish & $2.06 \mathrm{E}-04$ & $9.61 E-03$ & $6.50 \mathrm{E}-02$ & $1.04 \mathrm{E}-03$ & $5.07 E-04$ & $7.63 E-02$ \\
\hline & Panfish & $1.32 \mathrm{E}-04$ & $4.40 \mathrm{E}-02$ & $3.21 E-02$ & $2.12 \mathrm{E}-03$ & $0.00 \mathrm{E}+00$ & $7.83 E-02$ \\
\hline \multirow[t]{3}{*}{ FMC Mouth } & Bass & $8.83 E-04$ & $3.16 \mathrm{E}-02$ & $1.91 \mathrm{E}-01$ & $0.00 \mathrm{E}+00$ & $0.00 E+00$ & $2.23 E-01$ \\
\hline & Catfish & $1.54 E-03$ & $8.69 \mathrm{E}-03$ & 8.77E-02 & $1.43 E-03$ & $0.00 \mathrm{E}+00$ & $9.94 \mathrm{E}-02$ \\
\hline & Panfish & $8.19 E-04$ & $4.96 \mathrm{E}-02$ & 2.87E-02 & $1.87 E-03$ & $0.00 E+00$ & $8.10 E-02$ \\
\hline \multirow[t]{3}{*}{ SC Mouth } & Bass & $6.02 E-04$ & $3.63 E-02$ & $5.68 E-01$ & $2.17 E-05$ & $0.00 \mathrm{E}+00$ & $6.05 E+01$ \\
\hline & Catfish & $1.09 E-03$ & $9.41 E-03$ & $1.05 \mathrm{E}-01$ & $0.00 E+00$ & $1.55 E-04$ & $1.15 E-01$ \\
\hline & Panfish & $7.57 E-04$ & $3.95 \mathrm{E}-02$ & 5.77E-02 & $0.00 E+00$ & $5.64 \mathrm{E}-05$ & $9.80 E-02$ \\
\hline \multirow[t]{3}{*}{ L3R Mouth } & Bass & $3.04 E-04$ & $9.39 E-03$ & $7.74 \mathrm{E}-02$ & $0.00 \mathrm{E}+00$ & $0.00 E+00$ & 8.71E-02 \\
\hline & Catfish & $2.42 E-04$ & $2.05 E-03$ & $1.11 E-01$ & $0.00 E+00$ & $0.00 \mathrm{E}+00$ & $1.13 E-01$ \\
\hline & Panfish & $3.02 E-04$ & $8.92 E-02$ & $3.00 \mathrm{E}-02$ & $2.32 E-03$ & $0.00 \mathrm{E}+00^{\circ}$ & $1.22 E-01$ \\
\hline \multirow[t]{3}{*}{$\mathrm{RM} 120$} & Bass & $2.67 E-04$ & $1.65 \mathrm{E}-02$ & $3.04 \mathrm{E}-01$ & $0.00 E+00$ & $1.47 E-04$ & $3.21 E-01$ \\
\hline & Catfish & $3.06 E-04$ & $2.02 E-02$ & $4.93 \mathrm{E}-02$ & $0.00 E+00$ & $0.00 E+00$ & $6.98 E-02$ \\
\hline & Panfish & $3.05 E-04$ & $2.74 E-02$ & $3.47 E-02$ & $0.00 E+00$ & $0.00 \mathrm{E}+00$ & $6.24 E-02$ \\
\hline
\end{tabular}

a Dose was based on maximum consumption of $19 \mathrm{~kg} /$ year; to obtain dose from average consumption of fish (9 kg/year), multiply doses in table by 0.474 .

b Dose based on negative concentration of individual radionuclides in fish was assumed to be $0.00 \mathrm{mrem}$.

c New Savannah Bluff Lock and Dam (formerly Augusta Lock and Dam) 
Table 51

Calculated Doses to Aquatic Biota from SRS Releases

Page 1 of 1

Dose to Biota, $\mathrm{rad} / \mathrm{d}^{\mathrm{a}, \mathrm{b}}$

\begin{tabular}{lccccccc} 
Stream & Location & Flow, cfs & Fish & Shellfish & Algae & Raccoon & Duck \\
\hline Upper Three Runs & U3R-4 & 202 & $1.16 \mathrm{E}-05$ & $1.44 \mathrm{E}-05$ & $8.52 \mathrm{E}-06$ & $6.52 \mathrm{E}-06$ & $8.85 \mathrm{E}-05$ \\
Beaver Dam Creek & $400-\mathrm{D}$ & 5 & $1.43 \mathrm{E}-04$ & $2.32 \mathrm{E}-05$ & $5.53 \mathrm{E}-05$ & $2.44 \mathrm{E}-04$ & $5.84 \mathrm{E}-04$ \\
Four Mile Creek & FM-3 & 3 & $3.34 \mathrm{E}-03$ & $4.14 \mathrm{E}-03$ & $2.79 \mathrm{E}-03$ & $2.21 \mathrm{E}-03$ & $3.92 \mathrm{E}-02$ \\
Pen Branch & PB-3 & 14 & $2.51 \mathrm{E}-05$ & $2.61 \mathrm{E}-05$ & $3.01 \mathrm{E}-05$ & $6.30 \mathrm{E}-06$ & $9.45 \mathrm{E}-05$ \\
Steel Creek & SC-2A & 2 & $4.99 \mathrm{E}-05$ & $1.18 \mathrm{E}-04$ & $4.90 \mathrm{E}-04$ & $2.45 \mathrm{E}-04$ & $7.18 \mathrm{E}-03$ \\
Lower Three Runs & L3R-2 & 22 & $3.75 \mathrm{E}-06$ & $6.19 \mathrm{E}-06$ & $1.53 \mathrm{E}-05$ & $8.06 \mathrm{E}-06$ & $2.19 \mathrm{E}-04$
\end{tabular}


Table 52

Toxic Air Pollutant Emissions (1998)

Page 1 of 7

\begin{tabular}{|c|c|c|c|}
\hline \multicolumn{2}{|c|}{ Pollutant } & $\begin{array}{l}\text { Chemical Abstract } \\
\text { Number (CAS) }\end{array}$ & $\begin{array}{l}\text { Actual Emissions } \\
\text { (Tons/Year) }\end{array}$ \\
\hline Note: & \multicolumn{3}{|c|}{$\begin{array}{l}\text { Emissions are calculated each year as part of an annual emissions inventory. In 1999, operating data } \\
\text { were compiled and emissions calculated for } 1998 \text { operations for all site air emission sources. Because } \\
\text { this process, which begins in January, requires up to } 6 \text { months to complete, this report provides } 1998 \\
\text { emissions only. Actual emissions for } 1999 \text { will be compiled and reported in depth in the SRS } \\
\text { Environmental Report for } 2000 \text { and in SRS Environmental Data for } 2000 \text {. }\end{array}$} \\
\hline \multicolumn{2}{|c|}{ ACETALDEHYDE } & $75-7-0$ & $2.215 E-01$ \\
\hline \multicolumn{2}{|c|}{ ACETAMIDE } & $60-35-5$ & 7.727E-05 \\
\hline \multicolumn{2}{|c|}{ ACETONITRILE } & $75-5-8$ & $1.525 \mathrm{E}+00$ \\
\hline \multicolumn{2}{|c|}{ 2-ACETYLAMINOFLUORINE } & $53-96-3$ & 7.727E-05 \\
\hline \multicolumn{2}{|c|}{ ACROLEIN } & $107-2-8$ & $9.470 E-03$ \\
\hline \multicolumn{2}{|c|}{ ALIPHATIC POLYISOCYANATE } & $28182-81-2$ & $7.930 E-04$ \\
\hline \multicolumn{2}{|c|}{ ALLYL CHLORIDE } & $107-5-1$ & 7.727E-03 \\
\hline \multicolumn{2}{|c|}{ P-AMINODIPHENYL } & $92-67-1$ & 7.727E-05 \\
\hline \multicolumn{2}{|c|}{ AMMONIUM CHLORIDE } & $12125-2-9$ & $4.254 \mathrm{E}-04$ \\
\hline \multicolumn{2}{|c|}{ AMMONIUM THIOCYANATE } & $1762-95-4$ & $5.500 E-04$ \\
\hline \multicolumn{2}{|c|}{ ANILINE } & $62-53-3$ & 1.117E-02 \\
\hline \multicolumn{2}{|c|}{ O-ANISIDINE } & $90-4-0$ & 7.727E-05 \\
\hline \multicolumn{2}{|c|}{ P-ANISIDINE } & $104-94-9$ & 7.727E-05 \\
\hline \multicolumn{2}{|c|}{ ANTIMONY } & $7440-36-0$ & $1.667 E-03$ \\
\hline \multicolumn{2}{|c|}{ ANTIMONY COMPOUNDS } & a & $2.809 E-04$ \\
\hline \multicolumn{2}{|c|}{ ANTIMONY OXIDE } & $1327-33-9$ & $1.609 \mathrm{E}-04$ \\
\hline \multicolumn{2}{|c|}{ ARSENIC } & $7440-38-2$ & $6.692 E-03$ \\
\hline \multicolumn{2}{|c|}{ BENZENE } & $71-43-2$ & $6.381 E+00$ \\
\hline \multicolumn{2}{|c|}{ BERYLLIUM } & $7440-41-7$ & $9.943 E-04$ \\
\hline \multicolumn{2}{|c|}{ BERYLLIUM OXIDE } & $1304-56-9$ & $1.190 E-06$ \\
\hline \multicolumn{2}{|c|}{ BIPHENYL } & $92-52-4$ & $1.211 E-02$ \\
\hline \multicolumn{2}{|c|}{ BIS(2-ETHYLHEXYL)PHTHALATE } & $117-81-7$ & $9.851 E-03$ \\
\hline \multicolumn{2}{|c|}{ BROMOFORM } & $75-25-2$ & $5.913 E-01$ \\
\hline \multicolumn{2}{|c|}{ 1,3-BUTADIENE } & $106-99-0$ & $6.073 E-02$ \\
\hline \multicolumn{2}{|c|}{ BUTANETHIOL } & $109-79-5$ & 7.727E-03 \\
\hline \multicolumn{2}{|c|}{ CADMIUM } & $7440-43-9$ & $1.495 E-03$ \\
\hline
\end{tabular}

a No CAS number 
Table 52

Toxic Air Pollutant Emissions (1998)

Page 2 of 7

\begin{tabular}{|c|c|c|}
\hline Pollutant & $\begin{array}{c}\text { Chemical Abstract } \\
\text { Number (CAS) }\end{array}$ & $\begin{array}{c}\text { Actual Emissions } \\
\text { (Tons/Year) }\end{array}$ \\
\hline CADMIUM OXIDE & $1306-19-0$ & $2.125 E-04$ \\
\hline CALCIUM CYANAMIDE & $156-62-7$ & 7.727E-05 \\
\hline CAPROLACTAM, VAPOR & $105-60-2$ & $1.538 E-03$ \\
\hline CAPTAN & $133-6-2$ & 7.727E-05 \\
\hline CARBARYL & $63-25-2$ & 7.727E-05 \\
\hline CARBON DISULFIDE & $75-15-0$ & 3.417E-03 \\
\hline CARBON TETRACHLORIDE & $56-23-5$ & 2.474E-03 \\
\hline CATECHOL & $120-80-9$ & 7.727E-05 \\
\hline CHLORDANE & $57-74-9$ & 1.487E-03 \\
\hline CHLORINE & $7782-50-5$ & $2.172 E-02$ \\
\hline CHLOROACETIC ACID & $79-11-8$ & 7.727E-05 \\
\hline CHLOROBENZENE & $108-90-7$ & $1.403 E-03$ \\
\hline CHLOROBENZILATE & $510-15-6$ & 7.727E-05 \\
\hline CHLOROFORM & $67-66-3$ & $7.547 E+00$ \\
\hline P-CHLORONITROBENZENE & $100-0-5$ & 7.727E-05 \\
\hline CHLOROPRENE & $126-99-8$ & 7.727E-03 \\
\hline CHROMIUM $(+6)$ COMPOUNDS & a & 5.587E-05 \\
\hline CHROMIUM COMPOUNDS & a & $7.746 \mathrm{E}-04$ \\
\hline COBALT & $7440-48-4$ & $6.365 E-04$ \\
\hline COBALT COMPOUNDS & $\mathbf{a}$ & $6.239 E-06$ \\
\hline COBALT NITRATE HEXAHYDRATE & $10026-22-9$ & $4.843 E-15$ \\
\hline COBALTOUS FORMATE & $544-18-3$ & $1.228 \mathrm{E}-12$ \\
\hline COBALTOUS OXIDE & $1307-96-6$ & $3.285 E-10$ \\
\hline CRESOL & $1319-77-3$ & $7.251 \mathrm{E}-04$ \\
\hline CUMENE & $98-82-8$ & $1.566 \mathrm{E}-02$ \\
\hline CYANAMIDE & $420-4-2$ & 7.727E-03 \\
\hline CYANIDE & $57-12-5$ & $1.079 E-03$ \\
\hline CYANIDE COMPOUNDS & a & $1.157 E-03$ \\
\hline CYANOACETAMIDE & $107-91-5$ & 7.727E-05 \\
\hline
\end{tabular}


Table 52

Toxic Air Pollutant Emissions (1998)

Page 3 of 7

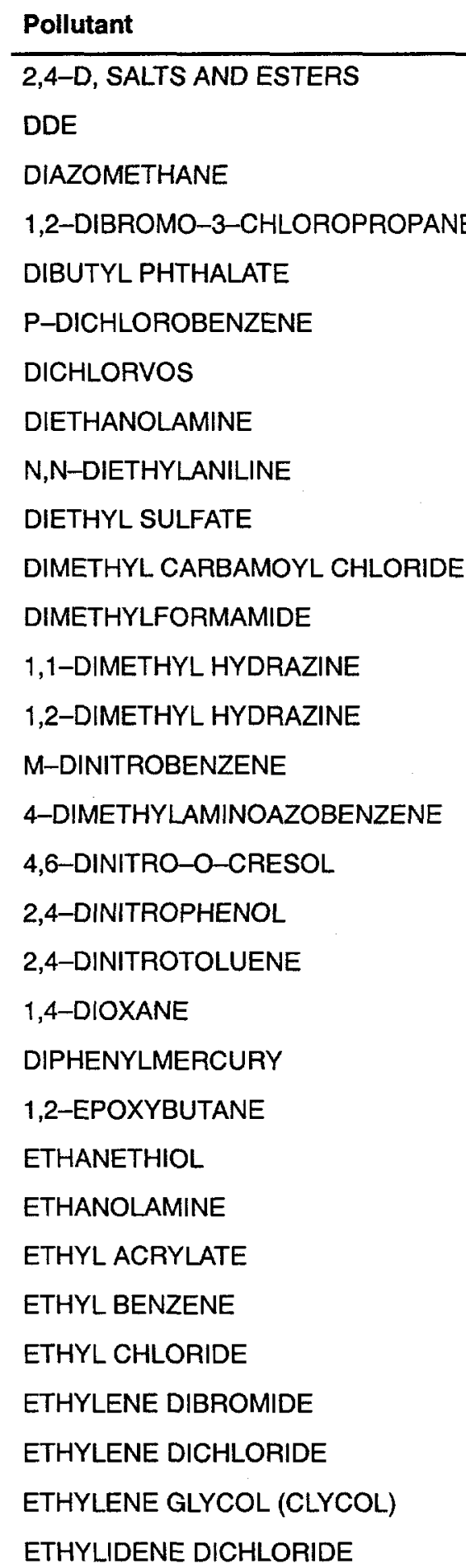

Chemical Abstract Number (CAS)

94-75-7

72-55-9

334-88-3

$96-12-8$

84-74-2

106-46-7

62-73-7

$111-42-2$

$121-69-7$

$64-67-5$

$79-44-7$

68-12-2

57-14-7

540-73-8

99-65-0

$60-11-7$

534-52-1

51-28-5

121-14-2

123-91-1

587-85-9

106-88-7

75-8-1

141-43-5

140-88-5

$100-41-4$

$75-0-3$

106-93-4

107-6-2

107-21-1

75-34-3
Actual Emissions (Tons/Year)

4.123E-04

7.727E-05

7.727E-05

7.727E-05

4.762E-04

$6.495 \mathrm{E}-11$

7.727E-05

1.003E-03

7.727E-05

7.727E-05

7.727E-03

2.081E-05

7.727E-03

7.727E-03

7.777E-05

7.727E-05

7.727E-05

3.790E-07

7.195E-04

$6.920 \mathrm{E}-04$

5.047E-03

7.727E-03

7.727E-03

1.663E-02

7.727E-03

$6.528 \mathrm{E}-01$

8.624E-13

2.816E-06

1.141E-04

2.382E-01

2.595E-03 


\section{Table 52}

Toxic Air Pollutant Emissions (1998)

Page 4 of 7

\begin{tabular}{|c|c|c|}
\hline Pollutant & $\begin{array}{l}\text { Chemical Abstract } \\
\text { Number (CAS) }\end{array}$ & $\begin{array}{l}\text { Actual Emissions } \\
\text { (Tons/Year) }\end{array}$ \\
\hline FORMALDEHYDE & $50-0-0$ & $1.578 E+00$ \\
\hline FORMIC ACID & $64-18-6$ & $3.766 E+00$ \\
\hline FURFURYL ALCOHOL & $98-0-0$ & 7.727E-05 \\
\hline GLYCIDALDEHYDE & $765-34-4$ & $7.727 \mathrm{E}-03$ \\
\hline HEPTACHLOR & $76-44-8$ & $1.455 E-05$ \\
\hline HEPTACHLOR EPOXIDE & $1024-57-3$ & $9.410 \mathrm{E}-08$ \\
\hline HEXACHLOROBENZENE & $118-74-1$ & $1.300 E-04$ \\
\hline HEXACHLOROBUTADIENE & $87-68-3$ & 4.378E-04 \\
\hline HEXACHLOROCYCLOHEXANE & $608-73-1$ & $7.727 E-05$ \\
\hline HEXACHLOROCYLOPENTADIENE & $77-47-4$ & $5.396 \mathrm{E}-04$ \\
\hline HEXACHLOROETHANE & $67-72-1$ & $2.348 E-05$ \\
\hline HEXACHLORONAPTHALENE & $1335-87-1$ & 7.727E-05 \\
\hline HEXAMETHYLENE-1,6-DIISOCYANATOHEXANE & $822-6-0$ & $2.764 E-04$ \\
\hline HEXAMETHYLPHOSPHORAMIDE & $680-31-9$ & $7.727 E-05$ \\
\hline HEXANE & $110-54-3$ & $4.925 E+00$ \\
\hline HYDRAZINE & $302-1-2$ & $6.949 E-04$ \\
\hline HYDROCHLORIC ACID (HYDROGEN CHLORIDE) & $7647-1-0$ & $3.954 E+01$ \\
\hline HYDROGEN CYANIDE & $74-90-8$ & $5.430 E-01$ \\
\hline HYDROGEN SULFIDE & $7783-6-4$ & $5.100 E+00$ \\
\hline HYDROQUINONE & $123-31-9$ & $6.500 E-02$ \\
\hline ISOPHORONE & $78-59-1$ & $7.027 E-03$ \\
\hline KEPONE & $143-50-0$ & 7.727E-05 \\
\hline LEAD ARSENATE & $7645-25-2$ & $7.727 \mathrm{E}-05$ \\
\hline LEAD(+2) ARSENATE & $7784-40-9$ & $7.727 E-05$ \\
\hline LINDANE & $58-89-9$ & $3.742 E-06$ \\
\hline MALATHION & $121-75-5$ & $5.303 E-04$ \\
\hline MALEIC ANHYDRIDE & $108-31-6$ & $5.580 \mathrm{E}-07$ \\
\hline MANGANESE & $7439-96-5$ & $5.137 E-01$ \\
\hline MANGANESE COMPOUNDS & a & $1.212 \mathrm{E}-04$ \\
\hline
\end{tabular}


Table 52

Toxic Air Pollutant Emissions (1998)

Page 5 of 7

\begin{tabular}{|c|c|c|}
\hline Pollutant & $\begin{array}{c}\text { Chemical Abstract } \\
\text { Number (CAS) }\end{array}$ & $\begin{array}{l}\text { Actual Emissions } \\
\text { (Tons/Year) }\end{array}$ \\
\hline MANGANOUS NITRATE & $10377-66-9$ & $5.251 E-16$ \\
\hline MERCURY (ELEMENTAL) & $7439-97-6$ & $5.007 E-02$ \\
\hline METHOXYCHLOR & $72-43-5$ & $2.895 \mathrm{E}-02$ \\
\hline METHYL ALCOHOL & $67-56-1$ & $7.321 E+\infty 0$ \\
\hline METHYL BROMIDE & $74-83-9$ & $3.990 E-10$ \\
\hline METHYL CHLORIDE & $74-87-3$ & $2.491 E-04$ \\
\hline 4,4-METHYLENEDIANILINE & $101-77-9$ & $7.727 E-05$ \\
\hline METHYL ETHYL KETONE (2-BUTANONE) & $78-93-3$ & $1.516 \mathrm{E}-01$ \\
\hline METHYL IODIDE & $74-88-4$ & $3.110 \mathrm{E}-08$ \\
\hline METHYL ISOBUTYL KETONE & $108-10-1$ & $6.504 E-01$ \\
\hline METHYL METHACRYLATE & $80-62-6$ & $1.848 \mathrm{E}-03$ \\
\hline METHYL TERT-BUTYL ETHER & $1634-4-4$ & $7.814 \mathrm{E}-01$ \\
\hline METHYLENE CHLORIDE & $75-9-2$ & $7.533 \mathrm{E}+00$ \\
\hline METHYL CHLOROFORM $(1,1,1-T R I C H L O R O E T H A N E)$ & $71-55-6$ & $5.814 \mathrm{E}-02$ \\
\hline MINERAL OIL & $8012-95-1$ & $3.706 \mathrm{E}-04$ \\
\hline MIREX & $2385-85-5$ & $8.984 \mathrm{E}-05$ \\
\hline NAPHTHALENE & $91-20-3$ & $8.673 E-03$ \\
\hline A-NAPHTHYLAMINE & $134-32-7$ & $7.736 \mathrm{E}-05$ \\
\hline B-NAPHTHYLAMINE & $91-59-8$ & 7.727E-05 \\
\hline NICKEL & $7440-2-0$ & $9.390 \mathrm{E}-02$ \\
\hline NICKEL OXIDE & $1313-99-1$ & $6.153 E-04$ \\
\hline NICKEL SULFATE & $7786-81-4$ & 7.727E-05 \\
\hline NITRIC ACID & $7697-37-2$ & $1.043 E+01$ \\
\hline P-NITROANILINE & $100-1-6$ & $7.852 E-05$ \\
\hline NITROBENZENE & $98-95-3$ & $5.004 \mathrm{E}-03$ \\
\hline 4-NITROBIPHENYL & $92-93-3$ & $7.727 E-05$ \\
\hline NITROGLYCERIN & $55-63-0$ & $7.727 \mathrm{E}-05$ \\
\hline P-NITROPHENOL & $100-2-7$ & $1.192 E-05$ \\
\hline 1-NITROPROPANE & $108-3-2$ & $5.409 E-04$ \\
\hline 2-NITROPROPANE & $79-46-9$ & $5.463 E-04$ \\
\hline N-NITROSO-N-METHYLUREA & $684-93-5$ & $1.545 \mathrm{E}-04$ \\
\hline
\end{tabular}


Table 52

Toxic Air Pollutant Emissions (1998)

Page 6 of 7

\begin{tabular}{|c|c|c|}
\hline Pollutant & $\begin{array}{l}\text { Chemical Abstract } \\
\text { Number (CAS) }\end{array}$ & $\begin{array}{c}\text { Actual Emissions } \\
\text { (Tons/Year) }\end{array}$ \\
\hline N-NITROSODIMETHYLAMINE & $62-75-9$ & $1.545 \mathrm{E}-04$ \\
\hline N-NITROSOMORPHOLINE & $59-89-2$ & 7.727E-05 \\
\hline P-NITROSOPHENOL & $104-91-6$ & $1.961 E-04$ \\
\hline P-NITROTOLUENE & $99-99-0$ & $7.727 E-05$ \\
\hline OCTACHLORONAPHTHALENE & $2234-13-1$ & 7.727E-05 \\
\hline OXALIC ACID & $144-62-7$ & $8.186 \mathrm{E}-03$ \\
\hline PARAQUAT & $1910-42-5$ & $7.727 E-05$ \\
\hline PHENOL & $108-95-2$ & $1.179 \mathrm{E}-01$ \\
\hline P-PHENYLENEDIAMINE & $106-50-3$ & $7.727 E-05$ \\
\hline PHOSPHORIC ACID & $7664-38-2$ & $8.637 E-01$ \\
\hline PICRIC ACID & $88-89-1$ & $7.727 E-05$ \\
\hline POLYCHLORINATED BIPHENYLS (PCB) & a & $1.630 E-03$ \\
\hline POLYCYCLIC ORGANIC MATTER & a & $2.060 \mathrm{E}-02$ \\
\hline POTASSIUM PERMANGANATE & $7722-64-7$ & $1.653 E-04$ \\
\hline POTASSIUM THIOCYANATE & $333-20-0$ & $2.590 \mathrm{E}-04$ \\
\hline PROPANE SULTONE & $1120-71-4$ & $7.727 \mathrm{E}-05$ \\
\hline PROPIONALDEHYDE & $123-38-6$ & $7.727 E-03$ \\
\hline PROPOXUR & $114-26-1$ & $9.727 E-05$ \\
\hline PROPYLENE DICHLORIDE & $78-87-5$ & 7.727E-03 \\
\hline PROPYLENE OXIDE & $75-56-9$ & $4.500 E-07$ \\
\hline 1,2-PROPYLENIMINE & $75-55-8$ & $4.636 \mathrm{E}-04$ \\
\hline PYRETHRUM & $8003-34-7$ & $6.940 E-09$ \\
\hline QUINOLINE & $91-22-5$ & 7.727E-05 \\
\hline QUINONE & $106-51-4$ & $7.727 \mathrm{E}-05$ \\
\hline ROTENONE & $83-79-4$ & $7.727 E-05$ \\
\hline SELENIUM & $7782-49-2$ & $1.171 E-03$ \\
\hline SELENIUM COMPOUNDS & a & 2.707E-05 \\
\hline SELENIUM DIOXIDE & $7446-8-4$ & $6.100 E-04$ \\
\hline SELENIUM SULFIDE & $7446-34-6$ & $8.499 \mathrm{E}-07$ \\
\hline
\end{tabular}

a No CAS number 
Table 52

Toxic Air Pollutant Emissions (1998)

Page 7 of 7

\section{Pollutant}

SODIUM HYDROXIDE (CAUSTIC SODA)

STYRENE

SULFURIC ACID

1,1,2,2-TETRACHLOROETHANE

TETRACHLOROETHYLENE (PERCHLOROETHYLENE)

TITANIUM TETRACHLORIDE

TOLUENE

2,4-TOLUENEDIAMINE

TOLUENE-2,4-DIISOCYNANATE

O-TOLUIDINE

TOXAPHENE

1,2,4-TRICHLOROBENZENE

1,1,2-TRICHLOROETHANE

TRICHLOROETHYLENE (TCE)

2,4,5-TRICHLOROPHENOL

TRIETHYLAMINE

TRIFLURALIN

2,2,4-TRIMETHYLPENTANE

VINYL ACETATE

VINYL CHLORIDE

VINYLIDENE CHLORIDE

(M-XYLENE

XYLENE (MIXED ISOMERS)

O-XYLENE

P-XYLENE

XYLIDINE
Chemical Abstract

Number (CAS)

1310-73-2

$100-42-5$

7664-93-9

79-34-5

$127-18-4$

$7550-45-0$

108-88-3

95-80-7

584-84-9

95-53-4

8001-35-2

120-82-1

79-00-5

$79-1-6$

95-95-4

$121-44-8$

1582-9-8

540-84-1

108-5-4

$75-1-4$

75-35-4

108-38-3

$1330-20-7$

95-47-6

$106-42-3$

$1300-73-8$
Actual Emissions

(Tons/Year)

1.329E+00

1.734E-02

7.648E-02

6.320E-04

1.289E+01

6.954E-04

2.143E+00

7.727E-05

5.319E-06

7.727E-05

8.702E-02

$1.345 \mathrm{E}-04$

6.028E-03

$2.371 E+00$

1.073E-04

2.000E-05

7.727E-05

5.150E-02

7.919E-03

2.268E-03

7.731E-09

2.074E-01

$5.575 \mathrm{E}+00$

5.783E-01

3.022E-01

7.727E-05 
Table 53

National Pollutant Discharge Elimination System Monitoring Data

Page 1 of 10

\section{Permit SC0000175}

Note: Permit levels can be found in WSRC NPDES Environmental Monitoring Program (ESH-EMS-910110) or in the permit itself. The permit is available from the South Carolina Department of Health and Environmental Control.

\begin{tabular}{|c|c|c|c|c|c|c|}
\hline Measurement & Units & $\begin{array}{c}\text { No. of } \\
\text { Samples }\end{array}$ & Maximum & Minimum & $\begin{array}{l}\text { No. in } \\
\text { Average }\end{array}$ & Average \\
\hline
\end{tabular}

\section{Outfall A-01}

Effluent consists of the following types of wastewater: noncontact cooling water, steam condensate, laboratory drain water, cooling tower overflow, steam-cleaning-rack wastewater, well flush water, groundwater air stripper effluent from outfall $A-1 A$, and stormwater.

\begin{tabular}{|c|c|c|c|c|c|c|}
\hline $\mathrm{BOD}$ & $\mathrm{mg} / \mathrm{L}$ & 22 & $6.40 E+00$ & $0.00 E+00$ & 22 & $8.27 E-01$ \\
\hline $\mathrm{CHL}$ & $\mathrm{mg} / \mathrm{L}$ & 42 & 8.00E-02 & $0.00 \mathrm{E}+00$ & 42 & $1.90 E-03$ \\
\hline $\mathrm{CU}$ & $\mathrm{mg} / \mathrm{L}$ & 93 & $1.10 E-01$ & $0.00 E+00$ & 93 & $2.40 \mathrm{E}-02$ \\
\hline FLO & MGD & 30 & $2.59 E+00$ & $1.73 E-01$ & 30 & $1.22 E+00$ \\
\hline$H G$ & $\mathrm{mg} / \mathrm{L}$ & 94 & $2.30 E-04$ & $0.00 \mathrm{E}+00$ & 93 & $4.01 E-05$ \\
\hline $\mathrm{O} \& \mathrm{G}$ & $\mathrm{mg} / \mathrm{L}$ & 12 & $2.10 E+00$ & $0.00 E+00$ & 12 & 7.17E-01 \\
\hline PB & $\mathrm{mg} / \mathrm{L}$ & 93 & $1.10 E-02$ & $0.00 E+00$ & 93 & $1.72 E-04$ \\
\hline PERCL & $\mu g / L$ & 3 & $0.00 E+00$ & $0.00 E+00$ & 3 & $0.00 E+00$ \\
\hline $\mathrm{PH}$ & SU & 12 & $8.29 E+00$ & $6.68 E+00$ & & \\
\hline TMP & DEG C & 12 & $2.30 E+01$ & $1.52 E+01$ & 12 & $1.93 E+01$ \\
\hline TRICL & $\mu g / L$ & 3 & $0.00 E+00$ & $0.00 E+00$ & 3 & $0.00 E+00$ \\
\hline TSS & $\mathrm{mg} / \mathrm{L}$ & 60 & $1.30 E+01$ & $0.00 E+00$ & 60 & $2.15 E+00$ \\
\hline
\end{tabular}

Outfall A-1A

Effluent consists of the following type of wastewater: groundwater air stripper effluent.

$\begin{array}{lllllrr}\text { FLO } & \text { MGD } & 15 & 4.51 E-01 & 7.92 E-02 & 15 & 4.12 E-01 \\ \text { PERCL } & \mu g / L & 15 & 0.00 E+00 & 0.00 E+00 & 15 & 0.00 E+00 \\ \text { TRICL } & \mu g / L & 15 & 2.40 E+00 & 0.00 E+00 & 15 & 1.60 E-01\end{array}$

\section{Outfall A-11}

Effluent consists of the following types of wastewater: fire station building drains, air conditioner condensate, well flush water,noncontact cooling water, steam condensate, liquid effluent treatment facility process effluent from Outfall M-04, air stripper effluent from outfall M-05, and stormwater.

$\begin{array}{lllllll}\text { BOD } & \mathrm{mg} / \mathrm{L} & 12 & 3.53 \mathrm{E}+01 & 0.00 \mathrm{E}+00 & 12 & 3.36 \mathrm{E}+00 \\ \mathrm{CU} & \mathrm{mg} / \mathrm{L} & 12 & 0.00 \mathrm{E}+00 & 0.00 \mathrm{E}+00 & 12 & 0.00 \mathrm{E}+00 \\ \mathrm{FLO} & \mathrm{MGD} & 49 & 2.40 \mathrm{E}+00 & 7.76 \mathrm{E}-02 & 49 & 7.88 \mathrm{E}-01 \\ \mathrm{HG} & \mathrm{mg} / \mathrm{L} & 13 & 1.40 \mathrm{E}-04 & 0.00 \mathrm{E}+00 & 13 & 1.08 \mathrm{E}-05 \\ \mathrm{~PB} & \mathrm{mg} / \mathrm{L} & 12 & 0.00 \mathrm{E}+00 & 0.00 \mathrm{E}+00 & 12 & 0.00 \mathrm{E}+00\end{array}$




\section{Table 53}

National Pollutant Discharge Elimination System Monitoring Data

Page 2 of 10

\section{Permit SC0000175}

\begin{tabular}{|c|c|c|c|c|c|c|}
\hline Measurement & Units & $\begin{array}{c}\text { No. of } \\
\text { Samples }\end{array}$ & Maximum & Minimum & $\begin{array}{c}\text { No. in } \\
\text { Average }\end{array}$ & Average \\
\hline \multicolumn{7}{|c|}{ Outfall A-11 (cont.) } \\
\hline $\mathrm{PH}$ & SU & 12 & $7.46 \mathrm{E}+00$ & $6.47 E+00$ & & \\
\hline TRICL & $\mathrm{mg} / \mathrm{L}$ & 5 & $0.00 \mathrm{E}+00$ & $0.00 E+00$ & 5 & $0.00 E+00$ \\
\hline TSS & mg/L & 12 & $1.00 E+01$ & $1.00 \mathrm{E}+00$ & 12 & $3.58 E+00$ \\
\hline
\end{tabular}

Outfall D-1A

Effluent consists of the following type of wastewater: sanitary wastewater.

\begin{tabular}{|c|c|c|c|c|c|c|}
\hline BOD & $\mathrm{mg} / \mathrm{L}$ & 12 & $1.07 E+01$ & $0.00 E+00$ & 12 & $4.19 E+00$ \\
\hline DO & $\mathrm{mg} / \mathrm{L}$ & 12 & $8.70 E+00$ & $2.50 \mathrm{E}+00$ & 12 & $5.68 \mathrm{E}+00$ \\
\hline FEC & $\mathrm{mg} / \mathrm{L}$ & 12 & $1.40 \mathrm{E}+01$ & $0.00 E+00$ & 12 & $1.67 E+00$ \\
\hline FLO & MGD & 365 & $2.84 E-02$ & $3.04 E-04$ & 365 & $5.63 \mathrm{E}-03$ \\
\hline $\mathrm{PH}$ & SU & 12 & $7.40 \mathrm{E}+00$ & $6.90 E+00$ & & \\
\hline TSS & $\mathrm{mg} / \mathrm{L}$ & 12 & $1.45 \mathrm{E}+01$ & $1.20 E+00$ & 12 & $6.17 \mathrm{E}+00$ \\
\hline
\end{tabular}

Outfall F-01

Effluent consists of the following types of wastewater: nonprocess cooling water, cooling tower blowdown, and stormwater.

$\begin{array}{lllllll}\text { CHL } & \mathrm{mg} / \mathrm{L} & 14 & 1.09 \mathrm{E}+00 & 1.90 \mathrm{E}-01 & 14 & 6.36 \mathrm{E}-01 \\ \mathrm{FLO} & \mathrm{MGD} & 13 & 1.01 \mathrm{E}-01 & 4.32 \mathrm{E}-02 & 13 & 6.99 \mathrm{E}-02 \\ \mathrm{PH} & \mathrm{mg} / \mathrm{L} & 14 & 8.39 \mathrm{E}+00 & 7.11 \mathrm{E}+00 & & \\ \text { TMP } & \mathrm{DEG} \mathrm{C} & 13 & 2.80 \mathrm{E}+01 & 1.66 \mathrm{E}+01 & 13 & 2.25 \mathrm{E}+01 \\ \text { TSS } & \mathrm{mg} / \mathrm{L} & 15 & 3.90 \mathrm{E}+01 & 0.00 \mathrm{E}+00 & 15 & 3.00 \mathrm{E}+00\end{array}$

\section{Outfall F-02}

Effluent consists of the following types of wastewater: nonprocess cooling water, cooling tower blowdown, and stormwater.

$\begin{array}{lllllrr}\text { FLO } & \text { MGD } & 15 & 1.15 \mathrm{E}-01 & 1.44 \mathrm{E}-02 & 15 & 6.11 \mathrm{E}-02 \\ \text { PH } & \text { SU } & 15 & 8.42 \mathrm{E}+00 & 7.22 \mathrm{E}+00 & & \\ \text { TMP } & \text { DEG C } & 15 & 2.80 \mathrm{E}+01 & 1.83 \mathrm{E}+01 & 15 & 2.29 \mathrm{E}+01 \\ \text { TSS } & \mathrm{mg} / \mathrm{L} & 15 & 1.00 \mathrm{E}+00 & 0.00 \mathrm{E}+00 & 15 & 2.00 \mathrm{E}-01\end{array}$

\section{Outfall F-03}

Effluent consists of the following types of wastewater: nonprocess cooling water, steam condensate, cooling tower blowdown, process water, and stormwater.

$\begin{array}{lllllll}\text { FLO } & \text { MGD } & 12 & 0.00 E+00 & 0.00 E+00 & 12 & 0.00 E+00\end{array}$


Table 53

National Pollutant Discharge Elimination System Monitoring Data

Page 3 of 10

Permit SC0000175

\begin{tabular}{|c|c|c|c|c|c|c|}
\hline Measurement & Units & $\begin{array}{c}\text { No. of } \\
\text { Samples }\end{array}$ & Maximum & Minimum & $\begin{array}{c}\text { No. in } \\
\text { Average }\end{array}$ & Average \\
\hline
\end{tabular}

\section{Outfall F-05}

Effluent consists of the following types of wastewater: nonprocess cooling water and steam condensate from the plutonium fabrication facility, well flush water, and stormwater.

$\begin{array}{lllllll}\text { FLO } & \text { MGD } & 15 & 9.79 E-02 & 1.01 E-02 & 15 & 3.76 E-02 \\ \text { O\&G } & \mathrm{mg} / \mathrm{L} & 15 & 7.60 \mathrm{E}+00 & 0.00 \mathrm{E}+00 & 15 & 6.13 \mathrm{E}-01 \\ \mathrm{PH} & \mathrm{SU} & 15 & 8.08 \mathrm{E}+00 & 7.18 \mathrm{E}+00 & & \\ \text { TMP } & \mathrm{DEG} C & 15 & 2.70 \mathrm{E}+01 & 1.69 \mathrm{E}+01 & 15 & 2.22 \mathrm{E}+01 \\ \text { TSS } & \mathrm{MG} / \mathrm{L} & 16 & 1.50 \mathrm{E}+01 & 0.00 \mathrm{E}+00 & 16 & 3.13 \mathrm{E}+00\end{array}$

Outfall F-08

Effluent consists of the following types of wastewater: water from power operations and waste management cooling towers, separations cooling water, steam condensate, process wastewater, laundry effluent, water tank overflow, stormwater, and radiological retention basin cooling water.

$\begin{array}{llccccc}\text { CU } & \mathrm{mg} / \mathrm{L} & 12 & 8.70 \mathrm{E}-03 & 0.00 \mathrm{E}+00 & 12 & 3.06 \mathrm{E}-03 \\ \mathrm{FLO} & \mathrm{MGD} & 365 & 5.56 \mathrm{E}+00 & 5.04 \mathrm{E}-01 & 365 & 1.54 \mathrm{E}+00 \\ \mathrm{HG} & \mathrm{mg} / \mathrm{L} & 12 & 1.00 \mathrm{E}-04 & 0.00 \mathrm{E}+00 & 12 & 1.67 \mathrm{E}-05 \\ \mathrm{~PB} & \mathrm{mg} / \mathrm{L} & 12 & 0.00 \mathrm{E}+00 & 0.00 \mathrm{E}+00 & 12 & 0.00 \mathrm{E}+00 \\ \mathrm{PH} & \mathrm{SU} & 12 & 7.78 \mathrm{E}+00 & 6.71 \mathrm{E}+00 & & \\ \mathrm{TMP} & \mathrm{DEG} \mathrm{C} & 12 & 2.90 \mathrm{E}+01 & 1.80 \mathrm{E}+01 & 12 & 2.43 \mathrm{E}+01 \\ \mathrm{TSS} & \mathrm{mg} / \mathrm{L} & 12 & 4.00 \mathrm{E}+00 & 0.00 \mathrm{E}+00 & 12 & 2.17 \mathrm{E}+00\end{array}$

\section{Outfall G-10}

Effluent consists of the following type of wastewater: Centralized Sanitary Wastewater Treatment Facility wastewater.

$\begin{array}{lcccccc}\text { AN-Summer } & \mathrm{mg} / \mathrm{L} & 8 & 6.96 \mathrm{E}+00 & 1.83 \mathrm{E}-01 & 8 & 1.40 \mathrm{E}+00 \\ \text { AN-Winter } & \mathrm{mg} / \mathrm{L} & 16 & 1.64 \mathrm{E}+00 & 1.24 \mathrm{E}-01 & 16 & 4.37 \mathrm{E}-01 \\ \text { BOD } & \mathrm{mg} / \mathrm{L} & 24 & 1.23 \mathrm{E}+01 & 3.40 \mathrm{E}+00 & 24 & 6.29 \mathrm{E}+00 \\ \mathrm{CU} & \mathrm{mg} / \mathrm{L} & 13 & 5.20 \mathrm{E}-02 & 1.70 \mathrm{E}-02 & 13 & 2.75 \mathrm{E}-02 \\ \text { DO } & \mathrm{mg} / \mathrm{L} & 26 & 9.90 \mathrm{E}+00 & 5.30 \mathrm{E}+00 & 26 & 6.96 \mathrm{E}+00 \\ \mathrm{FEC} & \mathrm{mg} / \mathrm{L} & 24 & 1.20 \mathrm{E}+02 & 0.00 \mathrm{E}+00 & 24 & 1.53 \mathrm{E}+01 \\ \text { FLO } & \mathrm{MGD} & 365 & 4.99 \mathrm{E}-01 & 1.73 \mathrm{E}-02 & 365 & 1.81 \mathrm{E}-01 \\ \mathrm{~PB} & \mathrm{MG} / \mathrm{L} & 13 & 3.90 \mathrm{E}-02 & 0.00 \mathrm{E}+00 & 13 & 3.00 \mathrm{E}-03 \\ \mathrm{PH} & \mathrm{SU} & 26 & 7.80 \mathrm{E}+00 & 6.80 \mathrm{E}+00 & & \\ \text { TSS } & \mathrm{mg} / \mathrm{L} & 24 & 3.04 \mathrm{E}+01 & 3.20 \mathrm{E}+00 & 24 & 1.56 \mathrm{E}+01\end{array}$


Table 53

National Pollutant Discharge Elimination System Monitoring Data

Page 4 of 10

Permit SC0000175

\begin{tabular}{|c|c|c|c|c|c|c|}
\hline Measurement & Units & $\begin{array}{c}\text { No. of } \\
\text { Samples }\end{array}$ & Maximum & Minimum & $\begin{array}{c}\text { No. in } \\
\text { Average }\end{array}$ & Average \\
\hline
\end{tabular}

Outfall H-02

Effluent consists of the following types of wastewater: nonprocess cooling water and stormwater.

$\begin{array}{llcllll}\text { FLO } & \text { MGD } & 365 & 7.76 E-01 & 6.46 E-03 & 365 & 7.36 \mathrm{E}-02 \\ \text { O\&G } & \mathrm{mg} / \mathrm{L} & 14 & 1.00 \mathrm{E}+00 & 0.00 \mathrm{E}+00 & 14 & 7.14 \mathrm{E}-02 \\ \mathrm{PH} & \text { SU } & 13 & 8.41 \mathrm{E}+00 & 6.64 \mathrm{E}+00 & & \\ \text { TMP } & \text { DEG C } & 14 & 2.60 \mathrm{E}+01 & 9.00 \mathrm{E}+00 & 14 & 1.98 \mathrm{E}+01 \\ \text { TSS } & \mathrm{mg} / \mathrm{L} & 14 & 3.00 \mathrm{E}+00 & 0.00 \mathrm{E}+00 & 14 & 1.57 \mathrm{E}+00\end{array}$

Outfall $\mathrm{H}-04$

Effluent consists of the following types of wastewater: nonprocess cooling water, CIF nonprocess wastewater, steam condensate, and stormwater.

$\begin{array}{lllllll}\text { FLO } & \text { MGD } & 15 & 1.58 \mathrm{E}-02 & 2.88 \mathrm{E}-03 & 15 & 7.18 \mathrm{E}-03 \\ \mathrm{PH} & \mathrm{SU} & 15 & 7.52 \mathrm{E}+00 & 6.48 \mathrm{E}+00 & 15 & 6.84 \mathrm{E}+00 \\ \mathrm{TMP} & \mathrm{mg} / \mathrm{L} & 15 & 2.60 \mathrm{E}+01 & 9.00 \mathrm{E}+00 & 15 & 2.00 \mathrm{E}+01 \\ \mathrm{TSS} & \mathrm{mg} / \mathrm{L} & 14 & 2.00 \mathrm{E}+00 & 0.00 \mathrm{E}+00 & 14 & 6.43 \mathrm{E}-01\end{array}$

Outfall H-07

Effluent consists of the following types of wastewater: air compressor cooling water, cooling tower blowdown, and stormwater.

$\begin{array}{llcllll}\text { FLO } & \mathrm{mg} / \mathrm{L} & 13 & 6.52 \mathrm{E}-03 & 0.00 \mathrm{E}+00 & 13 & 6.76 \mathrm{E}-04 \\ \mathrm{O} \& \mathrm{G} & \mathrm{mg} / \mathrm{L} & 2 & 0.00 \mathrm{E}+00 & 0.00 \mathrm{E}+00 & 2 & 0.00 \mathrm{E}+00 \\ \mathrm{PH} & \mathrm{SU} & 2 & 6.15 \mathrm{E}+00 & 5.79 \mathrm{E}+00 & & \\ \text { TSS } & \mathrm{mg} / \mathrm{L} & 2 & 3.00 \mathrm{E}+00 & 3.00 \mathrm{E}+00 & 2 & 3.00 \mathrm{E}+00\end{array}$

Outfall H-08

Effluent consists of the following types of wastewater: water from power operations and waste management cooling towers, steam condensate, ash basin overflow, water tank overflow, neutralization system discharge, water laboratory discharge, well-flush water, powerhouse drains, and stormwater.

FLO

$\mathrm{PB}$

$\mathrm{PH}$

TSS

\begin{abstract}
MGD
$2.45 \mathrm{E}+00$
\end{abstract}

$\mathrm{mg} / \mathrm{L}$

$\mathrm{mg} / \mathrm{L}$

$\mathrm{mg} / \mathrm{L}$
$0.00 E+00$

$8.41 \mathrm{E}+00$

$3.00 \mathrm{E}+00$
3.17E-01

$6.36 \mathrm{E}+00$

$0.00 \mathrm{E}+00$
49

12

8.87E-01

$0.00 E+00$

12

$2.00 E+00$ 
Table 53

National Pollutant Discharge Elimination System Monitoring Data

Page 5 of 10

Permit SC0000175

\begin{tabular}{|c|c|c|c|c|c|c|}
\hline Measurement & Units & $\begin{array}{l}\text { No. of } \\
\text { Samples }\end{array}$ & Maximum & Minimum & $\begin{array}{l}\text { No. in } \\
\text { Average }\end{array}$ & Average \\
\hline
\end{tabular}

Outfall $\mathrm{H}-12$

Effluent consists of the following types of wastewater: nonprocess cooling water, cooling tower and air compressor blowdown, stormwater, In-tank Precipitation Facility neutralized flush water, and radiological retention basin cooling water.

$\mathrm{CU}$

FLO

PB

$\mathrm{PH}$

TMP

TSS $\mathrm{mg} / \mathrm{L} \quad 12$

$\mathrm{mg} / \mathrm{L}$

$\mathrm{mg} / \mathrm{L}$

SU

$\mathrm{mg} / \mathrm{L}$

$\mathrm{mg} / \mathrm{L}$
1.50E-02

$4.43 E+00$

$0.00 E+00$

$7.69 \mathrm{E}+00$

$2.70 \mathrm{E}+01$

$2.00 \mathrm{E}+00$
$0.00 E+00$

5.49E-02

$0.00 \mathrm{E}+00$

$6.61 E+00$

$1.20 \mathrm{E}+01$

$0.00 E+00$
12

41

12

12

12
4.63E-03

5.79E-01

$0.00 E+00$

2.06E+01

6.67E-01

Outfall H-16

Effluent consists of the following type of wastewater: F-Area/H-Area Effluent Treatment Facility process wastewater.

$\begin{array}{lllllll}\text { AG } & \mathrm{mg} / \mathrm{L} & 47 & 0.00 \mathrm{E}+00 & 0.00 \mathrm{E}+00 & 47 & 0.00 \mathrm{E}+00 \\ \mathrm{BOD} & \mathrm{mg} / \mathrm{L} & 47 & 3.07 \mathrm{E}+01 & 0.00 \mathrm{E}+00 & 47 & 2.75 \mathrm{E}+00 \\ \mathrm{CD} & \mathrm{mg} / \mathrm{L} & 47 & 0.00 \mathrm{E}+00 & 0.00 \mathrm{E}+00 & 47 & 0.00 \mathrm{E}+00 \\ \mathrm{CR} & \mathrm{mg} / \mathrm{L} & 47 & 1.90 \mathrm{E}-02 & 0.00 \mathrm{E}+00 & 47 & 5.72 \mathrm{E}-04 \\ \mathrm{CU} & \mathrm{mg} / \mathrm{L} & 47 & 9.10 \mathrm{E}-03 & 0.00 \mathrm{E}+00 & 47 & 5.70 \mathrm{E}-04 \\ \mathrm{FLO} & \mathrm{MGD} & 47 & 1.58 \mathrm{E}-01 & 5.31 \mathrm{E}-02 & 47 & 1.12 \mathrm{E}-01 \\ \mathrm{HG} & \mathrm{mg} / \mathrm{L} & 47 & 3.00 \mathrm{E}-03 & 0.00 \mathrm{E}+00 & 47 & 1.56 \mathrm{E}-04 \\ \mathrm{NI} & \mathrm{mg} / \mathrm{L} & 47 & 1.20 \mathrm{E}+00 & 0.00 \mathrm{E}+00 & 47 & 2.55 \mathrm{E}-02 \\ \mathrm{O} \& \mathrm{G} & \mathrm{mg} / \mathrm{L} & 47 & 5.30 \mathrm{E}+00 & 0.00 \mathrm{E}+00 & 47 & 4.40 \mathrm{E}-01 \\ \mathrm{~PB} & \mathrm{mg} / \mathrm{L} & 47 & 0.00 \mathrm{E}+00 & 0.00 \mathrm{E}+00 & 47 & 0.00 \mathrm{E}+00 \\ \mathrm{PH} & \mathrm{SU} & 47 & 8.05 \mathrm{E}+00 & 6.23 \mathrm{E}+00 & 0.00 & \\ \text { TSS } & \mathrm{mg} / \mathrm{L} & 47 & 1.30 \mathrm{E}+01 & 0.00 \mathrm{E}+00 & 47 & 2.17 \mathrm{E}+00 \\ \text { ZN } & \mathrm{mg} / \mathrm{L} & 47 & 9.90 \mathrm{E}-02 & 0.00 \mathrm{E}+00 & 47 & 2.28 \mathrm{E}-02\end{array}$

Outfall K-06

Effluent consists of the following types of wastewater: powerhouse wastewater, package boiler blowdown, cooling tower overflow, and stormwater.

$\begin{array}{llcccrr}\text { CHL } & \mathrm{mg} / \mathrm{L} & 4 & 5.00 \mathrm{E}-02 & 0.00 \mathrm{E}+00 & 4 & 1.25 \mathrm{E}-02 \\ \mathrm{FLO} & \mathrm{MGD} & 13 & 1.12 \mathrm{E}-01 & 0.00 \mathrm{E}+00 & 13 & 1.68 \mathrm{E}-02 \\ \mathrm{O} \& \mathrm{G} & \mathrm{mg} / \mathrm{L} & 5 & 1.40 \mathrm{E}+00 & 0.00 \mathrm{E}+00 & 5 & 7.40 \mathrm{E}-01 \\ \mathrm{PH} & \mathrm{SU} & 5 & 8.01 \mathrm{E}+00 & 7.12 \mathrm{E}+00 & & \end{array}$


Table 53

National Pollutant Discharge Elimination System Monitoring Data

Page 6 of 10

\section{Permit SC0000175}

\begin{tabular}{lcccccc}
\hline Measurement & Units & $\begin{array}{c}\text { No. of } \\
\text { Samples }\end{array}$ & Maximum & Minimum & $\begin{array}{c}\text { No. in } \\
\text { Average }\end{array}$ & Average \\
\hline $\begin{array}{l}\text { Outfall K-06(cont.) } \\
\text { TSS }\end{array}$ & $\mathrm{mg} / \mathrm{L}$ & 5 & $9.00 \mathrm{E}+00$ & $0.00 \mathrm{E}+00$ & 5 & $2.60 \mathrm{E}+00$
\end{tabular}

Outfall K-10

Effluent consists of the following type of wastewater: Infrequent diversion from outfall K-18.
FLO
MGD
12
$0.00 E+00$
$0.00 E+00$
12
$0.00 E+00$

Outfall K-12

Effluent consists of the following type of wastewater: sanitary wastewater.

$\begin{array}{llccccc}\text { BOD } & \mathrm{mg} / \mathrm{L} & 11 & 8.40 \mathrm{E}+00 & 0.00 \mathrm{E}+00 & 11 & 4.46 \mathrm{E}+00 \\ \text { DO } & \mathrm{mg} / \mathrm{L} & 11 & 7.80 \mathrm{E}+00 & 1.20 \mathrm{E}+00 & 11 & 3.70 \mathrm{E}+00 \\ \text { FEC } & \mathrm{mg} / \mathrm{L} & 11 & 6.00 \mathrm{E}+00 & 0.00 \mathrm{E}+00 & 11 & 1.27 \mathrm{E}+00 \\ \text { FLO } & \mathrm{MGD} & 365 & 1.42 \mathrm{E}-02 & 0.00 \mathrm{E}+00 & 365 & 3.76 \mathrm{E}-03 \\ \mathrm{PH} & \mathrm{SU} & 12 & 7.60 \mathrm{E}+00 & 7.00 \mathrm{E}+00 & & \\ \text { TSS } & \mathrm{mg} / \mathrm{L} & 12 & 1.06 \mathrm{E}+01 & 8.00 \mathrm{E}-01 & 12 & 5.09 \mathrm{E}+00\end{array}$

\section{Outfall K-18}

Effluent consists of the following types of wastewater: building 105-K noncontact cooling water and process sewer flow, 186-K Basin overflow, and water plant wastewater and treated sanitary wastewater from outfall $K-12$.

$\begin{array}{lllllll}\mathrm{AL} & \mathrm{mg} / \mathrm{L} & 6 & 2.40 \mathrm{E}-01 & 0.00 \mathrm{E}+00 & 6 & 1.31 \mathrm{E}-01 \\ \mathrm{FLO} & \mathrm{MGD} & 6 & 4.52 \mathrm{E}-01 & 2.87 \mathrm{E}-01 & 6 & 4.00 \mathrm{E}-01 \\ \mathrm{O} \& \mathrm{G} & \mathrm{mg} / \mathrm{L} & 6 & 0.00 \mathrm{E}+00 & 0.00 \mathrm{E}+00 & 6 & 0.00 \mathrm{E}+00 \\ \mathrm{PH} & \mathrm{SU} & 6 & 7.74 \mathrm{E}+00 & 6.98 \mathrm{E}+00 & & \\ \mathrm{TSS} & \mathrm{mg} / \mathrm{L} & 6 & 5.00 \mathrm{E}+00 & 1.00 \mathrm{E}+00 & 6 & 2.83 \mathrm{E}+00\end{array}$

\section{Outfall $L-07$}

Effluent consists of the following types of wastewater: 186-L Basin overflow, treated sanitary wastewater from outfall $L-7 A$, and process sewer/reactor building drains.

$\begin{array}{llccccr}\text { FLO } & \mathrm{MGD} & 365 & 9.05 \mathrm{E}+00 & 6.01 \mathrm{E}+00 & 365 & 6.90 \mathrm{E}+00 \\ \text { O\&G } & \mathrm{mg} / \mathrm{L} & 6 & 1.50 \mathrm{E}+00 & 0.00 \mathrm{E}+00 & 6 & 2.50 \mathrm{E}-01 \\ \mathrm{PH} & \mathrm{SU} & 6 & 8.19 \mathrm{E}+00 & 7.01 \mathrm{E}+00 & & \\ \mathrm{TSS} & \mathrm{mg} / \mathrm{L} & 6 & 1.20 \mathrm{E}+01 & 0.00 \mathrm{E}+00 & 6 & 3.83 \mathrm{E}+00\end{array}$


Table 53

National Pollutant Discharge Elimination System Monitoring Data

Page 7 of 10

Permit SC0000175

\begin{tabular}{lcccccc}
\hline Measurement & Units & $\begin{array}{c}\text { No. of } \\
\text { Samples }\end{array}$ & Maximum & Minimum & $\begin{array}{c}\text { No. in } \\
\text { Average }\end{array}$ & Average \\
\hline Outfall L-7A & \multicolumn{7}{c}{ Effluent consists of the following type of wastewater: sanitary wastewater. } \\
BOD & $\mathrm{mg} / \mathrm{L}$ & 12 & $6.60 \mathrm{E}+00$ & $0.00 \mathrm{E}+00$ & 12 & $2.36 \mathrm{E}+00$ \\
DO & $\mathrm{mg} / \mathrm{L}$ & 12 & $8.90 \mathrm{E}+00$ & $3.80 \mathrm{E}+00$ & 12 & $5.66 \mathrm{E}+00$ \\
FEC & $\# / 100 \mathrm{~mL}$ & 12 & $8.00 \mathrm{E}+00$ & $0.00 \mathrm{E}+00$ & 12 & $1.00 \mathrm{E}+00$ \\
FLO & $\mathrm{MGD}$ & 365 & $3.37 \mathrm{E}-02$ & $0.00 \mathrm{E}+00$ & 365 & $2.63 \mathrm{E}-03$ \\
$\mathrm{PH}$ & $\mathrm{SU}$ & 12 & $7.40 \mathrm{E}+00$ & $6.70 \mathrm{E}+00$ & & \\
TSS & $\mathrm{mg} / \mathrm{L}$ & 12 & $1.72 \mathrm{E}+01$ & $3.60 \mathrm{E}+00$ & 12 & $7.83 \mathrm{E}+00$
\end{tabular}

Outfall L-08

Effluent consists of the following types of wastewater: engine house cooling water and stormwater.

$\begin{array}{lllllll}\text { FLO } & \text { MGD } & 6 & 8.35 \mathrm{E}-02 & 2.26 \mathrm{E}-02 & 6 & 5.72 \mathrm{E}-02 \\ \mathrm{O \& G} & \mathrm{mg} / \mathrm{L} & 6 & 1.40 \mathrm{E}+00 & 0.00 \mathrm{E}+00 & 6 & 2.33 \mathrm{E}-01 \\ \mathrm{PH} & \mathrm{SU} & 6 & 7.70 \mathrm{E}+00 & 6.60 \mathrm{E}+00 & & \\ \text { TSS } & \mathrm{mg} / \mathrm{L} & 6 & 7.00 \mathrm{E}+00 & 0.00 \mathrm{E}+00 & 6 & 1.67 \mathrm{E}+00\end{array}$

Outfall M-04

Effluent consists of the following types of wastewater: Liquid Effluent Treatment Facility process wastewater.

$\begin{array}{lllllll}\mathrm{AL} & \mathrm{mg} / \mathrm{L} & 8 & 2.50 \mathrm{E}-01 & 0.00 \mathrm{E}+00 & 8 & 1.16 \mathrm{E}-01 \\ \mathrm{AN} & \mathrm{mg} / \mathrm{L} & 8 & 4.53 \mathrm{E}+00 & 0.00 \mathrm{E}+00 & 8 & 1.61 \mathrm{E}+00 \\ \mathrm{CN} & \mathrm{mg} / \mathrm{L} & 8 & 1.00 \mathrm{E}-02 & 0.00 \mathrm{E}+00 & 8 & 2.50 \mathrm{E}-03 \\ \mathrm{CR} & \mathrm{mg} / \mathrm{L} & 8 & 0.00 \mathrm{E}+00 & 0.00 \mathrm{E}+00 & 8 & 0.00 \mathrm{E}+00 \\ \mathrm{FLO} & \mathrm{MGD} & 8 & 7.20 \mathrm{E}-03 & 4.31 \mathrm{E}-03 & 8 & 5.84 \mathrm{E}-03 \\ \mathrm{NI} & \mathrm{mg} / \mathrm{L} & 8 & 7.60 \mathrm{E}-02 & 3.80 \mathrm{E}-02 & 8 & 5.15 \mathrm{E}-02 \\ \mathrm{~PB} & \mathrm{mg} / \mathrm{L} & 8 & 1.50 \mathrm{E}-02 & 0.00 \mathrm{E}+00 & 8 & 1.88 \mathrm{E}-03 \\ \mathrm{PH} & \mathrm{sU} & 8 & 7.37 \mathrm{E}+00 & 6.47 \mathrm{E}+00 & & \\ \mathrm{TSS} & \mathrm{mg} / \mathrm{L} & 8 & 4.00 \mathrm{E}+00 & 0.00 \mathrm{E}+00 & 8 & 1.13 \mathrm{E}+00\end{array}$

Outfall M-05

Effluent consists of the following type of wastewater: groundwater air stripper effluent.

$\begin{array}{lllllll}\text { FLO } & \text { MGD } & 15 & 7.20 \mathrm{E}-01 & 5.76 \mathrm{E}-01 & 15 & 6.18 \mathrm{E}-01 \\ \text { PERCL } & \mu \mathrm{g} / \mathrm{L} & 15 & 0.00 \mathrm{E}+00 & 0.00 \mathrm{E}+00 & 15 & 0.00 \mathrm{E}+00 \\ \text { TRICL } & \mu \mathrm{g} / \mathrm{L} & 15 & 0.00 \mathrm{E}+00 & 0.00 \mathrm{E}+00 & 15 & 0.00 \mathrm{E}+00\end{array}$




\section{Table 53}

\section{National Pollutant Discharge Elimination System Monitoring Data}

Page 8 of 10

\section{Permit SC0000175}

\begin{tabular}{|c|c|c|c|c|c|c|}
\hline Measurement & Units & $\begin{array}{c}\text { No. of } \\
\text { Samples }\end{array}$ & Maximum & Minimum & $\begin{array}{l}\text { No. in } \\
\text { Average }\end{array}$ & Average \\
\hline
\end{tabular}

\section{Outfall PP-1}

Effluent consists of the following type of wastewater: manganese greensand filter backwash/rinse water.

$\begin{array}{lllllll}\text { FE } & \mathrm{mg} / \mathrm{L} & 4 & 2.91 \mathrm{E}-01 & 9.90 \mathrm{E}-02 & 4 & 2.10 \mathrm{E}-01 \\ \text { FLO } & \text { MGD } & 5 & 1.22 \mathrm{E}-03 & 3.28 \mathrm{E}-04 & 5 & 8.02 \mathrm{E}-04 \\ \mathrm{MN} & \mathrm{mg} / \mathrm{L} & 5 & 1.26 \mathrm{E}+01 & 1.70 \mathrm{E}+00 & 5 & 7.34 \mathrm{E}+00 \\ \mathrm{PH} & \mathrm{SU} & 6 & 6.89 \mathrm{E}+00 & 6.29 \mathrm{E}+00 & & \\ \text { TSS } & \mathrm{mg} / \mathrm{L} & 5 & 3.00 \mathrm{E}+00 & 0.00 \mathrm{E}+00 & 5 & 1.40 \mathrm{E}+00\end{array}$

\section{Outfall S-04}

Effluent consists of the following types of wastewater: DWPF nonprocess wastewater-including cooling tower blowdown, neutralization wastewater, and infrequent flushing/rinsing activities.

$\begin{array}{lllllll}\text { FLO } & \text { MGD } & 12 & 1.24 \mathrm{E}-01 & 7.49 \mathrm{E}-02 & 12 & 9.45 \mathrm{E}-02 \\ \mathrm{PH} & \mathrm{SU} & 12 & 8.22 \mathrm{E}+00 & 7.29 \mathrm{E}+00 & & \\ \mathrm{TSS} & \mathrm{mg} / \mathrm{L} & 12 & 1.40 \mathrm{E}+01 & 0.00 \mathrm{E}+00 & 12 & 2.92 \mathrm{E}+00 \\ \mathrm{ZN} & \mathrm{mg} / \mathrm{L} & 12 & 3.70 \mathrm{E}+00 & 7.40 \mathrm{E}-02 & 12 & 5.61 \mathrm{E}-01\end{array}$

\section{Outfall $X-04$}

Effluent consists of the following types of wastewater: welding quench sink water, steam condensate, and stormwater.

$\begin{array}{lllllll}\text { FLO } & \text { MGD } & 13 & 1.96 E-02 & 2.59 E-04 & 13 & 4.08 E-03 \\ \text { PH } & \text { SU } & 14 & 7.50 E+00 & 4.23 E+00 & & \\ \text { TSS } & \mathrm{mg} / \mathrm{L} & 14 & 4.00 \mathrm{E}+00 & 0.00 \mathrm{E}+00 & 14 & 1.00 \mathrm{E}+00\end{array}$

\section{Outfall $X-08$}

Effluent consists of the following types of wastewater: noncontact cooling water, domestic well overflow, treated sanitary wastewater from outfall $X-8 A$, treated process water from outfall $X-8 B$, treated groundwater from outfall $X-8 C$, and stormwater.

$\begin{array}{lllllll}\text { FLO } & \text { MGD } & 14 & 5.90 E-01 & 1.73 E-01 & 14 & 3.39 E-01 \\ \text { PH } & \text { SU } & 14 & 7.47 E+00 & 6.15 E+00 & & \\ \text { TSS } & \mathrm{mg} / \mathrm{L} & 14 & 3.00 \mathrm{E}+00 & 0.00 \mathrm{E}+00 & 14 & 1.21 \mathrm{E}+00\end{array}$

\section{Outfall X-8A}

Effluent consists of the following type of wastewater: sanitary wastewater. (Note: one data point, for fecal coliform, was a > value and was used in the computation at face value.)

$\begin{array}{lllllll}\text { BOD } & \mathrm{mg} / \mathrm{L} & 11 & 1.07 \mathrm{E}+01 & 0.00 \mathrm{E}+00 & 11 & 4.88 \mathrm{E}+00 \\ \text { DO } & \mathrm{mg} / \mathrm{L} & 11 & 10.0 \mathrm{E}+00 & 4.00 \mathrm{E}+00 & 11 & 7.54 \mathrm{E}+00 \\ \text { FEC } & \# / 100 \mathrm{~mL} & 11 & 2.00 \mathrm{E}+00 & 0.00 \mathrm{E}+00 & 11 & 1.82 \mathrm{E}-01\end{array}$


Table 53

National Pollutant Discharge Elimination System Monitoring Data

Page 9 of 10

Permit SC0000175

\begin{tabular}{lcccccc}
\hline Measurement & Units & $\begin{array}{c}\text { No. of } \\
\text { Samples }\end{array}$ & Maximum & Minimum & $\begin{array}{c}\text { No. in } \\
\text { Average }\end{array}$ & Average \\
\hline Outfall X-8A (cont.) & & & & & & \\
FLO & MGD & 365 & $4.75 \mathrm{E}-03$ & $0.00 \mathrm{E}+00$ & 365 & $1.62 \mathrm{E}-03$ \\
PH & SU & 11 & $7.40 \mathrm{E}+00$ & $6.40 \mathrm{E}+00$ & & \\
TSS & $\mathrm{mg} / \mathrm{L}$ & 11 & $1.60 \mathrm{E}+01$ & $0.00 \mathrm{E}+00$ & 11 & $7.85 \mathrm{E}+00$
\end{tabular}

Outfall X-8B

Effluent consists of the following type of wastewater: treated process water from the TNX Effluent Treatment Plant.

$\begin{array}{lllllll}\text { BEN } & \mu \mathrm{g} / \mathrm{L} & 22 & 1.10 \mathrm{E}+00 & 0.00 \mathrm{E}+00 & 22 & 5.00 \mathrm{E}-02 \\ \mathrm{BOD} & \mathrm{mg} / \mathrm{L} & 22 & 3.70 \mathrm{E}+00 & 0.00 \mathrm{E}+00 & 22 & 4.86 \mathrm{E}-01 \\ \mathrm{C} & \mathrm{mg} / \mathrm{L} & 22 & 1.16 \mathrm{E}+01 & 0.00 \mathrm{E}+00 & 22 & 3.90 \mathrm{E}+00 \\ \mathrm{FLO} & \mathrm{MGD} & 23 & 2.40 \mathrm{E}-02 & 1.00 \mathrm{E}-03 & 23 & 2.07 \mathrm{E}-02 \\ \mathrm{HG} & \mathrm{mg} / \mathrm{L} & 22 & 5.80 \mathrm{E}-03 & 0.00 \mathrm{E}+00 & 22 & 9.54 \mathrm{E}-04 \\ \mathrm{PH} & \mathrm{SU} & 22 & 7.56 \mathrm{E}+00 & 7.02 \mathrm{E}+00 & & \\ \mathrm{PHE} & \mathrm{mg} / \mathrm{L} & 21 & 2.30 \mathrm{E}-02 & 0.00 \mathrm{E}+00 & 21 & 4.47 \mathrm{E}-03 \\ \mathrm{TSS} & \mathrm{mg} / \mathrm{L} & 22 & 1.20 \mathrm{E}+01 & 1.00 \mathrm{E}+00 & 22 & 4.68 \mathrm{E}+00\end{array}$

Outfall X-8C

Effluent consists of the following type of wastewater: treated groundwater air stripper effluent.

$\begin{array}{lllllll}\text { FLO } & \text { MGD } & 12 & 1.17 E-01 & 0.00 E+00 & 12 & 6.84 E-02 \\ \text { PERCL } & \mu g / L & 12 & 0.00 E+00 & 0.00 E+00 & 12 & 0.00 E+00 \\ \text { TRICL } & \mu g / L & 12 & 0.00 E+00 & 0.00 E+00 & 12 & 0.00 E+00\end{array}$

Outfall X-19

Effluent consists of the following type of wastewater: treated groundwater.

$\begin{array}{llcllll}1,2-D C E & \mu g / L & 2 & 0.00 E+00 & 0.00 E+00 & 2 & 0.00 E+00 \\ \text { FLO } & M G D & 12 & 0.00 E+00 & 0.00 E+00 & 12 & 8.88 E-04 \\ \text { PERCL } & \mu g / L & 2 & 0.00 E+00 & 0.00 E+00 & 2 & 0.00 E+00 \\ \text { TRICL } & \mu g / L & 2 & 0.00 E+00 & 0.00 E+00 & 2 & 2.85 E+00 \\ V C & \mu g / L & 2 & 0.00 E+00 & 0.00 E+00 & 2 & 0.00 E+00\end{array}$


Table 53

National Pollutant Discharge Elimination System Monitoring Data

Page 10 of 10

\begin{tabular}{|c|c|c|c|c|c|c|}
\hline Measurement & Units & $\begin{array}{c}\text { No. of } \\
\text { Samples }\end{array}$ & Maximum & Minimum & $\begin{array}{c}\text { No. in } \\
\text { Average }\end{array}$ & Average \\
\hline
\end{tabular}

Permit SCG250162

Note: Permit levels can be found in WSRC NPDES Environmental Monitoring Program (ESH-EMS-910110) or in the permit itself. The permit is available from the South Carolina Department of Health and Environmental Control.

\begin{tabular}{|c|c|c|c|c|c|c|}
\hline Measurement & Units & $\begin{array}{c}\text { No. of } \\
\text { Samples }\end{array}$ & Maximum & Minimum & $\begin{array}{l}\text { No. in } \\
\text { Average }\end{array}$ & Average \\
\hline
\end{tabular}

\section{Outfall 001}

Effluent consists of the following types of wastewater: once-through noncontact cooling water.
FLO
MGD
5
$0.00 \mathrm{E}+00$
$0.00 \mathrm{E}+00$
5
$0.00 E+00$ 
Table 54

National Pollutant Discharge Elimination System Toxicity Monitoring Data

Page 1 of 1

\begin{tabular}{llll}
\hline Measurement & Number of Samples & Pass & Fail \\
\hline
\end{tabular}

\section{Permit SC0000175}

Note: Toxicity requirements can be found in WSRC's NPDES industrial discharge permit, which is available from the South Carolina Department of Health and Environmental Control. Toxicity analysis is a pass/fail test; failure indicates that the tested water was toxic to the test organisms.

\section{Outfall A-01}

Effluent consists of the following types of wastewater: noncontact cooling water, steam condensate, laboratory drain water, cooling tower overflow, steam cleaning oil/water separation, well-flushing operations, air conditioning condensate, A-1A air stripper discharge, and stormwater.

Chronic Toxicity

12

0

12

\section{Outfall A-11}

Effluent consists of the following types of wastewater: fire station floor drains, air conditioning condensate, well flushing operations, cooling water, steam condensate, Liquid Effluent Treatment Facility discharge, M-Area air stripper discharge, and stormwater.

Chronic Toxicity

5

7

\section{Outfall G-10}

Effluent consists of the following types of wastewater: sanitary treatment facility.

Acute Toxicity

Chronic Toxicity
5

2
5

2
0

0

\section{Outfall X-08}

Effluent consists of the following types of wastewater: noncontact cooling water, domestic well overflow water, treated sanitary wastewater from outfall $X-08 A$, treated process water from outfall $X-8 B$, treated groundwater from outfall $X-8 C$, and stormwater.

Acute Toxicity

\section{Outfall $X-19$}

Effluent consists of the following types of wastewater: treated groundwater. Acute Toxicity 
Table 55

National Pollutant Discharge Elimination System Stormwater Monitoring Data

Page 1 of 17

\begin{tabular}{|c|c|c|c|c|c|c|}
\hline Measurement & Units & & Type Sample/ & centration & & \\
\hline \multicolumn{7}{|c|}{ Permit SCR000000 } \\
\hline Outfall N-006 & & Background Grab & Composite & Composite & Grab & Grab \\
\hline$O \& G$ & $\mathrm{mg} / \mathrm{L}$ & No Flow & & & $<1.0$ & $<1.0$ \\
\hline TSS & $\mathrm{mg} / \mathrm{L}$ & No Flow & 21 & 70 & 57 & 11 \\
\hline BOD & $\mathrm{mg} / \mathrm{L}$ & No Flow & $<2.0$ & 2.1 & 2.2 & $<2.0$ \\
\hline $\mathrm{NO}_{2}, \mathrm{NO}_{3}$ & $\mathrm{mg} / \mathrm{L}$ & No Flow & 0.053 & 0.024 & $<0.020$ & $<0.020$ \\
\hline $\mathrm{PO}_{4}-\mathrm{P}$ & $\mathrm{mg} / \mathrm{L}$ & No Flow & 0.155 & 0.017 & 0.295 & 0.065 \\
\hline COD & $\mathrm{mg} / \mathrm{L}$ & No Flow & 19.5 & 23.3 & 55.2 & 26.1 \\
\hline TOC & $\mathrm{mg} / \mathrm{L}$ & No Flow & 8.9 & 6.6 & 16 & 6.2 \\
\hline TKN & $\mathrm{mg} / \mathrm{L}$ & No Flow & 0.489 & 1.400 & 1.010 & 1.140 \\
\hline Phenol & $\mathrm{mg} / \mathrm{L}$ & No Flow & & & $<0.0060$ & $<0.0060$ \\
\hline AL & $\mathrm{mg} / \mathrm{L}$ & No Flow & 0.82 & 0.66 & 2.3 & 0.5 \\
\hline Boron & $\mathrm{mg} / \mathrm{L}$ & No Flow & 0.27 & $<0.050$ & $<0.050$ & 0.15 \\
\hline CR & $\mathrm{mg} / \mathrm{L}$ & No Flow & $<0.0050$ & $<0.0050$ & $<0.0050$ & $<0.0050$ \\
\hline $\mathrm{CU}$ & $\mathrm{mg} / \mathrm{L}$ & No Flow & $<0.0050$ & 0.007 & 0.0078 & $<0.0050$ \\
\hline $\mathrm{FE}$ & $\mathrm{mg} / \mathrm{L}$ & No Flow & 2.5 & 0.77 & 9.10 & 2.2 \\
\hline PB & $\mathrm{mg} / \mathrm{L}$ & No Flow & $<0.0030$ & $<0.0030$ & $<0.0030$ & $<0.0030$ \\
\hline MN & $\mathrm{mg} / \mathrm{L}$ & No Flow & 0.32 & 0.082 & 1.2 & 0.12 \\
\hline $\mathrm{NI}$ & $\mathrm{mg} / \mathrm{L}$ & No Flow & $<0.010$ & $<0.010$ & $<0.010$ & $<0.010$ \\
\hline$A G$ & $\mathrm{mg} / \mathrm{L}$ & No Flow & $<0.0050$ & $<0.0050$ & $<0.0050$ & $<0.0050$ \\
\hline$Z N$ & $\mathrm{mg} / \mathrm{L}$ & No Flow & 0.35 & 0.2 & 1.4 & 0.075 \\
\hline
\end{tabular}




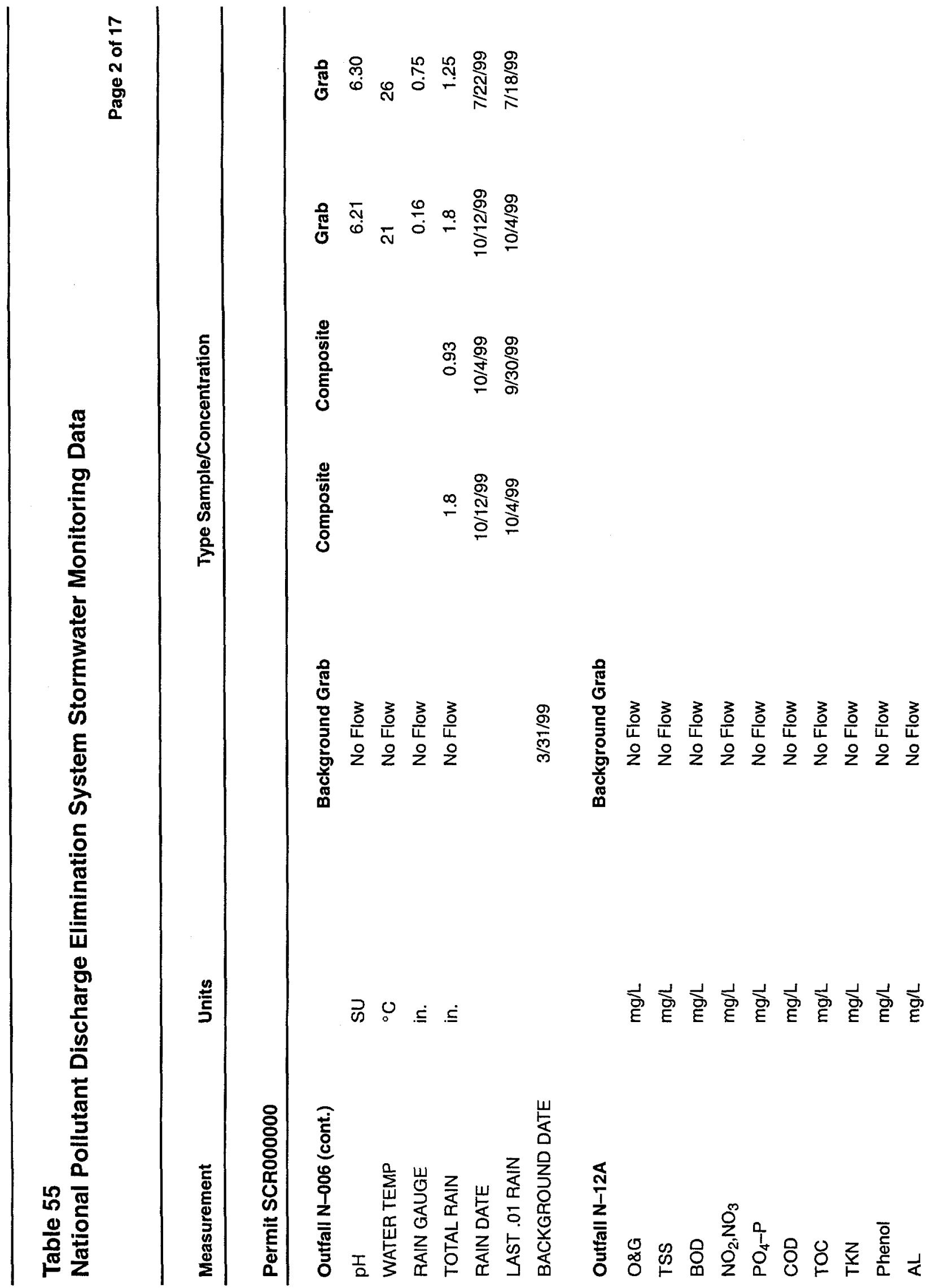




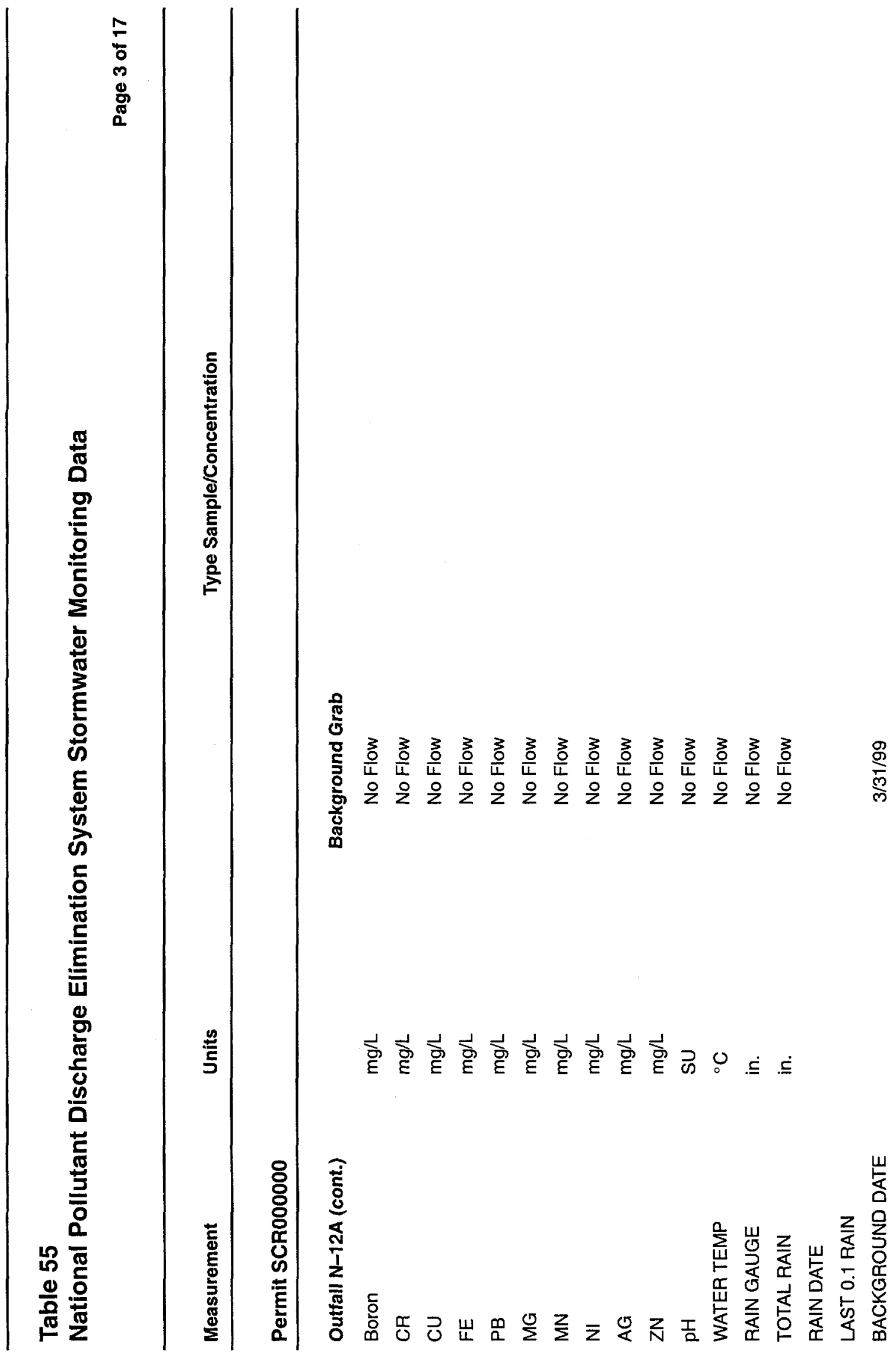


Table 55

National Pollutant Discharge Elimination System Stormwater Monitoring Data

Page 4 of 17

Measurement

Units

Type Sample/Concentration

\section{Permit SCR000000}

\begin{tabular}{|c|c|c|c|c|}
\hline Outfall E-001 & & Background Grab & Grab & Grab \\
\hline O\&G & $\mathrm{mg} / \mathrm{L}$ & No Flow & $<1.0$ & $<1.0$ \\
\hline TSS & $\mathrm{mg} / \mathrm{L}$ & No Flow & 10 & 13 \\
\hline BOD & $\mathrm{mg} / \mathrm{L}$ & No Flow & 6.6 & 5.7 \\
\hline TDS & $\mathrm{mg} / \mathrm{L}$ & No Flow & 60 & 64 \\
\hline $\mathrm{NH} 3-\mathrm{N}$ & $\mathrm{mg} / \mathrm{L}$ & No Flow & 0.109 & 0.233 \\
\hline $\mathrm{NO} 2 / \mathrm{NO}_{3}$ & $\mathrm{mg} / \mathrm{L}$ & No Flow & 0.084 & 0.173 \\
\hline $\mathrm{PO}_{4}-\mathrm{P}$ & $\mathrm{mg} / \mathrm{L}$ & No Flow & 0.261 & 0.024 \\
\hline COD & $\mathrm{mg} / \mathrm{L}$ & No Flow & 46.3 & 39.5 \\
\hline TOC & $\mathrm{mg} / \mathrm{L}$ & No Flow & 16 & 16 \\
\hline TKN & $\mathrm{mg} / \mathrm{L}$ & No Flow & 0.464 & 1.43 \\
\hline Phenol & $\mathrm{mg} / \mathrm{L}$ & No Flow & 0.008 & $<0.0060$ \\
\hline $\mathrm{SO} 4$ & $\mathrm{mg} / \mathrm{L}$ & No Flow & 3.57 & 9.83 \\
\hline Bromide & $\mathrm{mg} / \mathrm{L}$ & No Flow & $<1.00$ & $<1.00$ \\
\hline $\mathrm{CN}$ & $\mathrm{mg} / \mathrm{L}$ & No Flow & 0.011 & $<0.010$ \\
\hline$A L$ & $\mathrm{mg} / \mathrm{L}$ & No Flow & 0.13 & 0.86 \\
\hline SB & $\mathrm{mg} / \mathrm{L}$ & No Flow & $<0.0050$ & $<0.0050$ \\
\hline AS & $\mathrm{mg} / \mathrm{L}$ & No Flow & $<0.0050$ & $<0.0050$ \\
\hline BA & $\mathrm{mg} / \mathrm{L}$ & No Flow & 0.05 & 0.038 \\
\hline Boron & $\mathrm{mg} / \mathrm{L}$ & No Flow & $<0.050$ & 0.22 \\
\hline
\end{tabular}




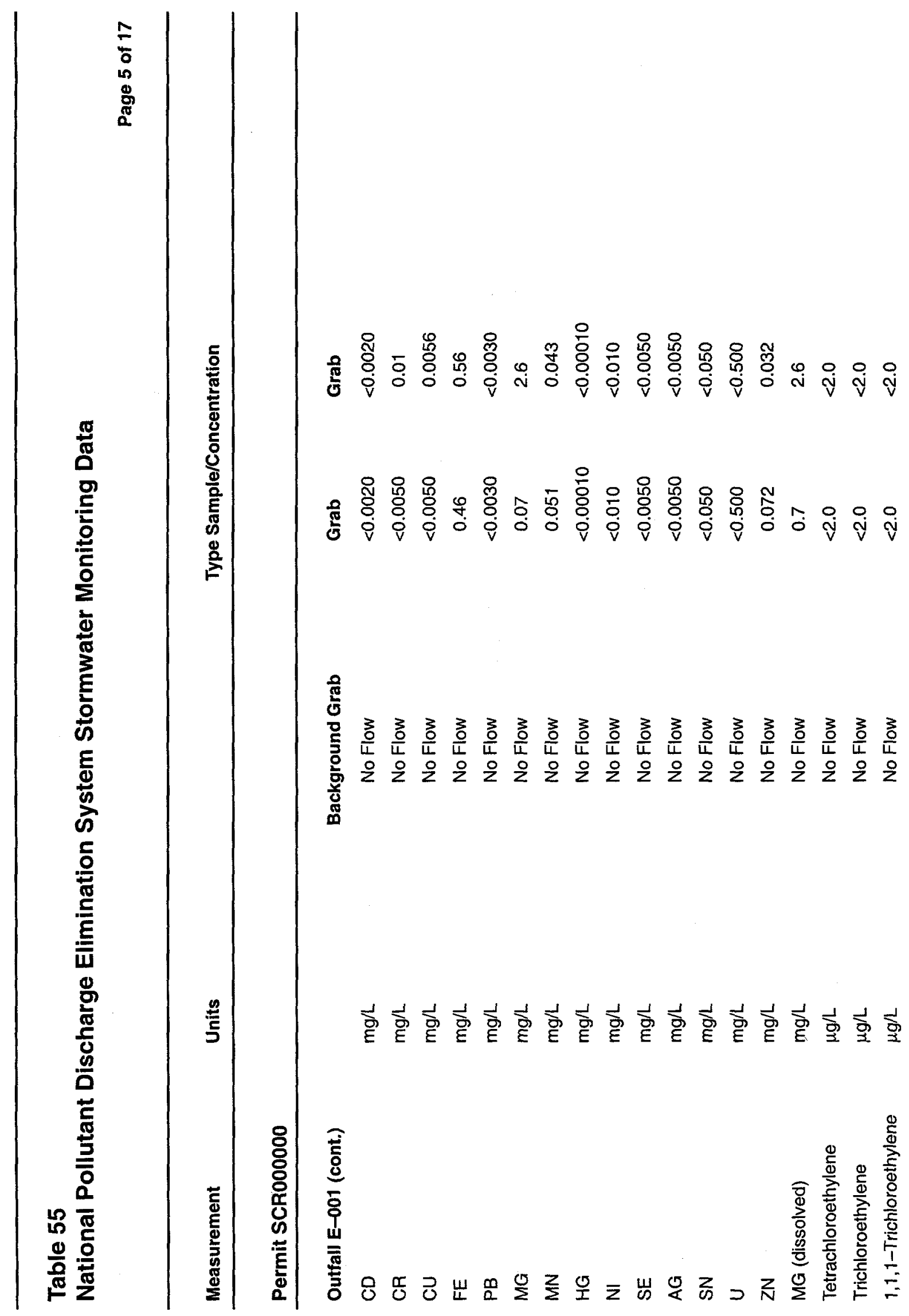


璦

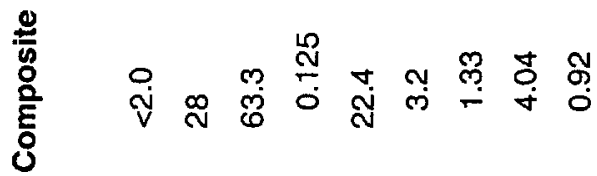

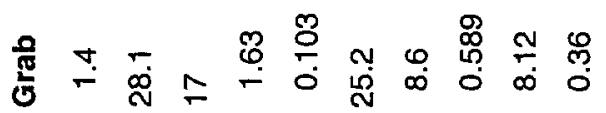

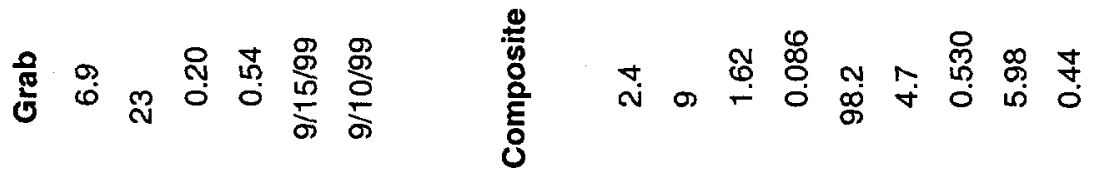

온

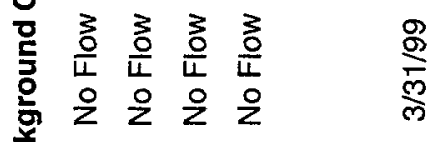

은

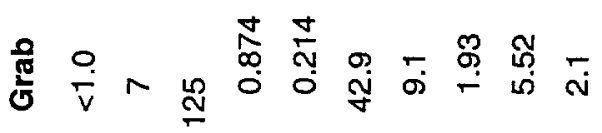

总

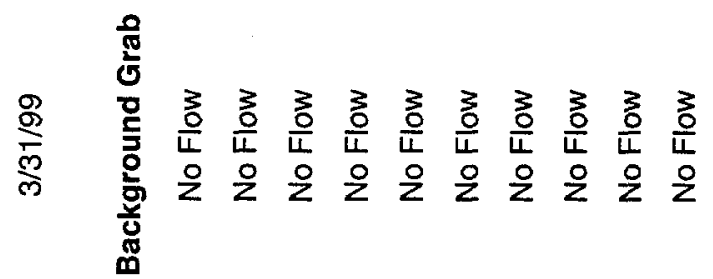

क ० $\doteq$.

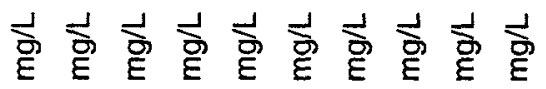

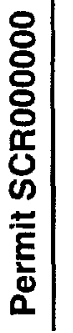

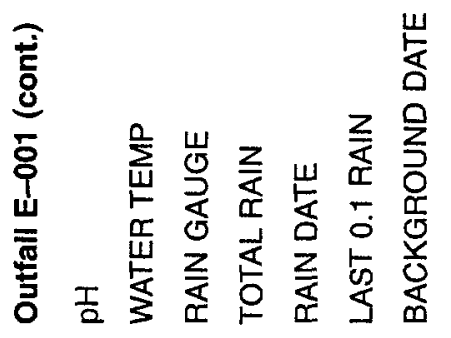

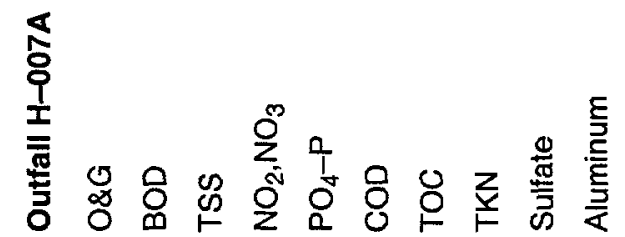


Table 55

National Pollutant Discharge Elimination System Stormwater Monitoring Data

Page 7 of 17

$\begin{array}{lll}\text { Measurement Units } & \text { Type Sample/Concentration }\end{array}$

Permit SCR000000

\begin{tabular}{|c|c|c|c|c|}
\hline Outfall H-007A (cont.) & & Background Grab & Composite & Composite \\
\hline Copper & $\mathrm{mg} / \mathrm{L}$ & No Flow & 0.012 & 0.0086 \\
\hline Iron & $\mathrm{mg} / \mathrm{L}$ & No Flow & 0.36 & 0.92 \\
\hline $\mathrm{pH}$ & SU & No Flow & & \\
\hline WATER TEMP & ${ }^{\circ} \mathrm{C}$ & No Flow & & \\
\hline RAIN GAUGE & in. & No Flow & & \\
\hline TOTAL RAIN & in. & No Flow & 1.4 & 1.25 \\
\hline RAIN DATE & & & $7 / 6 / 99$ & $7 / 22 / 99$ \\
\hline LAST 0.1 RAIN & & & $6 / 28 / 99$ & $7 / 18 / 99$ \\
\hline BACKGROUND DATE & & $3 / 31 / 99$ & & \\
\hline Outfall K-002 & & Background Grab & Composite & Grab \\
\hline O\&G & $\mathrm{mg} / \mathrm{L}$ & No Flow & & $<1.0$ \\
\hline BOD & $\mathrm{mg} / \mathrm{L}$ & No Flow & 17 & 2.9 \\
\hline TSS & $\mathrm{mg} / \mathrm{L}$ & No Flow & 37 & 7 \\
\hline$C O D$ & $\mathrm{mg} / \mathrm{L}$ & No Flow & $<10$ & 31.8 \\
\hline $\mathrm{NO}_{2}, \mathrm{NO}_{3}$ & $\mathrm{mg} / \mathrm{L}$ & No Flow & 1.41 & 0.394 \\
\hline $\mathrm{PO}_{4}-\mathrm{P}$ & $\mathrm{mg} / \mathrm{L}$ & No Flow & 0.058 & 0.106 \\
\hline TKN & $\mathrm{mg} / \mathrm{L}$ & No Flow & 0.413 & 0.501 \\
\hline TOC & $\mathrm{mg} / \mathrm{L}$ & No Flow & 8.3 & 8.4 \\
\hline
\end{tabular}




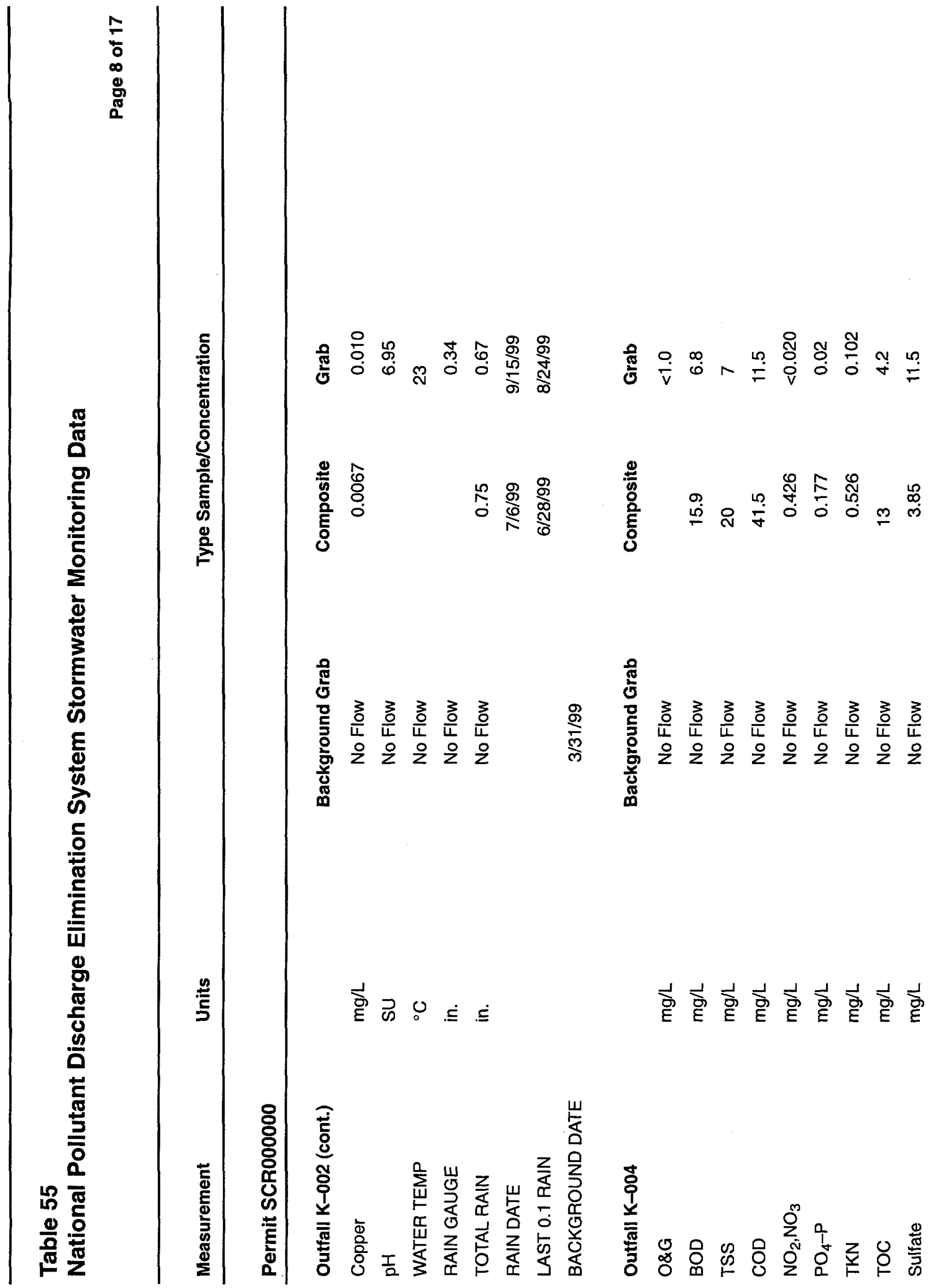




\section{Table 55 \\ National Pollutant Discharge Elimination System Stormwater Monitoring Data}

Page 9 of 17

\begin{tabular}{ll} 
Measurement Units $\quad$ Type Sample/Concentration \\
\hline
\end{tabular}

\section{Permit SCR000000}

Outfall K-004 (cont.)

Copper

$\mathrm{pH}$

WATER TEMP

RAIN GAUGE

TOTAL RAIN

RAIN DATE

LAST 0.1 RAIN

BACKGROUND DATE

Outfall S-005

O\&G

BOD

TSS

$\mathrm{NO}_{2}, \mathrm{NO}_{3}$

$\mathrm{PO}_{4}-\mathrm{P}$

COD

TOC

TKN

SO4

$\mathrm{mg} / \mathrm{L}$
$\mathrm{mg} / \mathrm{L}$
$\mathrm{mg} / \mathrm{L}$
$\mathrm{mg} / \mathrm{L}$
$\mathrm{mg} / \mathrm{L}$
$\mathrm{mg} / \mathrm{L}$
$\mathrm{mg} / \mathrm{L}$
$\mathrm{mg} / \mathrm{L}$
$\mathrm{mg} / \mathrm{L}$

$\mathrm{mg} / \mathrm{L}$
$\mathrm{mg} / \mathrm{L}$
$\mathrm{mg} / \mathrm{L}$
$\mathrm{mg} / \mathrm{L}$
$\mathrm{mg} / \mathrm{L}$
$\mathrm{mg} / \mathrm{L}$
$\mathrm{mg} / \mathrm{L}$
$\mathrm{mg} / \mathrm{L}$
$\mathrm{mg} / \mathrm{L}$

$\mathrm{mg} / \mathrm{L}$
$\mathrm{mg} / \mathrm{L}$
$\mathrm{mg} / \mathrm{L}$
$\mathrm{mg} / \mathrm{L}$
$\mathrm{mg} / \mathrm{L}$
$\mathrm{mg} / \mathrm{L}$
$\mathrm{mg} / \mathrm{L}$
$\mathrm{mg} / \mathrm{L}$
$\mathrm{mg} / \mathrm{L}$

$\mathrm{mg} / \mathrm{L}$
$\mathrm{mg} / \mathrm{L}$
$\mathrm{mg} / \mathrm{L}$
$\mathrm{mg} / \mathrm{L}$
$\mathrm{mg} / \mathrm{L}$
$\mathrm{mg} / \mathrm{L}$
$\mathrm{mg} / \mathrm{L}$
$\mathrm{mg} / \mathrm{L}$
$\mathrm{mg} / \mathrm{L}$

$\mathrm{mg} / \mathrm{L}$
$\mathrm{mg} / \mathrm{L}$
$\mathrm{mg} / \mathrm{L}$
$\mathrm{mg} / \mathrm{L}$
$\mathrm{mg} / \mathrm{L}$
$\mathrm{mg} / \mathrm{L}$
$\mathrm{mg} / \mathrm{L}$
$\mathrm{mg} / \mathrm{L}$
$\mathrm{mg} / \mathrm{L}$

$\mathrm{mg} / \mathrm{L}$
$\mathrm{mg} / \mathrm{L}$
$\mathrm{mg} / \mathrm{L}$
$\mathrm{mg} / \mathrm{L}$
$\mathrm{mg} / \mathrm{L}$
$\mathrm{mg} / \mathrm{L}$
$\mathrm{mg} / \mathrm{L}$
$\mathrm{mg} / \mathrm{L}$
$\mathrm{mg} / \mathrm{L}$

$\mathrm{mg} / \mathrm{L}$
$\mathrm{mg} / \mathrm{L}$
$\mathrm{mg} / \mathrm{L}$
$\mathrm{mg} / \mathrm{L}$
$\mathrm{mg} / \mathrm{L}$
$\mathrm{mg} / \mathrm{L}$
$\mathrm{mg} / \mathrm{L}$
$\mathrm{mg} / \mathrm{L}$
$\mathrm{mg} / \mathrm{L}$

$\mathrm{mg} / \mathrm{L}$
$\mathrm{mg} / \mathrm{L}$
$\mathrm{mg} / \mathrm{L}$
$\mathrm{mg} / \mathrm{L}$
$\mathrm{mg} / \mathrm{L}$
$\mathrm{mg} / \mathrm{L}$
$\mathrm{mg} / \mathrm{L}$
$\mathrm{mg} / \mathrm{L}$
$\mathrm{mg} / \mathrm{L}$

$\mathrm{mg} / \mathrm{L}$
$\mathrm{mg} / \mathrm{L}$
$\mathrm{mg} / \mathrm{L}$
$\mathrm{mg} / \mathrm{L}$
$\mathrm{mg} / \mathrm{L}$
$\mathrm{mg} / \mathrm{L}$
$\mathrm{mg} / \mathrm{L}$
$\mathrm{mg} / \mathrm{L}$
$\mathrm{mg} / \mathrm{L}$

$\mathrm{mg} / \mathrm{L}$

SU

in.

in.
Background Grab

No Flow

No Flow

No Flow

No Flow

No Flow

$3 / 31 / 99$

Background Grab

$<1.0$

3.7

10

$<0.020$

0.333

19.2

5.7

0.344

2.46
Type Sample/Concentration

$\begin{array}{rc}\text { Composite } & \text { Grab } \\ 0.0091 & 0.007 \\ & 6.93 \\ & 21.0 \\ & 0.3 \\ 1.05 & 9 / 29 / 99 \\ 7 / 6 / 99 & 10 / 4 / 99 \\ 6 / 27 / 99 & 9 / 28 / 99\end{array}$

Grab

Grab

Grab

$<1.0$

4

50

28.3

0.241

19.3

5.8

1.66

2.86 
Table 55

National Pollutant Discharge Elimination System Stormwater Monitoring Data

Page 10 of 17

\begin{tabular}{|c|c|c|c|c|c|}
\hline Measurement & Units & & Type Sample/C & centration & \\
\hline \multicolumn{6}{|l|}{ Permit SCR000000 } \\
\hline Outfall S-005 (cont.) & & Background Grab & Grab & Grab & Grab \\
\hline Benzene & $\mu g / L$ & $<0.5$ & & $<0.5$ & $<0.5$ \\
\hline CR & $m g / L$ & $<0.0050$ & $<0.0050$ & $<0.0050$ & \\
\hline $\mathrm{CU}$ & $\mathrm{mg} / \mathrm{L}$ & 0.015 & 0.0089 & 0.027 & \\
\hline$H G$ & $m g / L$ & $<0.00010$ & $<0.00010$ & $<0.00010$ & \\
\hline PB & $\mathrm{mg} / \mathrm{L}$ & $<0.0030$ & $<0.0030$ & 0.03 & \\
\hline ZN & $\mathrm{mg} / \mathrm{L}$ & 0.082 & 0.067 & 0.18 & \\
\hline $\mathrm{pH}$ & su & 6.85 & 6.57 & 6.9 & \\
\hline WATER TEMP & ${ }^{\circ} \mathrm{C}$ & 24 & 25 & 28 & \\
\hline RAIN GAUGE & in. & & 0.28 & 0.75 & 0.28 \\
\hline TOTAL RAIN & in. & & 1.35 & 1.25 & 1 \\
\hline RAIN DATE & & & $7 / 6 / 99$ & 7/22/99 & $10 / 4 / 99$ \\
\hline LAST 0.1 RAIN & & $6 / 17 / 99$ & $6 / 27 / 99$ & 7/19/99 & 9/30/99 \\
\hline BACKGROUND DATE & & $6 / 23 / 99$ & & & \\
\hline Outfall $x-001$ & & Background Grab & Composite & Composite & Grab \\
\hline$O \& G$ & $\mathrm{mg} / \mathrm{L}$ & No Flow & & & $<1.0$ \\
\hline$B O D$ & $\mathrm{mg} / \mathrm{L}$ & No Flow & 3.2 & 4.2 & 2.7 \\
\hline$C O D$ & $\mathrm{mg} / \mathrm{L}$ & No Flow & 32.5 & 18.6 & 23 \\
\hline $\mathrm{NO}_{2}, \mathrm{NO}_{3}$ & $\mathrm{mg} / \mathrm{L}$ & No Flow & 0.151 & 0.646 & 0.122 \\
\hline
\end{tabular}




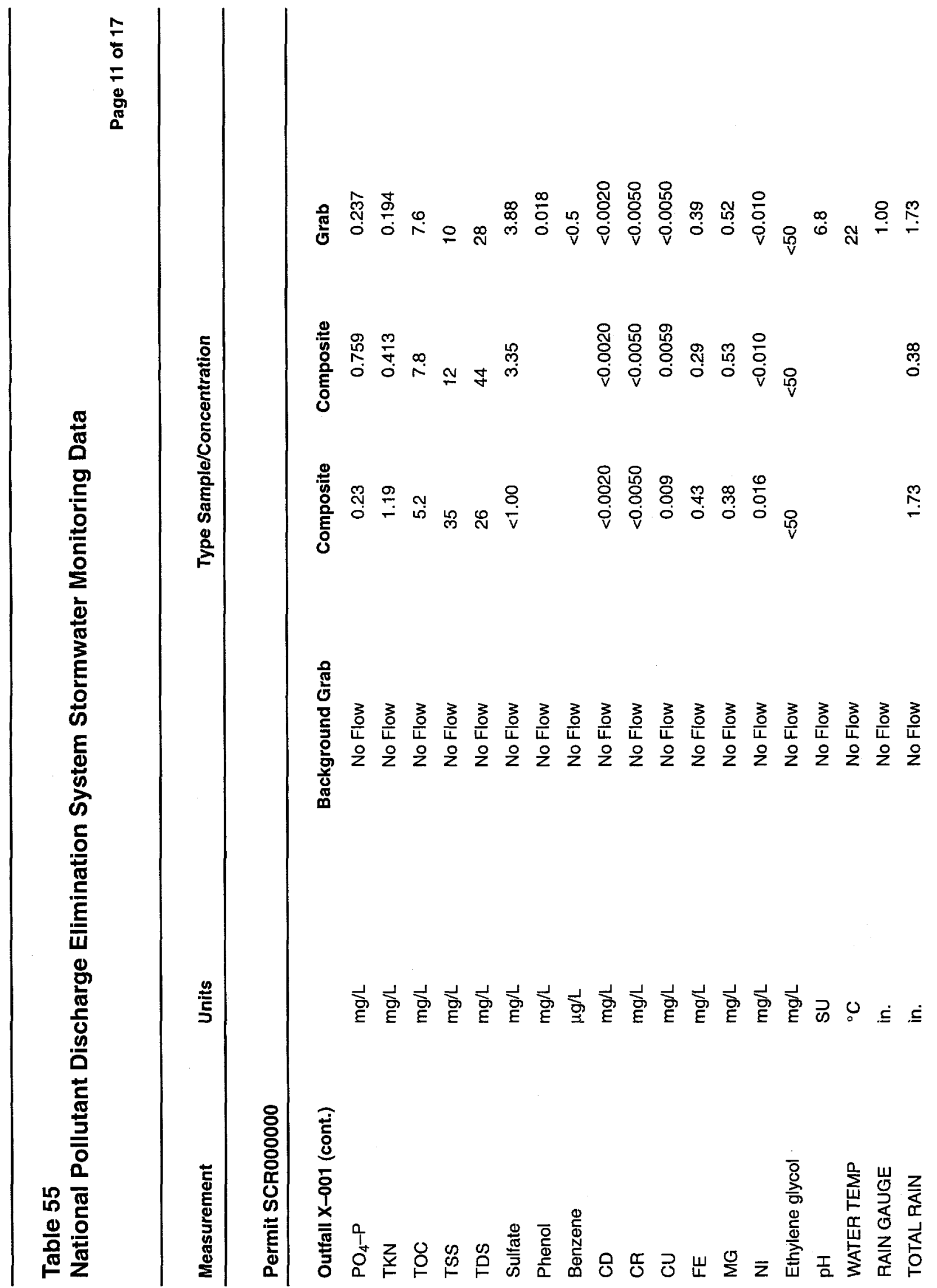


Table 55

National Pollutant Discharge Elimination System Stormwater Monitoring Data

Page 12 of 17

\begin{tabular}{|c|c|c|c|c|c|c|}
\hline Measurement & Units & & Type Sample/ & Icentration & & \\
\hline \multicolumn{7}{|l|}{ Permit SCR000000 } \\
\hline Outfall $X-001$ (cont.) & & Background Grab & Composite & Composite & Grab & \\
\hline RAIN DATE & & & $10 / 4 / 99$ & $7 / 11 / 99$ & $10 / 4 / 99$ & \\
\hline LAST 0.1 RAIN & & & $9 / 30 / 99$ & $6 / 27 / 99$ & $9 / 30 / 99$ & \\
\hline BACKGROUND DATE & & $3 / 31 / 99$ & & & & \\
\hline Outfall N-14 & & Background Grab & Composite & Composite & Grab & Grab \\
\hline BOD & $\mathrm{mg} / \mathrm{L}$ & No Flow & $<2.0$ & & 6 & 3.9 \\
\hline COD & $\mathrm{mg} / \mathrm{L}$ & No Flow & 12.9 & & 45.4 & 44.6 \\
\hline Nitrate & $\mathrm{mg} / \mathrm{L}$ & No Flow & 0.154 & & 0.571 & 0.275 \\
\hline O\&G & $\mathrm{mg} / \mathrm{L}$ & No Flow & & & $<1.0$ & $<1.0$ \\
\hline Phenol & $\mathrm{mg} / \mathrm{L}$ & No Flow & & & $<0.006$ & $<0.0060$ \\
\hline $\mathrm{PO}_{4}-\mathrm{P}$ & $\mathrm{mg} / \mathrm{L}$ & No Flow & 0.055 & & 0.151 & 0.146 \\
\hline TKN & $\mathrm{mg} / \mathrm{L}$ & No Flow & 0.701 & & 0.287 & 1.07 \\
\hline TOC & $\mathrm{mg} / \mathrm{L}$ & No Flow & 4.3 & & 15 & 6.1 \\
\hline TSS & $\mathrm{mg} / \mathrm{L}$ & No Flow & 23 & & 36 & 35 \\
\hline Arsenic & $\mathrm{mg} / \mathrm{L}$ & No Flow & $<0.0050$ & & $<0.0050$ & $<0.0050$ \\
\hline Copper & $\mathrm{mg} / \mathrm{L}$ & No Flow & $<0.050$ & & 0.0071 & $<0.0050$ \\
\hline Ehtylene Glycol & $\mathrm{mg} / \mathrm{L}$ & No Flow & $<50$ & & $<50$ & $<50$ \\
\hline $\mathrm{pH}$ & su & No Flow & & & 6.53 & 6.50 \\
\hline WATER TEMP & ${ }^{\circ} \mathrm{C}$ & No Flow & & & 23 & 28 \\
\hline
\end{tabular}


Table 55

National Pollutant Discharge Elimination System Stormwater Monitoring Data

Page 13 of 17

Measurement

Units

Type Sample/Concentration

Permit SCR000000

Outfall N-14 (cont.)

RAIN GAUGE

TOTAL RAIN

RAIN DATE

LAST 0.1 RAIN

BACKGROUND DATE

Outfall A-08

$O \& G$

$\mathrm{BOD}$

TSS

COD

Nitrate/Nitrite

$\mathrm{PO}_{4}-\mathrm{P}$

TKN

TOC

Arsenic

Copper

Nickel

Zinc

$\mathrm{mg} / \mathrm{L}$

$\mathrm{mg} / \mathrm{L}$

$\mathrm{mg} / \mathrm{L}$

$\mathrm{mg} / \mathrm{L}$

$\mathrm{mg} / \mathrm{L}$

$\mathrm{mg} / \mathrm{L}$

$\mathrm{mg} / \mathrm{L}$

$\mathrm{mg} / \mathrm{L}$

$\mathrm{mg} / \mathrm{L}$

$\mathrm{mg} / \mathrm{L}$

$\mathrm{mg} / \mathrm{L}$

$\mathrm{mg} / \mathrm{L}$

\section{Background Grab}

No Flow

No Flow

$3 / 31 / 99$

$3 / 31 / 99$

Background Grab

No Flow

No Flow

No Flow

No Flow

No Flow

No Flow

No Flow

No Flow

No Flow

No Flow

No Flow

No Flow
Composite

0.93

10/4/99

$9 / 29 / 99$

$\begin{array}{cc}\text { Composite } & \text { Composite } \\ 2.8 & <2.0 \\ 10 & 19 \\ 43.2 & 14.2 \\ 1.5 & 0.052 \\ 0.067 & 0.056 \\ 1.35 & <0.100 \\ 8.4 & 5.2 \\ 0.015 & <0.0050 \\ 0.029 & 0.008 \\ <0.010 & <0.010 \\ 1.2 & 0.61\end{array}$

Grab

0.3

0.56

9/15/99

$9 / 9 / 99$

Grab

0.25

4.1

7/22/99

$7 / 19 / 99$ 
Table 55

National Pollutant Discharge Elimination System Stormwater Monitoring Data

Page 14 of 17

\begin{tabular}{l|c|c|c|}
\hline Measurement & Units & Type Sample/Concentration \\
\hline
\end{tabular}

\section{Permit SCR000000}

\begin{tabular}{|c|c|c|c|c|c|c|}
\hline Outfall A-08 (cont.) & & Background Grab & Composite & Composite & Grab & Grab \\
\hline $\mathrm{pH}$ & su & No Flow & & & 6.23 & 4.45 \\
\hline WATER TEMP & ${ }^{\circ} \mathrm{C}$ & No Flow & & & 17 & 27 \\
\hline RAIN GAUGE & in. & No Flow & & & 0.15 & 0.16 \\
\hline TOTAL RAIN & in. & No Flow & 0.38 & 0.28 & 0.28 & 0.34 \\
\hline RAIN DATE & & & $12 / 13 / 99$ & $12 / 6 / 99$ & $12 / 6 / 99$ & $8 / 19 / 99$ \\
\hline LAST 0.1 RAIN & & & $12 / 10 / 99$ & $11 / 27 / 99$ & $11 / 27 / 99$ & $8 / 16 / 99$ \\
\hline BACKGROUND DATE & & $3 / 31 / 99$ & & & & \\
\hline Outfall Y-01 & & Background Grab & Composite & Composite & Grab & Grab \\
\hline O\&G & $\mathrm{mg} / \mathrm{L}$ & No Flow & & & 6.6 & 8.5 \\
\hline BOD & $\mathrm{mg} / \mathrm{L}$ & No Flow & 5.2 & 2.6 & 6.4 & 15 \\
\hline TSS & $\mathrm{mg} / \mathrm{L}$ & No Flow & 1 & 6 & 26 & 5 \\
\hline COD & $\mathrm{mg} / \mathrm{L}$ & No Flow & 22.7 & 30.3 & 12.3 & 17 \\
\hline Nitrate/Nitrite & $\mathrm{mg} / \mathrm{L}$ & No Flow & 0.203 & 0.11 & 0.02 & 0.022 \\
\hline $\mathrm{PO}_{4}-\mathrm{P}$ & $\mathrm{mg} / \mathrm{L}$ & No Flow & 0.063 & 0.071 & 0.023 & 0.061 \\
\hline TKN & $\mathrm{mg} / \mathrm{L}$ & No Flow & $<0.100$ & $<0.100$ & 1.73 & $<0.100$ \\
\hline TOC & $\mathrm{mg} / \mathrm{L}$ & No Flow & 6.8 & 7.1 & 2.6 & 6.6 \\
\hline Copper & $\mathrm{mg} / \mathrm{L}$ & No Flow & 0.11 & 0.075 & 0.13 & 0.085 \\
\hline Ethylene Glycol & $\mathrm{mg} / \mathrm{L}$ & No Flow & $<50$ & $<50$ & $<50$ & $<50$ \\
\hline
\end{tabular}


Table 55

National Pollutant Discharge Elimination System Stormwater Monitoring Data

Page 15 of 17

$\begin{array}{lll}\text { Measurement Units } & \text { Type Sample/Concentration }\end{array}$

Permit SCR000000

Outfall Y -01 (cont.)

$\mathrm{pH}$

SU

WATER TEMP

RAIN GAUGE

TOTAL RAIN

RAIN DATE

LAST 0.1 RAIN

BACKGROUND DATE

Outfall H-06

O\&G

BOD

TSS

COD

Nitrate/Nitrite

$\mathrm{PO}_{4}-\mathrm{P}$

TKN

TOC

Copper

Iron

$\mathrm{mg} / \mathrm{L}$

$\mathrm{mg} / \mathrm{L}$

$\mathrm{mg} / \mathrm{L}$

$\mathrm{mg} / \mathrm{L}$

$\mathrm{mg} / \mathrm{L}$

$\mathrm{mg} / \mathrm{L}$

$\mathrm{mg} / \mathrm{L}$

$\mathrm{mg} / \mathrm{L}$

$\mathrm{mg} / \mathrm{L}$

$\mathrm{mg} / \mathrm{L}$

${ }^{\circ} \mathrm{C} \quad$ No Flow

Background Grab

No Flow

No Flow

No Flow

Background Grab

No Flow

No Flow

No Flow

No Flow

No Flow

No Flow

No Flow

No Flow

No Flow

No Flow

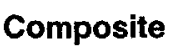

Composite

Grab

7.31

16

0.1

0.39

$12 / 13 / 99$

$12 / 6 / 99$

$$
12 / 6 / 99
$$

\section{$12 / 6 / 99$}

$11 / 26 / 99$

Composite

Composite

$$
<2.0
$$

19

26.5

0.11

$<0.010$

$<0.100$

7

$<0.0050$

1.2

$<2.0$
18
$<10$
0.658
0.212
0.542
6.6
$<0.0050$
0.48

$\begin{array}{cc}\text { Grab } & \text { Grab } \\ <1.0 & <1.0 \\ 2.8 & <2.0 \\ 14 & 25 \\ 19.8 & <10 \\ 0.618 & 0.373 \\ 0.13 & 0.035 \\ 0.561 & 0.126 \\ 7.2 & 2.6 \\ <0.0050 & <0.0050 \\ 0.41 & 0.45\end{array}$


Table 55

National Pollutant Discharge Elimination System Stormwater Monitoring Data

Page 16 of 17

Measurement

Units

Type Sample/Concentration

Permit SCR000000

\begin{tabular}{|c|c|c|c|c|c|c|}
\hline Outfall H-06 (cont.) & & Background Grab & Composite & Composite & Grab & Grab \\
\hline Manganese & $\mathrm{mg} / \mathrm{L}$ & No Flow & 0.019 & 0.021 & 0.013 & 0.02 \\
\hline Mercury & $\mathrm{mg} / \mathrm{L}$ & No Flow & 0.00012 & $<0.00010$ & $<0.00010$ & $<0.00010$ \\
\hline $\mathrm{pH}$ & su & No Flow & & & 7.21 & 7.02 \\
\hline WATER TEMP & ${ }^{\circ} \mathrm{C}$ & No Flow & & & 23 & 21 \\
\hline RAIN GAUGE & in. & No Flow & & & 0.7 & 0.3 \\
\hline TOTAL RAIN & in. & No Flow & 0.42 & 0.96 & 0.96 & 0.42 \\
\hline RAIN DATE & & & $12 / 6 / 99$ & $10 / 12 / 99$ & $10 / 12 / 99$ & $10 / 4 / 99$ \\
\hline LAST 0.1 RAIN & & & $11 / 28 / 99$ & $10 / 4 / 99$ & $10 / 4 / 99$ & $9 / 29 / 99$ \\
\hline BACKGROUND DATE & & $10 / 3 / 99$ & & & & \\
\hline Outfall $\mathrm{N}-02$ & & Background Grab & Composite & Grab & & \\
\hline $\mathrm{O \& G}$ & $\mathrm{mg} / \mathrm{L}$ & No Flow & & $<1.0$ & & \\
\hline $\mathrm{BOD}$ & $\mathrm{mg} / \mathrm{L}$ & No Flow & $<2.0$ & $<2.0$ & & \\
\hline TSS & $\mathrm{mg} / \mathrm{L}$ & No Flow & 9 & 66 & & \\
\hline Sulfate & $\mathrm{mg} / \mathrm{L}$ & No Flow & 11.9 & 5.36 & & \\
\hline COD & $\mathrm{mg} / \mathrm{L}$ & No Flow & $<10$ & 14.7 & & \\
\hline Nitrate/Nitrite & $\mathrm{mg} / \mathrm{L}$ & No Flow & 0.06 & 0.49 & & \\
\hline $\mathrm{PO}_{4}-\mathrm{P}$ & $\mathrm{mg} / \mathrm{L}$ & No Flow & 0.055 & 0.208 & & \\
\hline TKN & $\mathrm{mg} / \mathrm{L}$ & No Flow & 0.259 & 0.104 & & \\
\hline
\end{tabular}




\begin{tabular}{|c|c|c|c|c|c|}
\hline \multicolumn{6}{|c|}{$\begin{array}{l}\text { Table } 55 \\
\text { National Pollutant Discharge Elimination System Stormwater Monitoring Data }\end{array}$} \\
\hline Measurement & Units & & Type Sample// & intration & \\
\hline \multicolumn{6}{|l|}{ Permit SCR000000 } \\
\hline Outfall N-02 (cont.) & & Background Grab & Composite & Grab & \\
\hline TOC & $\mathrm{mg} / \mathrm{L}$ & No Flow & 3.7 & 3.2 & \\
\hline Copper & $\mathrm{mg} / \mathrm{L}$ & No Flow & $<0.0050$ & $<0.0050$ & \\
\hline Iron & $\mathrm{mg} / \mathrm{L}$ & No Flow & 0.27 & 1.9 & \\
\hline $\mathrm{pH}$ & SU & No Flow & & 7.10 & \\
\hline WATER TEMP & ${ }^{\circ} \mathrm{C}$ & No Flow & & 22 & \\
\hline RAIN GAUGE & in. & No Flow & & 0.25 & \\
\hline TOTAL RAIN & in. & No Flow & 1.68 & 1 & \\
\hline RAIN DATE & & & $10 / 13 / 99$ & $10 / 4 / 99$ & \\
\hline LAST 0.1 RAIN & & & $10 / 4 / 99$ & 9/29/99 & \\
\hline BACKGROUND DATE & & $3 / 31 / 99$ & & & \\
\hline
\end{tabular}


Table 56

National Pollutant Discharge Elimination System

Land Application Monitoring Data

Page 1 of 2

\section{Permit ND0072125}

Note: Permit levels can be found in WSRC NPDES Environmental Monitoring Program (ESH-EMS-910110) or in the permit itself. The permit is available from the South Carolina Department of Health and Environmental Control.

\section{Outfall ND-1}

\begin{tabular}{|c|c|c|c|}
\hline Analytical Laboratory & $\begin{array}{l}\text { Results } \\
\text { Shealy }\end{array}$ & $\begin{array}{c}\text { Results } \\
\text { Shealy }\end{array}$ & Units \\
\hline Date of Sample & $1 / 21 / 99$ & $6 / 29 / 99$ & \\
\hline Arsenic & 22.7 & 7.7 & $\mathrm{Mg} / \mathrm{Kg}$ \\
\hline Cadmium & 4.81 & 6.1 & $\mathrm{Mg} / \mathrm{Kg}$ \\
\hline Chromium & 25.6 & 25.0 & $\mathrm{Mg} / \mathrm{Kg}$ \\
\hline Copper & 449 & 460 & $\mathrm{Mg} / \mathrm{Kg}$ \\
\hline Lead & 173 & 140 & $\mathrm{Mg} / \mathrm{Kg}$ \\
\hline Mercury & 0.143 & 2.65 & $\mathrm{Mg} / \mathrm{Kg}$ \\
\hline Molybdenum & 34.2 & 40.0 & $\mathrm{Mg} / \mathrm{Kg}$ \\
\hline Nickel & 17.3 & 19 & $\mathrm{Mg} / \mathrm{Kg}$ \\
\hline Potassium & 980 & 600 & $\mathrm{Mg} / \mathrm{Kg}$ \\
\hline Selenium & 25.3 & 13 & $\mathrm{Mg} / \mathrm{Kg}$ \\
\hline Silver & 84.6 & 52 & $\mathrm{Mg} / \mathrm{Kg}$ \\
\hline Zinc & 3130 & 1500 & $\mathrm{Mg} / \mathrm{Kg}$ \\
\hline Percent Solids & 15.6 & 15.1 & $\%$ \\
\hline Percent Solids & 27.2 & 13.8 & $\%$ \\
\hline Percent Solids & 13.3 & 14.9 & $\%$ \\
\hline Percent Solids & 13.4 & 17.6 & $\%$ \\
\hline Percent Solids & 12.2 & 18.6 & $\%$ \\
\hline Percent Solids & 12.6 & 16.5 & $\%$ \\
\hline Percent Solids & 11.9 & 17.2 & $\%$ \\
\hline Percent Solids & 12.7 & 14.9 & $\%$ \\
\hline Percent Solids & 12.8 & 15.3 & $\%$ \\
\hline Percent Solids & & 14.5 & $\%$ \\
\hline Percent Solids & & 17.3 & $\%$ \\
\hline Fecal Coliform & 2790 & 418 & $\# / G$ \\
\hline Fecal Coliform & 11700 & $<105$ & $\# / G$ \\
\hline Fecal Coliform & 45800 & $<130$ & $\# / G$ \\
\hline
\end{tabular}


Table 56

National Pollutant Discharge Elimination System

Land Application Monitoring Data

Page 2 of 2

Permit ND0072125

Outfall ND-1

\begin{tabular}{lccc}
\hline Analytical Laboratory & $\begin{array}{c}\text { Results } \\
\text { Shealy }\end{array}$ & $\begin{array}{c}\text { Results } \\
\text { Shealy }\end{array}$ & Units \\
\hline Date of Sample & $1 / 21 / 99$ & $6 / 29 / 99$ & \\
\hline Fecal Coliform & 10300 & $<116$ & $\# / G$ \\
Fecal Coliform & 9430 & 360 & $\# / G$ \\
Fecal Coliform & 11600 & $<121$ & $\# / G$ \\
Fecal Coliform & 5130 & 1060 & $\# / G$ \\
Fecal Coliform & & 556 & $\mathrm{Mg} / \mathrm{Kg}$ \\
Ammonia as Nitrogen & 640 & 16.7 & $\mathrm{Mg} / \mathrm{Kg}$ \\
Total Kjeldahl Nitrogen & 640 & 732 & $\mathrm{SU}$ \\
Nitrate + Nitrite & 77.1 & 158 & $\%$ \\
pH & & 6.34 & $\%$ \\
Total Volatile Solids & 1.5 & 9.4 &
\end{tabular}


Table 57

Surface Water Surveillance - Inorganic Contaminants, Pesticides, and Herbicides

Page 1 of 32

\begin{tabular}{|c|c|c|c|c|c|c|c|c|c|c|c|c|c|}
\hline Parameter & Units & Jan & Feb & Mar & April & May & June & July & Aug & Sept & Oct & Nov & Dec \\
\hline “ND" dt & notes "not & tected." & & & & & & & & & & & \\
\hline \multicolumn{14}{|c|}{ Beaver Dam Creek (400-D) } \\
\hline Date & & $1 / 12 / 99$ & $2 / 9 / 99$ & $3 / 9 / 99$ & $4 / 7 / 99$ & $5 / 4 / 99$ & $6 / 8 / 99$ & $7 / 13 / 99$ & $8 / 10 / 99$ & $9 / 14 / 99$ & $10 / 26 / 99$ & $11 / 2 / 99$ & $12 / 7 / 99$ \\
\hline Temperature & ${ }^{\circ} \mathrm{C}$ & 13.4 & 17.3 & 14.6 & 23 & 21 & 28.7 & 25 & 29.3 & 26.7 & 19 & 21 & 18.2 \\
\hline DO & $\mathrm{mg} / \mathrm{L}$ & 10.3 & 8.6 & 8.1 & 7.9 & 7.9 & 4.6 & 5.5 & 6.8 & 6.6 & 9.4 & 8.3 & 9.1 \\
\hline $\mathrm{pH}$ & $\mathrm{pH}$ & 7.3 & 6.8 & 6.6 & 6.7 & 7.8 & 6.9 & 6.8 & 7 & 6.7 & 6.9 & 6.8 & 7 \\
\hline Conductivity & $\mu h o s / c m$ & 124 & 102 & 119 & 116 & 116 & 115 & 120 & 111 & 103 & 117 & 148 & 145 \\
\hline COD & $\mathrm{mg} / \mathrm{L}$ & ND & ND & ND & ND & ND & ND & ND & 22 & ND & ND & ND & ND \\
\hline Nitrite Nitrogen & $\mathrm{mg} / \mathrm{L}$ & ND & ND & ND & ND & ND & ND & ND & ND & ND & ND & ND & ND \\
\hline Nitrate Nitrogen & $\mathrm{mg} / \mathrm{L}$ & 0.43 & 0.40 & 0.38 & 0.39 & 0.51 & 0.50 & 0.49 & 0.50 & 0.37 & 0.37 & 0.49 & 0.32 \\
\hline TSS & $\mathrm{mg} / \mathrm{L}$ & 6 & 13 & 5 & 6 & 5 & 5 & 2 & 7 & 6 & 7 & 4 & 3 \\
\hline Total Phos & $\mathrm{mg} / \mathrm{L}$ & 0.236 & 0.303 & ND & 0.095 & 0.025 & 0.077 & 0.132 & 0.686 & 0.149 & 0.075 & ND & 0.122 \\
\hline TOC & $\mathrm{mg} / \mathrm{L}$ & ND & ND & 3.5 & 3.5 & 3 & 3.4 & 6 & 4.8 & 2.9 & 3.1 & 3.2 & 3.8 \\
\hline Aluminum & $\mathrm{mg} / \mathrm{L}$ & 0.355 & 0.299 & 0.391 & 0.109 & 0.32 & 0.27 & 0.32 & 0.47 & ND & 0.21 & 0.26 & 0.19 \\
\hline Cadmium & $\mathrm{mg} / \mathrm{L}$ & ND & ND & ND & ND & ND & ND & ND & ND & ND & ND & ND & ND \\
\hline Chromium & $\mathrm{mg} / \mathrm{L}$ & ND & ND & ND & ND & ND & ND & ND & ND & ND & ND & ND & ND \\
\hline Copper & $\mathrm{mg} / \mathrm{L}$ & ND & ND & 0.005 & ND & ND & ND & ND & ND & ND & ND & ND & ND \\
\hline Iron & $\mathrm{mg} / \mathrm{L}$ & 0.391 & 0.423 & 0.42 & 0.54 & 0.48 & 0.26 & 0.6 & 0.5 & 0.22 & 0.3 & 0.29 & 0.26 \\
\hline Lead & $\mathrm{mg} / \mathrm{L}$ & ND & ND & ND & ND & ND & ND & ND & ND & ND & ND & ND & ND \\
\hline Manganese & $\mathrm{mg} / \mathrm{L}$ & 0.056 & 0.112 & 0.064 & 0.069 & 0.066 & 0.05 & 0.09 & 0.074 & 0.052 & 0.058 & 0.047 & 0.05 \\
\hline Mercury & $\mathrm{mg} / \mathrm{L}$ & ND & ND & ND & ND & ND & ND & ND & ND & ND & ND & ND & ND \\
\hline Nickel & $\mathrm{mg} / \mathrm{L}$ & ND & ND & ND & ND & ND & ND & ND & ND & ND & ND & ND & ND \\
\hline Zinc & $\mathrm{mg} / \mathrm{L}$ & 0.009 & 0.005 & 0.035 & 0.013 & ND & ND & 0.011 & 0.0075 & 0.012 & 0.013 & 0.005 & 0.052 \\
\hline
\end{tabular}


Table 57

Surface Water Surveillance - Inorganic Contaminants, Pesticides, and Herbicides

Page 2 of 32

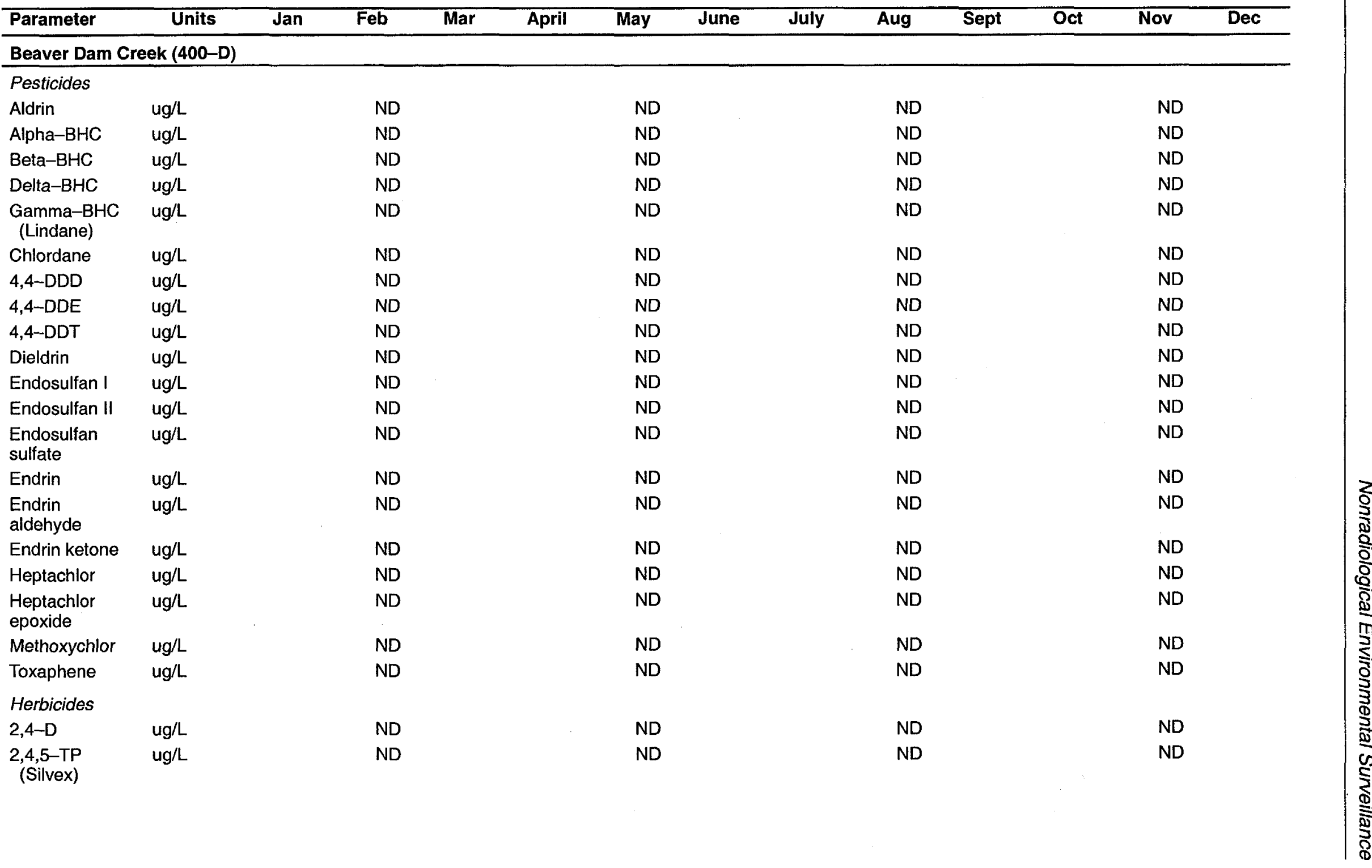


Table 57

Surface Water Surveillance — Inorganic Contaminants, Pesticides, and Herbicides

Page 3 of 32

\begin{tabular}{|c|c|c|c|c|c|c|c|c|c|c|c|c|c|}
\hline $\begin{array}{l}\text { Parameter } \\
\text { Four Mile Creek }\end{array}$ & $\begin{array}{c}\text { Units } \\
-2 \text { (FM-2; }\end{array}$ & $\begin{array}{l}\text { Jan } \\
-2)\end{array}$ & Feb & Mar & April & May & June & July & Aug & Sept & $\overline{\text { Oct }}$ & Nov & Dec \\
\hline Date & & $1 / 12 / 99$ & $2 / 9 / 99$ & $3 / 9 / 99$ & $4 / 7 / 99$ & $5 / 4 / 99$ & $6 / 8 / 99$ & $7 / 13 / 99$ & $8 / 10 / 99$ & $9 / 14 / 99$ & $10 / 26 / 99$ & $11 / 2 / 99$ & $12 / 7 / 99$ \\
\hline Temperature & ${ }^{\circ} \mathrm{C}$ & 6.7 & 13 & 10.8 & 20 & 19 & 22.8 & 22 & 25 & 20.5 & 14 & 19 & 15 \\
\hline DO & $\mathrm{mg} / \mathrm{L}$ & 7.9 & 6.7 & 8.5 & 5.2 & 5.9 & 4.7 & 4.1 & 5.1 & 5.3 & 7 & 6.3 & 8.3 \\
\hline $\mathrm{pH}$ & $\mathrm{pH}$ & 6.4 & 5.8 & 6.5 & 5.8 & 7.1 & 6.4 & 5.9 & 5.9 & 6.7 & 5.1 & 5.8 & 5.4 \\
\hline Conductivity & $\mu h o s / c m$ & 48 & 38 & 40 & 43 & 51 & 80 & 39 & 69 & 98 & 82 & 74 & 57 \\
\hline COD & $\mathrm{mg} / \mathrm{L}$ & ND & ND & ND & ND & ND & ND & 20 & ND & ND & ND & ND & ND \\
\hline Nitrite Nitrogen & $\mathrm{mg} / \mathrm{L}$ & ND & ND & ND & ND & ND & ND & ND & ND & ND & ND & ND & ND \\
\hline Nitrate Nitrogen & $\mathrm{mg} / \mathrm{L}$ & 0.31 & 0.32 & 0.41 & 0.25 & 0.39 & 0.36 & 0.21 & 0.49 & 0.30 & 0.33 & 0.29 & 0.18 \\
\hline TSS & $\mathrm{mg} / \mathrm{L}$ & 5 & 4 & 2 & 9 & 1 & 13 & 4 & ND & 66 & 3 & 1 & 5 \\
\hline Total Phos & $\mathrm{mg} / \mathrm{L}$ & 0.175 & ND & ND & 0.067 & 0.099 & 0.058 & 0.085 & 0.016 & 0.091 & ND & ND & ND \\
\hline TOC & $\mathrm{mg} / \mathrm{L}$ & 3.5 & ND & 3.1 & 4.6 & 3.7 & 1.8 & 7.3 & 4.3 & 2.8 & 1.4 & 1.9 & 2.5 \\
\hline Aluminum & $\mathrm{mg} / \mathrm{L}$ & 0.214 & 0.25 & 0.149 & ND & 0.31 & 0.36 & 0.21 & 0.44 & 0.082 & 0.18 & 0.22 & 0.26 \\
\hline Cadmium & $\mathrm{mg} / \mathrm{L}$ & ND & ND & ND & ND & ND & ND & ND & ND & ND & ND & ND & ND \\
\hline Chromium & $\mathrm{mg} / \mathrm{L}$ & ND & ND & ND & ND & ND & ND & ND & ND & ND & ND & ND & ND \\
\hline Copper & $\mathrm{mg} / \mathrm{L}$ & ND & ND & 0.008 & ND & 0.0085 & ND & ND & ND & ND & ND & ND & ND \\
\hline Iron & $\mathrm{mg} / \mathrm{L}$ & 0.734 & 0.699 & 0.76 & 1.8 & 1.6 & 1.3 & 1.5 & 1.6 & 1.8 & 0.84 & 0.66 & 0.64 \\
\hline Lead & $\mathrm{mg} / \mathrm{L}$ & ND & ND & ND & 0.014 & ND & ND & ND & ND & ND & ND & ND & ND \\
\hline Manganese & $\mathrm{mg} / \mathrm{L}$ & 0.04 & 0.048 & 0.039 & 0.096 & 0.083 & 0.15 & 0.13 & 0.12 & 0.19 & 0.055 & 0.069 & 0.061 \\
\hline Mercury & $\mathrm{mg} / \mathrm{L}$ & ND & ND & ND & ND & ND & ND & ND & ND & ND & ND & ND & ND \\
\hline Nickel & $\mathrm{mg} / \mathrm{L}$ & ND & ND & ND & ND & ND & ND & ND & ND & ND & ND & ND & ND \\
\hline Zinc & $\mathrm{mg} / \mathrm{L}$ & 0.011 & 0.009 & 0.06 & 0.022 & 0.078 & 0.016 & 0.026 & 0.018 & 0.03 & 0.021 & 0.049 & 0.082 \\
\hline
\end{tabular}


Table 57

Surface Water Surveillance - Inorganic Contaminants, Pesticides, and Herbicides

Page 4 of 32

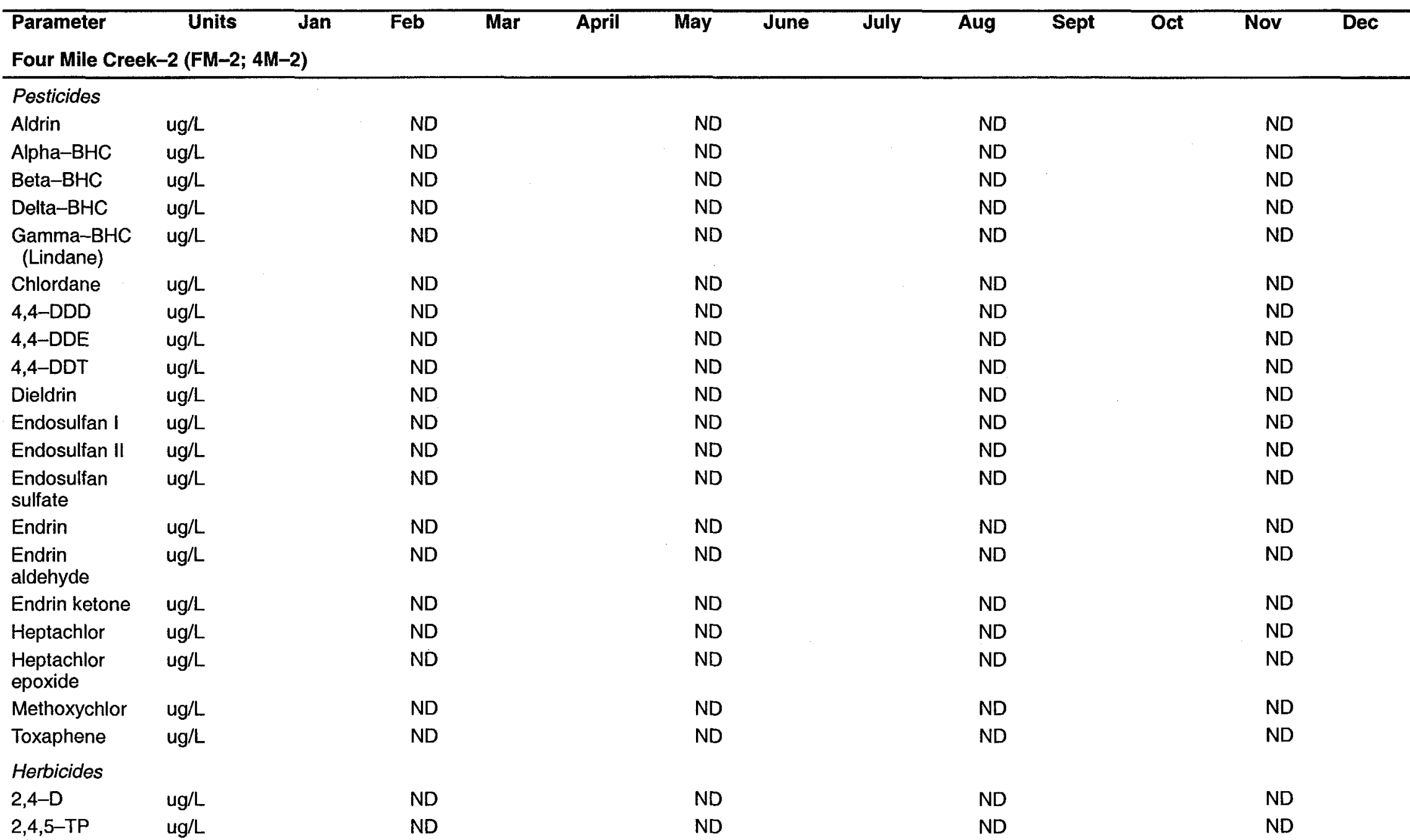

(Silvex)

ND

ND

ND 
Table 57

Surface Water Surveillance - Inorganic Contaminants, Pesticides, and Herbicides

Page 5 of 32

\begin{tabular}{|c|c|c|c|c|c|c|c|c|c|c|c|c|c|}
\hline $\begin{array}{l}\text { Parameter } \\
\text { Four Mile Creek }\end{array}$ & $\begin{array}{c}\text { Units } \\
-2 B(F M-2\end{array}$ & $\begin{array}{c}\text { Jan } \\
4 M-2 B)\end{array}$ & Feb & Mar & April & May & June & July & Aug & Sept & Oct & Nov & Dec \\
\hline Date & & $1 / 12 / 99$ & $2 / 9 / 99$ & $3 / 9 / 99$ & $4 / 7 / 99$ & $5 / 4 / 99$ & $6 / 8 / 99$ & $7 / 13 / 99$ & $8 / 10 / 99$ & $9 / 14 / 99$ & $10 / 26 / 99$ & $11 / 2 / 99$ & $12 / 7 / 99$ \\
\hline Temperature & ${ }^{\circ} \mathrm{C}$ & 5.5 & 12.7 & 9.2 & 20 & 18 & 23.1 & 23 & 26 & 19.8 & 11 & 17 & 10 \\
\hline DO & $\mathrm{mg} / \mathrm{L}$ & 8.3 & 5.8 & 7.6 & 3 & 5.2 & 2.4 & 2.7 & 1 & 3.4 & 7.3 & 5.7 & 7.9 \\
\hline $\mathrm{pH}$ & $\mathrm{pH}$ & 6.1 & 5.9 & 6.4 & 5.5 & 7 & 6.3 & 6.1 & 6.4 & 6.3 & 5.5 & 6.1 & 6.5 \\
\hline Conductivity & $\mu h o s / c m$ & 60 & 53 & 52 & 53 & 49 & 73 & 44 & 102 & 66 & 85 & 99 & 70 \\
\hline COD & $\mathrm{mg} / \mathrm{L}$ & ND & ND & ND & ND & ND & ND & 23 & ND & ND & ND & ND & ND \\
\hline Nitrite Nitrogen & $\mathrm{mg} / \mathrm{L}$ & ND & ND & ND & ND & ND & ND & ND & ND & ND & ND & ND & ND \\
\hline Nitrate Nitrogen & $\mathrm{mg} / \mathrm{L}$ & 0.49 & 0.35 & 0.46 & 0.27 & 0.27 & 0.17 & 0.19 & 0.15 & 0.17 & 0.27 & 0.24 & 0.17 \\
\hline TSS & $\mathrm{mg} / \mathrm{L}$ & 3 & 3 & 2 & 3 & 2 & 2 & 4 & 17 & 1 & 4 & ND & 2 \\
\hline Total Phos & $\mathrm{mg} / \mathrm{L}$ & 0.387 & ND & ND & 0.092 & ND & ND & 0.175 & 0.097 & 0.073 & ND & ND & ND \\
\hline TOC & $\mathrm{mg} / \mathrm{L}$ & 3.2 & ND & 2.7 & 4.4 & 3.3 & 3.6 & 6.7 & 7.9 & 2.2 & 2 & 4.2 & 2.7 \\
\hline Aluminum & $\mathrm{mg} / \mathrm{L}$ & 0.183 & 0.209 & 0.244 & ND & 0.19 & 0.12 & 0.18 & 0.19 & ND & 0.14 & 0.1 & ND \\
\hline Cadmium & $\mathrm{mg} / \mathrm{L}$ & ND & ND & ND & ND & ND & ND & ND & ND & ND & ND & ND & ND \\
\hline Chromium & $\mathrm{mg} / \mathrm{L}$ & ND & ND & ND & ND & ND & ND & ND & ND & ND & ND & ND & ND \\
\hline Copper & $\mathrm{mg} / \mathrm{L}$ & ND & ND & 0.012 & 0.006 & 0.0051 & ND & ND & ND & ND & ND & ND & ND \\
\hline Iron & $\mathrm{mg} / \mathrm{L}$ & 0.425 & 0.794 & 0.839 & 1.9 & 1.2 & 1.5 & 1.2 & 11 & 1.2 & 0.43 & 0.38 & 0.49 \\
\hline Lead & $\mathrm{mg} / \mathrm{L}$ & ND & ND & ND & 0.012 & ND & ND & ND & ND & ND & ND & ND & ND \\
\hline Manganese & $\mathrm{mg} / \mathrm{L}$ & 0.018 & 0.025 & 0.038 & 0.076 & 0.057 & 0.65 & 0.061 & 2 & 1.2 & 0.028 & 0.026 & 0.026 \\
\hline Mercury & $\mathrm{mg} / \mathrm{L}$ & ND & ND & ND & ND & ND & ND & ND & ND & ND & ND & ND & 0.0001 \\
\hline Nickel & $\mathrm{mg} / \mathrm{L}$ & ND & ND & ND & ND & ND & ND & ND & ND & ND & ND & ND & ND \\
\hline Zinc & $\mathrm{mg} / \mathrm{L}$ & 0.01 & 0.013 & 0.055 & 0.038 & 0.013 & 0.016 & 0.037 & ND & 0.0086 & 0.0064 & 0.022 & 0.059 \\
\hline
\end{tabular}


Table 57

Surface Water Surveillance — Inorganic Contaminants, Pesticides, and Herbicides

Page 6 of 32

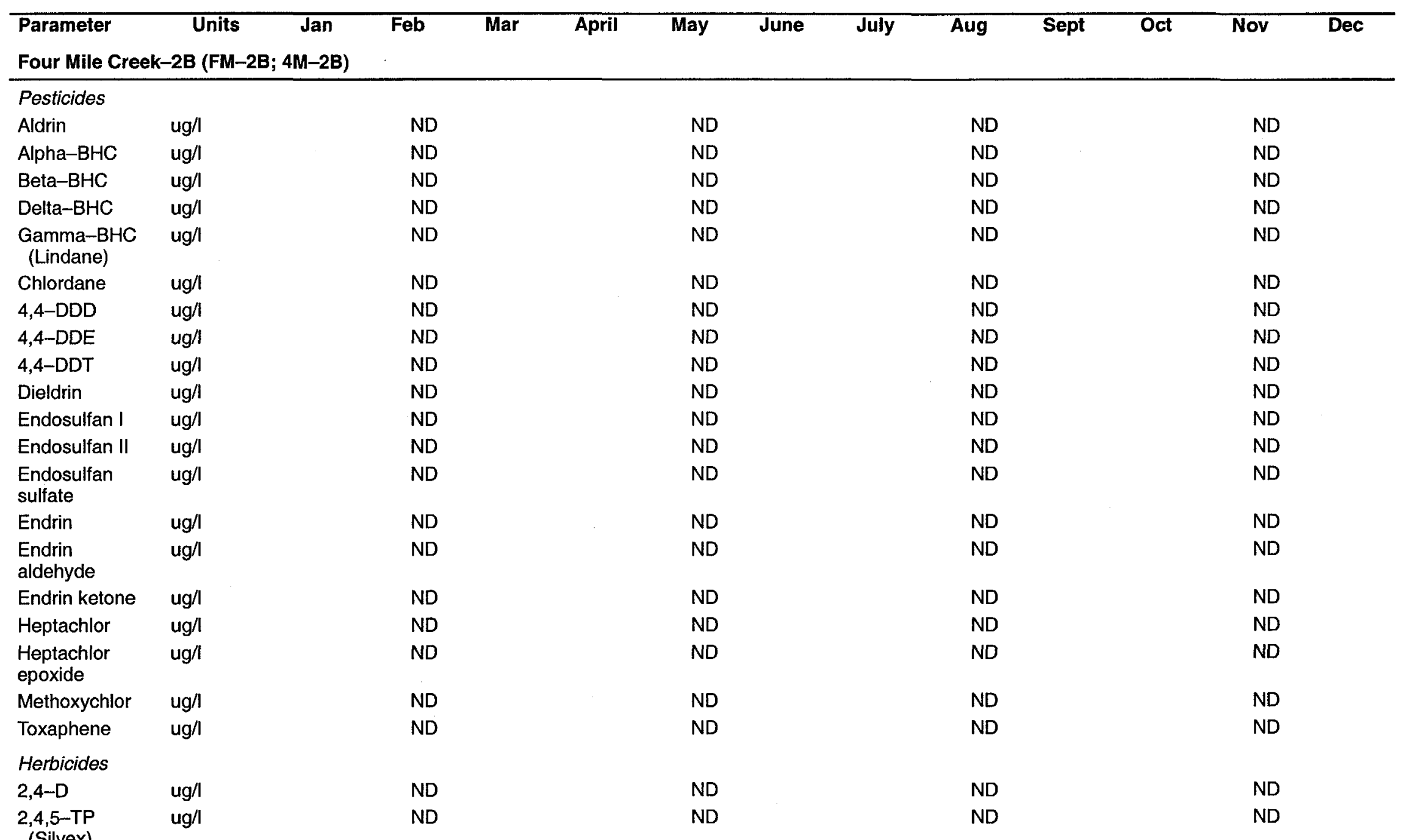


Table 57

Surface Water Surveillance - Inorganic Contaminants, Pesticides, and Herbicides

Page 7 of 32

\begin{tabular}{|c|c|c|c|c|c|c|c|c|c|c|c|c|c|}
\hline $\begin{array}{l}\text { Parameter } \\
\text { Four Mile Creek }\end{array}$ & $\begin{array}{c}\text { Units } \\
-6 \text { (FM-6; }\end{array}$ & $\begin{array}{l}\text { Jan } \\
-6)\end{array}$ & Feb & Mar & April & May & June & July & Aug & Sept & Oct & Nov & $\overline{D e c}$ \\
\hline Date & & $1 / 12 / 99$ & $2 / 9 / 99$ & $3 / 9 / 99$ & $4 / 7 / 99$ & $5 / 4 / 99$ & $6 / 8 / 99$ & $7 / 13 / 99$ & $8 / 10 / 99$ & $9 / 14 / 99$ & $10 / 26 / 99$ & $11 / 2 / 99$ & $12 / 7 / 99$ \\
\hline Temperature & ${ }^{\circ} \mathrm{C}$ & 9.8 & 15.8 & 10.3 & 23 & 21 & 26 & 22 & 28 & 23 & 14 & 18 & 12.3 \\
\hline DO & $\mathrm{mg} / \mathrm{L}$ & 9.5 & 8.5 & 10.7 & 8.3 & 8.2 & 6.3 & 5.1 & 7.1 & 6.7 & 9.9 & 8.5 & 10.5 \\
\hline $\mathrm{pH}$ & $\mathrm{pH}$ & 7 & 7 & 6.6 & 7 & 7.7 & 6.8 & 6.4 & 7.8 & 6.7 & 7 & 6.7 & 5.9 \\
\hline Conductivity & $\mu h o s / c m$ & 54 & 62 & 64 & 54 & 54 & 63 & 44 & 68 & 53 & 70 & 73 & 55 \\
\hline COD & $\mathrm{mg} / \mathrm{L}$ & ND & ND & ND & ND & ND & ND & ND & ND & ND & ND & ND & ND \\
\hline Nitrite Nitrogen & $\mathrm{mg} / \mathrm{L}$ & 0.13 & ND & ND & ND & ND & ND & ND & ND & ND & ND & ND & ND \\
\hline Nitrate Nitrogen & $\mathrm{mg} / \mathrm{L}$ & 1.61 & 2.11 & 1.99 & 1.35 & 1.49 & 1.08 & 0.85 & 1.05 & 0.32 & 1.28 & 1.13 & 0.68 \\
\hline TSS & $\mathrm{mg} / \mathrm{L}$ & 4 & 1 & 5 & 3 & 1 & 2 & 2 & ND & ND & 2 & 3 & 3 \\
\hline Total Phos & $\mathrm{mg} / \mathrm{L}$ & 0.402 & 0.043 & ND & 0.025 & ND & ND & 0.116 & ND & 0.098 & ND & ND & 0.318 \\
\hline TOC & $\mathrm{mg} / \mathrm{L}$ & 2.1 & ND & 2.9 & 3.7 & 2.8 & 2.5 & 9.9 & 2.7 & 4 & 1.5 & 3.2 & 3.6 \\
\hline Aluminum & $\mathrm{mg} / \mathrm{L}$ & 0.145 & 0.22 & 0.329 & ND & 0.24 & 0.16 & 0.25 & 0.17 & ND & 0.15 & 0.3 & 0.089 \\
\hline Cadmium & $\mathrm{mg} / \mathrm{L}$ & ND & ND & ND & ND & ND & ND & ND & ND & ND & ND & ND & ND \\
\hline Chromium & $\mathrm{mg} / \mathrm{L}$ & ND & ND & ND & ND & ND & ND & ND & ND & ND & ND & ND & ND \\
\hline Copper & $\mathrm{mg} / \mathrm{L}$ & ND & 0.009 & 0.007 & ND & ND & ND & ND & ND & ND & ND & ND & 0.046 \\
\hline Iron & $\mathrm{mg} / \mathrm{L}$ & 0.304 & 0.329 & 0.901 & 0.98 & 0.91 & 0.51 & 0.88 & 0.22 & 0.27 & 0.28 & 0.5 & 0.74 \\
\hline Lead & $\mathrm{mg} / \mathrm{L}$ & ND & ND & ND & ND & ND & ND & ND & ND & ND & ND & ND & ND \\
\hline Manganese & $\mathrm{mg} / \mathrm{L}$ & 0.026 & 0.028 & 0.073 & 0.046 & 0.036 & 0.042 & 0.055 & 0.015 & 0.017 & 0.017 & 0.023 & 0.048 \\
\hline Mercury & $\mathrm{mg} / \mathrm{L}$ & ND & ND & ND & 0.0001 & ND & ND & ND & ND & ND & ND & ND & ND \\
\hline Nickel & $\mathrm{mg} / \mathrm{L}$ & ND & ND & ND & ND & ND & ND & ND & ND & ND & ND & ND & ND \\
\hline Zinc & $\mathrm{mg} / \mathrm{L}$ & 0.011 & 0.021 & 0.053 & 0.033 & 0.032 & 0.01 & 0.017 & ND & 0.019 & 0.018 & 0.0089 & 0.098 \\
\hline
\end{tabular}




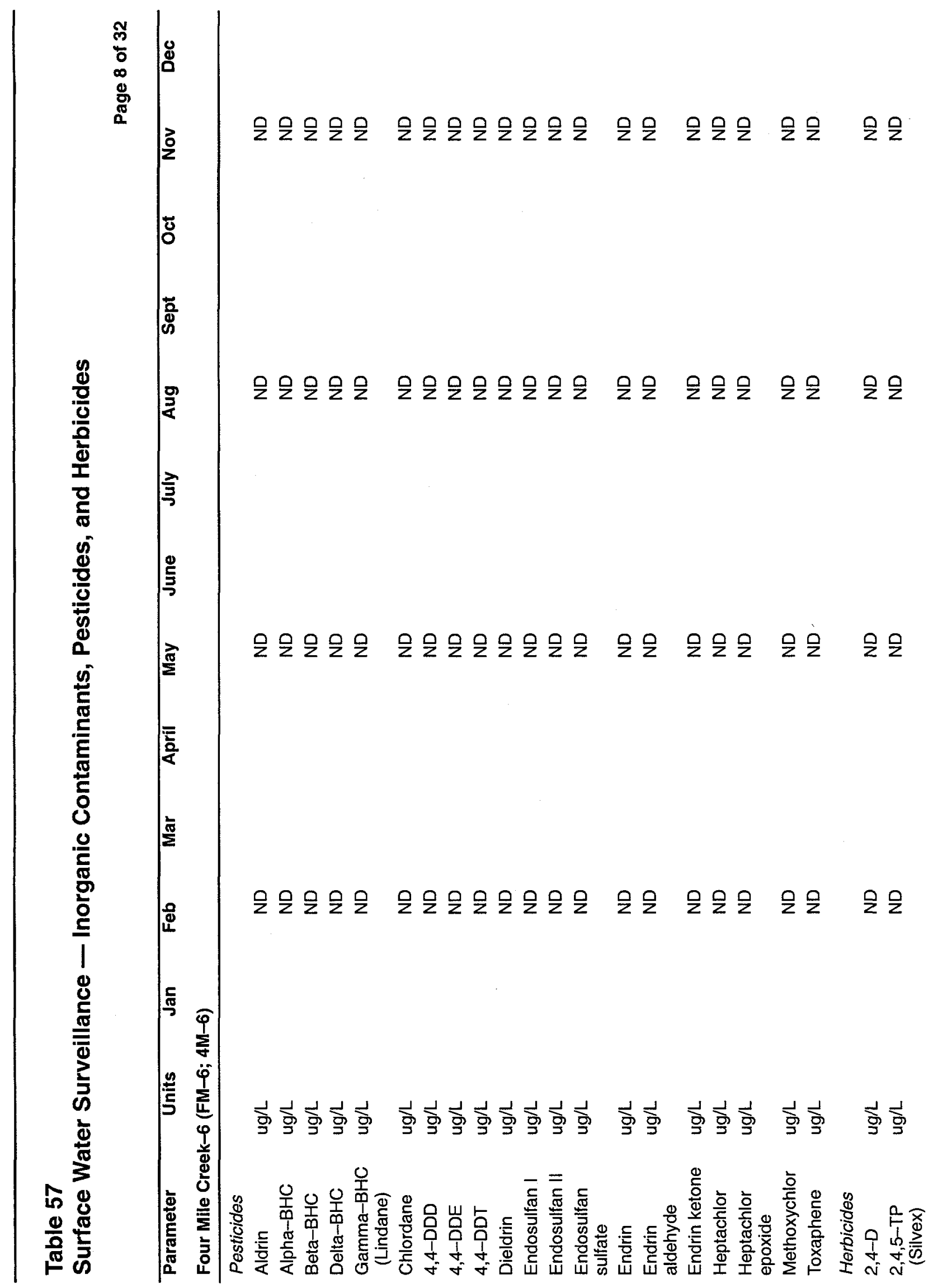


Table 57

Surface Water Surveillance - Inorganic Contaminants, Pesticides, and Herbicides

Page 9 of 32

\begin{tabular}{|c|c|c|c|c|c|c|c|c|c|c|c|c|c|}
\hline \multicolumn{13}{|c|}{ Lower Three Runs Creek-2 (L3R-2) } & Dec \\
\hline Date & & $1 / 12 / 99$ & $2 / 9 / 99$ & $3 / 9 / 99$ & $4 / 7 / 99$ & $5 / 4 / 99$ & $6 / 8 / 99$ & $7 / 13 / 99$ & $8 / 10 / 99$ & $9 / 14 / 99$ & $10 / 26 / 99$ & $11 / 2 / 99$ & $12 / 7 / 99$ \\
\hline Temperature & ${ }^{\circ} \mathrm{C}$ & 10 & 15.4 & 10.8 & 19 & 19 & 20 & 21 & 24 & 20 & 15 & 19 & 17 \\
\hline DO & $\mathrm{mg} / \mathrm{L}$ & 10.3 & 7.8 & 9.5 & 6.7 & 8.3 & 5.5 & 4.8 & 6.8 & 7.2 & 9 & 6.8 & 9.3 \\
\hline $\mathrm{pH}$ & $\mathrm{pH}$ & 7.2 & 6.8 & 6.9 & 6.5 & 7.5 & 6.5 & 6.5 & 7.5 & 6.5 & 6.7 & 6.9 & 7.2 \\
\hline Conductivity & $\mu \mathrm{hos} / \mathrm{cm}$ & 85 & 63 & 92 & 78 & 77 & 48 & 55 & 119 & 87 & 90 & 101 & 127 \\
\hline COD & $\mathrm{mg} / \mathrm{L}$ & ND & ND & ND & ND & ND & ND & ND & ND & ND & ND & ND & ND \\
\hline Nitrite Nitrogen & $\mathrm{mg} / \mathrm{L}$ & ND & ND & ND & ND & ND & ND & ND & ND & ND & ND & ND & ND \\
\hline Nitrate Nitrogen & $\mathrm{mg} / \mathrm{L}$ & 0.29 & 0.20 & 0.26 & 0.20 & 0.30 & 0.20 & 0.22 & 0.37 & 0.28 & 0.26 & 0.20 & 0.21 \\
\hline TSS & $\mathrm{mg} / \mathrm{L}$ & 3 & 3 & 8 & 3 & 3 & 3 & ND & 1 & 1 & 2 & ND & 2 \\
\hline Total Phos & $\mathrm{mg} / \mathrm{L}$ & 0.221 & ND & ND & 0.032 & ND & ND & 0.227 & ND & 0.098 & ND & ND & 0.079 \\
\hline TOC & $\mathrm{mg} / \mathrm{L}$ & 3.5 & ND & 4.7 & 3.8 & 2.9 & 4.9 & 15 & 1.9 & 4.7 & 5.5 & 6.1 & 3.4 \\
\hline Aluminum & $\mathrm{mg} / \mathrm{L}$ & 0.158 & 0.142 & 0.333 & ND & 0.19 & 0.14 & 0.22 & 0.25 & ND & 0.14 & ND & 0.067 \\
\hline Cadmium & $\mathrm{mg} / \mathrm{L}$ & ND & ND & ND & ND & ND & ND & ND & ND & ND & ND & ND & ND \\
\hline Chromium & $\mathrm{mg} / \mathrm{L}$ & ND & ND & ND & ND & ND & ND & ND & ND & 0.0062 & ND & ND & ND \\
\hline Copper & $\mathrm{mg} / \mathrm{L}$ & ND & ND & 0.029 & ND & ND & ND & 0.015 & ND & ND & ND & ND & 0.012 \\
\hline Iron & $\mathrm{mg} / \mathrm{L}$ & 0.191 & 0.232 & 0.298 & 0.37 & 0.33 & 0.15 & 0.61 & 0.41 & 2.3 & 3 & 0.51 & 0.26 \\
\hline Lead & $\mathrm{mg} / \mathrm{L}$ & ND & ND & ND & ND & ND & ND & ND & ND & ND & ND & ND & ND \\
\hline Manganese & $\mathrm{mg} / \mathrm{L}$ & 0.031 & 0.034 & 0.049 & 0.06 & 0.041 & 0.019 & 0.068 & 0.094 & 0.035 & 0.13 & 0.063 & 0.13 \\
\hline Mercury & $\mathrm{mg} / \mathrm{L}$ & ND & ND & ND & 0.0001 & ND & ND & ND & ND & ND & ND & ND & ND \\
\hline Nickel & $\mathrm{mg} / \mathrm{L}$ & ND & ND & ND & ND & ND & ND & ND & ND & ND & ND & ND & ND \\
\hline Zinc & $\mathrm{mg} / \mathrm{L}$ & 0.007 & 0.005 & 0.065 & 0.009 & 0.048 & 0.011 & 0.014 & ND & ND & 0.02 & 0.0085 & 0.068 \\
\hline
\end{tabular}


Table 57

Surface Water Surveillance — Inorganic Contaminants, Pesticides, and Herbicides

Page 10 of 32

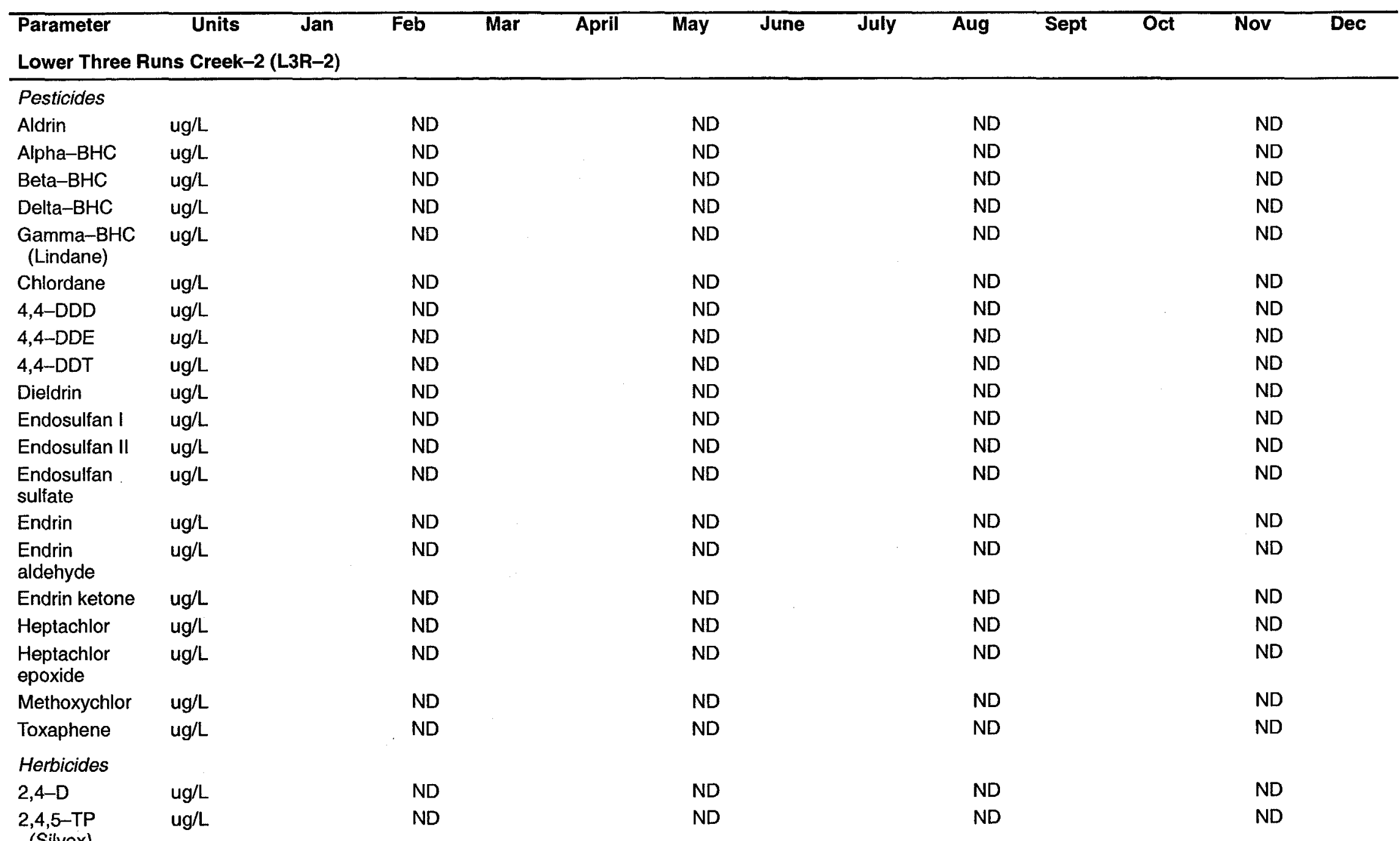


Table 57

Surface Water Surveillance - Inorganic Contaminants, Pesticides, and Herbicides

Page 11 of 32

\begin{tabular}{|c|c|c|c|c|c|c|c|c|c|c|c|c|c|}
\hline$\overline{\text { Parameter }}$ & Units & Jan & Feb & Mar & April & May & June & July & $\overline{\text { Aug }}$ & Sept & Oct & Nov & Dec \\
\hline \multicolumn{14}{|c|}{ Pen Branch-3 (PB-3) } \\
\hline Date & & $1 / 12 / 99$ & $2 / 9 / 99$ & $3 / 9 / 99$ & $4 / 7 / 99$ & $5 / 4 / 99$ & $6 / 8 / 99$ & $7 / 13 / 99$ & $8 / 10 / 99$ & $9 / 14 / 99$ & $10 / 26 / 99$ & $11 / 2 / 99$ & $12 / 7 / 99$ \\
\hline Temperature & ${ }^{\circ} \mathrm{C}$ & 9.9 & 15.3 & 10.3 & 22 & 21 & 26.6 & 22 & 28 & 23 & 14 & 18 & 11 \\
\hline DO & $\mathrm{mg} / \mathrm{L}$ & 9.7 & 8.8 & 9.1 & 7.1 & 6.8 & 5.5 & 5.5 & 6.5 & 7.2 & 10.2 & 8.4 & 11 \\
\hline $\mathrm{pH}$ & $\mathrm{pH}$ & 6.8 & 6.6 & 6.5 & 6.4 & 7.5 & 6.8 & 6.3 & 7.7 & 6.7 & 6.7 & 6.7 & 6.5 \\
\hline Conductivity & $\mu \mathrm{hos} / \mathrm{cm}$ & 72 & 56 & 55 & 59 & 55 & 68 & 43 & 69 & 64 & 64 & 64 & 68 \\
\hline COD & $\mathrm{mg} / \mathrm{L}$ & ND & ND & ND & ND & ND & ND & 21 & ND & ND & ND & ND & ND \\
\hline Nitrite Nitrogen & $\mathrm{mg} / \mathrm{L}$ & ND & ND & ND & ND & ND & ND & ND & ND & ND & ND & ND & ND \\
\hline Nitrate Nitrogen & $\mathrm{mg} / \mathrm{L}$ & 0.35 & 0.23 & 0.26 & 0.22 & 0.30 & 0.18 & 0.35 & 0.31 & 0.23 & 0.32 & 0.26 & 0.18 \\
\hline TSS & $\mathrm{mg} / \mathrm{L}$ & 3 & 1 & 3 & 2 & 2 & 2 & 2 & ND & ND & 2 & 2 & 1 \\
\hline Total Phos & $\mathrm{mg} / \mathrm{L}$ & 0.325 & ND & ND & 0.135 & ND & ND & 0.329 & 0.058 & 0.094 & ND & ND & 0.025 \\
\hline TOC & $\mathrm{mg} / \mathrm{L}$ & 1.8 & ND & 3.3 & 3.8 & 4.3 & 3.1 & 9.6 & 3.7 & 3.5 & 2.8 & 5.3 & 4.2 \\
\hline Aluminum & $\mathrm{mg} / \mathrm{L}$ & 0.154 & 0.161 & 0.171 & ND & 0.23 & 0.17 & 0.2 & 0.22 & ND & 0.15 & 0.28 & ND \\
\hline Cadmium & $\mathrm{mg} / \mathrm{L}$ & ND & ND & ND & ND & ND & ND & ND & ND & ND & ND & ND & ND \\
\hline Chromium & $\mathrm{mg} / \mathrm{L}$ & ND & ND & ND & ND & ND & ND & ND & ND & ND & ND & ND & ND \\
\hline Copper & $\mathrm{mg} / \mathrm{L}$ & ND & 0.008 & ND & ND & 0.0056 & ND & 0.0059 & ND & ND & ND & ND & 0.0077 \\
\hline Iron & $\mathrm{mg} / \mathrm{L}$ & 0.344 & 0.502 & 0.473 & 0.84 & 0.72 & 0.64 & 1.1 & 0.54 & 0.35 & 0.39 & 0.49 & 0.37 \\
\hline Lead & $\mathrm{mg} / \mathrm{L}$ & ND & ND & ND & ND & ND & ND & ND & ND & ND & ND & ND & ND \\
\hline Manganese & $\mathrm{mg} / \mathrm{L}$ & 0.031 & 0.047 & 0.047 & 0.059 & 0.056 & 0.077 & 0.059 & 0.038 & 0.024 & 0.033 & 0.07 & 0.038 \\
\hline Mercury & $\mathrm{mg} / \mathrm{L}$ & ND & ND & ND & 0.0001 & ND & ND & ND & ND & ND & ND & ND & ND \\
\hline Nickel & $\mathrm{mg} / \mathrm{L}$ & ND & ND & ND & ND & ND & ND & ND & ND & ND & ND & ND & ND \\
\hline Zinc & $\mathrm{mg} / \mathrm{L}$ & ND & 0.031 & 0.019 & 0.007 & 0.046 & ND & 0.0075 & ND & 0.012 & ND & ND & 0.057 \\
\hline
\end{tabular}




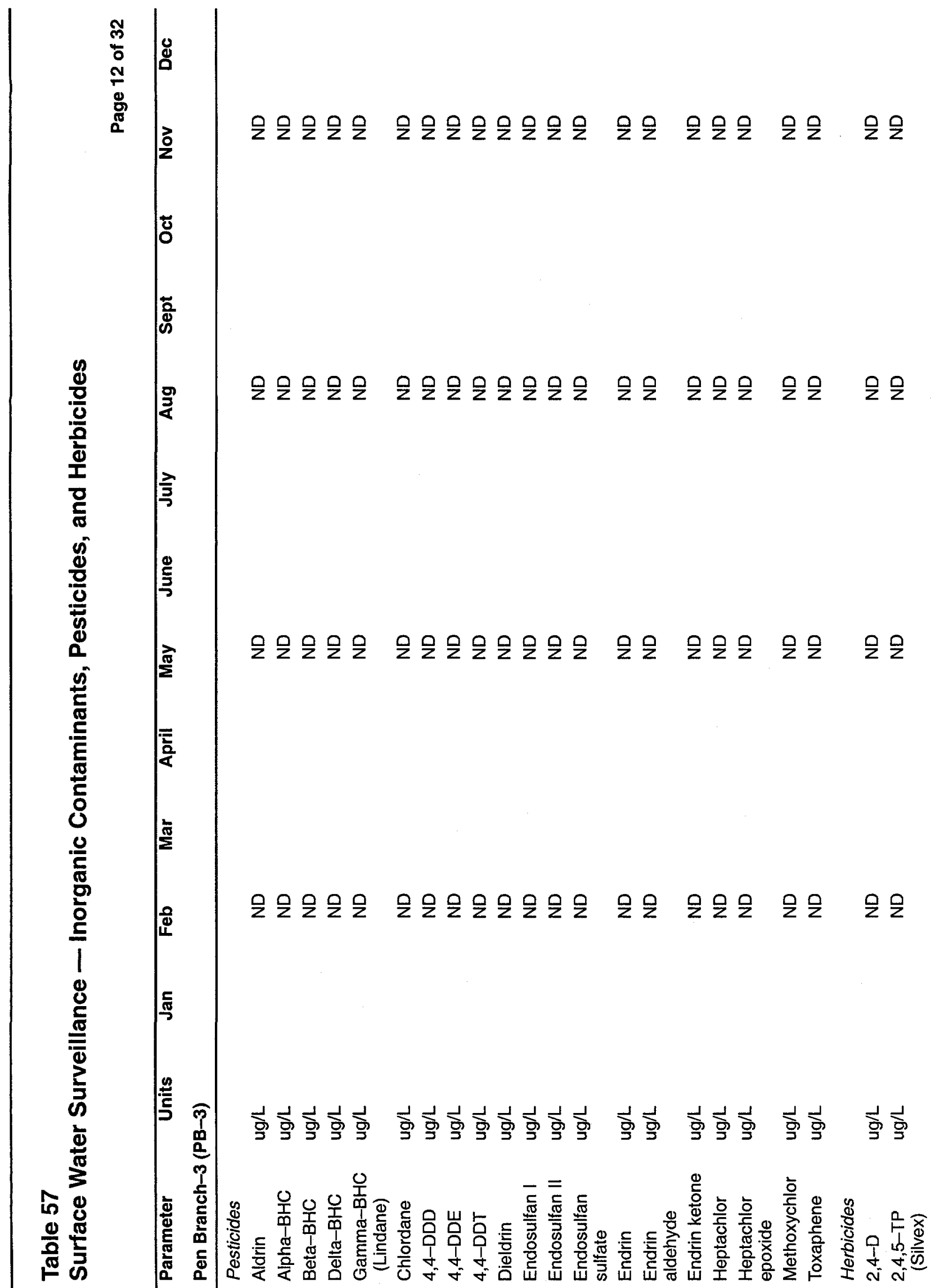


Table 57

Surface Water Surveillance - Inorganic Contaminants, Pesticides, and Herbicides

Page 13 of 32

\begin{tabular}{|c|c|c|c|c|c|c|c|c|c|c|c|c|c|}
\hline Parameter & Units & Jan & Feb & Mar & April & May & June & July & Aug & Sept & Oct & Nov & Dec \\
\hline \multicolumn{14}{|c|}{ River Mile 120 (RM-120; RM-10; Below SRS) } \\
\hline Date & & $1 / 12 / 99$ & $2 / 9 / 99$ & $3 / 9 / 99$ & $4 / 7 / 99$ & $5 / 4 / 99$ & $6 / 8 / 99$ & $7 / 13 / 99$ & $8 / 10 / 99$ & $9 / 14 / 99$ & $10 / 26 / 99$ & $11 / 2 / 99$ & $12 / 7 / 99$ \\
\hline Temperature & ${ }^{\circ} \mathrm{C}$ & 9.9 & 13.4 & 12.8 & 20 & 16.6 & 24 & 23 & 28 & 24 & 18.3 & 20 & 14 \\
\hline DO & $\mathrm{mg} / \mathrm{L}$ & 10.2 & $14.9^{a}$ & 7.7 & $10.3^{a}$ & 8.1 & 6.4 & 6.3 & 7 & 6.9 & 7.8 & 8.2 & 8.8 \\
\hline $\mathrm{pH}$ & $\mathrm{pH}$ & 7.11 & 6.7 & 7.3 & 6.5 & 6.7 & 7.1 & 7 & 6.8 & 6.7 & 7.2 & 6.7 & 6.4 \\
\hline Conductivity & $\mu h o s / c m$ & 107 & 96 & 125 & 105 & 102 & 119 & 96 & 111 & 99 & 104 & 129 & 128 \\
\hline COD & $\mathrm{mg} / \mathrm{L}$ & ND & ND & ND & ND & ND & ND & ND & ND & ND & ND & ND & ND \\
\hline Nitrite Nitrogen & $\mathrm{mg} / \mathrm{L}$ & ND & ND & ND & ND & ND & ND & ND & ND & ND & ND & ND & ND \\
\hline Nitrte Nitrogen & $\mathrm{mg} / \mathrm{L}$ & 0.39 & 0.42 & 0.41 & 0.44 & 0.58 & 0.49 & 0.46 & 0.48 & 0.42 & 0.43 & 0.44 & 0.38 \\
\hline TSS & $\mathrm{mg} / \mathrm{L}$ & 6 & 12 & 6 & 7 & 9 & 11 & ND & 7 & 8 & 5 & 3 & 4 \\
\hline Total Phos & $\mathrm{mg} / \mathrm{L}$ & 0.503 & 0.067 & ND & ND & 0.218 & 0.126 & 0.159 & 0.132 & 0.298 & 0.114 & 0.11 & 0.245 \\
\hline TOC & $\mathrm{mg} / \mathrm{L}$ & 3.6 & 2.8 & 3.7 & 3.1 & 4 & 3.2 & 7 & 3.5 & 2.6 & 2.9 & 3.1 & 3.6 \\
\hline Aluminum & $\mathrm{mg} / \mathrm{L}$ & 0.304 & 0.3 & 0.18 & 0.08 & 0.21 & 0.35 & 0.46 & 0.23 & ND & 0.2 & 0.27 & 0.19 \\
\hline Cadmium & $\mathrm{mg} / \mathrm{L}$ & ND & ND & ND & ND & ND & ND & ND & ND & ND & ND & ND & ND \\
\hline Chromium & $\mathrm{mg} / \mathrm{L}$ & ND & ND & ND & ND & ND & ND & ND & ND & ND & ND & ND & ND \\
\hline Copper & $\mathrm{mg} / \mathrm{L}$ & ND & ND & ND & 0.019 & ND & ND & ND & ND & ND & ND & ND & ND \\
\hline Iron & $\mathrm{mg} / \mathrm{L}$ & 0.365 & 0.562 & 0.415 & 0.48 & 0.47 & 0.44 & 0.84 & 0.41 & 0.24 & 0.29 & 0.21 & 0.29 \\
\hline Lead & $\mathrm{mg} / \mathrm{L}$ & ND & ND & ND & ND & ND & ND & ND & ND & ND & ND & ND & ND \\
\hline Manganese & $\mathrm{mg} / \mathrm{L}$ & 0.06 & 0.091 & 0.058 & 0.053 & 0.076 & 0.057 & 0.076 & 0.065 & 0.058 & 0.053 & 0.039 & 0.051 \\
\hline Mercury & $\mathrm{mg} / \mathrm{L}$ & ND & ND & ND & ND & ND & ND & ND & ND & ND & ND & ND & ND \\
\hline Nickel & $\mathrm{mg} / \mathrm{L}$ & ND & ND & ND & ND & ND & ND & ND & ND & ND & ND & ND & ND \\
\hline Zinc & $\mathrm{mg} / \mathrm{L}$ & 0.016 & ND & 0.015 & 0.019 & 0.012 & 0.011 & 0.017 & 0.021 & 0.0073 & ND & 0.007 & 0.036 \\
\hline
\end{tabular}

a Supersaturated, probably due to instream primary production 


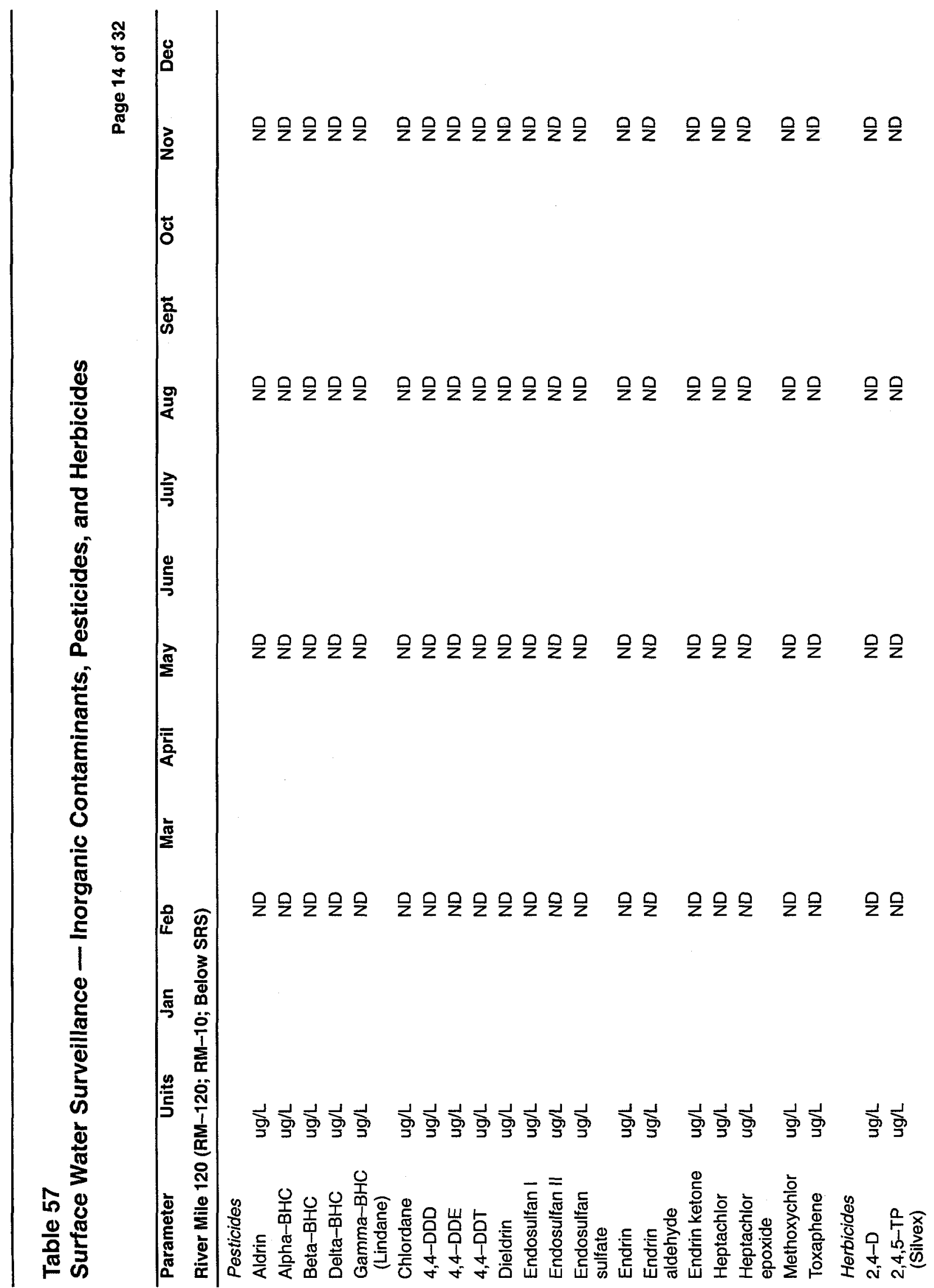


Table 57

Surface Water Surveillance - Inorganic Contaminants, Pesticides, and Herbicides

Page 15 of 32

\begin{tabular}{|c|c|c|c|c|c|c|c|c|c|c|c|c|c|}
\hline $\begin{array}{l}\text { Parameter } \\
\text { River Mile } 129 \text { ( }\end{array}$ & $\begin{array}{c}\text { Units } \\
\text { RM-129; B }\end{array}$ & $\begin{array}{c}\text { Jan } \\
\text { W SRS) }\end{array}$ & Feb & Mar & April & May & June & July & Aug & Sept & Oct & Nov & Dec \\
\hline Date & & $1 / 12 / 99$ & $2 / 9 / 99$ & $3 / 9 / 99$ & $4 / 7 / 99$ & $5 / 4 / 99$ & $6 / 8 / 99$ & $7 / 13 / 99$ & $8 / 10 / 99$ & $9 / 14 / 99$ & $10 / 26 / 99$ & $11 / 2 / 99$ & $12 / 7 / 99$ \\
\hline Temperature & ${ }^{\circ} \mathrm{C}$ & 6.1 & 13.6 & 10.9 & 19 & 15.2 & 21 & 22 & 26 & 24 & 18.3 & 20 & 14 \\
\hline DO & $\mathrm{mg} / \mathrm{L}$ & 11.5 & $14.1^{\mathrm{a}}$ & $11.7^{a}$ & 7.5 & 8.2 & 7.7 & 6.8 & 6.4 & 7.4 & 8.1 & 7.8 & 8.9 \\
\hline $\mathrm{pH}$ & $\mathrm{pH}$ & 7 & 6.8 & 7.2 & 6.8 & 7.1 & 7 & 7 & 6.9 & 6.8 & 7.3 & 8 & 6.5 \\
\hline Conductivity & $\mu h o s / c m$ & 84 & 68 & 82 & 90 & 72 & 109 & 94 & 114 & 99 & 110 & 124 & 112 \\
\hline COD & $\mathrm{mg} / \mathrm{L}$ & ND & ND & ND & ND & ND & ND & ND & ND & ND & ND & ND & ND \\
\hline Nitrite Nitrogen & $\mathrm{mg} / \mathrm{L}$ & ND & ND & ND & ND & ND & ND & ND & ND & ND & ND & ND & ND \\
\hline Nitrate Nitrogen & $\mathrm{mg} / \mathrm{L}$ & 0.37 & 0.26 & 0.29 & 0.41 & 0.38 & 0.48 & 0.51 & 0.43 & 0.40 & 0.43 & 0.39 & 0.33 \\
\hline TSS & $\mathrm{mg} / \mathrm{L}$ & 50 & 64 & 5 & 17 & 8 & 35 & ND & 7 & 7 & 7 & 4 & 6 \\
\hline Total Phos & $\mathrm{mg} / \mathrm{L}$ & 0.215 & ND & ND & 0.194 & 0.173 & 0.516 & 0.149 & 0.082 & 0.18 & 0.073 & 0.075 & 0.116 \\
\hline TOC & $\mathrm{mg} / \mathrm{L}$ & 3.5 & 2.7 & 3.4 & 3.5 & 4.3 & 2.9 & 7.1 & 4.1 & 3.5 & 2.6 & 3.9 & 3.4 \\
\hline Aluminum & $\mathrm{mg} / \mathrm{L}$ & 0.726 & 0.337 & 0.238 & 0.17 & 0.55 & 0.27 & 0.39 & 0.2 & ND & 0.24 & 0.14 & 0.22 \\
\hline Cadmium & $\mathrm{mg} / \mathrm{L}$ & ND & ND & ND & ND & ND & ND & ND & ND & ND & ND & ND & ND \\
\hline Chromium & $\mathrm{mg} / \mathrm{L}$ & ND & ND & ND & ND & 0.009 & ND & ND & ND & ND & ND & ND & ND \\
\hline Copper & $\mathrm{mg} / \mathrm{L}$ & ND & ND & ND & 0.006 & 0.0067 & ND & ND & ND & ND & ND & ND & ND \\
\hline Iron & $m g / L$ & 1.06 & 1.04 & 0.305 & 0.75 & 1.5 & 0.59 & 0.71 & 0.24 & 0.24 & 0.28 & 0.23 & 0.47 \\
\hline Lead & $\mathrm{mg} / \mathrm{L}$ & ND & ND & ND & ND & ND & ND & ND & ND & ND & ND & ND & ND \\
\hline Manganese & $\mathrm{mg} / \mathrm{L}$ & 0.124 & 0.353 & 0.024 & 0.081 & 0.28 & 0.09 & 0.068 & 0.06 & 0.052 & 0.055 & 0.043 & 0.063 \\
\hline Mercury & $\mathrm{mg} / \mathrm{L}$ & ND & ND & ND & ND & ND & ND & ND & ND & ND & ND & ND & ND \\
\hline Nickel & $\mathrm{mg} / \mathrm{L}$ & ND & ND & ND & ND & ND & ND & ND & ND & ND & ND & ND & ND \\
\hline Zinc & $\mathrm{mg} / \mathrm{L}$ & 0.015 & ND & 0.008 & 0.019 & 0.037 & 0.006 & 0.011 & 0.0054 & ND & ND & 0.016 & 0.0061 \\
\hline
\end{tabular}

a Supersaturated, probably due to instream primary production 
Table 57

Surface Water Surveillance - Inorganic Contaminants, Pesticides, and Herbicides

Page 16 of 32

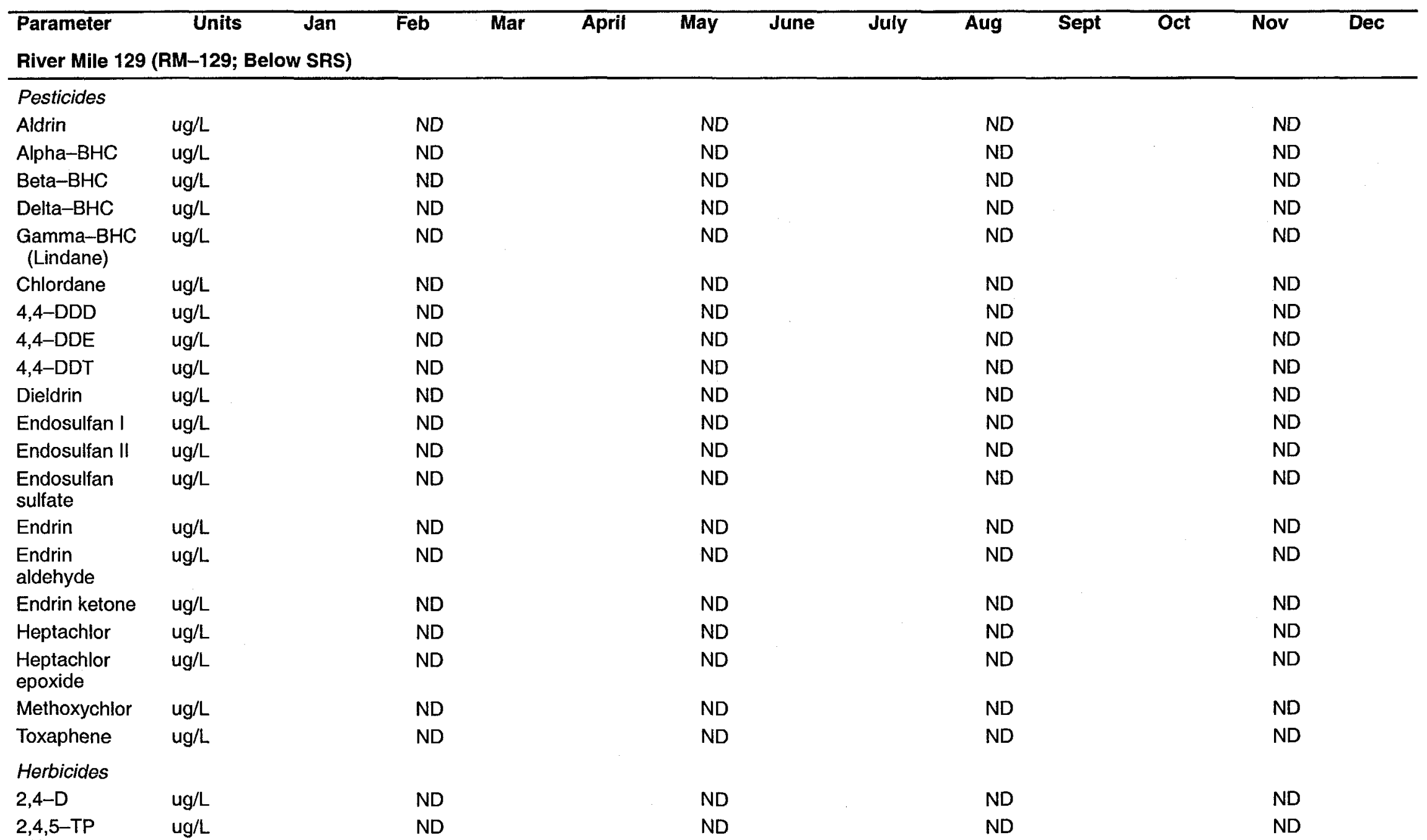


Table 57

Surface Water Surveillance - Inorganic Contaminants, Pesticides, and Herbicides

Page 17 of 32

\begin{tabular}{|c|c|c|c|c|c|c|c|c|c|c|c|c|c|}
\hline $\begin{array}{l}\text { Parameter } \\
\text { River Mile } 140 \text { ( }\end{array}$ & $\begin{array}{r}\text { Units } \\
\text { (M-140) }\end{array}$ & Jan & Feb & Mar & April & May & June & July & Aug & Sept & $\overline{\text { Oct }}$ & Nov & $\overline{\text { Dec }}$ \\
\hline Date & & $1 / 12 / 99$ & $2 / 9 / 99$ & $3 / 9 / 99$ & $4 / 7 / 99$ & $5 / 4 / 99$ & $6 / 8 / 99$ & $7 / 13 / 99$ & $8 / 10 / 99$ & $9 / 14 / 99$ & $10 / 26 / 99$ & $11 / 2 / 99$ & $12 / 7 / 99$ \\
\hline Temperature & ${ }^{\circ} \mathrm{C}$ & 10.2 & 13.3 & 12.2 & 19.2 & 16.7 & 24 & 22 & 27 & 24 & 18.3 & 20 & 14.2 \\
\hline DO & $\mathrm{mg} / \mathrm{L}$ & 10.04 & $10.68^{a}$ & $11.1^{a}$ & 8 & 8.2 & 7.4 & 6.4 & 6.2 & 7.1 & 8 & 7.77 & 8.76 \\
\hline $\mathrm{pH}$ & $\mathrm{pH}$ & 6.9 & 6.7 & 7.2 & 6.6 & 7 & 6.7 & 6.9 & 6.8 & 6.5 & 7.2 & 6.8 & 6.9 \\
\hline Conductivity & uhos/cm & 105 & 95 & 117 & 102 & 98 & 99 & 92 & 105 & 93 & 106 & 129 & 115 \\
\hline COD & $\mathrm{mg} / \mathrm{L}$ & ND & ND & ND & ND & ND & 20 & ND & ND & ND & ND & ND & ND \\
\hline Nitrite Nitrogen & $\mathrm{mg} / \mathrm{L}$ & ND & ND & ND & ND & ND & ND & ND & ND & ND & ND & ND & ND \\
\hline Nitrate Nitrogen & $\mathrm{mg} / \mathrm{L}$ & 0.42 & 0.40 & 0.52 & 0.45 & 0.53 & 0.47 & 0.45 & 0.49 & 0.38 & 0.40 & 0.42 & 0.34 \\
\hline TSS & $\mathrm{mg} / \mathrm{L}$ & 5 & 11 & 6 & 6 & 7 & 8 & ND & 6 & 7 & 8 & 3 & 4 \\
\hline Total Phos & $\mathrm{mg} / \mathrm{L}$ & 0.242 & 0.037 & ND & 0.02 & 0.262 & 0.268 & 0.223 & 0.051 & 0.172 & 0.075 & 0.047 & 0.023 \\
\hline TOC & $\mathrm{mg} / \mathrm{L}$ & 3.8 & ND & 4.1 & 3.2 & 3.2 & 3.2 & 5.1 & 3.1 & 2.7 & 2.9 & 3.1 & 4 \\
\hline Aluminum & $\mathrm{mg} / \mathrm{L}$ & 0.185 & 0.233 & 0.413 & ND & 0.18 & 0.25 & 0.44 & 0.26 & ND & 0.19 & 0.12 & 0.14 \\
\hline Cadmium & $\mathrm{mg} / \mathrm{L}$ & ND & ND & ND & ND & ND & ND & ND & ND & ND & ND & ND & ND \\
\hline Chromium & $\mathrm{mg} / \mathrm{L}$ & ND & ND & ND & ND & 0.041 & ND & ND & ND & ND & ND & ND & ND \\
\hline Copper & $\mathrm{mg} / \mathrm{L}$ & ND & ND & ND & 0.009 & ND & ND & ND & ND & ND & ND & ND & ND \\
\hline Iron & $\mathrm{mg} / \mathrm{L}$ & 0.198 & 0.562 & 0.477 & 0.49 & 0.46 & 0.28 & 0.88 & 0.35 & 0.19 & 0.24 & 0.22 & 0.36 \\
\hline Lead & $\mathrm{mg} / \mathrm{L}$ & ND & ND & ND & ND & ND & ND & ND & ND & ND & ND & ND & ND \\
\hline Manganese & $\mathrm{mg} / \mathrm{L}$ & 0.045 & 0.08 & 0.056 & 0.058 & 0.11 & 0.056 & 0.1 & 0.06 & 0.057 & 0.064 & 0.051 & 0.068 \\
\hline Mercury & $\mathrm{mg} / \mathrm{L}$ & ND & ND & ND & ND & ND & ND & ND & ND & ND & ND & ND & ND \\
\hline Nickel & $\mathrm{mg} / \mathrm{L}$ & ND & ND & ND & ND & 0.03 & ND & ND & ND & ND & ND & ND & ND \\
\hline Zinc & $\mathrm{mg} / \mathrm{L}$ & 0.023 & ND & 0.009 & 0.022 & 0.023 & 0.057 & 0.01 & 0.007 & ND & 0.006 & 0.0073 & 0.027 \\
\hline
\end{tabular}

a Supersaturated, probably due to instream primary production 


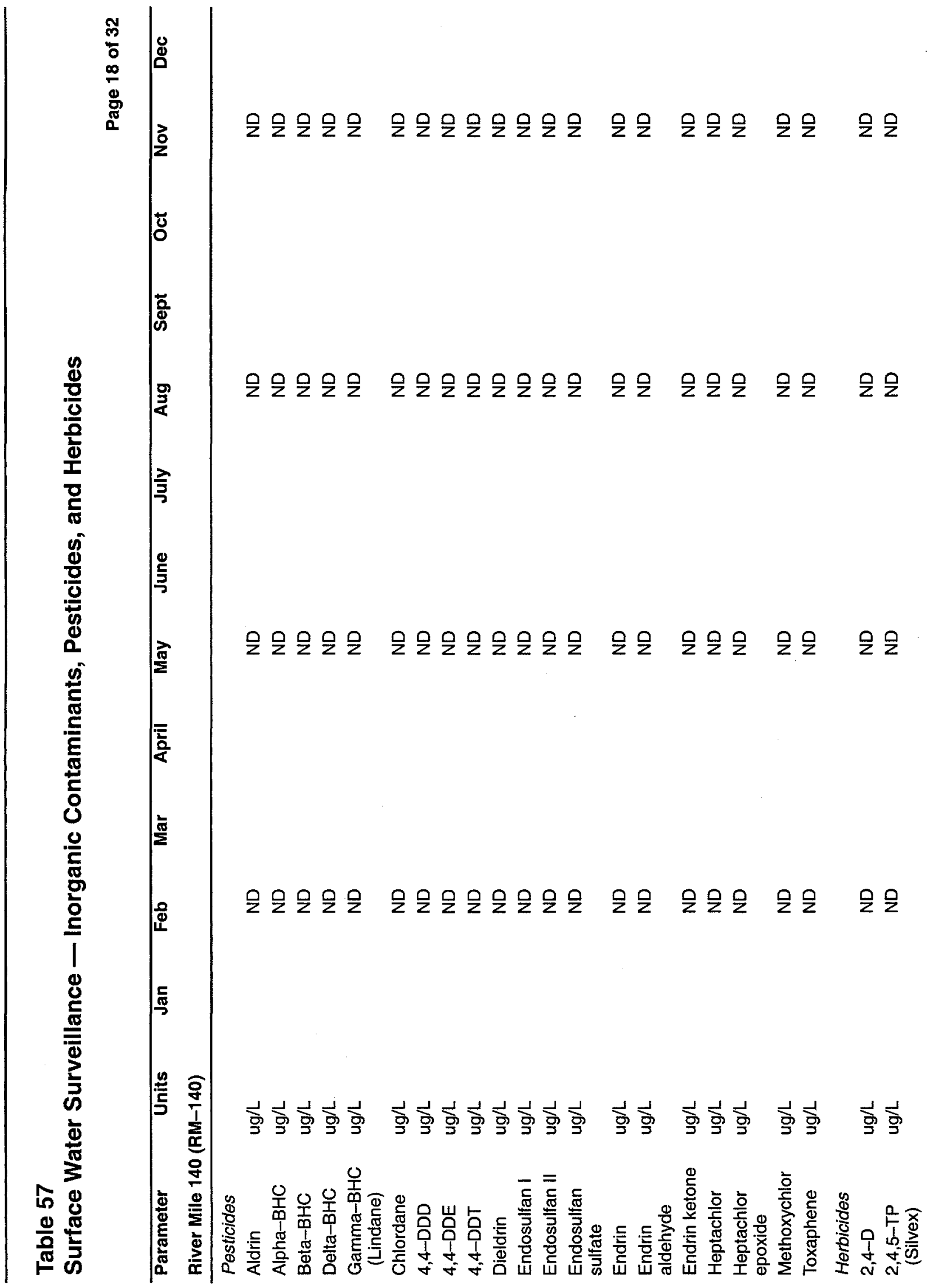


Table 57

Surface Water Surveillance - Inorganic Contaminants, Pesticides, and Herbicides

Page 19 of 32

\begin{tabular}{|c|c|c|c|c|c|c|c|c|c|c|c|c|c|}
\hline Parameter & Units & Jan & Feb & Mar & April & May & June & July & Aug & Sept & Oct & Nov & $\overline{\text { Dec }}$ \\
\hline \multicolumn{14}{|c|}{$R M-150.4(R-3 B)$} \\
\hline Date & & $1 / 12 / 99$ & $2 / 9 / 99$ & $3 / 9 / 99$ & $4 / 7 / 99$ & $5 / 4 / 99$ & $6 / 8 / 99$ & $7 / 13 / 99$ & $8 / 10 / 99$ & $9 / 14 / 99$ & $10 / 26 / 99$ & $11 / 2 / 99$ & $12 / 7 / 99$ \\
\hline Temperature & ${ }^{\circ} \mathrm{C}$ & 10.3 & 13.5 & 12.1 & 18.7 & 17.4 & 25 & 22 & 26 & 24 & 18 & 20 & 14.4 \\
\hline DO & $\mathrm{mg} / \mathrm{L}$ & $11.7^{\mathrm{a}}$ & $11.7^{a}$ & 10.4 & 8.6 & 7.3 & 7.2 & 6.1 & 6.3 & 6.5 & 7.7 & 7.7 & 8.9 \\
\hline $\mathrm{pH}$ & $\mathrm{pH}$ & 7 & 6.5 & 7.2 & 6.7 & 7.1 & 7 & 7 & 6.8 & 6.8 & 7.2 & 6.8 & 7 \\
\hline Conductivity & $\mu \mathrm{hos} / \mathrm{cm}$ & 106 & 83 & 109 & 105 & 100 & 115 & 100 & 106 & 98 & 116 & 130 & 129 \\
\hline COD & $\mathrm{mg} / \mathrm{L}$ & ND & ND & ND & ND & ND & 21 & ND & ND & ND & ND & ND & ND \\
\hline Nitrite Nitrogen & $\mathrm{mg} / \mathrm{L}$ & ND & ND & ND & ND & ND & ND & ND & ND & ND & ND & ND & ND \\
\hline Nitrate Nitrogen & $\mathrm{mg} / \mathrm{L}$ & 0.46 & 0.37 & 0.39 & 0.43 & 0.52 & 0.47 & 0.47 & 0.49 & 0.40 & 0.37 & 0.41 & 0.36 \\
\hline TSS & $\mathrm{mg} / \mathrm{L}$ & 7 & 25 & 7 & 8 & 7 & 5 & 1 & 5 & 10 & 6 & ND & 5 \\
\hline Total Phos & $\mathrm{mg} / \mathrm{L}$ & 0.359 & 0.086 & ND & 0.202 & 0.151 & 0.157 & 0.391 & 0.202 & 0.232 & 0.199 & 1.64 & 0.162 \\
\hline TOC & $\mathrm{mg} / \mathrm{L}$ & 3.5 & ND & 3.7 & 3.6 & 3.7 & 3.6 & 6.1 & 3.7 & 2.8 & 3 & 3.5 & 3.4 \\
\hline Aluminum & $\mathrm{mg} / \mathrm{L}$ & 0.236 & 0.249 & 0.268 & ND & 0.2 & 0.25 & 0.4 & 0.29 & ND & 0.3 & 0.17 & 0.18 \\
\hline Cadmium & $\mathrm{mg} / \mathrm{L}$ & ND & ND & ND & ND & ND & ND & ND & ND & ND & ND & ND & ND \\
\hline Chromium & $\mathrm{mg} / \mathrm{L}$ & ND & ND & ND & ND & ND & ND & ND & ND & ND & ND & ND & ND \\
\hline Copper & $\mathrm{mg} / \mathrm{L}$ & ND & ND & ND & 0.007 & ND & ND & ND & ND & ND & ND & ND & ND \\
\hline Iron & $\mathrm{mg} / \mathrm{L}$ & 0.319 & 0.352 & 0.33 & 0.42 & 0.4 & 0.31 & 0.77 & 0.37 & 0.2 & 0.38 & 0.22 & 0.31 \\
\hline Lead & $\mathrm{mg} / \mathrm{L}$ & ND & ND & ND & ND & ND & ND & ND & ND & ND & ND & ND & ND \\
\hline Manganese & $\mathrm{mg} / \mathrm{L}$ & 0.098 & 0.124 & 0.076 & 0.065 & 0.072 & 0.068 & 0.074 & 0.061 & 0.059 & 0.059 & 0.049 & 0.058 \\
\hline Mercury & $\mathrm{mg} / \mathrm{L}$ & ND & ND & ND & ND & ND & ND & ND & ND & ND & ND & ND & ND \\
\hline Nickel & $\mathrm{mg} / \mathrm{L}$ & ND & ND & ND & ND & ND & ND & ND & ND & ND & ND & ND & ND \\
\hline Zinc & $\mathrm{mg} / \mathrm{L}$ & 0.029 & 0.006 & 0.039 & 0.023 & 0.018 & 0.021 & 0.022 & 0.015 & 0.011 & 0.0057 & 0.0094 & 0.039 \\
\hline
\end{tabular}

a Supersaturated, probably due to instream primary production 


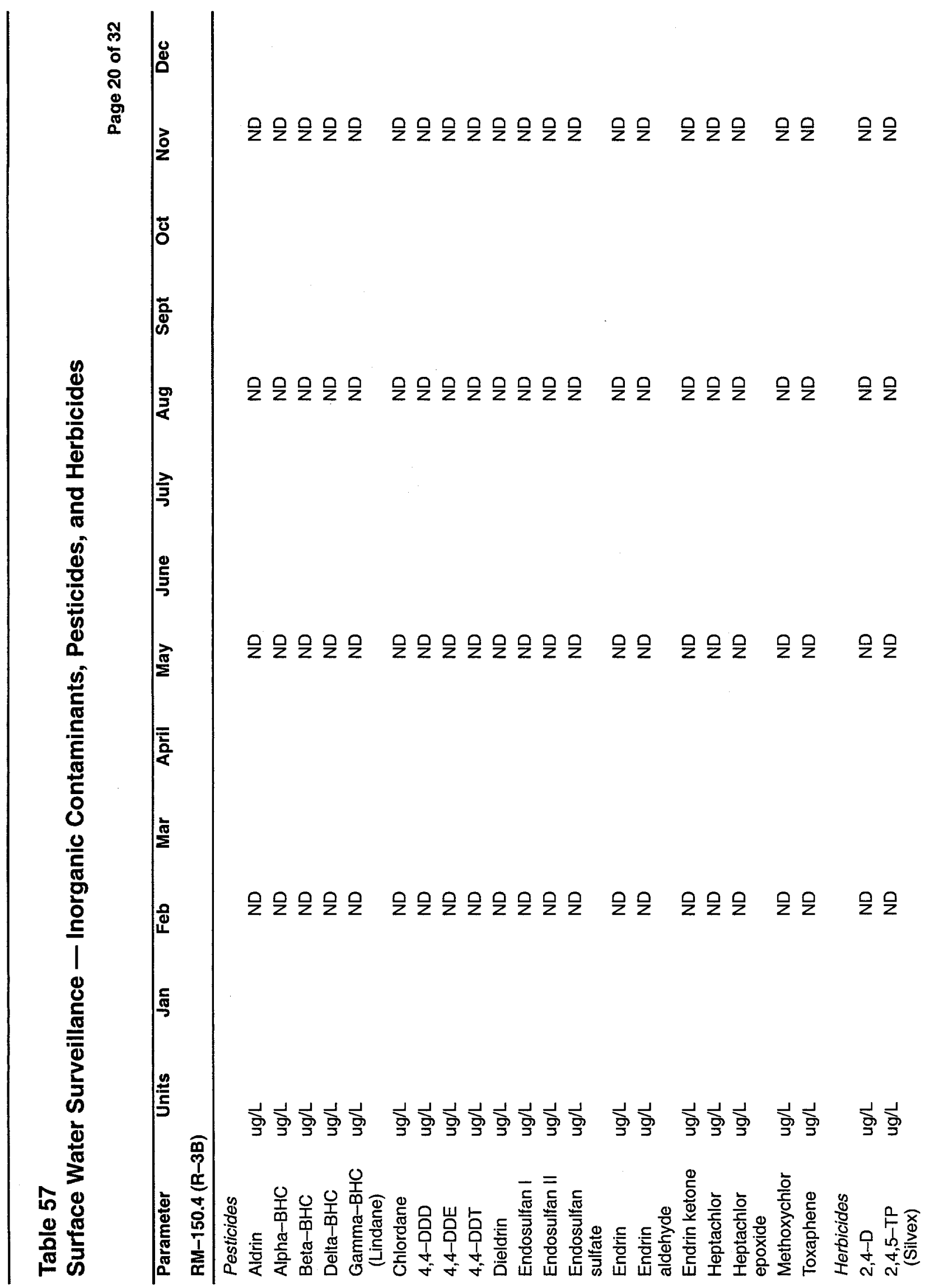


Table 57

Surface Water Surveillance - Inorganic Contaminants, Pesticides, and Herbicides

Page 21 of 32

\begin{tabular}{|c|c|c|c|c|c|c|c|c|c|c|c|c|c|}
\hline Parameter & Units & Jan & Feb & Mar & April & May & June & July & Aug & Sept & Oct & Nov & Dec \\
\hline \multicolumn{14}{|c|}{ River Mile $160(R M-160)$} \\
\hline Date & & $1 / 12 / 99$ & $2 / 9 / 99$ & $3 / 9 / 99$ & $4 / 7 / 99$ & $5 / 4 / 99$ & $6 / 8 / 99$ & $7 / 13 / 99$ & $8 / 10 / 99$ & $9 / 14 / 99$ & $10 / 26 / 99$ & $11 / 2 / 99$ & $12 / 7 / 99$ \\
\hline Temperature & ${ }^{\circ} \mathrm{C}$ & 10.3 & 13.1 & 12.2 & 18 & 17.9 & 24 & 22 & 25 & 23 & 17 & 20 & 14.8 \\
\hline DO & $\mathrm{mg} / \mathrm{L}$ & $11.7^{\mathrm{a}}$ & $11.3^{\mathrm{a}}$ & $11.2^{\mathrm{a}}$ & 8.8 & 8.1 & 8.1 & 6.7 & 6.6 & 6.4 & 8.5 & 7.6 & 9 \\
\hline $\mathrm{pH}$ & $\mathrm{pH}$ & 7.1 & 7 & 7.3 & 6.6 & 7 & 7.1 & 7 & 6.7 & 6.6 & 7.1 & 6.6 & 7 \\
\hline Conductivity & $\mu h o s / c m$ & 102 & 74 & 99 & 99 & 115 & 103 & 102 & 96 & 88 & 113 & 125 & 120 \\
\hline COD & $\mathrm{mg} / \mathrm{L}$ & ND & ND & ND & ND & ND & 20 & ND & ND & ND & ND & ND & ND \\
\hline Nitrite Nitrogen & $\mathrm{mg} / \mathrm{L}$ & ND & ND & ND & ND & ND & ND & ND & ND & ND & ND & ND & ND \\
\hline Nitrate Nitrogen & $\mathrm{mg} / \mathrm{L}$ & 0.40 & 0.40 & 0.37 & 0.46 & 0.48 & 0.42 & 0.52 & 0.46 & 0.32 & 0.33 & 0.39 & 0.34 \\
\hline TSS & $\mathrm{mg} / \mathrm{L}$ & 6 & 18 & 4 & 4 & 5 & 6 & ND & 5 & 5 & 4 & 2 & 3 \\
\hline Total Phos & $\mathrm{mg} / \mathrm{L}$ & 0.175 & 0.283 & ND & 0.115 & 0.243 & 0.107 & 0.154 & 0.056 & 0.124 & 0.081 & 0.102 & 0.134 \\
\hline TOC & $\mathrm{mg} / \mathrm{L}$ & 3.2 & ND & 3.4 & 3.2 & 3.9 & 3.4 & 5.3 & 3.8 & 2.5 & 3.2 & 3.7 & 3.5 \\
\hline Aluminum & $\mathrm{mg} / \mathrm{L}$ & 0.24 & 0.222 & 0.29 & ND & 0.31 & 0.15 & 0.33 & 0.5 & ND & 0.19 & 0.12 & ND \\
\hline Cadmium & $\mathrm{mg} / \mathrm{L}$ & ND & ND & ND & ND & ND & ND & ND & ND & ND & ND & ND & ND \\
\hline Chromium & $\mathrm{mg} / \mathrm{L}$ & ND & ND & ND & ND & ND & ND & ND & ND & ND & ND & ND & ND \\
\hline Copper & $\mathrm{mg} / \mathrm{L}$ & ND & ND & ND & 0.006 & ND & ND & ND & ND & ND & ND & ND & ND \\
\hline Iron & $\mathrm{mg} / \mathrm{L}$ & 0.232 & 0.314 & 0.361 & 0.43 & 0.51 & 0.11 & 0.6 & 0.46 & 0.16 & 0.3 & 0.21 & 0.14 \\
\hline Lead & $\mathrm{mg} / \mathrm{L}$ & ND & ND & ND & ND & ND & ND & ND & ND & ND & ND & ND & ND \\
\hline Manganese & $\mathrm{mg} / \mathrm{L}$ & 0.054 & 0.119 & 0.083 & 0.067 & 0.084 & 0.053 & 0.073 & 0.069 & 0.068 & 0.068 & 0.052 & 0.057 \\
\hline Mercury & $\mathrm{mg} / \mathrm{L}$ & ND & ND & ND & ND & ND & ND & ND & ND & ND & ND & ND & ND \\
\hline Nickel & $\mathrm{mg} / \mathrm{L}$ & ND & ND & ND & ND & ND & ND & ND & ND & ND & ND & ND & ND \\
\hline Zinc & $\mathrm{mg} / \mathrm{L}$ & 0.025 & ND & 0.017 & 0.01 & 0.013 & ND & 0.012 & 0.025 & ND & 0.0068 & ND & ND \\
\hline
\end{tabular}

a Supersaturated, probably due to instream primary production 


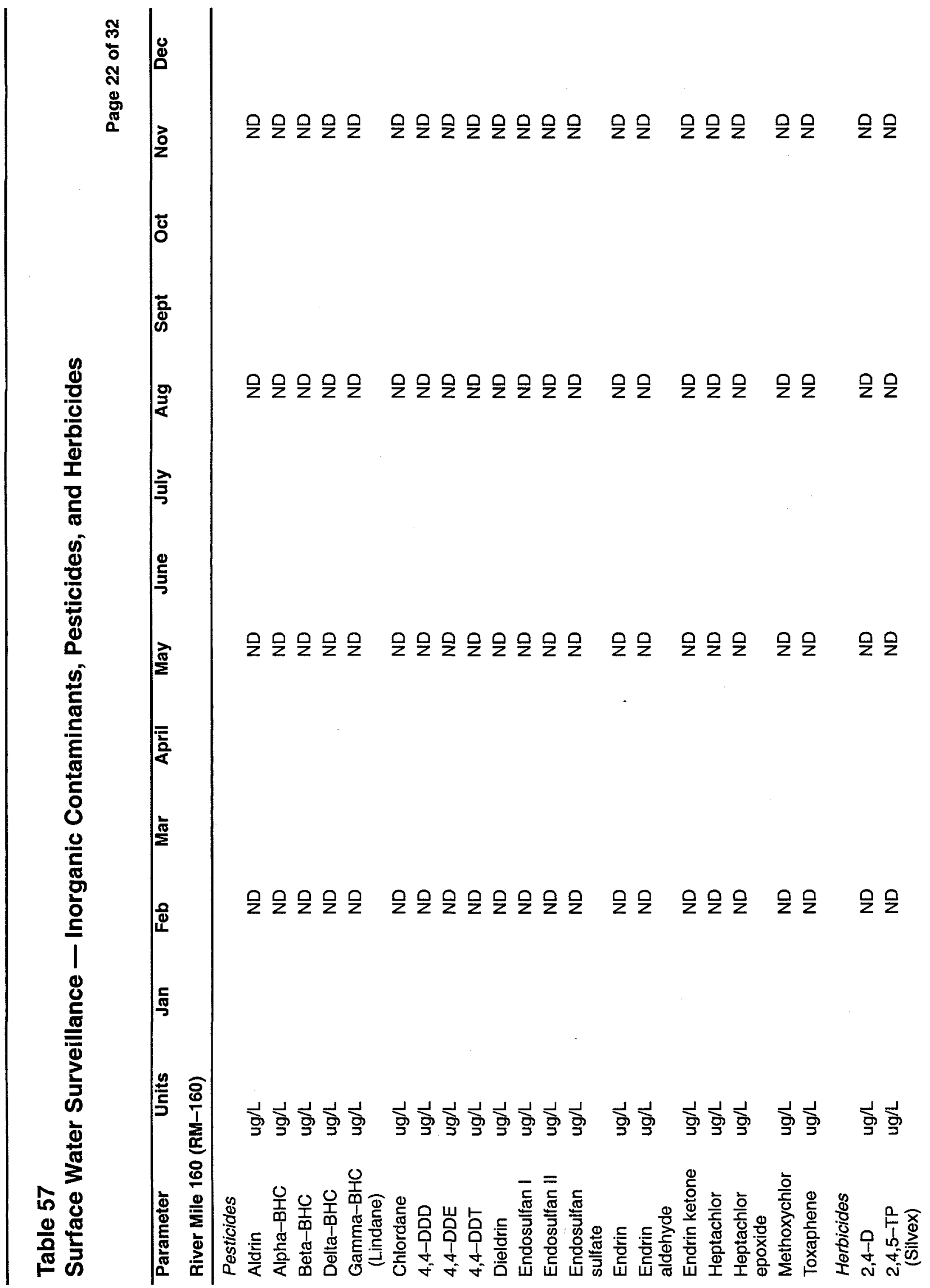


Table 57

Surface Water Surveillance - Inorganic Contaminants, Pesticides, and Herbicides

Page 23 of 32

\begin{tabular}{|c|c|c|c|c|c|c|c|c|c|c|c|c|c|}
\hline $\begin{array}{l}\text { Parameter } \\
\text { Steel Creek-4 a }\end{array}$ & $\begin{array}{c}\text { Units } \\
\text { Road A }\end{array}$ & $\begin{array}{l}\text { Jan } \\
-4)\end{array}$ & Feb & Mar & April & May & June & July & Aug & Sept & Oct & Nov & Dec \\
\hline Date & & $1 / 12 / 99$ & $2 / 9 / 99$ & $3 / 9 / 99$ & $4 / 7 / 99$ & $5 / 4 / 99$ & $6 / 8 / 99$ & $7 / 13 / 99$ & $8 / 10 / 99$ & $9 / 14 / 99$ & $10 / 26 / 99$ & $11 / 2 / 99$ & $12 / 7 / 99$ \\
\hline Temperature & ${ }^{\circ} \mathrm{C}$ & 10.5 & 16 & 10.9 & 21 & 19 & 24 & 23 & 26 & 23 & 15 & 17 & 12 \\
\hline DO & $\mathrm{mg} / \mathrm{L}$ & 9.4 & 8.6 & 9.9 & 7.8 & 7.9 & 5.2 & 5.1 & 5.9 & 6.5 & 9.3 & 8 & 9.3 \\
\hline $\mathrm{pH}$ & $\mathrm{pH}$ & 7 & 7 & 6.3 & 6.6 & 7.4 & 6.9 & 6.3 & 7.3 & 6.7 & 6.9 & 6.5 & 6.2 \\
\hline Conductivity & $\mu h o s / c m$ & 54 & 57 & 56 & 65 & 53 & 59 & 42 & 58 & 58 & 61 & 62 & 64 \\
\hline COD & $\mathrm{mg} / \mathrm{L}$ & ND & ND & ND & ND & ND & ND & 23 & ND & ND & ND & ND & ND \\
\hline Nitrite Nitrogen & $\mathrm{mg} / \mathrm{L}$ & ND & ND & ND & ND & ND & ND & ND & ND & ND & ND & ND & ND \\
\hline Nitrate Nitrogen & $\mathrm{mg} / \mathrm{L}$ & 0.27 & 0.24 & 0.26 & 0.16 & 0.27 & 0.24 & 0.21 & 0.28 & 0.22 & 0.28 & 0.29 & 0.21 \\
\hline TSS & $\mathrm{mg} / \mathrm{L}$ & 3 & 3 & 5 & 4 & 1 & 4 & 1 & ND & 2 & 7 & 3 & 2 \\
\hline Total Phos & $\mathrm{mg} / \mathrm{L}$ & 0.147 & ND & ND & 0.023 & ND & ND & 0.077 & ND & 0.065 & ND & ND & 0.045 \\
\hline TOC & $\mathrm{mg} / \mathrm{L}$ & 3.4 & ND & 4.4 & 4.2 & 3.2 & 3.2 & 10 & 2.6 & 2.6 & 2.7 & 4.8 & 4.6 \\
\hline Aluminum & $\mathrm{mg} / \mathrm{L}$ & 0.157 & 0.16 & 0.147 & ND & 0.17 & 0.2 & 0.18 & 0.17 & ND & 0.14 & 0.12 & ND \\
\hline Cadmium & $\mathrm{mg} / \mathrm{L}$ & ND & ND & ND & ND & ND & ND & ND & ND & ND & ND & ND & ND \\
\hline Chromium & $\mathrm{mg} / \mathrm{L}$ & ND & ND & ND & ND & ND & ND & ND & ND & ND & ND & ND & ND \\
\hline Copper & $\mathrm{mg} / \mathrm{L}$ & ND & ND & ND & ND & ND & ND & ND & ND & ND & ND & ND & ND \\
\hline Iron & $\mathrm{mg} / \mathrm{L}$ & 0.29 & 0.324 & 0.4 & 0.42 & 0.33 & 0.53 & 0.46 & 0.45 & 0.2 & 0.95 & 1 & 1 \\
\hline Lead & $\mathrm{mg} / \mathrm{L}$ & ND & ND & ND & ND & ND & ND & ND & ND & ND & ND & ND & ND \\
\hline Manganese & $\mathrm{mg} / \mathrm{L}$ & 0.028 & . $\quad 0.039$ & 0.05 & 0.044 & 0.041 & 0.06 & 0.029 & 0.036 & 0.023 & 0.34 & 0.32 & 0.32 \\
\hline Mercury & $\mathrm{mg} / \mathrm{L}$ & ND & ND & ND & 0.0001 & ND & ND & ND & 0.00016 & ND & ND & ND & ND \\
\hline Nickel & $\mathrm{mg} / \mathrm{L}$ & ND & ND & ND & ND & ND & ND & ND & ND & ND & ND & ND & ND \\
\hline Zinc & $\mathrm{mg} / \mathrm{L}$ & 0.006 & 0.013 & 0.079 & 0.02 & 0.0093 & 0.026 & ND & ND & 0.0087 & ND & ND & 0.063 \\
\hline
\end{tabular}


Table 57

Surface Water Surveillance - Inorganic Contaminants, Pesticides, and Herbicides

Page 24 of 32

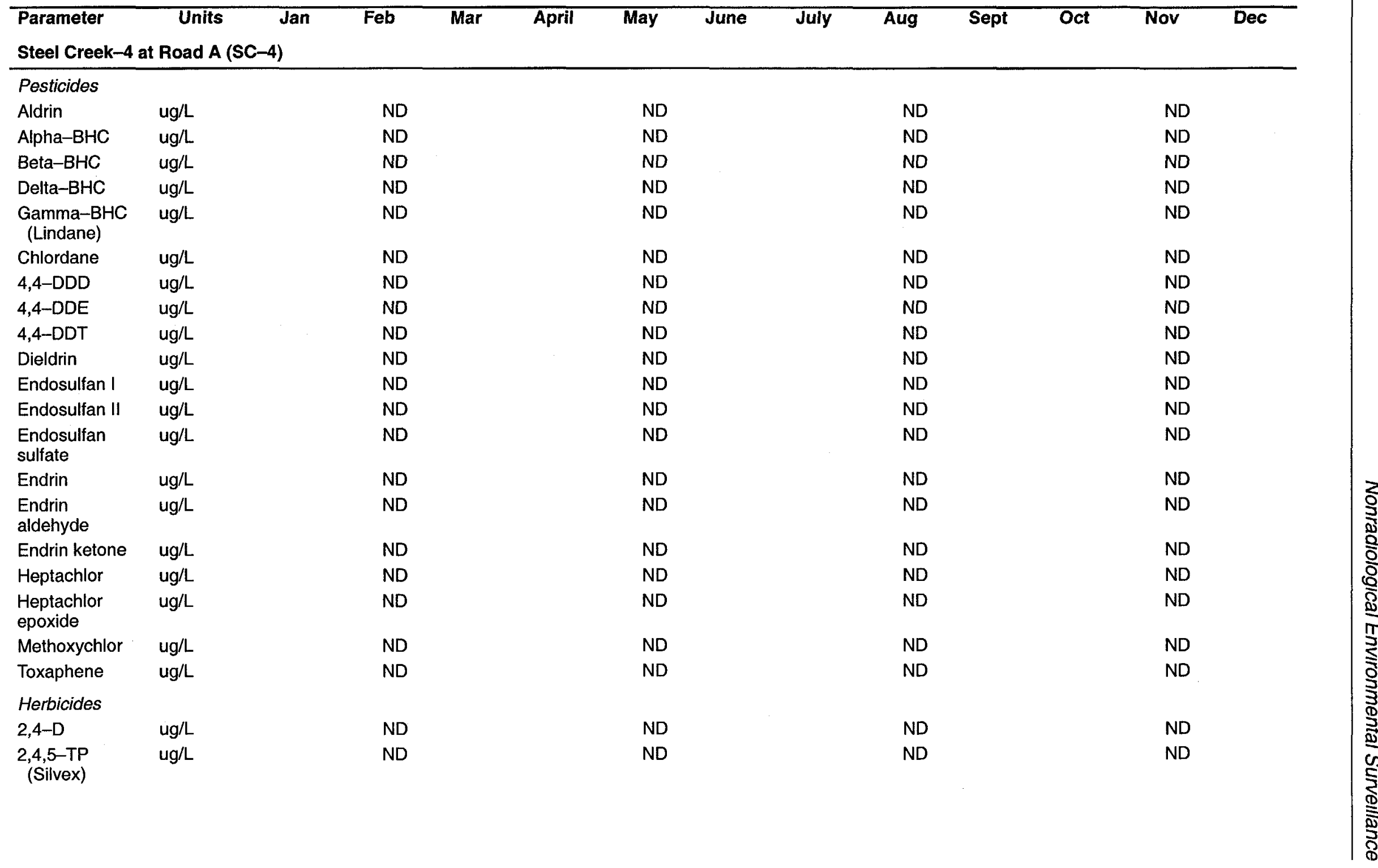


Table 57

Surface Water Surveillance — Inorganic Contaminants, Pesticides, and Herbicides

Page 25 of 32

\begin{tabular}{|c|c|c|c|c|c|c|c|c|c|c|c|c|c|}
\hline $\begin{array}{l}\text { Parameter } \\
\text { Tims Branch-5 }\end{array}$ & $\begin{array}{l}\text { Units } \\
\text { (TB-5) }\end{array}$ & Jan & Feb & Mar & April & May & June & July & $\overline{\text { Aug }}$ & Sept & Oct & Nov & Dec \\
\hline Date & & $1 / 12 / 99$ & $2 / 9 / 99$ & $3 / 9 / 99$ & $4 / 7 / 99$ & $5 / 4 / 99$ & $6 / 8 / 99$ & $7 / 13 / 99$ & $8 / 10 / 99$ & $9 / 14 / 99$ & $10 / 26 / 99$ & $11 / 2 / 99$ & $12 / 7 / 99$ \\
\hline Temperature & ${ }^{\circ} \mathrm{C}$ & 7 & 13.2 & 9.6 & 18.1 & 16.5 & 20.2 & 22 & 24 & 19 & 11 & 17 & 10 \\
\hline DO & $\mathrm{mg} / \mathrm{L}$ & 8.2 & 8.2 & 8.1 & 7.3 & 9.2 & 5.5 & 5.1 & 5.9 & 7.5 & 9.8 & 8.2 & 10.2 \\
\hline $\mathrm{pH}$ & $\mathrm{pH}$ & 6.1 & 5.7 & 6.9 & 5.7 & 7.4 & 6.5 & 6 & 6.8 & 6.6 & 6.7 & 6 & 7.1 \\
\hline Conductivity & $\mu h o s / c m$ & 34 & 33 & 42 & 34 & 31 & 27 & 30 & 29 & 26 & 26 & 35 & 30 \\
\hline COD & $\mathrm{mg} / \mathrm{L}$ & ND & ND & ND & ND & ND & ND & ND & ND & ND & ND & ND & ND \\
\hline Nitrite Nitrogen & $\mathrm{mg} / \mathrm{L}$ & ND & ND & ND & ND & ND & ND & ND & ND & ND & ND & ND & ND \\
\hline Nitrate Nitrogen & $\mathrm{mg} / \mathrm{L}$ & 0.53 & 0.36 & 0.38 & 0.31 & 0.37 & 0.26 & 0.30 & 0.21 & 0.19 & 0.30 & 0.25 & 0.26 \\
\hline TSS & $\mathrm{mg} / \mathrm{L}$ & 5 & 4 & 6 & 8 & 4 & 4 & 6 & 3 & 3 & 4 & 3 & 5 \\
\hline Total Phos & $\mathrm{mg} / \mathrm{L}$ & 0.12 & 0.029 & ND & 0.045 & ND & 0.044 & 0.458 & 0.066 & 0.106 & ND & ND & 0.063 \\
\hline TOC & $\mathrm{mg} / \mathrm{L}$ & 3.3 & ND & 3 & 4.1 & 4.1 & 3.9 & 7.9 & 5.8 & 3 & 1.9 & 5.5 & 2.9 \\
\hline Aluminum & $\mathrm{mg} / \mathrm{L}$ & 0.205 & 0.213 & 0.152 & 0.057 & 0.35 & 0.17 & 0.37 & 0.26 & ND & 0.17 & 0.18 & 0.12 \\
\hline Cadmium & $\mathrm{mg} / \mathrm{L}$ & ND & ND & ND & ND & ND & ND & ND & ND & ND & ND & ND & ND \\
\hline Chromium & $\mathrm{mg} / \mathrm{L}$ & ND & ND & ND & ND & ND & ND & ND & ND & ND & ND & ND & ND \\
\hline Copper & $\mathrm{mg} / \mathrm{L}$ & ND & 0.005 & 0.005 & 0.009 & 0.0071 & ND & ND & 0.005 & ND & ND & ND & ND \\
\hline Iron & $\mathrm{mg} / \mathrm{L}$ & 0.656 & 0.749 & 0.678 & 1.5 & 1.7 & 1.3 & 2.5 & 1.8 & 1.1 & 0.91 & 1 & 1.1 \\
\hline Lead & $\mathrm{mg} / \mathrm{L}$ & ND & ND & ND & ND & ND & ND & ND & ND & ND & ND & ND & ND \\
\hline Manganese & $\mathrm{mg} / \mathrm{L}$ & 0.029 & 0.03 & 0.038 & 0.05 & 0.045 & 0.048 & 0.14 & 0.077 & 0.026 & 0.022 & 0.048 & 0.028 \\
\hline Mercury & $\mathrm{mg} / \mathrm{L}$ & ND & ND & ND & ND & ND & ND & ND & ND & ND & ND & ND & ND \\
\hline Nickel & $\mathrm{mg} / \mathrm{L}$ & ND & 0.012 & ND & ND & ND & ND & 0.013 & ND & ND & ND & ND & ND \\
\hline Zinc & $\mathrm{mg} / \mathrm{L}$ & 0.008 & 0.019 & 0.076 & 0.016 & 0.036 & 0.0064 & 0.0069 & 0.042 & 0.01 & 0.016 & ND & 0.051 \\
\hline
\end{tabular}


Table 57

Surface Water Surveillance - Inorganic Contaminants, Pesticides, and Herbicides

Page 26 of 32

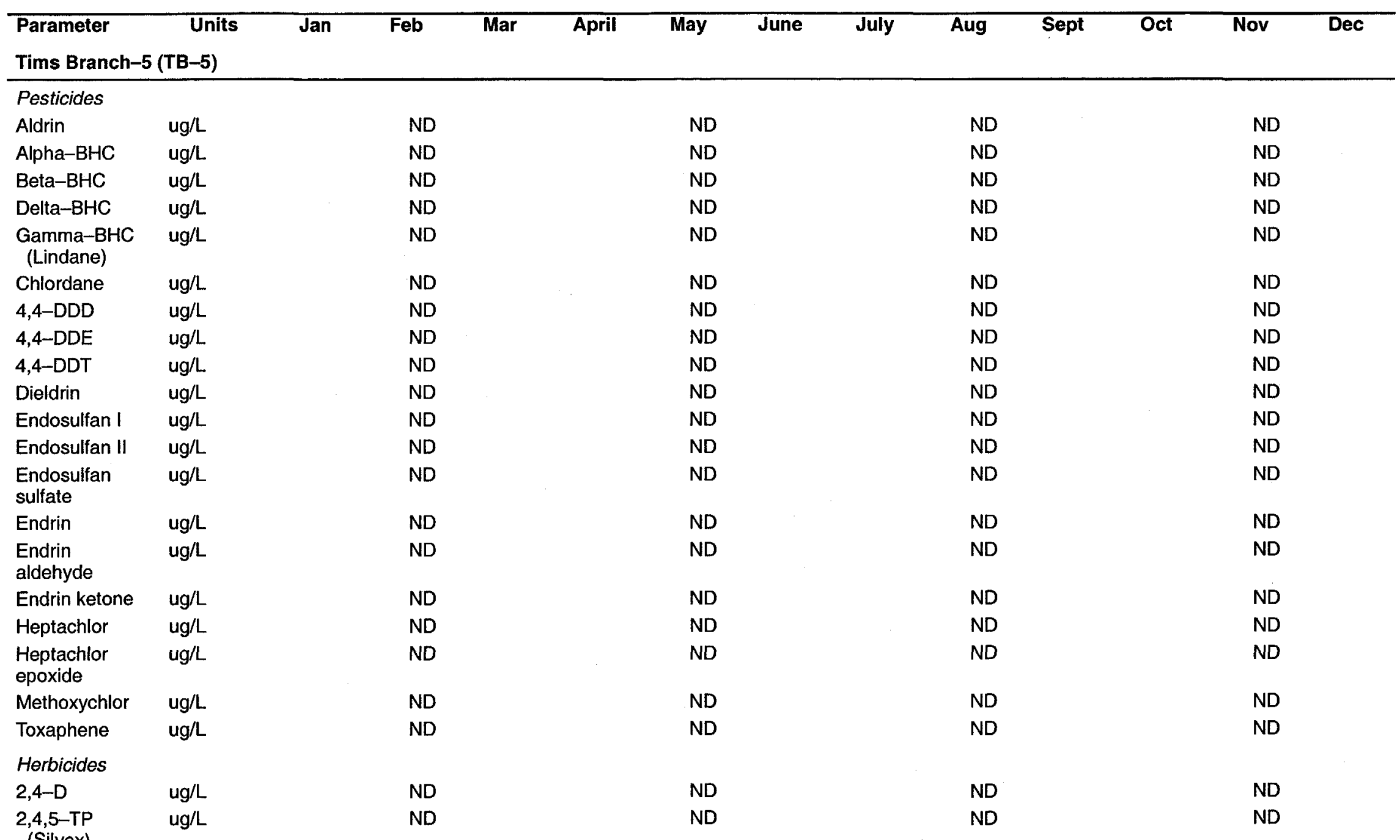


Table 57

Surface Water Surveillance - Inorganic Contaminants, Pesticides, and Herbicides

Page 27 of 32

\begin{tabular}{|c|c|c|c|c|c|c|c|c|c|c|c|c|c|}
\hline \multicolumn{14}{|c|}{ Tinker Creek-I (TC-1; TCKP) } \\
\hline Date & & $1 / 12 / 99$ & $2 / 9 / 99$ & $3 / 9 / 99$ & $4 / 7 / 99$ & $5 / 4 / 99$ & $6 / 8 / 99$ & $7 / 13 / 99$ & $8 / 10 / 99$ & $9 / 14 / 99$ & $10 / 26 / 99$ & $11 / 2 / 99$ & $12 / 7 / 99$ \\
\hline Temperature & ${ }^{\circ} \mathrm{C}$ & 6.8 & 13.5 & 8.9 & 19 & 18 & 21.3 & 22 & 24 & 19.1 & 10 & 18 & 9 \\
\hline DO & $\mathrm{mg} / \mathrm{L}$ & 10 & 9.1 & 10.6 & 7.1 & 8.5 & 5.8 & 4.4 & 5.8 & 6.3 & 10.4 & 7.4 & 10.4 \\
\hline $\mathrm{pH}$ & $\mathrm{pH}$ & 6.8 & 6.7 & 6.4 & 6 & 7.4 & 6.6 & 6.1 & 6.6 & 6.7 & 5.5 & 6.1 & 5.4 \\
\hline Conductivity & $\mu h o s / \mathrm{cm}$ & 30 & 28 & 31 & 31 & 24 & 34 & 28 & 34 & 31 & 29 & 34 & 30 \\
\hline COD & $\mathrm{mg} / \mathrm{L}$ & ND & ND & ND & ND & ND & ND & 20 & ND & ND & ND & ND & ND \\
\hline Nitrite Nitrogen & $\mathrm{mg} / \mathrm{L}$ & ND & ND & ND & ND & ND & ND & ND & ND & ND & ND & ND & ND \\
\hline Nitrate Nitrogen & $\mathrm{mg} / \mathrm{L}$ & 0.28 & 0.25 & 0.21 & 0.18 & 0.21 & 0.22 & 0.18 & 0.27 & 0.18 & 0.24 & 0.21 & 0.14 \\
\hline TSS & $\mathrm{mg} / \mathrm{L}$ & 3 & 4 & 8 & 5 & 3 & 4 & 1 & 5 & 3 & 2 & 3 & 2 \\
\hline Total Phos & $\mathrm{mg} / \mathrm{L}$ & 0.104 & ND & ND & ND & ND & 0.015 & 0.085 & 0.217 & 0.065 & ND & ND & 0.108 \\
\hline TOC & $\mathrm{mg} / \mathrm{L}$ & 2.7 & 5 & 4.2 & 3.2 & 3 & 3.5 & 8.1 & 5.3 & 3 & 2.3 & 6.1 & 3.3 \\
\hline Aluminum & $\mathrm{mg} / \mathrm{L}$ & 0.123 & 0.15 & 0.114 & ND & 0.2 & 0.17 & ND & 0.17 & ND & 0.13 & 0.18 & ND \\
\hline Cadmium & $\mathrm{mg} / \mathrm{L}$ & ND & ND & ND & ND & ND & ND & ND & ND & ND & ND & ND & ND \\
\hline Chromium & $\mathrm{mg} / \mathrm{L}$ & ND & ND & ND & ND & ND & ND & ND & ND & ND & ND & ND & ND \\
\hline Copper & $\mathrm{mg} / \mathrm{L}$ & ND & 0.008 & ND & 0.005 & 0.0056 & ND & 0.01 & ND & ND & ND & ND & ND \\
\hline Iron & $\mathrm{mg} / \mathrm{L}$ & 0.101 & 0.142 & 0.243 & 0.36 & 0.26 & 0.82 & 0.8 & 0.89 & 0.44 & 0.17 & 0.33 & 0.06 \\
\hline Lead & $\mathrm{mg} / \mathrm{L}$ & ND & ND & ND & 0.016 & ND & ND & ND & ND & ND & ND & ND & ND \\
\hline Manganese & $\mathrm{mg} / \mathrm{L}$ & 0.006 & 0.012 & 0.022 & 0.024 & 0.018 & 0.013 & 0.038 & 0.046 & 0.026 & 0.011 & 0.027 & 0.011 \\
\hline Mercury & $\mathrm{mg} / \mathrm{L}$ & ND & ND & ND & 0.0001 & ND & ND & ND & ND & ND & ND & ND & ND \\
\hline Nickel & $\mathrm{mg} / \mathrm{L}$ & ND & ND & ND & ND & ND & ND & ND & ND & ND & ND & ND & ND \\
\hline Zinc & $\mathrm{mg} / \mathrm{L}$ & 0.01 & 0.012 & 0.05 & 0.008 & 0.041 & 0.01 & 0.03 & ND & 0.007 & ND & 0.0061 & 0.042 \\
\hline
\end{tabular}


Table 57

Surface Water Surveillance - Inorganic Contaminants, Pesticides, and Herbicides

Page 28 of 32

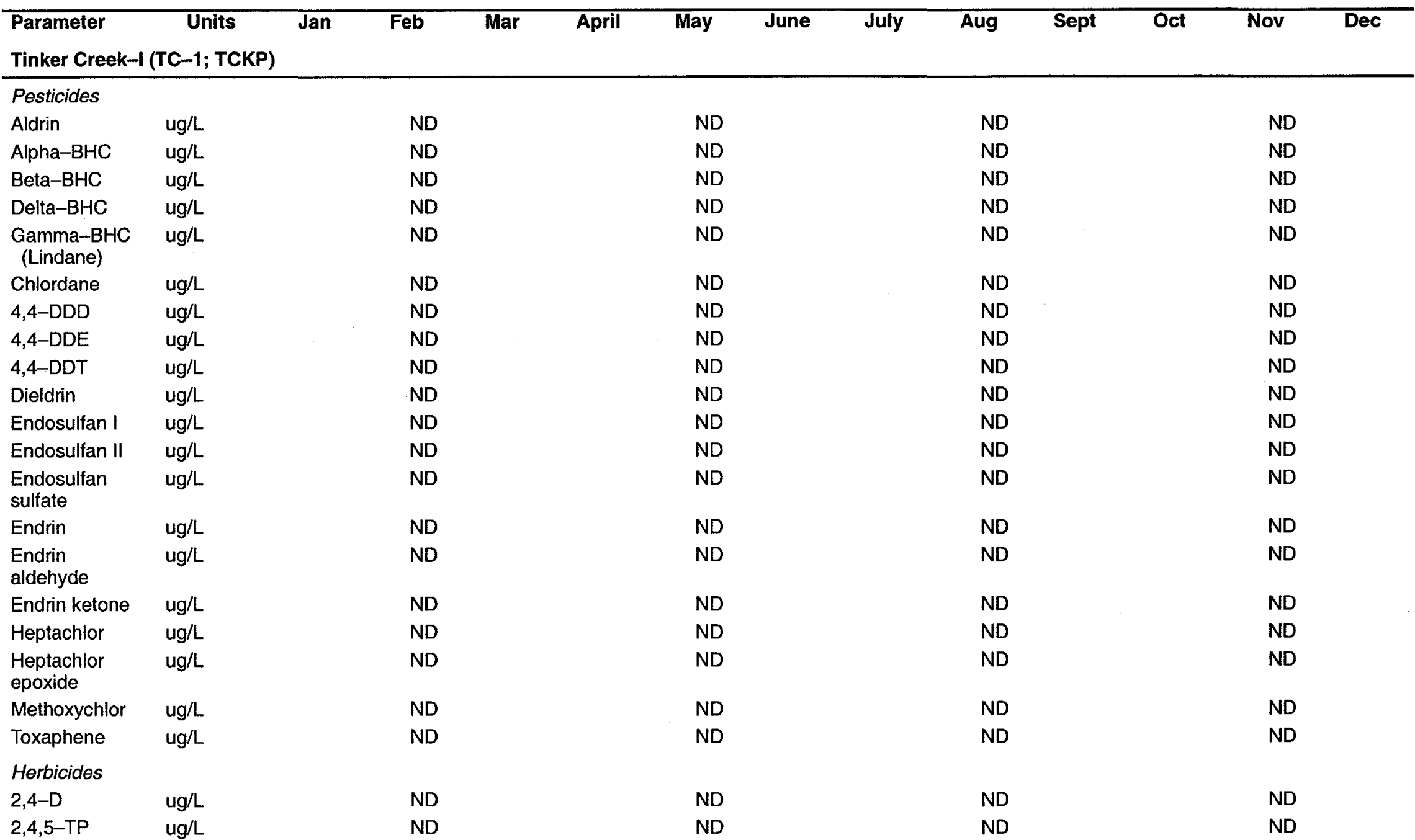

(Silvex) 
Table 57

Surface Water Surveillance - Inorganic Contaminants, Pesticides, and Herbicides

Page 29 of 32

\begin{tabular}{|c|c|c|c|c|c|c|c|c|c|c|c|c|c|}
\hline $\begin{array}{l}\text { Parameter } \\
\text { Upper Three Ru }\end{array}$ & $\begin{array}{c}\text { Units } \\
\text { ns-1A (U3 }\end{array}$ & $\begin{array}{l}\text { Jan } \\
\text {-1A) }\end{array}$ & Feb & Mar & April & May & June & July & Aug & Sept & Oct & Nov & $\overline{\text { Dec }}$ \\
\hline Date & & $1 / 12 / 99$ & $2 / 9 / 99$ & $3 / 9 / 99$ & $4 / 7 / 99$ & $5 / 4 / 99$ & $6 / 8 / 99$ & $7 / 13 / 99$ & $8 / 10 / 99$ & $9 / 14 / 99$ & $10 / 26 / 99$ & $11 / 2 / 99$ & $12 / 7 / 99$ \\
\hline Temperature & ${ }^{\circ} \mathrm{C}$ & 10.5 & 14.2 & 12 & 18 & 17 & 20 & 20 & 22 & 18.6 & 13 & 18 & 14 \\
\hline DO & $\mathrm{mg} / \mathrm{L}$ & 8.9 & 8 & 7.6 & 8 & 8.3 & 6.1 & 5.5 & 7.2 & 7 & 9.6 & 8 & 9.5 \\
\hline $\mathrm{pH}$ & $\mathrm{pH}$ & 5.7 & 5.8 & 6.2 & 5.2 & 7.2 & 6.9 & 5.1 & 5.5 & 6.2 & 6.2 & 5.5 & 4 \\
\hline Conductivity & $\mu h o s / c m$ & 16 & 16 & 15 & 15 & 14 & 14 & 21 & 15 & 15 & 15 & 16 & 15 \\
\hline COD & $\mathrm{mg} / \mathrm{L}$ & ND & ND & ND & ND & ND & ND & ND & ND & ND & ND & ND & ND \\
\hline Nitrite Nitrogen & $\mathrm{mg} / \mathrm{L}$ & ND & ND & ND & ND & ND & ND & ND & ND & ND & ND & ND & ND \\
\hline Nitrate Nitrogen & $\mathrm{mg} / \mathrm{L}$ & 0.42 & 0.39 & 0.37 & 0.32 & 0.39 & 0.35 & 0.36 & 0.26 & 0.37 & 0.39 & 0.44 & 0.34 \\
\hline TSS & $\mathrm{mg} / \mathrm{L}$ & 3 & 3 & 2 & 3 & 4 & 4 & 8 & 2 & 4 & 3 & 3 & 2 \\
\hline Total Phos & $\mathrm{mg} / \mathrm{L}$ & 0.126 & 0.227 & ND & ND & ND & ND & 0.154 & ND & 0.155 & ND & ND & 0.057 \\
\hline TOC & $\mathrm{mg} / \mathrm{L}$ & 2.2 & ND & 2.6 & 1.7 & 1.3 & 1.3 & 7.2 & 1.2 & 1.3 & 1.9 & 3.1 & 2.4 \\
\hline Aluminum & $\mathrm{mg} / \mathrm{L}$ & 0.161 & 0.216 & 0.11 & ND & 0.24 & 0.19 & 0.76 & 0.21 & ND & 0.14 & 0.16 & ND \\
\hline Cadmium & $\mathrm{mg} / \mathrm{L}$ & ND & ND & ND & ND & ND & ND & ND & ND & ND & ND & ND & ND \\
\hline Chromium & $\mathrm{mg} / \mathrm{L}$ & ND & ND & ND & ND & ND & ND & ND & ND & ND & ND & ND & ND \\
\hline Copper & $\mathrm{mg} / \mathrm{L}$ & ND & 0.009 & ND & ND & ND & ND & ND & ND & ND & ND & ND & 0.016 \\
\hline Iron & $\mathrm{mg} / \mathrm{L}$ & 0.165 & 0.285 & 0.187 & 0.33 & 0.29 & 0.3 & 1.1 & 0.28 & 0.21 & 0.18 & 0.3 & 0.11 \\
\hline Lead & $\mathrm{mg} / \mathrm{L}$ & ND & ND & ND & ND & ND & ND & ND & ND & ND & ND & ND & ND \\
\hline Manganese & $\mathrm{mg} / \mathrm{L}$ & 0.008 & 0.012 & 0.009 & 0.011 & 0.014 & 0.0093 & 0.034 & 0.0094 & ND & 0.007 & ND & 0.01 \\
\hline Mercury & $\mathrm{mg} / \mathrm{L}$ & ND & ND & ND & ND & ND & ND & ND & ND & ND & ND & ND & ND \\
\hline Nickel & $\mathrm{mg} / \mathrm{L}$ & ND & ND & ND & ND & ND & ND & ND & ND & ND & ND & ND & ND \\
\hline Zinc & $\mathrm{mg} / \mathrm{L}$ & ND & 0.019 & 0.046 & 0.009 & 0.072 & 0.05 & 0.022 & ND & 0.0078 & 0.0053 & 0.0053 & 0.082 \\
\hline
\end{tabular}


Table 57

Surface Water Surveillance - Inorganic Contaminants, Pesticides, and Herbicides

Page 30 of 32

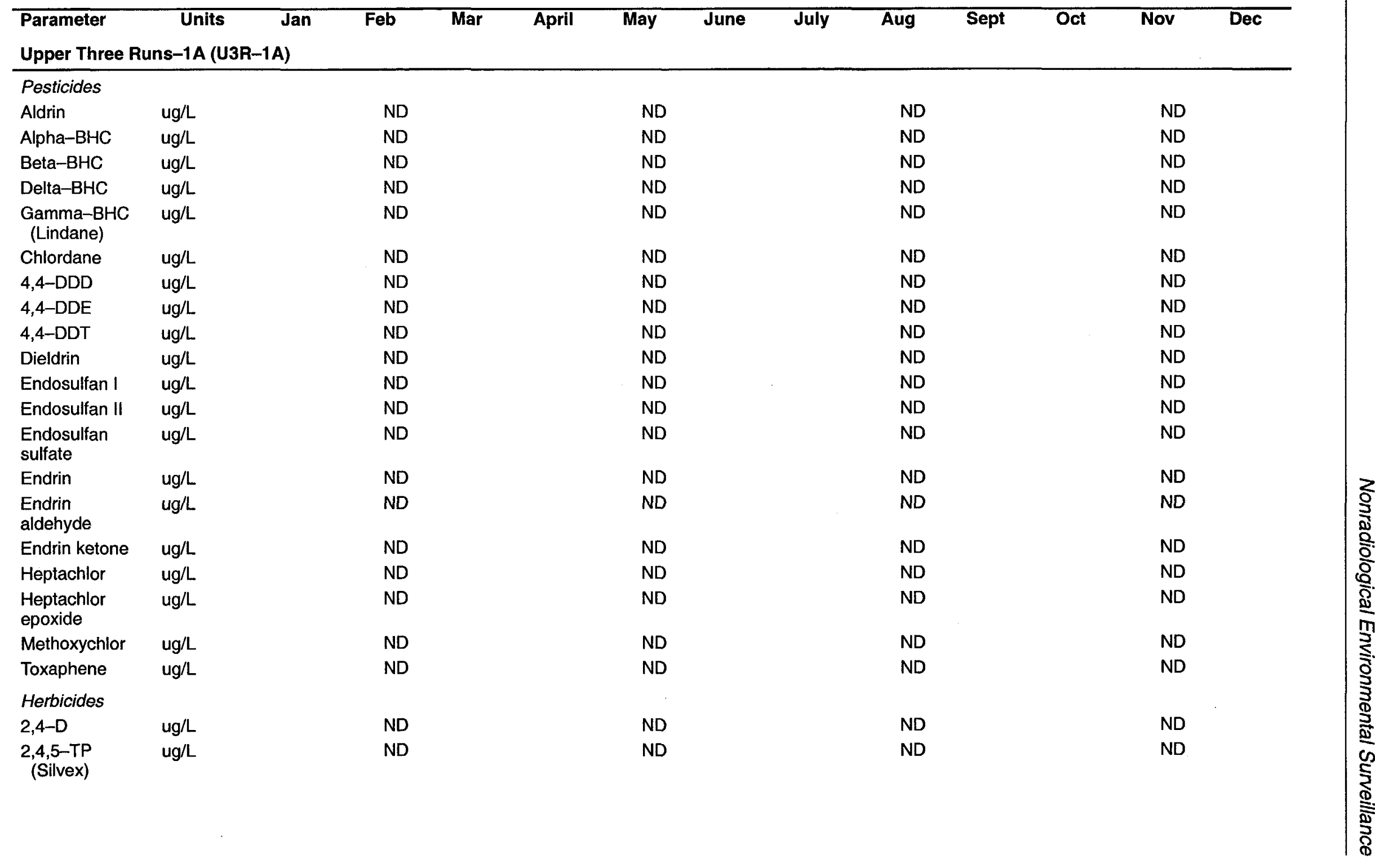


Table 57

Surface Water Surveillance — Inorganic Contaminants, Pesticides, and Herbicides

Page 31 of 32

\begin{tabular}{|c|c|c|c|c|c|c|c|c|c|c|c|c|c|}
\hline $\begin{array}{l}\text { Parameter } \\
\text { Upper Three Ru }\end{array}$ & $\begin{array}{c}\text { Units } \\
\text { ns-4 (U3R }\end{array}$ & Jan & Feb & Mar & April & May & June & July & Aug & Sept & Oct & Nov & $\overline{D e c}$ \\
\hline Date & & $1 / 12 / 99$ & $2 / 9 / 99$ & $3 / 9 / 99$ & $4 / 7 / 99$ & $5 / 4 / 99$ & $6 / 8 / 99$ & $7 / 13 / 99$ & $8 / 10 / 99$ & $9 / 14 / 99$ & $10 / 26 / 99$ & $11 / 2 / 99$ & $12 / 7 / 99$ \\
\hline Temperature & ${ }^{\circ} \mathrm{C}$ & 10.6 & 15.3 & 11 & 20 & 18.1 & 23.2 & 21 & 25 & 21 & 14 & 18 & 12.6 \\
\hline DO & $\mathrm{mg} / \mathrm{L}$ & 9.5 & 8.3 & 9.3 & 6.9 & 8.5 & 5.7 & 4.6 & 7.2 & 6.7 & 9.8 & 8.2 & 9.6 \\
\hline $\mathrm{pH}$ & $\mathrm{pH}$ & 6.5 & 6.1 & 6.4 & 5.5 & 6.7 & 6.4 & 6.3 & 6.3 & 6.8 & 6.7 & 6 & 6.3 \\
\hline Conductivity & $\mu h o s / c m$ & 23 & 23 & 23 & 24 & 22 & 21 & 27 & 21 & 21 & 23 & 23 & 25 \\
\hline COD & $\mathrm{mg} / \mathrm{L}$ & ND & ND & ND & ND & ND & ND & ND & ND & ND & ND & ND & ND \\
\hline Nitrite Nitrogen & $\mathrm{mg} / \mathrm{L}$ & ND & ND & ND & ND & ND & ND & ND & ND & ND & ND & ND & ND \\
\hline Nitrate Nitrogen & $\mathrm{mg} / \mathrm{L}$ & 0.35 & 0.27 & 0.37 & 0.25 & 0.35 & 0.29 & 0.20 & 0.38 & 0.26 & 0.27 & 0.29 & 0.19 \\
\hline TSS & $\mathrm{mg} / \mathrm{L}$ & 4 & 4 & 4 & 7 & 9 & 6 & 4 & 3 & 3 & 5 & ND & 3 \\
\hline Total Phos & $\mathrm{mg} / \mathrm{L}$ & 0.034 & ND & ND & 0.047 & ND & ND & 0.17 & ND & 0.093 & 0.029 & ND & ND \\
\hline TOC & $\mathrm{mg} / \mathrm{L}$ & 3.3 & ND & 2.6 & 2.9 & 2.6 & 1.5 & 15 & 1.6 & 2.5 & 1.1 & 2.9 & 4.6 \\
\hline Aluminum & $\mathrm{mg} / \mathrm{L}$ & 0.217 & 0.216 & 0.219 & ND & 0.23 & 0.24 & 0.53 & 0.24 & ND & 0.19 & 0.18 & 0.16 \\
\hline Cadmium & $\mathrm{mg} / \mathrm{L}$ & ND & ND & ND & ND & ND & ND & ND & ND & ND & ND & ND & ND \\
\hline Chromium & $\mathrm{mg} / \mathrm{L}$ & ND & ND & 0.006 & ND & ND & ND & ND & ND & ND & ND & ND & ND \\
\hline Copper & $\mathrm{mg} / \mathrm{L}$ & ND & 0.031 & ND & 0.005 & ND & ND & ND & ND & ND & ND & ND & 0.016 \\
\hline Iron & $\mathrm{mg} / \mathrm{L}$ & 0.246 & 0.783 & 0.337 & 0.5 & 0.36 & 0.34 & 0.71 & 0.33 & 0.22 & 0.27 & 0.33 & 0.32 \\
\hline Lead & $\mathrm{mg} / \mathrm{L}$ & ND & ND & ND & ND & ND & ND & ND & ND & ND & ND & ND & ND \\
\hline Manganese & $\mathrm{mg} / \mathrm{L}$ & 0.016 & 0.02 & 0.051 & 0.025 & 0.016 & 0.011 & 0.068 & 0.013 & 0.011 & 0.013 & 0.0091 & 0.016 \\
\hline Mercury & $\mathrm{mg} / \mathrm{L}$ & ND & ND & ND & 0.0001 & ND & ND & ND & ND & ND & ND & ND & ND \\
\hline Nickel & $\mathrm{mg} / \mathrm{L}$ & ND & ND & ND & ND & ND & ND & ND & ND & ND & ND & ND & ND \\
\hline Zinc & $\mathrm{mg} / \mathrm{L}$ & 0.005 & 0.039 & 0.073 & 0.015 & 0.0078 & 0.015 & 0.01 & ND & 0.027 & 0.013 & ND & 0.061 \\
\hline
\end{tabular}




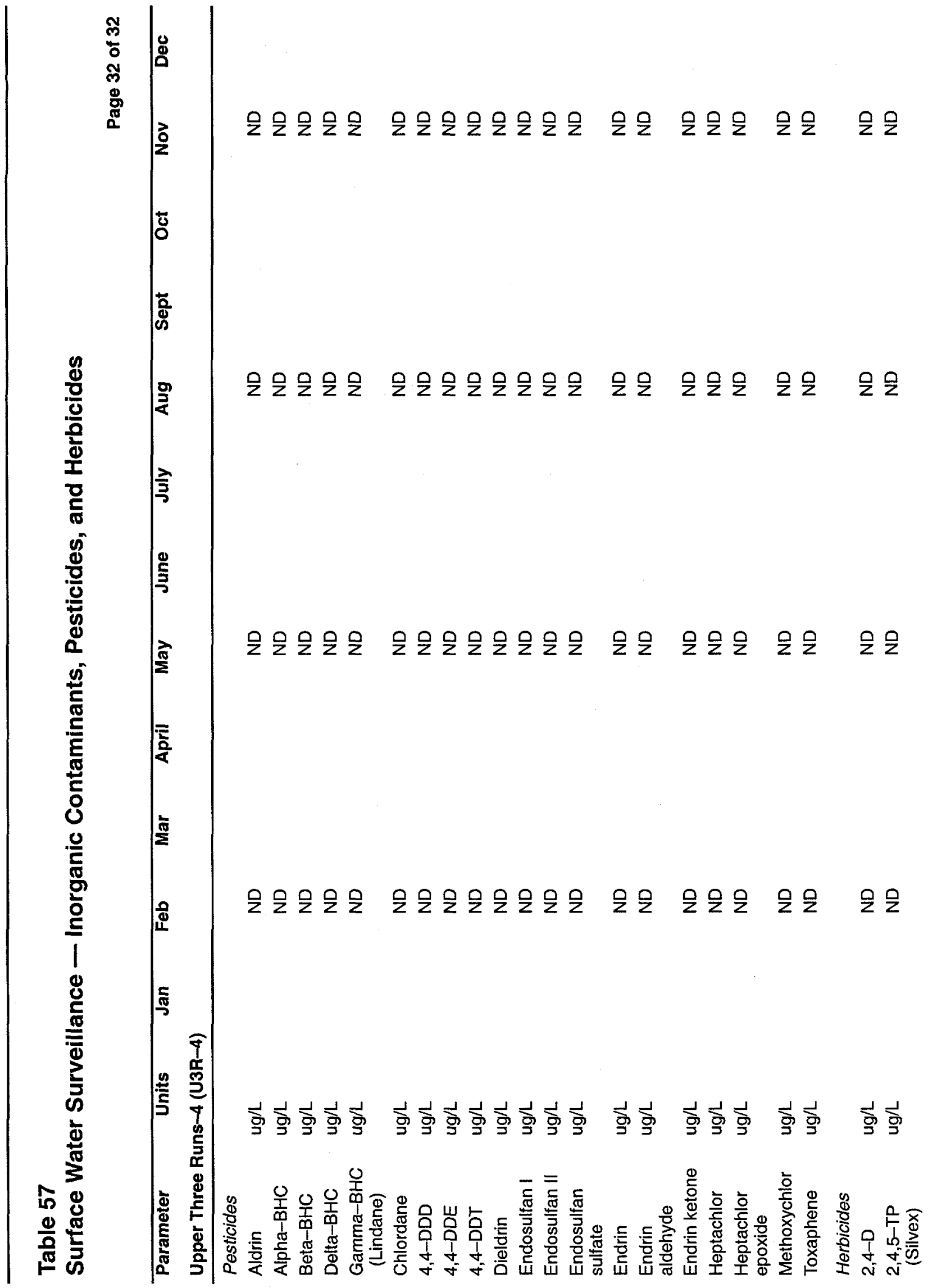


Table 58

Sediment Surveillance — Inorganic Contaminants, Pesticides, and Herbicides

Page 1 of 2

\begin{tabular}{|c|c|c|c|c|c|c|c|c|c|c|c|}
\hline \multirow[b]{2}{*}{ Constituent } & \multicolumn{11}{|c|}{$\mathrm{mg} / \mathrm{L}$} \\
\hline & $\begin{array}{c}\text { Upper } \\
\text { Three } \\
\text { Runs-4 } \\
\text { (U3R-4) }\end{array}$ & $\begin{array}{l}\text { Beaver } \\
\text { Dam } \\
\text { Creek } \\
\text { (400-D) }\end{array}$ & $\begin{array}{c}\text { Four Mile } \\
\text { Creek } \\
\text { (FMC/RdA) }\end{array}$ & $\begin{array}{c}\text { Pen } \\
\text { Branch } \\
\text { (PB/RdA) }\end{array}$ & $\begin{array}{c}\text { Steel } \\
\text { Creek-4 } \\
\text { (SC-4) }\end{array}$ & $\begin{array}{c}\text { Lower } \\
\text { Three } \\
\text { Runs-2 } \\
\text { (L3R-2) }\end{array}$ & $\begin{array}{c}\text { Upper } \\
\text { Three } \\
\text { Runs-1A } \\
\text { (U3R-1A) }\end{array}$ & $\begin{array}{c}\text { Tinker } \\
\text { Creek-1 } \\
\text { (TC-1) }\end{array}$ & $\begin{array}{c}\text { River Mile } \\
160 \\
(R M-160)\end{array}$ & $\begin{array}{c}\text { River Mile } \\
150.4\end{array}$ & $\begin{array}{c}\text { River Mile } \\
120 \\
(R M-120)\end{array}$ \\
\hline
\end{tabular}

Note: All analyses were performed by the Toxicity Characteristic Leaching Procedure (TCLP) method.

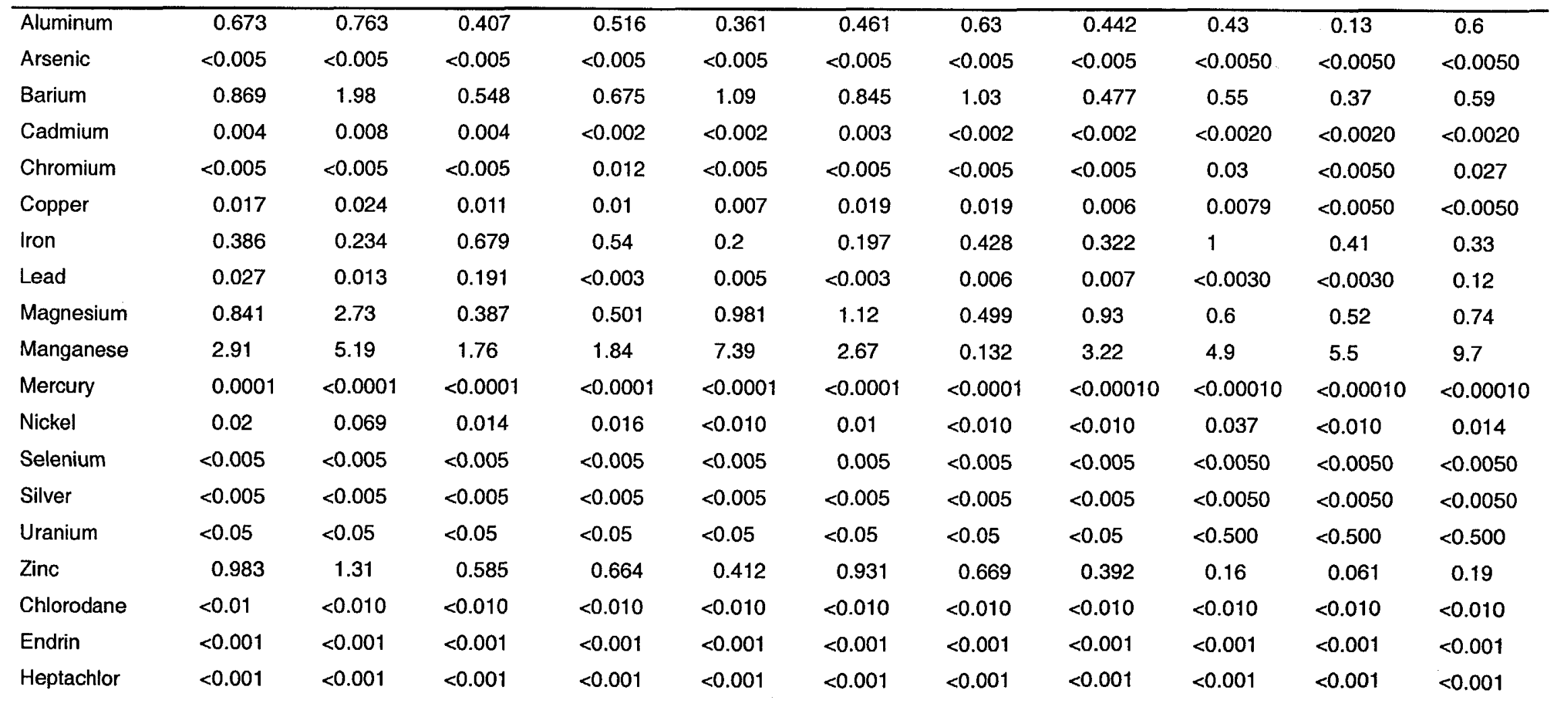


Table 58

Sediment Surveillance - Inorganic Contaminants, Pesticides, and Herbicides

Page 2 of 2

\begin{tabular}{|c|c|c|c|c|c|c|c|c|c|c|c|}
\hline \multirow[b]{2}{*}{ Constituent } & \multicolumn{11}{|c|}{$\mathrm{mg} / \mathrm{L}$} \\
\hline & $\begin{array}{c}\text { Upper } \\
\text { Three } \\
\text { Runs-4 } \\
\text { (U3R-4) }\end{array}$ & $\begin{array}{c}\text { Beaver } \\
\text { Dam } \\
\text { Creek } \\
\text { (400-D) }\end{array}$ & $\begin{array}{c}\text { Four Mile } \\
\text { Creek } \\
\text { (FMC/RdA) }\end{array}$ & $\begin{array}{c}\text { Pen } \\
\text { Branch } \\
\text { (PB/RdA) }\end{array}$ & $\begin{array}{c}\text { Steel } \\
\text { Creek-4 } \\
\text { (SC-4) }\end{array}$ & $\begin{array}{l}\text { Lower } \\
\text { Three } \\
\text { Runs-2 } \\
\text { (L3R-2) }\end{array}$ & $\begin{array}{c}\text { Upper } \\
\text { Three } \\
\text { Runs-1A } \\
\text { (U3R-1A) }\end{array}$ & $\begin{array}{c}\text { Tinker } \\
\text { Creek-1 } \\
\text { (TC-1) }\end{array}$ & $\begin{array}{c}\text { River Mile } \\
160 \\
\text { (RM-160) }\end{array}$ & $\begin{array}{c}\text { River Mile } \\
150.4\end{array}$ & $\begin{array}{c}\text { River Mile } \\
120 \\
(\mathbf{R M - 1 2 0})\end{array}$ \\
\hline $\begin{array}{l}\text { Heptachlor } \\
\text { Epoxide }\end{array}$ & $<0.001$ & $<0.001$ & $<0.001$ & $<0.001$ & $<0.001$ & $<0.001$ & $<0.001$ & $<0.001$ & $<0.001$ & $<0.001$ & $<0.001$ \\
\hline Lindane & $<0.001$ & $<0.001$ & $<0.001$ & $<0.001$ & $<0.001$ & $<0.001$ & $<0.001$ & $<0.001$ & $<0.001$ & $<0.001$ & $<0.001$ \\
\hline Methoxychlor & $<0.004$ & $<0.004$ & $<0.004$ & $<0.004$ & $<0.004$ & $<0.004$ & $<0.004$ & $<0.004$ & $<0.004$ & $<0.004$ & $<0.004$ \\
\hline Toxaphene & $<0.10$ & $<0.10$ & $<0.10$ & $<0.10$ & $<0.10$ & $<0.10$ & $<0.10$ & $<0.10$ & $<0.01$ & $<0.01$ & $<0.01$ \\
\hline 2,4-D & $<0.10$ & $<0.10$ & $<0.10$ & $<0.10$ & $<0.10$ & $<0.10$ & $<0.10$ & $<0.10$ & $<0.1$ & $<0.1$ & $<0.1$ \\
\hline Silvex & $<0.10$ & $<0.10$ & $<0.10$ & $<0.10$ & $<0.10$ & $<0.10$ & $<0.10$ & $<0.10$ & $<0.1$ & $<0.1$ & $<0.1$ \\
\hline Cyanide total & 0.134 & $<0.170$ & $<0.084$ & 0.357 & $<0.183$ & 0.258 & $<0.077$ & $<0.368$ & $<0.621$ & $<0.596$ & $<0.662$ \\
\hline
\end{tabular}


Table 59

Fish Surveillance - Mercury

Page 1 of 2

\begin{tabular}{|c|c|c|c|c|c|c|c|}
\hline \multirow[b]{2}{*}{ Location } & \multicolumn{7}{|c|}{ Mercury ( $\mu \mathrm{g} / \mathrm{g}$ - Parts Per Million) } \\
\hline & $\begin{array}{l}\text { Type } \\
\text { of Fish }\end{array}$ & \multicolumn{5}{|c|}{ Analytical Results } & \multirow[t]{2}{*}{ Maximum } \\
\hline \multicolumn{7}{|c|}{ Notes: "NC" denotes "not collected." The Practical Quantitation Limit (PQL) is $0.008 \mu \mathrm{g} / \mathrm{g}$." } & \\
\hline $\begin{array}{l}\text { New Savannah Bluff Lock \& } \\
\text { Dam }\end{array}$ & Bass & 0.26 & 0.26 & 0.23 & 0.22 & 0.23 & 0.26 \\
\hline $\begin{array}{l}\text { New Savannah Bluff Lock \& } \\
\text { Dam }\end{array}$ & Bream & 0.21 & 0.24 & 0.24 & 0.24 & 0.21 & 0.24 \\
\hline $\begin{array}{l}\text { New Savannah Bluff Lock \& } \\
\text { Dam }\end{array}$ & Catfish & 0.14 & 0.07 & 0.08 & 0.08 & 0.11 & 0.14 \\
\hline $\begin{array}{l}\text { New Savannah Bluff Lock \& } \\
\text { Dam }\end{array}$ & Shad & 0.23 & 0.23 & 0.23 & 0.22 & 0.25 & 0.25 \\
\hline $\begin{array}{l}\text { Upper Three Runs Creek } \\
\text { Mouth }\end{array}$ & Bass & 0.45 & 0.48 & 0.77 & 0.74 & 0.72 & 0.77 \\
\hline $\begin{array}{l}\text { Upper Three Runs Creek } \\
\text { Mouth }\end{array}$ & Bream & 0.22 & 0.20 & 0.23 & 0.16 & 0.27 & 0.27 \\
\hline $\begin{array}{l}\text { Upper Three Runs Creek } \\
\text { Mouth }\end{array}$ & Catfish & 0.24 & 0.12 & 0.10 & 0.52 & 0.27 & 0.52 \\
\hline Beaver Dam Creek Mouth & Bass & 0.35 & 0.62 & 0.74 & 0.35 & 0.91 & 0.91 \\
\hline Beaver Dam Creek Mouth & Bream & 0.33 & 0.32 & 0.23 & 0.40 & 0.17 & 0.40 \\
\hline Beaver Dam Creek Mouth & Cattish & 0.23 & 0.23 & 0.38 & 0.33 & 0.24 & 0.38 \\
\hline Four Mile Creek Mouth & Bass & 0.50 & 0.28 & 0.63 & 0.82 & 0.51 & 0.82 \\
\hline Four Mile Creek Mouth & Bream & 0.38 & 0.38 & 0.50 & 0.24 & 0.24 & 0.50 \\
\hline Four Mile Creek Mouth & Cattish & 0.37 & 0.23 & 0.23 & 0.55 & 0.23 & 0.55 \\
\hline Four Mile Creek Mouth & Shad & 0.09 & 0.80 & 0.10 & 0.16 & 0.10 & 0.80 \\
\hline Steel Ceek Mouth & Bass & 0.52 & 1.27 & 0.66 & 0.45 & 0.39 & 1.27 \\
\hline Steel Creek Mouth & Bream & 0.27 & 0.19 & 0.19 & 0.80 & 0.38 & 0.80 \\
\hline Steel Creek Mouth & Catfish & 0.29 & 0.67 & 0.35 & 0.12 & 0.42 & 0.67 \\
\hline Steel Creek Mouth & Shad & 0.26 & 0.21 & 0.27 & 0.22 & 0.22 & 0.27 \\
\hline $\begin{array}{l}\text { Lower Three Runs Creek } \\
\text { Mouth }\end{array}$ & Bass & 0.46 & 0.53 & 0.58 & 0.26 & 0.29 & 0.58 \\
\hline $\begin{array}{l}\text { Lower Three Runs Creek } \\
\text { Mouth }\end{array}$ & Bream & 0.24 & 0.27 & 0.29 & 0.26 & 0.11 & 0.29 \\
\hline $\begin{array}{l}\text { Lower Three Runs Creek } \\
\text { Mouth }\end{array}$ & Bream & 0.26 & 0.23 & 0.33 & 0.19 & 0.22 & 0.33 \\
\hline $\begin{array}{l}\text { Lower Three Runs Creek } \\
\text { Mouth }\end{array}$ & Catfish & 0.08 & 0.42 & 0.86 & 0.79 & 0.34 & 0.86 \\
\hline Highway 301 Bridge & Bass & 0.26 & 0.27 & 0.27 & 0.23 & 0.23 & 0.27 \\
\hline Highway 301 Bridge & Bream & 0.26 & 0.28 & 0.27 & 0.29 & 0.24 & 0.29 \\
\hline Highway 301 Bridge & Catfish & 0.43 & 0.59 & 0.23 & 0.83 & 0.92 & 0.92 \\
\hline Highway 301 Bridge & Shad & 0.24 & 0.28 & 0.24 & 0.24 & 0.24 & 0.28 \\
\hline
\end{tabular}


Table 59

Fish Surveillance - Mercury

Page 2 of 2

\begin{tabular}{|c|c|c|c|c|c|c|c|}
\hline \multirow{3}{*}{$\begin{array}{l}\text { Location } \\
\text { Stokes Bluff Landing }\end{array}$} & & \multicolumn{6}{|c|}{ Mercury ( $\mu \mathrm{g} / \mathrm{g}$ - Parts Per Million) } \\
\hline & \multicolumn{2}{|l|}{$\begin{array}{c}\text { Type } \\
\text { of Fish }\end{array}$} & \multicolumn{3}{|c|}{ Analytical Results } & & \multirow{2}{*}{$\frac{\text { Maximum }}{1.24}$} \\
\hline & Bass & 0.71 & 1.24 & 0.98 & 1.08 & 0.89 & \\
\hline Stokes Bluff Landing & Bream & 0.20 & 0.67 & 0.66 & 0.33 & 0.74 & 0.74 \\
\hline Stokes Bluff Landing & Catfish & 0.94 & 0.43 & 0.63 & 0.52 & 0.68 & 0.94 \\
\hline $\begin{array}{l}\text { Highway } 17 \text { Bridge Area } \\
\text { (upstream of River Mile 21.6) }\end{array}$ & Bass & 0.56 & 0.31 & 1.22 & 1.25 & 0.25 & 1.25 \\
\hline $\begin{array}{l}\text { Highway } 17 \text { Bridge Area } \\
\text { (upstream of River Mile 21.6) }\end{array}$ & Bream & 0.27 & 0.11 & 0.32 & 0.12 & 0.52 & 0.52 \\
\hline $\begin{array}{l}\text { Highway } 17 \text { Bridge Area } \\
\text { (upstream of River Mile 21.6) }\end{array}$ & Catfish & 0.48 & 0.64 & 0.24 & 0.54 & 0.12 & 0.64 \\
\hline $\begin{array}{l}\text { Highway } 17 \text { Bridge Area } \\
\text { (downstream, River Mile } \\
0-21.6 \text { ) }\end{array}$ & Mullet & 0.01 & 0.02 & 0.26 & 0.01 & 0.01 & 0.26 \\
\hline $\begin{array}{l}\text { Highway } 17 \text { Bridge } \\
\text { (downstream, River Mile } \\
0-21.6 \text { ) }\end{array}$ & Redfish & 0.32 & 0.25 & 0.24 & 0.23 & 0.21 & 0.32 \\
\hline $\begin{array}{l}\text { Highway } 17 \text { Bridge } \\
\text { (downstream, River Mile } \\
0-21.6 \text { ) }\end{array}$ & Sea Trout & 0.19 & 0.33 & 0.14 & NC & NC & 0.33 \\
\hline $\begin{array}{l}\text { Lower Three Runs Creek at } \\
\text { Patterson Mill Road }\end{array}$ & Bass & 1.68 & 2.02 & 1.67 & 1.38 & 1.63 & 2.02 \\
\hline $\begin{array}{l}\text { Lower Three Runs Creek at } \\
\text { Patterson Mill Road }\end{array}$ & Bream & 0.29 & 0.82 & 0.46 & 0.21 & 0.58 & 0.82 \\
\hline L-Lake & Bass & 1.22 & 0.77 & 0.93 & 0.53 & 0.37 & 1.22 \\
\hline L- Lake & Bream & 0.18 & 0.17 & 0.23 & 0.16 & 0.28 & 0.28 \\
\hline PAR Pond & Bass & 0.89 & 1.10 & 1.69 & 2.90 & 0.98 & 2.90 \\
\hline PAR Pond & Bream & 0.50 & 0.39 & 0.33 & 0.53 & 0.56 & 0.56 \\
\hline Pond B & Bass & 0.89 & 1.26 & 1.00 & 0.79 & 1.05 & 1.26 \\
\hline Pond B & Bream & 0.13 & 0.27 & 0.11 & 0.36 & 0.18 & 0.36 \\
\hline
\end{tabular}


Table 60

Blind Sample Results for pH Field Measurements

Page 1 of 1

pH Units

\begin{tabular}{lccl} 
& Measured Value & Known Value & Difference \\
\hline $\mathrm{npH}-01-1$ & 7.31 & 7.43 & 0.12 units \\
$\mathrm{BpH}-01-2$ & 6.91 & 6.88 & 0.03 units \\
$\mathrm{BpH}-02-1$ & 7.4 & 7.42 & 0.02 units \\
$\mathrm{BpH}-02-2$ & 6.78 & 6.87 & 0.09 units \\
$\mathrm{BpH}-03-1$ & 7.42 & 7.44 & 0.02 units \\
$\mathrm{BpH}-03-2$ & 4.10 & 4.04 & 0.06 units \\
$\mathrm{BpH}-04-1$ & 7.39 & 7.42 & 0.03 units \\
$\mathrm{BpH}-04-2$ & 3.75 & 4.05 & 0.30 units \\
$\mathrm{BpH}-05-1$ & 7.02 & 6.86 & 0.16 units \\
$\mathrm{BpH}-05-2$ & 6.92 & 6.87 & 0.05 units \\
$\mathrm{BpH}-06-1$ & 6.87 & 6.85 & 0.02 units \\
$\mathrm{BpH}-06-2$ & 6.86 & 6.85 & 0.01 units \\
$\mathrm{BpH}-07-1$ & 3.99 & 4.01 & 0.02 units \\
$\mathrm{BpH}-07-2$ & 6.79 & 6.84 & 0.05 units \\
$\mathrm{BpH}-08-1$ & 3.96 & 3.99 & 0.03 units \\
$\mathrm{BpH}-08-2$ & 6.91 & 6.87 & 0.04 units \\
$\mathrm{BpH}-09-1$ & 7.42 & 7.41 & 0.01 units \\
$\mathrm{BpH}-09-2$ & 4.02 & 4.02 & 0.00 units \\
$\mathrm{BpH}-10-1$ & 7.60 & 7.42 & 0.18 units \\
$\mathrm{BpH}-10-2$ & 4.04 & 4.04 & 0.00 units \\
$\mathrm{BpH}-11-1$ & 7.45 & 7.45 & 0.00 units \\
$\mathrm{BpH}-11-2$ & 3.94 & 4.06 & 0.12 units \\
$\mathrm{BpH}-12-1$ & 7.41 & 7.40 & 0.01 units \\
$\mathrm{BpH}-12-2$ & 3.85 & 4.03 & 0.18 units
\end{tabular}


Table 61

Blind Sample Results for Tritium

Page 1 of 1

\begin{tabular}{|c|c|c|c|c|c|c|}
\hline \multirow[b]{2}{*}{ Sample Date } & \multicolumn{2}{|c|}{$\mathrm{pCi} / \mathrm{mL}$} & \multirow[b]{2}{*}{ Ratio } & \multicolumn{2}{|c|}{ Range } & \multirow[b]{2}{*}{ Evaluation } \\
\hline & $\begin{array}{c}\text { Measured } \\
\text { Value }\end{array}$ & Known Value & & $\begin{array}{l}\text { Lower } \\
\text { Control } \\
\text { Limit }^{\mathrm{a}}\end{array}$ & $\begin{array}{l}\text { Upper } \\
\text { Control } \\
\text { Limita }\end{array}$ & \\
\hline \multicolumn{7}{|c|}{ Note: $\quad A$ is "acceptable." } \\
\hline $01-12-99$ & $9.800 \pm .483$ & 9.138 & 1.07 & 6.3 & 17.5 & $\bar{A}$ \\
\hline $02-12-99$ & $3.579 \pm .483$ & 3.421 & 1.05 & 2.4 & 6.5 & A \\
\hline $03-24-99$ & $7.227 \pm .419$ & 7.198 & 1.00 & 5.0 & 13.7 & A \\
\hline $04-14-99$ & $6.730 \pm .419$ & 6.095 & 1.10 & 4.2 & 11.6 & $A$ \\
\hline $05-12-99$ & $9.676 \pm .452$ & 8.673 & 1.12 & 6.0 & 16.6 & $A$ \\
\hline $06-16-99$ & $5.341 \pm .382$ & 5.828 & 0.92 & 4.0 & 11.1 & $A$ \\
\hline 07-19-99 & $5.838 \pm .419$ & 5.866 & 1.00 & 4.0 & 11.2 & $A$ \\
\hline $08-13-99$ & $11.03 \pm .42$ & 11.42 & 0.97 & 7.9 & 21.8 & A \\
\hline $09-16-99$ & $4.557 \pm .419$ & 4.581 & 0.99 & 3.2 & 8.7 & A \\
\hline $10-19-99$ & $3.481 \pm .452$ & 4.084 & 0.85 & 2.8 & 7.8 & A \\
\hline $11-10-99$ & $5.438 \pm .419$ & 5.631 & 0.97 & 3.9 & 10.8 & A \\
\hline $12-06-99$ & $5.892 \pm .382$ & 5.675 & 1.04 & 3.9 & 10.8 & A \\
\hline
\end{tabular}

a Control limits are based on Department of Energy's Environmental Measurements Laboratory (EML) historical data for tritium. 
Table 62

QAP Interlaboratory Comparison of Analytical Results

Page 1 of 5

\begin{tabular}{|c|c|c|c|c|c|c|c|c|}
\hline \multirow[b]{2}{*}{ Nuclide } & \multirow[b]{2}{*}{ SRS Value } & \multirow[b]{2}{*}{ QAPb Value } & \multirow[b]{2}{*}{$\begin{array}{c}\text { SRS/QAP } \\
\text { Ratio }\end{array}$} & \multirow[b]{2}{*}{$\begin{array}{c}\text { Number of } \\
\text { Labs }\end{array}$} & \multirow[b]{2}{*}{$\begin{array}{c}\text { Mean of } \\
\text { Labs }\end{array}$} & \multicolumn{2}{|c|}{ Range } & \multirow[b]{2}{*}{ Evaluation } \\
\hline & & & & & & $\begin{array}{l}\text { Lower Control } \\
\text { Limitc }\end{array}$ & $\begin{array}{l}\text { Upper Control } \\
\text { Limitc }^{c}\end{array}$ & \\
\hline \multicolumn{9}{|c|}{ Note: $\quad A$ is "acceptable," $W$ is "acceptable with warning," and $N$ is "not acceptable." } \\
\hline \multicolumn{9}{|c|}{ QAP Set \#50, June, 1999} \\
\hline \multicolumn{9}{|l|}{ Air } \\
\hline Alpha & $1.52 \pm 0.13$ & 1.61 & 0.94 & 72 & 1.691 & 0.805 & 2.496 & A \\
\hline Am-241 & $0.148 \pm 0.016$ & 0.134 & 1.11 & 65 & 0.154 & 0.098 & 0.345 & A \\
\hline Beta & $1.45 \pm 0.12$ & 1.56 & 0.93 & 76 & 1.576 & 1.123 & 2.605 & $A$ \\
\hline Co-60 & $5.11 \pm 0.74$ & 4.96 & 1.03 & 118 & 5.104 & 3.72 & 6.547 & A \\
\hline Cs-137 & $6.02 \pm 0.87$ & 6.05 & 1.00 & 120 & 6.268 & 4.417 & 8.289 & A \\
\hline Pu-238 & $0.299 \pm 0.033$ & 0.272 & 1.10 & 48 & 0.272 & 0.201 & 0.381 & $A$ \\
\hline Pu-239 & $0.139 \pm 0.017$ & 0.124 & 1.12 & 49 & 0.13 & 0.094 & 0.179 & $A$ \\
\hline Sb-125 & $4.31 \pm 0.63$ & 3.59 & 1.20 & 106 & 3.863 & 2.19 & 5.134 & $w$ \\
\hline Sr-90 & $0.63 \pm 0.12$ & 0.644 & 0.98 & 39 & 0.645 & 0.393 & 1.243 & A \\
\hline U-234 & $0.06 \pm 0.009$ & 0.06 & 1.00 & 40 & 0.07 & 0.05 & 0.115 & A \\
\hline$U-238$ & $0.061 \pm 0.009$ & 0.061 & 1.00 & 40 & 0.07 & 0.051 & 0.16 & $A$ \\
\hline \multicolumn{9}{|l|}{ Soil } \\
\hline Ac-228 & $57.9 \pm 4.5$ & 47.15 & 1.23 & 80 & 51.252 & 37.249 & 82.513 & A \\
\hline$A m-241$ & $4.58 \pm 0.76$ & 4.894 & 0.94 & 63 & 5.11 & 3.083 & 11.306 & $A$ \\
\hline Bi-214 & $74.8 \pm 6$ & 69.9 & 1.07 & 77 & 67.523 & 52.425 & 99.258 & A \\
\hline Cs-137 & $743 \pm 76$ & 659.5 & 1.13 & 115 & 693.794 & 547.385 & 870.54 & A \\
\hline $\mathrm{K}-40$ & $472 \pm 50$ & 362.75 & 1.30 & & 349.8 & 282.945 & 555.008 & $w$ \\
\hline
\end{tabular}


Table 62

QAP Interlaboratory Comparison of Analytical Results

Page 2 of 5

\begin{tabular}{|c|c|c|c|c|c|c|c|c|}
\hline \multirow{2}{*}{ Nuclide } & \multirow[b]{2}{*}{ SRS Valuea } & \multirow[b]{2}{*}{ QAPb Value $^{b}$} & \multirow[b]{2}{*}{$\begin{array}{c}\text { SRS/QAP } \\
\text { Ratio }\end{array}$} & \multirow[b]{2}{*}{$\begin{array}{c}\text { Number of } \\
\text { Labs }\end{array}$} & \multirow[b]{2}{*}{$\begin{array}{c}\text { Mean of } \\
\text { Labs }\end{array}$} & \multicolumn{2}{|c|}{ Range } & \multirow[b]{2}{*}{ Evaluation } \\
\hline & & & & & & $\begin{array}{l}\text { Lower Control } \\
\text { Limit }^{c}\end{array}$ & $\begin{array}{l}\text { Upper Control } \\
\text { Limitc }^{c}\end{array}$ & \\
\hline $\mathrm{Pb}-212$ & $53.7 \pm 5.5$ & 47.925 & 1.12 & 72 & 50.944 & 35.465 & 63.74 & $\bar{A}$ \\
\hline $\mathrm{Pb}-214$ & $76.1 \pm 5.9$ & 71. & 1.07 & 81 & 72.704 & 46.15 & 102.95 & $A$ \\
\hline Pu-239 & $10.83 \pm 1.85$ & 8.112 & 1.34 & 65 & 8.128 & 5.597 & 14.114 & $w$ \\
\hline Sr-90 & $41.2 \pm 19.6$ & 32.4 & 1.27 & 49 & 35.575 & 19.44 & 118.584 & $A$ \\
\hline Th-234 & $90.6 \pm 6.9$ & 138. & 0.66 & 50 & 155.112 & 81.42 & 255.3 & $w$ \\
\hline \multicolumn{9}{|c|}{ Vegetation } \\
\hline$A m-241$ & $3.34 \pm 0.43$ & 3.522 & 0.95 & 53 & 3.723 & 2.395 & 9.509 & A \\
\hline $\mathrm{Cm}-244$ & $1.71 \pm 0.31$ & 1.671 & 1.02 & 30 & 2.015 & 0.785 & 2.907 & $A$ \\
\hline Co-60 & $24.1 \pm 4.2$ & 21.45 & 1.12 & 85 & 23.166 & 14.801 & 31.317 & A \\
\hline Cs-137 & $529 \pm 55$ & 467. & 1.13 & 88 & 517.436 & 373.6 & 653.8 & A \\
\hline $\mathrm{K}-40$ & $718 \pm 108$ & 656.5 & 1.09 & & 511.939 & 518.635 & 932.23 & $A$ \\
\hline Pu-239 & $4.78 \pm 0.8$ & 5.204 & 0.92 & 48 & 5.033 & 3.539 & 8.275 & A \\
\hline Sr-90 & $822 \pm 99$ & 736.1 & 1.12 & 47 & 682.365 & 368.05 & 979.013 & A \\
\hline \multicolumn{9}{|l|}{ Water } \\
\hline Alpha & $940 \pm 103$ & 1090. & 0.86 & 68 & 1069.29 & 664.9 & 1438.8 & $A$ \\
\hline Am-241 & $1.33 \pm 0.15$ & 1.146 & 1.16 & 70 & 1.199 & 0.86 & 1.708 & $A$ \\
\hline Beta & $1130 \pm 100$ & 1100. & 1.03 & 75 & 1098.9 & 605. & 1694. & $A$ \\
\hline Co- 60 & $55 \pm 4$ & 51.1 & 1.08 & 124 & 54.268 & 40.88 & 61.32 & A \\
\hline Cs-137 & $41.5 \pm 2.7$ & 39.375 & 1.05 & 125 & 41.816 & 31.5 & 49.613 & A \\
\hline $\mathrm{H}-3$ & $128 \pm 18$ & 121.08 & 1.06 & 84 & 131.614 & 85.967 & 216.733 & A \\
\hline $\begin{array}{ll}\text { a } & \text { Value } \\
\text { b } & \text { Qualit } \\
\text { c } & \text { Contr }\end{array}$ & $\begin{array}{l}\mathrm{Kg} \text {, and } \mathrm{Bq} / \text { /filter. } \\
\text { Program conduc } \\
\text { stablished by EM }\end{array}$ & $\begin{array}{l}\text { partment of Ener } \\
\text { m historical data }\end{array}$ & Environmental & asurements La & tory (EML), & ew York & & \\
\hline
\end{tabular}


Table 62

QAP Interlaboratory Comparison of Analytical Results

Page 3 of 5

\begin{tabular}{|c|c|c|c|c|c|c|c|c|}
\hline \multirow[b]{2}{*}{ Nuclide } & \multirow[b]{2}{*}{ SRS Value ${ }^{a}$} & \multirow[b]{2}{*}{ QAPb Value } & \multirow[b]{2}{*}{$\begin{array}{c}\text { SRS/QAP } \\
\text { Ratio }\end{array}$} & \multirow[b]{2}{*}{$\begin{array}{l}\text { Number of } \\
\text { Labs }\end{array}$} & \multirow[b]{2}{*}{$\begin{array}{l}\text { Mean of } \\
\text { Labs }\end{array}$} & \multicolumn{2}{|c|}{ Range } & \multirow[b]{2}{*}{ Evaluation } \\
\hline & & & & & & $\begin{array}{l}\text { Lower Control } \\
\text { Limitc }\end{array}$ & $\begin{array}{l}\text { Upper Control } \\
\text { Limit }^{c}\end{array}$ & \\
\hline Pu-238 & $0.858 \pm 0.12$ & 0.772 & 1.11 & 59 & 0.789 & 0.602 & 0.965 & $\bar{w}$ \\
\hline Pu-239 & $1.106 \pm 0.13$ & 1.009 & 1.10 & 61 & 1.005 & 0.807 & 1.403 & A \\
\hline Sr-90 & $2.83 \pm 0.55$ & 4.104 & 0.69 & 62 & 3.85 & 3.078 & 6.156 & $N$ \\
\hline U-234 & $0.282 \pm 0.052$ & 0.269 & 1.05 & 50 & 0.288 & 0.215 & 0.376 & $A$ \\
\hline U-238 & $0.279 \pm 0.053$ & 0.262 & 1.07 & 50 & 0.282 & 0.21 & 0.33 & $A$ \\
\hline
\end{tabular}

QAP Set \#51, December, 1999

Air

\begin{tabular}{|c|c|c|c|c|c|c|c|c|}
\hline Alpha & $2.26 \pm 0.15$ & 2.77 & 0.82 & 85 & 2.764 & 1.385 & 4.294 & A \\
\hline$A m-241$ & $0.121 \pm 0.012$ & 0.127 & 0.95 & 63 & 0.127 & 0.093 & 0.328 & A \\
\hline Beta & $2.96 \pm 0.18$ & 2.66 & 1.11 & 86 & 2.873 & 1.915 & 4.442 & A \\
\hline Co-57 & $8.66 \pm 0.61$ & 7.73 & 1.12 & 115 & 7.885 & 5.025 & 10.745 & A \\
\hline Co-60 & $6.99 \pm 0.47$ & 6.35 & 1.10 & 119 & 6.661 & 4.763 & 8.382 & W \\
\hline Cs-137 & $7.08 \pm 0.79$ & 6.43 & 1.10 & 121 & 6.899 & 4.694 & 8.809 & A \\
\hline$M n-54$ & $8.8 \pm 0.91$ & 7.91 & 1.11 & 116 & 8.661 & 6.012 & 11.232 & $A$ \\
\hline Pu-238 & $0.097 \pm 0.011$ & 0.097 & 1.00 & 43 & 0.095 & 0.072 & 0.136 & A \\
\hline Pu-239 & $0.136 \pm 0.016$ & 0.136 & 1.00 & 43 & 0.132 & 0.103 & 0.196 & A \\
\hline$R u-106$ & $7.35 \pm 1.84$ & 5.5 & 1.34 & 80 & 5.693 & 3.245 & 7.15 & $\mathbf{N}$ \\
\hline Sr-90 & $0.38 \pm 0.09$ & 0.336 & 1.13 & 36 & 0.398 & 0.205 & 0.648 & A \\
\hline U-234 & $0.059 \pm 0.008$ & 0.066 & 0.89 & 36 & 0.072 & 0.055 & 0.127 & W \\
\hline U-238 & $0.059 \pm 0.008$ & 0.065 & 0.91 & 35 & 0.071 & 0.055 & 0.17 & $A$ \\
\hline
\end{tabular}

Values are $B q / L, B q / K g$, and $B q / f i l t e r$

b Quality Assessment Program conducted by Department of Energy's Environmental Measurements Laboratory (EML), New York

c Control limits were established by EML/QAP from historical data. 
Table 62

QAP Interlaboratory Comparison of Analytical Results

Page 4 of 5

\begin{tabular}{|c|c|c|c|c|c|c|c|c|}
\hline \multirow{2}{*}{ Nuclide } & \multirow[b]{2}{*}{ SRS Value } & \multirow[b]{2}{*}{ QAPb Value } & \multirow[b]{2}{*}{$\begin{array}{c}\text { SRS/QAP } \\
\text { Ratio }\end{array}$} & \multirow[b]{2}{*}{$\begin{array}{c}\text { Number of } \\
\text { Labs }\end{array}$} & \multirow[b]{2}{*}{$\begin{array}{l}\text { Mean of } \\
\text { Labs }\end{array}$} & \multicolumn{2}{|c|}{ Range } & \multirow[b]{2}{*}{ Evaluation } \\
\hline & & & & & & $\begin{array}{c}\text { Lower Control } \\
\text { Limit }^{c}\end{array}$ & $\begin{array}{c}\text { Upper Control } \\
\text { Limit }^{c}\end{array}$ & \\
\hline \multicolumn{9}{|l|}{ Soil } \\
\hline Ac-228 & $137 \pm 7$ & 124 & 1.10 & 79 & 134.044 & 97.96 & 217. & $A$ \\
\hline $\mathrm{Bi}-212$ & $90.3 \pm 13.9$ & 140 & 0.65 & 58 & 115.78 & 58.8 & 170.8 & A \\
\hline $\mathrm{Bi}-214$ & $78.7 \pm 6.2$ & 69.5 & 1.13 & 74 & 78.257 & 52.125 & 98.69 & A \\
\hline Cs-137 & $232 \pm 24$ & 204 & 1.14 & 117 & 220.728 & 169.32 & 269.28 & A \\
\hline $\mathrm{K}-40$ & $938 \pm 91$ & 780 & 1.20 & 104 & 854.88 & 608.4 & 1193.4 & A \\
\hline $\mathrm{Pb}-212$ & $122 \pm 8$ & 127 & 0.96 & 76 & 132.842 & 93.98 & 168.91 & $A$ \\
\hline $\mathrm{Pb}-214$ & $75.9 \pm 5.8$ & 72 & 1.05 & 72 & 82.224 & 46.8 & 104.4 & $A$ \\
\hline Pu-239 & $2.97 \pm 0.68$ & 3.2 & 0.93 & 49 & 3.014 & 2.208 & 5.568 & A \\
\hline Sr-90 & $21 \pm 12$ & 13 & 1.62 & 39 & 18.187 & 7.8 & 47.58 & $A$ \\
\hline Th-234 & $185 \pm 28$ & 198 & 0.93 & 42 & 213.444 & 116.82 & 366.3 & $A$ \\
\hline \multicolumn{9}{|c|}{ Vegetation } \\
\hline Am-241 & $3.6 \pm 0.51$ & 2.88 & 1.25 & 62 & 3.476 & 1.958 & 7.776 & $A$ \\
\hline $\mathrm{Cm}-244$ & $1.65 \pm 0.28$ & 1.61 & 1.02 & 30 & 1.882 & 0.757 & 2.801 & A \\
\hline Co-60 & $20.4 \pm 1.2$ & 17.6 & 1.16 & 102 & 18.814 & 12.144 & 25.696 & $A$ \\
\hline Cs-137 & $484 \pm 20$ & 440 & 1.10 & 107 & 472.12 & 352 & 616 & $A$ \\
\hline $\mathrm{K}-40$ & $550 \pm 32$ & 513 & 1.07 & 86 & 529.929 & 405.27 & 728.46 & A \\
\hline Pu-239 & $5.23 \pm 0.73$ & 4.3 & 1.22 & 48 & 4.459 & 2.924 & 6.837 & A \\
\hline Sr-90 & $693 \pm 51$ & 595 & 1.16 & 44 & 579.53 & 297.5 & 791.35 & $w$ \\
\hline \multicolumn{9}{|c|}{$\begin{array}{l}\text { a } \\
\text { b alues are Bq/L, Bq/Kg, and Bq/filter. } \\
\text { c Contry Assessment Program conducted by Department of Energy's } \\
\text { Core established by EML/QAP from historical data. }\end{array}$} \\
\hline
\end{tabular}




\section{Table 62}

QAP Interlaboratory Comparison of Analytical Results

Page 5 of 5

\begin{tabular}{|c|c|c|c|c|c|c|c|c|}
\hline \multirow[b]{2}{*}{ Nuclide } & \multirow[b]{2}{*}{ SRS Value ${ }^{a}$} & \multirow[b]{2}{*}{ QAPb Value } & \multirow[b]{2}{*}{$\begin{array}{c}\text { SRS/QAP } \\
\text { Ratio }\end{array}$} & \multirow[b]{2}{*}{$\begin{array}{c}\text { Number of } \\
\text { Labs }\end{array}$} & \multirow[b]{2}{*}{$\begin{array}{c}\text { Mean of } \\
\text { Labs }\end{array}$} & \multicolumn{2}{|c|}{ Range } & \multirow[b]{2}{*}{ Evaluation } \\
\hline & & & & & & $\begin{array}{l}\text { Lower Control } \\
\text { Limit }^{c}\end{array}$ & $\begin{array}{l}\text { Upper Control } \\
\text { Limitc }^{c}\end{array}$ & \\
\hline \multicolumn{9}{|l|}{ Water } \\
\hline Alpha & $1500 \pm 152$ & 1580 & 0.95 & 73 & 1488.36 & 963.8 & 2085.6 & A \\
\hline Am-241 & $0.95 \pm 0.033$ & 0.85 & 1.12 & 62 & 0.93 & 0.638 & 1.267 & A \\
\hline Beta & $863 . \pm 100$ & 740 & 1.17 & 78 & 885.78 & 407 & 1139.6 & A \\
\hline Co-60 & $56.5 \pm 4.3$ & 52.4 & 1.08 & 122 & 53.71 & 41.92 & 62.88 & $A$ \\
\hline Cs-137 & $78.7 \pm 8.2$ & 76 & 1.04 & 125 & 78.432 & 60.8 & 95.76 & A \\
\hline $\mathrm{H}-3$ & $86.1 \pm 10.1$ & 80.7 & 1.07 & 81 & 86.268 & 57.297 & 144.453 & A \\
\hline Pu-238 & $0.879 \pm 0.335$ & 0.79 & 1.11 & 55 & 0.82 & 0.616 & 0.988 & w \\
\hline Pu-239 & $0.962 \pm 0.036$ & 0.87 & 1.11 & 56 & 0.905 & 0.696 & 1.209 & A \\
\hline Sr-90 & $1.6 \pm 0.6$ & 1.72 & 0.93 & 62 & 1.71 & 1.29 & 2.58 & $A$ \\
\hline $\mathrm{U}-234$ & $0.362 \pm 0.019$ & 0.37 & 0.98 & 44 & 0.402 & 0.296 & 0.518 & $A$ \\
\hline U-238 & $0.377 \pm 0.019$ & 0.36 & 1.05 & 46 & 0.392 & 0.288 & 0.454 & A \\
\hline
\end{tabular}


Table 63

Quality Assurance/Quality Control Standards

Page 1 of 1

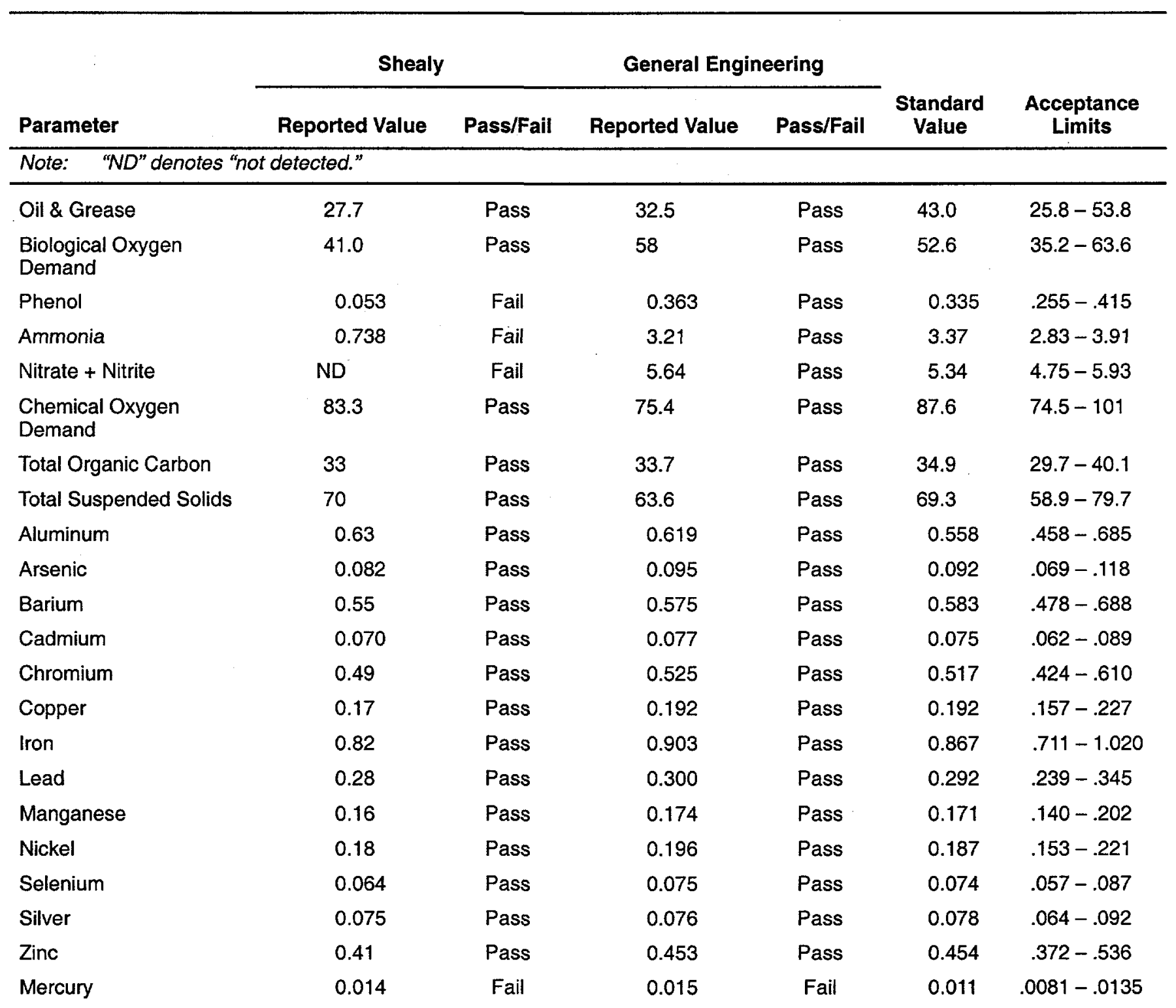


Table 64

NPDES Duplicate Sample Results

Page 1 of 5

\begin{tabular}{llllll}
\hline NPDES Site & Parameter Sampled & Units & SRS Reading & $\begin{array}{c}\text { Duplicate } \\
\text { Reading }\end{array}$ & Difference \\
\hline
\end{tabular}

Note: The Environmental Monitoring Section laboratory performs most analyses for total suspended solids. Other analyses, including some total suspended solids, are performed by Shealy Environmental Services.

\begin{tabular}{|c|c|c|c|c|c|}
\hline$\overline{X-8 C}$ & 1,1,1-Trichloroethane & ug/L & $<2.0$ & $<2.0$ & $\overline{0}$ \\
\hline$M-05$ & 1,1,1-Trichloroethane & $u g / L$ & $<2.0$ & $<2.0$ & 0 \\
\hline$A-1 A$ & 1,1,1-Trichloroethane & ug/L & $<2.0$ & $<2.0$ & 0 \\
\hline$X-8 C$ & 1,1,1-Trichloroethane & ug/L & $<2.0$ & $<2.0$ & 0 \\
\hline$A-1 A$ & 1,1,1-Trichloroethane & $u g / L$ & $<2.0$ & $<2.0$ & 0 \\
\hline$M-04$ & Aluminum & $\mathrm{mg} / \mathrm{L}$ & 0.25 & 0.12 & 0.13 \\
\hline$M-04$ & Ammonia & $\mathrm{mg} / \mathrm{L}$ & 0.97 & 1.04 & 0.07 \\
\hline$x-8 B$ & Benzene & $u g / L$ & $<0.5$ & $<0.5$ & 0 \\
\hline$Z-8 B$ & Benzene & $\mathrm{ug} / \mathrm{L}$ & $<0.5$ & $<0.5$ & 0 \\
\hline$x-8 B$ & Benzene & $\mathrm{ug} / \mathrm{L}$ & $<0.5$ & $<0.5$ & 0 \\
\hline A-01 & Biological Oxygen Demand & $\mathrm{mg} / \mathrm{L}$ & $<2.0$ & $<2.0$ & 0 \\
\hline$X-8 B$ & Biological Oxygen Demand & $\mathrm{mg} / \mathrm{L}$ & 3.7 & 3.5 & 0.2 \\
\hline$x-8 B$ & Biological Oxygen Demand & $\mathrm{mg} / \mathrm{L}$ & $<2.0$ & $<2.0$ & 0 \\
\hline$A-11$ & Biological Oxygen Demand & $\mathrm{mg} / \mathrm{L}$ & $<2.0$ & $<2.0$ & 0 \\
\hline $\mathrm{X}-8 \mathrm{~B}$ & Biological Oxygen Demand & $\mathrm{mg} / \mathrm{L}$ & $<2.0$ & $<2.0$ & 0 \\
\hline$A-01$ & Biological Oxygen Demand & $\mathrm{mg} / \mathrm{L}$ & $<2.0$ & $<2.0$ & 0 \\
\hline$A-11$ & Biological Oxygen Demand & $\mathrm{mg} / \mathrm{L}$ & $<2.0$ & $<2.0$ & 0 \\
\hline $\mathrm{H}-16$ & Cadmium & $\mathrm{mg} / \mathrm{L}$ & $<0.002$ & $<0.002$ & 0 \\
\hline$H-16$ & Cadmium & $\mathrm{mg} / \mathrm{L}$ & $<0.0020$ & $<0.0020$ & 0 \\
\hline $\mathrm{H}-16$ & Cadmium & $\mathrm{mg} / \mathrm{L}$ & $<0.0020$ & $<0.0020$ & 0 \\
\hline$H-16$ & Chromium & $\mathrm{mg} / \mathrm{L}$ & $<0.005$ & $<0.005$ & 0 \\
\hline$M-04$ & Chromium & $\mathrm{mg} / \mathrm{L}$ & $<0.0050$ & $<0.0050$ & 0 \\
\hline $\mathrm{H}-16$ & Chromium & $\mathrm{mg} / \mathrm{L}$ & $<0.0050$ & $<0.0050$ & 0 \\
\hline $\mathrm{H}-16$ & Chromium & $\mathrm{mg} / \mathrm{L}$ & $<0.0050$ & $<0.0050$ & 0 \\
\hline$x-8 C$ & cis-1,2-Dichloroethene & $u g / L$ & $<2.0$ & $<2.0$ & 0 \\
\hline$M-05$ & cis-1,2-Dichloroethene & $u g / L$ & $<2.0$ & $<2.0$ & 0 \\
\hline$A-1 A$ & cis-1,2-Dichloroethene & $u g / L$ & $<2.0$ & $<2.0$ & 0 \\
\hline$x-8 C$ & cis-1,2-Dichloroethene & $u g / L$ & $<2.0$ & $<2.0$ & 0 \\
\hline$A-1 A$ & cis-1,2-Dichloroethene & $u g / L$ & $<2.0$ & $<2.0$ & 0 \\
\hline $\mathrm{H}-16$ & Copper & $\mathrm{mg} / \mathrm{L}$ & $<0.005$ & $<0.005$ & 0 \\
\hline$F-08$ & Copper & $\mathrm{mg} / \mathrm{L}$ & $<0.005$ & $<0.005$ & 0 \\
\hline$A-01$ & Copper & $\mathrm{mg} / \mathrm{L}$ & 0.01 & 0.009 & 0.001 \\
\hline$A-11$ & Copper & $\mathrm{mg} / \mathrm{L}$ & $<0.005$ & 0.009 & $<0.009$ \\
\hline
\end{tabular}


Table 64

NPDES Duplicate Sample Results

Page 2 of 5

\begin{tabular}{|c|c|c|c|c|c|}
\hline NPDES Site & Parameter Sampled & Units & SRS Reading & $\begin{array}{c}\text { Duplicate } \\
\text { Reading }\end{array}$ & Difference \\
\hline $\mathrm{H}-12$ & Copper & $\mathrm{mg} / \mathrm{L}$ & $<0.005$ & $<0.005$ & 0 \\
\hline$A-01$ & Copper & $\mathrm{mg} / \mathrm{L}$ & 0.013 & 0.012 & 0.001 \\
\hline $\mathrm{H}-12$ & Copper & $\mathrm{mg} / \mathrm{L}$ & $<0.0050$ & 0.005 & $<0.005$ \\
\hline $\mathrm{H}-16$ & Copper & $\mathrm{mg} / \mathrm{L}$ & $<0.0050$ & $<0.0050$ & 0 \\
\hline$A-01$ & Copper & $\mathrm{mg} / \mathrm{L}$ & 0.012 & 0.011 & 0.001 \\
\hline$A-11$ & Copper & $\mathrm{mg} / \mathrm{L}$ & $<0.0050$ & $<0.0050$ & 0 \\
\hline $\mathrm{H}-16$ & Copper & $\mathrm{mg} / \mathrm{L}$ & $<0.0050$ & $<0.0050$ & 0 \\
\hline$F-08$ & Copper & mg/L & 0.0087 & 0.012 & 0 \\
\hline$M-04$ & Cyanide & $\mathrm{mg} / \mathrm{L}$ & $<0.010$ & $<0.010$ & 0 \\
\hline$M-04$ & Cyanide & $\mathrm{mg} / \mathrm{L}$ & $<0.010$ & $<0.010$ & 0 \\
\hline PP-1 & Iron & $\mathrm{mg} / \mathrm{L}$ & 0.19 & 0.22 & 0.03 \\
\hline $\mathrm{H}-16$ & Lead & $\mathrm{mg} / \mathrm{L}$ & $<0.003$ & $<0.003$ & 0 \\
\hline$F-08$ & Lead & $\mathrm{mg} / \mathrm{L}$ & $<0.003$ & $<0.003$ & 0 \\
\hline$A-01$ & Lead & $\mathrm{mg} / \mathrm{L}$ & $<0.003$ & $<0.003$ & 0 \\
\hline$A-11$ & Lead & $\mathrm{mg} / \mathrm{L}$ & $<0.003$ & $<0.003$ & 0 \\
\hline $\mathrm{H}-12$ & Lead & $\mathrm{mg} / \mathrm{L}$ & $<0.003$ & $<0.003$ & 0 \\
\hline$A-01$ & Lead & $\mathrm{mg} / \mathrm{L}$ & $<0.003$ & $<0.003$ & 0 \\
\hline$F-08$ & Lead & $\mathrm{mg} / \mathrm{L}$ & $<0.0030$ & $<0.0030$ & 0 \\
\hline $\mathrm{H}-08$ & Lead & $\mathrm{mg} / \mathrm{L}$ & $<0.0030$ & $<0.0030$ & 0 \\
\hline$M-04$ & Lead & $\mathrm{mg} / \mathrm{L}$ & $<0.0030$ & $<0.0030$ & 0 \\
\hline $\mathrm{H}-12$ & Lead & $\mathrm{mg} / \mathrm{L}$ & $<0.0030$ & $<0.0030$ & 0 \\
\hline $\mathrm{H}-16$ & Lead & $\mathrm{mg} / \mathrm{L}$ & $<0.0030$ & $<0.0030$ & 0 \\
\hline$A-01$ & Lead & $\mathrm{mg} / \mathrm{L}$ & $<0.0030$ & $<0.0030$ & 0 \\
\hline$A-11$ & Lead & $\mathrm{mg} / \mathrm{L}$ & $<0.0030$ & $<0.0030$ & 0 \\
\hline $\mathrm{H}-16$ & Lead & $\mathrm{mg} / \mathrm{L}$ & $<0.0030$ & $<0.0030$ & 0 \\
\hline$F-08$ & Lead & $\mathrm{mg} / \mathrm{L}$ & $<0.0030$ & $<0.0030$ & 0 \\
\hline PP-1 & Manganese & $m g / L$ & 6.3 & 6.7 & 0.4 \\
\hline $\mathrm{H}-16$ & Mercury & $\mathrm{mg} / \mathrm{L}$ & $<0.0001$ & $<0.0001$ & 0 \\
\hline$F-08$ & Mercury & $\mathrm{mg} / \mathrm{L}$ & $<0.0001$ & $<0.0001$ & 0 \\
\hline$X-8 B$ & Mercury & $\mathrm{mg} / \mathrm{L}$ & 0.0003 & 0.0005 & 0.0002 \\
\hline A-01 & Mercury & $\mathrm{mg} / \mathrm{L}$ & $<0.0001$ & $<0.0001$ & 0 \\
\hline$A-11$ & Mercury & $\mathrm{mg} / \mathrm{L}$ & $<0.0001$ & $<0.0001$ & 0 \\
\hline$A-11$ & Mercury & $\mathrm{mg} / \mathrm{L}$ & $<0.0001$ & $<0.0001$ & 0 \\
\hline$A-01$ & Mercury & $\mathrm{mg} / \mathrm{L}$ & 0.0001 & 0.0001 & 0 \\
\hline$Z-8 B$ & Mercury & $\mathrm{mg} / \mathrm{L}$ & 0.0058 & 0.0059 & 0.0001 \\
\hline
\end{tabular}


Table 64

NPDES Duplicate Sample Results

Page 3 of 5

\begin{tabular}{|c|c|c|c|c|c|}
\hline NPDES Site & Parameter Sampled & Units & SRS Reading & $\begin{array}{l}\text { Duplicate } \\
\text { Reading }\end{array}$ & Difference \\
\hline $\mathrm{H}-16$ & Mercury & $\mathrm{mg} / \mathrm{L}$ & $<0.00010$ & $<0.00010$ & 0 \\
\hline$A-01$ & Mercury & $\mathrm{mg} / \mathrm{L}$ & 0.00011 & 0.00012 & 0.0001 \\
\hline$x-8 B$ & Mercury & $\mathrm{mg} / \mathrm{L}$ & 0.00016 & 0.00016 & 0 \\
\hline$A-11$ & Mercury & $\mathrm{mg} / \mathrm{L}$ & $<0.00010$ & $<0.00010$ & 0 \\
\hline $\mathrm{H}-16$ & Mercury & $\mathrm{mg} / \mathrm{L}$ & 0.00013 & 0.00011 & 0 \\
\hline$F-08$ & Mercury & $\mathrm{mg} / \mathrm{L}$ & $<0.00010$ & $<0.00010$ & 0 \\
\hline $\mathrm{H}-16$ & Nickel & $\mathrm{mg} / \mathrm{L}$ & $<0.010$ & $<0.010$ & 0 \\
\hline$M-04$ & Nickel & $\mathrm{mg} / \mathrm{L}$ & 0.042 & 0.04 & 0.002 \\
\hline $\mathrm{H}-16$ & Nickel & $\mathrm{mg} / \mathrm{L}$ & $<0.010$ & $<0.010$ & 0 \\
\hline $\mathrm{H}-16$ & Nickel & $\mathrm{mg} / \mathrm{L}$ & $<0.010$ & $<0.010$ & 0 \\
\hline$A-01$ & Oil \& Grease & $\mathrm{mg} / \mathrm{L}$ & $<1.0$ & $<1.0$ & 0 \\
\hline $\mathrm{H}-02$ & Oil \& Grease & $\mathrm{mg} / \mathrm{L}$ & $<1.0$ & 4.7 & $<4.7$ \\
\hline$L-07$ & Oil \& Grease & $\mathrm{mg} / \mathrm{L}$ & $<1.0$ & $<1.0$ & 0 \\
\hline$L-08$ & Oil \& Grease & $\mathrm{mg} / \mathrm{L}$ & $<1.0$ & $<1.0$ & 0 \\
\hline $\mathrm{H}-02$ & Oil \& Grease & $\mathrm{mg} / \mathrm{L}$ & $<1.0$ & 2.8 & $<2.8$ \\
\hline$A-01$ & Oil \& Grease & $\mathrm{mg} / \mathrm{L}$ & $<1.0$ & $<1.0$ & 0 \\
\hline $\mathrm{H}-07$ & Oil \& Grease & $\mathrm{mg} / \mathrm{L}$ & $<1.0$ & $<1.0$ & 0 \\
\hline$K-06$ & Oil \& Grease & $\mathrm{mg} / \mathrm{L}$ & 1.4 & 4 & 0 \\
\hline$x-8 B$ & Phenol & $\mathrm{mg} / \mathrm{L}$ & $<0.006$ & $<0.006$ & 0 \\
\hline$Z-8 B$ & Phenol & $\mathrm{mg} / \mathrm{L}$ & 0.02 & 0.022 & 0.002 \\
\hline$x-8 B$ & Phenol & $\mathrm{mg} / \mathrm{L}$ & 0.006 & $<0.0060$ & $<0.006$ \\
\hline $\mathrm{H}-16$ & Silver & $\mathrm{mg} / \mathrm{L}$ & $<0.005$ & $<0.005$ & 0 \\
\hline$H-16$ & Silver & $\mathrm{mg} / \mathrm{L}$ & $<0.0050$ & $<0.0050$ & 0 \\
\hline$H-16$ & Silver & $\mathrm{mg} / \mathrm{L}$ & $<0.0050$ & $<0.0050$ & 0 \\
\hline$x-8 C$ & Tetrachloroethene & $u g / L$ & $<2.0$ & $<2.0$ & 0 \\
\hline$M-05$ & Tetrachloroethene & $u g / L$ & $<2.0$ & $<2.0$ & 0 \\
\hline$A-1 A$ & Tetrachloroethene & $\mathrm{ug} / \mathrm{L}$ & $<2.0$ & $<2.0$ & 0 \\
\hline$x-8 C$ & Tetrachloroethene & $u g / L$ & $<2.0$ & $<2.0$ & 0 \\
\hline$A-1 A$ & Tetrachloroethene & $u g / L$ & $<2.0$ & $<2.0$ & 0 \\
\hline$X-8 B$ & Total Organic Carbon & $\mathrm{mg} / \mathrm{L}$ & 6.07 & $<1.00$ & $<6.07$ \\
\hline$x-8 B$ & Total Organic Carbon & $\mathrm{mg} / \mathrm{L}$ & 3.7 & 3.8 & 0.1 \\
\hline$x-8 B$ & Total Organic Carbon & $\mathrm{mg} / \mathrm{L}$ & 2.7 & 2.9 & 0.2 \\
\hline$s-04$ & Total Suspended Solids & $\mathrm{mg} / \mathrm{L}$ & 2 & 2 & 0 \\
\hline$x-04$ & Total Suspended Solids & $\mathrm{mg} / \mathrm{L}$ & $<1$ & $<1$ & 0 \\
\hline$A-01$ & Total Suspended Solids & $\mathrm{mg} / \mathrm{L}$ & 1 & 1 & 0 \\
\hline
\end{tabular}


Table 64

NPDES Duplicate Sample Results

Page 4 of 5

\begin{tabular}{|c|c|c|c|c|c|}
\hline NPDES Site & Parameter Sampled & Units & SRS Reading & $\begin{array}{l}\text { Duplicate } \\
\text { Reading }\end{array}$ & Difference \\
\hline $\mathrm{H}-02$ & Total Suspended Solids & $\mathrm{mg} / \mathrm{L}$ & 3 & 3 & $\overline{0}$ \\
\hline$x-08$ & Total Suspended Solids & $\mathrm{mg} / \mathrm{L}$ & 1 & 1 & 0 \\
\hline$X-8 B$ & Total Suspended Solids & $\mathrm{mg} / \mathrm{L}$ & 3 & 3 & 0 \\
\hline $\mathrm{H}-04$ & Total Suspended Solids & $\mathrm{mg} / \mathrm{L}$ & $<1$ & $<1$ & 0 \\
\hline$X-8 B$ & Total Suspended Solids & $\mathrm{mg} / \mathrm{L}$ & 6 & 6 & 0 \\
\hline$A-11$ & Total Suspended Solids & $\mathrm{mg} / \mathrm{L}$ & 2 & 2 & 0 \\
\hline $\mathrm{H}-08$ & Total Suspended Solids & $\mathrm{mg} / \mathrm{L}$ & 2 & 2 & 0 \\
\hline$x-04$ & Total Suspended Solids & $\mathrm{mg} / \mathrm{L}$ & $<1$ & $<1$ & 0 \\
\hline$F-01$ & Total Suspended Solids & $\mathrm{mg} / \mathrm{L}$ & $<1$ & 1 & $<1$ \\
\hline $\mathrm{H}-12$ & Total Suspended Solids & $\mathrm{mg} / \mathrm{L}$ & $<1$ & 1 & $<1$ \\
\hline S-04 & Total Suspended Solids & $\mathrm{mg} / \mathrm{L}$ & 1 & 2 & 1 \\
\hline$M-04$ & Total Suspended Solids & $\mathrm{mg} / \mathrm{L}$ & 1 & 1 & 0 \\
\hline $\mathrm{L}-07$ & Total Suspended Solids & $\mathrm{mg} / \mathrm{L}$ & 2 & 1 & 1 \\
\hline$L-08$ & Total Suspended Solids & $\mathrm{mg} / \mathrm{L}$ & 1 & 1 & 0 \\
\hline PP-1 & Total Suspended Solids & $\mathrm{mg} / \mathrm{L}$ & 1 & 2 & 1 \\
\hline $\mathrm{F}-02$ & Total Suspended Solids & $\mathrm{mg} / \mathrm{L}$ & $<1$ & $<1$ & 0 \\
\hline$x-04$ & Total Suspended Solids & $\mathrm{mg} / \mathrm{L}$ & 1 & 1 & 0 \\
\hline$x-8 B$ & Total Suspended Solids & $\mathrm{mg} / \mathrm{L}$ & 3 & 3 & 0 \\
\hline$S-04$ & Total Suspended Solids & $\mathrm{mg} / \mathrm{L}$ & 5 & 3 & 2 \\
\hline $\mathrm{H}-02$ & Total Suspended Solids & $\mathrm{mg} / \mathrm{L}$ & 3 & 2 & 1 \\
\hline$A-01$ & Total Suspended Solids & $\mathrm{mg} / \mathrm{L}$ & $<1$ & 1 & $<1$ \\
\hline$x-8 B$ & Total Suspended Solids & $\mathrm{mg} / \mathrm{L}$ & 2 & 1 & 0 \\
\hline $\mathrm{H}-04$ & Total Suspended Solids & $\mathrm{mg} / \mathrm{L}$ & $<1$ & $<1$ & 0 \\
\hline$A-11$ & Total Suspended Solids & $\mathrm{mg} / \mathrm{L}$ & 2 & 2 & 0 \\
\hline $\mathrm{H}-07$ & Total Suspended Solids & $\mathrm{mg} / \mathrm{L}$ & 3 & 2 & 0 \\
\hline $\mathrm{H}-08$ & Total Suspended Solids & $\mathrm{mg} / \mathrm{L}$ & 3 & 3 & 0 \\
\hline$x-04$ & Total Suspended Solids & $\mathrm{mg} / \mathrm{L}$ & 2 & 1 & 0 \\
\hline$F-01$ & Total Suspended Solids & $\mathrm{mg} / \mathrm{L}$ & 1 & 1 & 0 \\
\hline $\mathrm{K}-06$ & Total Suspended Solids & $\mathrm{mg} / \mathrm{L}$ & 2 & 1 & 0 \\
\hline$x-8 C$ & Trichloroethene & $u g / L$ & $<2.0$ & $<2.0$ & 0 \\
\hline$M-05$ & Trichloroethene & $u g / L$ & $<2.0$ & $<2.0$ & 0 \\
\hline$A-1 A$ & Trichloroethene & $u g / L$ & $<2.0$ & $<2.0$ & 0 \\
\hline $\mathrm{X}-8 \mathrm{C}$ & Trichloroethene & ug $/ \mathrm{L}$ & $<2.0$ & $<2.0$ & 0 \\
\hline$A-1 A$ & Trichloroethene & $u g / L$ & $<2.0$ & $<2.0$ & 0 \\
\hline$x-8 C$ & Vinyl Chloride & $u g / L$ & $<2.0$ & $<2.0$ & 0 \\
\hline
\end{tabular}


Table 64

NPDES Duplicate Sample Results

Page 5 of 5

\begin{tabular}{llcccc}
\hline NPDES Site & Parameter Sampled & Units & SRS Reading & $\begin{array}{c}\text { Duplicate } \\
\text { Reading }\end{array}$ & Difference \\
\hline M-05 & Vinyl Chloride & ug/L & $<2.0$ & $<2.0$ & 0 \\
$\mathrm{~A}-1 \mathrm{~A}$ & Vinyl Chloride & $\mathrm{ug} / \mathrm{L}$ & $<2.0$ & $<2.0$ & 0 \\
$\mathrm{X}-8 \mathrm{C}$ & Vinyl Chloride & $\mathrm{ug} / \mathrm{L}$ & $<2.0$ & $<2.0$ & 0 \\
$\mathrm{~A}-1 \mathrm{~A}$ & Vinyl Chloride & $\mathrm{ug} / \mathrm{L}$ & $<2.0$ & $<2.0$ & 0 \\
$\mathrm{~S}-04$ & Zinc & $\mathrm{mg} / \mathrm{L}$ & 0.074 & 0.069 & 0.005 \\
$\mathrm{H}-16$ & Zinc & $\mathrm{mg} / \mathrm{L}$ & 0.024 & 0.009 & 0.015 \\
$\mathrm{~S}-04$ & Zinc & $\mathrm{mg} / \mathrm{L}$ & 0.19 & 0.17 & 0.02 \\
$\mathrm{~S}-04$ & Zinc & $\mathrm{mg} / \mathrm{L}$ & 0.35 & 0.34 & 0.01 \\
$\mathrm{H}-16$ & Zinc & $\mathrm{mg} / \mathrm{L}$ & 0.045 & 0.042 & 0.003 \\
$\mathrm{H}-16$ & Zinc & $\mathrm{mg} / \mathrm{L}$ & 0.06 & 0.021 & 0.039
\end{tabular}


Table 65

NPDES Blind Sample Results

Page 1 of 4

\begin{tabular}{llllll}
\hline NPDES Site & Parameter Sampled & Units & Result 1 & Result 2 & Dlfference
\end{tabular}

Note: The Environmental Monitoring Section laboratory performs most analyses for total suspended solids. Other analyses, including some total suspended solids, are performed by Shealy Environmental Services.

\begin{tabular}{|c|c|c|c|c|c|}
\hline$M-05$ & 1,1,1-Trichloroethane & $u g / L$ & $<2.0$ & $<2.0$ & 0 \\
\hline$x-8 C$ & 1,1,1-Trichloroethane & $u g / L$ & $<2.0$ & $<2.0$ & 0 \\
\hline$M-05$ & 1,1,1-Trichloroethane & $\mathrm{ug} / \mathrm{L}$ & $<2.0$ & $<2.0$ & 0 \\
\hline$A-1 A$ & 1,1,1-Trichloroethane & $u g / L$ & $<2.0$ & $<2.0$ & 0 \\
\hline $\mathrm{K}-18$ & Aluminum & $\mathrm{mg} / \mathrm{L}$ & $<0.050$ & 0.072 & $<0.072$ \\
\hline$M-04$ & Ammonia & $\mathrm{mg} / \mathrm{L}$ & 0.99 & 0.504 & 0.486 \\
\hline$x-8 B$ & Benzene & $u g / L$ & $<0.5$ & $<0.5$ & 0 \\
\hline$x-8 B$ & Benzene & $u g / L$ & 1.1 & $<0.5$ & $<1.1$ \\
\hline$x-8 B$ & Benzene & $u g / L$ & $<0.5$ & $<0.5$ & 0 \\
\hline$x-8 B$ & Biological Oxygen Demand & $\mathrm{mg} / \mathrm{L}$ & 2 & $<2.0$ & $<2.0$ \\
\hline$x-8 B$ & Biological Oxygen Demand & $\mathrm{mg} / \mathrm{L}$ & $<2.0$ & $<2.0$ & 0 \\
\hline$A-11$ & Biological Oxygen Demand & $\mathrm{mg} / \mathrm{L}$ & $<2.0$ & $<2.0$ & 0 \\
\hline$X-8 B$ & Biological Oxygen Demand & $\mathrm{mg} / \mathrm{L}$ & $<2.0$ & $<2.0$ & 0 \\
\hline$A-11$ & Biological Oxygen Demand & $\mathrm{mg} / \mathrm{L}$ & $<2.0$ & $<2.0$ & 0 \\
\hline$A-01$ & Biological Oxygen Demand & $\mathrm{mg} / \mathrm{L}$ & $<2.0$ & $<2.0$ & 0 \\
\hline $\mathrm{H}-16$ & Biological Oxygen Demand & $\mathrm{mg} / \mathrm{L}$ & 4.7 & 2.1 & 2.6 \\
\hline$H-16$ & Cadmium & $\mathrm{mg} / \mathrm{L}$ & $<0.002$ & $<0.002$ & 0 \\
\hline $\mathrm{H}-16$ & Cadmium & $\mathrm{mg} / \mathrm{L}$ & $<0.002$ & $<0.002$ & 0 \\
\hline $\mathrm{H}-16$ & Cadmium & $\mathrm{mg} / \mathrm{L}$ & $<0.0020$ & $<0.0020$ & 0 \\
\hline $\mathrm{H}-16$ & Chromium & $\mathrm{mg} / \mathrm{L}$ & $<0.005$ & $<0.005$ & 0 \\
\hline $\mathrm{H}-16$ & Chromium & $\mathrm{mg} / \mathrm{L}$ & $<0.005$ & $<0.005$ & 0 \\
\hline $\mathrm{H}-16$ & Chromium & $\mathrm{mg} / \mathrm{L}$ & $<0.0050$ & $<0.0050$ & 0 \\
\hline$M-05$ & cis-1,2-Dichloroethene & ug/L & $<2.0$ & $<2.0$ & 0 \\
\hline$x-8 c$ & cis-1,2-Dichloroethene & ug/L & $<2.0$ & $<2.0$ & 0 \\
\hline$M-05$ & cis-1,2-Dichloroethene & $u g / L$ & $<2.0$ & $<2.0$ & 0 \\
\hline$A-1 A$ & cis-1,2-Dichloroethene & ug/L & $<2.0$ & $<2.0$ & 0 \\
\hline $\mathrm{H}-16$ & Copper & $\mathrm{mg} / \mathrm{L}$ & $<0.005$ & $<0.005$ & 0 \\
\hline $\mathrm{H}-16$ & Copper & $\mathrm{mg} / \mathrm{L}$ & $<0.005$ & $<0.005$ & 0 \\
\hline$F-08$ & Copper & $\mathrm{mg} / \mathrm{L}$ & $<0.0050$ & $<0.0050$ & 0 \\
\hline $\mathrm{H}-12$ & Copper & $\mathrm{mg} / \mathrm{L}$ & $<0.0050$ & $<0.0050$ & 0 \\
\hline$F-08$ & Copper & $\mathrm{mg} / \mathrm{L}$ & $<0.0050$ & $<0.0050$ & 0 \\
\hline$A-11$ & Copper & $\mathrm{mg} / \mathrm{L}$ & $<0.0050$ & $<0.0050$ & 0 \\
\hline $\mathrm{H}-16$ & Copper & $\mathrm{mg} / \mathrm{L}$ & $<0.0050$ & $<0.0050$ & 0 \\
\hline
\end{tabular}


Table 65

NPDES Blind Sample Results

Page 2 of 4

\begin{tabular}{|c|c|c|c|c|c|}
\hline NPDES Site & Parameter Sampled & Units & Result 1 & Result 2 & Dlfference \\
\hline$\overline{A-01}$ & Copper & $\mathrm{mg} / \mathrm{L}$ & 0.015 & 0.017 & 0.002 \\
\hline$M-04$ & Cyanide & $\mathrm{mg} / \mathrm{L}$ & $<0.010$ & $<0.010$ & 0 \\
\hline $\mathrm{H}-16$ & Lead & $\mathrm{mg} / \mathrm{L}$ & $<0.003$ & $<0.003$ & 0 \\
\hline $\mathrm{H}-08$ & Lead & $\mathrm{mg} / \mathrm{L}$ & $<0.003$ & $<0.003$ & 0 \\
\hline $\mathrm{H}-16$ & Lead & $\mathrm{mg} / \mathrm{L}$ & $<0.003$ & $<0.003$ & 0 \\
\hline $\mathrm{F}-08$ & Lead & $\mathrm{mg} / \mathrm{L}$ & $<0.0030$ & $<0.0030$ & 0 \\
\hline $\mathrm{H}-12$ & Lead & $\mathrm{mg} / \mathrm{L}$ & $<0.0030$ & $<0.0030$ & 0 \\
\hline$F-08$ & Lead & $\mathrm{mg} / \mathrm{L}$ & $<0.0030$ & $<0.0030$ & 0 \\
\hline A-11 & Lead & $\mathrm{mg} / \mathrm{L}$ & $<0.0030$ & $<0.0030$ & 0 \\
\hline $\mathrm{H}-16$ & Lead & $\mathrm{mg} / \mathrm{L}$ & $<0.0030$ & $<0.0030$ & 0 \\
\hline$A-01$ & Lead & $\mathrm{mg} / \mathrm{L}$ & $<0.0030$ & $<0.0030$ & 0 \\
\hline $\mathrm{H}-16$ & Mercury & $\mathrm{mg} / \mathrm{L}$ & $<0.0001$ & $<0.0001$ & 0 \\
\hline$X-8 B$ & Mercury & $\mathrm{mg} / \mathrm{L}$ & 0.0003 & 0.0003 & 0 \\
\hline $\mathrm{H}-16$ & Mercury & $\mathrm{mg} / \mathrm{L}$ & 0.0003 & 0.0002 & 0.0001 \\
\hline$x-8 B$ & Mercury & $\mathrm{mg} / \mathrm{L}$ & 0.004 & 0.003 & 0.001 \\
\hline$F-08$ & Mercury & $m g / L$ & $<0.00010$ & $<0.00010$ & 0 \\
\hline$X-8 B$ & Mercury & $\mathrm{mg} / \mathrm{L}$ & 0.0004 & 0.00031 & 0.00009 \\
\hline$F-08$ & Mercury & $\mathrm{mg} / \mathrm{L}$ & $<0.00010$ & $<0.00010$ & 0 \\
\hline$A-11$ & Mercury & $\mathrm{mg} / \mathrm{L}$ & $<0.00010$ & $<0.00010$ & 0 \\
\hline $\mathrm{H}-16$ & Mercury & $\mathrm{mg} / \mathrm{L}$ & 0.00013 & 0.00021 & 0.00008 \\
\hline$A-01$ & Mercury & $\mathrm{mg} / \mathrm{L}$ & 0.00015 & 0.00013 & 0.00002 \\
\hline $\mathrm{H}-16$ & Nickel & $\mathrm{mg} / \mathrm{L}$ & $<0.010$ & $<0.010$ & 0 \\
\hline$H-16$ & Nickel & $\mathrm{mg} / \mathrm{L}$ & $<0.010$ & $<0.010$ & 0 \\
\hline $\mathrm{H}-16$ & Nickel & $\mathrm{mg} / \mathrm{L}$ & $<0.010$ & $<0.010$ & 0 \\
\hline$F-05$ & Oil \& Grease & $\mathrm{mg} / \mathrm{L}$ & $<1.0$ & $<1.0$ & 0 \\
\hline$A-01$ & Oil \& Grease & $\mathrm{mg} / \mathrm{L}$ & 2.1 & $<1.0$ & $<2.1$ \\
\hline $\mathrm{F}-05$ & Oil \& Grease & $\mathrm{mg} / \mathrm{L}$ & $<1.0$ & $<1.0$ & 0 \\
\hline$K-18$ & Oil \& Grease & $\mathrm{mg} / \mathrm{L}$ & $<1.0$ & 1.4 & $<1.4$ \\
\hline $\mathrm{F}-05$ & Oil \& Grease & $\mathrm{mg} / \mathrm{L}$ & $<1.0$ & $<1.0$ & 0 \\
\hline$A-01$ & Oil \& Grease & $\mathrm{mg} / \mathrm{L}$ & 1 & 4.8 & 3.8 \\
\hline $\mathrm{H}-02$ & Oil \& Grease & $\mathrm{mg} / \mathrm{L}$ & $<1.0$ & 1.7 & $<1.7$ \\
\hline $\mathrm{K}-06$ & Oil \& Grease & $\mathrm{mg} / \mathrm{L}$ & 1.4 & $<1.0$ & $<1.4$ \\
\hline$X-8 B$ & Phenol & $\mathrm{mg} / \mathrm{L}$ & $<0.006$ & 0.011 & $<0.011$ \\
\hline$x-8 B$ & Phenol & $\mathrm{mg} / \mathrm{L}$ & $<0.006$ & $<0.006$ & 0 \\
\hline$x-8 B$ & Phenol & $\mathrm{mg} / \mathrm{L}$ & $<0.006$ & $<0.006$ & 0 \\
\hline $\mathrm{H}-16$ & Silver & $\mathrm{mg} / \mathrm{L}$ & $<0.005$ & $<0.005$ & 0 \\
\hline
\end{tabular}


Table 65

NPDES Blind Sample Results

Page 3 of 4

\begin{tabular}{|c|c|c|c|c|c|}
\hline NPDES Site & Parameter Sampled & Units & Result 1 & Result 2 & Dlfference \\
\hline $\mathrm{H}-16$ & Silver & $\mathrm{mg} / \mathrm{L}$ & $<0.005$ & $<0.005$ & 0 \\
\hline $\mathrm{H}-16$ & Silver & $\mathrm{mg} / \mathrm{L}$ & $<0.0050$ & $<0.0050$ & 0 \\
\hline$M-05$ & Tetrachloroethene & $u g / L$ & $<2.0$ & $<2.0$ & 0 \\
\hline$x-8 C$ & Tetrachloroethene & $u g / L$ & $<2.0$ & $<2.0$ & 0 \\
\hline$M-05$ & Tetrachloroethene & $u g / L$ & $<2.0$ & $<2.0$ & 0 \\
\hline$A-1 A$ & Tetrachloroethene & $\mathrm{ug} / \mathrm{L}$ & $<2.0$ & $<2.0$ & 0 \\
\hline $\mathrm{X}-8 \mathrm{~B}$ & Total Organic Carbon & $\mathrm{mg} / \mathrm{L}$ & 3.2 & 3.4 & 0.2 \\
\hline$x-8 B$ & Total Organic Carbon & $\mathrm{mg} / \mathrm{L}$ & 2.9 & 3.2 & 0.3 \\
\hline$X-8 B$ & Total Organic Carbon & $\mathrm{mg} / \mathrm{L}$ & 3.5 & 4.8 & 1.3 \\
\hline$F-01$ & Total Suspended Solids & mg/L & 1 & $<1$ & $<1$ \\
\hline$A-01$ & Total Suspended Solids & $\mathrm{mg} / \mathrm{L}$ & $<1$ & $<1$ & 0 \\
\hline$A-11$ & Total Suspended Solids & mg/L & 10 & 7 & 3 \\
\hline $\mathrm{F}-05$ & Total Suspended Solids & $\mathrm{mg} / \mathrm{L}$ & 15 & 16 & 1 \\
\hline$F-08$ & Total Suspended Solids & $\mathrm{mg} / \mathrm{L}$ & 2 & 2 & 0 \\
\hline $\mathrm{F}-02$ & Total Suspended Solids & mg/L & $<1$ & $<1$ & 0 \\
\hline $\mathrm{H}-02$ & Total Suspended Solids & $\mathrm{mg} / \mathrm{L}$ & 1 & 1 & 0 \\
\hline $\mathrm{H}-04$ & Total Suspended Solids & $\mathrm{mg} / \mathrm{L}$ & $<1$ & $<1$ & 0 \\
\hline $\mathrm{H}-08$ & Total Suspended Solids & $\mathrm{mg} / \mathrm{L}$ & 1 & 1 & 0 \\
\hline $\mathrm{H}-16$ & Total Suspended Solids & $\mathrm{mg} / \mathrm{L}$ & 5 & 6 & 1 \\
\hline$x-04$ & Total Suspended Solids & $\mathrm{mg} / \mathrm{L}$ & 1 & 1 & 0 \\
\hline$x-08$ & Total Suspended Solids & $\mathrm{mg} / \mathrm{L}$ & 1 & 1 & 0 \\
\hline S-04 & Total Suspended Solids & $\mathrm{mg} / \mathrm{L}$ & $<1$ & $<1$ & 0 \\
\hline$x-8 B$ & Total Suspended Solids & mg/L & 12 & 12 & 0 \\
\hline$A-01$ & Total Suspended Solids & mg/L & 5 & 4 & 1 \\
\hline$A-11$ & Total Suspended Solids & $\mathrm{mg} / \mathrm{L}$ & 3 & 4 & 1 \\
\hline$K-18$ & Total Suspended Solids & $\mathrm{mg} / \mathrm{L}$ & 3 & 3 & 0 \\
\hline $\mathrm{F}-01$ & Total Suspended Solids & $\mathrm{mg} / \mathrm{L}$ & $<1$ & $<1$ & 0 \\
\hline$F-02$ & Total Suspended Solids & $\mathrm{mg} / \mathrm{L}$ & $<1$ & $<1$ & 0 \\
\hline $\mathrm{F}-05$ & Total Suspended Solids & $\mathrm{mg} / \mathrm{L}$ & $<1$ & 2 & $<2$ \\
\hline$F-08$ & Total Suspended Solids & $\mathrm{mg} / \mathrm{L}$ & $<1$ & 1 & $<1$ \\
\hline $\mathrm{H}-02$ & Total Suspended Solids & $\mathrm{mg} / \mathrm{L}$ & $<1$ & $<1$ & 0 \\
\hline $\mathrm{H}-04$ & Total Suspended Solids & mg/L & $<1$ & 1 & $<1$ \\
\hline $\mathrm{H}-08$ & Total Suspended Solids & $\mathrm{mg} / \mathrm{L}$ & $<1$ & 1 & $<1$ \\
\hline $\mathrm{H}-12$ & Total Suspended Solids & $\mathrm{mg} / \mathrm{L}$ & $<1$ & $<1$ & 0 \\
\hline$s-04$ & Total Suspended Solids & mg/L & 1 & 2 & 1 \\
\hline$X-8 B$ & Total Suspended Solids & $\mathrm{mg} / \mathrm{L}$ & 1 & 2 & 1 \\
\hline
\end{tabular}




\section{Table 65}

\section{NPDES Blind Sample Results}

Page 4 of 4

\begin{tabular}{lllccc}
\hline NPDES Site & Parameter Sampled & Units & Result 1 & Result 2 & Difference \\
\hline X-08 & Total Suspended Solids & $\mathrm{mg} / \mathrm{L}$ & 1 & 2 & 1 \\
X-04 & Total Suspended Solids & $\mathrm{mg} / \mathrm{L}$ & $<1$ & $<1$ & 0 \\
K-06 & Total Suspended Solids & $\mathrm{mg} / \mathrm{L}$ & 2 & 1 & 1 \\
$\mathrm{H}-16$ & Total Suspended Solids & $\mathrm{mg} / \mathrm{L}$ & $<1$ & $<1$ & 0 \\
M-05 & Trichloroethene & $\mathrm{ug} / \mathrm{L}$ & $<2.0$ & $<2.0$ & 0 \\
X-8C & Trichloroethene & $\mathrm{ug} / \mathrm{L}$ & $<2.0$ & $<2.0$ & 0 \\
M-05 & Trichloroethene & $\mathrm{ug} / \mathrm{L}$ & $<2.0$ & $<2.0$ & 0 \\
$\mathrm{~A}-1 \mathrm{~A}$ & Trichloroethene & $\mathrm{ug} / \mathrm{L}$ & $<2.0$ & $<2.0$ & 0 \\
M-05 & Vinyl Chloride & $\mathrm{ug} / \mathrm{L}$ & $<2.0$ & $<2.0$ & 0 \\
X-8C & Vinyl Chloride & $\mathrm{ug} / \mathrm{L}$ & $<2.0$ & $<2.0$ & 0 \\
M-05 & Vinyl Chloride & $\mathrm{ug} / \mathrm{L}$ & $<2.0$ & $<2.0$ & 0 \\
$\mathrm{~A}-1 \mathrm{~A}$ & Vinyl Chloride & $\mathrm{ug} / \mathrm{L}$ & $<2.0$ & $<2.0$ & 0 \\
$\mathrm{H}-16$ & Zinc & $\mathrm{mg} / \mathrm{L}$ & 0.024 & 0.006 & 0.018 \\
$\mathrm{H}-16$ & Zinc & $\mathrm{mg} / \mathrm{L}$ & 0.02 & 0.024 & 0.004 \\
$\mathrm{~S}-04$ & Zinc & $\mathrm{mg} / \mathrm{L}$ & 0.19 & 0.17 & 0.02 \\
$\mathrm{H}-16$ & Zinc & $\mathrm{mg} / \mathrm{L}$ & 0.06 & 0.066 & 0.006 \\
S-04 & Zinc & $\mathrm{mg} / \mathrm{L}$ & 1.1 & 1.1 & 0
\end{tabular}


Table 66

SRS Stream and Savannah River Water Quality Duplicate Sample Results

Page 1 of 15

\begin{tabular}{|c|c|c|c|c|c|}
\hline Location & Parameter Sampled & Units & $\begin{array}{c}\text { SRS } \\
\text { Reading }\end{array}$ & $\begin{array}{l}\text { Duplicate } \\
\text { Reading }\end{array}$ & Difference \\
\hline $\mathrm{RM}-120$ & Iron & $\mathrm{mg} / \mathrm{L}$ & 0.365 & 0.416 & 0.051 \\
\hline BDC & Iron & $\mathrm{mg} / \mathrm{L}$ & 0.391 & 0.388 & -0.003 \\
\hline FM-2 & Iron & $\mathrm{mg} / \mathrm{L}$ & 0.699 & 0.87 & 0.171 \\
\hline $\mathrm{RM}-129$ & Iron & $\mathrm{mg} / \mathrm{L}$ & 1.04 & 1.11 & 0.07 \\
\hline $4 M-6$ & Iron & $\mathrm{mg} / \mathrm{L}$ & 0.901 & 0.673 & -0.228 \\
\hline $\mathrm{RM}-140$ & Iron & $\mathrm{mg} / \mathrm{L}$ & 0.477 & 1.1 & 0.623 \\
\hline $\mathrm{RM}-160$ & Iron & $\mathrm{mg} / \mathrm{L}$ & 0.43 & 0.36 & -0.07 \\
\hline$S C-4$ & Iron & $\mathrm{mg} / \mathrm{L}$ & 0.42 & 0.41 & -0.01 \\
\hline U3R-4 & Iron & $\mathrm{mg} / \mathrm{L}$ & 0.36 & 0.34 & -0.02 \\
\hline Vogtle & Iron & $\mathrm{mg} / \mathrm{L}$ & 0.4 & 0.54 & 0.14 \\
\hline $\mathrm{RM}-120$ & Iron & $\mathrm{mg} / \mathrm{L}$ & 0.44 & 0.1 & -0.34 \\
\hline $4 M-2 B$ & Iron & $\mathrm{mg} / \mathrm{L}$ & 1.2 & 1.5 & 0.3 \\
\hline $\mathrm{RM}-129$ & Iron & $\mathrm{mg} / \mathrm{L}$ & 0.71 & 0.58 & -0.13 \\
\hline $\mathrm{BDC}$ & Iron & $\mathrm{mg} / \mathrm{L}$ & 0.5 & 0.45 & -0.05 \\
\hline $\mathrm{RM}-140$ & Iron & $\mathrm{mg} / \mathrm{L}$ & 0.35 & 0.25 & -0.1 \\
\hline $4 M-2$ & Iron & $\mathrm{mg} / \mathrm{L}$ & 1.8 & 1.7 & -0.1 \\
\hline $\mathrm{RM}-160$ & Iron & $\mathrm{mg} / \mathrm{L}$ & 0.16 & 0.12 & -0.04 \\
\hline VOGTLE & Iron & $\mathrm{mg} / \mathrm{L}$ & 0.38 & 0.39 & 0.01 \\
\hline PB-3 & Iron & $\mathrm{mg} / \mathrm{L}$ & 0.39 & 0.35 & -0.04 \\
\hline TC-1 & Iron & $\mathrm{mg} / \mathrm{L}$ & 0.33 & 0.29 & -0.04 \\
\hline $\mathrm{RM}-120$ & Iron & $\mathrm{mg} / \mathrm{L}$ & 0.21 & 0.28 & 0.07 \\
\hline U3R-1A & Iron & $\mathrm{mg} / \mathrm{L}$ & 0.11 & 0.18 & 0.07 \\
\hline $\mathrm{RM}-129$ & Iron & $\mathrm{mg} / \mathrm{L}$ & 0.47 & 0.3 & -0.17 \\
\hline FM-2 & $2,4-D$ & $u g / L$ & $<2.00$ & $<2.00$ & 0 \\
\hline $\mathrm{RM}-129$ & 2,4-D & $u g / L$ & $<2.00$ & $<2.00$ & 0 \\
\hline U3R-4 & 2,4-D & $u g / L$ & $<2.00$ & $<2.00$ & 0 \\
\hline Vogtle & $2,4-D$ & $u g / L$ & $<2.00$ & $<2.00$ & 0 \\
\hline $\mathrm{BDC}$ & $2,4-D$ & $u g / L$ & $<2.4$ & $<2.4$ & 0 \\
\hline$R M-140$ & $2,4-D$ & $u g / L$ & $<2.4$ & $<2.4$ & 0 \\
\hline $\mathrm{TC}-1$ & 2,4-D & ug/L & $<2.4$ & $<2.5$ & 0 \\
\hline$R M-120$ & $2,4-D$ & $\mathrm{ug} / \mathrm{L}$ & $<2.4$ & $<2.4$ & 0 \\
\hline FM-2 & 4,4'-DDD & $\mathrm{ug} / \mathrm{L}$ & $<0.05$ & $<0.05$ & 0 \\
\hline $\mathrm{RM}-129$ & 4,4'-DDD & $\mathrm{ug} / \mathrm{L}$ & $<0.05$ & $<0.05$ & 0 \\
\hline U3R-4 & 4,4'-DDD & $\mathrm{ug} / \mathrm{L}$ & $<0.05$ & $<0.05$ & 0 \\
\hline Vogtle & 4,4'-DDD & $\mathrm{ug} / \mathrm{L}$ & $<0.05$ & $<0.05$ & 0 \\
\hline
\end{tabular}


Table 66

SRS Stream and Savannah River Water Quality Duplicate Sample Results

Page 2 of 15

\begin{tabular}{|c|c|c|c|c|c|}
\hline Location & Parameter Sampled & Units & $\begin{array}{c}\text { SRS } \\
\text { Reading }\end{array}$ & $\begin{array}{l}\text { Duplicate } \\
\text { Reading }\end{array}$ & Difference \\
\hline $\mathrm{BDC}$ & $4,4^{\prime}-\mathrm{DDD}$ & $u g / L$ & $<0.03$ & $<0.03$ & 0 \\
\hline $\mathrm{RM}-140$ & 4,4'-DDD & $u g / L$ & $<0.03$ & $<0.03$ & 0 \\
\hline TC-1 & 4,4'-DDD & $u g / L$ & $<0.03$ & $<0.03$ & 0 \\
\hline $\mathrm{RM}-120$ & 4,4'-DDD & $u g / L$ & $<0.03$ & $<0.03$ & 0 \\
\hline FM-2 & 4,4'-DDE & $u g / L$ & $<0.05$ & $<0.05$ & 0 \\
\hline $\mathrm{RM}-129$ & $4,4^{\prime}-\mathrm{DDE}$ & $u g / L$ & $<0.05$ & $<0.05$ & 0 \\
\hline U3R-4 & 4,4'-DDE & $u g / L$ & $<0.05$ & $<0.05$ & 0 \\
\hline Vogtle & $4,4^{\prime}-\mathrm{DDE}$ & $u g / L$ & $<0.05$ & $<0.05$ & 0 \\
\hline BDC & $4,4^{\prime}-\mathrm{DDE}$ & $u g / L$ & $<0.03$ & $<0.03$ & 0 \\
\hline $\mathrm{RM}-140$ & $4,4^{\prime}-\mathrm{DDE}$ & $\mathrm{ug} / \mathrm{L}$ & $<0.03$ & $<0.03$ & 0 \\
\hline TC-1 & 4,4'-DDE & $\mathrm{ug} / \mathrm{L}$ & $<0.03$ & $<0.03$ & 0 \\
\hline $\mathrm{RM}-120$ & 4,4'-DDE & $\mathrm{ug} / \mathrm{L}$ & $<0.03$ & $<0.03$ & 0 \\
\hline FM-2 & 4,4'-DDT & $u g / L$ & $<0.05$ & $<0.05$ & 0 \\
\hline $\mathrm{RM}-129$ & 4,4'-DDT & $u g / L$ & $<0.05$ & $<0.05$ & 0 \\
\hline U3R-4 & $4,4^{\prime}-\mathrm{DDT}$ & $\mathrm{ug} / \mathrm{L}$ & $<0.05$ & $<0.05$ & 0 \\
\hline Vogtle & 4,4'-DDT & $u g / L$ & $<0.05$ & $<0.05$ & 0 \\
\hline BDC & $4,4^{\prime}-\mathrm{DDT}$ & $u g / L$ & $<0.03$ & $<0.03$ & 0 \\
\hline$R M-140$ & 4,4'-DDT & $u g / L$ & $<0.03$ & $<0.03$ & 0 \\
\hline TC-1 & 4,4'-DDT & ug/L & $<0.03$ & $<0.03$ & 0 \\
\hline$R M-120$ & 4,4'-DDT & ug/L & $<0.03$ & $<0.03$ & 0 \\
\hline $\mathrm{FM}-2$ & Aldrin & $\mathrm{mg} / \mathrm{L}$ & $<0.05$ & $<0.05$ & 0 \\
\hline$R M-129$ & Aldrin & ug/L & $<0.05$ & $<0.05$ & 0 \\
\hline U3R-4 & Aldrin & $u g / L$ & $<0.05$ & $<0.05$ & 0 \\
\hline Vogtle & Aldrin & $u g / L$ & $<0.05$ & $<0.05$ & 0 \\
\hline $\mathrm{BDC}$ & Aldrin & ug/L & $<0.03$ & $<0.03$ & 0 \\
\hline $\mathrm{RM}-140$ & Aldrin & $u g / L$ & $<0.03$ & $<0.03$ & 0 \\
\hline TC-1 & Aldrin & $u g / L$ & $<0.03$ & $<0.03$ & 0 \\
\hline RM-120 & Aldrin & $\mathrm{ug} / \mathrm{L}$ & $<0.03$ & $<0.03$ & 0 \\
\hline FM-2 & alpha-BHC & $\mathrm{ug} / \mathrm{L}$ & $<0.05$ & $<0.05$ & 0 \\
\hline RM-129 & alpha-BHC & $u g / L$ & $<0.05$ & $<0.05$ & 0 \\
\hline U3R-4 & alpha-BHC & $u g / L$ & $<0.05$ & $<0.05$ & 0 \\
\hline Vogtle & alpha-BHC & $\mathrm{ug} / \mathrm{L}$ & $<0.05$ & $<0.05$ & 0 \\
\hline $\mathrm{BDC}$ & alpha-BHC & $\mathrm{ug} / \mathrm{L}$ & $<0.03$ & $<0.03$ & 0 \\
\hline$R M-140$ & alpha-BHC & $u g / L$ & $<0.03$ & $<0.03$ & 0 \\
\hline $\mathrm{TC}-1$ & alpha-BHC & $u g / L$ & $<0.03$ & $<0.03$ & 0 \\
\hline
\end{tabular}


Table 66

SRS Stream and Savannah River Water Quality Duplicate Sample Results

Page 3 of 15

\begin{tabular}{|c|c|c|c|c|c|}
\hline Location & Parameter Sampled & Units & $\begin{array}{c}\text { SRS } \\
\text { Reading }\end{array}$ & $\begin{array}{l}\text { Duplicate } \\
\text { Reading }\end{array}$ & Difference \\
\hline $\mathrm{RM}-120$ & alpha-BHC & $u g / L$ & $<0.03$ & $<0.03$ & 0 \\
\hline $\mathrm{RM}-120$ & Aluminum & $m g / L$ & 0.304 & 0.305 & 0.001 \\
\hline $\mathrm{BDC}$ & Aluminum & $\mathrm{mg} / \mathrm{L}$ & 0.355 & 0.332 & -0.023 \\
\hline FM-2 & Aluminum & $\mathrm{mg} / \mathrm{L}$ & 0.25 & 0.278 & 0.028 \\
\hline $\mathrm{RM}-129$ & Aluminum & $\mathrm{mg} / \mathrm{L}$ & 0.337 & 0.356 & 0.019 \\
\hline $4 M-6$ & Aluminum & $\mathrm{mg} / \mathrm{L}$ & 0.329 & 0.243 & -0.086 \\
\hline RM-140 & Aluminum & $\mathrm{mg} / \mathrm{L}$ & 0.413 & 0.927 & 0.514 \\
\hline $\mathrm{RM}-160$ & Aluminum & $\mathrm{mg} / \mathrm{L}$ & $<0.050$ & $<0.050$ & 0 \\
\hline $\mathrm{sc}-4$ & Aluminum & $\mathrm{mg} / \mathrm{L}$ & $<0.050$ & $<0.050$ & 0 \\
\hline U3R-4 & Aluminum & $\mathrm{mg} / \mathrm{L}$ & 0.23 & 0.2 & -0.03 \\
\hline Vogtle & Aluminum & $\mathrm{mg} / \mathrm{L}$ & 0.2 & 0.38 & 0.18 \\
\hline $\mathrm{RM}-120$ & Aluminum & $\mathrm{mg} / \mathrm{L}$ & 0.35 & 0.16 & -0.19 \\
\hline $4 M-2 B$ & Aluminum & $\mathrm{mg} / \mathrm{L}$ & 0.18 & 0.34 & 0.16 \\
\hline $\mathrm{RM}-129$ & Aluminum & $\mathrm{mg} / \mathrm{L}$ & 0.39 & 0.33 & -0.06 \\
\hline $\mathrm{BDC}$ & Aluminum & $\mathrm{mg} / \mathrm{L}$ & 0.47 & 0.38 & -0.09 \\
\hline $\mathrm{RM}-140$ & Aluminum & $\mathrm{mg} / \mathrm{L}$ & 0.26 & 0.25 & -0.01 \\
\hline $4 M-2$ & Aluminum & $\mathrm{mg} / \mathrm{L}$ & 0.082 & $<0.050$ & $<0.082$ \\
\hline $\mathrm{RM}-160$ & Aluminum & $\mathrm{mg} / \mathrm{L}$ & $<0.050$ & $<0.050$ & 0 \\
\hline VOGTLE & Aluminum & $\mathrm{mg} / \mathrm{L}$ & 0.3 & 0.28 & -0.02 \\
\hline PB-3 & Aluminum & $\mathrm{mg} / \mathrm{L}$ & 0.15 & 0.16 & 0.01 \\
\hline TC-1 & Aluminum & $\mathrm{mg} / \mathrm{L}$ & 0.18 & 0.14 & -0.04 \\
\hline $\mathrm{RM}-120$ & Aluminum & $\mathrm{mg} / \mathrm{L}$ & 0.27 & 0.17 & -0.1 \\
\hline U3R-1A & Aluminum & $\mathrm{mg} / \mathrm{L}$ & $<0.050$ & 0.069 & $<0.069$ \\
\hline$R M-129$ & Aluminum & $\mathrm{mg} / \mathrm{L}$ & 0.22 & 0.071 & -0.149 \\
\hline$F M-2$ & betaOBHC & $\mathrm{ug} / \mathrm{L}$ & $<0.05$ & $<0.05$ & 0 \\
\hline $\mathrm{AM}-129$ & beta0BHC & $u g / L$ & $<0.05$ & $<0.05$ & 0 \\
\hline U3R-4 & betaOBHC & $\mathrm{ug} / \mathrm{L}$ & $<0.05$ & $<0.05$ & 0 \\
\hline Vogtle & betaOBHC & $\mathrm{ug} / \mathrm{L}$ & $<0.05$ & $<0.05$ & 0 \\
\hline $\mathrm{BDC}$ & beta0BHC & $\mathrm{ug} / \mathrm{L}$ & $<0.03$ & $<0.03$ & 0 \\
\hline $\mathrm{RM}-140$ & beta0BHC & ug/L & $<0.03$ & $<0.03$ & 0 \\
\hline TC-1 & beta0BHC & $u g / L$ & $<0.03$ & $<0.03$ & 0 \\
\hline $\mathrm{RM}-120$ & betaOBHC & ug/L & $<0.03$ & $<0.03$ & 0 \\
\hline$R M-120$ & Cadmium & $\mathrm{mg} / \mathrm{L}$ & $<0.002$ & $<0.002$ & 0 \\
\hline $\mathrm{BDC}$ & Cadmium & $\mathrm{mg} / \mathrm{L}$ & $<0.002$ & $<0.002$ & 0 \\
\hline FM-2 & Cadmium & $\mathrm{mg} / \mathrm{L}$ & $<0.002$ & $<0.002$ & 0 \\
\hline
\end{tabular}


Table 66

SRS Stream and Savannah River Water Quality Duplicate Sample Results

Page 4 of 15

\begin{tabular}{|c|c|c|c|c|c|}
\hline Location & Parameter Sampled & Units & $\begin{array}{c}\text { SRS } \\
\text { Reading }\end{array}$ & $\begin{array}{l}\text { Duplicate } \\
\text { Reading }\end{array}$ & Difference \\
\hline $\mathrm{RM}-129$ & Cadmium & $\mathrm{mg} / \mathrm{L}$ & $<0.002$ & $<0.002$ & 0 \\
\hline $4 M-6$ & Cadmium & $\mathrm{mg} / \mathrm{L}$ & $<0.002$ & $<0.002$ & 0 \\
\hline $\mathrm{RM}-140$ & Cadmium & $\mathrm{mg} / \mathrm{L}$ & $<0.002$ & $<0.002$ & 0 \\
\hline RM-160 & Cadmium & $\mathrm{mg} / \mathrm{L}$ & $<0.002$ & $<0.002$ & 0 \\
\hline$s c-4$ & Cadmium & $\mathrm{mg} / \mathrm{L}$ & $<0.002$ & $<0.002$ & 0 \\
\hline U3R-4 & Cadmium & $\mathrm{mg} / \mathrm{L}$ & $<0.0020$ & $<0.0020$ & 0 \\
\hline Vogtle & Cadmium & $\mathrm{mg} / \mathrm{L}$ & $<0.0020$ & $<0.0020$ & 0 \\
\hline $\mathrm{RM}-120$ & Cadmium & $\mathrm{mg} / \mathrm{L}$ & $<0.0020$ & $<0.0020$ & 0 \\
\hline $4 M-2 B$ & Cadmium & $\mathrm{mg} / \mathrm{L}$ & $<0.0020$ & $<0.0020$ & 0 \\
\hline $\mathrm{RM}-129$ & Cadmium & $\mathrm{mg} / \mathrm{L}$ & $<0.0020$ & $<0.0020$ & 0 \\
\hline $\mathrm{BDC}$ & Cadmium & $\mathrm{mg} / \mathrm{L}$ & $<0.0020$ & $<0.0020$ & 0 \\
\hline$R M-140$ & Cadmium & $\mathrm{mg} / \mathrm{L}$ & $<0.0020$ & $<0.0020$ & 0 \\
\hline $4 \mathrm{M}-2$ & Cadmium & $\mathrm{mg} / \mathrm{L}$ & $<0.0020$ & $<0.0020$ & 0 \\
\hline $\mathrm{RM}-160$ & Cadmium & $\mathrm{mg} / \mathrm{L}$ & $<0.0020$ & $<0.0020$ & 0 \\
\hline VOGTLE & Cadmium & $\mathrm{mg} / \mathrm{L}$ & $<0.0020$ & $<0.0020$ & 0 \\
\hline PB-3 & Cadmium & $\mathrm{mg} / \mathrm{L}$ & $<0.0020$ & $<0.0020$ & 0 \\
\hline TC-1 & Cadmium & $\mathrm{mg} / \mathrm{L}$ & $<0.0020$ & $<0.0020$ & 0 \\
\hline $\mathrm{RM}-120$ & Cadmium & $\mathrm{mg} / \mathrm{L}$ & $<0.0020$ & $<0.0020$ & 0 \\
\hline U3R-1A & Cadmium & $\mathrm{mg} / \mathrm{L}$ & $<0.0020$ & $<0.0020$ & 0 \\
\hline RM-129 & Cadmium & $\mathrm{mg} / \mathrm{L}$ & $<0.0020$ & $<0.0020$ & 0 \\
\hline $\mathrm{FM}-2$ & Chlordane & $u g / L$ & $<0.50$ & $<0.50$ & 0 \\
\hline $\mathrm{RM}-129$ & Chlordane & $\mathrm{ug} / \mathrm{L}$ & $<0.50$ & $<0.50$ & 0 \\
\hline U3R-4 & Chlordane & $\mathrm{ug} / \mathrm{L}$ & $<0.50$ & $<0.50$ & 0 \\
\hline Vogtle & Chlordane & $\mathrm{ug} / \mathrm{L}$ & $<0.50$ & $<0.50$ & 0 \\
\hline BDC & Chlordane & $u g / L$ & $<0.06$ & $<0.06$ & 0 \\
\hline RM-140 & Chlordane & $u g / L$ & $<0.06$ & $<0.06$ & 0 \\
\hline TC-1 & Chlordane & $u g / L$ & $<0.06$ & $<0.05$ & 0 \\
\hline$R M-120$ & Chlordane & ug/L & $<0.06$ & $<0.06$ & 0 \\
\hline $\mathrm{RM}-120$ & Chromium & $\mathrm{mg} / \mathrm{L}$ & $<0.005$ & $<0.005$ & 0 \\
\hline BDC & Chromium & $\mathrm{mg} / \mathrm{L}$ & $<0.005$ & $<0.005$ & 0 \\
\hline FM-2 & Chromium & $\mathrm{mg} / \mathrm{L}$ & $<0.005$ & $<0.005$ & 0 \\
\hline RM-129 & Chromium & $\mathrm{mg} / \mathrm{L}$ & $<0.005$ & $<0.005$ & 0 \\
\hline $4 M-6$ & Chromium & $\mathrm{mg} / \mathrm{L}$ & $<0.005$ & $<0.005$ & 0 \\
\hline RM-140 & Chromium & $\mathrm{mg} / \mathrm{L}$ & $<0.005$ & $<0.005$ & 0 \\
\hline RM-160 & Chromium & $\mathrm{mg} / \mathrm{L}$ & $<0.005$ & $<0.005$ & 0 \\
\hline
\end{tabular}


Table 66

SRS Stream and Savannah River Water Quality Duplicate Sample Results

Page 5 of 15

\begin{tabular}{|c|c|c|c|c|c|}
\hline Location & Parameter Sampled & Units & $\begin{array}{c}\text { SRS } \\
\text { Reading }\end{array}$ & $\begin{array}{l}\text { Duplicate } \\
\text { Reading }\end{array}$ & Difference \\
\hline$\overline{S C-4}$ & Chromium & $\mathrm{mg} / \mathrm{L}$ & $<0.005$ & $<0.005$ & 0 \\
\hline U3R-4 & Chromium & $\mathrm{mg} / \mathrm{L}$ & $<0.0050$ & $<0.0050$ & 0 \\
\hline Vogtle & Chromium & $\mathrm{mg} / \mathrm{L}$ & $<0.0050$ & $<0.0050$ & 0 \\
\hline $\mathrm{RM}-120$ & Chromium & $\mathrm{mg} / \mathrm{L}$ & $<0.0050$ & $<0.0050$ & 0 \\
\hline $4 M-2 B$ & Chromium & $\mathrm{mg} / \mathrm{L}$ & $<0.0050$ & $<0.0050$ & 0 \\
\hline $\mathrm{RM}-129$ & Chromium & $\mathrm{mg} / \mathrm{L}$ & $<0.0050$ & $<0.0050$ & 0 \\
\hline $\mathrm{BDC}$ & Chromium & $\mathrm{mg} / \mathrm{L}$ & $<0.0050$ & $<0.0050$ & 0 \\
\hline $\mathrm{RM}-140$ & Chromium & $\mathrm{mg} / \mathrm{L}$ & $<0.0050$ & $<0.0050$ & 0 \\
\hline $4 M-2$ & Chromium & $\mathrm{mg} / \mathrm{L}$ & $<0.0050$ & $<0.0050$ & 0 \\
\hline RM-160 & Chromium & $\mathrm{mg} / \mathrm{L}$ & $<0.0050$ & $<0.0050$ & 0 \\
\hline VOGTLE & Chromium & $\mathrm{mg} / \mathrm{L}$ & $<0.0050$ & $<0.0050$ & 0 \\
\hline PB-3 & Chromium & $\mathrm{mg} / \mathrm{L}$ & $<0.0050$ & $<0.0050$ & 0 \\
\hline TC-1 & Chromium & $\mathrm{mg} / \mathrm{L}$ & $<0.0050$ & $<0.0050$ & 0 \\
\hline $\mathrm{RM}-120$ & Chromium & $\mathrm{mg} / \mathrm{L}$ & $<0.0050$ & $<0.0050$ & 0 \\
\hline U3R-1A & Chromium & $\mathrm{mg} / \mathrm{L}$ & $<0.0050$ & $<0.0050$ & 0 \\
\hline$R M-129$ & Chromium & $\mathrm{mg} / \mathrm{L}$ & $<0.0050$ & $<0.0050$ & 0 \\
\hline $\mathrm{RM}-120$ & Copper & $\mathrm{mg} / \mathrm{L}$ & $<0.005$ & $<0.005$ & 0 \\
\hline$B D C$ & Copper & $\mathrm{mg} / \mathrm{L}$ & $<0.005$ & $<0.005$ & 0 \\
\hline FM-2 & Copper & $\mathrm{mg} / \mathrm{L}$ & $<0.005$ & 0.005 & 0 \\
\hline $\mathrm{RM}-129$ & Copper & $\mathrm{mg} / \mathrm{L}$ & $<0.005$ & $<0.005$ & 0 \\
\hline $4 M-6$ & Copper & $\mathrm{mg} / \mathrm{L}$ & 0.007 & $<0.005$ & $<0.007$ \\
\hline$R M-140$ & Copper & $\mathrm{mg} / \mathrm{L}$ & $<0.005$ & $<0.005$ & 0 \\
\hline$R M-160$ & Copper & $\mathrm{mg} / \mathrm{L}$ & 0.006 & 0.008 & 0.002 \\
\hline sc-4 & Copper & $\mathrm{mg} / \mathrm{L}$ & $<0.005$ & 0.005 & 0 \\
\hline U3R-4 & Copper & $\mathrm{mg} / \mathrm{L}$ & $<0.0050$ & $<0.0050$ & 0 \\
\hline Vogtle & Copper & $\mathrm{mg} / \mathrm{L}$ & $<0.0050$ & $<0.0050$ & 0 \\
\hline$R M-120$ & Copper & $\mathrm{mg} / \mathrm{L}$ & $<0.0050$ & $<0.0050$ & 0 \\
\hline $4 M-2 B$ & Copper & $\mathrm{mg} / \mathrm{L}$ & $<0.0050$ & 0.0057 & $<0.0057$ \\
\hline RM-129 & Copper & $\mathrm{mg} / \mathrm{L}$ & $<0.0050$ & $<0.0050$ & 0 \\
\hline BDC & Copper & $\mathrm{mg} / \mathrm{L}$ & $<0.0050$ & $<0.0050$ & 0 \\
\hline$R M-140$ & Copper & $\mathrm{mg} / \mathrm{L}$ & $<0.0050$ & $<0.0050$ & 0 \\
\hline $4 M-2$ & Copper & $\mathrm{mg} / \mathrm{L}$ & $<0.0050$ & $<0.0050$ & 0 \\
\hline$R M-160$ & Copper & $\mathrm{mg} / \mathrm{L}$ & $<0.0050$ & $<0.0050$ & 0 \\
\hline VOGTLE & Copper & $\mathrm{mg} / \mathrm{L}$ & $<0.0050$ & $<0.0050$ & 0 \\
\hline PB-3 & Copper & $\mathrm{mg} / \mathrm{L}$ & $<0.0050$ & $<0.0050$ & 0 \\
\hline
\end{tabular}


Table 66

SRS Stream and Savannah River Water Quality Duplicate Sample Results

Page 6 of 15

\begin{tabular}{|c|c|c|c|c|c|}
\hline Location & Parameter Sampled & Units & $\begin{array}{c}\text { SRS } \\
\text { Reading }\end{array}$ & $\begin{array}{l}\text { Duplicate } \\
\text { Reading }\end{array}$ & Difference \\
\hline TC-1 & Copper & $\mathrm{mg} / \mathrm{L}$ & $<0.0050$ & $<0.0050$ & 0 \\
\hline $\mathrm{RM}-120$ & Copper & $\mathrm{mg} / \mathrm{L}$ & $<0.0050$ & $<0.0050$ & 0 \\
\hline U3R-1A & Copper & $\mathrm{mg} / \mathrm{L}$ & 0.016 & 0.028 & 0.012 \\
\hline RM-129 & Copper & $\mathrm{mg} / \mathrm{L}$ & $<0.0050$ & 0.0098 & $<0.0098$ \\
\hline FM-2 & delta-BHC & $\mathrm{ug} / \mathrm{L}$ & $<0.05$ & $<0.05$ & 0 \\
\hline $\mathrm{RM}-129$ & delta-BHC & $u g / L$ & $<0.05$ & $<0.05$ & 0 \\
\hline U3R-4 & delta-BHC & $u g / L$ & $<0.05$ & $<0.05$ & 0 \\
\hline Vogtle & delta-BHC & $u g / L$ & $<0.05$ & $<0.05$ & 0 \\
\hline $\mathrm{BDC}$ & delta-BHC & $\mathrm{ug} / \mathrm{L}$ & $<0.03$ & $<0.03$ & 0 \\
\hline $\mathrm{RM}-140$ & delta-BHC & $\mathrm{ug} / \mathrm{L}$ & $<0.03$ & $<0.03$ & 0 \\
\hline TC-1 & delta-BHC & $\mathrm{ug} / \mathrm{L}$ & $<0.03$ & $<0.03$ & 0 \\
\hline$R M-120$ & delta-BHC & $u g / L$ & $<0.03$ & $<0.03$ & 0 \\
\hline FM-2 & Dieldrin & ug/L & $<0.05$ & $<0.05$ & 0 \\
\hline $\mathrm{RM}-129$ & Dieldrin & $u g / L$ & $<0.05$ & $<0.05$ & 0 \\
\hline U3R-4 & Dieldrin & $\mathrm{ug} / \mathrm{L}$ & $<0.05$ & $<0.05$ & 0 \\
\hline Vogtle & Dieldrin & $u g / L$ & $<0.05$ & $<0.05$ & 0 \\
\hline $\mathrm{BDC}$ & Dieldrin & $\mathrm{ug} / \mathrm{L}$ & $<0.03$ & $<0.03$ & 0 \\
\hline $\mathrm{RM}-140$ & Dieldrin & $u g / L$ & $<0.03$ & $<0.03$ & 0 \\
\hline TC-1 & Dieldrin & $u g / L$ & $<0.03$ & $<0.03$ & 0 \\
\hline $\mathrm{RM}-120$ & Dieldrin & $\mathrm{ug} / \mathrm{L}$ & $<0.03$ & $<0.03$ & 0 \\
\hline$F M-2$ & Endosulfan I & $u g / L$ & $<0.05$ & $<0.05$ & 0 \\
\hline $\mathrm{RM}-129$ & Endosulfan I & $u g / L$ & $<0.05$ & $<0.05$ & 0 \\
\hline U3R-4 & Endosulfan I & $u g / L$ & $<0.05$ & $<0.05$ & 0 \\
\hline Vogtle & Endosulfan I & $u g / L$ & $<0.05$ & $<0.05$ & 0 \\
\hline $\mathrm{BDC}$ & Endosulfan I & $u g / L$ & $<0.03$ & $<0.03$ & 0 \\
\hline $\mathrm{RM}-140$ & Endosulfan I & $u g / L$ & $<0.03$ & $<0.03$ & 0 \\
\hline TC-1 & Endosulfan I & ug/L & $<0.03$ & $<0.03$ & 0 \\
\hline $\mathrm{RM}-120$ & Endosulfan I & $u g / L$ & $<0.03$ & $<0.03$ & 0 \\
\hline FM-2 & Endosulfan II & $u g / L$ & $<0.05$ & $<0.05$ & 0 \\
\hline$R M-129$ & Endosulfan II & $\mathrm{ug} / \mathrm{L}$ & $<0.05$ & $<0.05$ & 0 \\
\hline U3R-4 & Endosulfan II & ug/L & $<0.05$ & $<0.05$ & 0 \\
\hline Vogtle & Endosulfan II & $\mathrm{ug} / \mathrm{L}$ & $<0.05$ & $<0.05$ & 0 \\
\hline $\mathrm{BDC}$ & Endosulfan II & $\mathrm{ug} / \mathrm{L}$ & $<0.03$ & $<0.03$ & 0 \\
\hline $\mathrm{RM}-140$ & Endosulfan II & $u g / L$ & $<0.03$ & $<0.03$ & 0 \\
\hline TC-1 & Endosulfan II & $u g / L$ & $<0.03$ & $<0.03$ & 0 \\
\hline
\end{tabular}


Table 66

SRS Stream and Savannah River Water Quality Duplicate Sample Results

Page 7 of 15

\begin{tabular}{|c|c|c|c|c|c|}
\hline Location & Parameter Sampled & Units & $\begin{array}{c}\text { SRS } \\
\text { Reading }\end{array}$ & $\begin{array}{l}\text { Duplicate } \\
\text { Reading }\end{array}$ & Difference \\
\hline $\mathrm{RM}-120$ & Endosulfan II & $u g / L$ & $<0.03$ & $<0.03$ & 0 \\
\hline FM-2 & Endosulfan sulfate & $\mathrm{ug} / \mathrm{L}$ & $<0.05$ & $<0.05$ & 0 \\
\hline $\mathrm{RM}-129$ & Endosulfan sulfate & $u g / L$ & $<0.05$ & $<0.05$ & 0 \\
\hline U3R-4 & Endosulfan sulfate & ug/L & $<0.05$ & $<0.05$ & 0 \\
\hline Vogtle & Endosulfan sulfate & $u g / L$ & $<0.05$ & $<0.05$ & 0 \\
\hline $\mathrm{BDC}$ & Endosulfan sulfate & $u g / L$ & $<0.03$ & $<0.03$ & 0 \\
\hline $\mathrm{RM}-140$ & Endosulfan sulfate & $\mathrm{ug} / \mathrm{L}$ & $<0.03$ & $<0.03$ & 0 \\
\hline $\mathrm{TC}-1$ & Endosulfan sulfate & $u g / L$ & $<0.03$ & $<0.03$ & 0 \\
\hline $\mathrm{RM}-120$ & Endosulfan sulfate & $u g / L$ & $<0.03$ & $<0.03$ & 0 \\
\hline FM-2 & Endrin & $u g / L$ & $<0.05$ & $<0.05$ & 0 \\
\hline RM-129 & Endrin & $u g / L$ & $<0.05$ & $<0.05$ & 0 \\
\hline U3R-4 & Endrin & $\mathrm{ug} / \mathrm{L}$ & $<0.05$ & $<0.05$ & 0 \\
\hline Vogtle & Endrin & $\mathrm{ug} / \mathrm{L}$ & $<0.05$ & $<0.05$ & 0 \\
\hline $\mathrm{BDC}$ & Endrin & ug/L & $<0.03$ & $<0.03$ & 0 \\
\hline $\mathrm{RM}-140$ & Endrin & ug/L & $<0.03$ & $<0.03$ & 0 \\
\hline TC-1 & Endrin & $u g / L$ & $<0.03$ & $<0.03$ & 0 \\
\hline $\mathrm{RM}-120$ & Endrin & $u g / L$ & $<0.03$ & $<0.03$ & 0 \\
\hline FM-2 & Endrin aldehyde & ug/L & $<0.05$ & $<0.05$ & 0 \\
\hline RM-129 & Endrin aldehyde & $u g / L$ & $<0.05$ & $<0.05$ & 0 \\
\hline U3R-4 & Endrin aldehyde & $\mathrm{ug} / \mathrm{L}$ & $<0.05$ & $<0.05$ & 0 \\
\hline Vogtle & Endrin aldehyde & ug/L & $<0.05$ & $<0.05$ & 0 \\
\hline $\mathrm{BDC}$ & Endrin aldehyde & $u g / L$ & $<0.03$ & $<0.03$ & 0 \\
\hline$R M-140$ & Endrin aldehyde & $u g / L$ & $<0.03$ & $<0.03$ & 0 \\
\hline TC-1 & Endrin aldehyde & $u g / L$ & $<0.03$ & $<0.03$ & 0 \\
\hline$R M-120$ & Endrin aldehyde & ug/L & $<0.03$ & $<0.03$ & 0 \\
\hline FM-2 & Endrin ketone & $\mathrm{ug} / \mathrm{L}$ & $<0.05$ & $<0.05$ & 0 \\
\hline $\mathrm{RM}-129$ & Endrin ketone & $u g / L$ & $<0.05$ & $<0.05$ & 0 \\
\hline U3R-4 & Endrin ketone & $u g / L$ & $<0.05$ & $<0.05$ & 0 \\
\hline Vogtle & Endrin ketone & $u g / L$ & $<0.05$ & $<0.05$ & 0 \\
\hline $\mathrm{BDC}$ & Endrin ketone & $\mathrm{ug} / \mathrm{L}$ & $<0.03$ & $<0.03$ & 0 \\
\hline $\mathrm{RM}-140$ & Endrin ketone & $\mathrm{ug} / \mathrm{L}$ & $<0.03$ & $<0.03$ & 0 \\
\hline TC-1 & Endrin ketone & ug/L & $<0.03$ & $<0.03$ & 0 \\
\hline$R M-120$ & Endrin ketone & ug/L & $<0.03$ & $<0.03$ & 0 \\
\hline FM-2 & gamma-BHC (Lindane) & $u g / L$ & $<0.05$ & $<0.05$ & 0 \\
\hline RM-129 & gamma-BHC (Lindane) & ug/L & $<0.05$ & $<0.05$ & 0 \\
\hline
\end{tabular}


Table 66

SRS Stream and Savannah River Water Quality Duplicate Sample Results

Page 8 of 15

\begin{tabular}{|c|c|c|c|c|c|}
\hline Location & Parameter Sampled & Units & $\begin{array}{c}\text { SRS } \\
\text { Reading }\end{array}$ & $\begin{array}{l}\text { Duplicate } \\
\text { Reading }\end{array}$ & Difference \\
\hline U3R-4 & gamma-BHC (Lindane) & ug/L & $<0.05$ & $<0.05$ & 0 \\
\hline Vogtle & gamma-BHC (Lindane) & ug/L & $<0.05$ & $<0.05$ & 0 \\
\hline $\mathrm{BDC}$ & gamma-BHC (Lindane) & $u g / L$ & $<0.03$ & $<0.03$ & 0 \\
\hline $\mathrm{RM}-140$ & gamma-BHC (Lindane) & $u g / L$ & $<0.03$ & $<0.03$ & 0 \\
\hline TC-1 & gamma-BHC (Lindane) & $u g / L$ & $<0.03$ & $<0.03$ & 0 \\
\hline $\mathrm{RM}-120$ & gamma-BHC (Lindane) & $u g / L$ & $<0.03$ & $<0.03$ & 0 \\
\hline FM-2 & Heptachlor & ug/L & $<0.05$ & $<0.05$ & 0 \\
\hline RM-129 & Heptachlor & $u g / L$ & $<0.05$ & $<0.05$ & 0 \\
\hline U3R-4 & Heptachlor & $u g / L$ & $<0.05$ & $<0.05$ & 0 \\
\hline Vogtle & Heptachlor & $u g / L$ & $<0.05$ & $<0.05$ & 0 \\
\hline $\mathrm{BDC}$ & Heptachlor & $u g / L$ & $<0.03$ & $<0.03$ & 0 \\
\hline $\mathrm{RM}-140$ & Heptachlor & ug/L & $<0.03$ & $<0.03$ & 0 \\
\hline TC-1 & Heptachlor & $\mathrm{ug} / \mathrm{L}$ & $<0.03$ & $<0.03$ & 0 \\
\hline $\mathrm{RM}-120$ & Heptachlor & ug/L & $<0.03$ & $<0.03$ & 0 \\
\hline FM-2 & Heptachlor epoxide & $u g / L$ & $<0.05$ & $<0.05$ & 0 \\
\hline $\mathrm{RM}-129$ & Heptachlor epoxide & $u g / L$ & $<0.05$ & $<0.05$ & 0 \\
\hline U3R-4 & Heptachlor epoxide & $u g / L$ & $<0.05$ & $<0.05$ & 0 \\
\hline Vogtle & Heptachlor epoxide & $u g / L$ & $<0.05$ & $<0.05$ & 0 \\
\hline $\mathrm{BDC}$ & Heptachlor epoxide & $u g / L$ & $<0.03$ & $<0.03$ & 0 \\
\hline $\mathrm{RM}-140$ & Heptachlor epoxide & $u g / L$ & $<0.03$ & $<0.03$ & 0 \\
\hline TC-1 & Heptachlor epoxide & $u g / L$ & $<0.03$ & $<0.03$ & 0 \\
\hline $\mathrm{RM}-120$ & Heptachlor epoxide & $\mathrm{ug} / \mathrm{L}$ & $<0.03$ & $<0.03$ & 0 \\
\hline $\mathrm{RM}-120$ & Lead & $\mathrm{mg} / \mathrm{L}$ & $<0.003$ & $<0.003$ & 0 \\
\hline $\mathrm{BDC}$ & Lead & $\mathrm{mg} / \mathrm{L}$ & $<0.003$ & $<0.003$ & 0 \\
\hline $\mathrm{FM}-2$ & Lead & $\mathrm{mg} / \mathrm{L}$ & $<0.003$ & $<0.003$ & 0 \\
\hline RM-129 & Lead & $\mathrm{mg} / \mathrm{L}$ & $<0.003$ & $<0.003$ & 0 \\
\hline $4 M-6$ & Lead & $\mathrm{mg} / \mathrm{L}$ & $<0.003$ & $<0.003$ & 0 \\
\hline $\mathrm{RM}-140$ & Lead & $\mathrm{mg} / \mathrm{L}$ & $<0.003$ & $<0.003$ & 0 \\
\hline $\mathrm{RM}-160$ & Lead & $\mathrm{mg} / \mathrm{L}$ & $<0.003$ & $<0.003$ & 0 \\
\hline$s c-4$ & Lead & $\mathrm{mg} / \mathrm{L}$ & $<0.003$ & $<0.003$ & 0 \\
\hline U3R-4 & Lead & $\mathrm{mg} / \mathrm{L}$ & $<0.0030$ & $<0.0030$ & 0 \\
\hline Vogtle & Lead & $\mathrm{mg} / \mathrm{L}$ & $<0.0030$ & $<0.0030$ & 0 \\
\hline $\mathrm{RM}-120$ & Lead & $\mathrm{mg} / \mathrm{L}$ & $<0.0030$ & $<0.0030$ & 0 \\
\hline $4 M-2 B$ & Lead & $\mathrm{mg} / \mathrm{L}$ & $<0.0030$ & $<0.0030$ & 0 \\
\hline $\mathrm{RM}-129$ & Lead & $\mathrm{mg} / \mathrm{L}$ & $<0.0030$ & $<0.0030$ & 0 \\
\hline
\end{tabular}


Table 66

SRS Stream and Savannah River Water Quality Duplicate Sample Results

Page 9 of 15

\begin{tabular}{|c|c|c|c|c|c|}
\hline Location & Parameter Sampled & Units & $\begin{array}{c}\text { SRS } \\
\text { Reading }\end{array}$ & $\begin{array}{l}\text { Duplicate } \\
\text { Reading }\end{array}$ & Difference \\
\hline $\mathrm{BDC}$ & Lead & $\mathrm{mg} / \mathrm{L}$ & $<0.0030$ & $<0.0030$ & 0 \\
\hline $\mathrm{RM}-140$ & Lead & $\mathrm{mg} / \mathrm{L}$ & $<0.0030$ & $<0.0030$ & 0 \\
\hline $4 M-2$ & Lead & mg/L & $<0.0030$ & $<0.0030$ & 0 \\
\hline RM-160 & Lead & $\mathrm{mg} / \mathrm{L}$ & $<0.0030$ & $<0.0030$ & 0 \\
\hline VOGTLE & Lead & $\mathrm{mg} / \mathrm{L}$ & $<0.0030$ & $<0.0030$ & 0 \\
\hline PB-3 & Lead & $\mathrm{mg} / \mathrm{L}$ & $<0.0030$ & $<0.0030$ & 0 \\
\hline TC-1 & Lead & $\mathrm{mg} / \mathrm{L}$ & $<0.0030$ & $<0.0030$ & 0 \\
\hline $\mathrm{RM}-120$ & Lead & $\mathrm{mg} / \mathrm{L}$ & $<0.0030$ & $<0.0030$ & 0 \\
\hline U3R-1A & Lead & $\mathrm{mg} / \mathrm{L}$ & $<0.0030$ & $<0.0030$ & 0 \\
\hline $\mathrm{RM}-129$ & Lead & $\mathrm{mg} / \mathrm{L}$ & $<0.0030$ & $<0.0030$ & 0 \\
\hline $\mathrm{RM}-120$ & Manganese & $\mathrm{mg} / \mathrm{L}$ & 0.06 & 0.068 & 0.008 \\
\hline$B D C$ & Manganese & $\mathrm{mg} / \mathrm{L}$ & 0.056 & 0.058 & 0.002 \\
\hline$F M-2$ & Manganese & $\mathrm{mg} / \mathrm{L}$ & 0.048 & 0.056 & 0.008 \\
\hline $\mathrm{RM}-129$ & Manganese & $\mathrm{mg} / \mathrm{L}$ & 0.353 & 0.365 & 0.012 \\
\hline $4 M-6$ & Manganese & $\mathrm{mg} / \mathrm{L}$ & 0.073 & 0.045 & -0.028 \\
\hline$R M-140$ & Manganese & mg/L & 0.056 & 0.067 & 0.011 \\
\hline $\mathrm{RM}-160$ & Manganese & $\mathrm{mg} / \mathrm{L}$ & 0.067 & 0.053 & -0.014 \\
\hline$S C-4$ & Manganese & $\mathrm{mg} / \mathrm{L}$ & 0.044 & 0.049 & 0.005 \\
\hline U3R-4 & Manganese & $\mathrm{mg} / \mathrm{L}$ & 0.016 & 0.017 & 0.001 \\
\hline Vogtle & Manganese & $\mathrm{mg} / \mathrm{L}$ & 0.072 & 0.078 & 0.006 \\
\hline $\mathrm{RM}-120$ & Manganese & $\mathrm{mg} / \mathrm{L}$ & 0.057 & 0.037 & -0.02 \\
\hline $4 M-2 B$ & Manganese & $m g / L$ & 0.061 & 0.076 & 0.015 \\
\hline $\mathrm{RM}-129$ & Manganese & mg/L & 0.068 & 0.066 & -0.002 \\
\hline $\mathrm{BDC}$ & Manganese & $\mathrm{mg} / \mathrm{L}$ & 0.074 & 0.075 & 0.001 \\
\hline $\mathrm{RM}-140$ & Manganese & $\mathrm{mg} / \mathrm{L}$ & 0.06 & 0.0066 & -0.0534 \\
\hline $4 M-2$ & Manganese & $\mathrm{mg} / \mathrm{L}$ & 0.19 & 0.19 & 0 \\
\hline $\mathrm{RM}-160$ & Manganese & $m g / L$ & 0.068 & 0.055 & -0.013 \\
\hline VOGTLE & Manganese & $\mathrm{mg} / \mathrm{L}$ & 0.059 & 0.065 & 0.006 \\
\hline PB-3 & Manganese & $\mathrm{mg} / \mathrm{L}$ & 0.033 & 0.03 & -0.003 \\
\hline $\mathrm{TC}-1$ & Manganese & $\mathrm{mg} / \mathrm{L}$ & 0.027 & 0.024 & -0.003 \\
\hline $\mathrm{RM}-120$ & Manganese & $\mathrm{mg} / \mathrm{L}$ & 0.039 & 0.042 & 0.003 \\
\hline U3R-1A & Manganese & $\mathrm{mg} / \mathrm{L}$ & 0.01 & 0.012 & 0.002 \\
\hline $\mathrm{RM}-129$ & Manganese & $\mathrm{mg} / \mathrm{L}$ & 0.063 & 0.065 & 0.002 \\
\hline $\mathrm{RM}-120$ & Mercury & $\mathrm{mg} / \mathrm{L}$ & $<0.0001$ & $<0.0001$ & 0 \\
\hline BDC & Mercury & $\mathrm{mg} / \mathrm{L}$ & $<0.0001$ & $<0.0001$ & 0 \\
\hline
\end{tabular}


Table 66

SRS Stream and Savannah River Water Quality Duplicate Sample Results

Page 10 of 15

\begin{tabular}{|c|c|c|c|c|c|}
\hline Location & Parameter Sampled & Units & $\begin{array}{c}\text { SRS } \\
\text { Reading }\end{array}$ & $\begin{array}{l}\text { Duplicate } \\
\text { Reading }\end{array}$ & Difference \\
\hline FM-2 & Mercury & $\mathrm{mg} / \mathrm{L}$ & $<0.0001$ & $<0.00001$ & 0 \\
\hline $\mathrm{RM}-129$ & Mercury & $\mathrm{mg} / \mathrm{L}$ & $<0.0001$ & $<0.0001$ & 0 \\
\hline $4 M-6$ & Mercury & $\mathrm{mg} / \mathrm{L}$ & $<0.0001$ & $<0.0001$ & 0 \\
\hline $\mathrm{RM}-140$ & Mercury & $\mathrm{mg} / \mathrm{L}$ & $<0.0001$ & $<0.0001$ & 0 \\
\hline $\mathrm{RM}-160$ & Mercury & $m g / L$ & $<0.0001$ & $<0.0001$ & 0 \\
\hline$S C-4$ & Mercury & $\mathrm{mg} / \mathrm{L}$ & 0.0001 & 0.0001 & 0 \\
\hline U3R-4 & Mercury & $\mathrm{mg} / \mathrm{L}$ & $<0.00010$ & $<0.00010$ & 0 \\
\hline Vogtle & Mercury & $\mathrm{mg} / \mathrm{L}$ & $<0.00010$ & $<0.00010$ & 0 \\
\hline$R M-120$ & Mercury & $\mathrm{mg} / \mathrm{L}$ & $<0.00010$ & $<0.00010$ & 0 \\
\hline $4 M-2 B$ & Mercury & $m g / L$ & $<0.00010$ & $<0.00010$ & 0 \\
\hline$R M-129$ & Mercury & $\mathrm{mg} / \mathrm{L}$ & $<0.00010$ & $<0.00010$ & 0 \\
\hline $\mathrm{BDC}$ & Mercury & $\mathrm{mg} / \mathrm{L}$ & $<0.00010$ & $<0.00010$ & 0 \\
\hline$R M-140$ & Mercury & $\mathrm{mg} / \mathrm{L}$ & $<0.00010$ & $<0.00010$ & 0 \\
\hline $4 \mathrm{M}-2$ & Mercury & $\mathrm{mg} / \mathrm{L}$ & $<0.00010$ & $<0.00010$ & 0 \\
\hline$R M-160$ & Mercury & $\mathrm{mg} / \mathrm{L}$ & $<0.00010$ & $<0.00010$ & 0 \\
\hline VOGTLE & Mercury & $\mathrm{mg} / \mathrm{L}$ & $<0.00010$ & $<0.00010$ & 0 \\
\hline PB-3 & Mercury & $\mathrm{mg} / \mathrm{L}$ & $<0.00010$ & $<0.00010$ & 0 \\
\hline $\mathrm{TC}-1$ & Mercury & $\mathrm{mg} / \mathrm{L}$ & $<0.00010$ & $<0.00010$ & 0 \\
\hline RM-120 & Mercury & $\mathrm{mg} / \mathrm{L}$ & $<0.00010$ & $<0.00010$ & 0 \\
\hline U3R-1A & Mercury & $\mathrm{mg} / \mathrm{L}$ & $<0.00010$ & $<0.00010$ & 0 \\
\hline $\mathrm{RM}-129$ & Mercury & $\mathrm{mg} / \mathrm{L}$ & $<0.00010$ & $<0.00010$ & 0 \\
\hline FM-2 & Methoxychlor & $u g / L$ & $<0.20$ & $<0.20$ & 0 \\
\hline$R M-129$ & Methoxychlor & $\mathrm{ug} / \mathrm{L}$ & $<0.20$ & $<0.20$ & 0 \\
\hline U3R-4 & Methoxychlor & $u g / L$ & $<0.20$ & $<0.20$ & 0 \\
\hline Vogtle & Methoxychlor & $u g / L$ & $<0.20$ & $<0.20$ & 0 \\
\hline BDC & Methoxychlor & $u g / L$ & $<0.12$ & $<0.12$ & 0 \\
\hline$R M-140$ & Methoxychlor & $u g / L$ & $<0.12$ & $<0.12$ & 0 \\
\hline $\mathrm{TC}-1$ & Methoxychlor & $u g / L$ & $<0.12$ & $<0.10$ & 0 \\
\hline$R M-120$ & Methoxychlor & $\mathrm{ug} / \mathrm{L}$ & $<0.12$ & $<0.12$ & 0 \\
\hline RM-120 & Nickel & $\mathrm{mg} / \mathrm{L}$ & $<0.010$ & $<0.010$ & 0 \\
\hline $\mathrm{BDC}$ & Nickel & $\mathrm{mg} / \mathrm{L}$ & $<0.010$ & $<0.010$ & 0 \\
\hline$F M-2$ & Nickel & $\mathrm{mg} / \mathrm{L}$ & $<0.10$ & $<0.010$ & 0 \\
\hline $\mathrm{RM}-129$ & Nickel & $\mathrm{mg} / \mathrm{L}$ & $<0.010$ & $<0.010$ & 0 \\
\hline $4 M-6$ & Nickel & $\mathrm{mg} / \mathrm{L}$ & $<0.010$ & $<0.010$ & 0 \\
\hline $\mathrm{RM}-140$ & Nickel & $\mathrm{mg} / \mathrm{L}$ & $<0.010$ & $<0.010$ & 0 \\
\hline
\end{tabular}


Table 66

SRS Stream and Savannah River Water Quality Duplicate Sample Results

Page 11 of 15

\begin{tabular}{|c|c|c|c|c|c|}
\hline Location & Parameter Sampled & Units & $\begin{array}{c}\text { SRS } \\
\text { Reading }\end{array}$ & $\begin{array}{l}\text { Duplicate } \\
\text { Reading }\end{array}$ & Difference \\
\hline $\mathrm{RM}-160$ & Nickel & $\mathrm{mg} / \mathrm{L}$ & $<0.010$ & $<0.010$ & 0 \\
\hline$s c-4$ & Nickel & $\mathrm{mg} / \mathrm{L}$ & $<0.010$ & $<0.010$ & 0 \\
\hline U3R-4 & Nickel & $\mathrm{mg} / \mathrm{L}$ & $<0.010$ & $<0.010$ & 0 \\
\hline Vogtle & Nickel & $\mathrm{mg} / \mathrm{L}$ & $<0.010$ & $<0.010$ & 0 \\
\hline $\mathrm{RM}-120$ & Nickel & $\mathrm{mg} / \mathrm{L}$ & $<0.010$ & $<0.010$ & 0 \\
\hline $4 \mathrm{M}-2 \mathrm{~B}$ & Nickel & $\mathrm{mg} / \mathrm{L}$ & $<0.010$ & 0.011 & $<0.011$ \\
\hline $\mathrm{RM}-129$ & Nickel & $\mathrm{mg} / \mathrm{L}$ & $<0.010$ & $<0.010$ & 0 \\
\hline $\mathrm{BDC}$ & Nickel & $\mathrm{mg} / \mathrm{L}$ & $<0.010$ & $<0.010$ & 0 \\
\hline $\mathrm{RM}-140$ & Nickel & $\mathrm{mg} / \mathrm{L}$ & $<0.010$ & $<0.010$ & 0 \\
\hline $4 \mathrm{M}-2$ & Nickel & $\mathrm{mg} / \mathrm{L}$ & $<0.010$ & $<0.010$ & 0 \\
\hline RM-160 & Nickel & $\mathrm{mg} / \mathrm{L}$ & $<0.010$ & $<0.010$ & 0 \\
\hline VOGTLE & Nickel & $\mathrm{mg} / \mathrm{L}$ & $<0.010$ & $<0.010$ & 0 \\
\hline PB-3 & Nickel & $\mathrm{mg} / \mathrm{L}$ & $<0.010$ & $<0.010$ & 0 \\
\hline TC-1 & Nickel & $\mathrm{mg} / \mathrm{L}$ & $<0.010$ & $<0.010$ & 0 \\
\hline $\mathrm{RM}-120$ & Nickel & $\mathrm{mg} / \mathrm{L}$ & $<0.010$ & $<0.010$ & 0 \\
\hline U3R-1A & Nickel & $\mathrm{mg} / \mathrm{L}$ & $<0.010$ & $<0.010$ & 0 \\
\hline$R M-129$ & Nickel & $\mathrm{mg} / \mathrm{L}$ & $<0.010$ & $<0.010$ & 0 \\
\hline FM-2 & РCB 1016 & $u g / L$ & $<0.50$ & $<0.50$ & 0 \\
\hline$R M-129$ & РCB 1016 & $\mathrm{ug} / \mathrm{L}$ & $<0.50$ & $<0.50$ & 0 \\
\hline U3R-4 & РСB 1016 & $u g / L$ & $<0.50$ & $<0.50$ & 0 \\
\hline Vogtle & РCB 1016 & $u g / L$ & $<0.50$ & $<0.50$ & 0 \\
\hline $\mathrm{BDC}$ & РCB 1016 & $u g / L$ & $<0.31$ & $<0.31$ & 0 \\
\hline $\mathrm{RM}-140$ & РСВ 1016 & $u g / L$ & $<0.31$ & $<0.31$ & 0 \\
\hline $\mathrm{TC}-1$ & РСВ 1016 & $u g / L$ & $<0.29$ & $<0.25$ & 0 \\
\hline $\mathrm{RM}-120$ & PCB 1016 & $u g / L$ & $<0.29$ & $<0.29$ & 0 \\
\hline$F M-2$ & PCB 1221 & $\mathrm{ug} / \mathrm{L}$ & $<0.50$ & $<0.50$ & 0 \\
\hline$R M-129$ & PCB 1221 & $u g / L$ & $<0.50$ & $<0.50$ & 0 \\
\hline U3R-4 & РCB 1221 & $u g / L$ & $<0.50$ & $<0.50$ & 0 \\
\hline Vogtle & PCB 1221 & $\mathrm{ug} / \mathrm{L}$ & $<0.50$ & $<0.50$ & 0 \\
\hline $\mathrm{BDC}$ & РCB 1221 & ug/L & $<0.31$ & $<0.31$ & 0 \\
\hline$R M-140$ & РCB 1221 & ug/L & $<0.31$ & $<0.31$ & 0 \\
\hline TC-1 & РCB 1221 & $\mathrm{ug} / \mathrm{L}$ & $<0.29$ & $<0.25$ & 0 \\
\hline $\mathrm{RM}-120$ & PCB 1221 & $\mathrm{ug} / \mathrm{L}$ & $<0.29$ & $<0.29$ & 0 \\
\hline $\mathrm{FM}-2$ & PCB 1232 & $\mathrm{ug} / \mathrm{L}$ & $<0.50$ & $<0.50$ & 0 \\
\hline $\mathrm{RM}-129$ & PCB 1232 & $u g / L$ & $<0.50$ & $<0.50$ & 0 \\
\hline
\end{tabular}


Table 66

SRS Stream and Savannah River Water Quality Duplicate Sample Results

Page 12 of 15

\begin{tabular}{|c|c|c|c|c|c|}
\hline Location & Parameter Sampled & Units & $\begin{array}{c}\text { SRS } \\
\text { Reading }\end{array}$ & $\begin{array}{l}\text { Duplicate } \\
\text { Reading }\end{array}$ & Difference \\
\hline U3R-4 & PCB 1232 & $\mathrm{ug} / \mathrm{L}$ & $<0.50$ & $<0.50$ & 0 \\
\hline Vogtle & PCB 1232 & $\mathrm{ug} / \mathrm{L}$ & $<0.50$ & $<0.50$ & 0 \\
\hline$B D C$ & РCB 1232 & $\mathrm{ug} / \mathrm{L}$ & $<0.31$ & $<0.31$ & 0 \\
\hline $\mathrm{RM}-140$ & PCB 1232 & $\mathrm{ug} / \mathrm{L}$ & $<0.31$ & $<0.31$ & 0 \\
\hline $\mathrm{TC}-1$ & PCB 1232 & $\mathrm{ug} / \mathrm{L}$ & $<0.29$ & $<0.25$ & 0 \\
\hline $\mathrm{RM}-120$ & PCB 1232 & ug/L & $<0.29$ & $<0.29$ & 0 \\
\hline $\mathrm{FM}-2$ & PCB 1242 & $u g / L$ & $<0.50$ & $<0.50$ & 0 \\
\hline $\mathrm{RM}-129$ & PCB 1242 & $\mathrm{ug} / \mathrm{L}$ & $<0.50$ & $<0.50$ & 0 \\
\hline U3R-4 & PCB 1242 & $u g / L$ & $<0.50$ & $<0.50$ & 0 \\
\hline Vogtle & РCB 1242 & $\mathrm{ug} / \mathrm{L}$ & $<0.50$ & $<0.50$ & 0 \\
\hline $\mathrm{BDC}$ & РCB 1242 & $u g / L$ & $<0.31$ & $<0.31$ & 0 \\
\hline $\mathrm{RM}-140$ & РСB 1242 & ug/L & $<0.31$ & $<0.31$ & 0 \\
\hline $\mathrm{TC}-1$ & PCB 1242 & $u g / L$ & $<0.29$ & $<0.25$ & 0 \\
\hline $\mathrm{RM}-120$ & PCB 1242 & $u g / L$ & $<0.29$ & $<0.29$ & 0 \\
\hline$F M-2$ & РCB 1248 & ug/L & $<0.50$ & $<0.50$ & 0 \\
\hline $\mathrm{RM}-129$ & PCB 1248 & $\mathrm{ug} / \mathrm{L}$ & $<0.50$ & $<0.50$ & 0 \\
\hline U3R-4 & РСВ 1248 & $\mathrm{ug} / \mathrm{L}$ & $<0.50$ & $<0.50$ & 0 \\
\hline Vogtle & РСВ 1248 & ug/L & $<0.50$ & $<0.50$ & 0 \\
\hline $\mathrm{BDC}$ & РСB 1248 & ug/L & $<0.31$ & $<0.31$ & 0 \\
\hline $\mathrm{RM}-140$ & РCB 1248 & $\mathrm{ug} / \mathrm{L}$ & $<0.31$ & $<0.31$ & 0 \\
\hline $\mathrm{TC}-1$ & PCB 1248 & ug/L & $<0.29$ & $<0.25$ & 0 \\
\hline $\mathrm{RM}-120$ & РСВ 1248 & ug/L & $<0.29$ & $<0.29$ & 0 \\
\hline$F M-2$ & PCB 1254 & $u g / L$ & $<0.50$ & $<0.50$ & 0 \\
\hline $\mathrm{RM}-129$ & PCB 1254 & $u g / L$ & $<0.50$ & $<0.50$ & 0 \\
\hline U3R-4 & PCB 1254 & $\mathrm{ug} / \mathrm{L}$ & $<0.50$ & $<0.50$ & 0 \\
\hline Vogtle & PCB 1254 & $u g / L$ & $<0.50$ & $<0.50$ & 0 \\
\hline$B D C$ & РCB 1254 & ug/L & $<0.31$ & $<0.31$ & 0 \\
\hline $\mathrm{RM}-140$ & PCB 1254 & ug/L & $<0.31$ & $<0.31$ & 0 \\
\hline $\mathrm{TC}-1$ & РСВ 1254 & ug/L & $<0.29$ & $<0.25$ & 0 \\
\hline $\mathrm{RM}-120$ & PCB 1254 & ug/L & $<0.29$ & $<0.29$ & 0 \\
\hline FM-2 & PCB 1260 & $u g / L$ & $<0.50$ & $<0.50$ & 0 \\
\hline $\mathrm{RM}-129$ & PCB 1260 & ug/L & $<0.50$ & $<0.50$ & 0 \\
\hline U3R-4 & PCB 1260 & ug/L & $<0.50$ & $<0.50$ & 0 \\
\hline Vogtle & РСВ 1260 & $u g / L$ & $<0.50$ & $<0.50$ & 0 \\
\hline $\mathrm{BDC}$ & РСВ 1260 & $u g / L$ & $<0.31$ & $<0.31$ & 0 \\
\hline
\end{tabular}


Table 66

SRS Stream and Savannah River Water Quality Duplicate Sample Results

Page 13 of 15

\begin{tabular}{|c|c|c|c|c|c|}
\hline Location & Parameter Sampled & Units & $\begin{array}{c}\text { SRS } \\
\text { Reading }\end{array}$ & $\begin{array}{l}\text { Duplicate } \\
\text { Reading }\end{array}$ & Difference \\
\hline $\mathrm{RM}-140$ & PCB 1260 & $\mathrm{ug} / \mathrm{L}$ & $<0.31$ & $<0.31$ & 0 \\
\hline$T C-1$ & PCB 1260 & $\mathrm{ug} / \mathrm{L}$ & $<0.29$ & $<0.25$ & 0 \\
\hline $\mathrm{RM}-120$ & PCB 1260 & ug/L & $<0.29$ & $<0.29$ & 0 \\
\hline $\mathrm{RM}-120$ & Phosphorus & $\mathrm{mg} / \mathrm{L}$ & 0.503 & 0.276 & -0.227 \\
\hline $\mathrm{BDC}$ & Phosphorus & $\mathrm{mg} / \mathrm{L}$ & 0.236 & 0.298 & 0.062 \\
\hline$F M-2$ & Phosphorus & $\mathrm{mg} / \mathrm{L}$ & $<0.010$ & $<0.010$ & 0 \\
\hline $\mathrm{RM}-129$ & Phosphorus & $\mathrm{mg} / \mathrm{L}$ & $<0.010$ & $<0.010$ & 0 \\
\hline $4 M-6$ & Phosphorus & $\mathrm{mg} / \mathrm{L}$ & $<0.010$ & $<0.010$ & 0 \\
\hline $\mathrm{RM}-140$ & Phosphorus & $\mathrm{mg} / \mathrm{L}$ & $<0.010$ & $<0.010$ & 0 \\
\hline $\mathrm{RM}-160$ & Phosphorus & $\mathrm{mg} / \mathrm{L}$ & 0.115 & 0.097 & -0.018 \\
\hline$S C-4$ & Phosphorus & $\mathrm{mg} / \mathrm{L}$ & 0.023 & 0.041 & 0.018 \\
\hline U3R-4 & Phosphorus & $\mathrm{mg} / \mathrm{L}$ & $<0.010$ & $<0.010$ & 0 \\
\hline Vogtle & Phosphorus & $\mathrm{mg} / \mathrm{L}$ & 0.151 & 0.132 & -0.019 \\
\hline $\mathrm{RM}-120$ & Phosphorus & $\mathrm{mg} / \mathrm{L}$ & 0.126 & 0.149 & 0.023 \\
\hline $4 M-2 B$ & Phosphorus & $\mathrm{mg} / \mathrm{L}$ & 0.175 & 0.072 & -0.103 \\
\hline $\mathrm{RM}-129$ & Phosphorus & $\mathrm{mg} / \mathrm{L}$ & 0.149 & 0.14 & -0.00899999 \\
\hline $4 M-2 B$ & Phosphorus & $\mathrm{mg} / \mathrm{L}$ & 0.686 & 0.589 & -0.097 \\
\hline $\mathrm{RM}-140$ & Phosphorus & $\mathrm{mg} / \mathrm{L}$ & 0.051 & 0.07 & 0.019 \\
\hline $4 M-2$ & Phosphorus & $\mathrm{mg} / \mathrm{L}$ & 0.091 & 0.135 & 0.044 \\
\hline $\mathrm{RM}-160$ & Phosphorus & $\mathrm{mg} / \mathrm{L}$ & 0.124 & 0.158 & 0.034 \\
\hline VOGTLE & Phosphorus & $\mathrm{mg} / \mathrm{L}$ & 0.199 & 0.055 & -0.144 \\
\hline PB-3 & Phosphorus & $\mathrm{mg} / \mathrm{L}$ & $<0.010$ & 0.067 & $<0.067$ \\
\hline$T C-1$ & Phosphorus & $\mathrm{mg} / \mathrm{L}$ & $<0.010$ & $<0.010$ & 0 \\
\hline $\mathrm{RM}-120$ & Phosphorus & $-m g / L$ & 0.11 & 0.033 & -0.077 \\
\hline U3R-1A & Phosphorus & $\mathrm{mg} / \mathrm{L}$ & 0.057 & 0.025 & -0.032 \\
\hline $\mathrm{RM}-129$ & Phosphorus & $\mathrm{mg} / \mathrm{L}$ & 0.116 & 0.063 & -0.053 \\
\hline FM-2 & Silvex $(2,4,5-T P)$ & $u g / L$ & $<0.50$ & $<0.50$ & 0 \\
\hline $\mathrm{RM}-129$ & Silvex $(2,4,5-T P)$ & $\mathrm{ug} / \mathrm{L}$ & $<0.50$ & $<0.50$ & 0 \\
\hline U3R-4 & Silvex $(2,4,5-T P)$ & $\mathrm{ug} / \mathrm{L}$ & $<0.50$ & $<0.50$ & 0 \\
\hline Vogtle & Silvex $(2,4,5-T P)$ & ug/L & $<0.50$ & $<0.50$ & 0 \\
\hline $\mathrm{BDC}$ & Silvex $(2,4,5-T P)$ & $\mathrm{ug} / \mathrm{L}$ & $<0.59$ & $<0.59$ & 0 \\
\hline$R M-140$ & Silvex $(2,4,5-T P)$ & $\mathrm{ug} / \mathrm{L}$ & $<0.59$ & $<0.59$ & 0 \\
\hline TC-1 & Silvex $(2,4,5-T P)$ & ug/L & $<0.60$ & $<0.63$ & 0 \\
\hline $\mathrm{RM}-120$ & Silvex $(2,4,5-T P)$ & ug/L & $<0.61$ & $<0.60$ & 0 \\
\hline $\mathrm{RM}-120$ & Total Organic Carbon & $\mathrm{mg} / \mathrm{L}$ & 3.6 & 3.3 & -0.3 \\
\hline
\end{tabular}




\section{Table 66}

SRS Stream and Savannah River Water Quality Duplicate Sample Results

Page 14 of 15

\begin{tabular}{|c|c|c|c|c|c|}
\hline Location & Parameter Sampled & Units & $\begin{array}{c}\text { SRS } \\
\text { Reading }\end{array}$ & $\begin{array}{l}\text { Duplicate } \\
\text { Reading }\end{array}$ & Difference \\
\hline$B D C$ & Total Organic Carbon & $\mathrm{mg} / \mathrm{L}$ & $<1.0$ & 5.1 & $<5.1$ \\
\hline FM-2 & Total Organic Carbon & $\mathrm{mg} / \mathrm{L}$ & $<1.00$ & $<1.00$ & 0 \\
\hline RM-129 & Total Organic Carbon & $\mathrm{mg} / \mathrm{L}$ & 2.7 & 3.1 & 0.4 \\
\hline $4 M-6$ & Total Organic Carbon & $\mathrm{mg} / \mathrm{L}$ & 2.9 & 3.2 & 0.3 \\
\hline$R M-140$ & Total Organic Carbon & $\mathrm{mg} / \mathrm{L}$ & 4.1 & 3.8 & -0.3 \\
\hline$R M-160$ & Total Organic Carbon & $\mathrm{mg} / \mathrm{L}$ & 3.2 & 3.5 & 0.3 \\
\hline SC-4 & Total Organic Carbon & $\mathrm{mg} / \mathrm{L}$ & 4.2 & 3.9 & -0.3 \\
\hline U3R-4 & Total Organic Carbon & $\mathrm{mg} / \mathrm{L}$ & 2.6 & 3.2 & 0.6 \\
\hline Vogtle & Total Organic Carbon & $\mathrm{mg} / \mathrm{L}$ & 3.7 & 3.6 & -0.1 \\
\hline RM-120 & Total Organic Carbon & $\mathrm{mg} / \mathrm{L}$ & 3.2 & 3.1 & -0.1 \\
\hline $4 M-2 B$ & Total Organic Carbon & $\mathrm{mg} / \mathrm{L}$ & 6.7 & 7.1 & 0.39999999 \\
\hline $\mathrm{RM}-129$ & Total Organic Carbon & $\mathrm{mg} / \mathrm{L}$ & 7.1 & 6 & -1.1 \\
\hline$B D C$ & Total Organic Carbon & $\mathrm{mg} / \mathrm{L}$ & 4.8 & 3.9 & -0.9 \\
\hline$R M-140$ & Total Organic Carbon & $\mathrm{mg} / \mathrm{L}$ & 3.1 & 3.1 & 0 \\
\hline $4 M-2$ & Total Organic Carbon & $\mathrm{mg} / \mathrm{L}$ & 2.8 & 1.8 & -1 \\
\hline$R M-160$ & Total Organic Carbon & $\mathrm{mg} / \mathrm{L}$ & 2.5 & 2.4 & -0.1 \\
\hline VOGTLE & Total Organic Carbon & $\mathrm{mg} / \mathrm{L}$ & 3 & 2.6 & -0.4 \\
\hline PB-3 & Total Organic Carbon & $\mathrm{mg} / \mathrm{L}$ & 2.8 & 2.1 & -0.7 \\
\hline TC-1 & Total Organic Carbon & $\mathrm{mg} / \mathrm{L}$ & 6.1 & 5.5 & -0.6 \\
\hline $\mathrm{RM}-120$ & Total Organic Carbon & $\mathrm{mg} / \mathrm{L}$ & 3.1 & 3.1 & 0 \\
\hline U3R-1A & Total Organic Carbon & $\mathrm{mg} / \mathrm{L}$ & 2.4 & 1.7 & -0.7 \\
\hline $\mathrm{RM}-129$ & Total Organic Carbon & $\mathrm{mg} / \mathrm{L}$ & 3.4 & 4.2 & 0.8 \\
\hline FM-2 & Toxaphene & ug/L & $<2.00$ & $<2.00$ & 0 \\
\hline$R M-129$ & Toxaphene & ug/L & $<2.00$ & $<2.00$ & 0 \\
\hline U3R-4 & Toxaphene & $u g / L$ & $<2.00$ & $<2.00$ & 0 \\
\hline Vogtle & Toxaphene & $u g / L$ & $<2.00$ & $<2.00$ & 0 \\
\hline$B D C$ & Toxaphene & ug/L & $<0.31$ & $<0.31$ & 0 \\
\hline $\mathrm{RM}-140$ & Toxaphene & $u g / L$ & $<0.31$ & $<0.31$ & 0 \\
\hline TC-1 & Toxaphene & ug/L & $<0.29$ & $<0.25$ & 0 \\
\hline$R M-120$ & Toxaphene & $\mathrm{ug} / \mathrm{L}$ & $<0.29$ & $<0.29$ & 0 \\
\hline $\mathrm{RM}-120$ & Zinc & $\mathrm{mg} / \mathrm{L}$ & 0.016 & 0.013 & -0.003 \\
\hline $\mathrm{BDC}$ & Zinc & $\mathrm{mg} / \mathrm{L}$ & 0.009 & 0.009 & 0 \\
\hline$F M-2$ & Zinc & $\mathrm{mg} / \mathrm{L}$ & 0.009 & 0.02 & 0.011 \\
\hline $\mathrm{RM}-129$ & Zinc & $\mathrm{mg} / \mathrm{L}$ & $<0.005$ & $<0.005$ & 0 \\
\hline $4 M-6$ & Zinc & $\mathrm{mg} / \mathrm{L}$ & 0.053 & 0.044 & -0.009 \\
\hline
\end{tabular}


Table 66

SRS Stream and Savannah River Water Quality Duplicate Sample Results

Page 15 of 15

\begin{tabular}{llcccc}
\hline Location & Parameter Sampled & Units & $\begin{array}{c}\text { SRS } \\
\text { Reading }\end{array}$ & $\begin{array}{c}\text { Duplicate } \\
\text { Reading }\end{array}$ & Difference \\
\hline RM-140 & Zinc & $\mathrm{mg} / \mathrm{L}$ & 0.009 & 0.009 & 0 \\
RM-160 & Zinc & $\mathrm{mg} / \mathrm{L}$ & 0.01 & 0.019 & 0.009 \\
SC-4 & Zinc & $\mathrm{mg} / \mathrm{L}$ & 0.02 & 0.009 & -0.011 \\
U3R-4 & Zinc & $\mathrm{mg} / \mathrm{L}$ & 0.0078 & 0.012 & 0.0042 \\
Vogtle & Zinc & $\mathrm{mg} / \mathrm{L}$ & 0.018 & 0.013 & -0.005 \\
RM-120 & Zinc & $\mathrm{mg} / \mathrm{L}$ & 0.011 & 0.36 & 0.349 \\
4M-2B & Zinc & $\mathrm{mg} / \mathrm{L}$ & 0.037 & 0.049 & 0.012 \\
RM-129 & Zinc & $\mathrm{mg} / \mathrm{L}$ & 0.011 & 0.01 & -0.00099999 \\
BDC & Zinc & $\mathrm{mg} / \mathrm{L}$ & 0.0075 & 0.0074 & -0.0001 \\
RM-140 & Zinc & $\mathrm{mg} / \mathrm{L}$ & 0.007 & 0.037 & 0.03 \\
$4 M-2$ & Zinc & $\mathrm{mg} / \mathrm{L}$ & 0.03 & 0.031 & 0.001 \\
RM-160 & Zinc & $\mathrm{mg} / \mathrm{L}$ & $<0.0050$ & $<0.0050$ & 0 \\
VOGTLE & Zinc & $\mathrm{mg} / \mathrm{L}$ & 0.0057 & 0.0052 & -0.0005 \\
PB-3 & Zinc & $\mathrm{mg} / \mathrm{L}$ & $<0.0050$ & $<0.0050$ & 0 \\
TC-1 & Zinc & $\mathrm{mg} / \mathrm{L}$ & 0.0061 & $<0.0050$ & $<0.0061$ \\
RM-120 & Zinc & $\mathrm{mg} / \mathrm{L}$ & 0.007 & $<0.0050$ & $<0.007$ \\
U3R-1A & Zinc & $\mathrm{mg} / \mathrm{L}$ & 0.082 & 0.12 & 0.038 \\
RM-129 & Zinc & $\mathrm{mg} / \mathrm{L}$ & 0.0061 & 0.013 & 0.0069
\end{tabular}

